University of Tennessee Health Science Center

UTHSC Digital Commons

\title{
Dental Maturation in Children Treated for Acute Lymphocytic Leukemia
}

\author{
Michael Grey Weathersby \\ University of Tennessee Health Science Center
}

Follow this and additional works at: https://dc.uthsc.edu/dissertations

Part of the Neoplasms Commons, and the Orthodontics and Orthodontology Commons

\section{Recommended Citation}

Weathersby, Michael Grey , "Dental Maturation in Children Treated for Acute Lymphocytic Leukemia" (2006). Theses and Dissertations (ETD). Paper 294. http://dx.doi.org/10.21007/etd.cghs.2006.0346. 


\title{
Dental Maturation in Children Treated for Acute Lymphocytic Leukemia
}

\begin{abstract}
Acute lymphocytic leukemia (ALL) is a life-threatening disease primarily affecting children. Treatment currently consists of chemotherapy sometimes in combination with head irradiation. The literature documents the damage of treatment to the teeth (e.g., congenital absence, microdontia, abnormal crownroot ratios). The present study assessed the effects of treatment for ALL on the tempos of dental maturation (i.e., dental age) in a mixed longitudinal sample of 72 children treated for ALL at St Jude's Children's' Research Hospital, Memphis, Tennessee. Panoramic radiographs had been taken as indicated to assess and maintain dental health in this retrospective study. Dental age was quantified both on a tooth-specific basis and averaged across all scorable teeth using published standards for American whites. The tempo of toothformation was gauged as dental age (DA) minus chronological age (CA). Children were developmentally normal $(D A=C A)$ at the onset of $A L L$, and there was no sex difference in response to treatment. No statistically significant developmental delay was found during the first two years from the onset of treatment, perhaps because tooth mineralization progresses slowly enough that effects were obscured. From two years onward, DA was significantly depressed. The effect of chemotherapy alone only had a slight negative effect on DA. The addition of irradiation to chemotherapy had a significant additive effect on DA, as well as increasing the incidence of developmental variants such as stunted roots (i.e., roots with apical closure prior to achieving normal length). Importantly, there was no evidence of a compensatory increase in the tempo of growth following treatment. The chemotherapeutic treatment for ALL is a serious stressor to the body's tempo of growth, and the addition of irradiation therapy significantly adds to the negative effect on the tempo of dental maturation. Significant developmental delay was not seen until two years after the start of treatment, either because the dentition is buffered from the stresses of treatment or because the morphological changes are slow to occur. The end result of treatment for ALL is delayed tooth maturation in addition to the previously documented risks of developmental dental anomalies.
\end{abstract}

\section{Document Type}

Thesis

Degree Name

Master of Dental Science (MDS)

Program

Orthodontics

Research Advisor

Edward F. Harris, Ph.D.

\section{Keywords}

Acute Lymphocytic Leukemia, tooth formation, dental age, amelogenesis, odontogenesis

\section{Subject Categories}

Dentistry | Diseases | Medicine and Health Sciences | Neoplasms | Orthodontics and Orthodontology 


\title{
DENTAL MATURATION IN CHILDREN TREATED FOR ACUTE LYMPHOCYTIC LEUKEMIA
}

\author{
A Thesis \\ Presented for \\ The Graduate Studies Council \\ The University of Tennessee \\ Health Science Center
}

In Partial Fulfillment

Of the Requirements for the Degree

Master of Dental Science

From The University of Tennessee

By

Michael Grey Weathersby , D.D.S.

May 2006 
Copyright $(\subseteq$ Michael Grey Weathersby, 2006 All rights reserved 


\section{ACKNOWLEDGEMENTS}

I would like to thank everyone who has helped me in my process of becoming an orthodontist. I would especially like to thank Dr. Harris. Without his hard work and knowledge this thesis would not have been finished. I would like to thank Drs. Sue Kaste, Christopher Rowland and William Parris for all their devoted time and help with this monumental project and for serving on my committee. I want to thank Dr. Vaden for providing me with the opportunity to get the greatest orthodontic education in the country. I would like to thank the entire faculty who has shared their time and knowledge to train and develop my

skills. Lastly, I must thank my parents, for without their guidance, love and support, I would not have been able to accomplish everything that I have. 


\begin{abstract}
Acute lymphocytic leukemia (ALL) is a life-threatening disease primarily affecting children. Treatment currently consists of chemotherapy sometimes in combination with head irradiation. The literature documents the damage of treatment to the teeth (e.g., congenital absence, microdontia, abnormal crownroot ratios). The present study assessed the effects of treatment for ALL on the tempos of dental maturation (i.e., dental age) in a mixed longitudinal sample of 72 children treated for ALL at St Jude's Children's' Research Hospital, Memphis, Tennessee. Panoramic radiographs had been taken as indicated to assess and maintain dental health in this retrospective study. Dental age was quantified both on a tooth-specific basis and averaged across all scorable teeth using published standards for American whites. The tempo of tooth formation was gauged as dental age (DA) minus chronological age (CA). Children were developmentally normal $(\mathrm{DA}=\mathrm{CA})$ at the onset of $\mathrm{ALL}$, and there was no sex difference in response to treatment. No statistically significant developmental delay was found during the first two years from the onset of treatment, perhaps because tooth mineralization progresses slowly enough that effects were obscured. From two years onward, DA was significantly depressed. The effect of chemotherapy alone only had a slight negative effect on DA. The addition of irradiation to chemotherapy had a significant additive effect on DA, as well as
\end{abstract}


increasing the incidence of developmental variants such as stunted roots (i.e., roots with apical closure prior to achieving normal length). Importantly, there was no evidence of a compensatory increase in the tempo of growth following treatment. The chemotherapeutic treatment for ALL is a serious stressor to the body's tempo of growth, and the addition of irradiation therapy significantly adds to the negative effect on the tempo of dental maturation. Significant developmental delay was not seen until two years after the start of treatment, either because the dentition is buffered from the stresses of treatment or because the morphological changes are slow to occur. The end result of treatment for ALL is delayed tooth maturation in addition to the previously documented risks of developmental dental anomalies. 


\section{TABLE OF CONTENTS}

CHAPTER PAGE

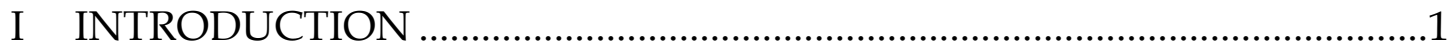

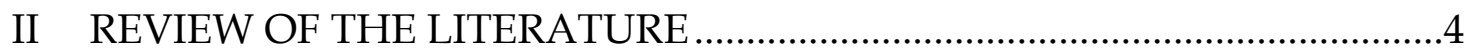

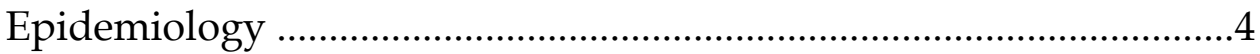

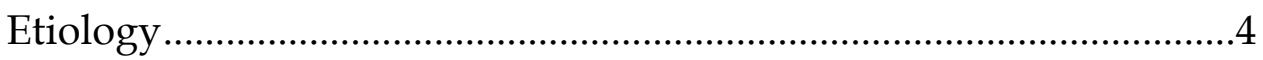

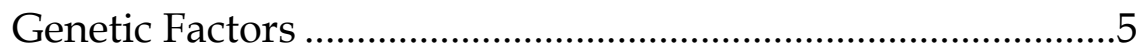

Environmental Factors ...............................................................

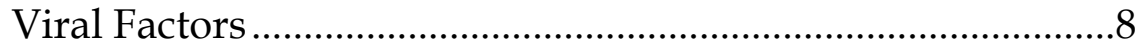

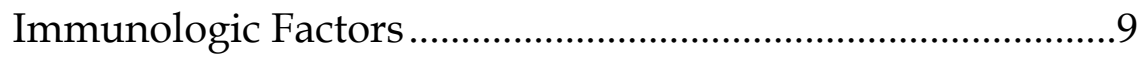

Types of Acute Lymphocytic Leukemia ...............................................9

Morphological Classification.......................................................

Immunophenotyping ...........................................................10

Clinical Manifestations...................................................................11

Laboratory Manifestations...............................................................13

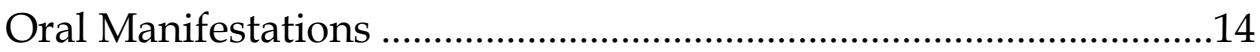

Basic Chemotherapeutic Principles .......................................................17

Current Therapy for ALL.....................................................................18

Tooth Formation.................................................................................19

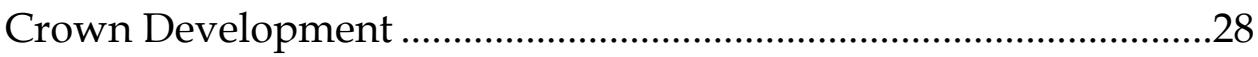


Root Formation..................................................................................

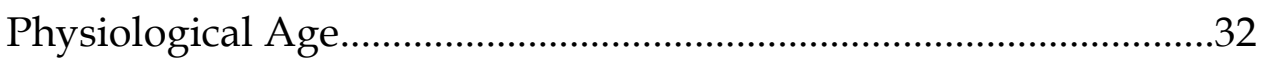

Maturity in Relation to Age.....................................................................33

Assessment of Dental Age ....................................................................34

Stages of Tooth Formation Seen Radiographically ............................43

Left-Right Asymmetry and Delayed Dental Development...............48

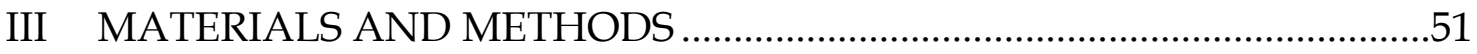

Sample Selection.................................................................................51

Sample Distribution............................................................................51

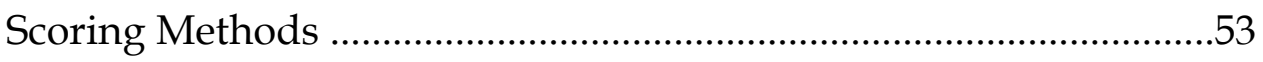

Assessment of Intraobserver Reliability ..............................................56

Appropriateness of Tooth Formation Standards ................................59

Statistical Methods ................................................................................65

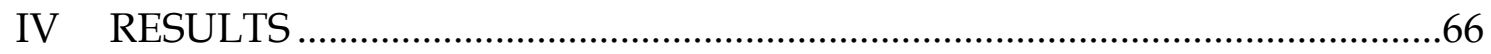

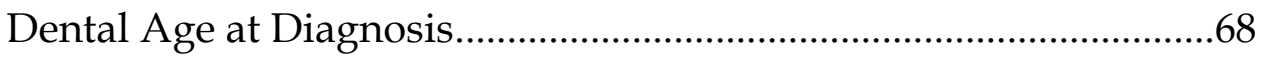

Individual Responses to Treatment ....................................................69

Left-Right Symmetry ………………………………………..............71

Tooth Type and DA-CA ........................................................................73

Tooth Development During Treatment ...............................................95

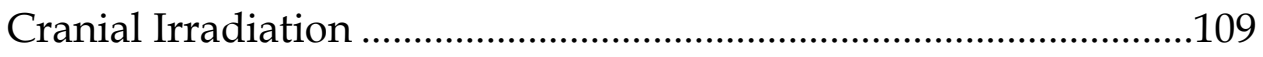


CHAPTER PAGE

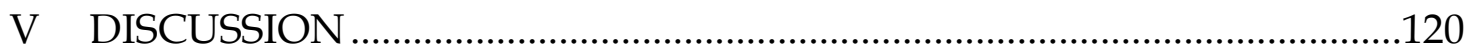

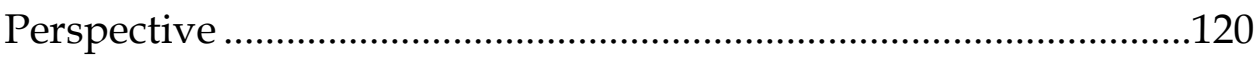

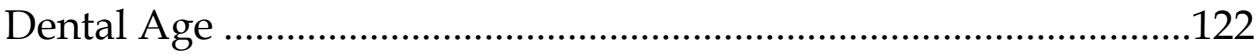

Response Between Sexes................................................................125

Chemotherapy Treatment Alone ......................................................125

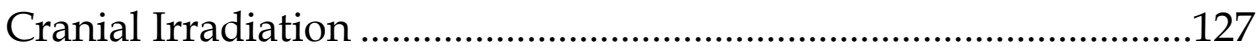

Onset of Dental Age Delay ……………….......................................130

Orthodontic Treatment of Survivors.................................................130

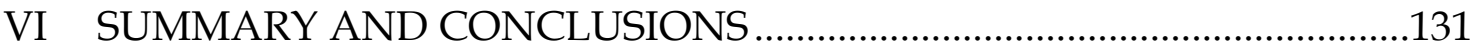

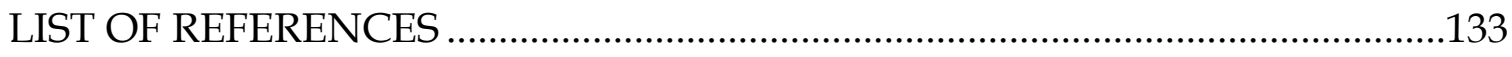

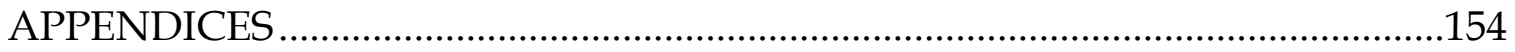

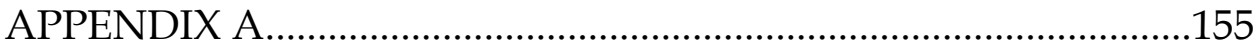

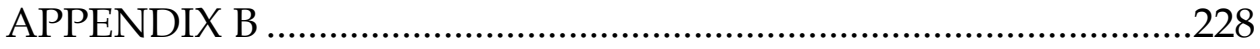

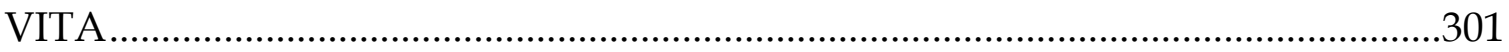




\section{LIST OF TABLES}

TABLE

PAGE

1. Approximate relative frequency of leukemia in children ................................2

2. Frequency of symptoms associated with acute

lymphocytic leukemia

3. Comparative table of mineralization stages used

by different authors

4. Definitions of the 15 stages of tooth formation used by

Gleiser and Hunt (1955) to grade the mineralization of the mandibular first permanent molar

5. Definitions of the ten stages of tooth formation used by

Nolla (1960) to grade the mineralization of all tooth types

6. Definitions of the 20 stages of tooth formation used by

Fanning (1961) to grade the mineralization of the mandibular canines, premolars and molars

7. Definitions of the tooth formation stages ………….......................................54

8. One-sample t-test of DA-CA based on tooth formation stages of patients within 1.0 year of diagnosis for ALL

9. Results of paired t-test assessing whether formation stages were systematically more advanced on one side of the mouth compared to the other

10. Pearson product-moment correlation coefficients between

DA-CA between homologous left and right teeth

11. Pearson product-moment correlation coefficients among

DA-CA of the 12 tooth types on the right side of the mouth

12. Resulting of testing for a statistically significant association between DA-CA against time in treatment on a tooth-specific basis 
13. Results of one-way analysis of variance testing for differences in DA-CA among four intervals of time in treatment...

14. Descriptive statistics for DA-CA grouped into four time intervals based on when the examination was made relative to the start of treatment for ALL 106

15. ANOVA results assessing the effect of time-in-treatment on DA-CA partitioned by whether the child was treated with cranial irradiation

16. ANOVA results assessing the effect of time-in-treatment on DA-CA in the subset of the sample that was treated with chemotherapy alone

17. ANOVA results assessing the effect of time-in-treatment on DA-CA in the subset of the sample that was treated with chemotherapy and cranial irradiation 


\section{LIST OF FIGURES}

FIGURE

PAGE

1. Constitutional disorders with high risk of leukemia........................................6

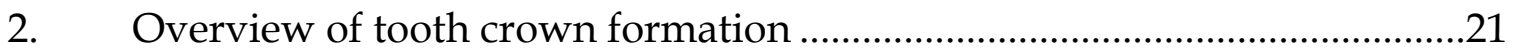

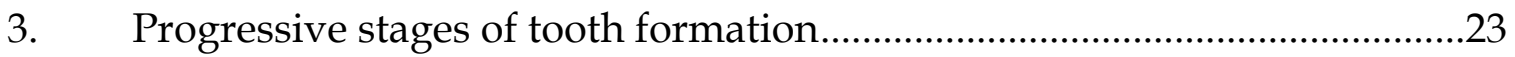

4. Schematic drawings of the stages of tooth mineralization used by Moorrees, Fanning and Hunt (1963)

5. Schematic drawings along with the textual cues for discriminating between Demirjian's 8 grades of tooth formation

6. Radiographic examples of the single-rooted grades of tooth mineralization

7. Radiographic examples of the multi-rooted grades of tooth mineralization

8. Scatterplot showing the negative linear dependency between time in treatment and the degree of dental developmental delay, measured as DA-CA

9. Scatterplot between time in treatment on the horizontal axis and DA-CA on the vertical axis for the maxillary right canine

10. Scatterplot between time in treatment on the horizontal axis and DA-CA on the vertical axis for the maxillary right first premolar

11. Scatterplot between time in treatment on the horizontal axis and DA-CA on the vertical axis for the maxillary right second premolar 
12. Scatterplot between time in treatment on the horizontal axis and DA-CA on the vertical axis for the maxillary right first molar

13. Scatterplot between time in treatment on the horizontal axis and DA-CA on the vertical axis for the maxillary right second molar.

14. Scatterplot between time in treatment on the horizontal axis and DA-CA on the vertical axis for the maxillary right third molar

15. Scatterplot between time in treatment on the horizontal axis and DA-CA on the vertical axis for the mandibular right canine

16. Scatterplot between time in treatment on the horizontal axis and DA-CA on the vertical axis for the mandibular right first premolar

17. Scatterplot between time in treatment on the horizontal axis and DA-CA on the vertical axis for the mandibular right second premolar

18. Scatterplot between time in treatment on the horizontal axis and DA-CA on the vertical axis for the mandibular right first molar

19. Scatterplot between time in treatment on the horizontal axis and DA-CA on the vertical axis for the mandibular right second molar.

20. Scatterplot between time in treatment on the horizontal axis and DA-CA on the vertical axis for the mandibular right third molar

21. Bar chart of the distribution of panoramic radiographs in the present study as a function of time into treatment 
22. Plot of the mean values of DA-CA for the mandibular canine where time in treatment is partitioned into four intervals

23. Plot of the mean values of DA-CA for the mandibular first premolar where time in treatment is partitioned into four intervals

24. Plot of the mean values of DA-CA for the mandibular second premolar where time in treatment is partitioned into four intervals .101

25. Plot of the mean values of DA-CA for the mandibular first molar where time in treatment is partitioned into four intervals

26. Plot of the mean values of DA-CA for the mandibular second molar where time in treatment is partitioned into four intervals

27. Plot of the mean values of DA-CA for the mandibular third molar where time in treatment is partitioned into four intervals

28. Plot of the mean values of DA-CA for the average of all scorable teeth where time in treatment is partitioned into four intervals

29. Plot of time in treatment against DA-CA, with the data (mandibular canine) partitioned by whether the child was treated with cranial irradiation or chemotherapy alone

30. Plot of time in treatment against DA-CA, with the data (mandibular first premolar) partitioned by whether the child was treated with cranial irradiation or chemotherapy alone. 
31. Plot of time in treatment against DA-CA, with the data (mandibular second premolar) partitioned by whether the child was treated with cranial irradiation or chemotherapy alone

32. Plot of time in treatment against DA-CA, with the data (mandibular first molar) partitioned by whether the child was treated with cranial irradiation or chemotherapy alone.

33. Plot of time in treatment against DA-CA, with the data (mandibular second molar) partitioned by whether the child was treated with cranial irradiation or chemotherapy alone.

34. Plot of time in treatment against DA-CA, with the data (mandibular third molar) partitioned by whether the child was treated with cranial irradiation or chemotherapy alone

35. Plot of time in treatment against DA-CA, with the data (average of all scorable teeth) partitioned by whether the child was treated with cranial irradiation or chemotherapy alone

A-1. Plot of chronological age against dental age averaged across all scorable teeth (symbols) for a male (pseudo-record \#1).

A-2. Plot of chronological age against dental age averaged across all scorable teeth (symbols) for a male (pseudo-record \#2)

A-3. Plot of chronological age against dental age averaged across all scorable teeth (symbols) for a female (pseudo-record \#3). .158

A-4. Plot of chronological age against dental age averaged across all scorable teeth (symbols) for a male (pseudo-record \#4)

A-5. Plot of chronological age against dental age averaged across all scorable teeth (symbols) for a male (pseudo-record \# 5) 
FIGURE

PAGE

A-6. Plot of chronological age against dental age averaged across all scorable teeth (symbols) for a male (pseudo-record \#6)

A-7. Plot of chronological age against dental age averaged across all scorable teeth (symbols) for a female (pseudo-record \#7)

A-8. Plot of chronological age against dental age averaged across all scorable teeth (symbols) for a female (pseudo-record \#8).

A-9. Plot of chronological age against dental age averaged across all scorable teeth (symbols) for a female (pseudo-record \#9)

A-10. Plot of chronological age against dental age averaged across all scorable teeth (symbols) for a male (pseudo-record \#10)

A-11. Plot of chronological age against dental age averaged across all scorable teeth (symbols) for a female (pseudo-record \#11)

A-12. Plot of chronological age against dental age averaged across all scorable teeth (symbols) for a male (pseudo-record \#12)

A-13. Plot of chronological age against dental age averaged across all scorable teeth (symbols) for a male (pseudo-record \#13) 168

A-14. Plot of chronological age against dental age averaged across all scorable teeth (symbols) for a male (pseudo-record \#14)

A-15. Plot of chronological age against dental age averaged across all scorable teeth (symbols) for a male (pseudo-record \#15)

A-16. Plot of chronological age against dental age averaged across all scorable teeth (symbols) for a female (pseudo-record \#16)

A-17. Plot of chronological age against dental age averaged across all scorable teeth (symbols) for a female (pseudo-record \#17)

A-18. Plot of chronological age against dental age averaged across all scorable teeth (symbols) for a female (pseudo-record \#18). .173 
FIGURE

PAGE

A-19. Plot of chronological age against dental age averaged across all scorable teeth (symbols) for a male (pseudo-record \#19)

A-20. Plot of chronological age against dental age averaged across all scorable teeth (symbols) for a male (pseudo-record \#20)

A-21. Plot of chronological age against dental age averaged across all scorable teeth (symbols) for a male (pseudo-record \#21)

A-22. Plot of chronological age against dental age averaged across all scorable teeth (symbols) for a female (pseudo-record \#22).

A-23. Plot of chronological age against dental age averaged across all scorable teeth (symbols) for a male (pseudo-record \#23)

A-24. Plot of chronological age against dental age averaged across all scorable teeth (symbols) for a male (pseudo-record \#24)

A-25. Plot of chronological age against dental age averaged across all scorable teeth (symbols) for a female (pseudo-record \#25) 180

A-26. Plot of chronological age against dental age averaged across all scorable teeth (symbols) for a female (pseudo-record \#26)

A-27. Plot of chronological age against dental age averaged across all scorable teeth (symbols) for a male (pseudo-record \#27)

A-28. Plot of chronological age against dental age averaged across all scorable teeth (symbols) for a female (pseudo-record \#28).

A-29. Plot of chronological age against dental age averaged across all scorable teeth (symbols) for a female (pseudo-record \#29)

A-30. Plot of chronological age against dental age averaged across all scorable teeth (symbols) for a female (pseudo-record \#30)

A-31. Plot of chronological age against dental age averaged across all scorable teeth (symbols) for a male (pseudo-record \#31) 186 
A-32. Plot of chronological age against dental age averaged across all scorable teeth (symbols) for a male (pseudo-record \#32)

A-33. Plot of chronological age against dental age averaged across all scorable teeth (symbols) for a male (pseudo-record \#33)

A-34. Plot of chronological age against dental age averaged across all scorable teeth (symbols) for a male (pseudo-record \#34)

A-35. Plot of chronological age against dental age averaged across all scorable teeth (symbols) for a male (pseudo-record \#35)

A-36. Plot of chronological age against dental age averaged across all scorable teeth (symbols) for a male (pseudo-record \#36)

A-37. Plot of chronological age against dental age averaged across all scorable teeth (symbols) for a female (pseudo-record \#37).

A-38. Plot of chronological age against dental age averaged across all scorable teeth (symbols) for a male (pseudo-record \#38)

A-39. Plot of chronological age against dental age averaged across all scorable teeth (symbols) for a male (pseudo-record \#39)

A-40. Plot of chronological age against dental age averaged across all scorable teeth (symbols) for a male (pseudo-record \#40)

A-41. Plot of chronological age against dental age averaged across all scorable teeth (symbols) for a female (pseudo-record \#41)

A-42. Plot of chronological age against dental age averaged across all scorable teeth (symbols) for a male (pseudo-record \#42)

A-43. Plot of chronological age against dental age averaged across all scorable teeth (symbols) for a male (pseudo-record \#43

A-44. Plot of chronological age against dental age averaged across all scorable teeth (symbols) for a female (pseudo-record \#44). 199 
FIGURE

PAGE

A-45. Plot of chronological age against dental age averaged across all scorable teeth (symbols) for a female (pseudo-record \#45) 200

A-46. Plot of chronological age against dental age averaged across all scorable teeth (symbols) for a female (pseudo-record \#46) 201

A-47. Plot of chronological age against dental age averaged across all scorable teeth (symbols) for a female (pseudo-record \#47)

A-48. Plot of chronological age against dental age averaged across all scorable teeth (symbols) for a female (pseudo-record \#48).

A-49. Plot of chronological age against dental age averaged across all scorable teeth (symbols) for a male (pseudo-record \#49)

A-50. Plot of chronological age against dental age averaged across all scorable teeth (symbols) for a female (pseudo-record \#50) .205

A-51. Plot of chronological age against dental age averaged across all scorable teeth (symbols) for a male (pseudo-record \#51) .206

A-52. Plot of chronological age against dental age averaged across all scorable teeth (symbols) for a male (pseudo-record \#52) 207

A-53. Plot of chronological age against dental age averaged across all scorable teeth (symbols) for a female (pseudo-record \#53) 208

A-54. Plot of chronological age against dental age averaged across all scorable teeth (symbols) for a male (pseudo-record \#54)

A-55. Plot of chronological age against dental age averaged across all scorable teeth (symbols) for a female (pseudo-record \#55)

A-56. Plot of chronological age against dental age averaged across all scorable teeth (symbols) for a female (pseudo-record \#56)

A-57. Plot of chronological age against dental age averaged across all scorable teeth (symbols) for a male (pseudo-record \#57) 
FIGURE

PAGE

A-58. Plot of chronological age against dental age averaged across all scorable teeth (symbols) for a male (pseudo-record \#58) .213

A-59. Plot of chronological age against dental age averaged across all scorable teeth (symbols) for a female (pseudo-record \#59)

A-60. Plot of chronological age against dental age averaged across all scorable teeth (symbols) for a male (pseudo-record \#60)

A-61. Plot of chronological age against dental age averaged across all scorable teeth (symbols) for a male (pseudo-record \#61)

A-62. Plot of chronological age against dental age averaged across all scorable teeth (symbols) for a male (pseudo-record \#62)

A-63. Plot of chronological age against dental age averaged across all scorable teeth (symbols) for a male (pseudo-record \#63)

A-64. Plot of chronological age against dental age averaged across all scorable teeth (symbols) for a male (pseudo-record \#64)

A-65. Plot of chronological age against dental age averaged across all scorable teeth (symbols) for a female (pseudo-record \#65) .220

A-66. Plot of chronological age against dental age averaged across all scorable teeth (symbols) for a male (pseudo-record \#66)

A-67. Plot of chronological age against dental age averaged across all scorable teeth (symbols) for a male (pseudo-record \#67)

A-68. Plot of chronological age against dental age averaged across all scorable teeth (symbols) for a male (pseudo-record \#68)

A-69. Plot of chronological age against dental age averaged across all scorable teeth (symbols) for a female (pseudo-record \#69)

A-70. Plot of chronological age against dental age averaged across all scorable teeth (symbols) for a female (pseudo-record \#70) .225 
FIGURE

PAGE

A-71. Plot of chronological age against dental age averaged across all scorable teeth (symbols) for a female (pseudo-record \#71) .226

A-72. Plot of chronological age against dental age averaged across all scorable teeth (symbols) for a female (pseudo-record \#72)

B-1. Plot of tooth-specific DA-CA values at each dental examination for a male (pseudo-record \#1) whose age at diagnosis was 2.35 years.

B-2. Plot of tooth-specific DA-CA values at each dental examination for a male (pseudo-record \#2) whose age at diagnosis was 6.02 years

B-3. Plot of tooth-specific DA-CA values at each dental examination for a female (pseudo-record \#3) whose age at diagnosis was 5.35 years.

B-4. Plot of tooth-specific DA-CA values at each dental examination for a male (pseudo-record \#4) whose age at diagnosis was 5.77 years.

B-5. Plot of tooth-specific DA-CA values at each dental examination for a male (pseudo-record \#5) whose age at diagnosis was 6.06 years.

B-6. Plot of tooth-specific DA-CA values at each dental examination for a male (pseudo-record \#6) whose age at diagnosis was 4.51 years.

B-7. Plot of tooth-specific DA-CA values at each dental examination for a female (pseudo-record \#7) whose age at diagnosis was 2.26 years.

B-8. Plot of tooth-specific DA-CA values at each dental examination for a female (pseudo-record \#8) whose age at diagnosis was 3.72 years. 
FIGURE

PAGE

B-9. Plot of tooth-specific DA-CA values at each dental examination for a female (pseudo-record \#9) whose age at diagnosis was 2.74 years.

B-10. Plot of tooth-specific DA-CA values at each dental examination for a male (pseudo-record \#10) whose age at diagnosis was 4.52 years.

B-11. Plot of tooth-specific DA-CA values at each dental examination for a female (pseudo-record \#11) whose age at diagnosis was 4.25 years.

B-12. Plot of tooth-specific DA-CA values at each dental examination for a male (pseudo-record \#12) whose age at diagnosis was 2.24 years.

B-13. Plot of tooth-specific DA-CA values at each dental examination for a male (pseudo-record \#13) whose age at diagnosis was 6.64 years.

B-14. Plot of tooth-specific DA-CA values at each dental examination for a male (pseudo-record \#14) whose age at diagnosis was 9.74 years.

B-15. Plot of tooth-specific DA-CA values at each dental examination for a male (pseudo-record \#15) whose age at diagnosis was 6.48 years.

B-16. Plot of tooth-specific DA-CA values at each dental examination for a female (pseudo-record \#16) whose age at diagnosis was 3.72 years.

B-17. Plot of tooth-specific DA-CA values at each dental examination for a female (pseudo-record \#17) whose age at diagnosis was 5.34 years.

B-18. Plot of tooth-specific DA-CA values at each dental examination for a female (pseudo-record \#18) whose age at diagnosis was 4.11 years. 
B-19. Plot of tooth-specific DA-CA values at each dental examination for a male (pseudo-record \#19) whose age at diagnosis was 0.65 years.

B-20. Plot of tooth-specific DA-CA values at each dental examination for a male (pseudo-record \#20) whose age at diagnosis was 2.90 years.

B-21. Plot of tooth-specific DA-CA values at each dental examination for a male (pseudo-record \#21) whose age at diagnosis was 5.37 years.

B-22. Plot of tooth-specific DA-CA values at each dental examination for a female (pseudo-record \#22) whose age at diagnosis was 1.84 years. .250

B-23. Plot of tooth-specific DA-CA values at each dental examination for a male (pseudo-record \#23) whose age at diagnosis was 10.02 years.

B-24. Plot of tooth-specific DA-CA values at each dental examination for a male (pseudo-record \#24) whose age at diagnosis was 3.01 years.

B-25. Plot of tooth-specific DA-CA values at each dental examination for a female (pseudo-record \#25) whose age at diagnosis was 3.34 years.

B-26. Plot of tooth-specific DA-CA values at each dental examination for a female (pseudo-record \#26) whose age at diagnosis was 4.73 years.

B-27. Plot of tooth-specific DA-CA values at each dental examination for a male (pseudo-record \#27) whose age at diagnosis was 3.82 years.

B-28. Plot of tooth-specific DA-CA values at each dental examination for a female (pseudo-record \#28) whose age at diagnosis was 10.08 years. 
FIGURE

PAGE

B-29. Plot of tooth-specific DA-CA values at each dental examination for a female (pseudo-record \#29) whose age at diagnosis was 5.99 years

B-30. Plot of tooth-specific DA-CA values at each dental examination for a female (pseudo-record \#30) whose age at diagnosis was 6.09 years. .258

B-31. Plot of tooth-specific DA-CA values at each dental examination for a male (pseudo-record \#31) whose age at diagnosis was 5.52 years.

B-32. Plot of tooth-specific DA-CA values at each dental examination for a male (pseudo-record \#32) whose age at diagnosis was 5.94 years. .260

B-33. Plot of tooth-specific DA-CA values at each dental examination for a male (pseudo-record \#33) whose age at diagnosis was 6.40 years.

B-34. Plot of tooth-specific DA-CA values at each dental examination for a male (pseudo-record \#34) whose age at diagnosis was 8.75 years.

B-35. Plot of tooth-specific DA-CA values at each dental examination for a male (pseudo-record \#35) whose age at diagnosis was 7.33 years.

B-36. Plot of tooth-specific DA-CA values at each dental examination for a male (pseudo-record \#36) whose age at diagnosis was 5.87 years.

B-37. Plot of tooth-specific DA-CA values at each dental examination for a female (pseudo-record \#37) whose age at diagnosis was 9.75 years.

B-38. Plot of tooth-specific DA-CA values at each dental examination for a male (pseudo-record \#38) whose age at diagnosis was 4.70 years. 
B-39. Plot of tooth-specific DA-CA values at each dental examination for a male (pseudo-record \#39) whose age at diagnosis was 4.53 years

B-40. Plot of tooth-specific DA-CA values at each dental examination for a male (pseudo-record \#40) whose age at diagnosis was 7.12 years. .268

B-41. Plot of tooth-specific DA-CA values at each dental examination for a female (pseudo-record \#41) whose age at diagnosis was 5.95 years.

B-42. Plot of tooth-specific DA-CA values at each dental examination for a male (pseudo-record \#42) whose age at diagnosis was 3.88 years.

B-43. Plot of tooth-specific DA-CA values at each dental examination for a male (pseudo-record \#43) whose age at diagnosis was 2.69 years.

B-44. Plot of tooth-specific DA-CA values at each dental examination for a female (pseudo-record \#44) whose age at diagnosis was 3.58 years.

B-45. Plot of tooth-specific DA-CA values at each dental examination for a female (pseudo-record \#45) whose age at diagnosis was 3.95 years.

B-46. Plot of tooth-specific DA-CA values at each dental examination for a female (pseudo-record \#46) whose age at diagnosis was 6.56 years.

B-47. Plot of tooth-specific DA-CA values at each dental examination for a female (pseudo-record \#47) whose age at diagnosis was 5.79 years.

B-48. Plot of tooth-specific DA-CA values at each dental examination for a female (pseudo-record \#48) whose age at diagnosis was 5.16 years. 
FIGURE

PAGE

B-49. Plot of tooth-specific DA-CA values at each dental examination for a male (pseudo-record \#49) whose age at diagnosis was 5.05 years

B-50. Plot of tooth-specific DA-CA values at each dental examination for a female (pseudo-record \#50) whose age at diagnosis was 6.07 years. .278

B-51. Plot of tooth-specific DA-CA values at each dental examination for a male (pseudo-record \#51) whose age at diagnosis was 7.63 years.

B-52. Plot of tooth-specific DA-CA values at each dental examination for a male (pseudo-record \#52) whose age at diagnosis was 3.93 years. .280

B-53. Plot of tooth-specific DA-CA values at each dental examination for a female (pseudo-record \#53) whose age at diagnosis was 6.09 years.

B-54. Plot of tooth-specific DA-CA values at each dental examination for a male (pseudo-record \#54) whose age at diagnosis was 3.60 years.

B-55. Plot of tooth-specific DA-CA values at each dental examination for a female (pseudo-record \#55) whose age at diagnosis was 8.01 years.

B-56. Plot of tooth-specific DA-CA values at each dental examination for a female (pseudo-record \#56) whose age at diagnosis was 8.00 years.

B-57. Plot of tooth-specific DA-CA values at each dental examination for a male (pseudo-record \#57) whose age at diagnosis was 6.58 years.

B-58. Plot of tooth-specific DA-CA values at each dental examination for a male (pseudo-record \#58) whose age at diagnosis was 8.43 years. 
B-59. Plot of tooth-specific DA-CA values at each dental examination for a female (pseudo-record \#59) whose age at diagnosis was 2.73 years

B-60. Plot of tooth-specific DA-CA values at each dental examination for a male (pseudo-record \#60) whose age at diagnosis was 8.30 years. .288

B-61. Plot of tooth-specific DA-CA values at each dental examination for a male (pseudo-record \#61) whose age at diagnosis was 7.66 years.

B-62. Plot of tooth-specific DA-CA values at each dental examination for a male (pseudo-record \#62) whose age at diagnosis was 9.68 years.

B-63. Plot of tooth-specific DA-CA values at each dental examination for a male (pseudo-record \#63) whose age at diagnosis was 4.90 years.

B-64. Plot of tooth-specific DA-CA values at each dental examination for a male (pseudo-record \#64) whose age at diagnosis was 3.38 years.

B-65. Plot of tooth-specific DA-CA values at each dental examination for a female (pseudo-record \#65) whose age at diagnosis was 8.55 years.

B-66. Plot of tooth-specific DA-CA values at each dental examination for a male (pseudo-record \#66) whose age at diagnosis was 3.68 years.

B-67. Plot of tooth-specific DA-CA values at each dental examination for a male (pseudo-record \#67) whose age at diagnosis was 4.27 years.

B-68. Plot of tooth-specific DA-CA values at each dental examination for a male (pseudo-record \#68) whose age at diagnosis was 7.10 years. 
B-69. Plot of tooth-specific DA-CA values at each dental examination for a female (pseudo-record \#69) whose age at diagnosis was 9.64 years

B-70. Plot of tooth-specific DA-CA values at each dental examination for a female (pseudo-record \#70) whose age at diagnosis was 8.54 years.

B-71. Plot of tooth-specific DA-CA values at each dental examination for a female (pseudo-record \#71) whose age at diagnosis was 3.49 years

B-72. Plot of tooth-specific DA-CA values at each dental examination for a female (pseudo-record \#72) whose age at diagnosis was 7.18 years .300 


\section{CHAPTER I}

\section{INTRODUCTION}

Leukemia is a malignant disease of the blood and its formative organs, in which there is an overproduction of leukocytes. Leukemia results from the

clonal proliferation of lymphoid precursors with arrested maturation (Cortes and Kantarjian 1995). It can be classified as either lymphocytic or myelocytic leukemia depending on the type of cell that is altered, and can be further subdivided as detailed in Table 1. Contemporary classification techniques use morphology, cytochemistry, differentiation antigens, cytogenetics, and molecular genetics to classify the type of leukemia.

Acute lymphoblastic leukemia (ALL) comprises approximately 80 percent of all childhood leukemias. The remaining 20 percent are divided among acute myeloblastic leukemia (AML), acute myelommonocytic leukemia (AMMoL), acute monocytic leukemia (AMoL), acute progranulocytic leukemia (APL), acute megakaryocytic leukemia (AMkL), and juvenile myelomonocytic leukemia (JMML) (Zipf et al. 2000).

Leukemia is the most frequent form of childhood cancer. It comprises about one third of the cancers in children under the age of 15 . Leukemia was the leading cause of death among children in the United States until the early 1980s. 
Table 1. Approximate relative frequency of leukemia in children.

\begin{tabular}{lr}
\hline Leukemia type & Percentage \\
\hline Lymphoid & 72 \\
& \\
B-precursor & 10 \\
B-precursor - monocytic and/or & \\
myloid & 16 \\
T-cell & 2 \\
B-cell &
\end{tabular}

Myeloid

Myeloid 50

Progranulocytic 7

Myelomonocytic 16

Monocytoid 16

Erythroid 2

Megakaryocytic 6

Juvenile myelomoocytic 3

Source: Zipf TF, Berg SL, Roberts WM, Poplack DG, Steuber CP, Bleyer WA. Childhood

leukemias. In: Abeloff MD, Armitage JO, Lichter AS, Niederhuber JE, editors.

Clinical oncology, 2nd ed. New York:

Churchill Livingstone, 2000, p 2402-34. 
Great progress has been made in improving event-free survival rates of the children affected by this tragic disease (Pui and Evans 1998). Until every child can be free of leukemia, much more research is needed to increase our knowledge of this disease.

The purpose of the present study was to assess the affects of ALL treatment on the tempos of dental maturation evaluated as dental age. This project primarily addressed this question: To what degree is the tempo of tooth mineralization suppressed in children following treatment for ALL? 


\section{CHAPTER II}

\section{REVIEW OF THE LITERATURE}

\section{Epidemiology}

Although the overall incidence of ALL has remained constant over the past three decades, new information concerning subgroups continues to be uncovered (Smith et al. 1997). On the order of 2,500 to 3,500 children are diagnosed with ALL each year in the United States, with an incidence of 3 to 4 cases per 100,000 white children (Gurney et al. 1995; Greenlee et al. 2000). The peak incidence of ALL occurs between age 2 and 5 years, and it may be trending downward in the United States (Swensen et al. 1997). ALL is more common among white children than black children in the United States. It also is more common in boys than in girls, and this sex difference is greatest among pubertal children (Swensen et al. 1997; Haddy 1982).

\section{Etiology}

The molecular basis for leukemic transformation from a normal hematopoietic cell to a malignant cell in humans is unknown, but it is known to be a genetic alteration of a hematopoietic cell (Zipf et al. 2000). In normal bone marrow, undifferentiated pluripotent progenitor cells have the ability for self- 
renewal, and they give rise to committed progenitor cells. These are morphologically recognizable cells that differentiate into erythroid, myeloid, megakaryocytic, eosinophilic, and monocytic-macrophage cell types. In the clonal expansion theory (Mahoney 1999), leukemia arises from a damaged progenitor cell that has the tendency for unlimited self-renewal or has lost the ability to differentiate along the lines of normally differentiated progenitor cells. The modal age of onset seems to coincide with children's growth and proliferation of their lymphoid tissue and first exposures to infection (Zipf et al. 2000).

Although the cause of ALL is unknown, there are a number of genetic, environmental, viral and immunologic factors that may contribute to the development of the disease.

\section{Genetic Factors}

An unusual susceptibility to leukemia has been associated with certain heritable diseases, chromosomal disorders, and constitutional syndromes (Figure 1) (Mahoney 1999). Children with Down's syndrome (trisomy 21), Bloom's syndrome, Fanconi's anemia, Klinefelter's syndrome (gonadal dysgenesis) and ataxia telangiectasia are all at higher risk than the general population for developing leukemia (Zipf et al. 2000). 
Down's syndrome (trisomy 21)

Bloom's syndrome

Ataxia-telangiectasia

Wiskott-Aldrich syndrome

Fanconi's anemia

Diamond-Blackfan syndrome

Scwachman's syndrome

Klinefelter's syndrome (gonadaldysgenesis)

Turner's syndrome $(45, \mathrm{XO})$

Multiple neurofibromatosis

Severe combined immunodeficiency syndrome

Fig. 1. Constitutional disorders with high risk of leukemia.

Source: Zipf TF, Berg SL, Roberts WM, Poplack DG, Steuber CP, Bleyer WA. Childhood leukemias. In: Abeloff MD, Armitage JO, Lichter AS, Niederhuber JE, editors. Clinical oncology, 2nd ed. New York: Churchill Livingstone, 2000, p 2402-34. 
Reports have identified families with multiple members affected by leukemia (Gunz et al. 1978). Children with an affected sibling and, especially, twins are at greater risk of developing leukemia, although this risk may be only approximately twice that of the general population and applies to twins whose affected sibling was diagnosed before age 5 to 7 years of age (Zipf et al. 2000). Some cases of ALL may be related to hereditary or acquired mutations in the $p 53$ gene. ALL is the usual outcome in infants and preschool children with the myelodysplastic syndrome associated with hematopoietic cell monosomy 7 or deletion of the long arm of chromosome 5 (Zipf et al. 2000).

\section{Environmental Factors}

Exposure to radiation is a risk factor for ALL (Cortes and Kantarjian 1995). Ionizing radiation has been the most extensively studied of the various environmental factors leading to ALL (Mahoney 1999). Exposure in utero increases the risk of ALL, and exposure to therapeutic radiotherapy may also be leukemogenic (Cortes and Kantarjian 1995).

Stewart, Webb and Hewitt (1958) documented increased mortality due to leukemia among children of mothers who had received diagnostic radiographs during pregnancy.

The carcinogenic effects of low-level radiation were studied by Lyon et al. (1979). The study examined the population of Utah because of its exposure to 
fallout from 26 nuclear tests between 1951 to 1958, and the study reviewed all of the deaths from childhood cancer. The counties in Utah were categorized into either a low or high exposure group and matched with a county of the opposite group for control purposes. The high exposure areas showed a higher risk of developing leukemia than did the low exposure areas. The authors suggested that the increase in leukemia deaths could have been due to fallout or some other unidentified factor.

The relative risk of developing leukemia or other forms of cancer in persons exposed to electromagnetic fields has been investigated in several studies, but no definitive cause and effect has been established (Mahoney 1999).

\section{Viral Factors}

A viral etiology of leukemia has been explored for several years. Although viral agents have been accepted as a cause of leukemia in mice, the evidence in human leukemia for a viral agent is still incomplete (Anderson and Scotti, 1980). A small number of RNA viruses have known oncogenic potential. RNA viruses, such as the Rous sarcoma virus and the feline leukemia virus, have caused oncogenic transformation in a variety of animal species, but they have not been associated with cross-species transmission or with cancer in humans (Mahoney 1999). 
The human T-cell lymphotrophic virus (HTLV-I) is a retrovirus that has been isolated from a subset of patients with adult T-cell leukemia. Whether the HTLV-I agent is a cause of leukemia remains to be established (Mahoney 1999).

\section{Immunologic Factors}

Several immunodeficiency states have an associated increased risk for leukemia. These conditions include the syndromes of Wiskott-Aldrich, congenital hypogammaglobulinemia, $\mathrm{X}$-linked agammaglobulinemia, and severe combined immune deficiency (Mahoney 1999; Margolin et al. 2002).

\section{Types of Acute Lymphocytic Leukemia}

\section{Morphological Classification}

There have been multiple efforts to classify ALL cells morphologically using criteria such as cell size, nuclear to cytoplasmic ratio, nuclear shape, number and prominence of nucleoli, nature and intensity of cytoplasmic staining with a variety of staining agents, presence of cytoplasmic granules, prominence of cytoplasmic vacuoles, and the character of nuclear chromatin. Most of these efforts have been unsuccessful because they were technically difficult to reproduce or they lacked meaningful clinical correlations (Margolin et al. 2002). 
In 1976, a French-American-British (FAB) cooperative group developed a system for morphological classification of acute leukemias. The acute lymphoblastic leukemias were divided into three classes: L1, L2 and L3. L1 lymphoblasts are small, with scant cytoplasm and indistinct nucleoli. Cells of the L2 type are larger, more heterogeneous in size, and have more abundant cytoplasm, prominent nucleoli, and reniform nuclear membranes. Lymphoblasts of the L3 type are large, with a deep cytoplasmic basophilia, prominent vacuolation, one or more nucleoli and are morphologically identical to Burkitt's lymphoma cells (Bennett et al. 1976, 1981). Approximately 85\% of the children with ALL have lymphoblasts of L1 morphology. Fewer than $15 \%$ of patients have lymphoblasts of L2 morphology, and 1\% has L3 (Foon and Todd 1986). L1 morphology has been associated with a higher remission induction rate and better event-free survival (EFS) than L2 morphology, which appears to convey poor prognosis (Miller et al. 1981).

\section{Immunophenotyping}

Immunophenotyping plays an important role in the diagnosis of the acute leukemias. It is a more clinically relevant classification of ALL and is based on expression of certain antigens on the surface of leukemic cells (Cortes and Kantarjian 1995). The clinician is able to determine whether the leukemia is lymphoid or myeloid in origin through the use of monoclonal antibodies specific 
for various stages of B-cell, T-cell, and myeloid differentiation (Rubnitz and Look 2000). In most cases, immunophenotyping also permits assignment of the relative stage in the process of $\mathrm{B}$ - or T-cell differentiation from which the leukemic clone is believed to have arisen. Approximately $80 \%$ to $85 \%$ of cases of childhood ALL are supposed to develop from the monoclonal proliferation of Bcell precursors (Zipf et al. 2000).

Newly diagnosed patients with ALL occasionally show an abnormal immune system (Konioe and Leventhal 1976). Abnormally low serum immunoglobulin levels have been observed in as many as $30 \%$ of patients. It is unclear whether these abnormalities precede the development of leukemia or are a consequence of the disease (Margolin et al. 2002).

\section{Clinical Manifestations}

Patients with ALL present most frequently with signs and symptoms of the uncontrolled growth of leukemic cells in bone marrow, lymphoid structures, and other sites of extramedullary spread (Berg 2000). The most common presenting symptoms are fever, pallor, purpura, and pain (Mahoney 1999). The symptoms and the frequency of occurrence in patients of ALL are listed in Table 2 (Mahoney 1999). Bone marrow involvement results in varying degrees of anemia, thrombocytopenia, and granulocytopenia that may be manifested by pallor and fatigue, petechiae, purpura or bleeding, and fever (Berg 2000). Liver, 
Table 2. Frequency of symptoms associated with acute lymphocytic leukemia.

\begin{tabular}{lc}
\hline \multicolumn{1}{c}{ Symptoms } & Frequency $(\%)$ \\
\hline Fever & $43-61$ \\
Pallor & $39-55$ \\
Bleeding & $24-55$ \\
Bone/joint pain & $31-38$ \\
Abdominal pain & $9-19$ \\
Anorexia & $17-33$ \\
Fatigue & 30 \\
\hline
\end{tabular}

Source: Mahoney DH III. Acute lymphoblastic leukemia. In: McMillan JA, editors. Oski's pediatric principles and practice, 3rd ed. Philadelphia: Lippincott Williams \& Wilkins, 1999, p 1491-1501. 
spleen, and nodal enlargement is present in most patients and are the most common sites of extramedullary spread of the disease (Berg 2000). Symptoms may be present from a few days to several weeks before the diagnosis of ALL is made, although in some cases a relevant clinical history may precede diagnosis by several months (Berg 2000). At first, symptoms may be nonspecific and may mimic other nonmalignant conditions, and this nonspecific nature of the signs and symptoms of ALL occasionally leads to delay in diagnosis (Mahoney 1999; Berg 2000).

\section{$\underline{\text { Laboratory Manifestations }}$}

A wide variety of abnormalities can be observed in the clinical laboratory data obtained when the patient presents for diagnosis (Zipf et al. 2000). Over 90\% of patients with ALL have clinically evident hematologic abnormalities at diagnosis (Berg 2000). The white blood cell count is highly variable among patients and may be more than $1,000,000 / \mu \mathrm{l}$; whereas the range of normal is 4,500 to $100,000 / \mu l$. In approximately $50 \%$ of patients, the initial white blood cell count is elevated; in up to $25 \%$ it is greater than $50,000 / \mu l$ at initial presentation. Despite the elevation in leukocyte count at diagnosis, however, many patients present with severe neutropenia (defined as $<500$ granulocytes $/ \mathrm{mm}^{3}$ ) and are at significant risk of serious infection (Bodey et al. 1966). Thrombocytopenia is extremely common; over three fourths of patients present with platelet counts 
less than $100,000 / \mu \mathrm{l}$; whereas the range of normal for platelets is 150,000 to 400,000/ $\mu 1$. (Zipf et al. 2000). Only approximately one third of patients have a platelet count less than $50,000 / \mu l$ at diagnosis (Zipf et al. 2000). The majority of patients with ALL will have platelet counts below 100,000/ $\mu \mathrm{l}$, and a count below $10,000 / \mu l$ is not uncommon, yet the platelet size is usually normal (Zipf et al. 2000). Although petechiae and purpura are present in many patients, severe bleeding is unusual at initial presentation, even when the platelet count is less than $20,000 / \mu 1$, unless fever, infection, or an accompanying coagulopathy, such as disseminated intravascular coagulation (DIC), are also present (Zipf et al. 2000).

\section{Oral Manifestations}

Curtis (1971a) reviewed the records of 464 patients with a diagnosis of leukemia who had been admitted to St. Jude Children's Research Hospital, Memphis, Tennessee. Only patients who had been diagnosed within one week before admission and who had received no prior antineoplastic treatment were evaluated for initial oral manifestations of leukemia. Of this sample, $98 \%$ were diagnosed with ALL. Curtis found that in patients with ALL, petechiae and ecchymosis were the most frequent intraoral findings, followed by pallor, ulcers and bleeding. He reported that gingival hypertrophy was only seen in 3\% of children in this study prior to treatment. He found that only a small percentage 
of the patients with ALL had pronounced oral manifestations. Curtis stated that, "The early age at diagnosis within this group, when relatively little degeneration has taken place, may be a contributing factor" (Curtis 1971a:163).

Michaud et al. (1977) examined 77 patients with acute leukemia for oral manifestations of the disease. These children were examined in various stages of the disease and the examiners found lymph node enlargement in the submental, submandibular, anterior and posterior cervical and the preauricular and postauricular lymph nodes in 30 of the 77 patients. They noticed oral ulcerations in 28 patients and erosions of the oral mucosa in 23 patients. These erosions were present primarily on the buccal mucosa and hard palate. Punctate petechiae were among the most common findings and were encountered in 46 patients, mainly on the lips and buccal mucosa. Ecchymoses were also found, either isolated or interspersed between petechiae. Ecchymoses were not observed in patients whose platelet counts were greater than $50,000 / \mathrm{mm}^{3}$. Candidiasis of the oral mucosa was observed in 21 patients. Only three patients with ALL showed evidence of gingival hypertrophy, and three others had unilateral defects of the soft palate above the tonsillar pillars. These defects resembled a shallow traction diverticulum and varied from 2 to $5 \mathrm{~mm}$ in diameter.

Sonis, Sonis and Lieberman (1978) studied patients being treated for malignancies other than of the head and neck to determine associations between 
oral problems and the type of malignancy and therapy. The purpose of their study was to evaluate the distribution and type of oral problems in these patients. Of the 15 patients with leukemia, abnormal oral conditions developed in 10. The most frequent oral complications were ulceration, mucosistis and xerostomia, and these were seen most often in patients with leukemia at $67 \%$ and Hodgkin's disease at $100 \%$. Patients with leukemia also exhibited moniliasis, cellulites, abscesses, and acute necrotizing ulcerative gingivitis (ANUG). They also reported that younger patients (ages 1 to 20) had a higher prevalence of oral complications than older patients (ages 21 to $40 ; 41$ to 60 ; 61 or older).

Curtis (1971b) examined 374 panoramic dental radiographs of 214 children admitted to St. Jude Children's Hospital for the diagnosis or treatment of acute leukemia. The panoramic radiographs were studied for loss or thinning of the bony crypts of developing teeth and for loss of the lamina dura of the erupted teeth, and for the displacement of teeth. On dental radiographs, the earliest sign of acute leukemia (i.e., manifestations of the disease within the bone) was noted as destruction of the mandibular alveolar bone in the apical portion of the most distal developing molar crypt and the apical portions of the premolar and canine crypts. Alterations of the jaws were reported for $63 \%$ of the children with active leukemia. He stated that destruction of the apical portion of the most distal developing molar crypt was the abnormality most frequently encountered in the mandible. The apical portions of the premolar and canine crypts were the 
regions next most frequently involved. Curtis commented that active hematopoiesis continues in the jaws until puberty and thereafter usually decreases with age. Since leukemia is a disease of the hematopoietic tissues and affects the bone marrow in general, the alterations seen in the jaws of children are not unexpected.

Kaste et. al. (1997) examined panoramic radiographs for dental abnormalities in 423 children treated for ALL at St. Jude Children's Hospital. All patients were treated with multiagent chemotherapy and 243 of the 423 children received cranial irradiation in addition to the chemotherapy. Dental abnormalities includes root stunting, microdontia, hypodontia, taurodontia , and over-retention of primary teeth. The children that received the cranial irradiation developed more dental abnormalities than those who only received chemotherapy.

\section{$\underline{\text { Basic Chemotherapeutic Principles }}$}

After the identification of effective neoplastic drugs in the 1940s and 1950s the development of combination chemotherapy occurred in the 1960s at research hospitals across the country, including St. Jude Children's Research Hospital, Memphis, Tennessee. Since that time, refinements in combination chemotherapy have led to what is now referred to as risk-adapted therapy, which has increased remission rates to today's levels of around 80 percent (Pui 1997). The objective of 
this therapy is to eliminate the neoplastic cells with as little detriment as possible to the normal cells of the body. The protocol for risk-adapted therapy includes four major elements: remission induction, intensification-consolidation, prevention of overt CNS disease, and continuation of remission (Pui 1997).

\section{Current Therapy for ALL}

The beginning phase in treatment of ALL is induction therapy. The goal here is to induce complete remission with restoration of normal hematopoiesis (Pui and Evans 1998). The induction regimen includes doses of glucocorticoid (prednisone or dexamethasone), vincristine, and L-asparaginase (Pui and Evans 1998). This combination of chemotherapeutic agents should induce complete remission in $98 \%$ to $99 \%$ of children with newly diagnosed ALL (Pui 1997).

The next stage is intensification or consolidation therapy. This is designed to eradicate residual blast cells (Pui 1997). It consists of several drugs administered shortly after the induction of remission (Pui and Evans 1998). The drug regimen often includes high doses of methotrexate with or without 6mercaptopurine, high doses of asparaginase for an extended period and epipodophyllotoxin plus cytarabine. Alternatively, a combination of vincristine, dexamethasone, asparaginase, doxorubicin, and thioguanine may be given with or without cyclophosphamide (Pui and Evans 1998). This stage of therapy has been shown to improve outcomes, even in patients with low-risk ALL (Chessells 
et al. 1995). Research has suggested that intensive multidrug therapy begun after induction of remission results in longer lasting remissions (Cortes and Kantarjian 1995).

The control of subclinical CNS leukemia is the next step in treatment. Most children with leukemia have subclinical CNS involvement at the time of diagnosis (Pui 1995). This CNS leukemia can be eliminated by extended use of age-adjusted doses of intrathecal chemotherapy (Tubergen et al. 1993; Pullen et al. 1993). Studies have shown that additional doses of intrathecal chemotherapy and more intensive systemic treatment may replace cranial irradiation for prevention of CNS leukemia relapse (Conter et al. 1995).

\section{$\underline{\text { Tooth Formation }}$}

Many descriptions of the process of tooth formation as seen at the level of light microscopy have been written over the past several decades. Since the histological details have generally been worked out, these descriptions are concordant, and the fundamentals of tooth formation and maturation are considered common scientific knowledge today. The following description of tooth formation is a compilation of these works: Arey (1965), Slavkin (1974), Corliss (1976), Provenza (1986), Moss-Salentijn (1990), Bhaskar (1991), Melfi (1994), Ten Cate (1994), Sperber (2001), and Ash (2003). Except where noted, the description is invariant among these authors. 
Teeth develop from two of the primary germ layers, mesoderm and ectoderm, along with a neural crest contribution. The enamel of teeth is derived from oral ectoderm. The mesoderm forms the cementum and the periodontal membrane. Ectomesenchyme provides material for the dentine and pulp. The term ectomesenchyme is used to indicate that these mesenchymal cells originate from ectoderm (from the neural plate) and not from mesoderm, and these cells migrate from the neural plate ecotderm into their final position in the head. Ectomesenchymal-epithelial inductive interactions between neural crest tissue, pharyngeal endoderm and oral ectoderm induce ectodermal epithelial proliferation that produces the first morphologically identifiable manifestation of teeth, the odontogenic epithelial islands. The neural crest cells, in turn, are later induced to form the individual dental papillae, indicating the future number of teeth.

The first indication of tooth development is an epithelial plate (also called the dental lamina) about the 37th day of development (Fig. 2). In the maxilla this dental lamina is formed from the coalescence of four maxillary odontogenic epithelial islands and in the mandible there is a fusion of two odontogenic epithelial zones at the midline. The upper and lower dental laminae become a thickened horseshoe-shaped plate (band) that lies on the presumptive maxillary 

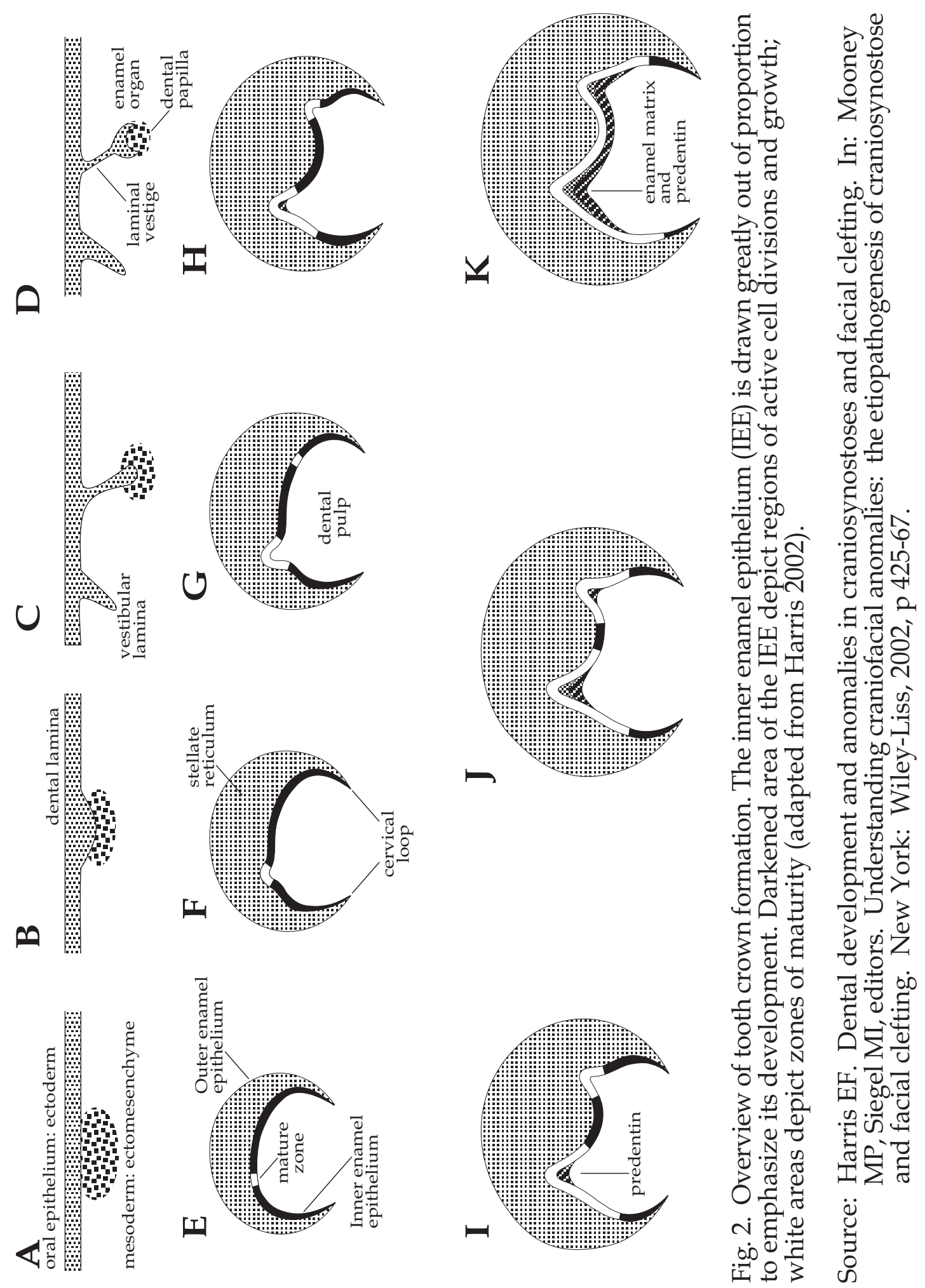
and mandibular alveolar ridges. This band of tissue invades the underlying mesenchyme and proliferates at an accelerated rate in ten places in both the maxillary and mandibular arches. These centers of increased mitosis will become the enamel organs (dental organs) for the developing primary teeth. The presumptive dental organs penetrate deeper into the underlying mesenchyme as they develop. The dental organs, which are knob-like thickenings along the dental lamina, also become submerged deeper into the mesenchyme because of occlusal growth of the maxillary and mandibular ridges.

Another, and later, subjacent proliferation of the oral epithelium occurs buccally and labially to the dental laminae and is known as the vestibular lamina. Degeneration (apoptosis) of the central epithelial cells of the vestibular laminae in the upper and lower jaws results in the formation of a sulcus, the vestibule, that will separate the lips and cheeks from the tooth-bearing gingival mucosa.

Early in the third month in-utero, the deeper side of each dental organ presses against a dense accumulation of mesenchyme and alters its initially small bud shape by enlarging and flattening out as a result of disproportionately rapid mitosis of the basal cells into a cap shape, and later cupping into a large bell shape (Fig. 3). The lumen of the cap (or cup) is filled with dense mesenchyme that will become the dental papilla. The dental organ will 

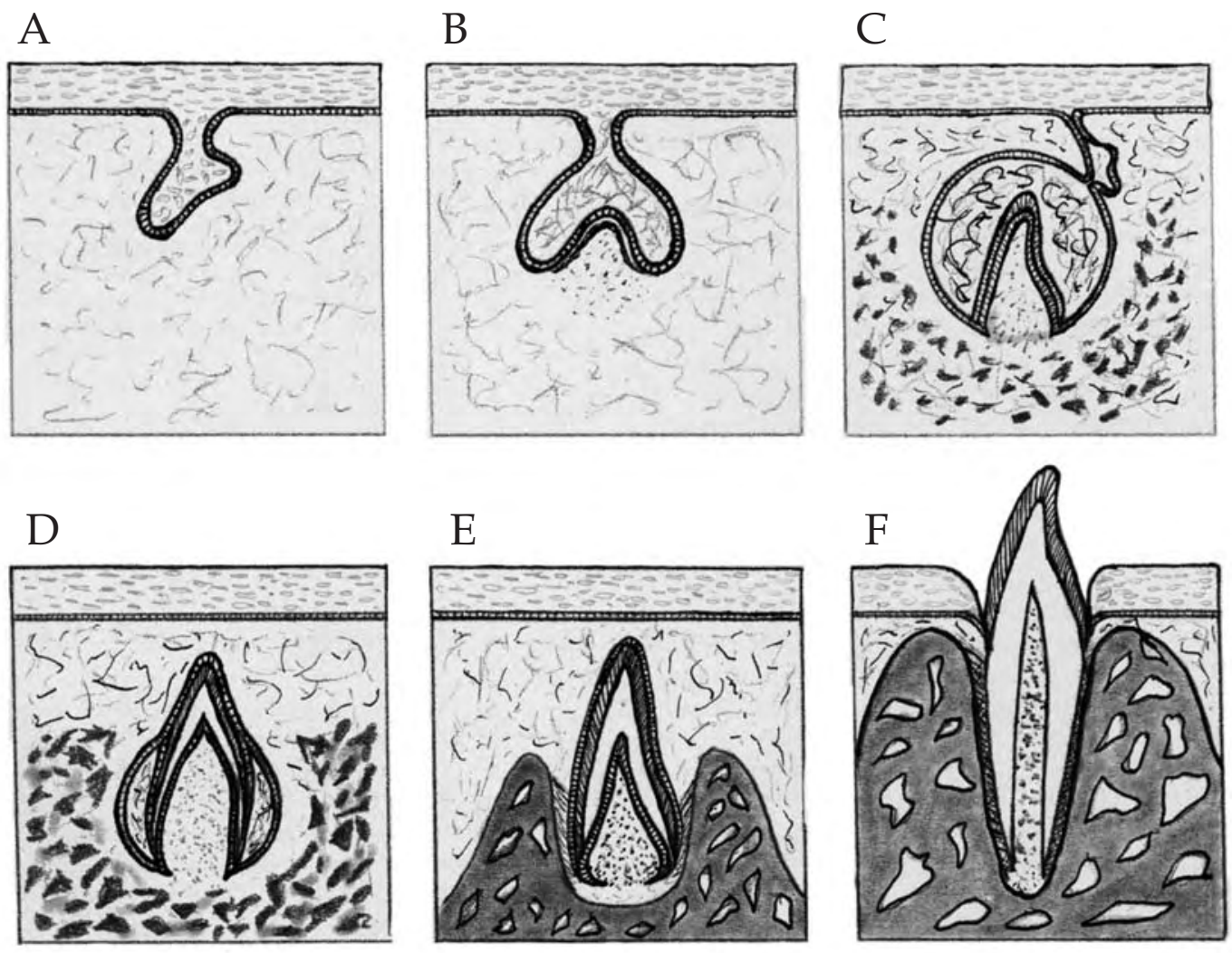

Fig. 3. Progressive stages of tooth formation: (A) bud; (B) cap; and (C) bell stages. (D) Early apposition stage. (E) Late apposition stage. (F) Eruption stage. (Diagram provided by Dr. E. F. Harris.) 
determine the shape of the crown, initiating dentin formation, establishing the dentogingival junction, and forming enamel, while the dental papilla will form the dentine and the pulp. The dental follicle is formed when the connective tissue beneath the dental papilla becomes fibrous and encircles the papilla and part of the enamel organ. The enamel organ, dental papilla and dental follicle together constitute the tooth germ. The tooth germ is still connected to the oral epithelium by means of the dental lamina that is attached to the dental organ. This tooth germ continues to enlarge and the cap becomes more bell shaped.

Concomitant with these morphological alterations, histodifferentiation occurs within the enamel organ and four distinct layers are discernible in its epithelium: the outer enamel epithelium (low cuboidal cells), the stellate reticulum (star shaped epithelial cells), the stratum intermedium (flat epithelial cells), and the inner enamel epithelium (cuboidal cells). The inner enamel epithelium is separated from the dental papilla by a basement membrane (i.e., acellular zone), which will ultimately become the dentoenamel junction.

The cells on either side of the basement membrane continue to develop in preparation for production of tooth structure. At the sites of the future cusp tips, where dentin will first be formed, mitotic activity ceases, and the small cuboidal cells of the inner enamel epithelium elongate and differentiate into ameloblasts (columnar cells) and prepare to lay down enamel. As these morphological changes occur in the cells of the internal enamel epithelium, changes also occur 
within the adjacent dental papilla. The cells of the dental papilla approximate the basement membrane and differentiate into columnar shaped odontoblasts (dentine forming cells). The formation of dentine by the odontoblast precedes and is necessary for the further induction of ameloblasts to produce enamel (reciprocal induction).

On the inner surface of the basement membrane, the odontoblasts begin the process of dentinogenesis at the incisal or cuspal area and progresses in an apical direction. The cells lay down a fibrillar matrix that mineralizes, but also leave an extension of the cell (odontoblastic process) imbedded in the material. The odontoblastic processes are vital cellular material and do not mineralize, but behave as if points of the cell membrane were stuck to the basement membrane. These processes elongate as the odontoblast move away from the basement membrane, toward the center of the pulp and lay down matrix. The end result is that the part of the cell with the nucleus comes to be some distance from the basement membrane, but it still is in physical contact with it through the odontoblastic processes.

The process of matrix formation of dentinogenesis begins at the time the odontoblasts differentiate form the mesenchyme of the pulp. Korff's fibers (heavy corkscrew shaped collagen type I fibers) are laid down at the time of differentiation and as the odontoblasts migrate away from the basement membrane, the fibers unravel and spread out among the odontoblastic processes. 
These processes and the ground substance are produced by the odontoblasts and this material is called predentine or dentinoid before it is mineralized. The predentine is mineralized progressively and the part proximal to the pulp is last to form and mineralize. Once dentine has been produced by the dental papilla it is then that the remnants of the dental papilla are termed the dental pulp.

Ameloblasts are unable to produce enamel matrix until some dentine has been lain down. They produce an organic matrix that facilitates the crystallization of mineral salts out of solution and creates enamel. Enamel formation begins at the incisal or cuspal surfaces and follows the same pattern as dentine formation. The ameloblasts move away from the basement membrane (now the dentoenamel junction) and leave enamel matrix behind. The cells lay down the matrix in such a way that it looks like a string of beads in close proximity to one another and flattened. This arrangement allows for the formation of the enamel rods and interrod substance that is apparent in mature enamel. It is thought that the beads compartmentalize the matrix and that the substance of each rod is contributed to by more than one ameloblast.

Once enamel matrix production has been completed, mineralization is complete and the enamel organ is reduced to a layer of ameloblasts and a few other epithelial cells. The ameloblasts eventually flatten as well and appear as normal epithelial cells covering the newly formed crown (called the reduced enamel epithelium). At this point, the reduced enamel epithelium produces the 
enamel cuticle (secondary enamel cuticle). The reduced enamel epithelium becomes the junctional epithelium upon emergence of the crown into the oral cavity.

Though enamel formation stops at the termination of the enamel organ, dentine formation continues apically to form the root of the tooth. The crown of the tooth begins to move occlusally during root formation, but the tooth will not emerge into the oral cavity until a considerable portion of the root has formed. Dentine production and root elongation continues from one to four years after emergence to complete the root. Production of dentine will enclose the pulp canal and eventually form the apical constriction (foramen) that signifies completion of the root, so-called root apexification.

Tooth development and bony growth and development of the jaws are interdependent and the timing is critical for proper positioning and eventual eruption of the teeth. The anterior primary tooth germs (central incisor, lateral incisor, canine and first molar) develop first, beginning in the sixth week postconception, while the second primary molar germs first appear at the seventh week. Also at this time, the dental lamina is beginning to break down except for the free edge (this secondary dental lamina develops lingual to the primary tooth germ) that persists to form the primordia of the succedaneous teeth. The tooth germs of the secondary teeth that do not replace primary teeth 
also form at this time from the primary dental lamina, but they are not associated with a developing primary tooth.

\section{Crown Development}

With the growth of the inner enamel epithelium (IEE), various regions of the formative crown respond allometrically to create the topography of cusps, ridges, pits and grooves (Korenhof 1960; Kraus and Jordan 1965; Ooë 1981). Attention now focuses on the primary and secondary enamel knots that direct the folding of the IEE that results in characteristic crown morphologies.

It has been long recognized as histological features that enamel knots begin to form in the cap stage of tooth development (reviewed in Butler 1956). Location of the primary enamel knot probably coincides with the presumptive apex of the first-forming cusp (Jernvall et al. 1994; Thesleff et al. 2001), which is the paracone (mesiolingual cusp) on the human upper molars. Subsequently, during the bell stage the ostensible secondary enamel knots develop and coincide with the number and positions of the other presumptive cusps.

Enamel knots are transitory condensations of the IEE that project toward the dental papilla (Butler 1956). They consist of non-dividing cells, which is significant since these knots seen to be control centers that stimulate rapid proliferation of adjacent dental epithelium though they themselves are nonproliferative (Jernvall et al. 1994; Luuko et al. 2003). This seems to be an 
essential relationship to the formation of tooth cusps (Jernvall et al. 2000).

Enamel knots define the number and modulate the spatial relationships of the presumptive cusp tips. Topographic differences in proliferation rates of the epithelium account for the angularity of the cusps - at least as expressed at the enamel-dentin interface (Butler 1967a,b, 1968) as well as the differences in cusp heights. The paracone begins mineralizing first on maxillary molars (Christensen and Kraus 1965; Kraus and Jordan 1965) so its apex is highest. The later-forming hypocone's apex is lower since occlusobasal development has progressed before its differentiation. This leaves the talon lower than the trigon (Gregory 1922; Ziesz and Nuckolls 1949; Swindler 2002).

The location of the enamel knots do not account for all of the variation in intercusp relationships because (1) there is significant allometric growth of the IEE before dentinogenesis "freezes" the size and shape of the DEJ (Turner 1963; Ooë and Nomata 1964; Butler 1967a,b) and (2) the accretion of enamel alters intercusp relationships because depositioin is not uniform (Kraus 1952; Hillson and Bond 1997; Smith et al. 1997; Peretz et al. 1988a,b).

A series of prenatal specimens at ages prior to mineralization of the intercuspal regions (dentinogenesis precedes amelogenesis, so it actually is dentine deposition that terminates intercuspal dimensions at the DEJ) was used by Butler (1967b) to quantify the intercusp distance of the human maxillary first molar. Butler shows that the paracone-metacone distance for UM1 is around 1 
$\mathrm{mm}$ when these cusp tips are first recognizable and this distance increases to about $4 \mathrm{~mm}$ before mineralization fixes the definitive distance. The pattern of growth, at least for this one distance, appears to be linear (Butler 1967b).

Intercuspal growth increases overall crown area, and the time- and rate-specific differences in growth rates produce species-specific crown morphologies as well as the inter-individual variation within a group (Peretz et al. 1997, 1998a,b; Salazar-Ciudad et al. 2002).

Generally, the cusp tip, which is specified as the enamel of a mature tooth, is typically to the side, buccal or lingually, of the apex defined at the DEJ. As the enamel thickens the cusp tip is shifted buccally on the paracone and metacone and lingually (palatally) on the protocone and hypocone in relation to the underlying prominences at the DEJ illustrating the moving of the cusps tips in opposite directions as amelogenesis proceeds (Kraus 1952).

\section{$\underline{\text { Root Formation }}$}

Following the completion of crown morphogenesis and the elaboration of coronal dentin and enamel extracellular matrix, the developing tooth germ begins to form its root, a process that will establish its connection to the surrounding bone. The mesenchyme that surrounds the enamel organ (dental sac) and that situated within the developing pulp is contiguous and derived from cranial neural crest ectomesenchyme. This mesenchyme and particularly that 
portion situated in the apical portion of the tooth germ, proliferates throughout the period of root development, generating not only cell populations that will contribute to the developing radicular pulp but also those that will form the developing periodontium. The epithelial root sheath, derived from cells of the cervical loop of the enamel organ, proliferates apically, thereby establishing the demarcation between pulp and periodontium (Thomas 1995).

Epithelial root sheath cells initiate the differentiation of odontoblasts that form root dentin (Ten Cate 1994). Distinct differences between crown and root dentine formation have been described (Ten Cate 1978; Beertsen and Niehof 1986), particularly during predentin secretion. Morphological differences are apparent between fully differentiated odontoblasts in the crown and root; coronal odontoblasts are columnar, root odontoblasts are cuboidal (Avery 1986). Differences have been described in the biochemical composition of root dentin when compared with crown dentine (Steinfort et al. 1989). Also the phosphoryn content of root dentine is less than that of coronal dentin, its degree of mineralization is slightly less, and the rate of deposition of root dentin is slower Ten Cate 1994).

The odontoblast moves centripetally, leaving behind the formed dentin. How such movement takes place has not been studied. The cell, as it migrates, leaves behind a process that comes to occupy a tubule within the mineralized dentin. Thus the dentinal tubule is a permanent record of the track of the 
odontoblasts, which in coronal dentin is S shaped and in root dentin straight (Ten Cate 1994).

\section{Physiological Age}

Physiological age (also called "biological age" or "developmental age") is the age of the subject as judged by normal functional developmental processes, that is, the degree of maturity of the biologic tissue and organ systems an individual has achieved (Moorrees et al. 1963). Most children are "average maturers" by definition; their physiological and chronological ages coincide fairly closely. Other children developmentally lag behind their calendar ages, and these are called "late maturers" and "delayed growers." Others develop faster than indicated by their chronological age; these are called "early maturers." Tempo of growth is the rapidity with which an individual achieves biological maturity. When the tempo of growth is faster or slower than average, the use of the child's chronological age can be misleading relative to his growth potential.

The link between growth and maturity has been described by Tanner et al. (1975) who stated that, "The normal growth process takes every individual from one common condition of being wholly immature to another of being wholly mature." Roche (1986) defined attaining maturity as the sequential alterations of 
a body, an organ or a tissue as it changes from an embryonic stage until it attains its adult form and functional level.

\section{Maturity in Relation to Age}

Tanner et al. (1975) stated that, in general, the use of an age scale for maturity measurements, and vice versa, is not advisable because of the following weaknesses. First, it fails at the extremes. For example, in skeletal maturity no particular age can be associated with an individual whose bones are all fully mature, although his status of $100 \%$ skeletal maturity is unequivocal. Second, and more important, the relationship between actual age and maturity is not necessarily constant from one population to another or from one time to another, so unless the maturity scale is continuously redefined and updated in relation to the actual age, the result will be that the maturity scale and age do not correspond to each other. Tanner et al. concluded that it is more proper to view maturity in a manner that does not refer directly to age, but rather study maturity and its relation to age as a second step in any given population, and produce "maturity standards" that can be updated.

Van Wieringen (1986) stated that maturity, in relation to age, varies from one ethnic group to another, from one individual in the same ethnic group to another, and from one generation to another in the same population, which is a phenomenon known as a secular trend. 
Maturational events associated with puberty can be very useful, but, of course, they are not applicable before or after the narrow range of a few years in early adolescence (Tanner 1962). Methods that are uniformly applicable throughout the span from birth to biological adulthood are more useful. Bone age and dental age are such methods. To be most useful, a method should meet four criteria: (1) the same technique should be uniformly applicable throughout growth; (2) the technique should be uniformly applicable on males and females; (3) the method should be available for routine clinical use, not be complex, expensive or difficult to perform as required; and (4) the technique should be based on an invariant sequence of developmental events.

\section{Assessment of Dental Age}

There are various methods of determining dental age. One common method uses eruption of teeth into the oral cavity (e.g., Filipsson 1975; Liversidge et al. 2003). Another method for determining dental age involves a determination of the degree of mineralization of the teeth using radiographs (Moorrees et al. 1963; Anderson et al. 1976) or histological sections (Kronfeld 1935a,b; Kraus and Jordan 1965). The histological method will reveal more details than the radiographic method, but it is invasive. Gleiser and Hunt (1955) suggested that, on an individual basis, tooth mineralization is superior to emergence for assessing dental age because of the greater spectrum of events involved in tooth formation. Clinical emergence is a fleeting event; in contrast mineralization can 
be evaluated at any time during the span of active growth of the tooth. The ease and frequency at which radiographs can be taken make this method the best approach to monitoring a child's dental development (Harris 2002). Fanning $(1961,1962)$ suggested that mineralization is superior to tooth emergence for assessing dental age because emergence can be influenced by local environmental factors such as injury, obstruction, crowding, infection, and extraction of deciduous or adjacent permanent teeth. Demirjian (1978) likewise noted several reasons why mineralization is preferred over emergence. He stated that tooth emergence is a transient event, timing this exact event is difficult, and emergence can be easily disturbed by environmental factors such as infection or crowding. He also stated that the emergence data is limited to the ages at which teeth actually emerge; for the primary dentition is 6 to 30 months of age and for the permanent dentition is 6 to 12 years of age.

The mineralization of a tooth is a continuous process that begins at the cusp tip and continues down the formative tooth in a regimented fashion. Mineralization of the root never begins until crown formation is complete. The last stage of tooth development is closure of the root apex. This continual mineralization process can be divided into distinguishable stages. Table 3 shows several systems that have been developed to separate this continuous process into discrete, identifiable stages to facilitate study (Gleiser and Hunt 1955; Garn et al. 1958; Nolla 1960; Fanning 1961; Moorrees et al. 1963; Demirjian et al. 1973). 
Table 3. Comparative table of mineralization stages used by different authors.

\begin{tabular}{|c|c|c|c|c|c|c|}
\hline & $\begin{array}{c}\text { Gleiser } \\
\text { \& Hunt } \\
(1955)\end{array}$ & $\begin{array}{l}\text { Garn } \\
\text { et al. } \\
(1958) \\
\end{array}$ & $\begin{array}{l}\text { Nolla } \\
(1960)\end{array}$ & $\begin{array}{c}\text { Fanning } \\
\text { (1961) }\end{array}$ & $\begin{array}{c}\text { Moorrees } \\
\text { et al. } \\
(1963)\end{array}$ & $\begin{array}{c}\text { Demirjian } \\
\text { et al. } \\
(1973) \\
\end{array}$ \\
\hline Presence of crypt & I & - & 1 & $\mathrm{O}$ & - & - \\
\hline Cusp formation & II & 1 & 2 & $\mathrm{C}_{1}$ & 1 & $\mathrm{~A}$ \\
\hline Cusp coalescence & III & - & - & $\mathrm{CCO}$ & 2 & - \\
\hline Cusp outline & IV & - & - & COC & 3 & B \\
\hline Crown $1 / 3$ & - & - & 3 & - & - & - \\
\hline Crown $1 / 2$ & V & - & - & $\mathrm{Cr}_{1 / 2}$ & 4 & $\mathrm{C}$ \\
\hline Crown $2 / 3$ & VI & - & 4 & $\mathrm{Cr}_{2 / 3}$ & - & - \\
\hline Crown $3 / 4$ & - & - & 5 & - & 5 & - \\
\hline Crown complete & VII & - & 6 & $\mathrm{Cr}_{\mathrm{C}}$ & 6 & $\mathrm{D}$ \\
\hline Initial root & VIII & 2 & - & $\mathrm{R}_{1}$ & 7 & - \\
\hline Initial cleft & VIII A & - & - & $\mathrm{Cl}_{1}$ & 8 & - \\
\hline Cleft enlarging & VIII B & - & - & Cle & 8 & - \\
\hline Root $1 / 4$ & IX & - & - & $\mathrm{R}_{1 / 4}$ & 9 & E \\
\hline $\operatorname{Root} 1 / 3$ & $x$ & - & 7 & $\mathrm{R}_{1 / 3}$ & - & - \\
\hline Root $1 / 2$ & XI & - & - & $\mathrm{R}_{1 / 2}$ & 10 & - \\
\hline Root $2 / 3$ & XII & - & 8 & $\mathrm{R}_{2 / 3}$ & - & F \\
\hline Root 3/4 & XIII & - & - & $\mathrm{R}_{3 / 4}$ & 11 & - \\
\hline Root complete & XIV & - & 9 & $\mathrm{R}_{\mathrm{C}}$ & 12 & G \\
\hline Apex 1/4 closed & - & - & - & $\mathrm{A}_{1} / 4$ & - & - \\
\hline Apex $1 / 2$ closed & - & - & - & $\mathrm{A}_{1 / 2}$ & 13 & - \\
\hline Apex 3/4 closed & - & - & - & $\mathrm{A} 3 / 4$ & - & - \\
\hline Apex closed & $X V$ & 3 & 10 & $\mathrm{AC}_{\mathrm{C}}$ & 14 & $\mathrm{H}$ \\
\hline
\end{tabular}

Source: Demirjian A. Dentition. In: Falkner F, Tanner JM, editors. Human growth 2. postnatal growth. New York and London: Plenum Press, 1978, p 412-44. 
Gleiser and Hunt (1955) made drawings of the outlines of the permanent mandibular first molar of the serial radiographs of 50 children ( 25 boys and 25 girls) that were taken at three month intervals from birth to 18 months, then at six month intervals from 18 months to 10 years of age. They chose 15 stages of mineralization (Table 4) from these drawings. The scoring of mineralization for individual teeth was accomplished by selection of the "closest" grade from the 16 possible choices.

Garn et al. (1958) used three mineralization stages and two stages of tooth eruption to evaluate sex differences in the formation of the mandibular premolar and molar teeth. The three stages of tooth mineralization were (1) initial mineralization, (2) beginning root mineralization, and (3) apical closure. The two stages of eruption were (1) beginning of alveolar eruption and (2) attainment of the occlusal level.

Nolla (1960) used a scale developed by Pinney (1939) and modified it for the study of tooth mineralization using serial radiographs of a group of 50 children. Her scale was comprised of a set of drawings of ten stages of mineralization for each permanent tooth type. The radiographic image was matched with the comparative drawing, which had a corresponding stage valued from one to ten (Table 5). If a tooth was between stages Nolla's solution was to use the lower stage and add 0.5 to the value assigned to the tooth. If a tooth was slightly less, then 0.3 was subtracted from the closest corresponding 
Table 4. Definitions of the 15 stages of tooth formation used by Gleiser and Hunt (1955) to grade the mineralization of the mandibular first permanent molar.

\begin{tabular}{cc}
\hline Stage & Definition \\
\hline I & no calcification: crypt formed but no mineralization yet \\
II & centers of calcification visible: amelogenesis has begun on the \\
& individual cusp tips \\
III & coalescence of centers: centers of calcification are merged \\
IV & cusp outline complete: the coronal outline is mineralized \\
V & $1 / 2$ crown: amelogenesis is half way to cervical margin \\
VI & 2/3 crown completed \\
VII & crown completed: morphologically, the crown has mineralized \\
& but root formation has not begun \\
VIII & minimal root formation: there is just a trace of root radiopacity \\
& below the crown outline \\
VIII A & cleft minimal: interradicular mineralization is evident \\
VIII B & cleft rapidly enlarging: significant mineralization evident in the \\
& interradicular area but root not $1 / 4$ formed \\
IX & $1 / 4$ root: the radiographic morphology of the root is $1 / 4$ of its \\
& projected final size \\
X & 1/3 root completed \\
XI & 1/2 root completed \\
XII & $2 / 3$ root completed \\
XIII & $3 / 4$ root completed \\
XIV & divergent root canal walls: full root length achieved but walls \\
& of root canal have not yet converged terminally \\
XV & convergent root canal walls: walls of root canal converged \\
& terminally and roots appear mature \\
&
\end{tabular}

Source: Gleiser I, Hunt EE Jr. The permanent mandibular first molar: its calcification, eruption and decay. Am J Phys Anthropol 1955;13:253-83. 
Table 5. Definitions of the ten stages of tooth formation used by Nolla (1960) to grade the mineralization of all tooth types.

\begin{tabular}{cl}
\hline Stage & \multicolumn{1}{c}{ Definition } \\
\hline 0 & $\begin{array}{c}\text { absence of crypt: no sign of tooth development is apparent } \\
\text { presence of crypt: crypt is formed but no mineralization has } \\
\text { begun }\end{array}$ \\
2 & $\begin{array}{c}\text { initial calcification: amelogenesis has begun on the cusp tips } \\
\text { one-third of crown completed: amelogenesis is 1/3 the way to } \\
\text { the cervical margin }\end{array}$ \\
3 & $\begin{array}{c}\text { two-thirds of crown completed } \\
\text { crown almost completed: morphologically, the crown has } \\
\text { mineralized to just short of the cervical margin } \\
5\end{array}$ \\
6 & $\begin{array}{c}\text { crown completed: morphologically, the crown has mineralized } \\
\text { but root formation has not begun } \\
\text { one-third of root completed: the radiographic morphology of } \\
\text { the root is 1/3 of its projected final size }\end{array}$ \\
8 & $\begin{array}{c}\text { two-thirds of root completed } \\
\text { root almost completed: full root length has been achieved but } \\
\text { apex is still open }\end{array}$ \\
9 & $\begin{array}{c}\text { root completed: apical end of root completed and apex is } \\
\text { closed }\end{array}$ \\
\hline
\end{tabular}

Source: Nolla CM. The development of the permanent teeth. J Dent Child 1960;27:254-66. 
grade. If a tooth was slightly more developed that the grade indicated, 0.2 was added to the grade indicated. The thought was to have a more accurate description of mineralization stage than just selection the closest full grade.

Fanning (1961) adapted the grading scheme of Gleiser and Hunt (1955) in her longitudinal study of tooth formation. Three apical stages were added for greater precision, to give a total of 20 gradable stages of tooth development (Table 6). The mesial and distal roots of molars were studied separately.

Moorrees et al. (1963) used the schemes of Fanning (1961) to develop a 13grade scale for single-rooted teeth and 14-grade scale for multi-rooted teeth (Fig. 4). These latest grading schemes by Moorrees et al. are a collaboration of information and conform with slight modifications to those used by other investigators (Gleiser and Hunt 1955; Demisch and Wartmann 1956; Garn et al. 1958; Nolla 1960; Fanning 1961). Normative data on the mineralization timing of ten permanent teeth, by sex, were calculated using these scales. Their method of estimating dental age was to score all of the available teeth and then use the graphs provided in their publication to obtain the expected chronological age from each stage of each tooth. The dental age for an individual was obtained by averaging across the scorable teeth. There was no weighting or selection of the tooth scores. Recently, Harris and Buck (2002) have published tables of values measured from Moorrees' graphs, which expedites calculating dental ages. 
Table 6. Definitions of the 20 stages of tooth formation used by Fanning (1961) to grade the mineralization of the mandibular canines, premolars and molars.

\begin{tabular}{|c|c|}
\hline Stage & Definition \\
\hline $\mathrm{O}$ & no calcification: crypt formed but no mineralization yet \\
\hline $\mathrm{C}_{1}$ & $\begin{array}{l}\text { centers of calcification visible: amelogenesis has begun on the } \\
\text { individual cusp tips }\end{array}$ \\
\hline $\mathrm{C}_{\mathrm{CO}}$ & coalescence of centers: centers of calcification are merged \\
\hline $\mathrm{COC}_{\mathrm{OC}}$ & cusp outline complete: the coronal outline is mineralized \\
\hline $\mathrm{Cr}_{1 / 2}$ & $1 / 2$ crown: amelogenesis is half way to cervical margin \\
\hline $\mathrm{Cr}_{2 / 3}$ & $2 / 3$ crown completed \\
\hline $\mathrm{Cr}_{\mathrm{C}}$ & $\begin{array}{l}\text { crown completed: morphologically, the crown has mineralized } \\
\text { but root formation has not begun }\end{array}$ \\
\hline $\mathrm{R}_{1}$ & $\begin{array}{l}\text { minimal root formation: there is just a trace of root radiopacity } \\
\text { below the crown outline }\end{array}$ \\
\hline $\mathrm{Cl}_{1}$ & cleft minimal: interradicular mineralization is evident \\
\hline $\mathrm{Cl}_{\mathrm{e}}$ & $\begin{array}{l}\text { cleft rapidly enlarging: significant mineralization evident in the } \\
\text { interradicular area but root not } 1 / 4 \text { formed }\end{array}$ \\
\hline $\mathrm{R}_{1 / 4}$ & $\begin{array}{l}1 / 4 \text { root: the radiographic morphology of the root is } 1 / 4 \text { of its } \\
\text { projected final size }\end{array}$ \\
\hline $\mathrm{R}_{1 / 3}$ & $1 / 3$ root completed \\
\hline $\mathrm{R}_{1 / 2}$ & $1 / 2$ root completed \\
\hline $\mathrm{R}_{2 / 3}$ & $2 / 3$ root completed \\
\hline $\mathrm{R}_{3 / 4}$ & $3 / 4$ root completed \\
\hline $\mathrm{R}_{\mathrm{C}}$ & $\begin{array}{l}\text { divergent root canal walls: full root length achieved but walls } \\
\text { of root canal have not yet converged terminally }\end{array}$ \\
\hline $\mathrm{A}_{1 / 4}$ & 1/4 apex closed \\
\hline $\mathrm{A}_{1 / 2}$ & $1 / 2$ apex closed \\
\hline $\mathrm{A}_{3 / 4}$ & 3/4 apex closed \\
\hline $\mathrm{A}_{\mathrm{C}}$ & $\begin{array}{l}\text { convergent root canal walls: walls of root canal converged } \\
\text { terminally and roots appear mature }\end{array}$ \\
\hline
\end{tabular}

Source: Fanning EA. A longitudinal study of tooth formation and root resorption. NZ Dent J 1961;57:202-17. 


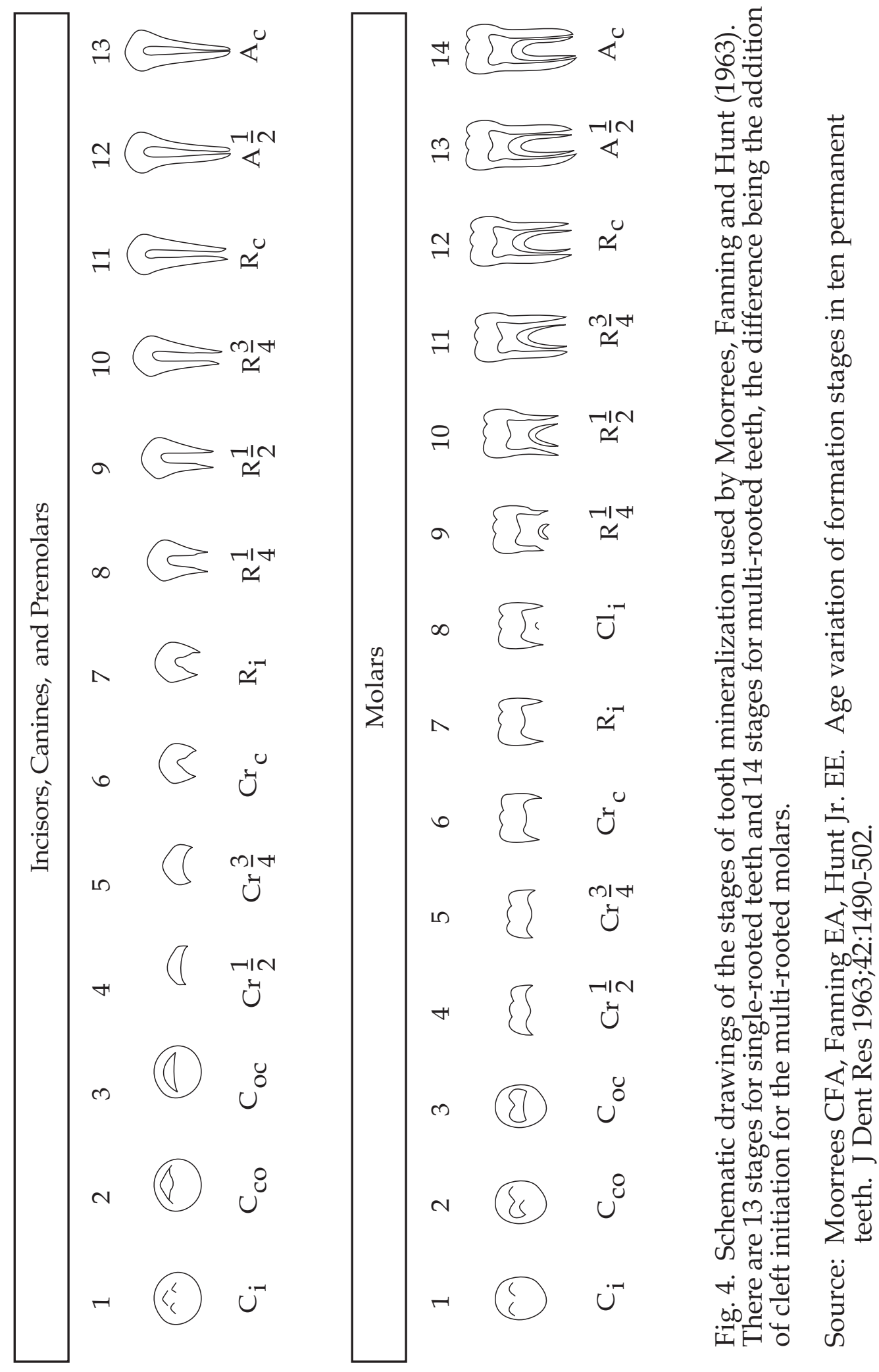


Demirjian et al. (1973) devised a different, statistically more sophisticated method of evaluating dental age. Demirjian et al. scored just the mandibular seven permanent teeth (omitting the third molar) because information from the maxillary teeth is redundant and they often are difficult to evaluate because of radiologically superimposed bony structures. In this new assessment, a letter grade is assigned to each tooth from $\mathrm{A}$ to $\mathrm{H}$, in relation to one, two or three written criteria (Fig. 5). If only one criterion was given for a particular stage, it must be met for the stage to be counted as attained; if two criteria were given, at least the first one has to be met; if three were given, at least the first two have to be met for the stage to be counted. To aid in the scoring, diagrams and pictures of radiographs corresponding to the eight stages were used. Letter scores were converted into self-weighted, sex-specific numerical scores that were then summed to give a total maturity score. The dental age could be calculated by converting the score using a table of standards.

\section{Stages of Tooth Formation Seen Radiographically}

The first radiographic evidence of tooth formation is the translucent follicle situated at or near the alveolar bone crest, which increases in size and becomes circular in shape. This image on the radiograph was not assigned a Moorrees et al.1-14 grade but an arbitrary grade assigned for crypt formation, but no crown formation. The follicle continues to enlarge while the crown 


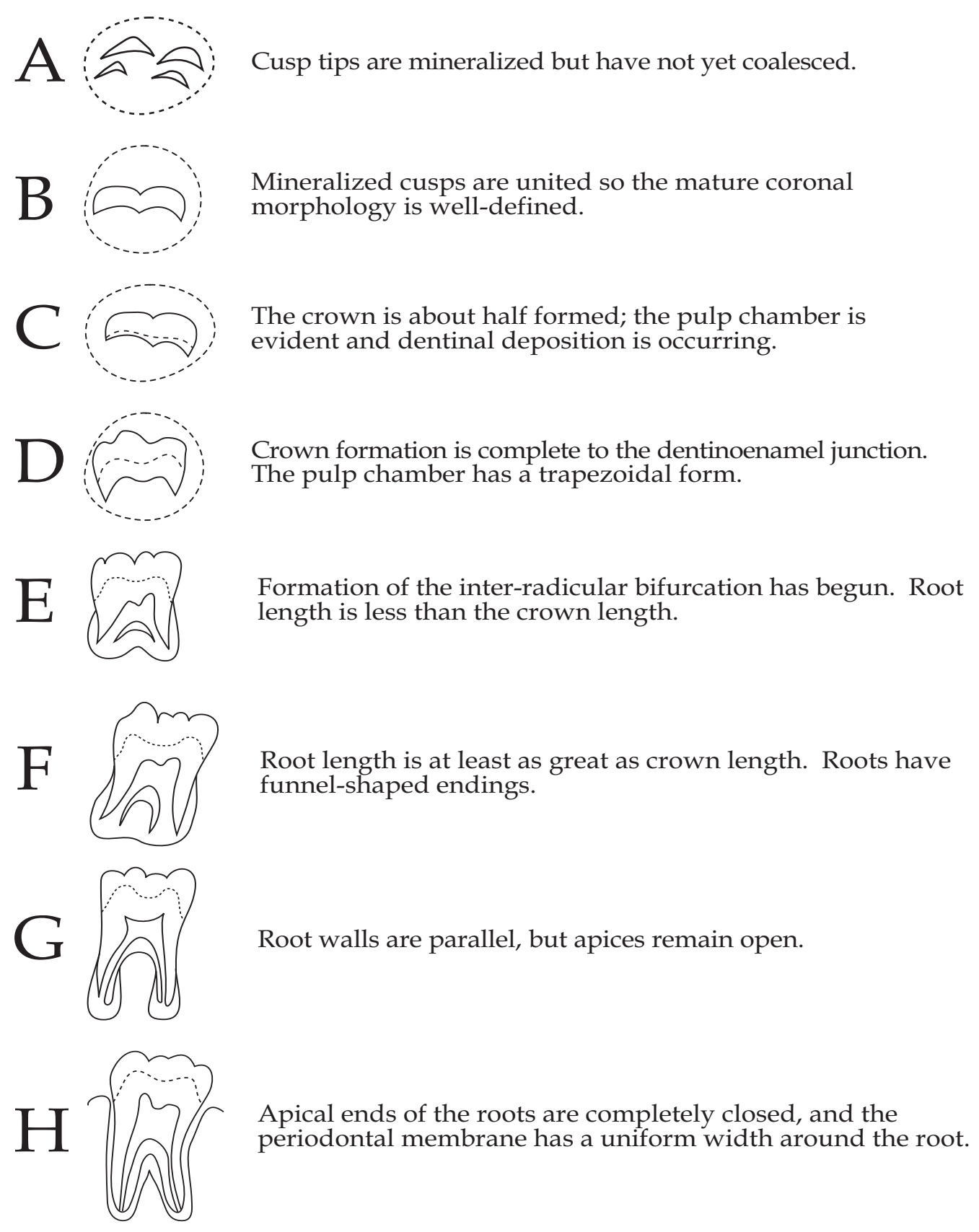

Fig. 5. Schematic drawings along with the textual cues for discriminating between Demirjian's 8 grades of tooth formation.
Source: Demirjian A, Goldstein H, Tanner JM. A new system of dental age assessment. Hum Biol 1973;45:211-27.


commences formation (Fanning 1961). Figures 6 and 7 are examples of the 13 and 14 stages seen radiographically. Stage one is termed initial cusp formation and defined as when amelogenesis has begun on the individual cusp tips. Stage two is termed coalescence of cusp and it is defined as when the centers of mineralization are merged but the border is not everywhere radio-opaque. Stage three is termed cusp outline complete and it is when the coronal outline of the tooth is mineralized. Stage four is termed crown one-half formed and it is when amelogenesis has proceeded half way to the crown-root as judged from morphology of the radio-opaque portion. The fifth stage is when one-half of the crown has completed mineralization. Stage six is termed crown complete and it is when morphologically, all the crown has mineralized but root formation has not begun. Stage seven is termed initial root formation and it is when there is a trace of root radiopacity below the crown outline. The next stage is only in multi-rooted teeth and it is defined as initial cleft formation and it is when mineralization is evident in the interradicular area. Stage eight in single rooted teeth and nine in multi-rooted teeth is termed root length one-forth and it is when the radiographic morphology of the root is one-forth its projected final size. As the root lengthens the stages progress to one-half (stages 9 and 10 for single and multi-rooted teeth, respectively), three-quarters (stages 10 and 11 for single and multi-rooted teeth, respectively), and then root completion (stages 11 and 12 for single and multi-rooted teeth, respectively). The last two stages are 


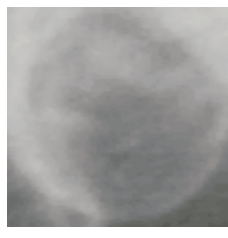

$$
1 C_{i}
$$

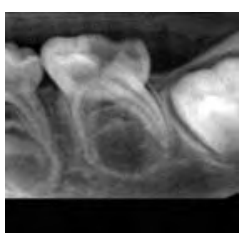

$2 \mathrm{C}_{\mathrm{CO}}$

$3 \mathrm{C}_{\mathrm{OC}}$

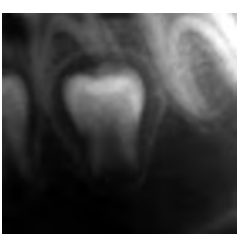

$8 \quad \mathrm{R}_{\frac{1}{4}}$
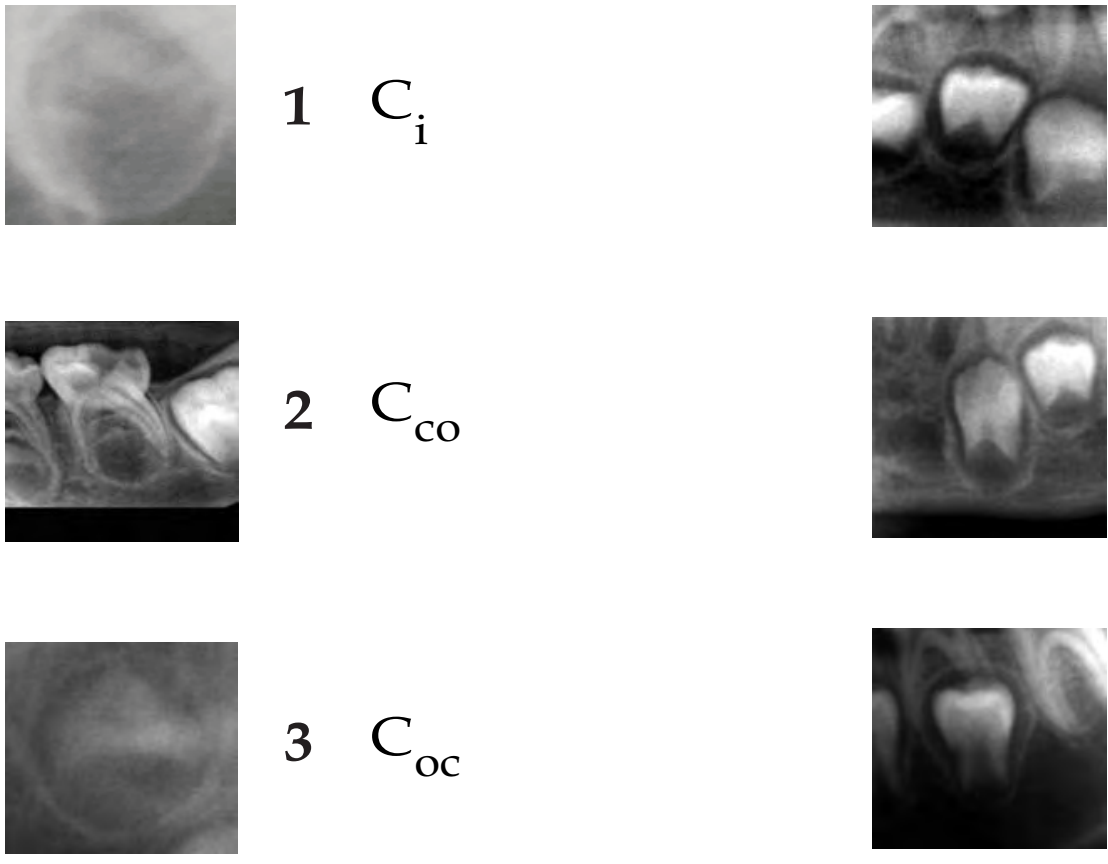

$\begin{array}{ll}7 & \mathrm{R}_{\mathrm{i}}\end{array}$

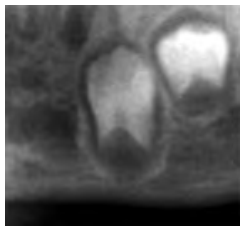

$9 \mathrm{R}$

$$
\frac{1}{2}
$$

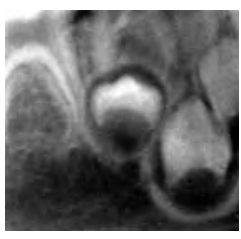

$4 \quad \mathrm{Cr}_{\frac{1}{2}}$

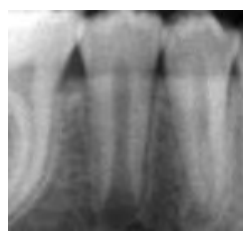

$10 \mathrm{R}_{\frac{3}{4}}$

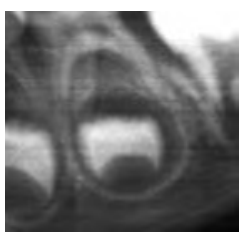

$5 \quad \mathrm{Cr}_{\frac{3}{4}}$

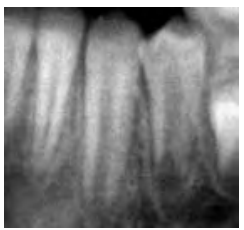

$11 R_{c}$

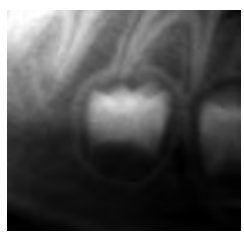

$6 \mathrm{Cr}_{\mathrm{C}}$

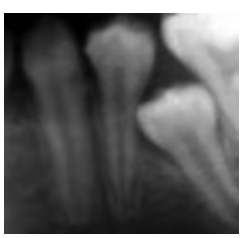

$12 \mathrm{~A}_{\frac{1}{2}}$

Fig. 6 Radiopgraphic examples of the single-rooted grades of tooth mineralization. 


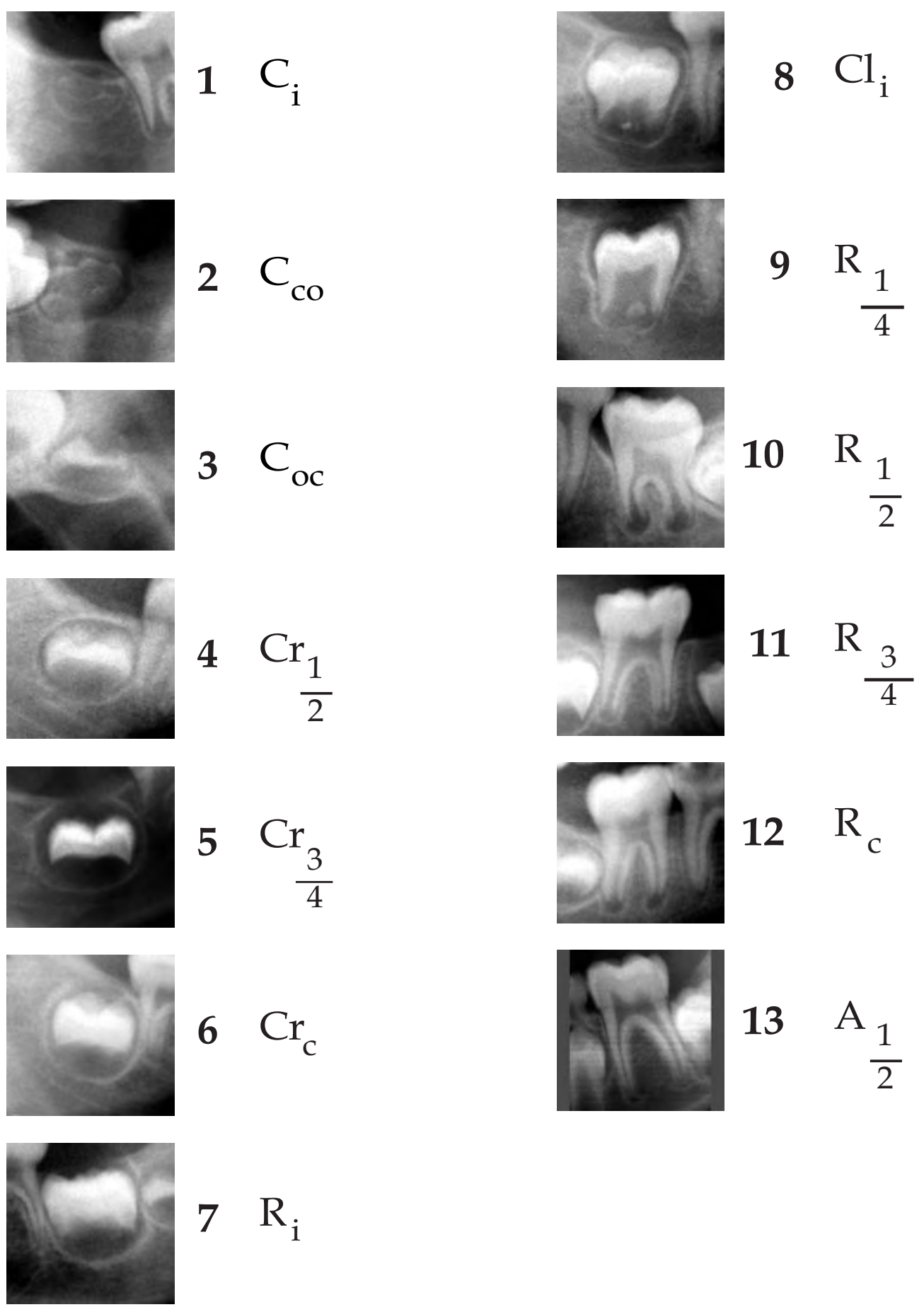

Fig. 7 Radiopgraphic examples of the multi-rooted grades of tooth mineralization. 
apex half closed and apical closure complete. The apex half closed is seen of the radiograph as the lateral borders for the root tip becomes convex rather than tapered as earlier (stages 12 and 13 for single and multi-rooted teeth, respectively). The final stage is apical closure complete and it is when the size of the apical foramen is reduced to its mature size (stages 13 and 14 for single and multi-rooted teeth, respectively) (Harris and McKee 1990).

\section{Left-Right Asymmetry and Delayed Dental Development}

It has been shown that children with a cleft lip and palate (CLP) are at risk for reduced size (Hunter and Dijkman 1977; Cooper, Harding and Krogman 1979; Jensen, Dahl, and Kreiborg 1983; Bowers et al. 1987), slower growth (Menius, Largent and Vincent 1966; Bailit, Doykos and Swanson 1968; Ranta 1984), and other postnatal deficiencies (Ross 1965; Dahl 1970; Ross and Johnston 1972). It is thought that the reduced growth is due to a debilitating environmental factors in which these children experience feeding difficulties, contract upper respiratory infections and undergo repeated hospitalizations for lip and palate surgeries (Cox 1960; Ross 1965; Drillien, Ingram and Wilkinson 1966; Hunter and Dijkman 1977). Harris and Hullings' (1990) study support the aforementioned statements, in that the teeth that begin to form in utero do not show any signs of delayed or altered dental development. It is a common clinical perception that teeth of children with CLP are slower to form and erupt. 
The stresses of CLP can also increase left-right asymmetry of the developing dentition (Harris and Hullings 1990; Ranta 1973a,b). Left-right asymmetry and delayed dental development were noted across all tooth types and throughout both arcades (Harris and Hullings 1990; Ranta 1973a,b). These dental sequelae yield insights into the developmental process of children with CLP and tooth formation constitutes a sensitive measure of overall health (Niswander 1963; Bailit 1975).

It is common knowledge that ALL itself is a debilitating disease and the stresses placed on the systems of the body are immense. Due to the nature of the disease and the quest to expeditiously diagnose and begin treatment yields little information on its effects and more specifically its effects on the dentition. Halton et al. (1995) have studied mineral homeostasis and bone mass at diagnosis in children with ALL before chemotherapy treatment begins. They found that most children with ALL have alterations in bone metabolism and bone mass when first seen. Abnormally low circulating 1,25(OH) sub 2 D3 levels associated with hypercalciuria; may result in calcium and phosphate depletion, limiting bone mineralization. Presently, there seems to be no research on the effect of ALL on tooth mineralization in the absence of treatment to cure the disease. Tooth mineralization is a relatively slow process compared to the quick onset of ALL. An expedited diagnosis followed by swift induction into treatment is desired, which leaves virtually no time to study the effects of ALL itself on the 
dental development and mineralization. Inferring from previous literature on bone mineralization we conclude that dental mineralization is also limited or delayed. Foote (1928:2) helps to reveal the link between bone and tooth mineralization with a passage from his book:

We are accustomed to think that dentinal substance was produced by one cell, the odontoblast, enamel substance by another cell, the ameloblast, and bone substance, by still another cell, the osteoblast, and we have erected impassable barriers between them, but the fact remains, however, that all cells of any individual were derived from one cell which possessed the original endowment of protoplasm and it only requires the presence of certain environmental conditions to cause the descendants of that cell to exercise their original prerogatives. Teeth and bond have something in common which is fundamental and we have reason for thinking that, since bone shows variations during its long biological history, teeth may also present similar analogous variations. 


\section{CHAPTER III}

\section{MATERIALS AND METHODS}

\section{$\underline{\text { Sample Selection }}$}

This was a retrospective mixed longitudinal study of panoramic radiographs taken by pediatric dentists for diagnostic purposes. The three selection criteria consisted of: (1) The children were enrolled at St. Jude Research Hospital, Memphis, Tennessee between August 12, 1982 and March 24, 2000 with a diagnosis of ALL. (2) Patients needed to have at least two panoramic radiographs separated by at least 22 months. (3) The children were between 4 and 10 years of age at the time of the first radiograph. Institutional Review Board approval was obtained and the examiner was certified to access the records and obtain all the necessary data.

\section{$\underline{\text { Sample Distribution }}$}

ALL is characterized by childhood onset, as is seen in the present sample. There were 72 subjects in the present study ( 31 girls and 41 boys). Mean chronological age at diagnosis of ALL was 6.76 years in both sexes (overall $\mathrm{sd}=$ 2.11 years), with a range of 3.1 to 10.5 years. 
Panoramic radiographs (Graber 1967) were taken of these children by the staff pediatric dentist as indicated for routine restorative dental work. As such, panoramic radiographs were not taken at any set interval, and the number of radiographs varied among the children. A total of 190 panoramic radiographs were available for the 72 children studied here. The distribution of radiographs was: 6 children had just one radiograph; 33 children had 2 radiographs; 20 children had 3 radiographs; 9 children had 4 radiographs; 2 children had 5 radiographs; and 2 children had 6 radiographs. There was a problem in the results of the records search using the selected criteria -6 of the children that were initially selected had only one radiograph available, however, they were included in the study.

At the time of diagnosis of ALL, patients are assigned to one of three risk categories based on the age at diagnosis, sex, type of leukemia, and prognosis. Cranial irradiation may be given to patients either with higher-risk leukemia or CNS leukemia at the time of diagnosis in addition to chemotherapy. Moreover, patients who did not respond favorably to the chemotherapy may have had irradiation added to their protocol. There were 25 of the 72 patients whose treatment protocol included the addition of radiation therapy.

Four of the 72 children were deceased from ALL when this study was performed. 


\section{$\underline{\text { Scoring Methods }}$}

The dental maturation of every tooth on each radiograph was examined and subsequently scored for the degree of crown-root formation. There were two types of panoramic radiographs examined: 143 conventional radiographs and 47 digital radiographs. The conventional radiographs were examined using a light box with no magnification. The digital radiographs were examined using the imaging software provided by St. Jude Research Hospital.

The Moorrees, Fanning and Hunt (1963) grading schemes were used in this study (Fig. 4). Single-rooted teeth are scored on a 13-grade scheme. Multirooted teeth are scored on a 14-grade scheme. The single difference between these two schemes is the addition of "cleft formation" for multi-rooted teeth. In our experience, this system provides finer discrimination than simpler morphological methods (Demirjian and Goldstein 1976), but the grades are not so numerous that confusion between adjacent grades is likely. Short descriptions of the grades are provided in Table 7. When the tooth development appears to fall between grades, the highest grade actually achieved was scored as suggested by Dahlberg and Menegaz-Bock (1958). All 32 permanent teeth were scored, so far as possible. Reasons for missing data include overlapping images of teeth, missing teeth, severely angulated or malpositioned teeth, unformed teeth, teeth diagnosis as being affected by irradiation, or underexposure or overexposure of the film. 
Table 7. Definitions of the tooth formation stages.

\begin{tabular}{|c|c|c|}
\hline $\begin{array}{l}\text { Single- } \\
\text { Rooted } \\
\text { Teeth }\end{array}$ & Definitions & $\begin{array}{l}\text { Multi- } \\
\text { Rooted } \\
\text { Teeth }\end{array}$ \\
\hline 1 & $\begin{array}{l}\text { Initial cusp formation: amelogenesis has begun } \\
\text { on the individual cusp tips. }\end{array}$ & 1 \\
\hline 2 & $\begin{array}{l}\text { Coalescence of cusps: centers of mineralization } \\
\text { are merged but the border is not everywhere } \\
\text { radiodense }\end{array}$ & 2 \\
\hline 3 & $\begin{array}{l}\text { Cusp outline complete: the coronal outline of } \\
\text { the tooth is mineralized. }\end{array}$ & 3 \\
\hline 4 & $\begin{array}{l}\text { Crown } 1 / 2 \text { formed: amelogenesis has } \\
\text { proceeded half way to the crown-root as } \\
\text { judged from morphology of the radiodense } \\
\text { portion }\end{array}$ & 4 \\
\hline 5 & Crown $3 / 4$ complete & 5 \\
\hline 6 & $\begin{array}{l}\text { Crown complete: morphologically, all the } \\
\text { crown has mineralized but root formation has } \\
\text { not begun. }\end{array}$ & 6 \\
\hline 7 & $\begin{array}{l}\text { Initial root formation: there is a trace of root } \\
\text { radiopacity below the crown outline. }\end{array}$ & 7 \\
\hline-- & $\begin{array}{l}\text { Initial cleft formation: mineralization is evident } \\
\text { in the interradicular area. }\end{array}$ & 8 \\
\hline 8 & $\begin{array}{l}\text { Root length } 1 / 4 \text { : the radiographic morphology } \\
\text { of the root is } 1 / 4 \text { its projected final size. }\end{array}$ & 9 \\
\hline 9 & Root length $1 / 2$ complete. & 10 \\
\hline 10 & Root length $3 / 4$ complete. & 11 \\
\hline 11 & Root length complete. & 12 \\
\hline 12 & $\begin{array}{l}\text { Apex half closed: the lateral borders of the root } \\
\text { tip become convex rather than tapered as } \\
\text { earlier. }\end{array}$ & 13 \\
\hline 13 & $\begin{array}{l}\text { Apical closure complete: size of the apical } \\
\text { foramen is reduced to its mature size. }\end{array}$ & 14 \\
\hline
\end{tabular}

Source: Harris EF, McKee JH. Tooth mineralization standards for blacks and whites from the middle southern United States. J Forensic Sci 1990;35:859-72. 
Comparisons were made to the sex-specific American white standards of Harris and McKee (1990). These data are based on children between the ages of about 3 and 13 years of age, which captures most intervals of permanent tooth formation. It does not, however, include the later formative stages of the third molar, so the standards were supplemented with data on mandibular third molars from Harris (1998). Dental age was computed for each permanent tooth that had not completed root apexification using the following method: (1) The stage of a tooth's formation was related along the 13-grade scheme for singlerooted teeth or the 14-grade scheme for multi-rooted teeth. (2) That grade was used to find the average chronological age (from the published standards) at which normal children of the appropriate sex achieved stage. This was termed the observed age. (3) The child's chronological age was used to find the stage of tooth formation expected if he or she were growing at a normal rate. The corresponding age (from the published norms) for that stage of formation was used as the expected age. (4) The difference between observed and expected age is, then, the extent to which ALL and subsequent treatment affect growth. This was done on a tooth-specific basis and then the results for all formative teeth were used to calculate the average, overall dental age.

Tooth crown and root mineralization is a continuous process beginning with mineralization of the cusp tips and progressing to root apex closure (Bhaskar 1991). This continuum can be artificially partitioned into several 
discrete "grades" in order to make it feasible to visually assess the degree of tooth completion. The grades - such as those devised by Nolla (1960), Moorrees et al. (1963), and Nielsen and Ravn (1976) - are ordinal-scale data; the intervals between the grades are unequal, but each grade of formation is morphologically discrete from others. In terms of chronological ages, there typically is about a one-year difference between one grade of formation and the next. Step 3 in the method of calculating dental age was used to account for these "jumps" in the data as tooth formation ages "lurch" from one stage to the next - rather than progressing smoothly and continuously if the data were ratio-scale.

\section{$\underline{\text { Assessment of Intraobserver Reliability }}$}

The value of the data collected in this study depends on the ability of the examiner to accurately distinguish among the grades of mineralization visualized for each tooth on the panoramic radiographs. Care was taken to ensure that the single examiner was experienced with the definitions of each grade and how to properly identify the grade obtained on the radiographs. Levesque and Demirjian (1980) reported on the inter-examiner variation in rating dental formation from radiographs. Their finding indicated that there was an inter-examiner reliability of $80 \%$ for molars and incisors; the reliability was lower for premolars (75\%) and canines (70\%). There is little research in the area of 
intra-examiner reliability to date, but some information is provided by Fanning (1961), Grøn (1962) and Harris and McKee (1990).

According to Fanning (1961:206-7) in her longitudinal study of tooth formation and root resorption certain stages could be graded with reasonable accuracy. Of note, her elaborate system contained more grades than used in the present study, and, indeed, Fanning deleted the more ambiguous grades to arrive at the grading system used here (Table 7).

Although the interpretation of dental development from radiographs is subjective, certain stages such as follicle and early crown formation, crown completion, initial root formation, and its apical closure could be evaluated accurately and served as a check in the assessment. Since the entire chronology was recorded in the longitudinal study, the stages of tooth formation or root resorption could be assigned with reasonable accuracy. Comparisons of developmental ratings with earlier and later films of the same tooth assisted in the assessment of the intermediate stages of crown and root formation that were the most difficult to evaluate.

Test for accuracy in assessment were made by independent ratings of incisor and premolar teeth in ten males. In 972 assessments, complete agreement occurred in $73 \%$ and disagreement of no more than one full stage in $27 \%$.

It can be concluded that one examiner can accurately assign the correct stages of tooth mineralization with adequate accuracy. It should be noted that only teeth imaged with good radiographic quality, not obstructed by superimposed bony structures or malpositioned enable the observer to obtain an accurate grading of the correct stage of mineralization. 
In Grøn's study (1962), 60 intraoral radiographs representing different teeth at different stages of formation were rated independently by two other observers who had no previous experience in assessing stages of root formation. Disagreement between these observers was never greater than one stage. Differences occurred in 2 out of 25 instances, except for the maxillary central incisor, where differences occurred in $50 \%$ of the double determinations. The evaluation of tooth development in this cross-sectional study was critical because the majority of the teeth had attained either one-half or three-quarters of their root length. In the absence of longitudinal records facilitating assessment, a test was made to determine the reliability of distinguishing between two stages of root development that were not far apart. Therefore, the formation of all teeth studied was rated twice by the author with an interval of at least 3 months. In approximately $10 \%$ of the two assessments the differences in the total material (omitting central incisors) never exceeded one stage. However, for both the maxillary and the mandibular central incisors, there was agreement in only $75 \%$ of the repeated assessments, but with the differences in assessment being still only one stage apart.

Harris and McKee (1990) reported a 96\% agreement in double determinations on 80 cases in their study, with no difference exceeding one stage.

The present study is in agreement with these previous reports of high repeatability. In $95 \%$ of the cases (8/160 teeth), the two assessments were in 
agreement, and of those eight, four were reported to be unscorable due to poor image quality, on the second assessment.

\section{Appropriateness of Tooth Formation Standards}

It is necessary to compare the tempos of crown-root formation in children with ALL against normative growing children in order to assess whether treatment impacts the rates of dental maturation. This creates some issues because (A) these children with ALL are of numerous ethnic and racial backgrounds and (B) this study is retrospective, so there is no opportunity to match children who are disease-free to those with ALL. Ideally, each participant with ALL could be matched with one or more siblings examined at the same chronological age (and adjusted for sex difference in growth tempos; (Demirjian 1978). This would match the children with ALL for race, ethnic differences and, importantly, make an effort at controlling for familial differences in the tempos of dental maturation. It also would match the children for cultural and socioeconomic parameters.

This desired design was not possible and, in fact, there are strikingly few "standards" of normative tooth formation available in the literature, and almost all of them are based on the development of caucasian population samples (e.g., Moorrees et al. 1963; Harris and McKee 1990; Haavikko 1974; Demirjian et al. 1973). Of these, the data from Harris and McKee (1990) seem the most 
appropriate inasmuch as (A) they are the most recent (from a cohort that grew up in the 1980s) and closest temporally to the subjects with ALL; (B) they were collected from a broad geographic region in the Southern United States, which may make them somewhat more generalized than studies of more localized groups; (C) they reflect a broader range of socioeconomic strata than some other previously published studies (e.g., Moorrees et al. 1963); and (D) we had access to the raw data for statistical manipulations. The Harris-McKee standards probably are far from a perfect fit, especially for the several children with ALL from foreign countries, but they seem to constitute the best-available standards. The subset of American caucasian standards was used for all comparisons (Harris and McKee also reported data for American Blacks).

It is important to recognize that tempos of development vary with a group's stages of growth (Nykanen et al. 1998; Farah et al. 1999; Olze et al. 2003; Liversidge 1999; Liversidge et al. 1999; Teivens and Mornstad 2001). It is unlikely that the growth tempos of two groups will simply parallel one another throughout childhood and adolescence. For present purposes, comparisons are limited to the start of treatment. The broad question is whether the HarrisMcKee standards for dental development are appropriate to the manner in which the sample with ALL matured? Once treatment for ALL was initiated, however, it was anticipated that the slowing effects would significantly impact the pretreatment tempos. At the start of treatment, the assumption is that DA-CA 
would be centered on zero. This assumes that ALL in itself does not alter the tempo of dental maturation. Analogously, there is some evidence that stature is somewhat greater in children with ALL at the time of diagnosis. Broomhall et al. (1983) performed a retrospective study of 236 children with ALL from the United Kingdom and found them to be significantly taller than their reference standards. There was no difference between the sexes, nor was there a difference between those older and younger than the median age. Griffin and Wadsworth (1980) also found the height at diagnosis to be greater than the reference sample in their study of 65 patients diagnosed with ALL. In contrast, other studies found no difference in height between children with ALL at the time of diagnosis and normal children (e.g., Bessho 1986; Holm e. al. 1994)

Table 8 lists the results of univariate one-sample t-tests of whether DA-CA differed significantly from zero. That is, if the Harris-McKee (1990) standards are representative of the manner in which the children with ALL were growing, then the distribution of DA-CA would be centered on zero. Rather arbitrarily, those dental examinations (panoramic radiographs) taken within 1.0 year of the onset of treatment for ALL were assessed. The two competing considerations with this analysis are (1) one would like to increase sample sizes (and, thus, statistical power) by including more scores of tooth development but 
Table 8. One-sample t-tests of DA-CA based on tooth formation stages of patients within 1.0 year of diagnosis for ALL. ${ }^{1}$

\begin{tabular}{rrrrrrc}
\hline Tooth & Mean & SD & SEM & $\mathrm{n}$ & $\mathrm{t}$-test & Prob > $|\mathrm{t}|$ \\
\hline C & -0.31 & 1.06 & 0.19 & 31 & -1.64 & 0.1096 \\
P1 & -0.95 & 1.34 & 0.95 & 2 & -1.00 & $(0.5000)$ \\
P2 & -1.07 & 0.15 & 0.09 & 3 & -12.10 & $(0.0068)$ \\
M1 & -0.23 & 0.98 & 0.16 & 36 & -1.39 & 0.1725 \\
M2 & 0.28 & 1.00 & 0.14 & 48 & 1.99 & 0.0523 \\
M3 & 1.13 & 0.84 & 0.42 & 4 & 2.68 & $0.0748)$ \\
& & & Mandible & & \\
C & -0.45 & 1.14 & 0.17 & 47 & -2.73 & 0.0089 \\
P1 & 0.36 & 1.04 & 0.14 & 52 & 2.49 & 0.0159 \\
P2 & 0.36 & 0.97 & 0.14 & 46 & 2.51 & 0.0156 \\
M1 & -0.40 & 1.03 & 0.15 & 44 & -2.57 & 0.0137 \\
M2 & 0.21 & 1.10 & 0.16 & 46 & 1.31 & 0.1958 \\
M3 & 1.13 & 0.90 & 0.29 & 10 & 3.96 & 0.0033 \\
& \multicolumn{7}{c}{ Arithmetic Average, All Teeth } & & \\
All teeth & 0.05 & 0.76 & 0.11 & 52 & 0.50 & 0.6200 \\
\hline
\end{tabular}

${ }^{1}$ Just teeth in the right jaws were used here. P-values based on unreliably small sample sizes are listed in parentheses. 
(2) there is some unknown point after the onset of treatment at which the slowing effects of treatment influence tooth development. A cut-off of 1.0 year seemed to be reasonable (other intervals were tested with comparable results).

Ignoring the three maxillary teeth with unreliably small sample sizes $(<5)$, it is striking that several differences are statistically significant (and two others are suggestive, with $0.10>\mathrm{P}>0.05$ ). Of note, too, the sign of the DA-CA differs among the tooth types. A negative value for DA-CA means that DA is less mature than expected from the Harris-McKee standards. Conversely, a positive DA-CA value indicates that the dental age of the tooth is advanced compared to the Harris-McKee standards developed from American whites.

This mix of positive and negative values is not surprising, but it means that the samples of children with ALL examined here exhibits a different pattern of tooth development than in the Harris-McKee standards (see, e.g., Tompkins 1996a,b). The significant differences for at least 5 of the 12 comparisons (Table 8) also is of concern, particularly since the DA-CA differences range up to one year (for mandibular M3) at the start of treatment.

Alternatively, there is no solution to the observed discrepancies, especially the mixture of positive and negative differences among the tooth-specific tempos of development. In fact, we explored the use of other dental "standards" notably the works of Moorrees et al. (1963) and of Demirjian et al. (1973) - but the 
DA-CA "discrepancies" were not resolved: Changing the standards merely altered the mixture of positive and negative differences.

Another solution might be to numerically adjust the data so, at least at the start of treatment, the two samples (the children with ALL and the published norms) would be comparable. For instance, mean DA-CA for the mandibular canine is -0.46 years. One approach would be to adjust the dental age of all mandibular canine scores by adding 0.46 years, so the resulting mean would be zero. There are at least two reasons not to do this. One, the data in Table 8 are composites of different children with teeth at different stages of formation. There is no practical way to adjust the mean ages - let alone the variabilities - of all stages of all teeth. Secondly, the results in Table 8 are in effect a snap-shot of the tempos of tooth development right after the age at diagnosis. There is no reason to suppose that the relation between the tempos of tooth formation between the two samples remained at all parallel with one another with advancing age.

Consequently, while noting the several statistically significant differences at the start of treatment for ALL, we made no adjustment in the data. Of note, the tooth-specific differences nullify one another: Inspection of DA-CA averaged over all scorable teeth (Table 8) shows that dental age for the individual is insignificantly different vis-à-vis the Harris-McKee standards. 


\section{Statistical Methods}

Data were compiled using Microsoft Excel (Microsoft Corporation, Redmond, WA). Data included the child's sex, birthdate, the age at diagnosis of ALL (which we used as being the age at the onset of treatment), and the date of each panoramic radiograph. Information also was available on the dates, if any, and dosages of irradiation received. The tooth formation scores from each panoramic radiograph also were entered. A separate custom-written program was used to calculate the dental age of each tooth as described earlier, and these data were merged into the Excel file.

Statistics all were generated using JMP version 5.0.02 (SAS Institute Inc., Cary, NC), which imports an Excel file directly.

The data treated here are all parametric continuous variables, and the conventional parametric tests are described in most statistical textbooks (e.g., Winer et al. 1991; Sokal and Rohlf 1995). The three major tests used here are (1) linear regression analysis, (2) factorial analysis of variance, and (3) Pearson product-moment correlation coefficients. The nature of the data prevented the use of a repeated measure model to follow the progress of DA through the course of treatment. Some use was made of a pairing-design t-test to test for intra-individual changes in DA-CA.

All tests were two-tail, and the conventional level of statistical significance $($ alpha $=0.05)$ was used. 


\section{CHAPTER IV}

\section{RESULTS}

The dental age of each scorable permanent tooth was calculated using the American-white standards of Harris and McKee (1990). As a preliminary, exploratory step, "average" dental age was calculated by (A) determining the difference between dental age and chronological age (DA-CA) for each tooth and, then, (B) taking the arithmetic average across all teeth. A potential issue is that there is appreciable redundant information in this average dental age (Demirjian et al. 1973), but it provides a practical starting point for the analysis. Regressing DA-CA on chronological age (using all available panoramic examinations) produces the scattergram in Figure 8. If dental age were unaffected by the treatment for ALL, the distribution of DA-CA would be centered on zero independent of chronological age. Instead, there is a highly significant negative association between $\mathrm{CA}$ and DA-CA $(b=-0.07 ; \mathrm{t}=2.91 ; \mathrm{P}<$ 0.0041) such that the older the individual, the more delayed DA is relative to CA. The effect is modest, however, explaining just $4 \%$ of the variance in DA-CA, and a geometric model did not improve the fit.

The dependency between age and DA - CA also was tested by sex. Results for females ( $\mathrm{n}=76$ films) were not significant $(\mathrm{b}=-0.02 ; \mathrm{t}=0.51 ; \mathrm{P}=$ 


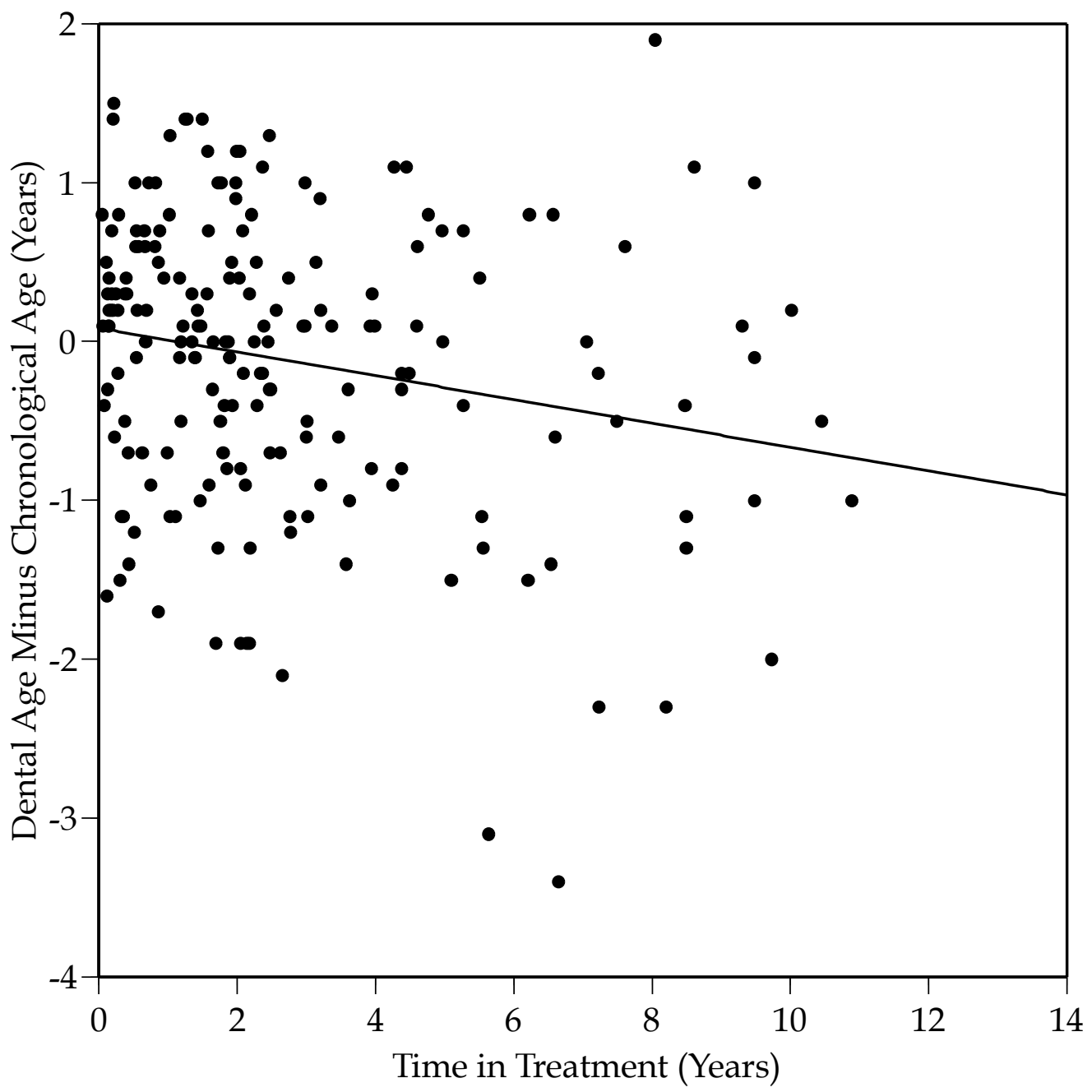

Fig. 8. Scatterplot showing the negative linear dependency between time in treatment and the degree of dental developmental delay, measured as DA-CA. The plot is for all scorable teeth from all radiographs of all children in this study treated for ALL. 
0.6115). In contrast, it can be seen that the data for the boys carried the sexespooled analysis (above) to significant, since the regression coefficient is -0.13 ( $\mathrm{df}$ $=113 ; \mathrm{t}=3.52 ; \mathrm{P}=0.0006)$. In other words, for the boys, DA drops an average of 0.13 years farther below CA for each year into treatment for ALL. This depression cannot go on indefinitely because physiological age ultimately achieves maturity in all subjects, but during this interval up to about a decade after the onset of treatment, there is a progressively increasing depression of DA behind CA. Test of a quadratic model for these males did not improve the model.

\section{$\underline{\text { Dental Age at Diagnosis }}$}

Our perspective in this study is that changes in dental age are iatrogenic responses to treatment, not to the disease itself. A key measure in support of this is that dental age is age-appropriate (i.e., $\mathrm{DA}=\mathrm{CA}$, so $\mathrm{DA}-\mathrm{CA}=0$ ) at the start of treatment. None of these children have dental radiographs prior to diagnosis and the onset of treatment, at least in the records at St. Jude Children's Hospital. Many were fairly young and probably had not yet had a routine pediatric dental radiograph. We tested the assumption of $\mathrm{DA}=\mathrm{CA}$ by selecting just those radiographs taken within a half-year of the onset of treatment. There were 37 radiographs, and we averaged DA - CA over all scorable teeth. The average DA 
- CA was 0.004 years $(s d=0.767)$. A one-sample $t$-test shows that there is no suggestion that $\mathrm{DA}$ is effected $(\mathrm{t}=0.03 ; \mathrm{df}=36 ; \mathrm{P}=0.51)$.

For completeness, this test of DA $=\mathrm{CA}$ was repeated by sex. There was no difference in DA - CA and the data for both sexes were insignificantly different from zero. In sum, one can infer that neither sex was demonstrably affected by ALL in terms of dental development.

Similarly, the sample was partitioned into those who were treated (A) with chemotherapy alone versus (B) with chemotherapy plus cranial irradiation. Again, there was no difference between DA - CA at the onset of treatment, and both means were insignificantly different from zero at the start of treatment. This suggests that criteria other than DA are involved in determining which children received cranial irradiation.

In sum, it seems safe to conclude that ALL itself does not affect DA - at least not around the time of diagnosis of this acute disease. Subsequently, the iatrogenic effects of treatment cannot be separated from any changes in DA - CA due to the disease per se.

\section{Individual Responses to Treatment}

In addition to the standard statistical treatment of these data, it is informative to inspect the responses of each child's dental maturation to the 
stressor of treatment. One perspective is that perusal of the data should both suggest and confirm the statistical relationships seen for the sample as a whole.

There are two appendices of graphs at the end of this work. Appendix I is a set of bivariate graphs, one for each child, plotting dental age against chronological age. Each plot shows the chronological age at diagnosis for ALL, which is virtually coincident with the onset of chemotherapy. The number of dental ages on a graph depends on how many radiographs were available for that child.

Notice the isochron line on each graph, which is the line where CA and DA are coincident. So, if a child's DA is equal to his CA, dental age will be on the isochron line. If DA falls behind CA as often happens because treatment depresses the tempo of dental maturation, then the symbol will be below the isochron line.

The second set of graphs (Appendix II) presents the same dental ages in a complementary format. Here, the difference between DA and CA (i.e., DA-CA) is plotted for each tooth. Each graph presents the information collected from one child, and different symbols code for the panoramic radiograph readings, showing the child's CA at each examination. The typical graph shows that all of the tooth types respond roughly the same and that DA-CA becomes a more negative value the farther the child is from the onset of treatment. 


\section{Left-Right Symmetry}

One supposes that treatment for ALL affects the dentition symmetrically. This seems true for those children treated with chemotherapy as well as those who also received radiation therapy.

Tests for systematic asymmetry involved assessing DA-CA on a toothspecific basis using paired t-tests. That is, for each tooth type, the left and right values for DA-CA were compared in a pairwise fashion. A significant difference would indicate that a tooth type on one side of the jaw was preferentially at risk. Results are listed in Table 9. Interest was on whether there was enough left-right difference in the data to warrant including measurements from both sides. Alternatively, one supposes that information on the left and right quadrants are so highly correlated that using both sides is redundant and duplicative.

There are 12 comparisons because the maxillary and mandibular incisors were seldom scorable because they form early and their development is less effected by therapy. Notice too that sample sizes (number of scorable pairs) tend to be small for the maxillary teeth. This is because details of the tooth structures often are obscured by the complexity of the overlying bony structures.

Of the 12 comparisons, only the maxillary canines achieved statistical significance, where some teeth on the left side were more severely affected (i.e., greater DA-CA) than on the corresponding right side. We attribute little importance to this finding since it is an isolated finding and only marginally 


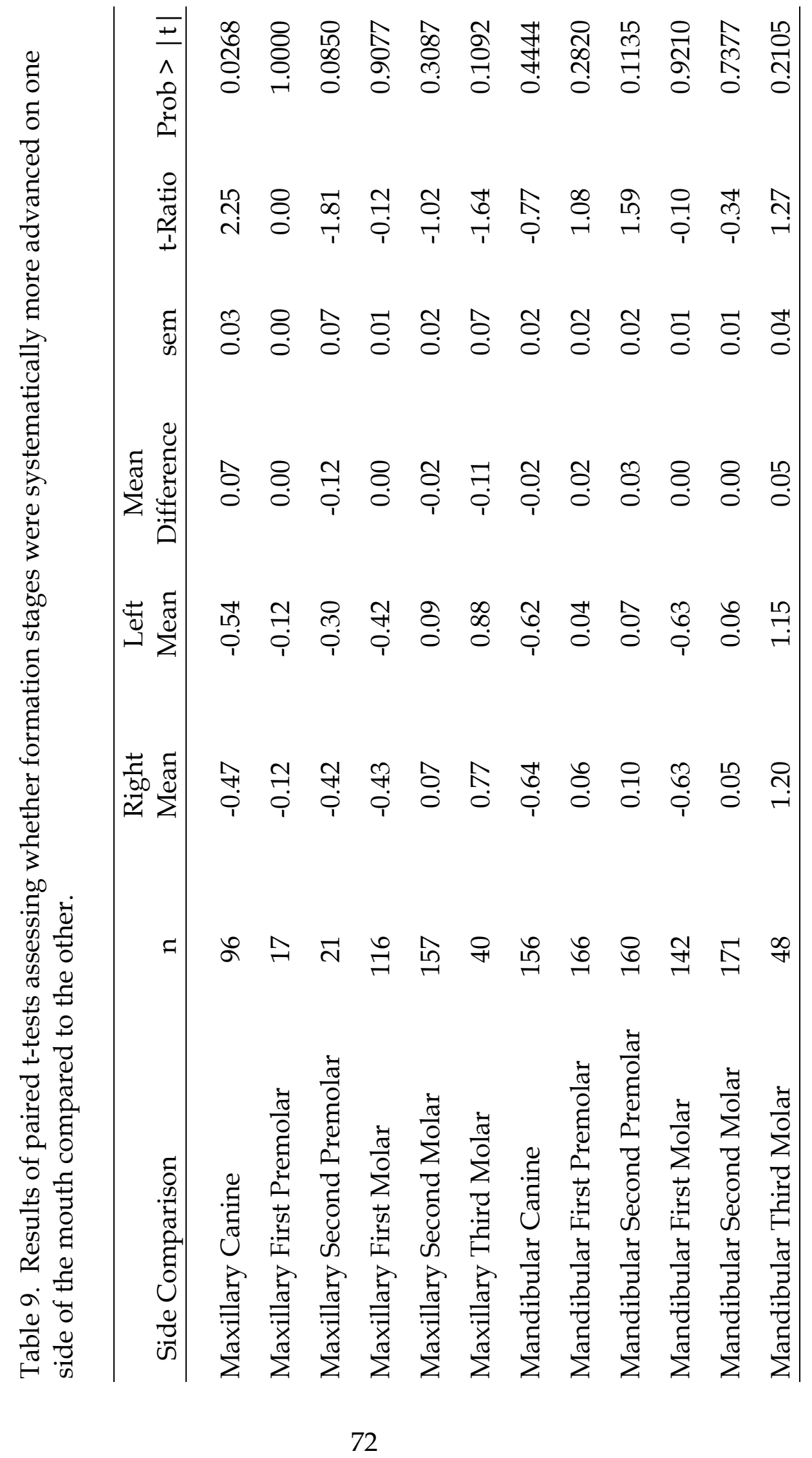


significant statistically $(\mathrm{P}=0.03)$. Moreover, inspection showed that the difference was due to just 3 cases out of the sample size of 95. Consequently, we ignored the redundancy of the data on homologous left and right teeth. The considerable redundancy in information also is shown by the results of calculating the correlation in DA-CA between homologous left and right teeth (Table 10). All 12 correlations are positive and in excess of 0.9. Subsequent analysis was therefore limited to the individual's right side.

\section{$\underline{\text { Tooth Type and DA-CA }}$}

Two related issues are described in this section. First, it is of interest whether DA-CA calculated from the various tooth types are positively interrelated or, alternatively, different tooth types - that develop at different ages (Moorrees et al. 1963) and that exhibit different variabilities (Dahlberg 1965) exhibit largely independent responses to the stress of treatment for ALL. The second, complementary issue is how labile the development of each tooth type may be to the stresses of ALL treatment. That is, does ALL produce a statistically discernible response in DA-CA for each tooth type? Alternatively, are some tooth types more resilient to these stresses such that the effects of treatment differ depending on which tooth type or set of teeth is examined?

To address the first question regarding the similarities of tooth formation to the stress of ALL, Pearson product-moment correlation coefficients were 
Table 10. Pearson product-moment correlation coefficients between DA-CA between homologous left and right teeth.

\begin{tabular}{ccccc}
\hline $\begin{array}{c}\text { Right } \\
\text { Variable }\end{array}$ & $\begin{array}{c}\text { Left } \\
\text { Variable }\end{array}$ & $\begin{array}{c}\text { Correlation } \\
\text { Coefficent }\end{array}$ & $\mathrm{n}$ & P-Value \\
\hline UC & UC & 0.9715 & 96 & 0.0000 \\
UP1 & UP1 & 1.0000 & 17 & 0.0000 \\
UP2 & UP2 & 0.9739 & 21 & 0.0000 \\
UM1 & UM1 & 0.9886 & 116 & 0.0000 \\
UM2 & UM2 & 0.9802 & 157 & 0.0000 \\
UM3 & UM3 & 0.9439 & 40 & 0.0000 \\
& & & & \\
LC & LC & 0.9708 & 156 & 0.0000 \\
LP1 & LP1 & 0.9825 & 167 & 0.0000 \\
LP2 & LP2 & 0.9766 & 160 & 0.0000 \\
LM1 & LM1 & 0.9888 & 142 & 0.0000 \\
LM2 & LM2 & 0.9948 & 171 & 0.0000 \\
LM3 & LM3 & 0.9719 & 48 & 0.0000 \\
\hline
\end{tabular}


calculated among all teeth on the right side of the dental arches. There are 12 tooth types because the early-forming incisors were omitted. The correlations were computed in a pairwise fashion to maximize sample sizes. Results (Table 11) show that all of the correlations are positive and the majority are significant statistically $(\mathrm{P}<0.05)$. Those associations that are not significant seem to be tied to small sample sizes; this always involves a maxillary tooth, where sample sizes are compromised by difficulties in viewing the tooth because of overlying bony structures. Taking the small sample sizes into account, there is no evidence that the sign or the magnitude of the correlations differs among the tooth types. Keeping in mind that different teeth undergo crown-root mineralization at different ages, there does not seem to be any differences in the strengths of the associations by tooth type.

Results of the complementary issue are shown in Table 12 . Here the question is whether each of the 12 tooth types (i.e., examining permanent teeth just on the right side of the mouth and omitting the incisors) exhibits a statistically significant association between (A) time in treatment for ALL and (B) the variable DA-CA. Treatment for ALL uses powerful chemotherapy with or without cranial irradiation. This is a substantial stressor to all growth processes. On a tooth-by-tooth basis, is there evidence that treatment discernibly impacts the growth rate? Here, the tempo of growth is measured by DA-CA. There are 
Table 11. Pearson product-moment correlation coefficients among DA-CA of the 12 tooth types on the right side of the mouth.

\begin{tabular}{rrrrc}
\hline Variable & Variable & $\begin{array}{c}\text { Correlation } \\
\text { Coefficient }\end{array}$ & $\mathrm{n}$ & P-Value \\
\hline RUP1 & RUC & 0.644 & 16 & 0.0071 \\
RUP2 & RUC & 0.609 & 19 & 0.0056 \\
RUP2 & RUP1 & 0.501 & 15 & 0.0571 \\
RUM1 & RUC & 0.534 & 65 & 0.0000 \\
RUM1 & RUP1 & 0.540 & 12 & 0.0700 \\
RUM1 & RUP2 & 0.680 & 15 & 0.0053 \\
RUM2 & RUC & 0.450 & 85 & 0.0000 \\
RUM2 & RUP1 & 0.563 & 15 & 0.0290 \\
RUM2 & RUP2 & 0.424 & 19 & 0.0704 \\
RUM2 & RUM1 & 0.461 & 111 & 0.0000 \\
RUM3 & RUC & 0.222 & 24 & 0.2983 \\
RUM3 & RUP1 & 0.496 & 8 & 0.2116 \\
RUM3 & RUP2 & 0.454 & 8 & 0.2581 \\
RUM3 & RUM1 & 0.314 & 13 & 0.2956 \\
RUM3 & RUM2 & 0.400 & 30 & 0.0288 \\
RLC & RUC & 0.618 & 86 & 0.0000 \\
RLC & RUP1 & 0.517 & 14 & 0.0581 \\
RLC & RUP2 & 0.339 & 19 & 0.1563 \\
RLC & RUM1 & 0.521 & 106 & 0.0000 \\
RLC & RUM2 & 0.397 & 140 & 0.0000 \\
RLC & RUM3 & 0.415 & 34 & 0.0146 \\
RLP1 & RUC & 0.685 & 90 & 0.0000 \\
RLP1 & RUP1 & 0.809 & 17 & 0.0001 \\
RLP1 & RUP2 & 0.503 & 21 & 0.0201 \\
RLP1 & RUM1 & 0.505 & 112 & 0.0000 \\
RLP1 & RUM2 & 0.528 & 147 & 0.0000 \\
RLP1 & RUM3 & 0.398 & 33 & 0.0219 \\
RLP1 & RLC & 0.600 & 150 & 0.0000 \\
RLP2 & RUC & 0.567 & 84 & 0.0000 \\
RLP2 & RUP1 & 0.543 & 16 & 0.0299 \\
RLP2 & RUP2 & 0.774 & 19 & 0.0001 \\
RLP2 & RUM1 & 0.562 & 104 & 0.0000 \\
RLP2 & RUM2 & 0.576 & 140 & 0.0000 \\
& & & &
\end{tabular}

Continued 
Table 11. Continued.

\begin{tabular}{|c|c|c|c|c|}
\hline Variable & Variable & $\begin{array}{l}\text { Correlation } \\
\text { Coefficient }\end{array}$ & $\mathrm{n}$ & P-Value \\
\hline RLP2 & RUM3 & 0.296 & 35 & 0.0845 \\
\hline RLP2 & RLC & 0.567 & 142 & 0.0000 \\
\hline RLP2 & RLP1 & 0.835 & 155 & 0.0000 \\
\hline RLM1 & RUC & 0.591 & 76 & 0.0000 \\
\hline RLM1 & RUP1 & 0.527 & 12 & 0.0784 \\
\hline RLM1 & RUP2 & 0.608 & 15 & 0.0162 \\
\hline RLM1 & RUM1 & 0.919 & 112 & 0.0000 \\
\hline RLM1 & RUM2 & 0.423 & 126 & 0.0000 \\
\hline RLM1 & RUM3 & 0.345 & 15 & 0.2086 \\
\hline RLM1 & RLC & 0.605 & 126 & 0.0000 \\
\hline RLM1 & RLP1 & 0.578 & 137 & 0.0000 \\
\hline RLM1 & RLP2 & 0.581 & 128 & 0.0000 \\
\hline RLM2 & RUC & 0.573 & 89 & 0.0000 \\
\hline RLM2 & RUP1 & 0.471 & 15 & 0.0766 \\
\hline RLM2 & RUP2 & 0.581 & 19 & 0.0090 \\
\hline RLM2 & RUM1 & 0.567 & 106 & 0.0000 \\
\hline RLM2 & RUM2 & 0.748 & 145 & 0.0000 \\
\hline RLM2 & RUM3 & 0.391 & 38 & 0.0153 \\
\hline RLM2 & RLC & 0.398 & 145 & 0.0000 \\
\hline RLM2 & RLP1 & 0.636 & 157 & 0.0000 \\
\hline RLM2 & RLP2 & 0.646 & 155 & 0.0000 \\
\hline RLM2 & RLM1 & 0.512 & 131 & 0.0000 \\
\hline RLM3 & RUC & 0.281 & 28 & 0.1475 \\
\hline RLM3 & RUP1 & 0.690 & 6 & 0.1293 \\
\hline RLM3 & RUP2 & 0.511 & 6 & 0.2998 \\
\hline RLM3 & RUM1 & 0.243 & 17 & 0.3477 \\
\hline RLM3 & RUM2 & 0.270 & 36 & 0.1112 \\
\hline RLM3 & RUM3 & 0.800 & 33 & 0.0000 \\
\hline RLM3 & RLC & 0.309 & 36 & 0.0671 \\
\hline RLM3 & RLP1 & 0.275 & 38 & 0.0951 \\
\hline RLM3 & RLP2 & 0.193 & 42 & 0.2214 \\
\hline RLM3 & RLM1 & 0.178 & 21 & 0.4461 \\
\hline RLM3 & RLM2 & 0.442 & 46 & 0.0021 \\
\hline
\end{tabular}


Table 12. Resulting of testing for a statistically significant association between DA-CA against time in treatment on a tooth-specific basis.

Bivariate fit of maxillary canine by time in treatment.

\begin{tabular}{lrccc}
\hline \multicolumn{1}{c}{ Source } & df & Mean Square & F Ratio & Prob > F \\
\hline Model & 1 & 4.91 & 3.93 & 0.0504 \\
Error & 94 & 1.24 & & \\
C. Total & 95 & & & \\
\hline
\end{tabular}

Bivariate fit of maxillary first premolar by time in treatment.

\begin{tabular}{lrccc}
\hline \multicolumn{1}{c}{ Source } & df & Mean Square & F Ratio & Prob > F \\
\hline Model & 1 & 1.14 & 1.00 & 0.3329 \\
Error & 15 & 1.14 & & \\
C. Total & 16 & & & \\
\hline
\end{tabular}

Bivariate fit of maxillary second premolar by time in treatment.

\begin{tabular}{lrccc}
\hline \multicolumn{1}{c}{ Source } & df & Mean Square & F Ratio & Prob > F \\
\hline Model & 1 & 0.00 & 0.00 & 0.9745 \\
Error & 19 & 1.14 & & \\
C. Total & 20 & & & \\
\hline
\end{tabular}

Bivariate fit of maxillary first molar by time in treatment.

\begin{tabular}{lrccc}
\hline \multicolumn{1}{c}{ Source } & df & Mean Square & F Ratio & Prob $>$ F \\
\hline Model & 1 & 18.11 & 19.67 & $<0.0001$ \\
Error & 114 & 0.92 & & \\
C. Total & 115 & & & \\
\hline
\end{tabular}

Bivariate fit of maxillary second molar by time in treatment.

\begin{tabular}{lrccc}
\hline \multicolumn{1}{c}{ Source } & df & Mean Square & F Ratio & Prob > F \\
\hline Model & 1 & 1.43 & 1.27 & 0.2622 \\
Error & 156 & 1.13 & & \\
C. Total & 157 & & & \\
\hline
\end{tabular}

\section{Continued}




\section{Table 12. Continued}

Bivariate fit of maxillary third molar by time in treatment.

\begin{tabular}{lrccc}
\hline \multicolumn{1}{c}{ Source } & df & Mean Square & F Ratio & Prob > F \\
\hline Model & 1 & 10.67 & 8.18 & 0.0068 \\
Error & 39 & 1.30 & & \\
C. Total & 40 & & & \\
\hline
\end{tabular}

Bivariate fit of mandibular canine by time in treatment.

\begin{tabular}{lrccc}
\hline \multicolumn{1}{c}{ Source } & df & Mean Square & F Ratio & Prob > F \\
\hline Model & 1 & 17.94 & 13.57 & 0.0003 \\
Error & 154 & 1.32 & & \\
C. Total & 155 & & & \\
\hline
\end{tabular}

Bivariate fit of mandibular first premolar by time in treatment.

\begin{tabular}{lrccc}
\hline \multicolumn{1}{c}{ Source } & df & Mean Square & F Ratio & Prob > F \\
\hline Model & 1 & 16.15 & 12.94 & 0.0004 \\
Error & 165 & 1.25 & & \\
C. Total & 166 & & & \\
\hline
\end{tabular}

Bivariate fit of mandibular second premolar by time in treatment.

\begin{tabular}{lrccc}
\hline \multicolumn{1}{c}{ Source } & df & Mean Square & F Ratio & Prob $>$ F \\
\hline Model & 1 & 19.71 & 17.18 & $<0.0001$ \\
Error & 158 & 1.15 & & \\
C. Total & 159 & & & \\
\hline
\end{tabular}

Bivariate fit of mandibular first molar by time in treatment.

\begin{tabular}{lrccc}
\hline \multicolumn{1}{c}{ Source } & df & Mean Square & F Ratio & Prob $>$ F \\
\hline Model & 1 & 29.78 & 27.68 & $<0.0001$ \\
Error & 140 & 1.08 & & \\
C. Total & 141 & & &
\end{tabular}

Continued 


\section{Table 12. Continued}

Bivariate fit of mandibular second molar by time in treatment.

\begin{tabular}{lrccc}
\hline \multicolumn{1}{c}{ Source } & df & Mean Square & F Ratio & Prob > F \\
\hline Model & 1 & 6.94 & 5.85 & 0.0166 \\
Error & 169 & 1.19 & & \\
C. Total & 170 & & & \\
\hline
\end{tabular}

Bivariate fit of mandibular third molar by time in treatment.

\begin{tabular}{lrccc}
\hline \multicolumn{1}{c}{ Source } & df & Mean Square & F Ratio & Prob > F \\
\hline Model & 1 & 0.98 & 0.73 & 0.3984 \\
Error & 46 & 1.35 & & \\
C. Total & 47 & & & \\
\hline
\end{tabular}


12 analysis of variance tables in Table 12, one for each of the regression analysis testing the dependency of DA-CA on time in treatment.

Nine of the 12 tests are statistically significant, confirming a positive association between (A) how long the child has been in treatment for ALL (and, thereby, the duration of time and the extent of growth that has been impacted by treatment) and (B) the magnitude of DA-CA, which is a measure of how developmentally delayed the child is relative to his chronological age. Most of these associations are highly significant statistically.

It is worth evaluating these results in some detail. Figures 9 through 20 are scatterplots showing these associations, along with the least-squares regression line for each set of data. There is a significant association for the UC between time in treatment and DA-CA (Fig. 9). The vertical scatter seems to be centered on zero at the start of treatment, and the regression line slopes downward (i.e., increasing developmental delay) as time progresses $(\mathrm{P}=0.007)$.

In contrast, neither maxillary premolar's development is significantly associated with time in treatment (Figs. 10,11), but these may well be artifacts of small sample sizes. Sample sizes for UP1 and UP2 are just 20 and 25, respectively. Neither association is significant statistically (Table 12).

There is a more reasonable sample size for UM1 $(n=121)$, and here the association is highly significant (Fig. 12). Inspection shows, however, that the association probably is leveraged by cases with long time since treatment. The 


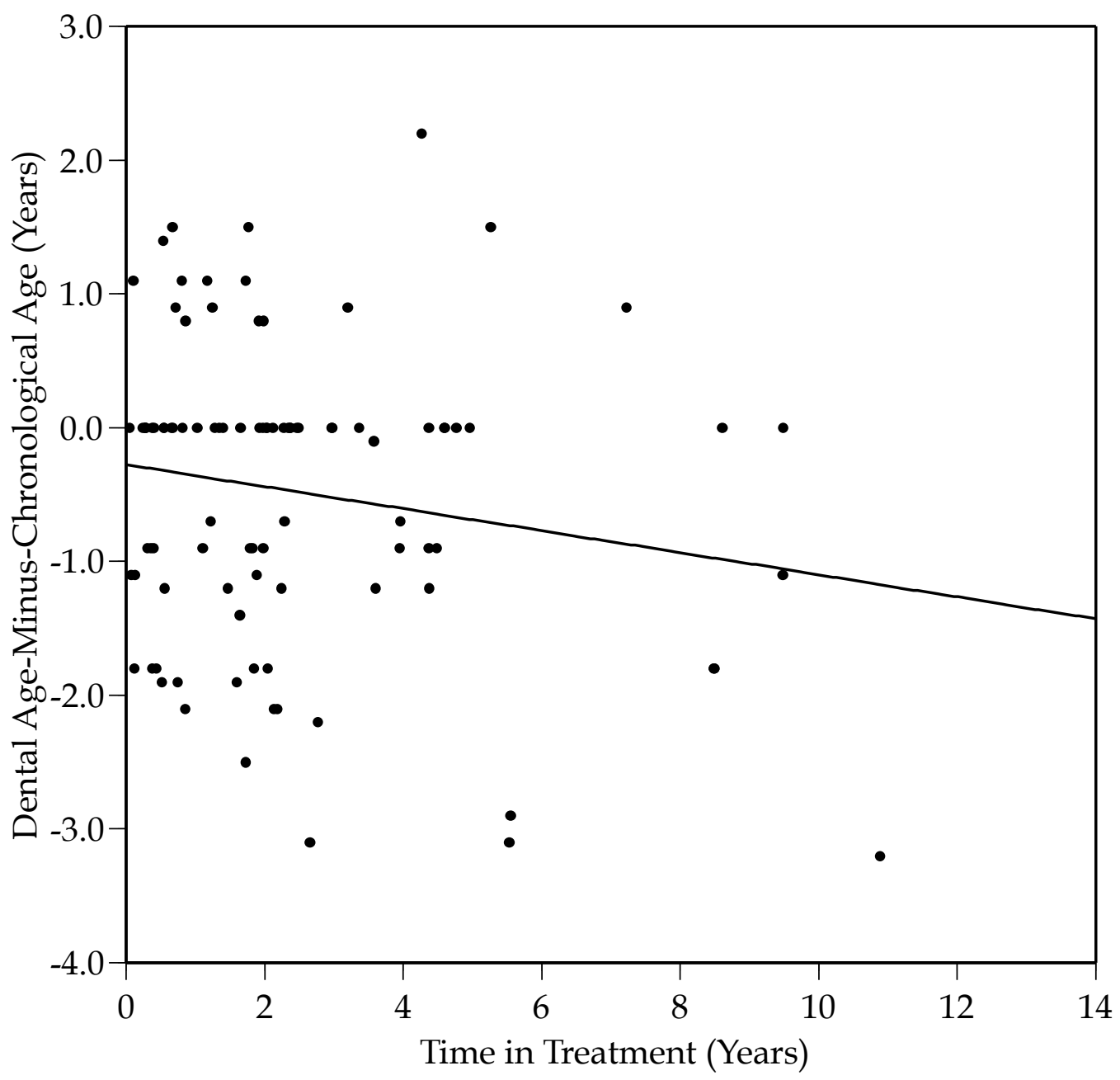

Fig. 9. Scatterplot between time in treatment on the horizontal axis and DA-CA on the vertical axis for the maxillary right canine. The least-squares regression line also is shown. 


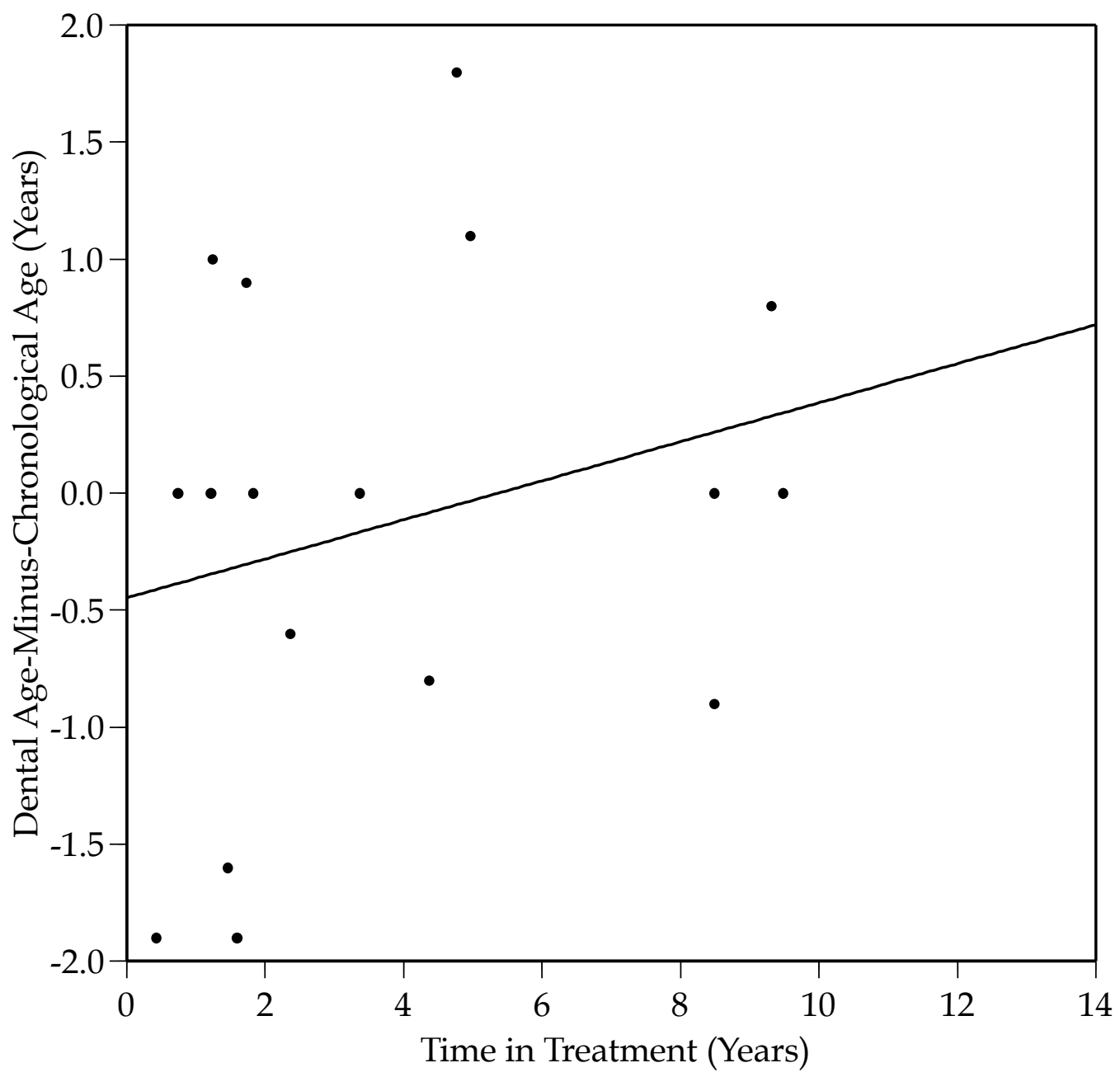

Fig. 10. Scatterplot between time in treatment on the horizontal axis and DA-CA on the vertical axis for the maxillary right first premolar. The least-squares regression line also is shown. 


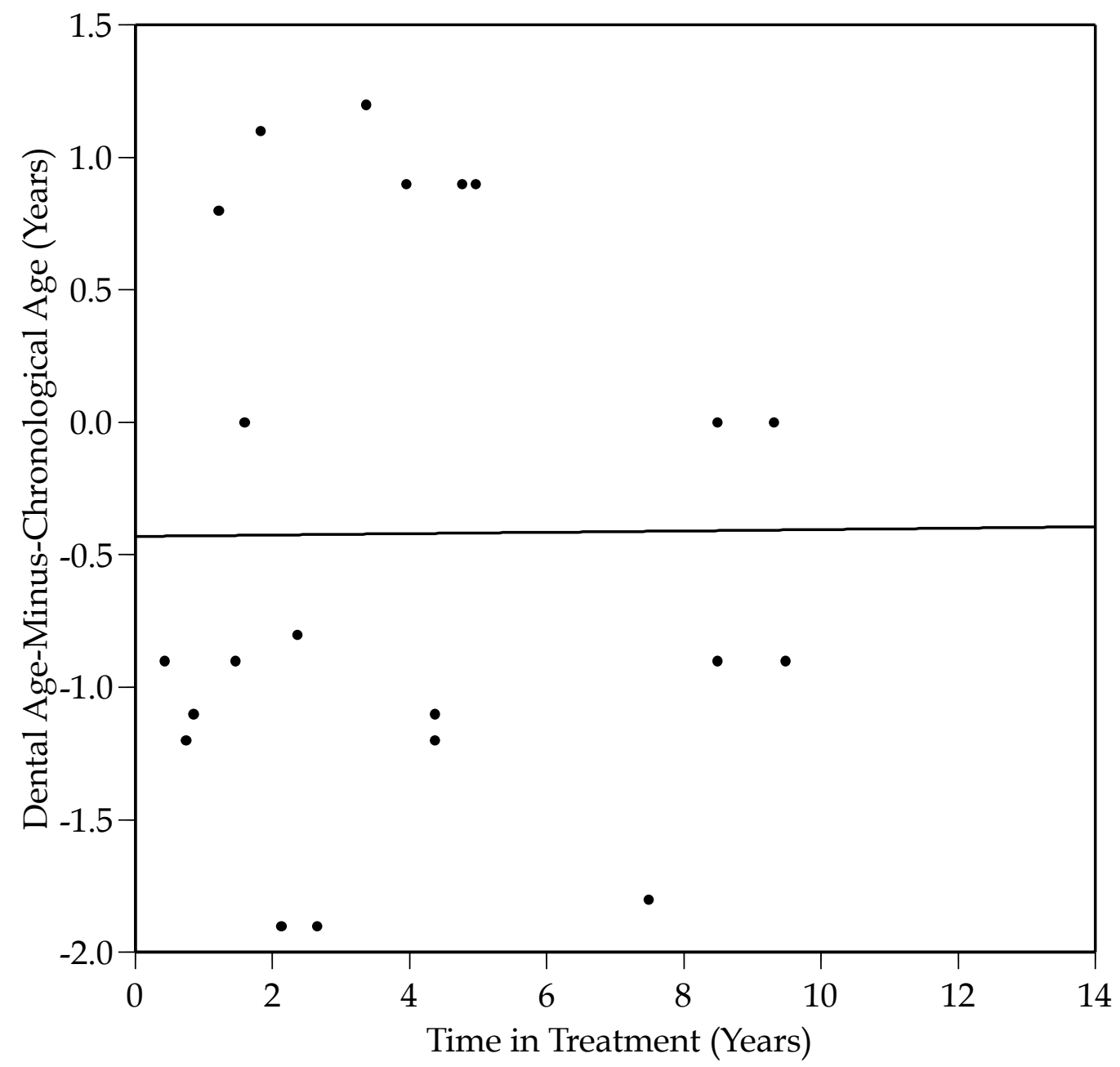

Fig. 11. Scatterplot between time in treatment on the horizontal axis and DA-CA on the vertical axis for the maxillary right second premolar. The least-squares regression line also is shown. 


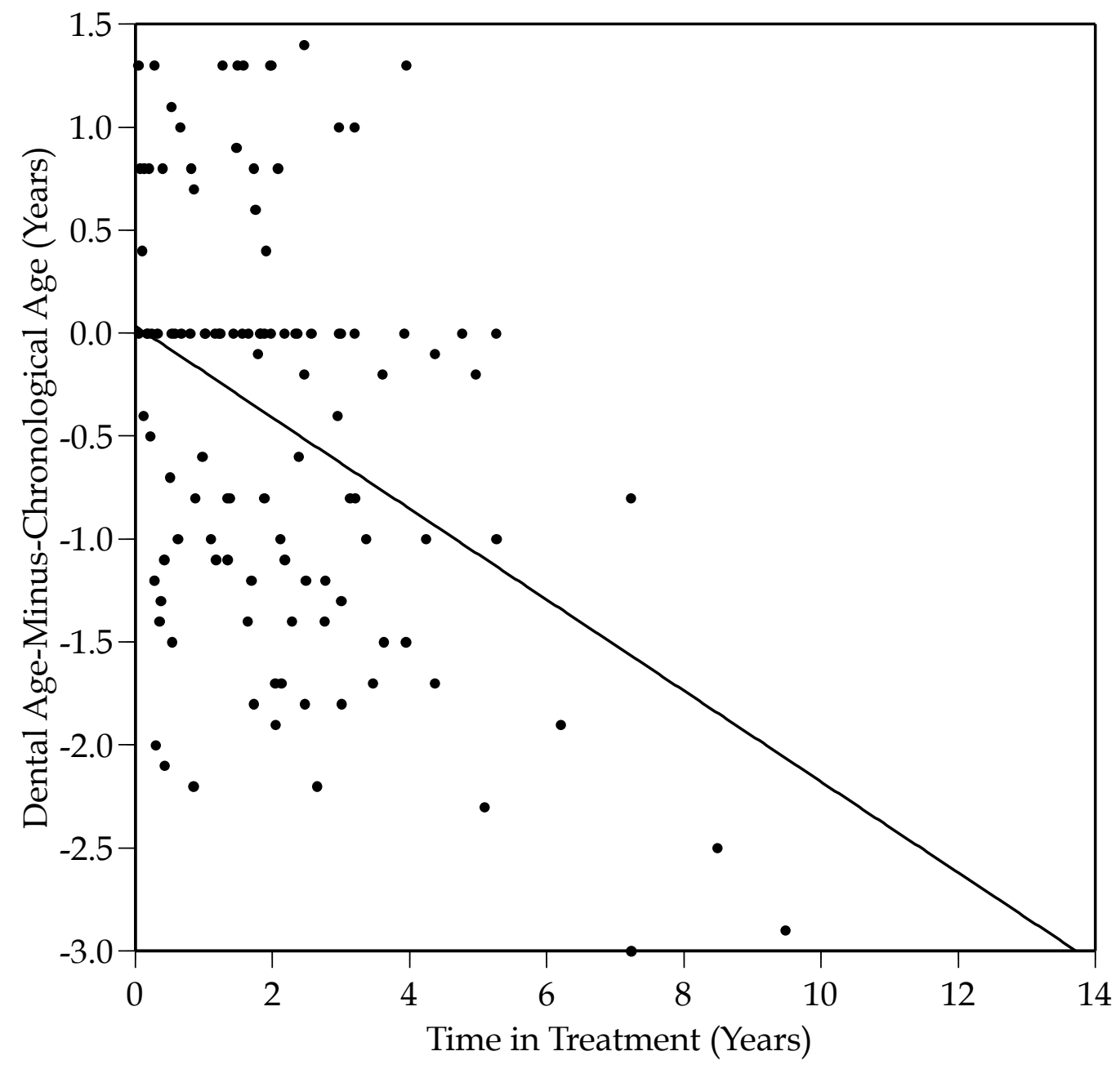

Fig. 12. Scatterplot between time in treatment on the horizontal axis and DA-CA on the vertical axis for the maxillary right first molar. The least-squares regression line also is shown. 


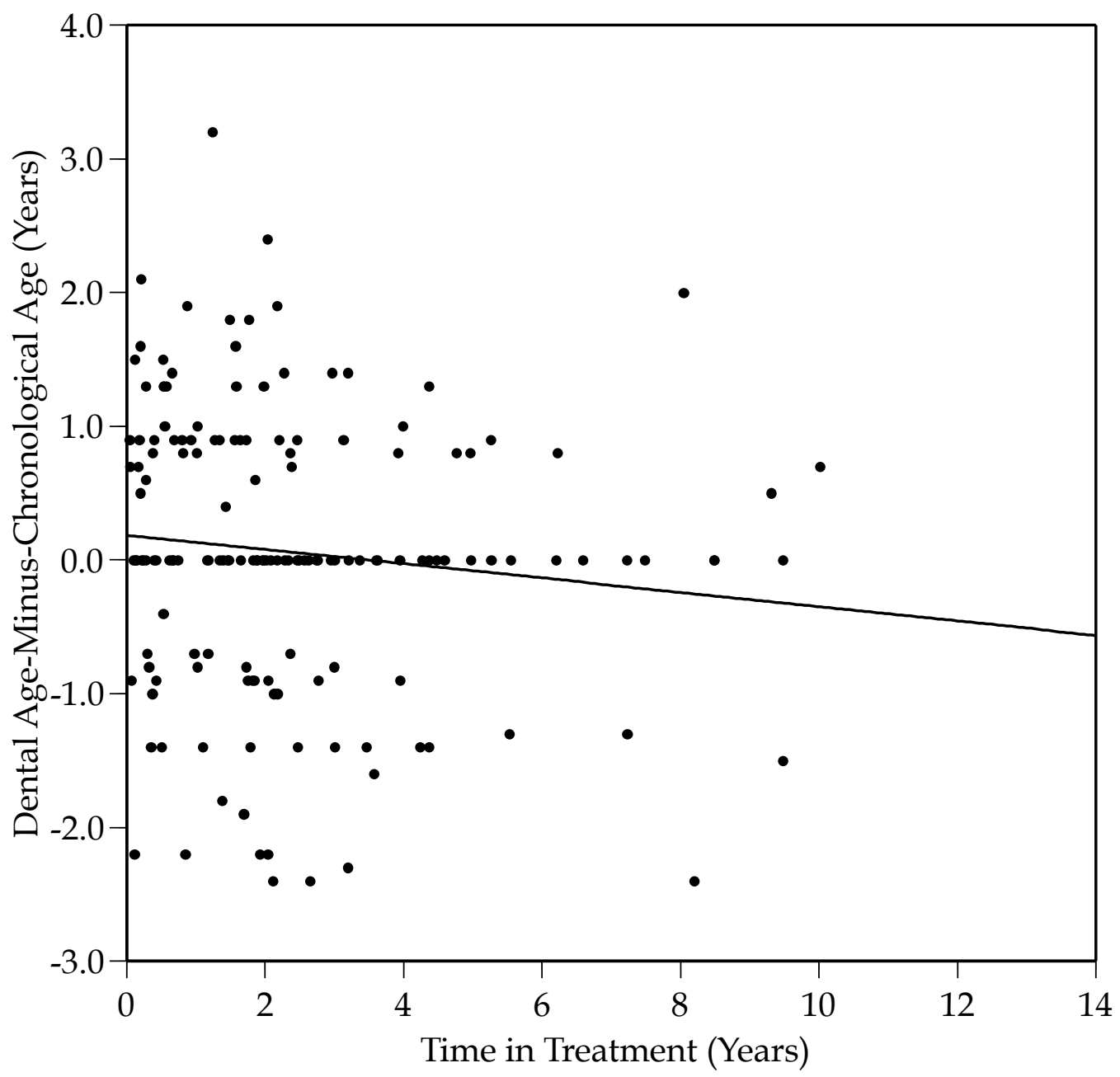

Fig. 13. Scatterplot between time in treatment on the horizontal axis and DA-CA on the vertical axis for the maxillary right second molar. The least-squares regression line also is shown. 


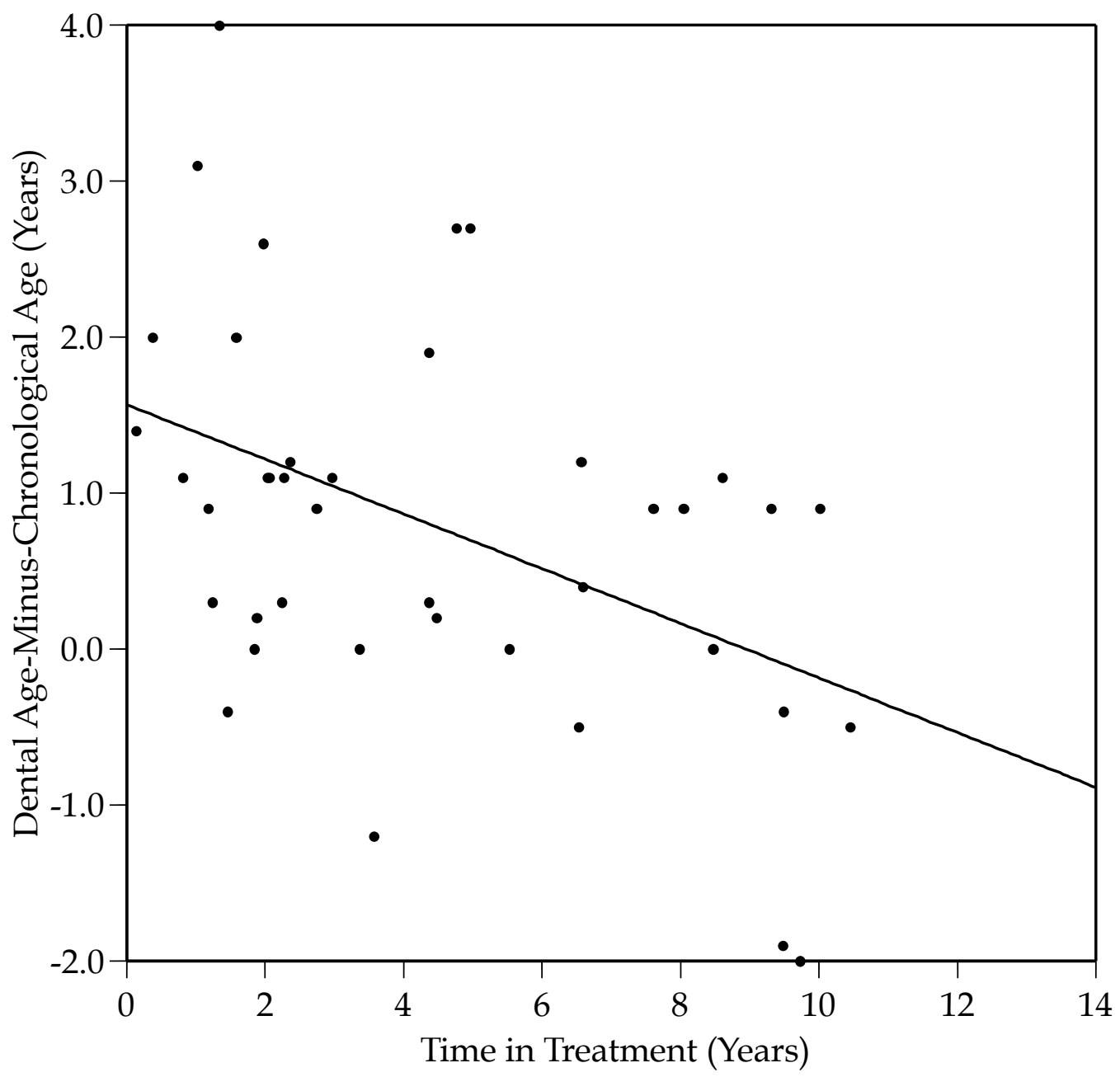

Fig. 14. Scatterplot between time in treatment on the horizontal axis and DA-CA on the vertical axis for the maxillary right third molar. The least-squares regression line also is shown. 


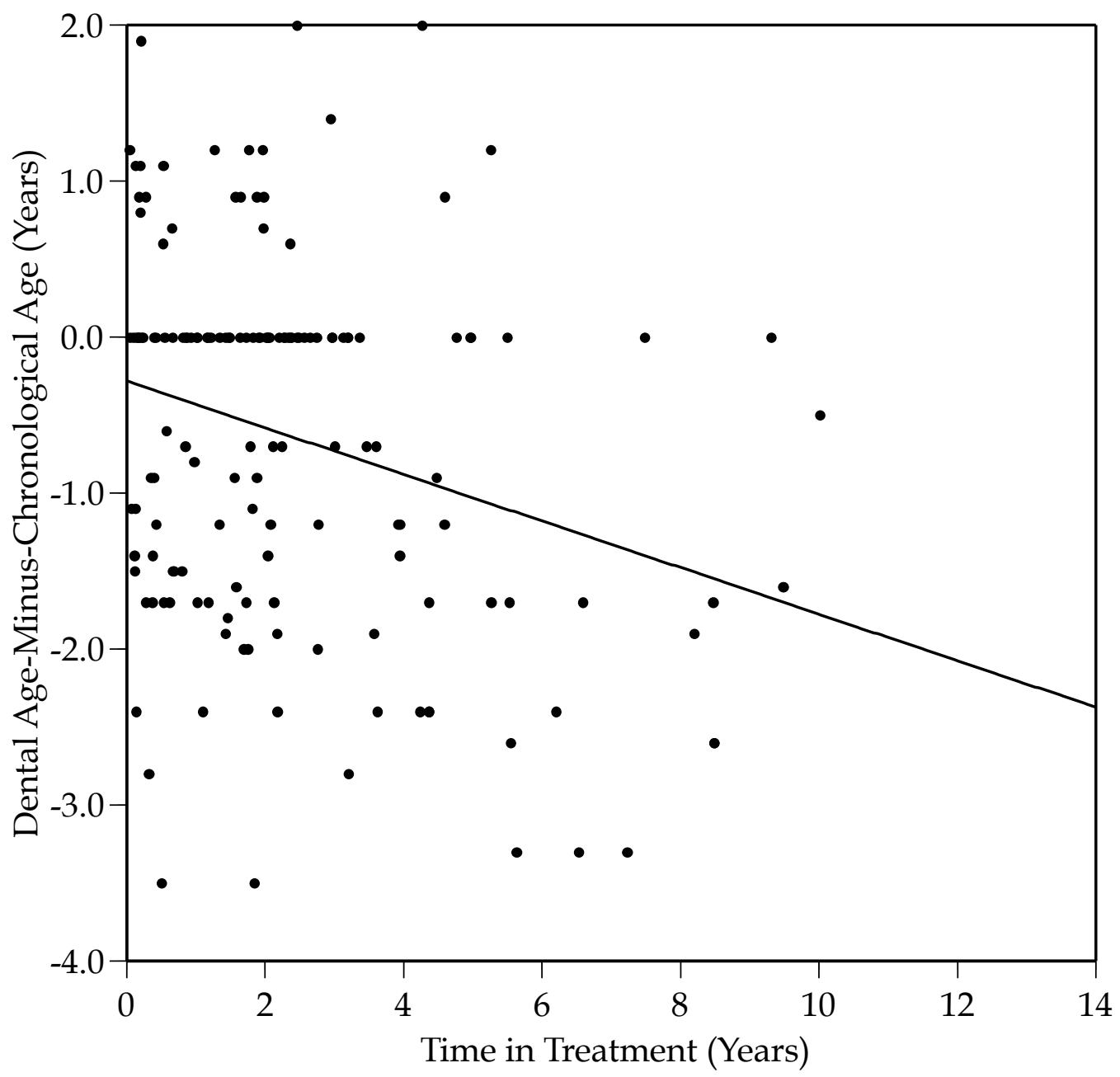

Fig. 15. Scatterplot between time in treatment on the horizontal axis and DA-CA on the vertical axis for the mandibular right canine. The least-squares regression line also is shown. 


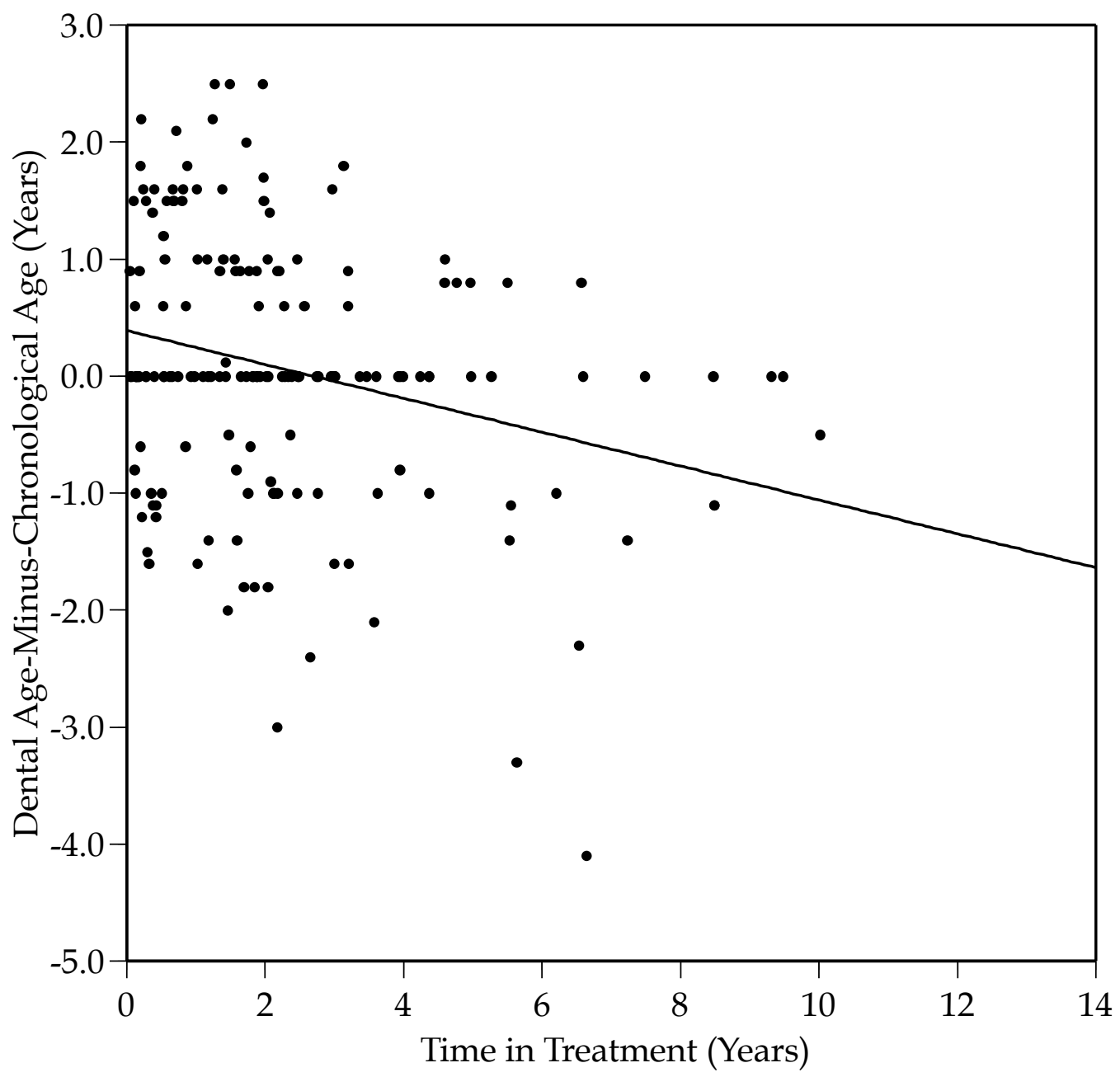

Fig. 16. Scatterplot between time in treatment on the horizontal axis and DA-CA on the vertical axis for the mandibular right first premolar. The least-squares regression line also is shown. 


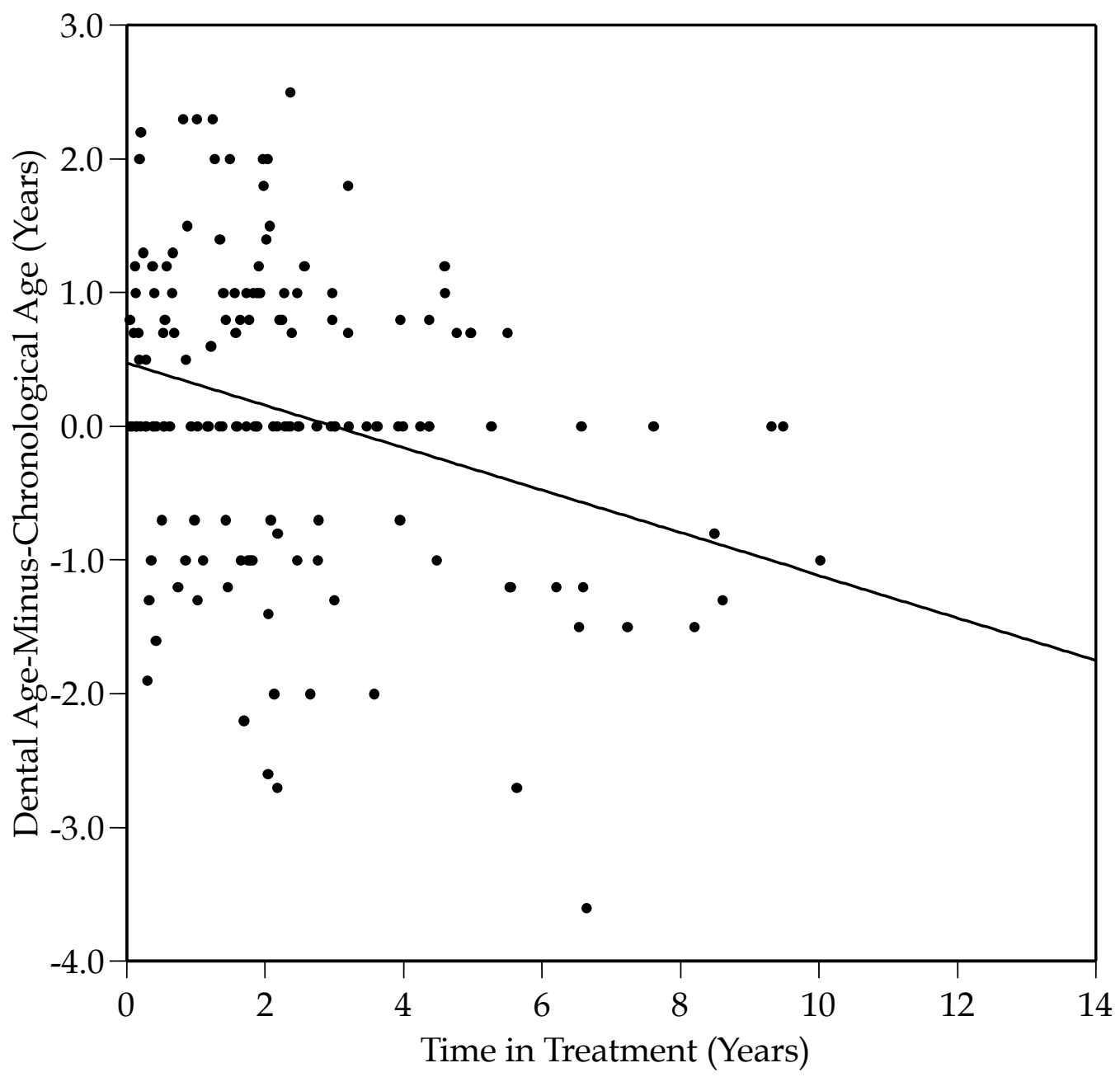

Fig. 17. Scatterplot between time in treatment on the horizontal axis and DA-CA on the vertical axis for the mandibular right second premolar. The least-squares regression line also is shown. 


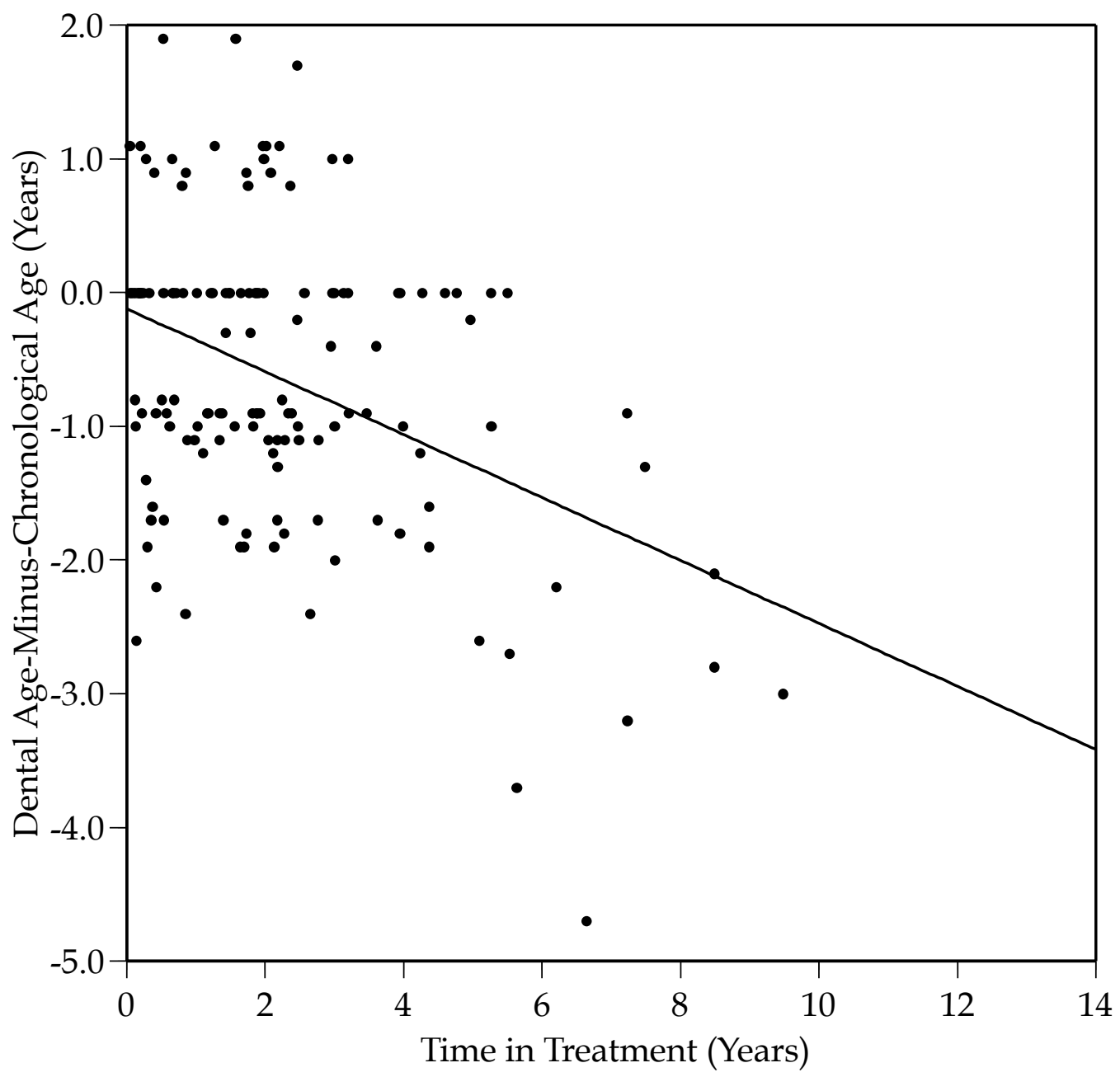

Fig. 18. Scatterplot between time in treatment on the horizontal axis and DA-CA on the vertical axis for the mandibular right first molar. The least-squares regression line also is shown. 


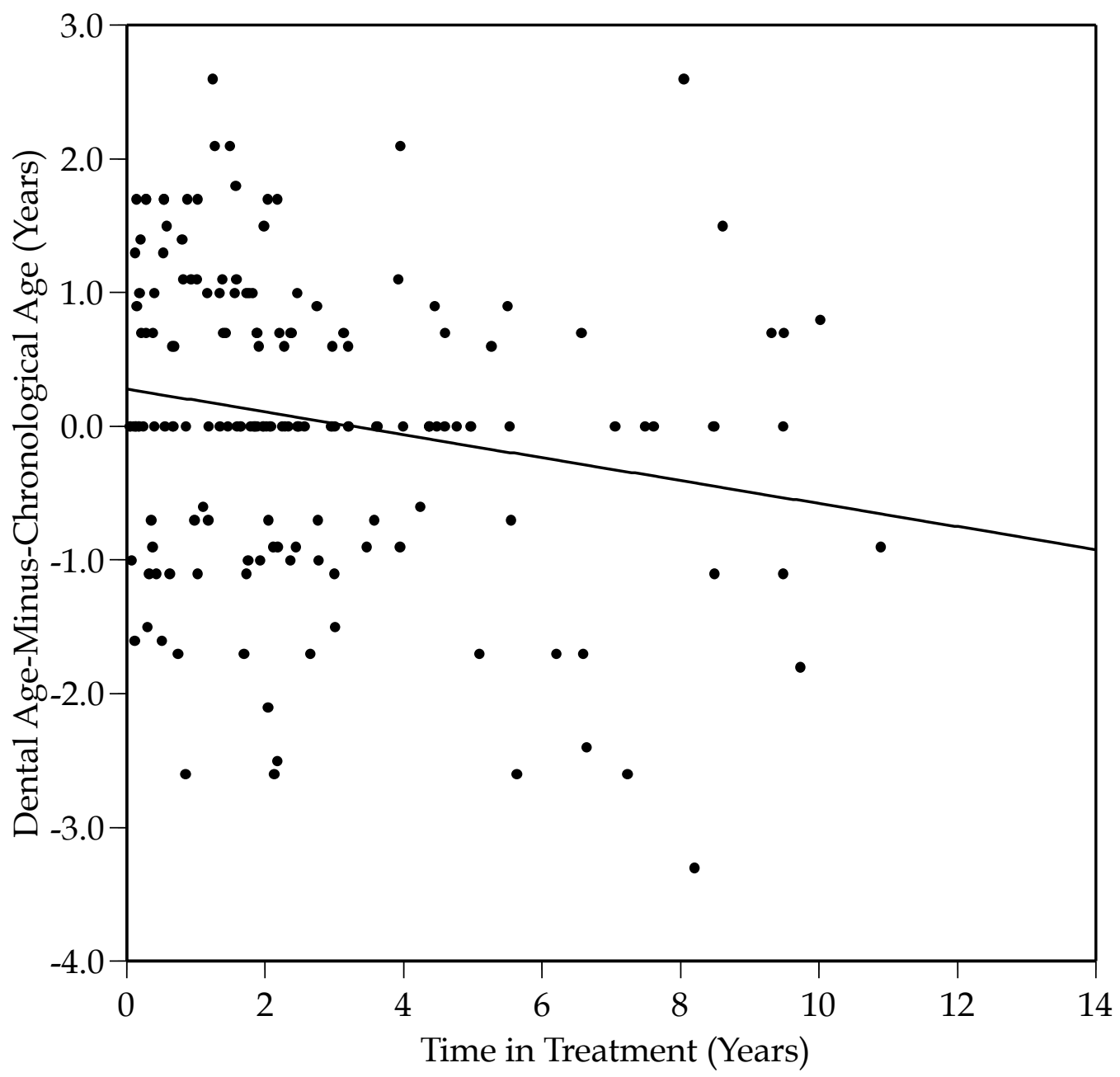

Fig. 19. Scatterplot between time in treatment on the horizontal axis and DA-CA on the vertical axis for the mandibular right second molar. The least-squares regression line also is shown. 


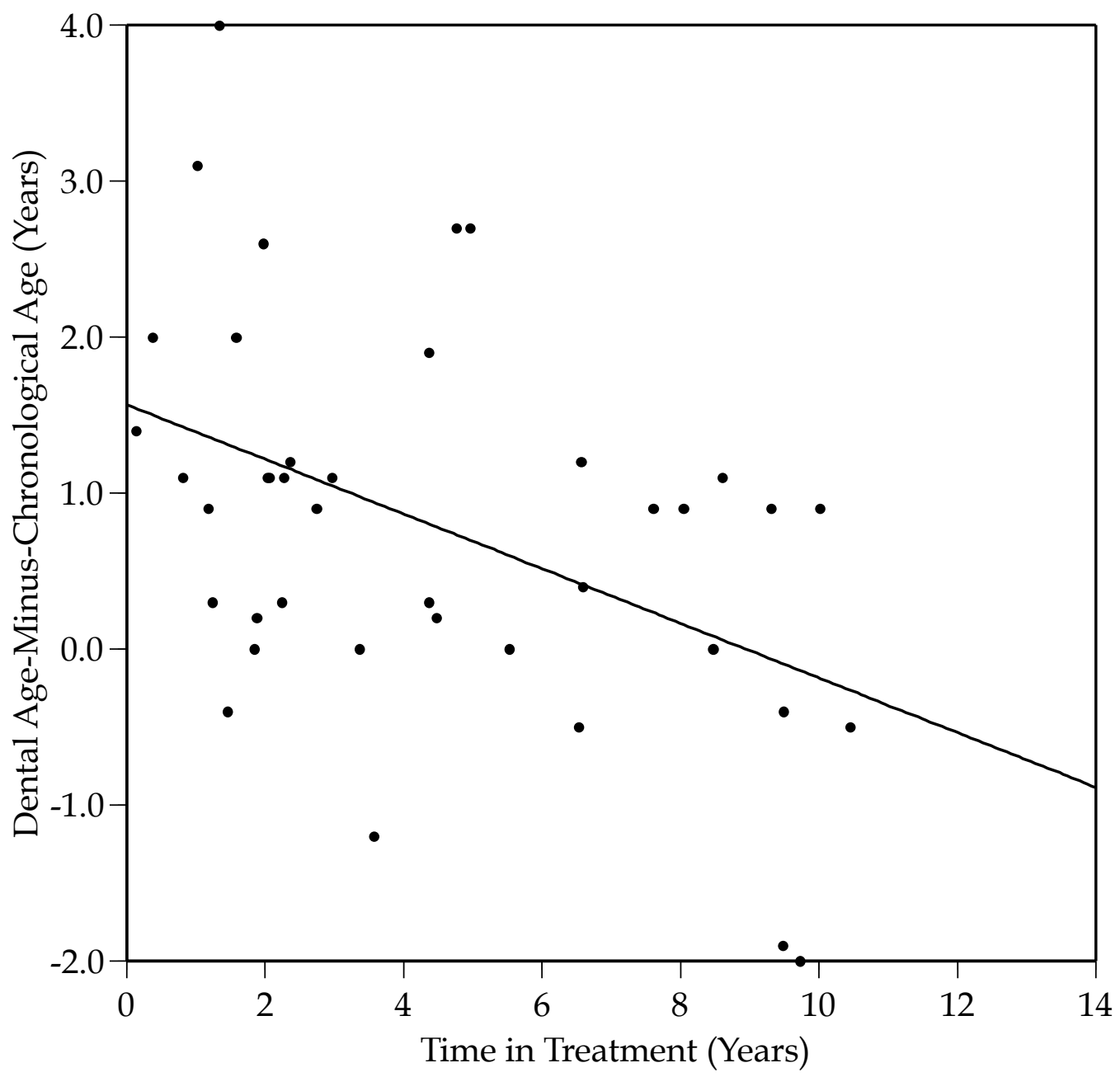

Fig. 20. Scatterplot between time in treatment on the horizontal axis and DA-CA on the vertical axis for the mandibular right third molar. The least-squares regression line also is shown. 
association between time and DA-CA is highly significant for UM2 $(\mathrm{P}=0.0063)$, as illustrated in Figure 13. As with these other relationships, DA-CA is centered around zero at the start of treatment, and it tends towards more negative values (i.e., greater developmental delays) as time progresses.

The same, highly significant negative relationship was found for UM3 $(\mathrm{P}=$ 0.0004) even though the sample size of scorable UM3 was only 43 . The association is shown in Figure. 14.

The sample sizes of scorable mandibular teeth are much improved compared to those in the maxilla. The association for the canine (Fig. 15) is highly significant $(\mathrm{P}=0.0031)$. Also, probably because of the much larger sample sizes, the associations for P1 and P2 both are highly significant statistically for these mandibular teeth (Figs. 16, 17), with P-values of 0.0006 and $<0.0001$, respectively.

The same, consistent highly significant negative association between time and DA-CA occurred for all these three mandibular molars (Figs. 18, 19, 20). Again, the greater consistency in these relationships compared to findings for the maxillary teeth seems to be attributable to the much larger scorable sample sizes for the mandibular teeth.

In summary, there is strong evidence (Table 12) that (A) the stress of treatment for ALL has a decided negative effect on developmental age as measured with the dentition (so-called "dental age") and (B) the longer the time 
since treatment initiation, the greater the DA-CA discrepancy. It also is readily evident that there is to be no compensatory growth after the termination of

treatment. At this exploratory level - without analysis of individual's patterns of dental development - there is no graphical suggestion that DA-CA in any way rebounds several years after diagnosis and treatment of ALL. As cautionary notes, these analyses have combined boys and girls (i.e., not yet tested for sexual dimorphism) and have not distinguished between children treated just with chemotherapy versus those who also received radiation therapy (Birkebaek and Clausen 1998; Halton et al. 1998; Jaruratanasirikul et al. 2004; Shusterman and Meadows 2000; Yamashita et al. 2003).

\section{Tooth Development During Treatment}

It was shown early in this chapter that DA-CA became a larger negative value as time from treatment progressed. The purpose in this section is to evaluate this trend more closely.

It needs to be appreciated that, strictly, the dental age data collected here constitutes a mixed longitudinal sample. There are multiple panoramic radiographs for most of the children in the study, though they were not taken at any set interval. Instead, they are the examinations that were obtained by the staff pediatric dentist to assess, restore, and maintain the oral health of these critically sick children. It was not intended at the time that these radiographs 
would be used to monitor the children's tempo of dental development. In consequence (Fig. 21), most examinations were obtained soon after the child arrived at St. Jude's, which coincides closely with the age at diagnosis and with the age at the start of treatment. The distribution of radiographs is strongly positively skewed; comparatively few images were taken years after diagnosis or after the cessation of treatment. For the present study, this means that, while there are multiple radiographs (and, thus, multiple assessments of dental age) for most of the 72 children, most of the data are clustered close to the start of treatment.

We investigated several ways of partitioning the data in order to produce longitudinal series of data representing the different phases of treatment, but with no success. Because of the strongly skewed distribution, it was not possible to generate sequences of observation where sample sizes were reliable. Even simply dichotomizing the radiographs into those that were less than 3 years into treatment versus those taken later after treatment provided sample sizes only on the order of 12 to 15 , which were too small to yield informative statistical results. In short, most data were clustered within too short a span near the start of treatment to provide informative longitudinal assessments of the effect of treatment on tooth development.

Instead, there is more statistical power in treating these data crosssectionally than if repeated-measures designs were used with critically small 


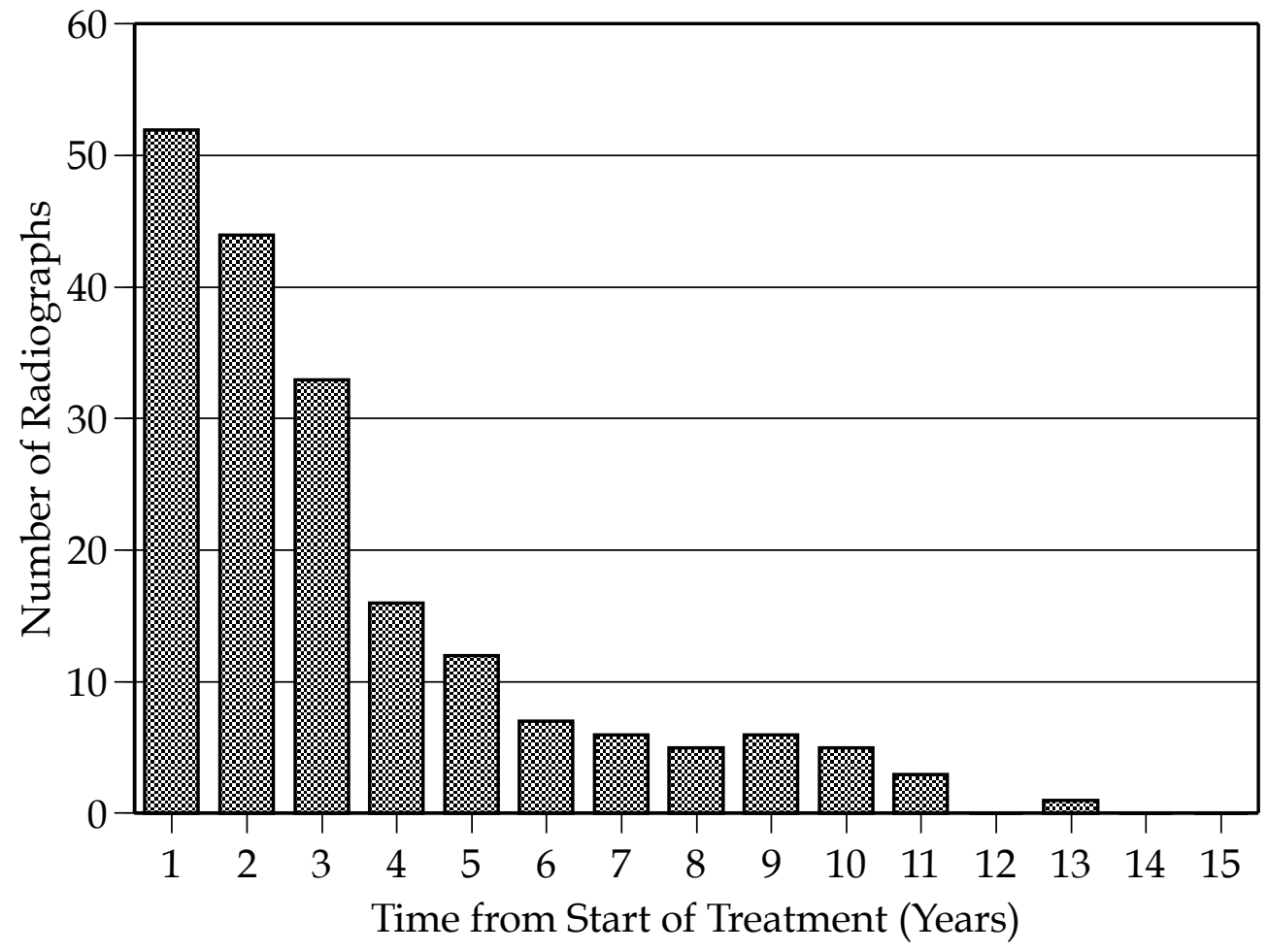

Fig. 21. Bar chart of the distribution of panoramic radiographs in the present study as a function of time into treatment. Most films were taken within 2 years of the initiation of treatment. 
sample sizes. Given the skewed distribution of the panoramic radiographs (most radiographs were taken within 2 years of the start of treatment) and the statistical need to obtain adequate sample sizes per group, four time intervals were developed, namely (1) radiographs within 1 year of the start of treatment, (2) radiographs between 1 and 2 years of the start, (3) radiographs between 2 and 3 years, and (4) radiographs taken more than 3 years after the start of treatment.

Then, for each mandibular tooth type (sample sizes were too small for the maxillary teeth), the DA-CA value from each radiograph was grouped into one of the four time intervals. Expectation was that DA-CA would be centered on zero at the start of treatment and that mean DA-CA would become a progressively larger negative number across the subsequent three time intervals as tooth development was retarded by treatment. The observed results were in concert with that expectation.

Results for each of the six mandibular tooth types (mandibular incisors were omitted) are graphed in Figures 22 though 27, and the corresponding statistical tests (from one-way analysis of variance) are listed in Table 13. Descriptive statistics for DA-CA partitioned by age interval are listed in Table 14. Notice that the sample sizes are proportional across the four time intervals. Sample sizes are small for the third molars. 


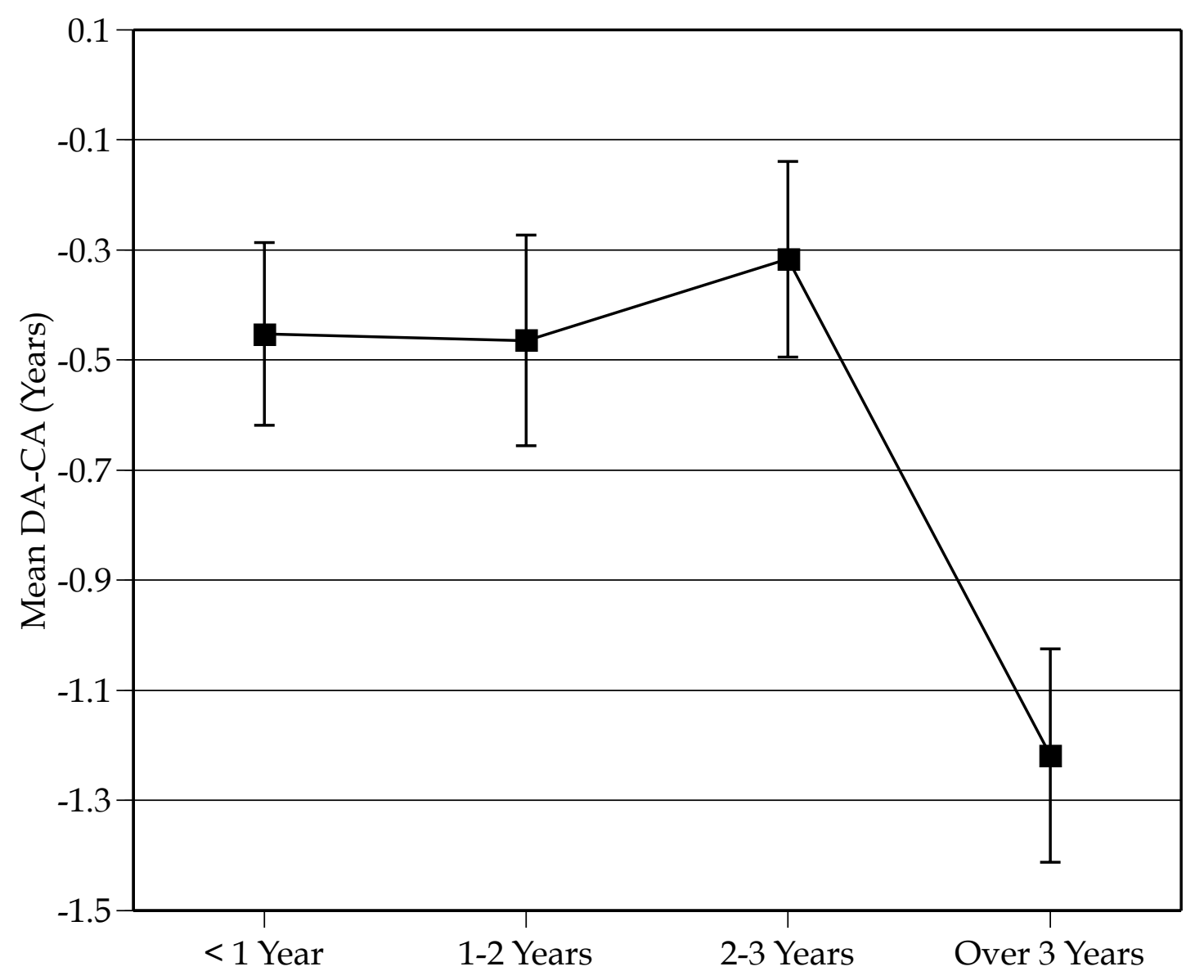

Fig. 22. Plot of the mean values of DA-CA for the mandibular canine where time in treatment is partitioned into four intervals. Error bars are the standard error of the mean. 


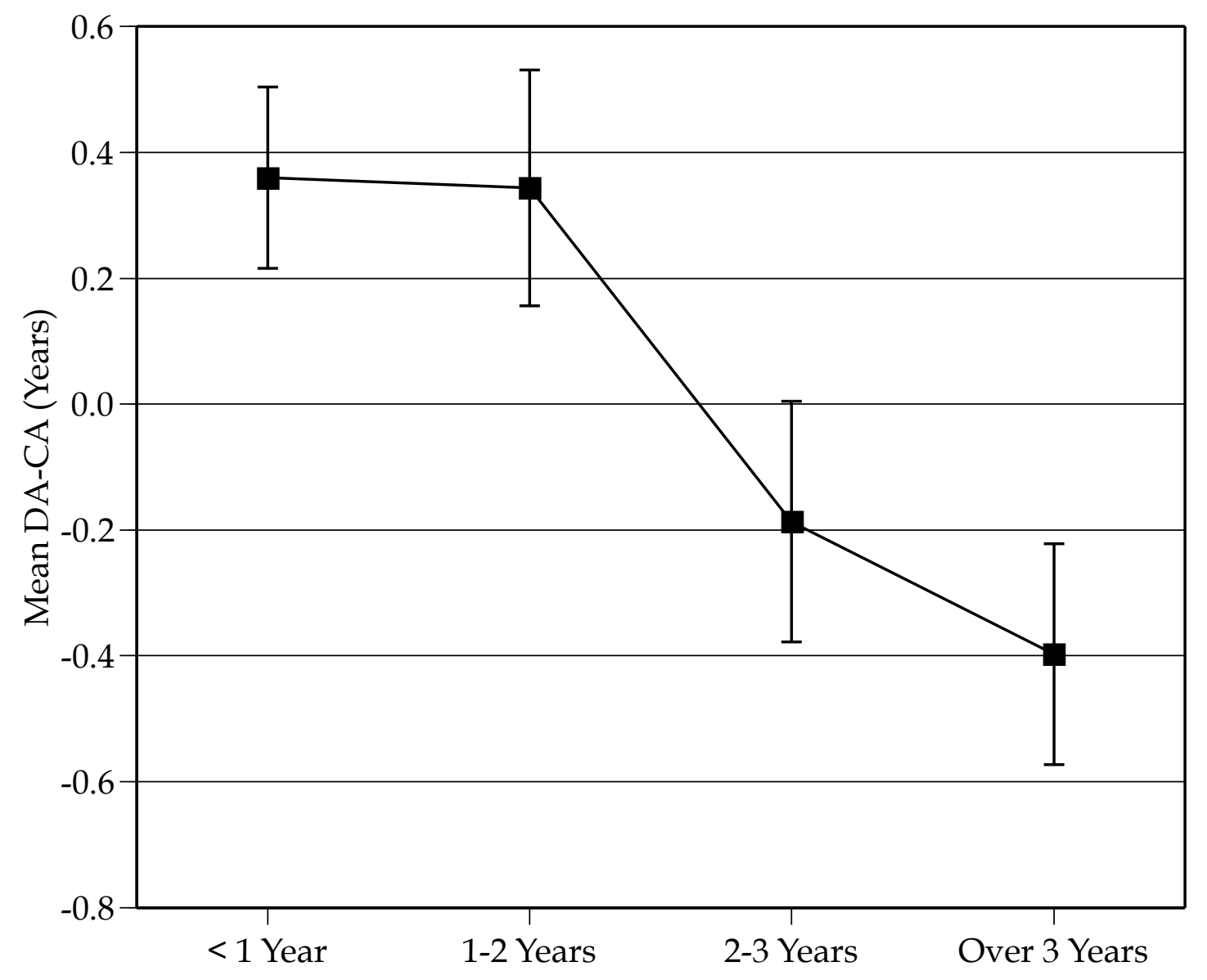

Fig. 23. Plot of the mean values of DA-CA for the mandibular first premolar where time in treatment is partitioned into four intervals. Error bars are the standard error of the mean. 


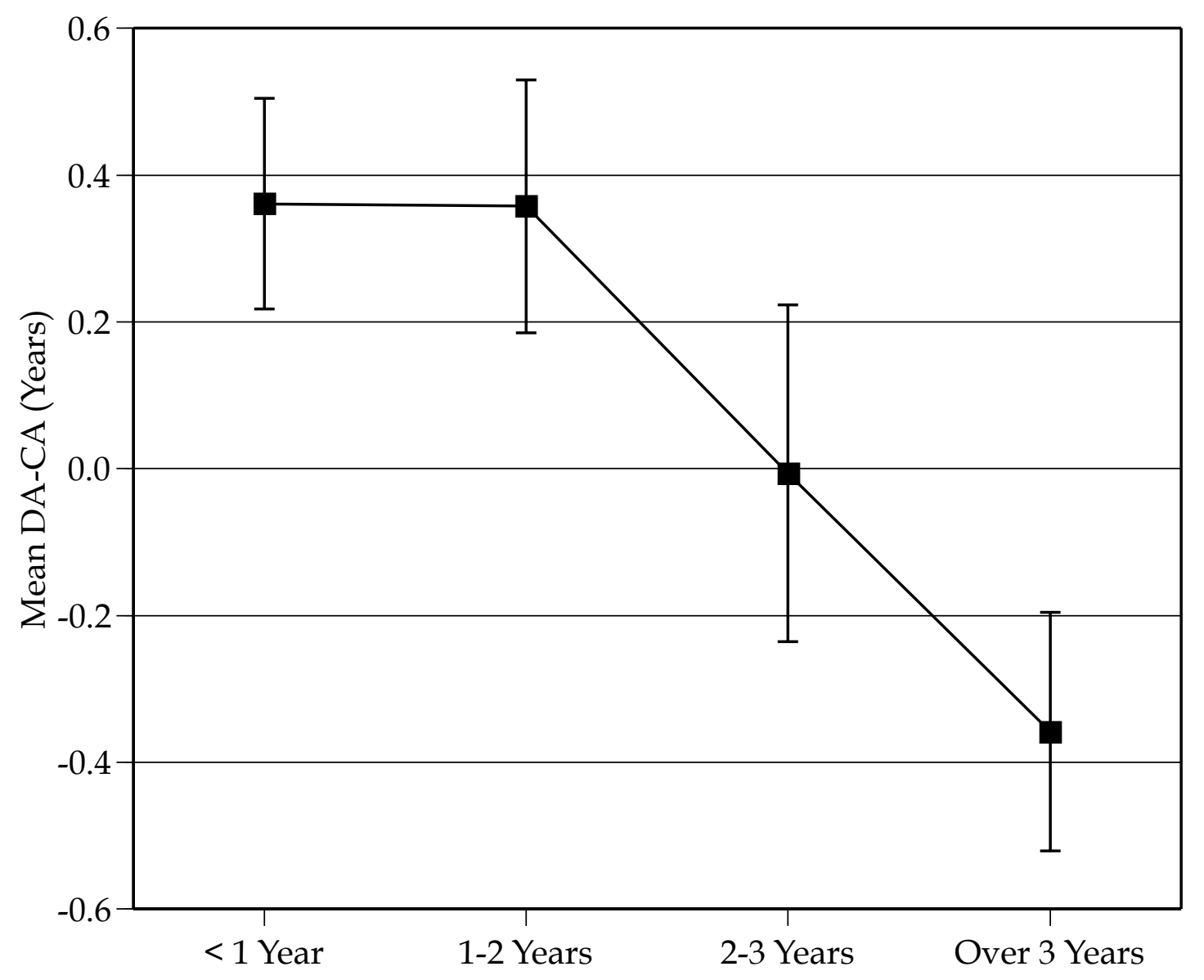

Fig. 24. Plot of the mean values of DA-CA for the mandibular second premolar where time in treatment is partitioned into four intervals. Error bars are the standard error of the mean. 


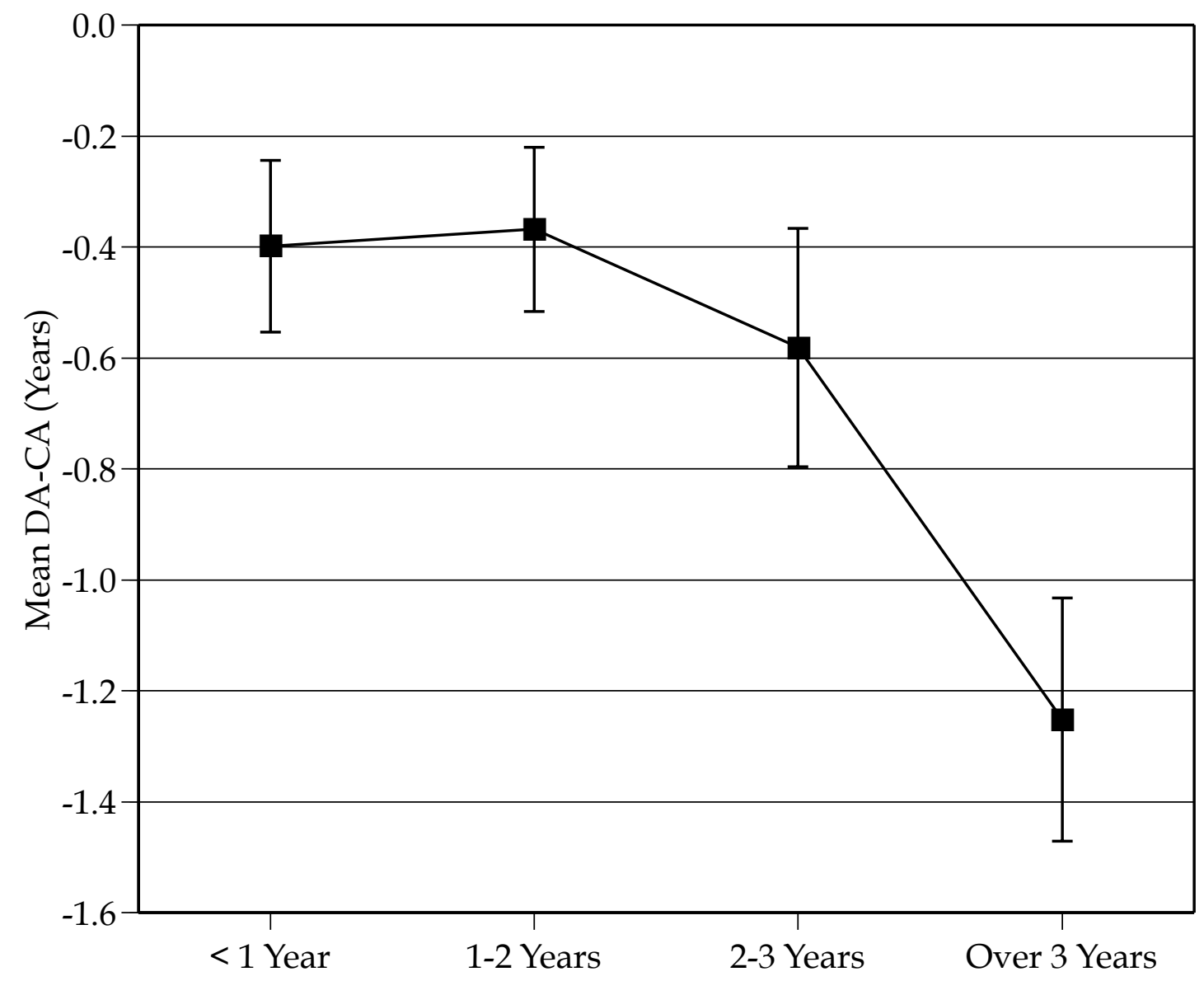

Fig. 25. Plot of the mean values of DA-CA for the mandibular first molar where time in treatment is partitioned into four intervals. Error bars are the standard error of the mean. 


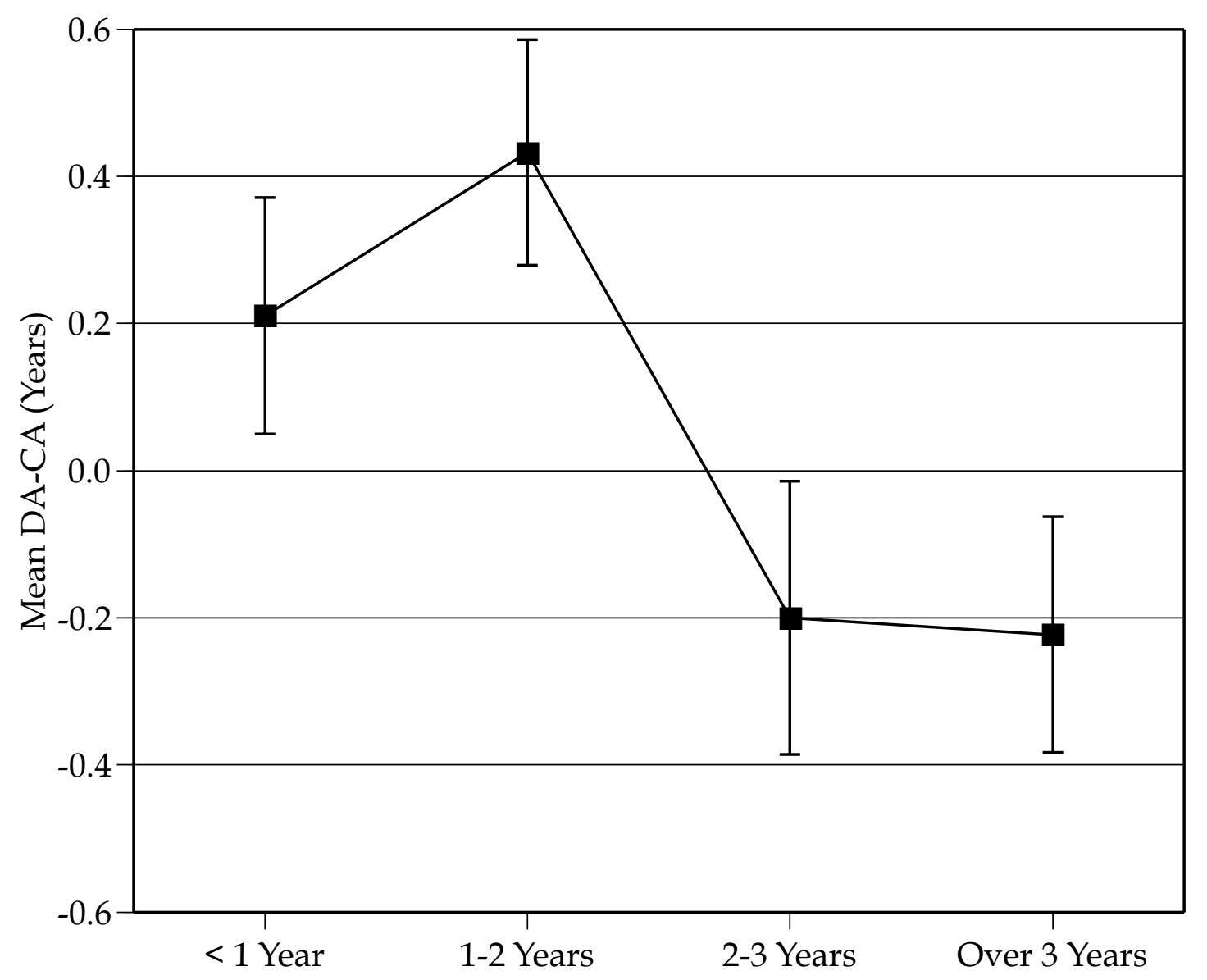

Fig. 26. Plot of the mean values of DA-CA for the mandibular second molar where time in treatment is partitioned into four intervals. Error bars are the standard error of the mean. 


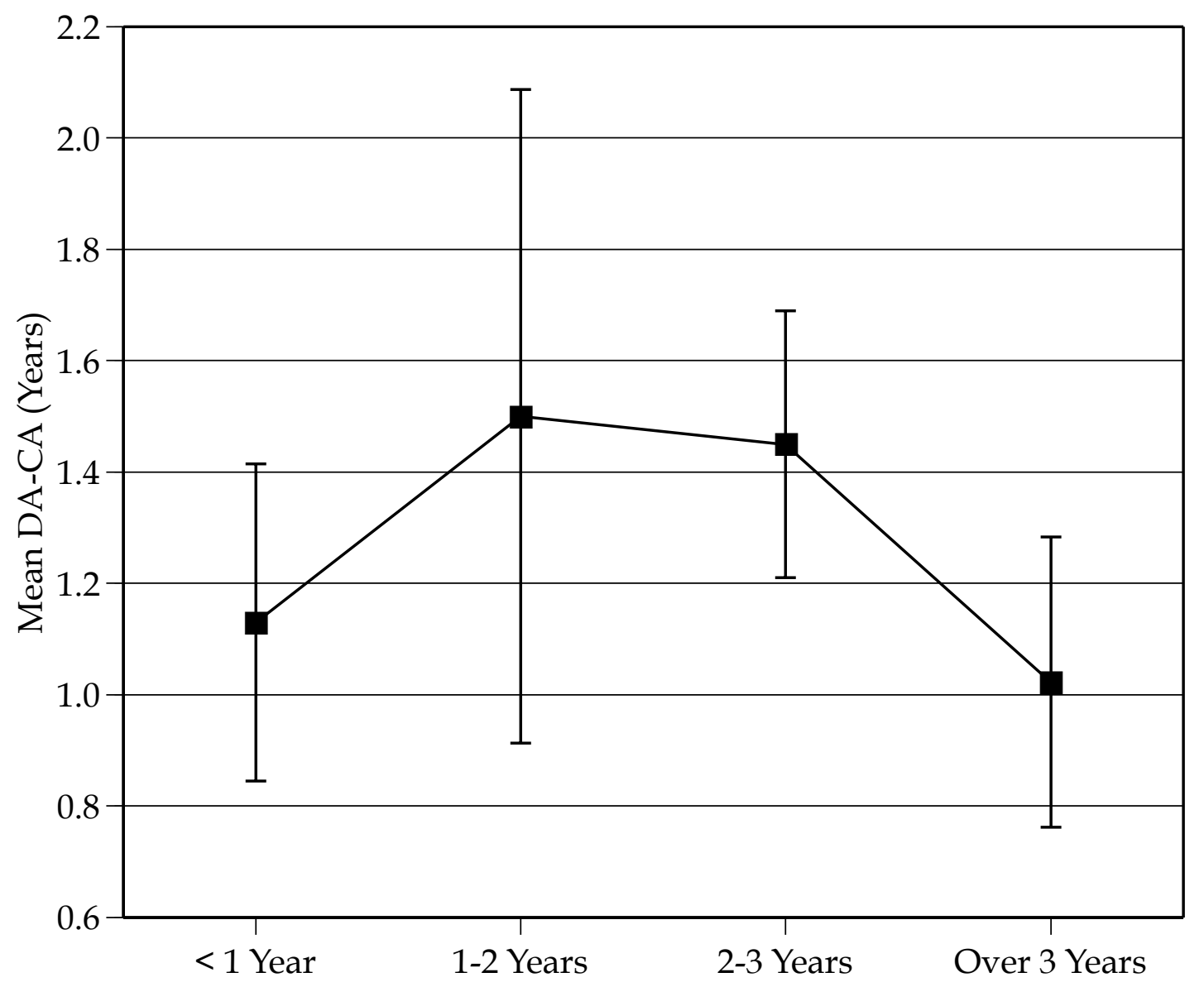

Fig. 27. Plot of the mean values of DA-CA for the mandibular third molar where time in treatment is partitioned into four intervals. Error bars are the standard error of the mean. 
Table 13. Results of one-way analysis of variance testing for differences in DA-CA among four intervals of time in treatment (and treating the data crosssectionally).

\begin{tabular}{|c|c|c|c|c|c|}
\hline Source & $\mathrm{df}$ & $\begin{array}{l}\text { Sum of } \\
\text { Squares }\end{array}$ & $\begin{array}{c}\text { Mean } \\
\text { Square }\end{array}$ & F Ratio & Prob $>$ F \\
\hline $\begin{array}{l}\text { Time in TX } \\
\text { Error }\end{array}$ & $\begin{array}{r}3 \\
152\end{array}$ & $\begin{array}{l}\text { Md Ca } \\
20.19 \\
201.41\end{array}$ & $\begin{array}{l}6.73 \\
1.33\end{array}$ & 5.08 & 0.0022 \\
\hline $\begin{array}{l}\text { Time in TX } \\
\text { Error }\end{array}$ & $\begin{array}{r}3 \\
163\end{array}$ & $\begin{array}{c}\text { Md First P } \\
18.87 \\
203.30\end{array}$ & $\begin{array}{l}\text { Iolar } \\
6.29 \\
1.25\end{array}$ & 5.04 & 0.0023 \\
\hline $\begin{array}{l}\text { Time in TX } \\
\text { Error }\end{array}$ & $\begin{array}{r}3 \\
156\end{array}$ & $\begin{array}{c}\text { Id Second } \\
15.16 \\
185.77\end{array}$ & $\begin{array}{c}\text { molar } \\
5.05 \\
1.19\end{array}$ & 4.24 & 0.0065 \\
\hline $\begin{array}{l}\text { Time in TX } \\
\text { Error }\end{array}$ & $\begin{array}{r}3 \\
138\end{array}$ & $\begin{array}{c}\text { Md First } \\
18.50 \\
161.90\end{array}$ & $\begin{array}{l}\text { lar } \\
6.16 \\
1.17\end{array}$ & 5.26 & 0.0018 \\
\hline $\begin{array}{l}\text { Time in TX } \\
\text { Error }\end{array}$ & $\begin{array}{r}3 \\
167\end{array}$ & $\begin{array}{c}\text { Md Secon } \\
12.94 \\
194.46\end{array}$ & $\begin{array}{l}\text { olar } \\
4.31 \\
1.16\end{array}$ & 3.70 & 0.0129 \\
\hline $\begin{array}{l}\text { Time in TX } \\
\text { Error }\end{array}$ & $\begin{array}{r}3 \\
44\end{array}$ & $\begin{array}{c}\text { Md Third } \\
1.96 \\
61.14\end{array}$ & $\begin{array}{l}\text { lar } \\
0.65 \\
1.39\end{array}$ & 0.47 & 0.7047 \\
\hline $\begin{array}{l}\text { Time in TX } \\
\text { Error }\end{array}$ & $\begin{array}{r}3 \\
184 \\
\end{array}$ & $\begin{array}{c}\text { All Scorab } \\
12.94 \\
176.77 \\
\end{array}$ & $\begin{array}{l}\text { eeth } \\
4.31 \\
0.96 \\
\end{array}$ & 4.49 & 0.0046 \\
\hline
\end{tabular}




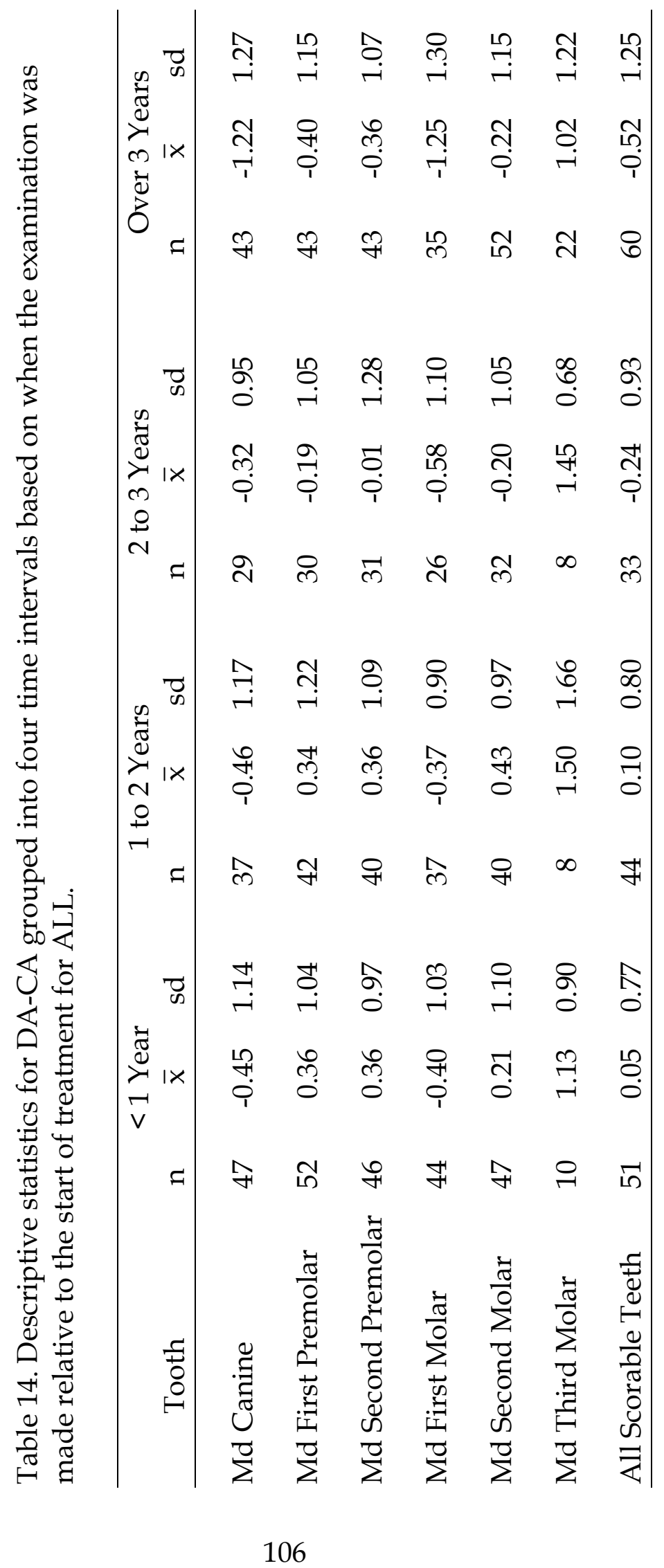


Treatment for ALL had no apparent effect on the rate of formation of the mandibular canine until 3 years after the start of treatment (Fig. 22), when DACA dropped significantly $(P=0.0025)$. The same pattern was found for the first and second premolars (Figs. 23, 24), but the drop in DA-CA was seen after 2 years of treatment instead of 3.

The pattern of response for M1 and M2 (Figs. 25 and 26) each shows a decrease in DA-CA after the second year, but, statistically, the single source of the significance is the obvious decrease after year 3 of treatment.

The mandibular third molar (Fig. 27) stands out as the one case of the six tested where there was no statistically significant difference across time $(\mathrm{P}=$ 0.73), but, as noted (Table 14), this lack of a statistical difference may be attributable to the small sample sizes.

To pursue this approach one step farther, Figure 28 is a graph of DA-CA across the four time intervals calculated from all scorable teeth (maxillary and mandibular). These data show that tooth development proceeded on a normal course during the first two years of treatment, but a developmental delay is evident during the third year, with a highly significant delay after the third year (Table 13; $\mathrm{P}=0.0046)$.

Noteworthy results that are consistent across these several analyses (Table 13) are (1) that tooth development appears to be undisturbed until the third year after the start of treatment-but this does not necessarily address 


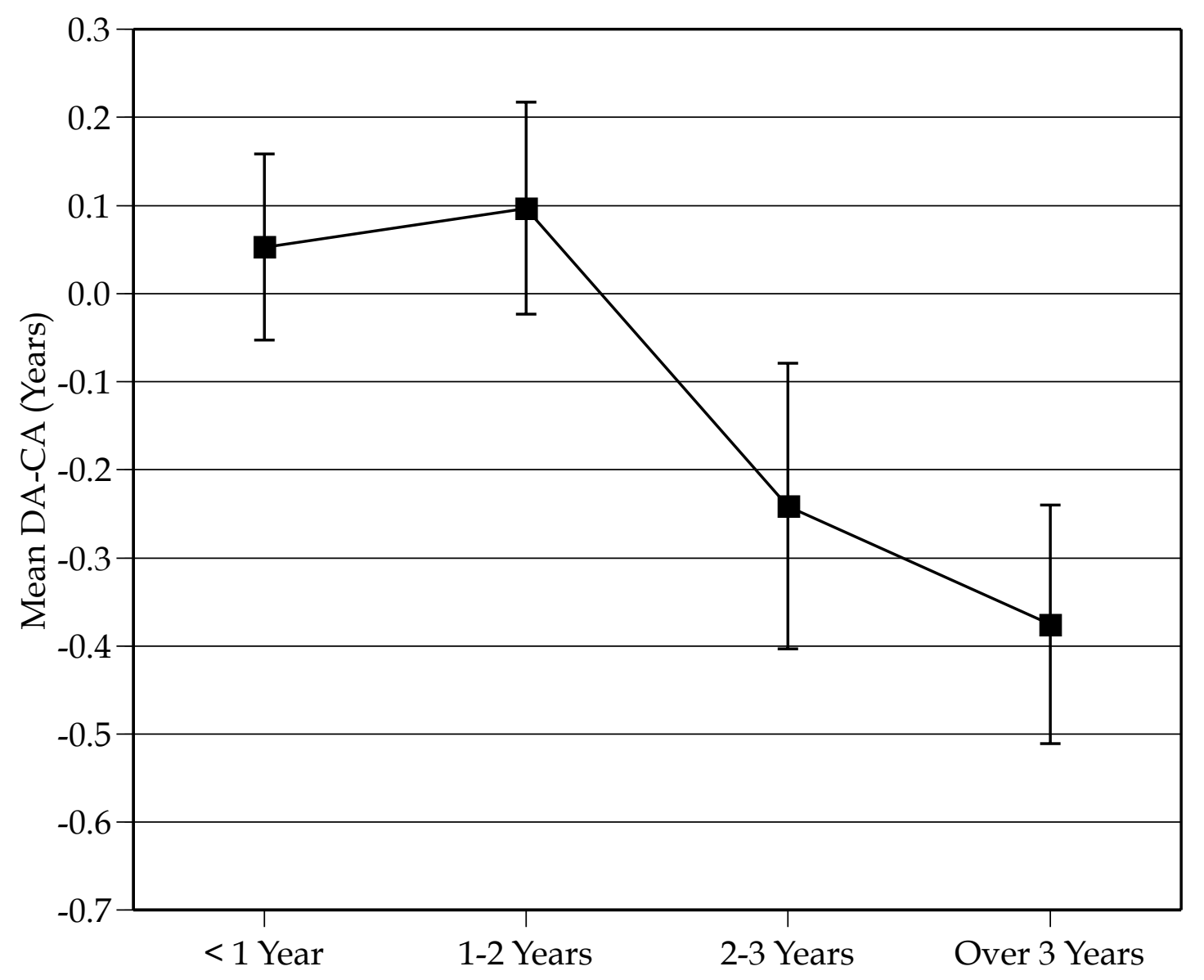

Fig. 28. Plot of the mean values of DA-CA for the average of all scorable teeth where time in treatment is partitioned into four intervals. Error bars are the standard error of the mean. 
when the damage was incurred - (2) the long-term effect (> 3 years) seems to be more dramatic, and (3) there is no suggestion of any sort of compensatory spurt in development after treatment. An additional point is that the considerable variability seen in the "over 3" category indicates that some children fare much better than others long-term and, as well, other children experience serious delays in dental development.

\section{Cranial Irradiation}

One-third of the 72 children studied (25/72) had been treated with cranial irradiation in addition to chemotherapy. The use of irradiation was just as common in boys $(26 / 41 ; 63 \%)$ as girls $(21 / 31 ; 68 \%)$, yielding a chi-square statistic of $0.150(\mathrm{P}=0.7021)$.

So too, the age at diagnosis was statistically independent whether the child was treated with irradiation. Mean age of children treated with irradiation $(\bar{x}=6.8)$ was the same as those treated with chemotherapy alone $(\bar{x}=6.7)$. By ANOVA, this difference between means was insignificant $(F=0.4$ with 1 and 71 $\mathrm{df} ; \mathrm{P}=0.8498)$.

Does cranial irradiation alter the rate of tooth development versus treating children diagnosed with ALL with chemotherapy alone? We looked at DA - CA against cranial irradiation (scored as yes or no), with time-in-treatment as a covariate. Results (Table 15) show, importantly, that there is significant 
Table 15. ANOVA results assessing the effect of time-intreatment on DA-CA partitioned by whether the child was treated with cranial irradiation. ${ }^{1}$

\begin{tabular}{ccccc}
\hline Source & df & Sum of Squares & F-Ratio & P-Value \\
\hline Irradiation & 1 & 0.555744 & 0.7264 & 0.3952 \\
Time in TX & 1 & 5.391796 & 7.0471 & 0.0086 \\
Interaction & 1 & 8.870618 & 11.5940 & 0.0008 \\
\hline
\end{tabular}

${ }^{1}$ Notice that the irradiation-by-time in treatment interaction effect is significant statistically. 
interaction between time-in-treatment and whether the patient received cranial irradiation $(P=0.03)$. This means that the effect of duration on CA -DA is different depending on whether treatment involved irradiation.

Without irradiation (Table 16), the dependency disappears $(\mathrm{n}=112$ examinations; $\mathrm{F}=0.26 ; \mathrm{P}=0.6138$ ). With irradiation, there is a highly significant association between time-in-treatment and the delay in DA behind CA $(F=20.31$; $\mathrm{P}<0.0001$ ) based on 78 examinations (Table 17). We also tested for an effect due to sex of the patient, but there was no difference in the response of boys and girls. Examining the data (DA - CA) for children who received cranial irradiation, the association was linear insofar as a quadratic equation did not significantly improve the fit. Tests were performed for the six mandibular teeth (canine through third molar) and for the summary measure of all scorable teeth. Data are too sparse to analyze the maxillary teeth individually. The fit of linear regression lines to the data are shown in Figures 29 through 35. In each instance, the regression coefficient has a significant negative slope for the subset of children treated with cranial irradiation, but the regression coefficient is always zero for the group receiving chemotherapy alone.

These results are important because they disclose (A) that the debilitating effect of treatment on dental development is predominately driven by the cranial irradiation and (B) chemotherapy alone has a nonsignificant effect on dental maturation. 
Table 16. ANOVA results assessing the effect of time-intreatment on DA-CA in the subset of the sample that was treated with chemotherapy alone.

\begin{tabular}{ccccc}
\hline Source & df & Sum of Squares & F-Ratio & P-Value \\
\hline Time in TX & 1 & .01929952 & 0.2561 & 0.6138 \\
\hline
\end{tabular}

Table 17. ANOVA results assessing the effect of time-intreatment on DA-CA in the subset of the sample that was treated with chemotherapy and cranial irradiation.

\begin{tabular}{ccccc}
\hline Source & df & Sum of Squares & F-Ratio & P-Value \\
\hline Time in TX & 1 & 15.890743 & 20.3058 & $<0.0001$ \\
\hline
\end{tabular}




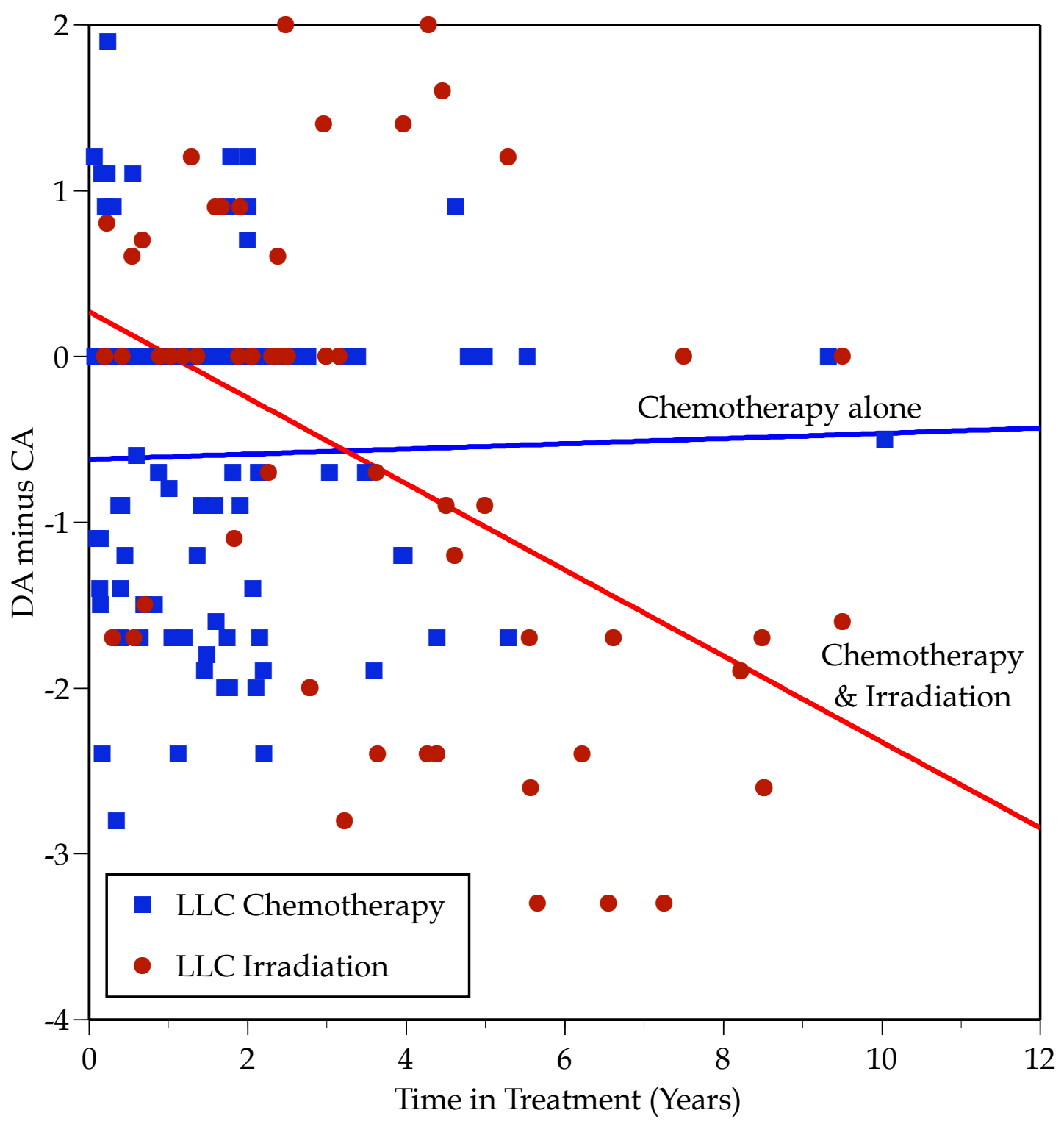

Fig. 29. Plot of time in treatment against DA-CA, with the data (mandibular canine) partitioned by whether the child was treated with cranial irradiation or chemotherapy alone. 


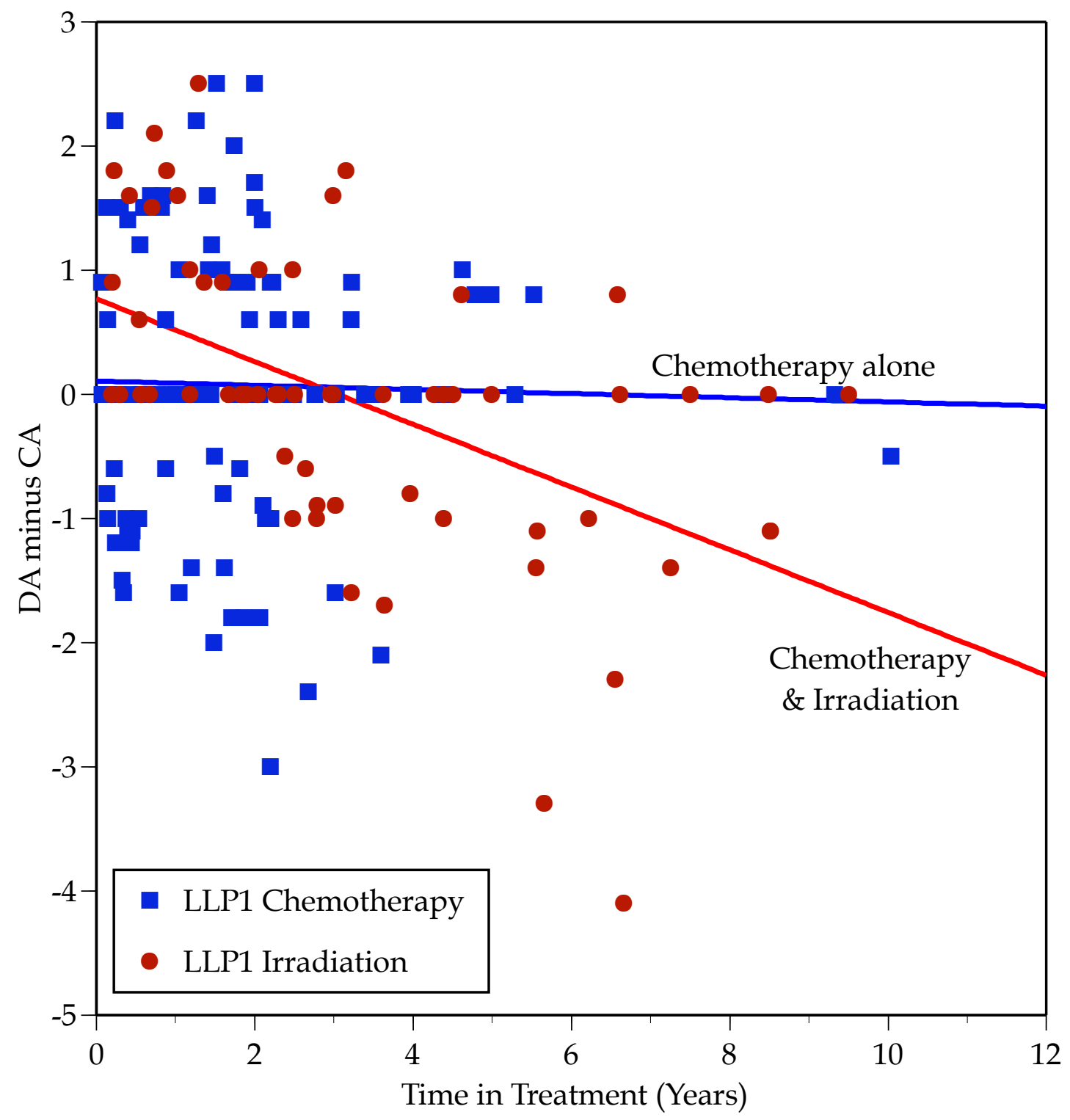

Fig. 30. Plot of time in treatment against DA-CA, with the data (mandibular first premolar) partitioned by whether the child was treated with cranial irradiation or chemotherapy alone. 


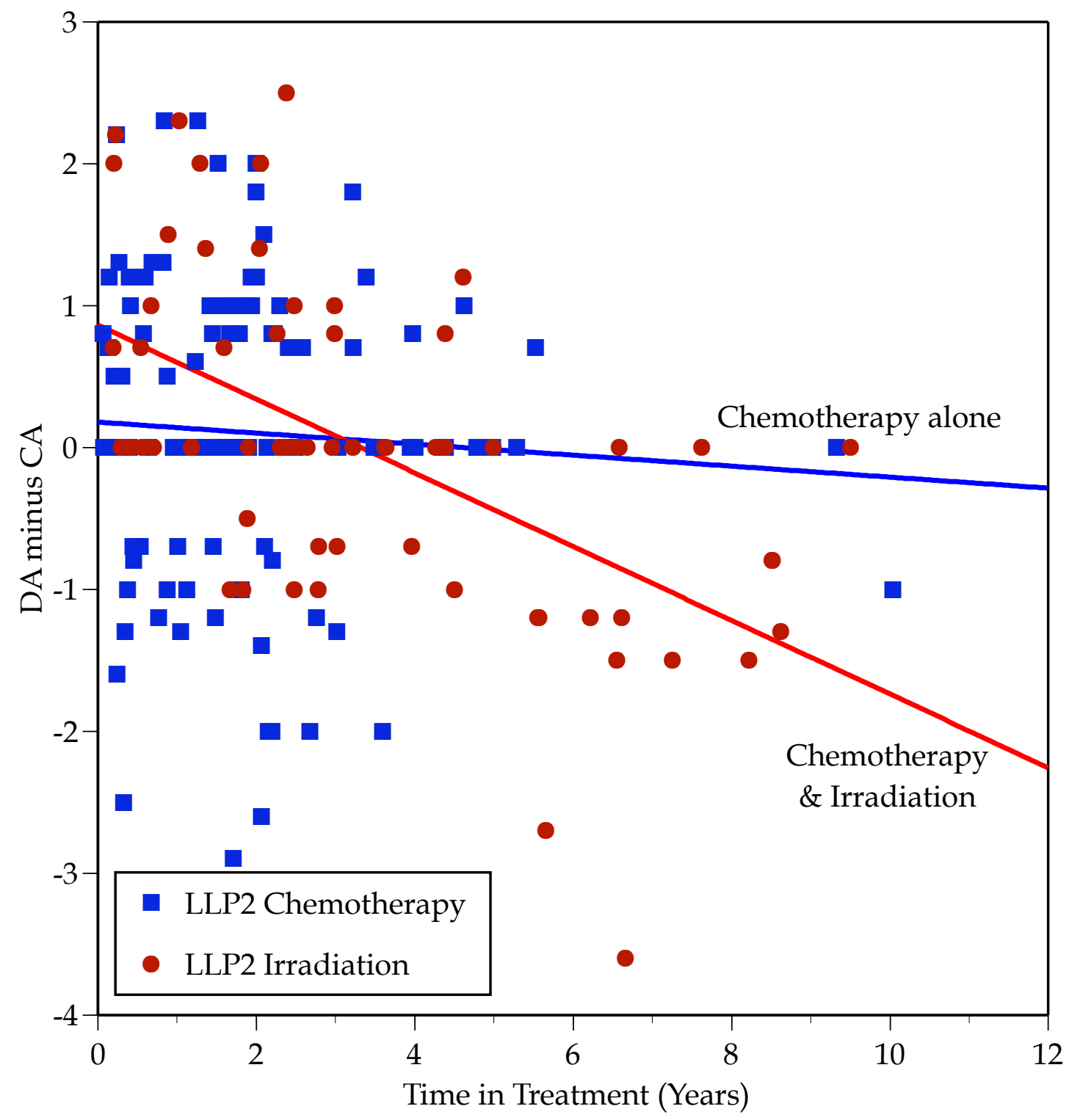

Fig. 31. Plot of time in treatment against DA-CA, with the data (mandibular second premolar) partitioned by whether the child was treated with cranial irradiation or chemotherapy alone. 


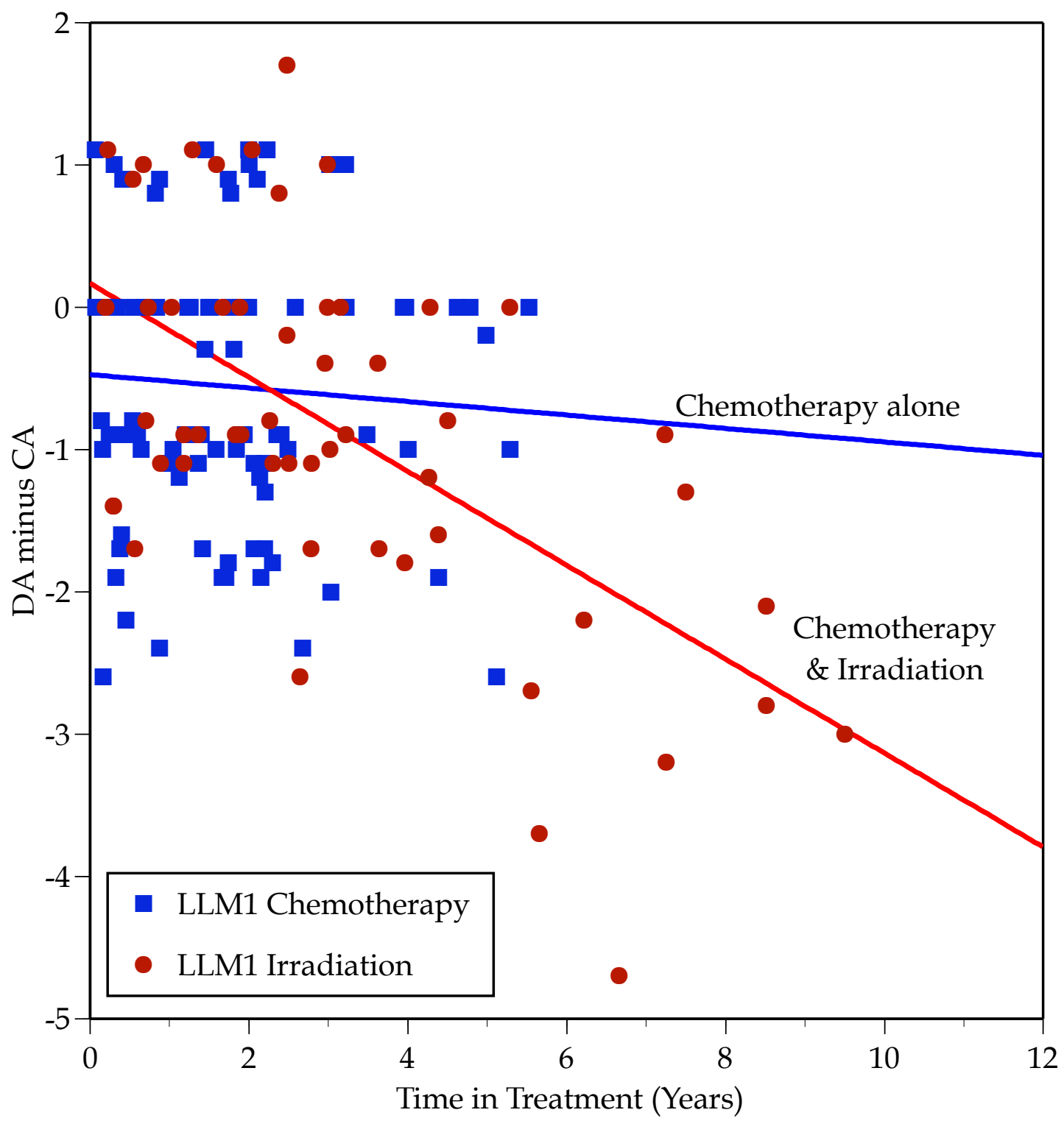

Fig. 32. Plot of time in treatment against DA-CA, with the data (mandibular first molar) partitioned by whether the child was treated with cranial irradiation or chemotherapy alone. 


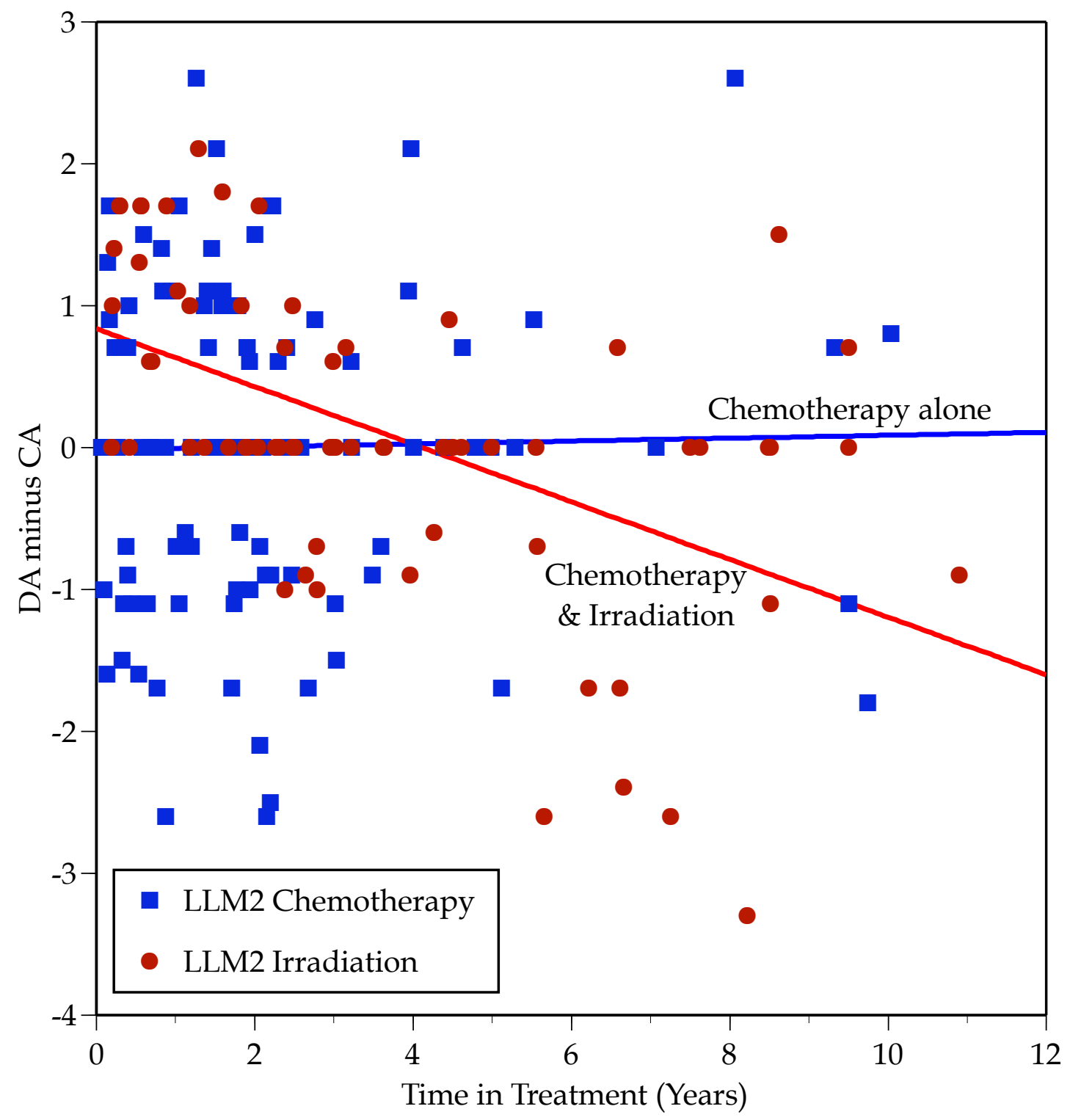

Fig. 33. Plot of time in treatment against DA-CA, with the data (mandibular second molar) partitioned by whether the child was treated with cranial irradiation or chemotherapy alone. 


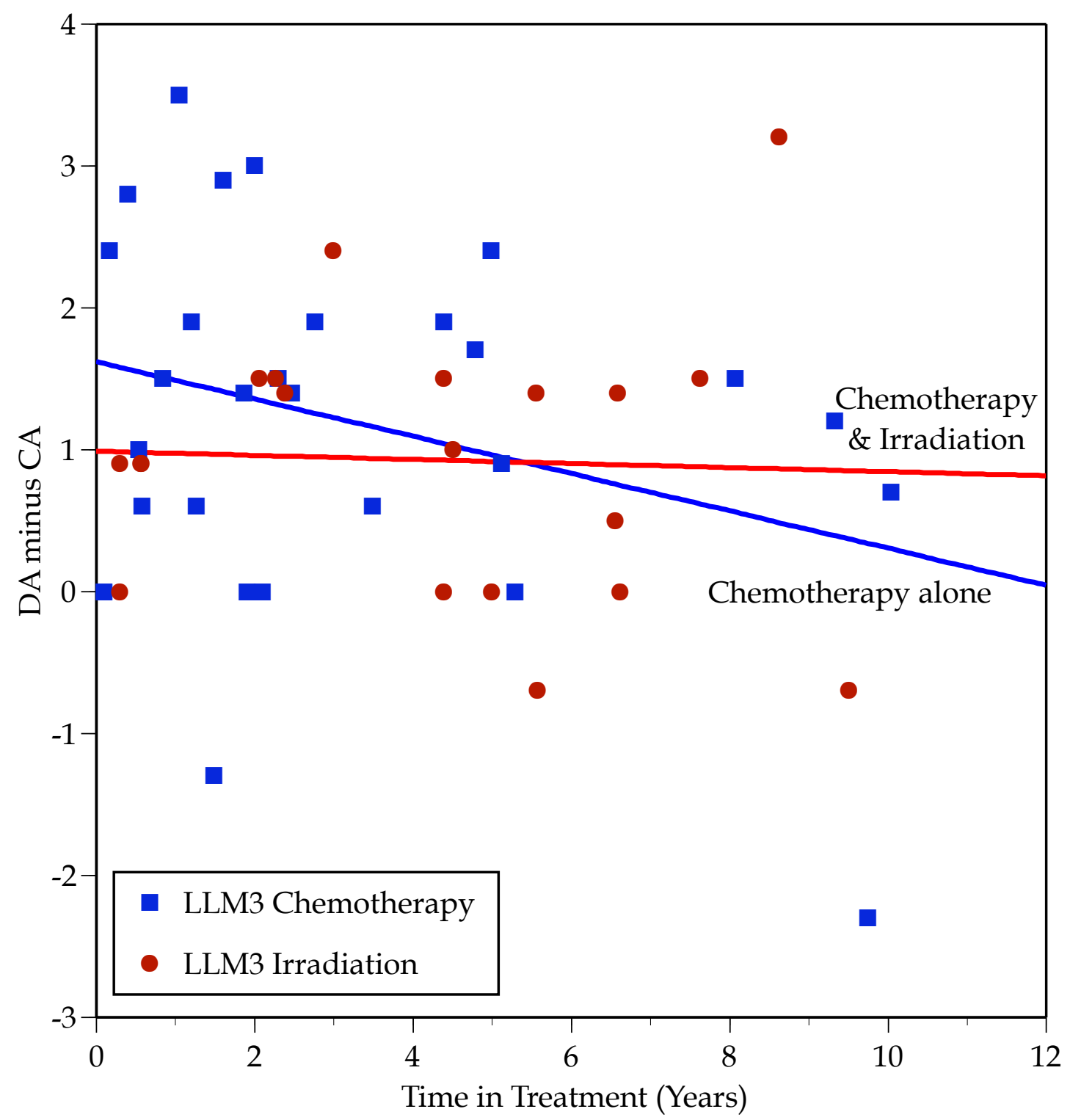

Fig. 34. Plot of time in treatment against DA-CA, with the data (mandibular third molar) partitioned by whether the child was treated with cranial irradiation or chemotherapy alone. 


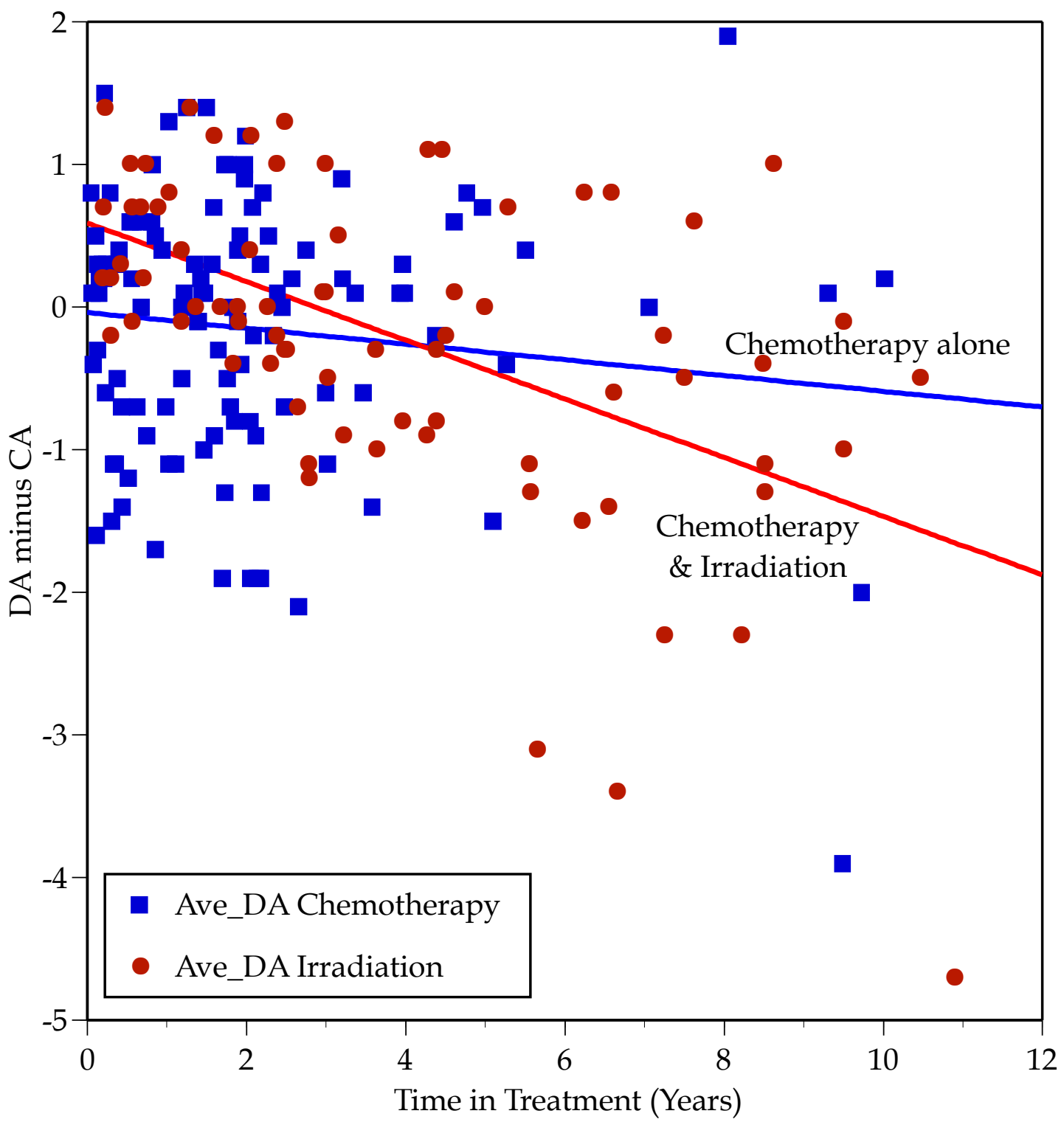

Fig. 35. Plot of time in treatment against DA-CA, with the data (average of all scorable teeth) partitioned by whether the child was treated with cranial irradiation or chemotherapy alone. 


\section{CHAPTER V}

\section{DISCUSSION}

\section{Perspective}

Leukemia is the most frequent form of childhood cancer, comprising about one-third of all cancers in children. Acute lymphoblastic leukemia (ALL) involves approximately 80 percent of all childhood leukemias (Zipf et al. 2000) and was the leading cause of death among children in the United States until the early 1980s (Pui and Evans 1998).

ALL is diagnosed in 2,500 to 3,500 children each year in the United States. This category of disease strikes children primarily between 2 and 10 years of age, with the peak incidence between age 2 and 5 years (Swensen et al. 1997). ALL is more common in white than black children in the United States. It also is more common in boys than girls, and this sex difference is greatest during puberty (Swensen et al. 1997; Haddy 1982).

Although the cause of ALL remains unknown, there are a number of genetic, environmental, viral, and immunological factors that may contribute to development of the disease. An heightened susceptibility to leukemia has been associated with certain heritable diseases, chromosomal disorders, and constitutional syndromes (Mahoney 1999). Exposure to ionizing radiation is an 
environmental factor shown to have carcinogenic effects (Lyon et al. 1979). Several viruses have exhibited associations with leukemia. These include the human T-cell lymphotrophic virus (HTLV-I), which is a retrovirus isolated from a subset of patients with adult T-cell leukemia, the Epstein-Barr virus with African Burkitt's lymphoma, and the HTLV-II virus with atypical hairy-cell leukemia (Perkins et al. 1997; Mahoney 1999). Several immunodeficiency states also carry increased risks for leukemia. These conditions include the syndromes of Wiskott-Aldrich, congenital hypogammaglobulinemia, X-linked agammaglobulinemia, and severe combined immune deficiency (Mahoney 1999; Margolin et al. 2002).

Patients with ALL present most frequently with signs of the uncontrolled growth of leukemic cells in bone marrow, lymphoid structures, and other sites of extramedullary spread (e.g., CNS, testes, lymph nodes, liver, spleen, and kidney) (Berg et al. 2000). The most common presenting symptoms are fever, pallor, purpura, and pain (Mahoney 1999). Bone marrow involvement results in varying degrees of anemia, thrombocytopenia, and granulocytopenia that may be manifested by pallor and fatigue, petechiae, purpura or bleeding, and fever (Berg et al. 2000). Bleeding problems range from mild complications such as petechiae, bruising, and mucosal bleeding, to severe problems such as gastrointestinal bleeding and CNS hemorrhage (Perkins et al. 1997). Oral manifestations of ALL include petechiae and ecchymosis, pallor, ulcers, and 
bleeding (Curtis 1971a). Overall signs and symptoms depend on a range of factors including age of onset, duration of treatment, and subtype of ALL. Since ALL is such a rapidly progressing disease, the radiographic signs are not detectable until later, usually further into the progression of the disease or well into treatment.

The overall prognosis of patients with ALL is promising as rates of remission continue to increase. Remission rates for children are currently near 80 percent, which reflects the obvious progress that has been made in the treatment of ALL (Pui 1997).

Treatments for ALL have negative effects on the normal cells of the body, so children's tempos of growth may be affected iatrogenically by treatment. We hypothesized in the present study that children's tempo of dental maturation would slow during treatment. The purpose of this study was to quantitatively assess the effects of treatment for ALL on the tempo of dental maturation evaluated as dental age (see concepts in Demirjian 1978), including the degree to which the tempo of tooth mineralization is altered during treatment.

\section{Dental Age}

The present retrospective study examined the panoramic radiographs of 72 children ( 31 girls and 41 boys) who had been treated for ALL at St. Jude Children's Research Hospital, Memphis, Tennessee. Mean chronological age at 
diagnosis was 6.8 years $(\mathrm{sd}=2.11)$, with a range of 3.1 to 10.5 years of age.

Panoramic radiographs were taken of these children by the staff pediatric dentist as indicated for routine restorative dental work. As such, radiographs were not taken at any set interval, and the timing and number of films varied appreciably among the children.

Few studies have assessed dental maturation in children treated for ALL. There are, however, many reports in the literature on the various morphological dental abnormalities associated with treatment of the disease (Kaste et al. 1997; Minicucci et al. 2003; Rosenberg et al. 1987), increased caries with chemotherapy (Bertolone et al. 1981; Purdell-Lewis et al. 1988; Pajari et al. 1988), delayed emergence in children treated with chemotherapy (Adatia 1968; Cetiner and Alpaslan 2004), and how these dental abnormalities can affect an orthodontist's treatment Dahllöf et al. (2001). All of these studies describe morphological dental sequelae that occur from the treatment of ALL. It does not appear that any of the aforementioned studies investigated whether treatment discernibly affects these children's tempo of dental development. The present study quantified the dental status (i.e., stage of crown-root formation) of all developing permanent teeth and compared them to sex-specific American white standards published by Harris and McKee (1990).

Statistical analysis showed (1) that DA is unaffected at the onset of treatment and that (2) DA-CA (the extent of delay) increases as treatment 
progressed. One anticipates that, as a child ages, his maturational indicators (e.g., dental age and bone age) should increase apace. A severe stress-like treatment for ALL-should, in turn, slow-down the child's growth tempos (e.g., Kirk et al. 1987; Dahllöf et al. 1989a; Birkebaek and Clausen 1998;). In turn, expectation is that, with resolution of the stressor, the tempos of growth ought to resume to a normal pace.

A study by Tamminga et al. (1993) showed that during treatment for ALL the tempo of bone maturation as well as height growth was retarded in relation to calendar age. A catch-up of bone age and of height was observed in the 2 years after cessation of treatment. Other investigators have used height and weight to assess the development of children treated for ALL. Dalton et al. (2003) found a slight decrease in height in children treated for ALL and received cranial irradiation in addition to chemotherapy. This decrease, however, was not statistically significant when these children were compared to non-irradiated children.

Kirk et al. (1987) reported several cases of diminished stature in children treated with chemotherapy and with irradiation for ALL. Stature had decreased by more than one standard deviation in 32 percent of survivors at 4 years postdiagnosis and in 71 percent at 6 years post-diagnosis. They also found that growth of younger children and those children especially tall for their age at diagnosis experienced greater depression in the rate of growth of stature. 
Holm et al. (1994) and Birkebaek and Clausen (1998) showed that the height standard deviation scores were decreased during treatment only to have catch-up growth and the height standard deviation scores return to normal at the final observation period. Holm et al. (1994) examined 28 patients that were treated for ALL with chemotherapy only and Birkebaek and Clausen (1998) sample included 11 patients with chemotherapy only and 22 that had added irradiation therapy.

\section{$\underline{\text { Response Between Sexes }}$}

There was no sex difference found in the dependency between age and DA-CA. It is known from the literature that boys have a worse prognosis than girls with ALL in terms of survival (Ishii et al. 2001; Shanta et al. 1996; Pui et al. 1999), but this sex difference does not appear to carry over to developmental delay within survivors.

\section{Chemotherapy Treatment Alone}

A study by Macleod et al. (1987) examined the cytotoxic effects of chemotherapy on the formation of dental tissues. Extracted teeth were collected from patients receiving various cytotoxic chemotherapeutic regimens for malignant disease. Children who had received radiotherapy to the jaws were excluded from the study. Slides were prepared to examine incremental lines, 
which correlated with intravenous drug administration, in both the dentine and enamel. The only formative disturbances that were found involved dentinogenesis. In teeth where amelogenesis would have been occurring at the time of treatment, the enamel appeared normal. Furthermore, the dentin that was formed during chemotherapy was hypermineralized compared to dentine formed before and after treatment and no related areas of hypomineralization were seen.

In the present study the data were partitioned into (1) children treated with only chemotherapy and (2) those treated with chemotherapy and irradiation. Examining the data for children treated with chemotherapy, there was no effect on the tempo of dental maturation. Our findings are based on a larger sample and in agreement with Dahllöf et al. (1989b) who reported no statistical difference of dental maturation in patients treated with just chemotherapy for ALL and AML. Their sample consisted of 44 Swedish patients who presented for bone marrow transplant. Each patient was matched with healthy controls for age and sex. Included in the 44 patients, were 28 with the diagnosis of ALL. The method described by Demirjian and Goldstein (1976) was used to score dental maturation in their study. Dahllöf et al. (1989b) compared their results to two studies, namely (1) by Purdell-Lewis et al. (1988) who reported a delay in tooth formation in 8 of 45 long term survivors of childhood cancer treated with chemotherapy and (2) by Adatia (1968) who reported 
delayed emergence eruption in one of 13 patients treated with cyclophosphamide, methotrexate, and vincristine for Burkitt's tumor.

Purdell-Lewis et al. (1988) studied 45 children treated with chemotherapy for various cancers (i.e., ALL, neuroblastoma, Wilm's tumor, rhabdomyosarcoma, histiocytosis $\mathrm{X}$, and acute non-lymphatic leukemia). It is important to note that the 23 ALL patients in the sample of 45 also had prophylactic CNS radiation therapy; the ALL patients studied by Purdell-Lewis et al. (1988) did not receive only chemotherapy. The results of Dahllöf et al. (1989b) should only be comparable to studies that include patients treated for ALL with chemotherapy only. Also, the Adatia (1968) study did not include any ALL patients and had one patient who exhibited delayed emergence (which is a weak indicator of dental maturity). This explains how the sample in the present study serves as the best comparable sample to the study by Dahllöf et al. (1989b).

\section{Cranial Irradiation}

When the data were separated for patients receiving cranial irradiation in addition to chemotherapy, a significant difference was seen. It is know that irradiation therapy targets actively dividing cells and those normal cells within the field of irradiation will be adversely affected. In order for a tooth root to develop the odontoblast must replicate along the long axis of the forming tooth root away from the newly formed crown. When the odontoblasts are irradiated, 
the mitotic activity is altered and the replication slows or ceases. Once the odontoblasts are altered or destroyed, the tooth root has lost the ability to form to its genetic potential.

The tooth crown differs in that the ameloblasts must replicate to form the outline of the crown, but once the initial outline is formed, mitosis no longer occurs. The ameloblasts merely secretes the matrix that calcifies to form enamel. Since the irradiation can affect the initial crown outline form, disturbances of crown formation is seen in the teeth in which crown formation was initiated during treatment with cranial irradiation.

If we examine dental development in normal children, the teeth will eventually reach maturity and, in an unaffected mature tooth, the morphology should resemble the final grade of apex closure shown in Figure 4. In children who received irradiation therapy the developing tooth root is malformed (e.g., stunted or blunted, tapered, open apices, microdont) or missing. These teeth have not had the opportunity to complete normal maturation. We speculate that this explains why we see that the longer a child is in treatment for ALL, with cranial irradiation, the more dentally delayed the child appears. The dental ages of the affected teeth are calculated using morphological grading schemes of normally developing teeth. This proposes a problem in that the affected teeth have, in a sense, matured to their fullest extent possible but they appear under 
developed according to the grades and descriptions in Figure 4 and Table 7, respectively.

Näsman et al. (1997) examined the dental radiographs of 16 children conditioned with total body irradiation and cyclophosphamide prior to bone marrow transplantation, and 52 children treated with multiagent chemotherapy. In the 16 children who had irradiation it was unclear as to how many had ALL. Also, of the 52 children treated with chemotherapy, only 19 had ALL. Additionally, 13 of the 52 children had irradiation along with chemotherapy. This resulted in a reduced sample size to 19 patients diagnosed with ALL and possibly including patients that received additional irradiation along with chemotherapy. Näsman et al. (1997) concluded that (1) when treatment was initiated in children below 12 years of age, all teeth are negatively affected by antineoplastic therapy, (2) children treated with irradiation exhibited significantly more disturbances in dental development than children treated with multiagent chemotherapy, and (3) there was a reduction in tooth size as a result of chemotherapy or radiation therapy, but the children receiving irradiation exhibited the most extensive reduction. Dahllöf et al. (1989b) and Näsman et al. (1997) seem to be the closest comparable studies to that conducted in this thesis. 


\section{Onset of Dental Age Delay}

Our results showed that dental maturation is only slightly affected by chemotherapy alone, whereas chemotherapy combined with irradiation has a decidedly negative effect on dental maturation. Results in the present study show that the delay does not become evident until two years after treatment. We hypothesize this delay is because of (1) the grading scale that was used has approximately six months between grades, (2) the delayed effects on mineralization takes time to become evident radiographically, and (3) the variability in the cross-sectional design of the analysis does not allow examination of intra-individual trends.

\section{Orthodontic Treatment of Survivors}

Dahllöf et al. (2001) conducted a survey of orthodontists who had performed treatment of post-cancer children. The orthodontists reported that (1) the patient's medical condition influenced their choice of treatment plan, (2) they used lighter forces in patients with severely disturbed root development, (3) one orthodontist reported root resorption, and (4) in 4 of 10 patients, the treatment result was judged to be unsatisfactory. They concluded that although ideal treatment results were not always achieved, orthodontic treatment did not produce harmful side effects in children who are long-term survivors of childhood cancer. 


\section{CHAPTER VI}

\section{SUMMARY AND CONCLUSIONS}

Leukemia is the most frequent form of childhood cancer, comprising about one-third of all cancers in children. This category of disease strikes children primarily between 2 and 10 years of age, with the peak incidence of ALL occurring between age 2 and 5 years. Treatment strategies for ALL consist of chemotherapy with or without irradiation of the neck and/or spine.

The present study used panoramic radiographs to determine the dental maturation of children treated for ALL. Dental age based on the degrees of crown-root development was assessed on a total of 190 panoramic radiographs from 72 children (41 boys, 31 girls) treated at St. Jude Children's Research Hospital, Memphis, Tennessee. Mean chronological age at diagnosis was 6.8 years $(\mathrm{sd}=2.11)$, with a range of 3.1 to 10.5 years. Dental ages for each radiograph were scored using the grading schemes of Moorrees, Fanning and Hunt (1963) and the published norms of Harris and McKee (1990).

The children's dental ages were normal at diagnosis for ALL and before commencement of treatment. We supposed that treatments for ALL would discernibly depress the children's tempos of growth, an analysis confirmed this hypothesis. 
In the children receiving only chemotherapy, treatment did not affect dental development to any statically significant extent. Due to the severe stress irradiation has on the dentition, children receiving both chemotherapy and irradiation experienced a decided delay in dental development, but the effects were not seen radiographically until 2 years after the start; the longer the children were into treatment the more the dental age was delayed.

Although orthodontic treatment might not produce any harmful side effects, the orthodontist must be aware of the dental conditions and disturbances which exist in patients treated for ALL. The orthodontist should also consider the dental sequelae of cranial irradiation, such as missing or malformed teeth, delayed development and eruption, and shortened or tapered roots that can alter the normal course of orthodontic treatment. 


\section{LIST OF REFERENCES}


Adatia AK. Response of dental elements to chemotherapy of Burkett's tumor. Int Dent J 1968;18:646-54.

Anderson DL, Thompson GW, Popovich F. Age of attainment of mineralization stages of the permanent dentition. J Forensic Sci 1976;21:191-200.

Anderson WAD, Scotti TM. Synopsis of pathology. St. Louis: CV Mosby, 1980.

Arey LB. Developmental anatomy: a textbook and laboratory manual of embryology, 7th ed. Philadelphia: WB Saunders Company, 1965.

Ash MM Jr. Wheeler's dental anatomy, physiology and occlusion, 8th ed. Philadelphia: WB Saunders Company, 2003.

Avery JK. Pulp. In: Bhasker SN, editor. Orban's Oral histology and embryology, 10th ed. St. Louis: CV Mosby Company, p 135-74.

Bailit HL. Dental variation among populations: an anthropologic view. Dent Clin N Am 1975;19:125-39.

Bailit HL; Doykos JD; Swanson LT. Dental development in children with cleft palates. J Dent Res 1968;47:664.

Bennett JM, Catovsky D, Daniel MT, Flandrin G, Galton DA, Gralnick HR, Sultan C. Proposals for the classification of the acute leukaemias. FrenchAmerican-British (FAB) co-operative group. Br J Haematol 1976;33:451-8.

Bennett JM, Catovsky D, Daniel MT. French-American-British (FAB) cooperative group: the morphological classification of acute leukemias-concordance among observers and clinical correlation. Br J Haematol 1981;47:553-61. 
Berg SL, Steuber CP, Poplack DG. Clinical manifestations of acute lymphoblastic leukemia. In: Hoffman R, Benz EJ, Shattil SJ, Furie B, Cohen HJ, Silberstein LE, McGlave P, editors. Hematology: basic principles and practices, 3rd ed. New York: Churchill Livingstone, 2000, p 1070-89.

Birkebaek NH, Clausen N. Height and weight pattern up to 20 years after treatment for acute lymphoblastic leukaemia. Arch Dis Child 1998;79:161-4.

Beertsen W, Niehof A. Root-analogue versus crown-analogue dentin: a radioautographic and ultrastructural investigation of the mouse incisor. Anat Rec 1986;215:106-18.

Bertolone SJ, Burzynski NJ, Borden D. Dental care in children with acute lymphocytic leukemia. South Med J 1981;74:976-8.

Bessho F. Height at diagnosis in acute lymphocytic leukaemia. Arch Dis Child 1986;61:296-8.

Bhaskar SN. Orban's oral histology and embryology, 11th ed. St. Louis: CV Mosby Company, 1991.

Birkebaek NH, Clausen N. Height and weight pattern up to 20 years after treatment for acute lymphoblastic leukaemia. Arch Dis Child 1998;79:161-4.

Bodey G, Buckley M, Sathe U. Quantitative relationships between circulating leukocytes and infections in patients with acute leukemia. Ann Intern Med 1966;64:328-40. 
Bowers EJ, Mayro RF, Whitaker LA, Pasquariello PS, Larossa D, Randall P.

General body growth in children with clefts of the lip, palate and craniofacial structures. Scand J Plast Reconstr Surg 1987;21:7-14.

Broomhall J, May R, Lilleyman JS, Milner RD. Height and lymphoblastic leukaemia. Arch Dis Child 1983;58:300-1.

Butler PM. Ontogeny of the molar pattern. Biol Rev 1956;31:30-70.

Butler PM. The prenatal development of the human upper permanent molar. Arch Oral Biol 1967a;12:551-63.

Butler PM. Relative growth within the human first upper permanent molar during the prenatal period. Arch Oral Biol 1967b;12:983-92.

Butler PM. Growth of the human second lower deciduous molar. Arch Oral Biol $1968 ; 13: 671-82$

Cetiner S, Alpaslan C. Long-term effects of cancer therapy on dental development: a case report. J Clin Pediatr Dent 2004;28:351-3.

Chessells JM, Bailey C, Richards SM. Intensification of treatment and survival in all children with lymphoblastic leukemia: results of UK Medical Research Council trial UKALL X. Lancet 1995;345:143-8.

Christensen GJ, Kraus BS. Initial calcification of the human permanent first molar. J Dent Res 1965;44:1338-42. 
Conter V, Aricò M, Valsecchi MG, et al. Extended intrathecal methotrexate may replace cranial irradiation for prevention of CNS relapse in children with intermediate-risk acute lymphoblastic leukemia treated with BerlinFrankfurt-Munster-based intensive chemotherapy. J Clin Oncol $1995 ; 13: 2497-2502$.

Cooper HK, Harding RL, Krogman WM, editors. Cleft palate and cleft lip: a team approach to clinical management and rehabilitation of he patient. Philadelphia: WB Saunders Company, 1979.

Corliss CE. Patton's human embryology: elements of clinical development. New York: McGraw-Hill Book Company, 1976.

Cortes JE, Kantarjian HM. Acute lymphoblastic leukemia: a comprehensive review with emphasis on biology and therapy. Cancer 1995;76:2393-417.

Cox MA, editor. Toronto research institute, the cleft lip and palate treatment center. A five year report, 1955-1959. Toronto: Hospital for Sick Children, 1960.

Curtis AB. Childhood leukemias: initial oral manifestations. J Am Dent Assoc 1971a;83:159-64.

Curtis AB. Childhood leukemias: osseous changes in jaws of panoramic dental radiographs. J Am Dent Assoc 1971b;83:844-7. 
Dahl E. Craniofacial morphology in congenital clefts of the lip and palate. Acta Odont Scand 1970;28:13-165.

Dahlberg AA. Evolutionary background of dental and facial growth. J Dent Res 1965;44:151-60.

Dahlberg AA, Menegaz-Bock RM. Emergence of the permanent teeth in Pima Indian children: a critical analysis of method and an estimate of population parameters. J Dent Res 1958;37:1123-40.

Dahllof G, Forsberg CM, Ringden O, Bolme P, Borgstrom B, Nasman M, Heimdahl A, Modeer T. Facial growth and morphology in long-term survivors after bone marrow transplantation. Eur J Orthod 1989a;11:332-40.

Dahllöf G, Jönsson A, Ulmner M, Huggare J. Orthodontic treatment in long-term survivors after pediatric bone marrow transplantation. Am J Orthod Dentofacial Orthop 2001;120:459-65.

Dahllöf G, Näsman M, Borgström A, Modéer T, Forsberg CM, Heimdahl A, Ringdén O. Effect of chemotherapy on dental maturity in children with hematological malignancies. Pediatr Dent 1989b;11:303-6.

Dalton VK, Rue M, Silverman LB, Gelber RD, Asselin BL, Barr RD, Clavell LA, Hurwitz CA, Moghrabi A, Samson Y, Schorin M, Tarbell NJ, Sallan SE, Cohen LE. Height and weight in children treated for acute lymphoblastic leukemia: relationship to CNS treatment. J Clin Oncol 2003;21:2953-60.

Demirjian A. Dentition. In: Falkner F, Tanner JM, editors. Human growth 2. postnatal growth. New York and London: Plenum Press, 1978, p 412-44. 
Demirjian A, Goldstein H. New systems for dental maturity based on seven and four teeth. Ann Hum Biol 1976;3:411-21.

Demirjian A, Goldstein H, Tanner JM. A new system of dental age assessment. Hum Biol 1973;45:211-27.

Demisch A, Wartmann P. Calcification of the mandibular third molar and its relation to skeletal and chronological age in children. Child Dev 1956;27:459-73.

Drillien CM, Ingram TTS, Wilkinson EM. The causes and natural history of cleft lip and palate. London: E \& S Livingstone, 1966.

Fanning EA. A longitudinal study of tooth formation and root resorption. NZ Dent J 1961;57:202-17.

Fanning EA. Effect of extraction of deciduous molars on the formation and eruption of their successor. Angle Orthod 1962;32:44-53.

Farah CS, Booth DR, Knott SC. Dental maturity of children in Perth, Western Australia, and its application in forensic age estimation. J Clin Forensic Med 1999;6:14-8.

Filipsson R. A new method for assessment of dental maturity using the individual curve of number of erupted permanent teeth. Ann Hum Biol $1975 ; 2: 13-24$.

Foon KA, Todd RF 3rd. Immunologic classification of leukemia and lymphoma. Blood 1986;68:1-31. 
Foote JS. When and how we acquired out teeth, part one-fishes. In: Bone as a measure of development when and how we acquired out teeth. Omaha: Press Douglas Printing Company, 1928, p 2.

Gallagher D, Phillips D, Heinrich S. Orthopedic manifestations of acute pediatric leukemia. Orthop Clin North Am 1996;27:635-44.

Garn SM, Lewis AB, Koski K, Polacheck DL. The sex difference in tooth calcification. J Dent Res 1958;37:561-7.

Garn SM, Lewis AB, Polacheck DL. Variability of tooth formation. J Dent Res 1959;38:135-48.

Gleiser I, Hunt EE Jr. The permanent mandibular first molar: its calcification, eruption and decay. Am J Phys Anthropol 1955;13:253-83.

Graber TM. Panoramic radiography in orthodontic diagnosis. Am J Orthod 1967;53:799-821.

Greenlee RT, Murray T, Bolden S, Wingo PA. Cancer statistics, 2000. CA Cancer J Clin 2000;50:7-34.

Gregory WK. The origin an evolution of the human dentition. Baltimore: Williams \& Wilkins Company, 1922.

Griffin NK, Wadsworth J. Effect of treatment of malignant disease on growth in children. Arch Dis Child 1980;55:600-3.

Grøn AM. Prediction of tooth emergence. J Dent Res 1962:41:573-85.

Gurney JG, Severson RK, Davis S, Robison LL. Incidence of cancer in children in the United States. Cancer 1995;75:2186-95. 
Gunz FW, Gunz JP, Vincent PC, Bergin M, Johnson FL, Bashir H, Kirk RL.

Thirteen cases of leukemia in a family. J Natl Cancer Inst 1978;60:1243-50.

Haddy TB. Cancer in black children. Am J Pediatr Hematol Oncol 1982;4:285-92.

Halton JM, Atkinson SA, Barr RD. Growth and body composition in response to chemotherapy in children with acute lymphoblastic leukemia. Int J Cancer Suppl 1998;11:81-4.

Halton JM, Atkinson SA, Fraher L, Webber CE, Cockshott WP, Tam C, Barr RD. Mineral homeostasis and bone mass at diagnosis in children with acute lymphoblastic leukemia. J Pediatr 1995;126:557-64.

Harris EF. Tooth mineralization standards for the mandibular third molar in American blacks and whites. Am J Phys Anthropol 1998;26S:90 [Abstract]

Harris EF. Dental development and anomalies in craniosynostoses and facial clefting. In: Mooney MP, Siegel MI, editors. Understanding craniofacial anomalies: the etiopathogenesis of craniosynostoses and facial clefting. New York: Wiley-Liss, 2002, p 425-67.

Harris EF, Buck AL. Tooth mineralization: a technical note on the moorreesfanning-hunt standards. Dent Anthropol 2002;16:15-20.

Harris EF, Hullings JG. Delayed dental development in children with isolated cleft lip and palate. Arch Oral Biol 1990;35:469-73.

Harris EF, McKee JH. Tooth mineralization standards for blacks and whites from the middle southern United States. J Forensic Sci 1990;35:859-72. 
Haavikko K. Tooth formation age estimated on a few selected teeth: a simple method for clinical use. Proc Finn Dent Soc 1974;70:15-9.

Hillson S, Bond S. Relationship of enamel hypoplasia to the pattern of tooth crown growth: a discussion. Am J Phys Anthropol 1997;104:89-103.

Holm K, Nysom K, Hertz H, Muller J. Normal final height after treatment for acute lymphoblastic leukemia without irradiation. Acta Paediatr 1994;83:1287-90.

Hunter WS, Dijkman DJ. The timing of height and weight deficits in twins discordant for cleft of the lip and/or palate. Cleft Palate 1977;14:158-66.

Ishii E, Eguchi H, Matsuzaki A, Koga H, Yanai F, Kuroda H, Kawakami K, Ayukawa H, Akiyoshi K, Kamizono J, Tamai Y, Kinukawa N, Okamura J. Outcome of acute lymphoblastic leukemia in children with AL90 regimen: impact of response to treatment and sex difference on prognostic factors. Med Pediatr Oncol 2001;37:10-9.

Jaruratanasirikul S, Owasith K, Wongchanchailert M, Laosombat V, Sriplung H. Growth patterns and final height of survivors of childhood leukemia. J Pediatr Endocrinol 2004;17:719-26.

Jensen BL, Dahl E, Kreiborg S. Longitudinal study of body height, radius length and skeletal maturity in Danish boys with cleft lip and palate. Scand J Dent Res 1983;91:473-81. 
Jernvall J, Keränen SV, Thesleff I. Evolutionary modification of development in mammalian teeth: quantifying gene expression patterns and topography. Proc Natl Acad Sci U S A 2000;97:14444-8.

Jernvall J, Kettunen P, Karavanova I, Martin LB, Thesleff I. Evidence for the role of the enamel knot as a control center in mammalian tooth cusp formation: non-dividing cells express growth stimulating Fgf-4 gene. Int J Dev Biol 1994;38:468-9.

Kaste SC, Hopkins KP, Jones D, Crom D, Greenwald CA, Santana VM. Dental abnormalities in children treated for acute lymphoblastic leukemia. Leukemia 1997;11:792-6.

Kirk JA, Raghupathy P, Stevens MM, Cowell CT, Menser MA, Bergin, M, Tink A, Vines RH, Silink M. Growth failure and growth-hormone deficiency after treatment for acute lymphoblastic leukemia. Lancet 1987;1:190-3.

Kraus BS, Jordan RE. The human dentition before birth. Philadelphia: Lea and Febiger, 1965.

Konior GS, Leventhal BG. Immunocompetence and prognosis in acute leukemia. Semin Oncol 1976;3:283-8.

Korenhof CAW. Morphogenetical aspects of the human upper molar. Utrecht: Uitgeversmaatschappij Neerlandia, 1960.

Kraus BS. Morphologic relationships between enamel and dentin surfaces of the lower first molar teeth. J Dent Res 1952;31:248-256. 
Kraus BS, Jordan RE. The human dentition before birth. Philadelphia: Lea and Febiger, 1965.

Kronfeld R. Development and calcification of the human decidous dentition. The Bur 1935a;15:18-25.

Kronfeld R. First permanent molar: its condition at birth and its postnatal development. J Am Dent Assoc 1935b;22:1131-55.

Levesque GY, Demirjian A. The inter-examiner variation in rating dental formation from radiographs. J Dent Res 1980;59:1123-6.

Liversidge HM. Dental maturation of 18th and 19th century British children using Demirjian's methods. Int J Paediatr Dent 1999;9:111-5.

Liversidge HM. Variation in modern human dental development. In: Thompson JL, Krovitz GE, Nelson AJ, eds. Patters of growth and development in the genus Homo. Cambridge: Cambridge University Press, 2003, p 73-113.

Liversidge HM, Speechly T, Hector MP. Dental maturation in British children: are Demirjian's standards applicable? Int J Paediatr Dent 1999;9:263-9.

Luukko K, Loes S, Furmanek T, Fjeld K, Kvinnsland IH, Kettunen P. Identification of a novel putative signaling center, the tertiary enamel knot in the postnatal mouse molar tooth. Mech Dev 2003;120:270-6.

Lyon JL, Klauber MR, Gardner JW, Udall KS. Childhood leukemias associated with fallout from nuclear testing. New Engl J Med 1979;300:397-402. 
Macleod RI, Welbury RR, Soames JV. Effects of cytotoxic chemotherapy on dental development. J R Soc Med 1987;80:207-9.

Mahoney DH III. Acute lymphoblastic leukemia. In: McMillan JA, editors. Oski's pediatric principles and practice, 3rd ed. Philadelphia: Lippincott Williams \& Wilkins, 1999, p 1491-1501.

Margolin JF, Steuber CP, Poplack DG. Acute lymphoblastic leukemia. In: Pizzo PA, Poplack DG, editors. Principles and practice of pediatric oncology, 4th ed. Philadelphia: Lippincott Williams \& Wilkins, 2002, p 489-544.

Melfi RC. Permar's oral embryology and microscopic anatomy. Philadephia: Lea and Febiger, 1994.

Menius JA, Largent MD, Vincent CJ. Skeletal development of cleft palate children as determined by hand-wrist roentgenographs, a preliminary study. Cleft Palate J 1966;3:67-75.

Michaud M, Baehner RL, Bixler D, Kafrawy AH. Oral manifestations of acute leukemia in children. J Am Dent Assoc 1977;95:1145-50.

Miller DR, Leikin S, Albo V, Sather H, Hammond D. Prognostic importance of morphology (FAB classification) in childhood acute lymphoblastic leukemia (ALL). Br J Haematol 1981;48:199-206.

Minicucci EM, Lopes LF, Crocci AJ. Dental abnormalities in children after chemotherapy treatment for acute lymphoid leukemia. Leuk Res 2003;27:45-50. 
Moore SM. Tooth mineralization in acute lymphocytic leukemia children undergoing chemotherapy. Masters Thesis, University of Tennessee Health Science Center, 1982.

Moorrees CFA, Fanning EA, Hunt Jr. EE. Age variation of formation stages in ten permanent teeth. J Dent Res 1963;42:1490-502.

Moss-Salentijn L. Dental and oral tissues. Philadelphia: Lea and Febiger, 1990.

Näsman M, Forsberg CM, Dahllöf G. Long-term dental development in children after treatment for malignant disease. Eur J Orthod 1997;19:151-9.

Nielsen HG, Ravn JJ. A radiographic study of mineralization of permanent teeth in a group of children aged 3-7 years. Scand J Dent Res 1976;84:109-18.

Niemeyer CM, Sallan SE. Acute lymphoblastic leukemia. In Nathan DG, Orkin SH. Nathan and Oski's hematology of infancy and childhood. Philadelphia: WB Saunders, 1998, p 1245-69.

Niswander JD. Effects of heredity and environment on development of dentition. J Dent Res 1963;42:1288-96.

Nolla CM. The development of the permanent teeth. J Dent Child 1960;27:25466.

Nykanen R, Espeland L, Kvaal SI, Krogstad O. Validity of the Demirjian method for dental age estimation when applied to Norwegian children. Acta Odontol Scand 1998;56:238-44. 
Olze A, Taniguchi M, Schmeling A. Zhu BL, Yamada Y, Maeda H, Geserick G. Comparative study on the chronology of third molar mineralization in a Japanese and a German population. Leg Med (Tokyo) 2003;5:S256-60.

Ooë T. Human tooth and dental arch development. Tokyo: Ishiyaku Publishers, Inc, 1981.

Ooë T, Nomata N. Mesio-distal growth of the deciduous front tooth germs in man. Bull Tokyo Med Dent Univ 1964;10:513-522.

Osborn JW, Ten Cate AR. Advanced dental histology, 3rd ed. Bristol: John Wright and Sons, 1976.

Pajari U, Lahtela P, Lanning M, Larmas M. Effect of anti-neoplastic therapy on dental maturity and tooth development. J Pedod 1988;12:266-274.

Peretz B, Nevis N, Smith P. Morphometric variables of developing primary maxillary first molar crowns in humans. Arch Oral Biol 1997;42:423-7.

Peretz B, Nevis N, Smith P. Morphometric analysis of developing crowns of maxillary primary second molars and permanent first molars in humans. Arch Oral Biol 1998a;43:525-33.

Peretz B, Shapira J, Farbstein H, Arieli E, Smith P. Modified cuspal relationships of mandibular molar teeth in children with Down's syndrome. J Anat 1998b;193:529-33. 
Perkins ML, Odell JM, Braziel RM. Introduction to leukemia and the acute leukemias. Hematopoiesis: morphology of human blood and marrow cells. In Harmening DM. Clinical hematology and fundamentals of hemostasis. Philadelphia: FA Davis Company, 1997, p 294-320.

Pinney LC. Calcification and development of mandibular teeth. Masters Thesis, University of Michigan, 1939;59.

Provenza DV. Oral histology, inheritance and development. Philadelphia: Lea and Febiger, 1986.

Pui CH. Childhood leukemias. N Engl J Med 1995;332:1618-30.

Pui CH. Acute lymphoblastic leukemia. Pediatr Clin North Am 1997;44:831-46.

Pui CH, Boyett JM, Relling MV, Harrison PL, Rivera GK, Behm FG, Sandlund JT, Ribeiro RC, Rubnitz JE, Gajjar A, Evans WE. Sex differences in prognosis for children with acute lymphoblastic leukemia. J Clin Oncol 1999;17:81824.

Pui CH, Evans WE. Acute lymphoblastic leukemia. N Engl J Med 1998;339:60515.

Pullen J, Boyett J, Shuster J, Crist W, Land V, Frankel L, Iyer R, Backstrom L, van Eys J, Harris M, et al. Extended triple intrathecal chemotherapy trial for prevention of CNS relapse in good-risk and poor-risk patients with Bprogenitor acute lymphoblastic leukemia: a Pediatric Oncology Group Study. J Clin Oncol 1993;11:839-49. 
Purdell-Lewis DJ, Stalman MS, Leeuw JA, Humphrey GB, Kalsbeek H. Long term results of chemotherapy on the developing dentition: caries risk and developmental aspects. Community Dent Oral Epidemiol 1988;16:68-71.

Ranta R. Asymmetric tooth formation in the permanent dentition of cleftaffected children. Scand J Plast Reconstr Surg 1973a;7:59-63.

Ranta R. Development of asymmetric tooth pairs in the permanent dentition of cleft-affected children. Proc Finn Dent Soc 1973b;69:71-5.

Ranta R. Associations of some variables to tooth formation in children with isolated cleft palate. Scand J Dent Res 1984;92:496-502.

Roche AF. Bone growth and maturation. In: Falkner F, Tanner JM. Human growth, postnatal growth. 2nd ed. New York: Plenum Press, 1986.

Rosenberg SW, Kolodney H, Wong GY, Murphy ML. Altered dental development in long-term survivors of pediatric acute lymphoblastic leukemia. Cancer 1987;59:1640-8.

Ross RB. Cranial base in children with lip and palate clefts. Cleft Palate J 1965;2:157-166.

Ross RB, Johnston MC. Cleft lip and palate. Baltimore: Williams and Wilkins, 1972.

Rubnitz JE, Look AT. Pathobiology of acute lymphoblastic leukemia. In: Hoffman R, Benz EJ, Shattil SJ, Furie B, Cohen HJ, Silberstein LE, McGlave P, editors. Hematology: basic principles and practice, 3rd ed. New York: Churchhill Livingstone, 2000, p 1052-89. 
Salazar-Ciudad I, Jernvall J, Newman SA. A gene network model accounting for development and evolution of mammalian teeth. Proc Natl Acad Sci U S A 2002;99:8116-20.

Shanta V, Maitreyan V, Sagar TG, Gajalakshmi CK, Rajalekshmy KR. Prognostic variables and survival in pediatric acute lymphoblastic leukemias: cancer institute experience. Pediatr Hematol Oncol 1996;13:205-16.

Shusterman S, Meadows AT. Long term survivors of childhood leukemia. Curr Opin Hematol 2000;7:217-22.

Slavkin HC. Embryonic tooth formation: a tool for developmental biology. Oral Sci Rev 1974:6-136.

Smith MA, Chen T, Simon R. Age-specific incidence of acute lymphoblastic leukemia in US children: in utero initiation model. J Natl Cancer Inst 1997;89:1542-44.

Sokal RR, Rohlf FJ. Statistical tables. New York: Freeman, 1995.

Sonis ST, Sonis AL, Lieberman A. Oral complications in patients receiving treatment for malignancies other than the head and neck. J Am Dent Assoc 1978;97:468-72.

Sperber GH. Craniofacial development. Hamilton, ON: BC Decker, Inc., 2001.

Steinfort J, van den Bos T, Beertsen W. Differences between enamel-related and cementum-related dentin in the rat incisor with special emphasis on the phosphoproteins. J Biol Chem 1989;264:2840-5. 
Stewart A, Webb J, Hewitt D. A survey of childhood malignancies. Br Med J 1958;30:1495-508.

Stokes ME, Davis CS, Koch GG. Categorical data analysis using the $\mathrm{SAS}^{\circledR}$ system. Cary, NC: SAS Institute Inc., 1995.

Swensen AR, Ross JA, Severson RK, Pollock BH, Robison LL. The age peak in childhood acute lymphoblastic leukemia: exploring the potential relationship with socioeconomic status. Cancer 1997;79:2045-51.

Swindler DR. Primate dentition: an introduction to the teeth of non-human primates. Cambridge: Cambridge University Press, 2002.

Tamminga RY, Zweens M, Kamps W, Drayer N. Longitudinal study of bone age in acute lymphoblastic leukaemia. Med Pediatr Oncol 1993;21:14-8.

Tanner JM. Growth at adolescence. 2nd ed. Oxford: Blackwell Scientific Publications, 1962.

Tanner JM, Whitehouse RH, Marshall WA, Healy MJ, Goldstein H. Assessment of skeletal maturity and prediction of adult height (tw2) method. London: Academic Press, 1975.

Teivens A, Mornstad H. A comparison between dental maturity rate in the Swedish and Korean populations using a modified Demirjian method. J Forensic Odontostomatol 2001;19:31-5.

Ten Cate AR. A fine structural study of coronal and root dentinogensis in the mouse: observations on the so-called 'von Korff fibres' and their contribution to mantle dentine. J Anat 1978;125:183-97. 
Ten Cate AR. Oral histology: development, structure, and function, 4th ed. St Louis: CV Mosby Company, 1994.

Thesleff I, Keranen S, Jernvall J. Enamel knots as signaling centers linking tooth morphogenesis and odontoblast differentiation. Adv Dent Res 2001;15:14-8.

Thomas HF. Root formation. Int J Dev Biol 1995;39:231-7.

Tompkins RL. Human poplulation variability in relative dental development. Am J Phys Anthropol 1996a;99:79-102.

Tompkins RL. Relative dental development of Upper Pleistocene hominids compared to human population variation. Am J Phys Anthropol 1996b;99:103-18.

Tubergen DG, Gilchrist GS, O'Brien RT, Coccia PF, Sather HN, Waskerwitz MJ, Hammond GD. Prevention of CNS disease in intermediate-risk acute lymphoblastic leukemia: comparison of cranial radiation and intrathecal methotrexate and the importance of systemic therapy: a Childrens Cancer Group report. J Clin Oncol 1993;11:520-6.

Turner EP. Crown development in human deciduous teeth. Arch Oral Biol 1963;8:523-50.

Van Wieringen JC. Secular growth changes. In: Falkner F, Tanner JM. Human growth, postnatal growth. 2nd ed. New York: Plenum Press, 1986.

Winer BJ, Brown DR, Michels KM. Statistical principles in experimental design. 3rd ed. New York: McGraw-Hill, 1991. 
Yamashita N, Tanaka J, Moriwake T, Nishiuchi R, Oda M, Seino Y. Analysis of linear growth in survivors of childhood acute lymphoblastic leukemia. J Bone Miner Metab 2003;21:172-8.

Zeisz RC, Nuckolls J. Dental anatomy. St Louis: CV Mosby Company, 1949.

Zipf TF, Berg SL, Roberts WM, Poplack DG, Steuber CP, Bleyer WA. Childhood leukemias. In: Abeloff MD, Armitage JO, Lichter AS, Niederhuber JE, editors. Clinical oncology, 2nd ed. New York: Churchill Livingstone, 2000, p 2402-34. 
APPENDICES 


\section{Appendix A}

Plots of dental age (averaged over all scorable teeth) against chronological age. 


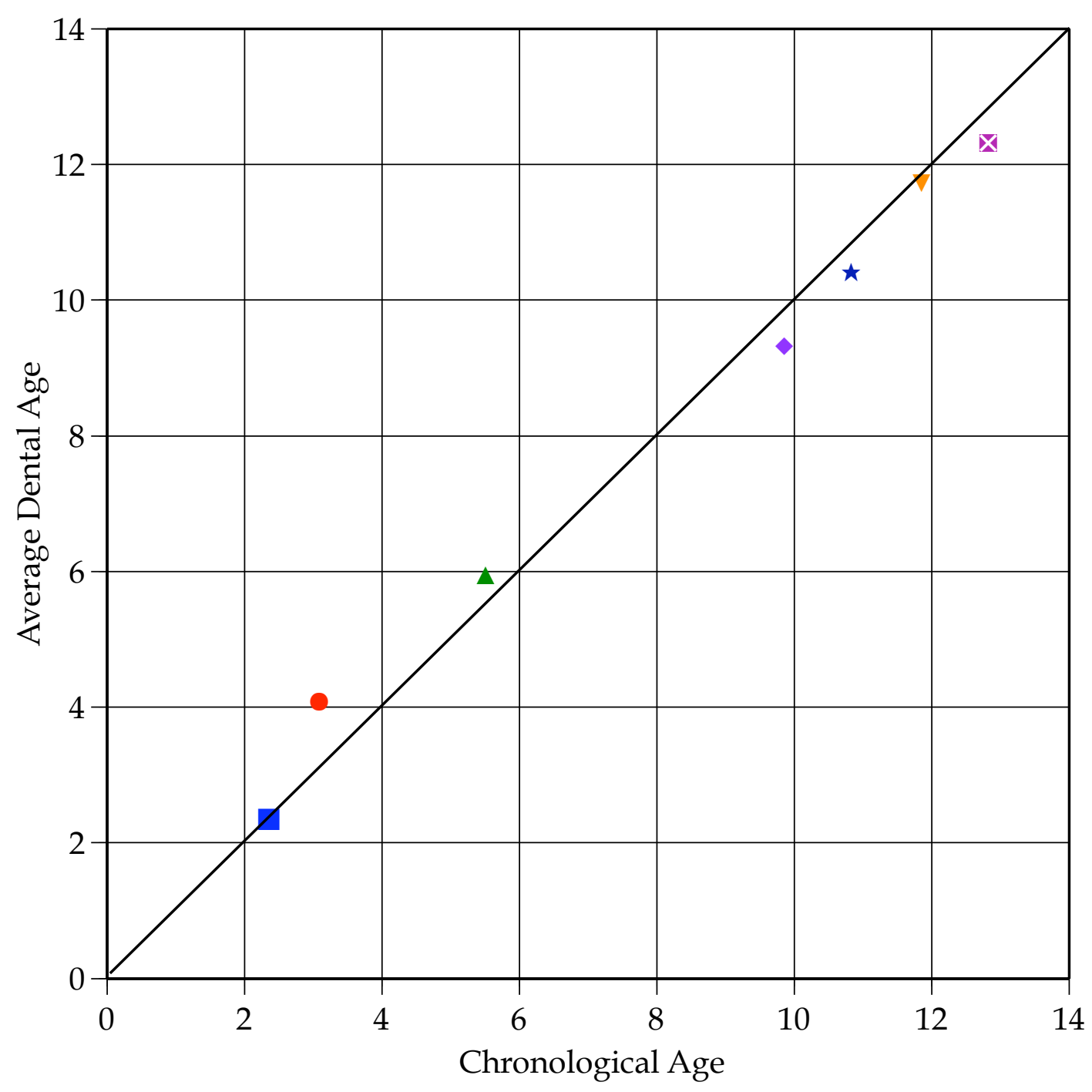

Fig. A-1. Plot of chronological age against dental age averaged across all scorable teeth (symbols) for a male (pseudo-record \#1). The square symbol denotes age at diagnosis of ALL. 


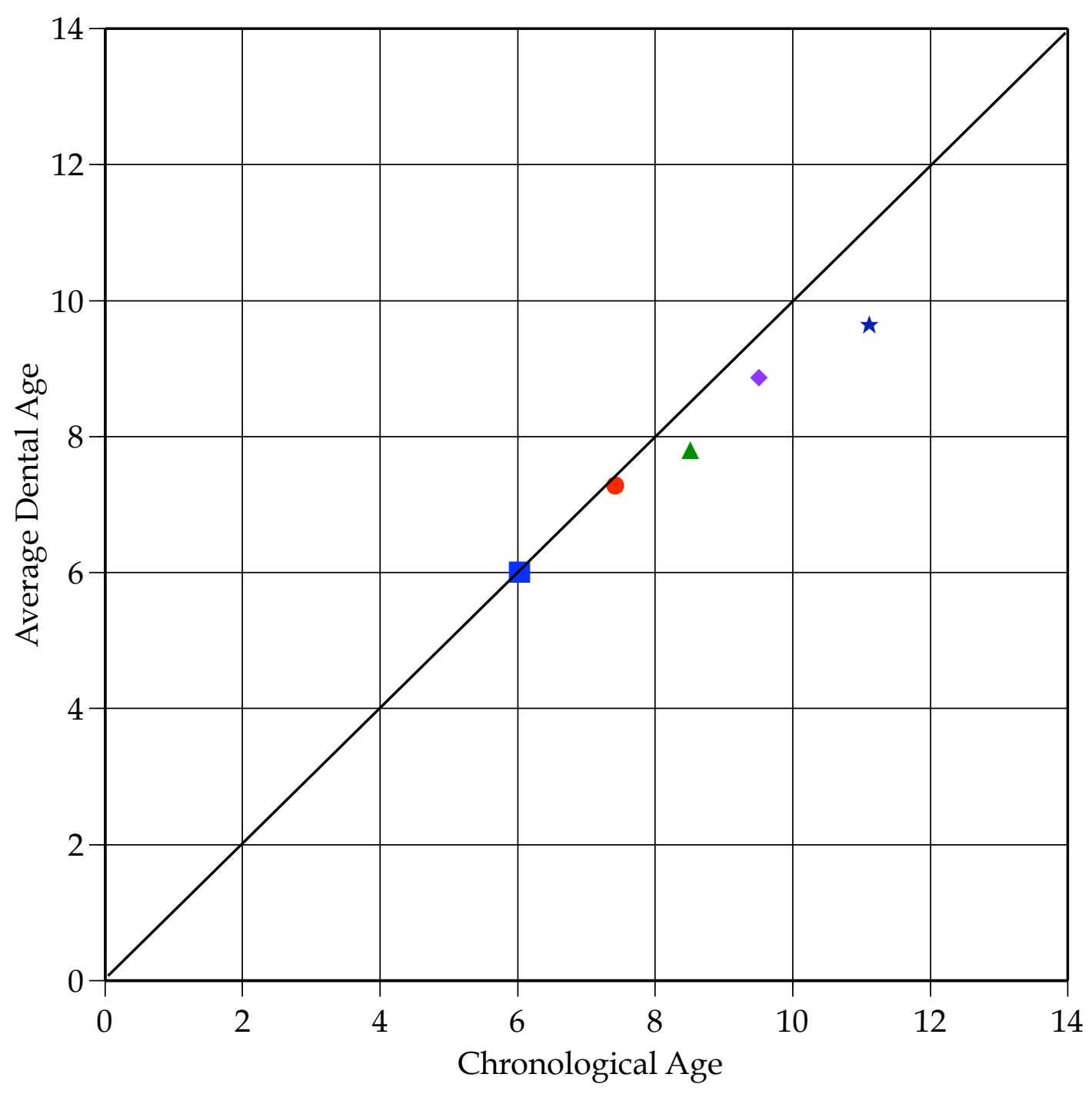

Fig. A-2. Plot of chronological age against dental age averaged across all scorable teeth (symbols) for a male (pseudo-record \#2). The square symbol denotes age at diagnosis of ALL. 


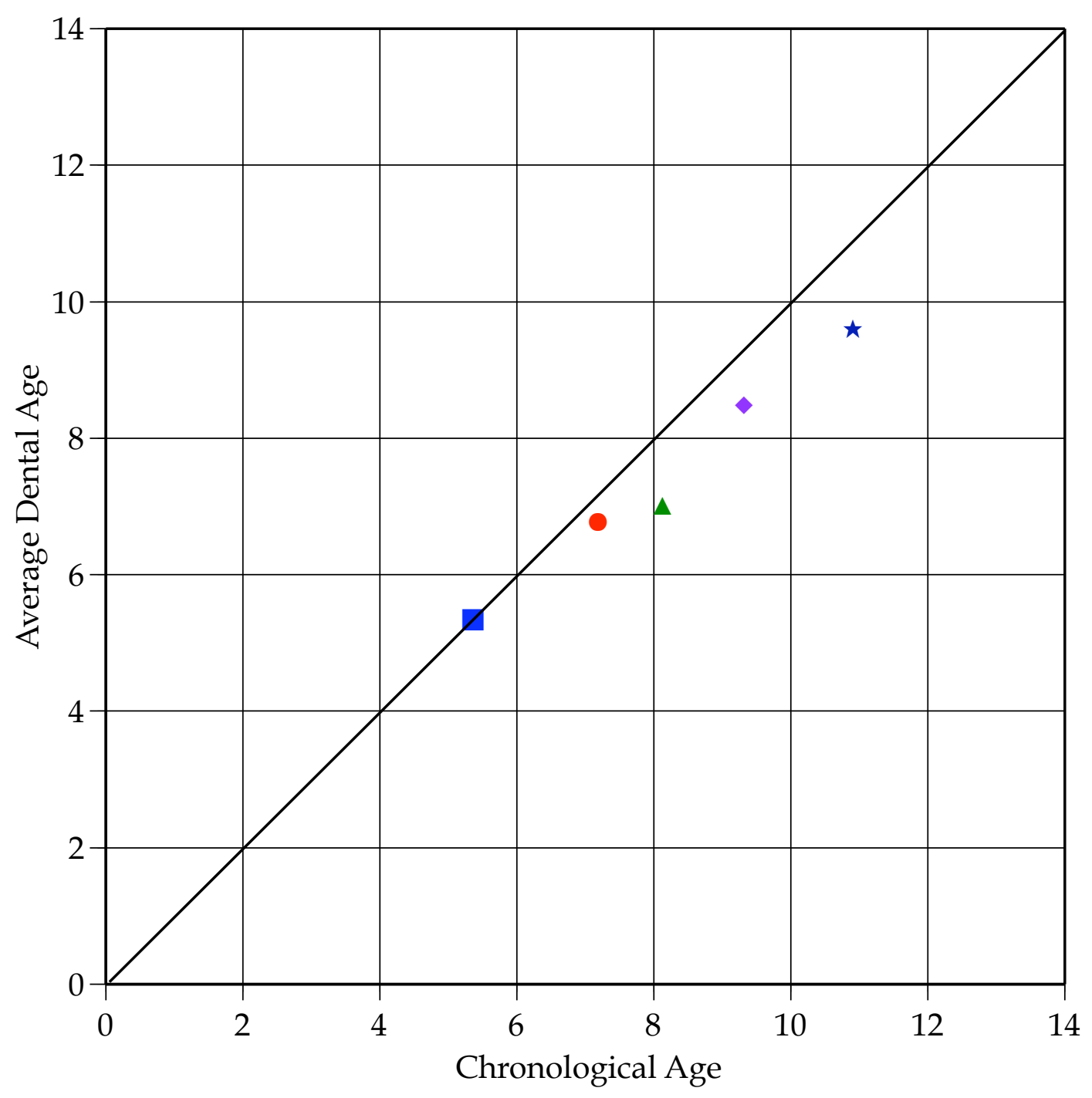

Fig. A-3. Plot of chronological age against dental age averaged across all scorable teeth (symbols) for a female (pseudo-record \#3). The square symbol denotes age at diagnosis of ALL. 


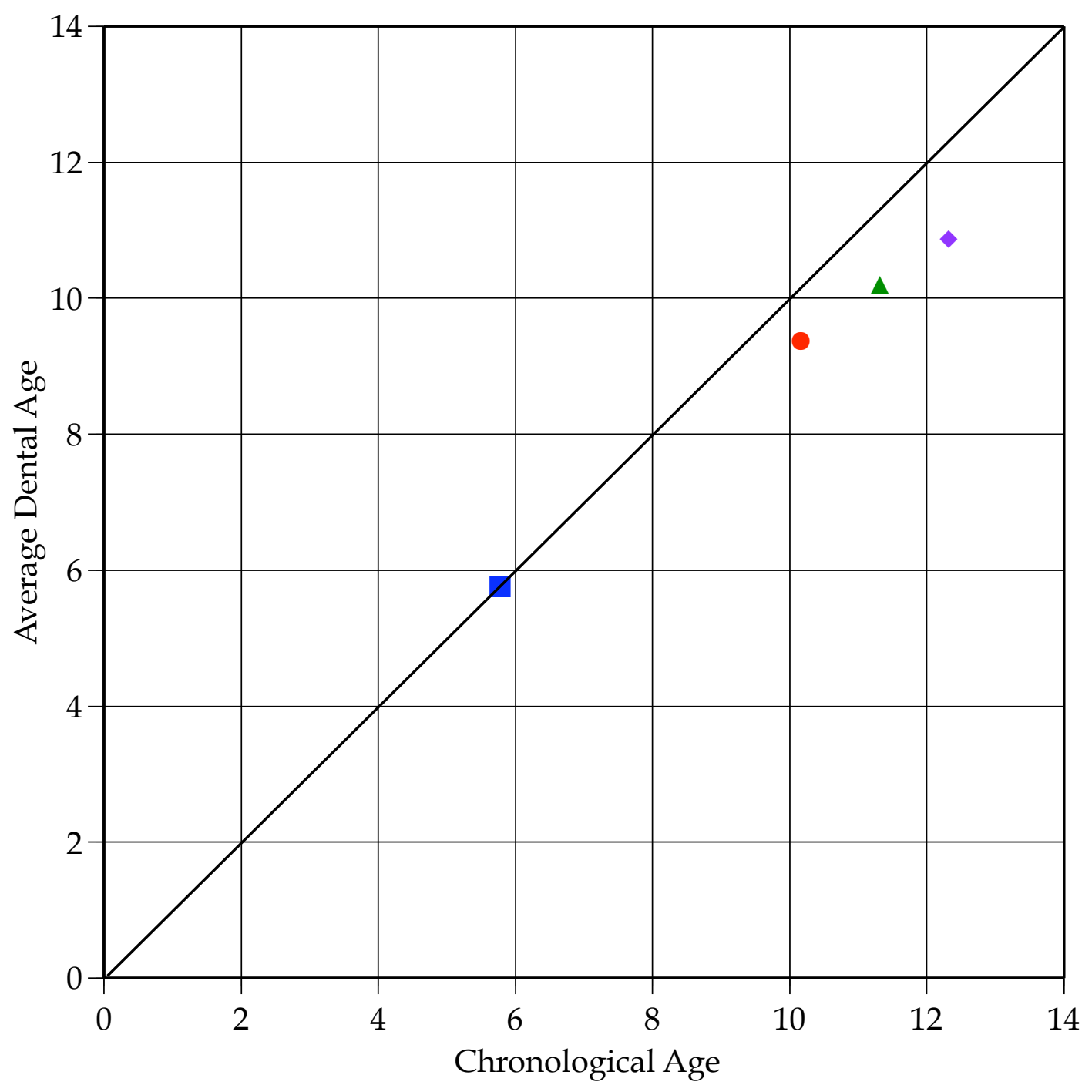

Fig. A-4. Plot of chronological age against dental age averaged across all scorable teeth (symbols) for a male (pseudo-record \#4). The square symbol denotes age at diagnosis of ALL. 


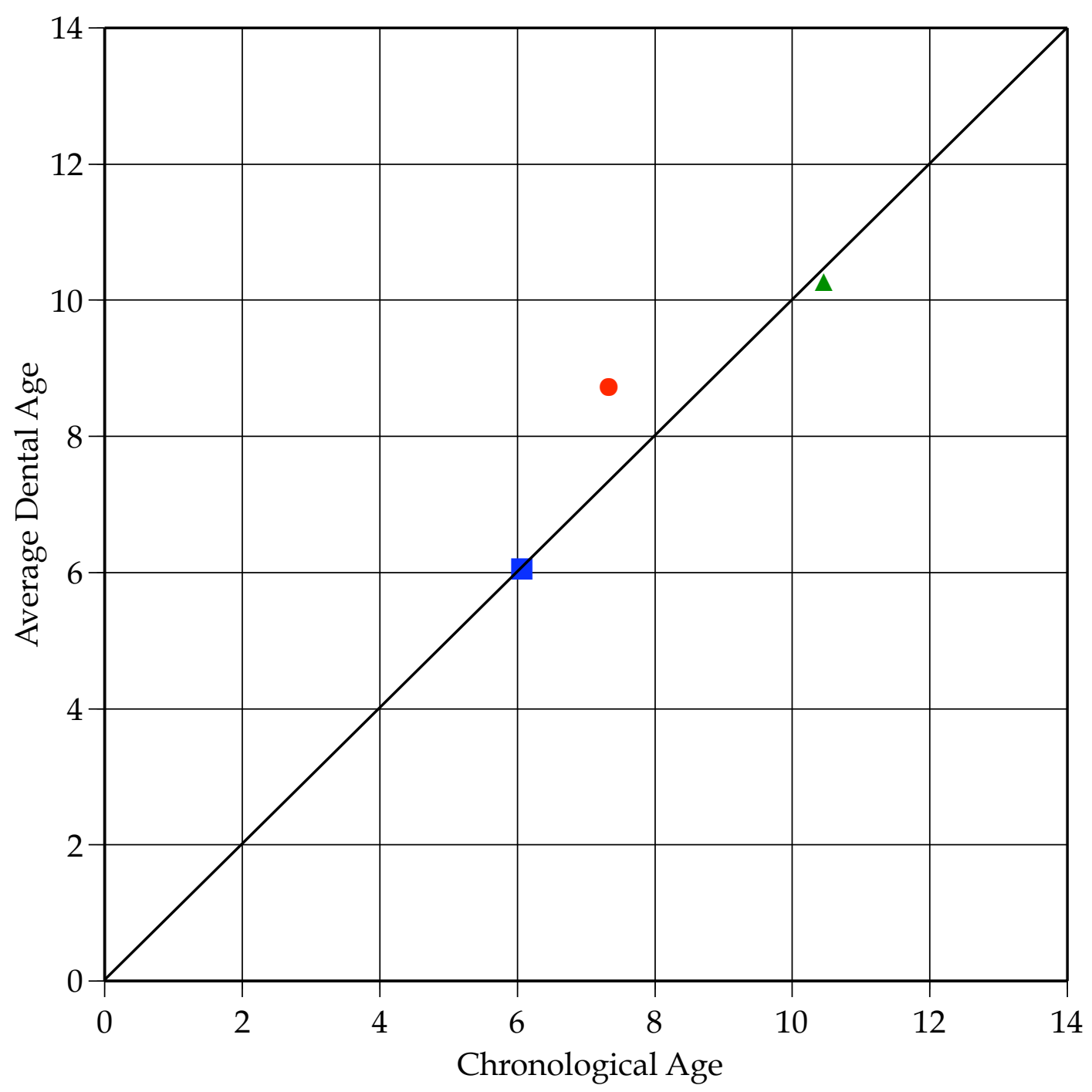

Fig. A-5. Plot of chronological age against dental age averaged across all scorable teeth (symbols) for a male (pseudo-record \#5). The square symbol denotes age at diagnosis of ALL. 


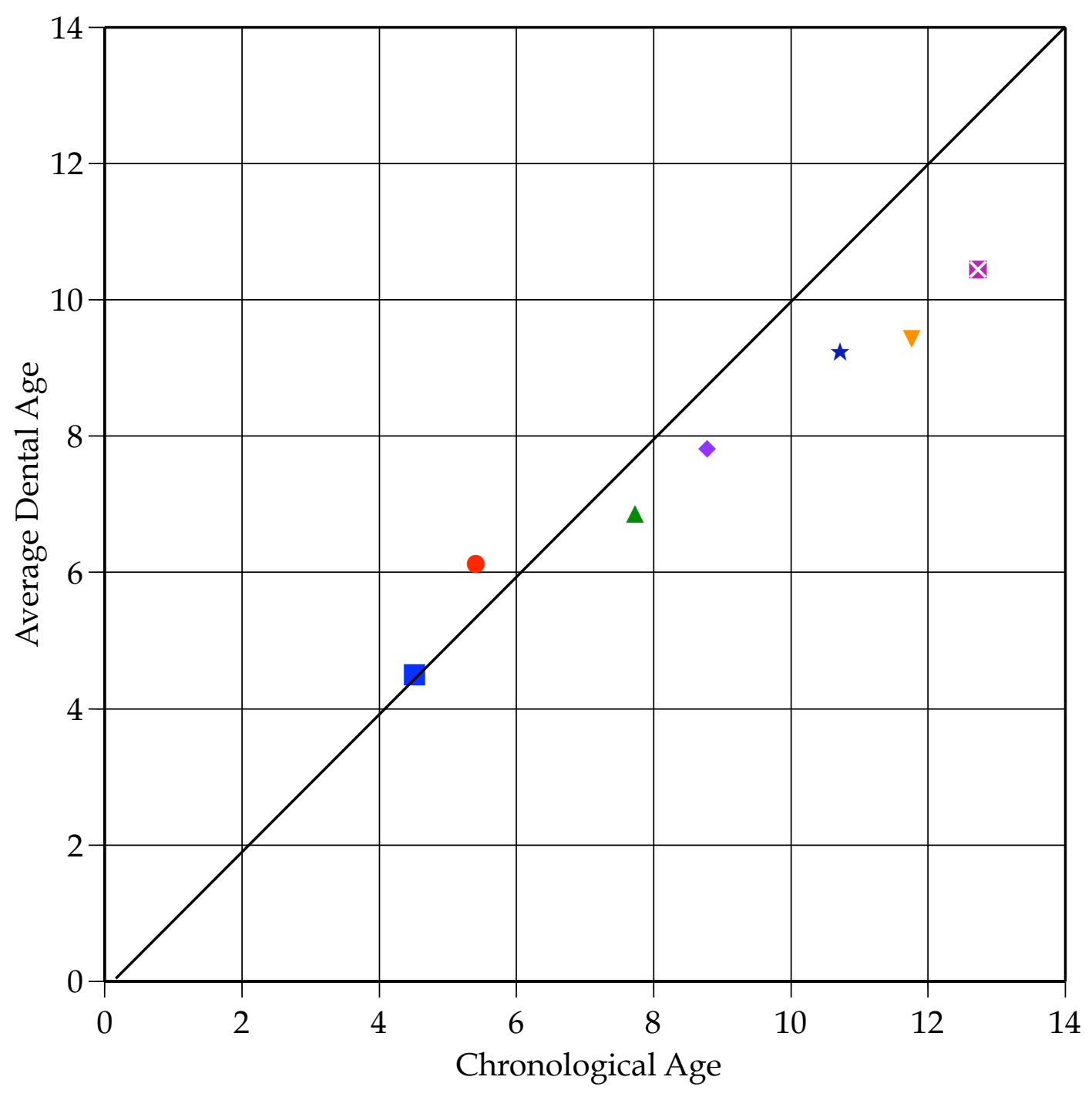

Fig. A-6. Plot of chronological age against dental age averaged across all scorable teeth (symbols) for a male (pseudo-record \#6). The square symbol denotes age at diagnosis of ALL. 


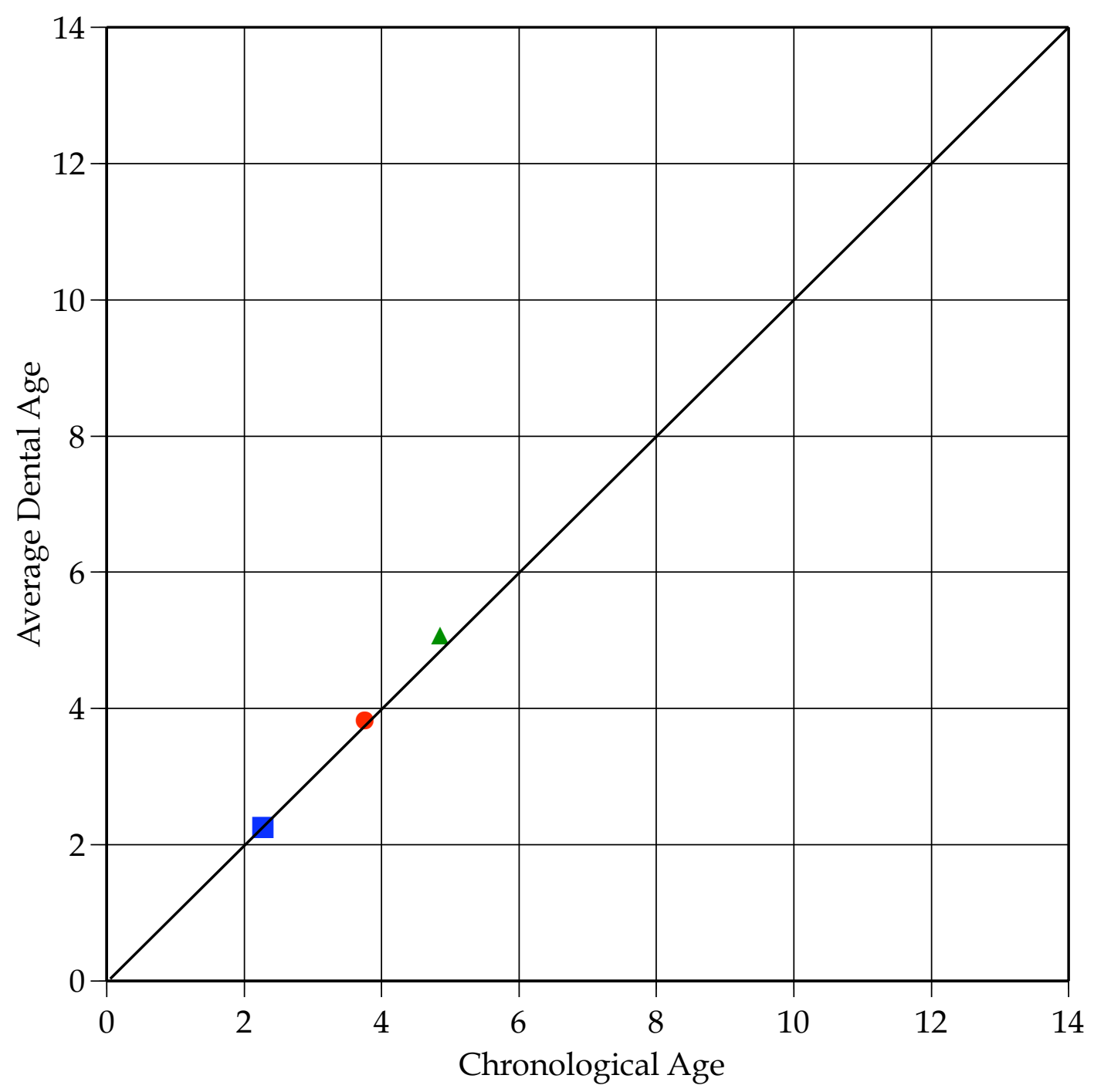

Fig. A-7. Plot of chronological age against dental age averaged across all scorable teeth (symbols) for a female (pseudo-record \#7). The square symbol denotes age at diagnosis of ALL. 


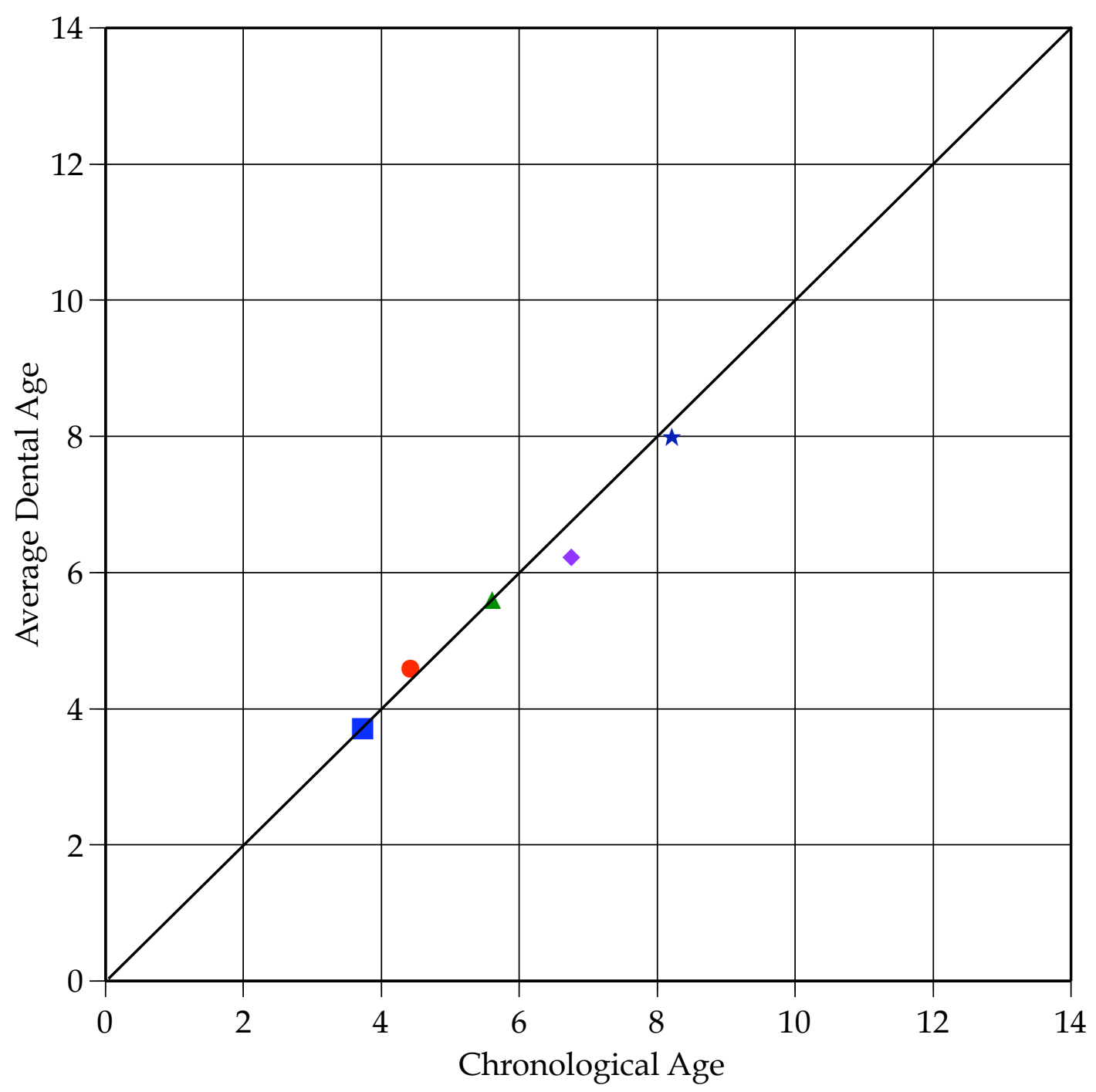

Fig. A-8. Plot of chronological age against dental age averaged across all scorable teeth (symbols) for a female (pseudo-record \#8). The square symbol denotes age at diagnosis of ALL. One examination that was beyond the age of 14 was omitted for consistency. 


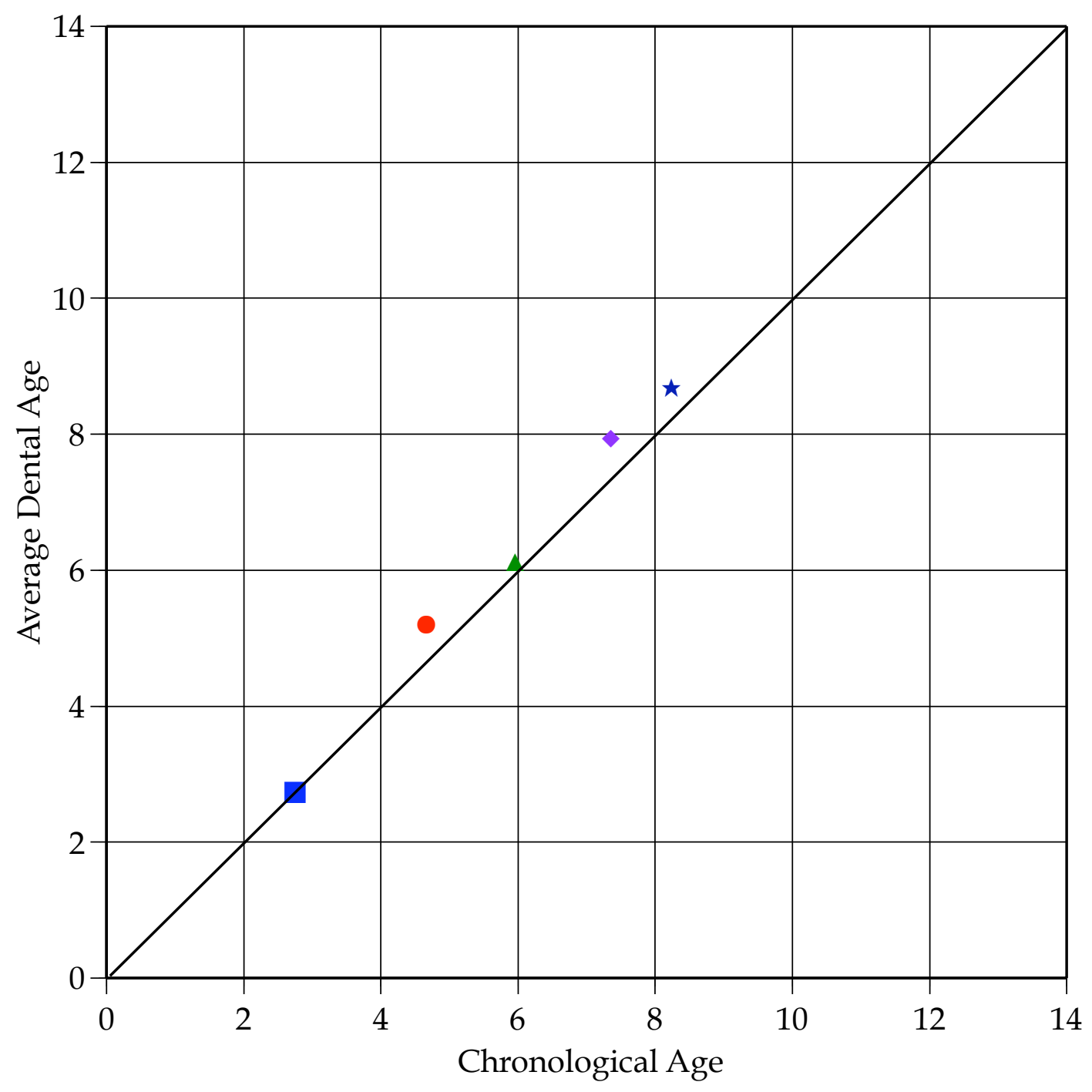

Fig. A-9. Plot of chronological age against dental age averaged across all scorable teeth (symbols) for a female (pseudo-record \#9). The square symbol denotes age at diagnosis of ALL. 


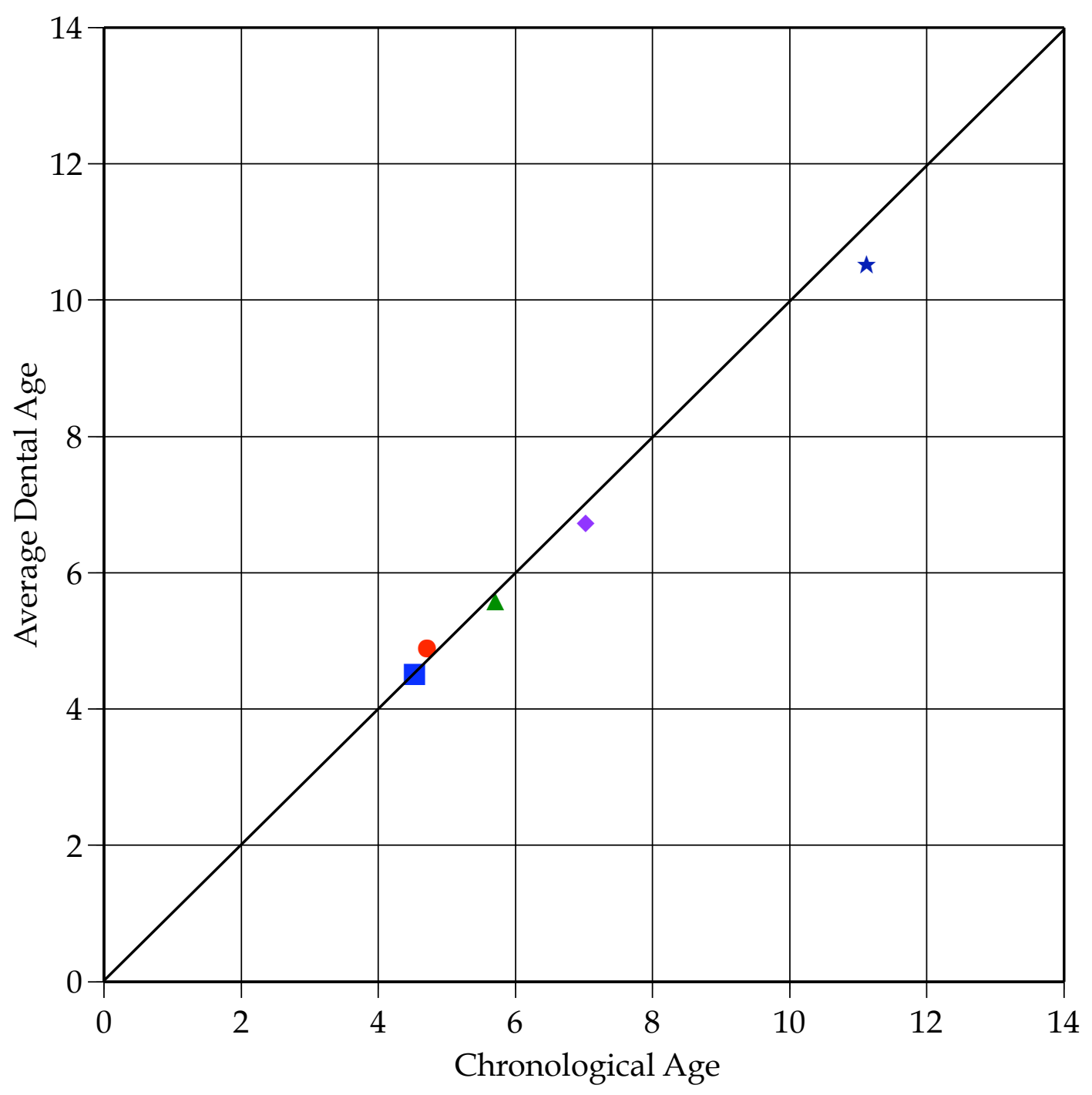

Fig. A-10. Plot of chronological age against dental age averaged across all scorable teeth (symbols) for a male (pseudo-record \#10). The square symbol denotes age at diagnosis of ALL. 


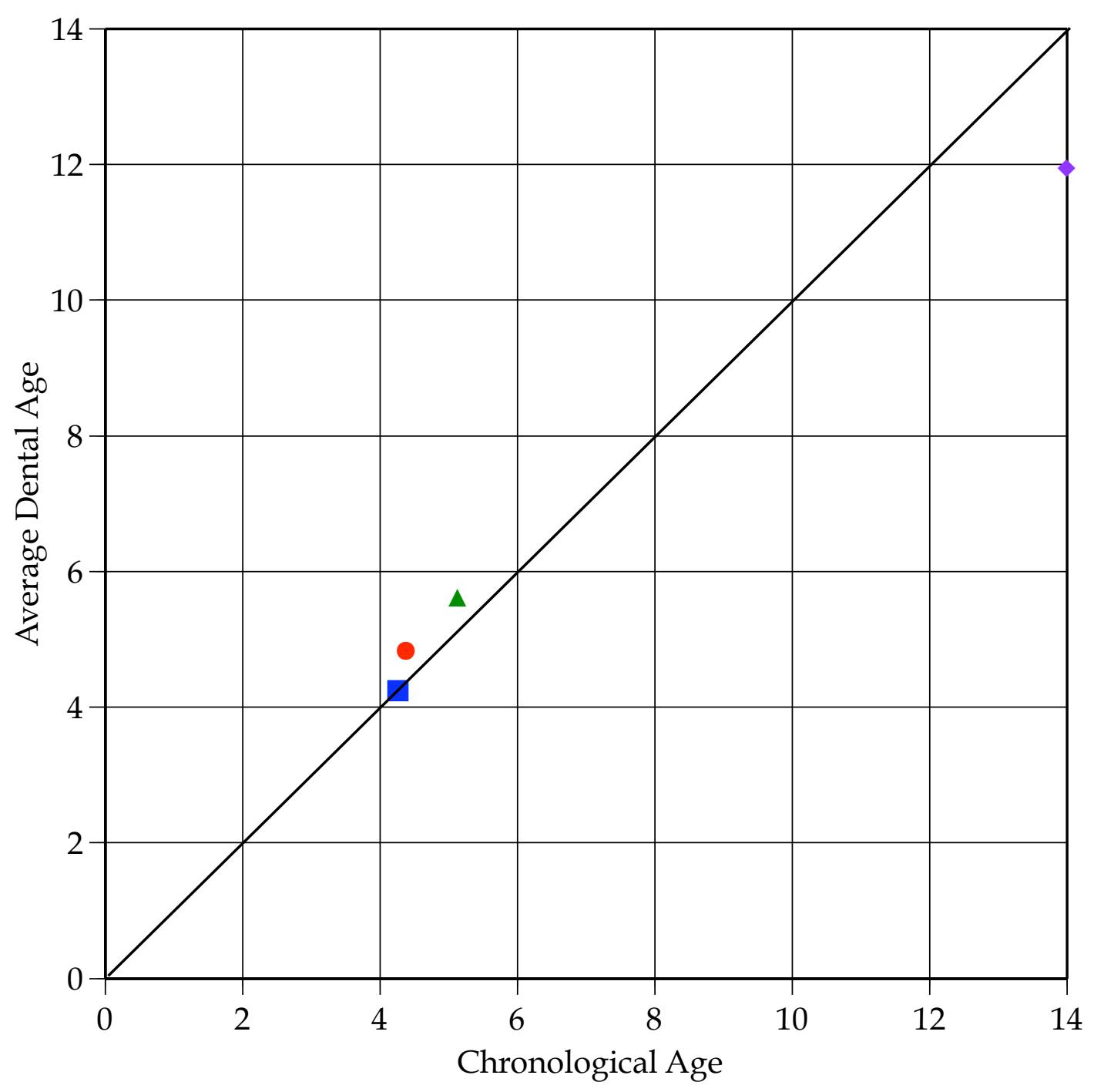

Fig. A-11. Plot of chronological age against dental age averaged across all scorable teeth (symbols) for a female (pseudo-record \#11). The square symbol denotes age at diagnosis of ALL. 


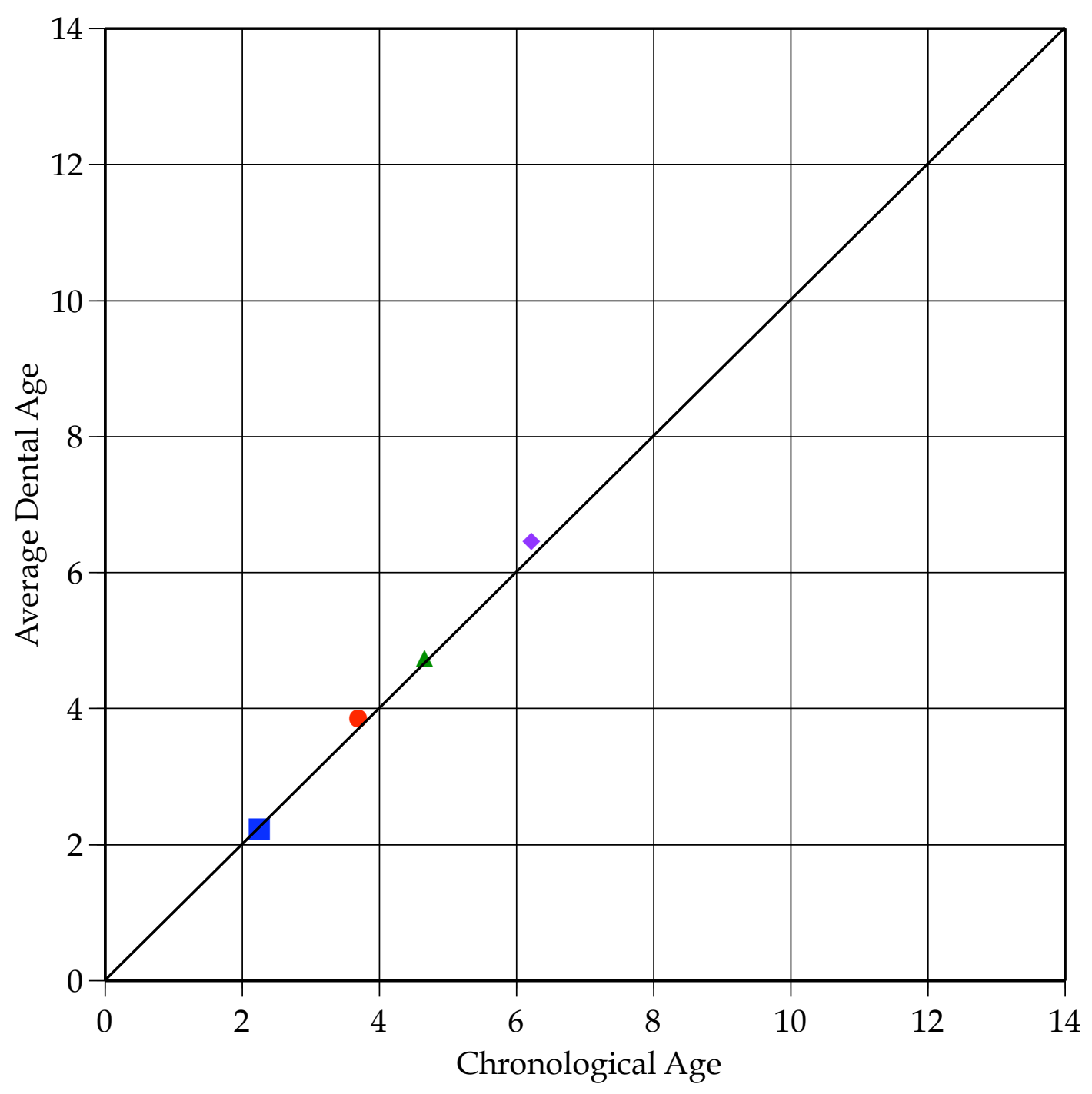

Fig. A-12. Plot of chronological age against dental age averaged across all scorable teeth (symbols) for a male (pseudo-record \#12). The square symbol denotes age at diagnosis of ALL. 


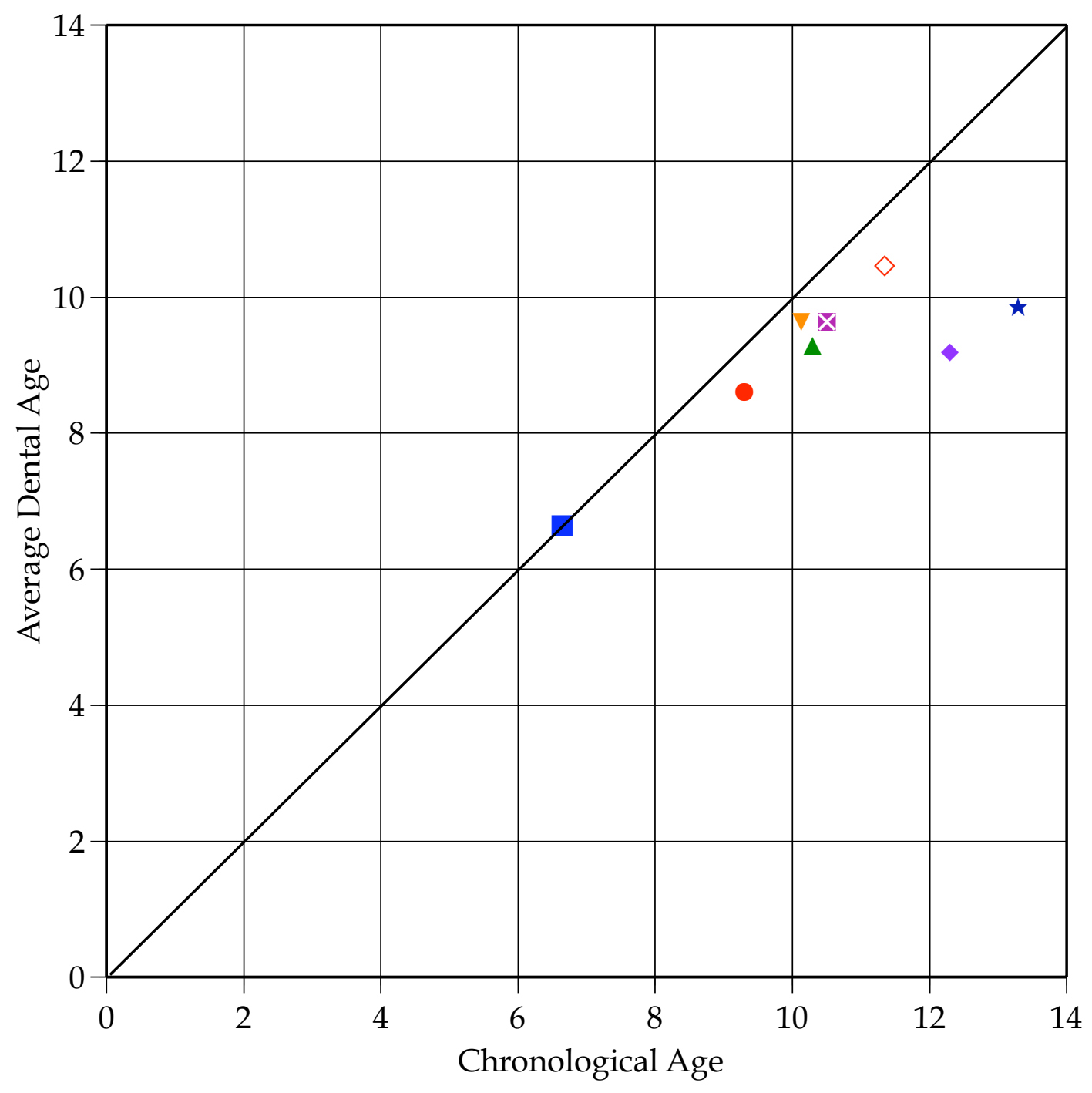

Fig. A-13. Plot of chronological age against dental age averaged across all scorable teeth (symbols) for a male (pseudo-record \#13). The square symbol denotes age at diagnosis of ALL. 


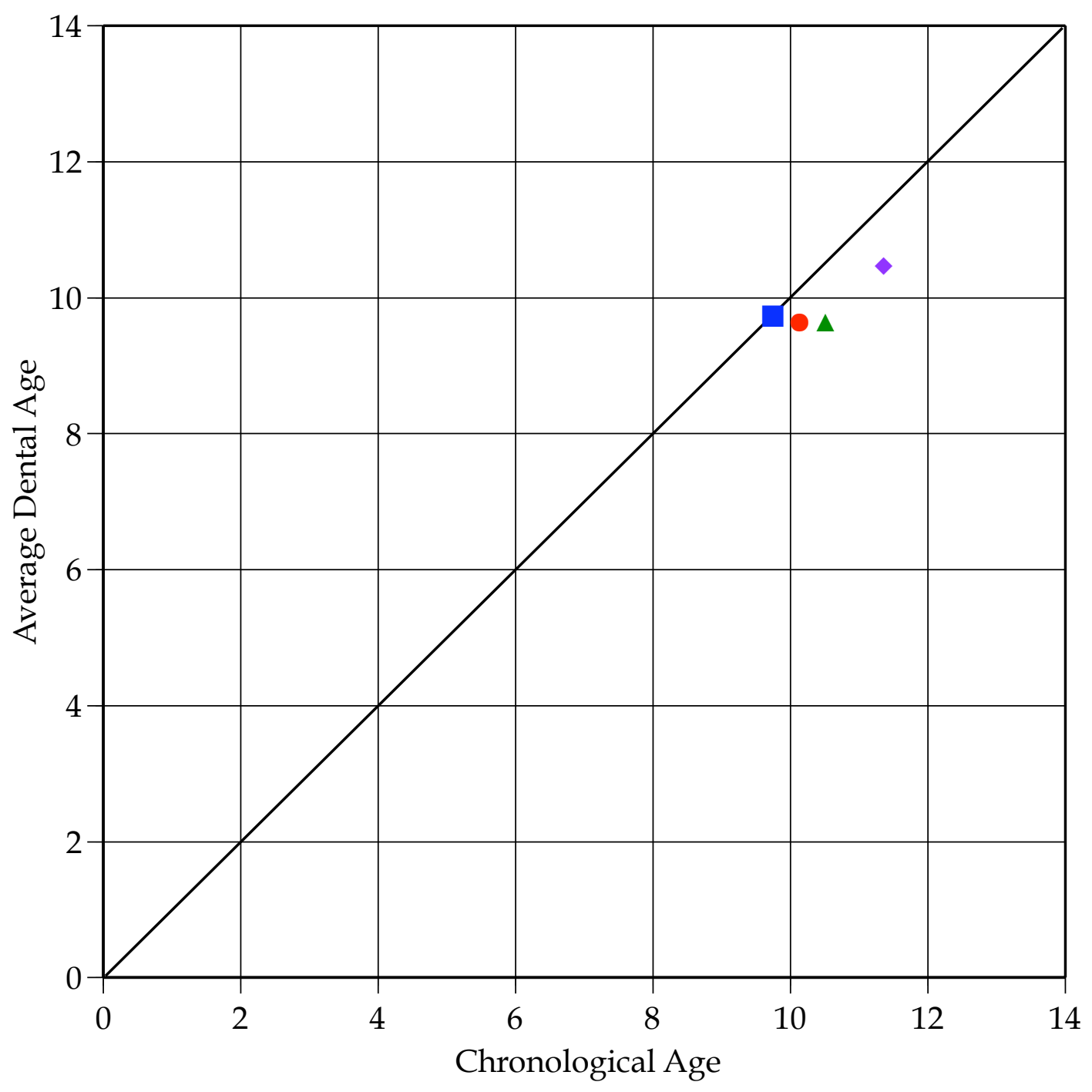

Fig. A-14. Plot of chronological age against dental age averaged across all scorable teeth (symbols) for a male (pseudo-record \#14). The square symbol denotes age at diagnosis of ALL. 


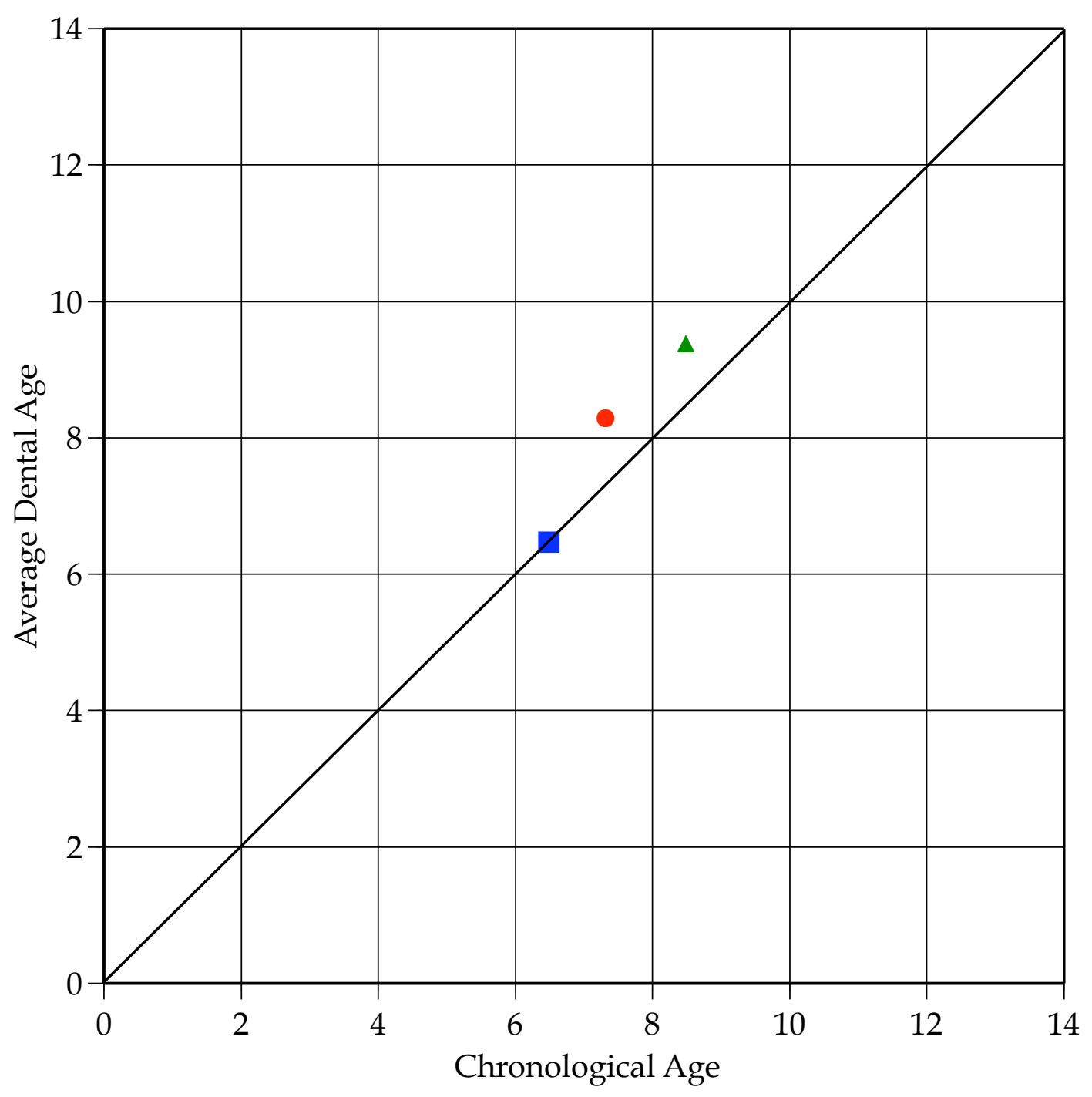

Fig. A-15. Plot of chronological age against dental age averaged across all scorable teeth (symbols) for a male (pseudo-record \#15). The square symbol denotes age at diagnosis of ALL. 


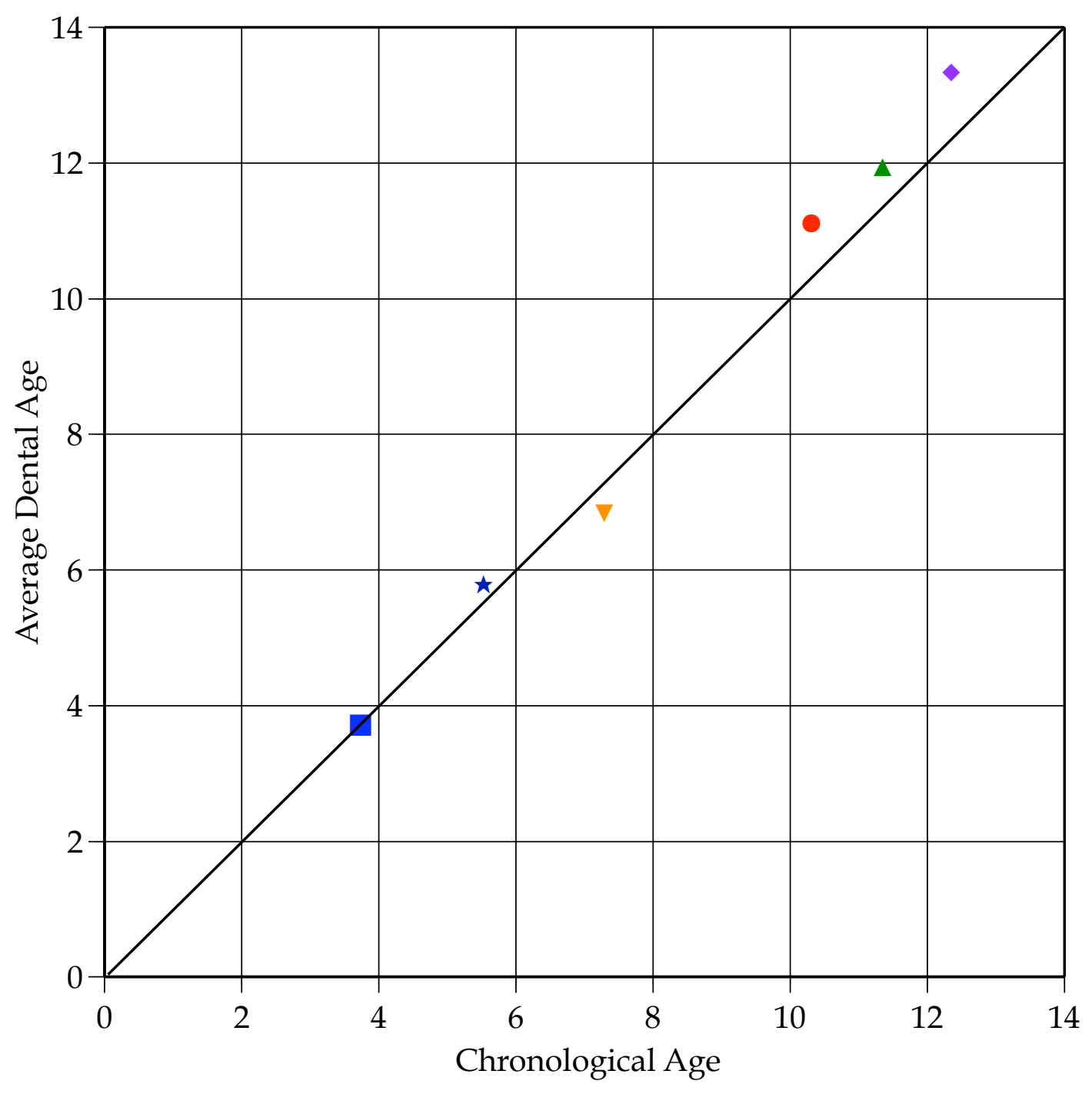

Fig. A-16. Plot of chronological age against dental age averaged across all scorable teeth (symbols) for a female (pseudo-record \#16). The square symbol denotes age at diagnosis of ALL. One examination that was beyond the age of 14 was omitted for consistency. 


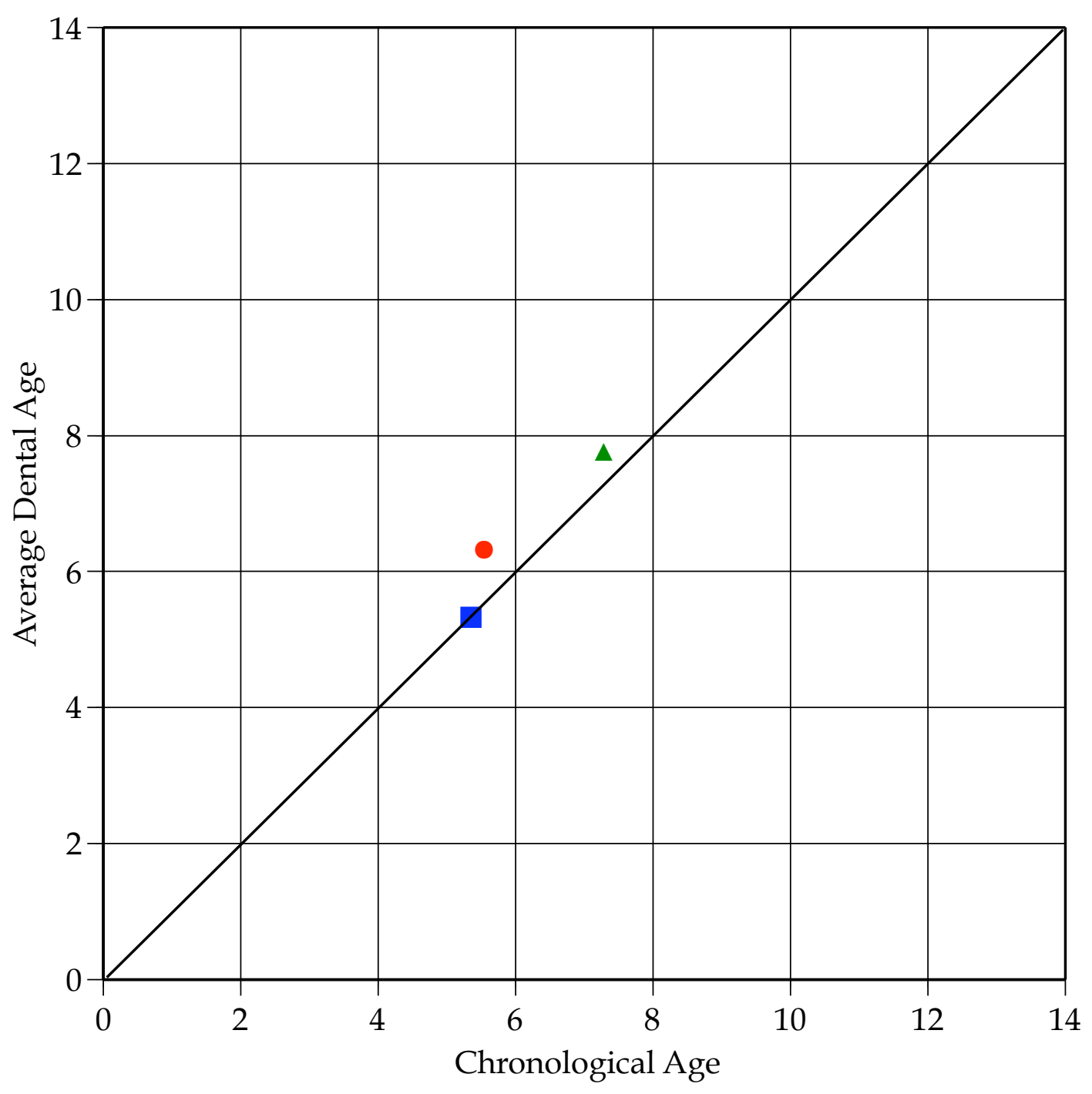

Fig. A-17. Plot of chronological age against dental age averaged across all scorable teeth (symbols) for a female (pseudo-record \#17). The square symbol denotes age at diagnosis of ALL. One examination that was beyond the age of 14 was omitted for consistency. 


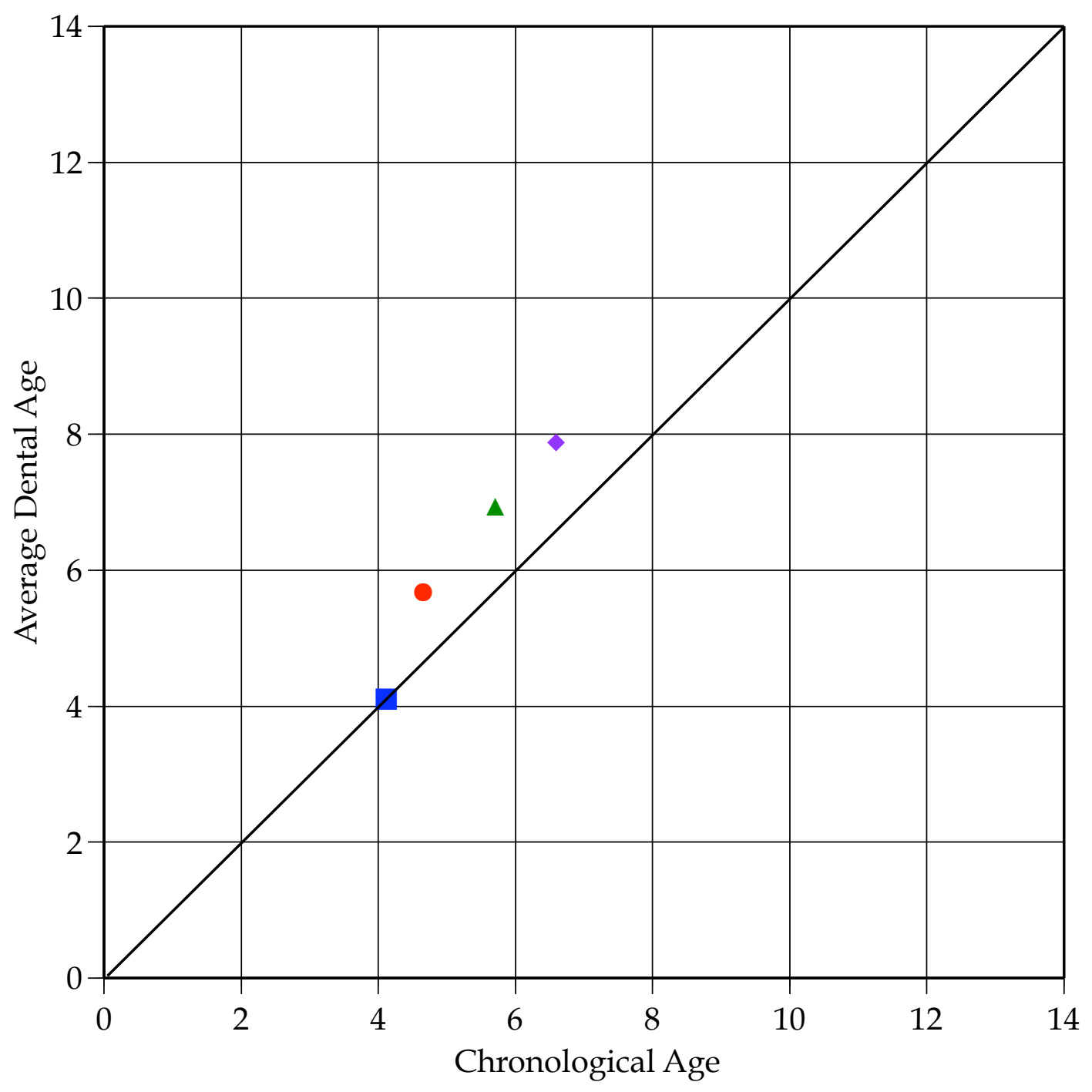

Fig. A-18. Plot of chronological age against dental age averaged across all scorable teeth (symbols) for a female (pseudo-record \#18). The square symbol denotes age at diagnosis of ALL. 


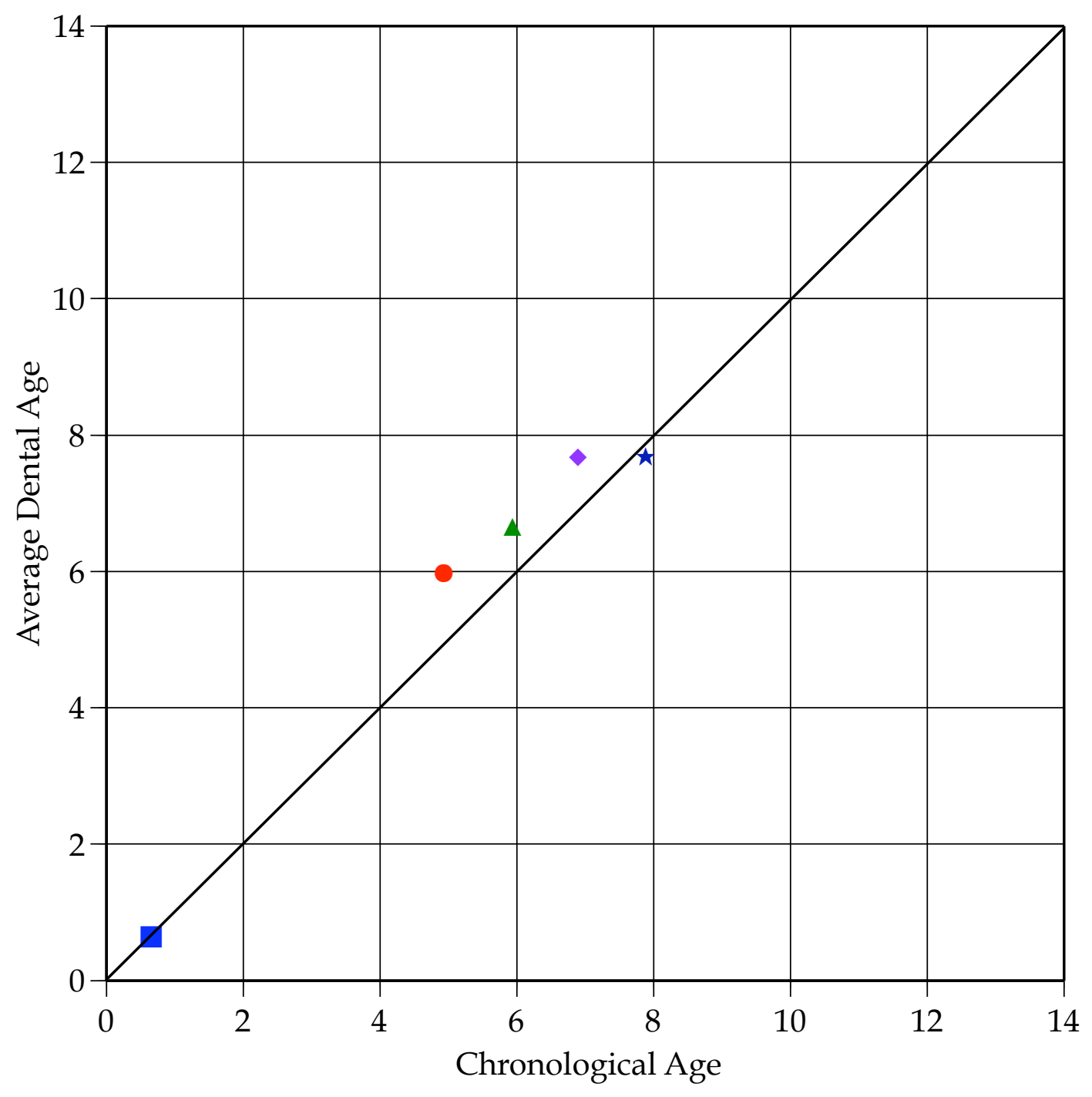

Fig. A-19. Plot of chronological age against dental age averaged across all scorable teeth (symbols) for a male (pseudo-record \#19). The square symbol denotes age at diagnosis of ALL. 


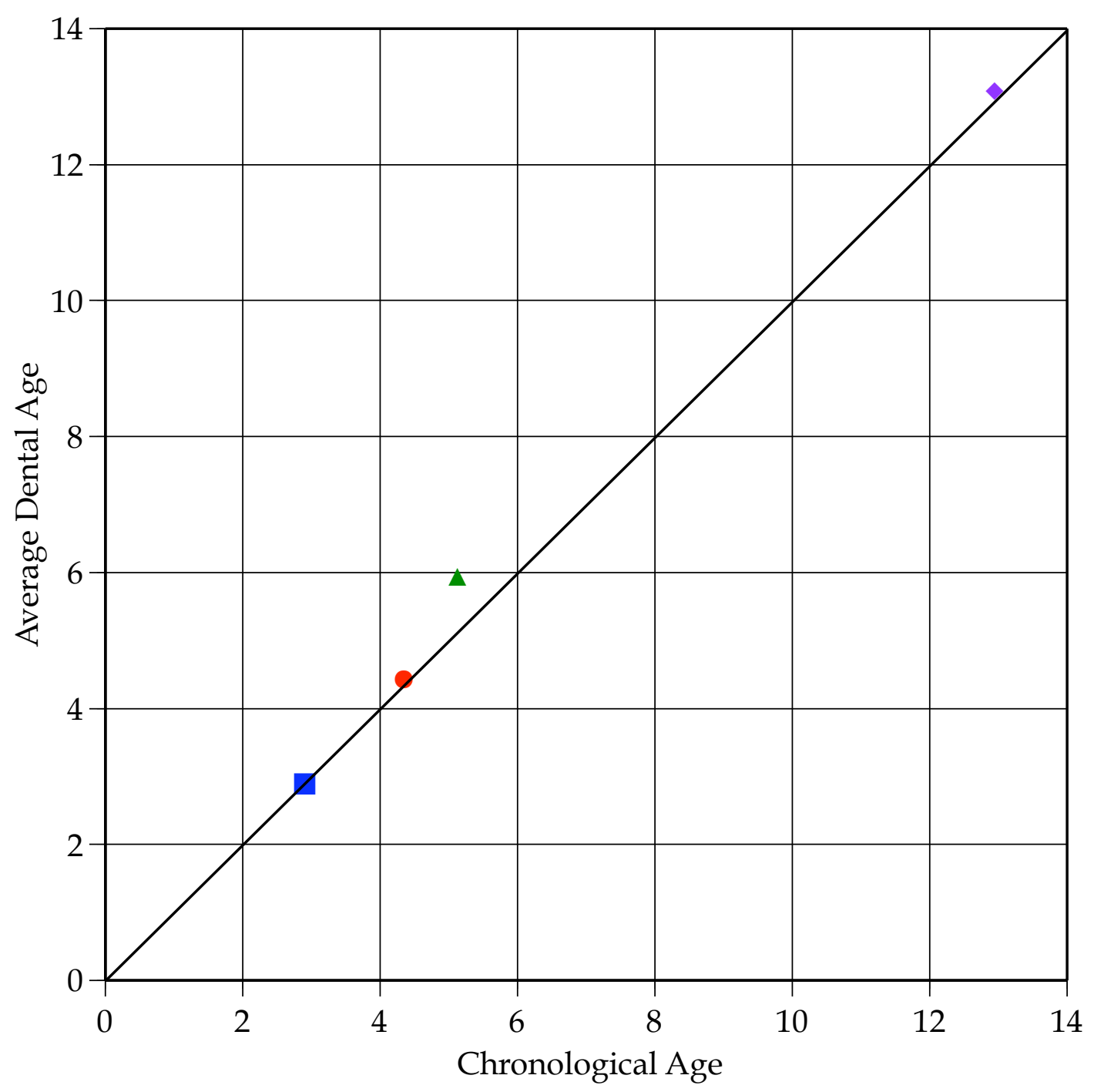

Fig. A-20. Plot of chronological age against dental age averaged across all scorable teeth (symbols) for a male (pseudo-record \#20). The square symbol denotes age at diagnosis of ALL. 


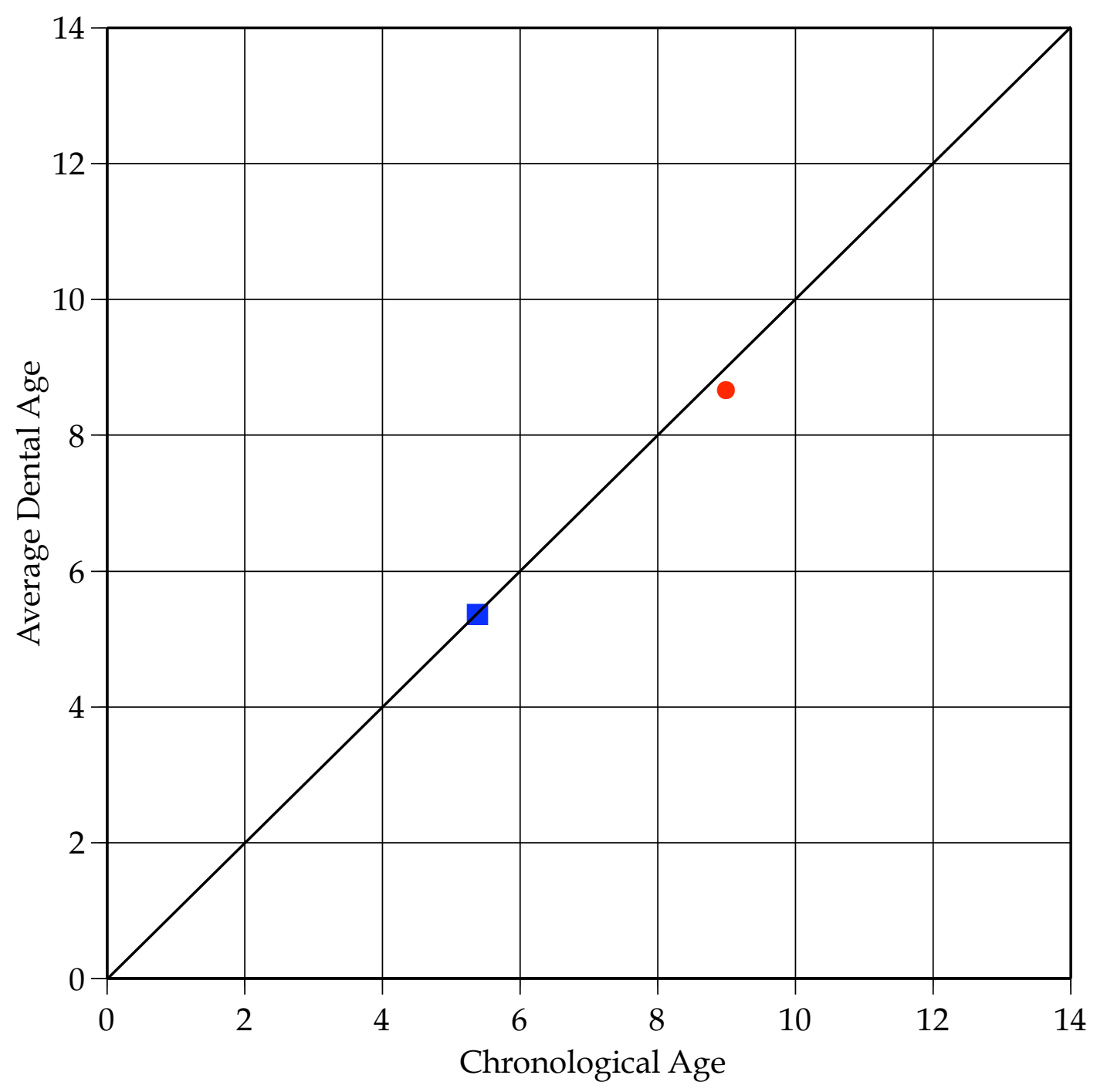

Fig. A-21. Plot of chronological age against dental age averaged across all scorable teeth (symbol) for a male (pseudo-record \#21). The square symbol denotes age at diagnosis of ALL. 


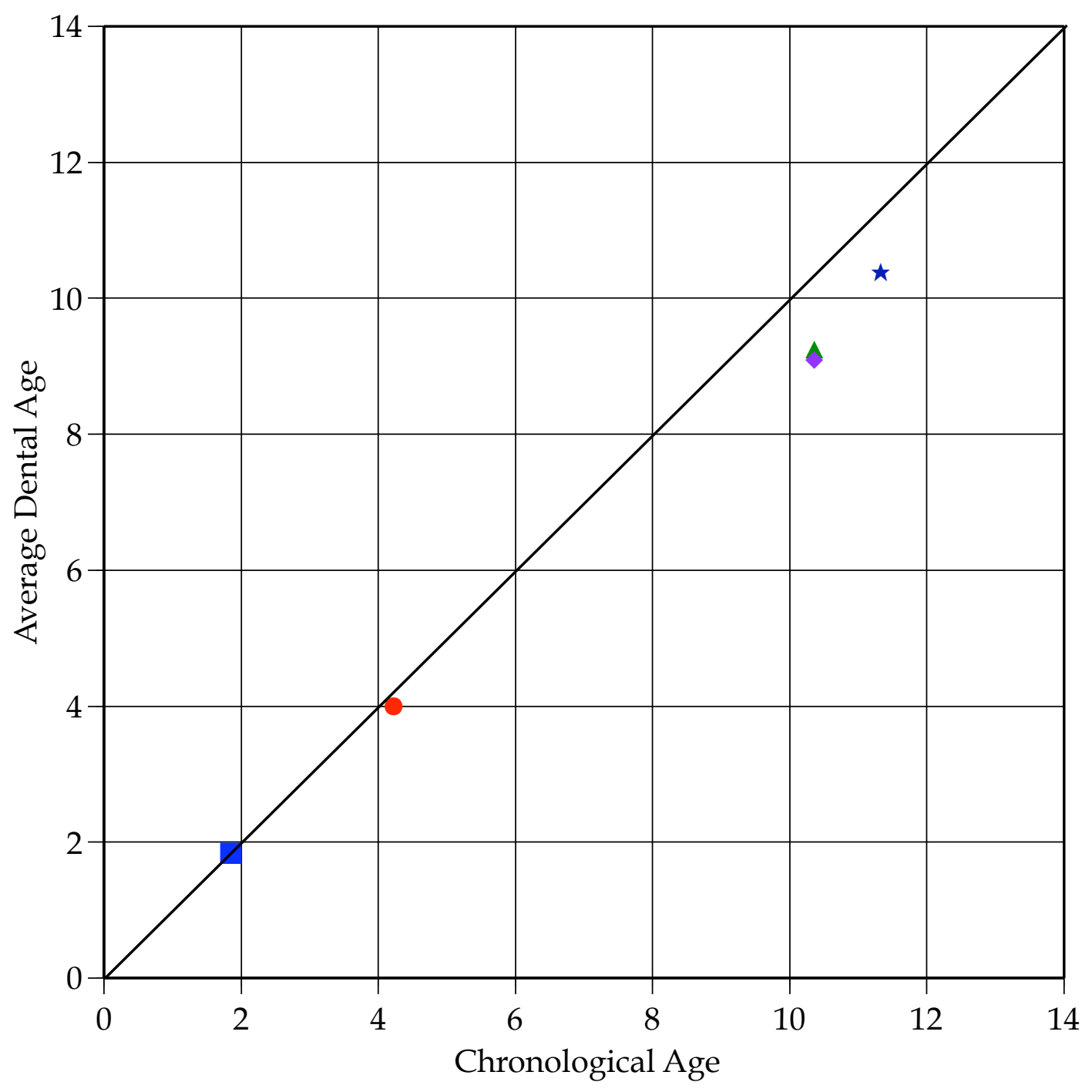

Fig. A-22. Plot of chronological age against dental age averaged across all scorable teeth (symbols) for a female (pseudo-record \#22). The square symbol denotes age at diagnosis of ALL. 


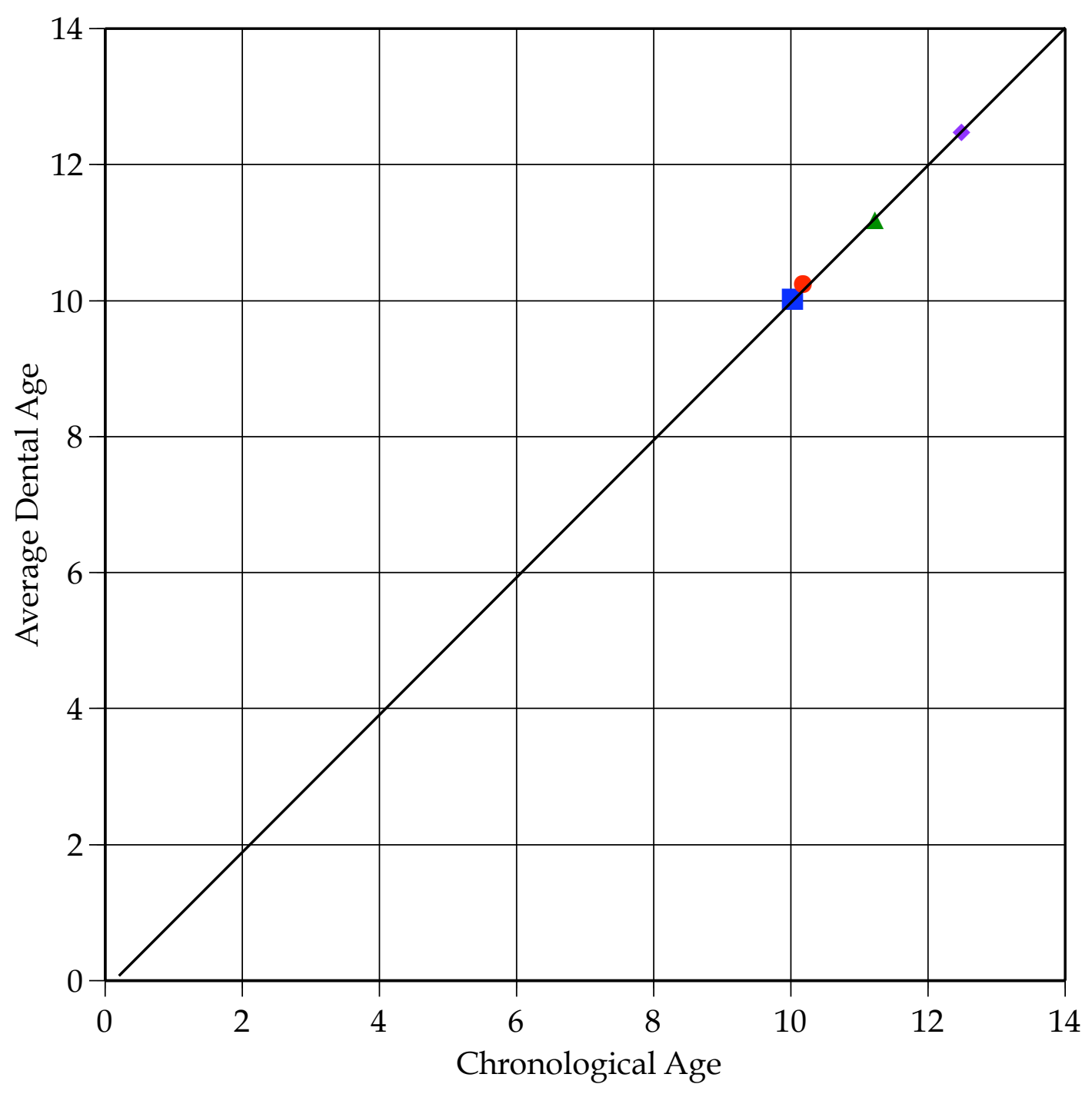

Fig. A-23. Plot of chronological age against dental age averaged across all scorable teeth (symbols) for a male (pseudo-record \#23). The square symbol denotes age at diagnosis of ALL. 


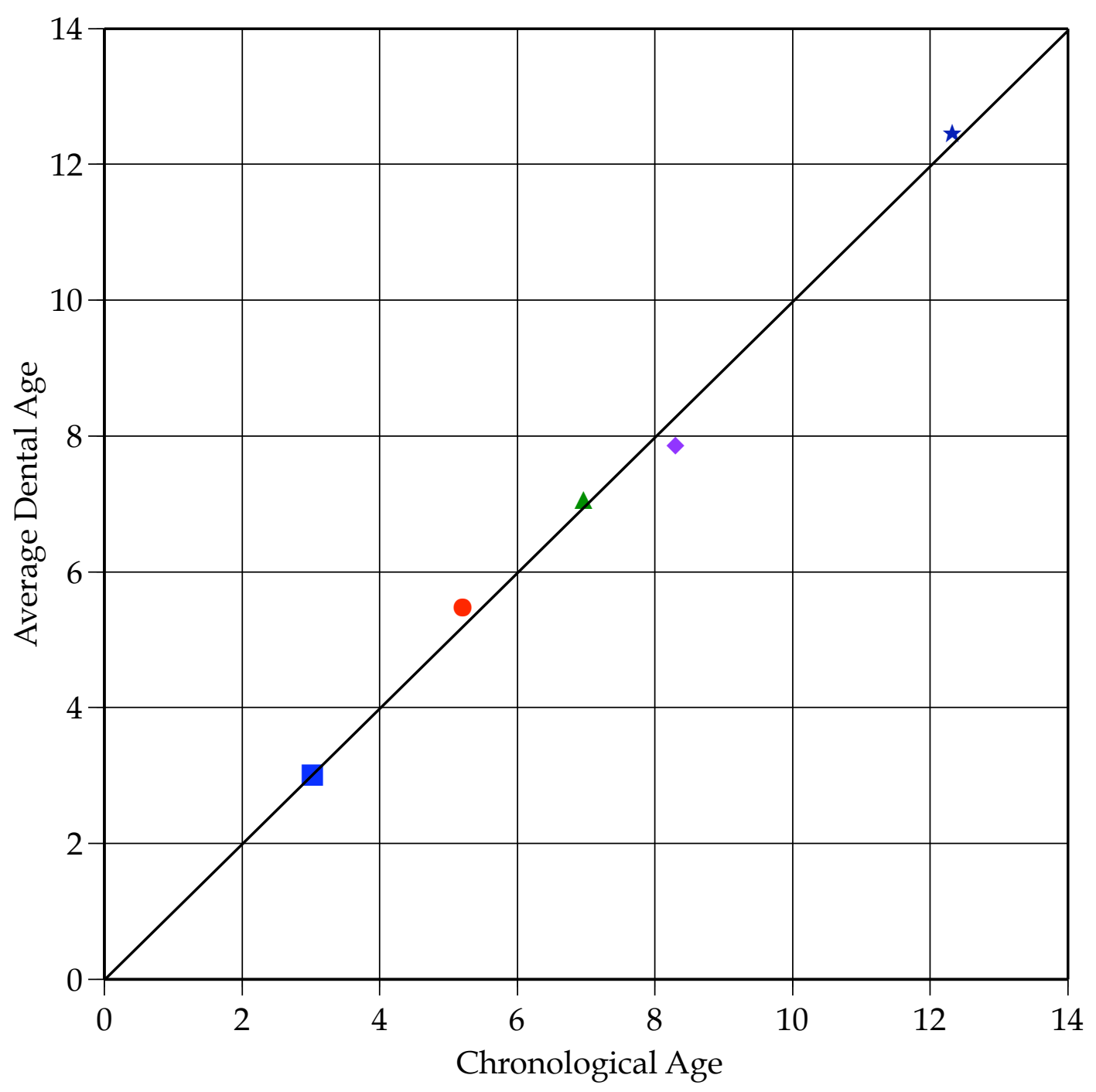

Fig. A-24. Plot of chronological age against dental age averaged across all scorable teeth (symbols) for a male (pseudo-record \#24). The square symbol denotes age at diagnosis of ALL. 


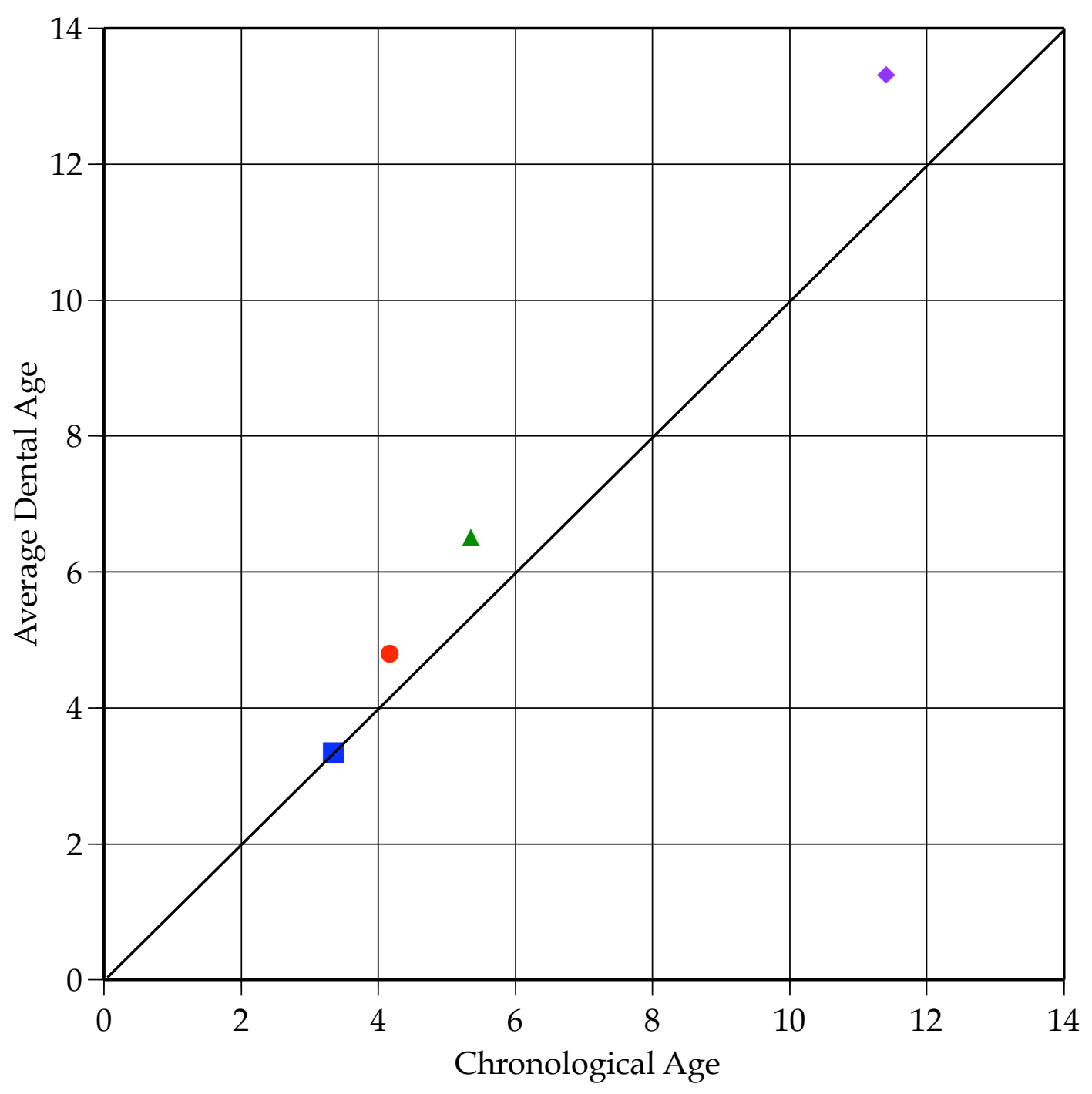

Fig. A-25. Plot of chronological age against dental age averaged across all scorable teeth (symbols) for a female (pseudo-record \#25). The square symbol denotes age at diagnosis of ALL. 


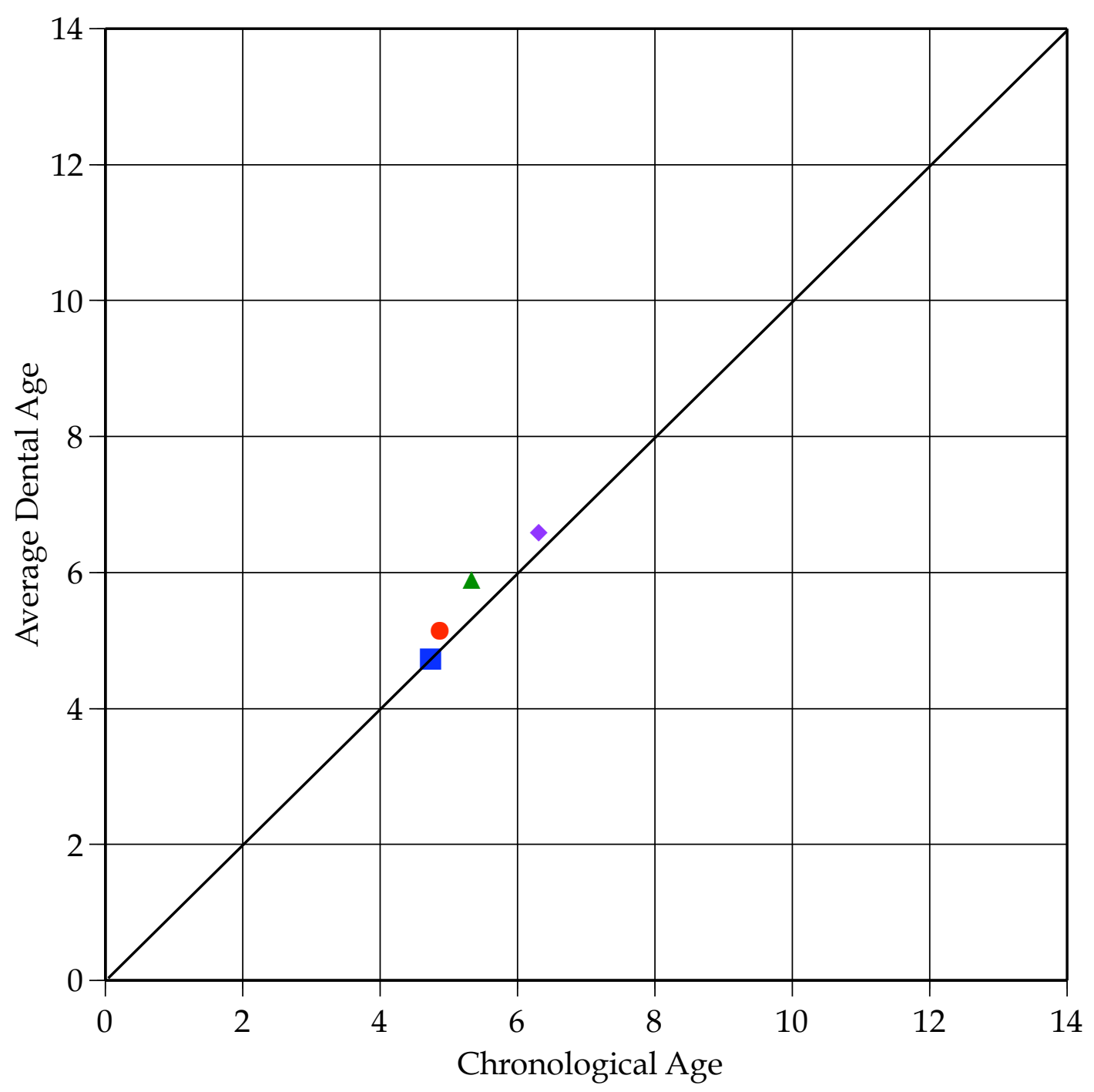

Fig. A-26. Plot of chronological age against dental age averaged across all scorable teeth (symbols) for a female (pseudo-record \#26). The square symbol denotes age at diagnosis of ALL. 


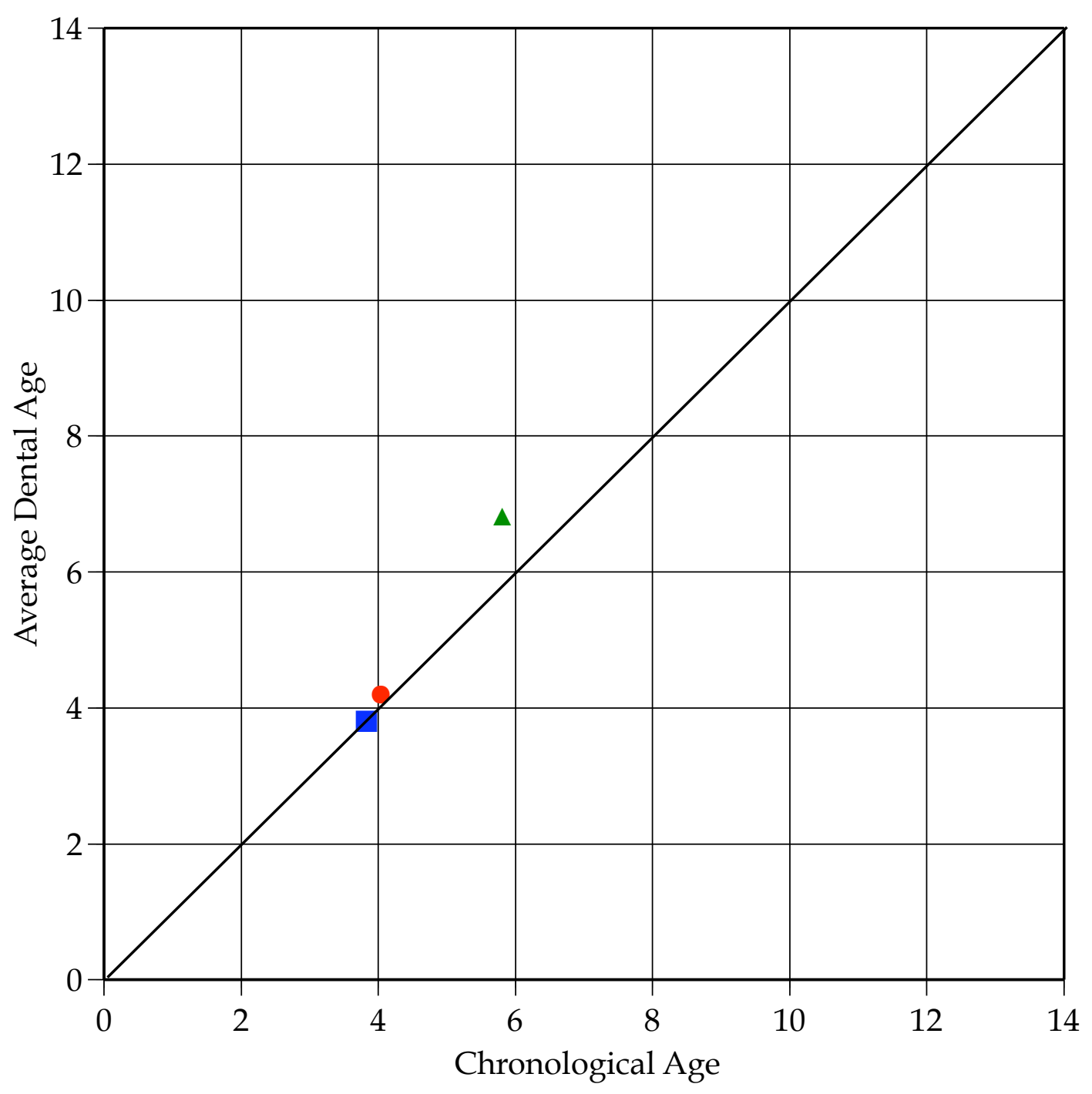

Fig. A-27. Plot of chronological age against dental age averaged across all scorable teeth (symbols) for a male (pseudo-record \#27). The square symbol denotes age at diagnosis of ALL. 


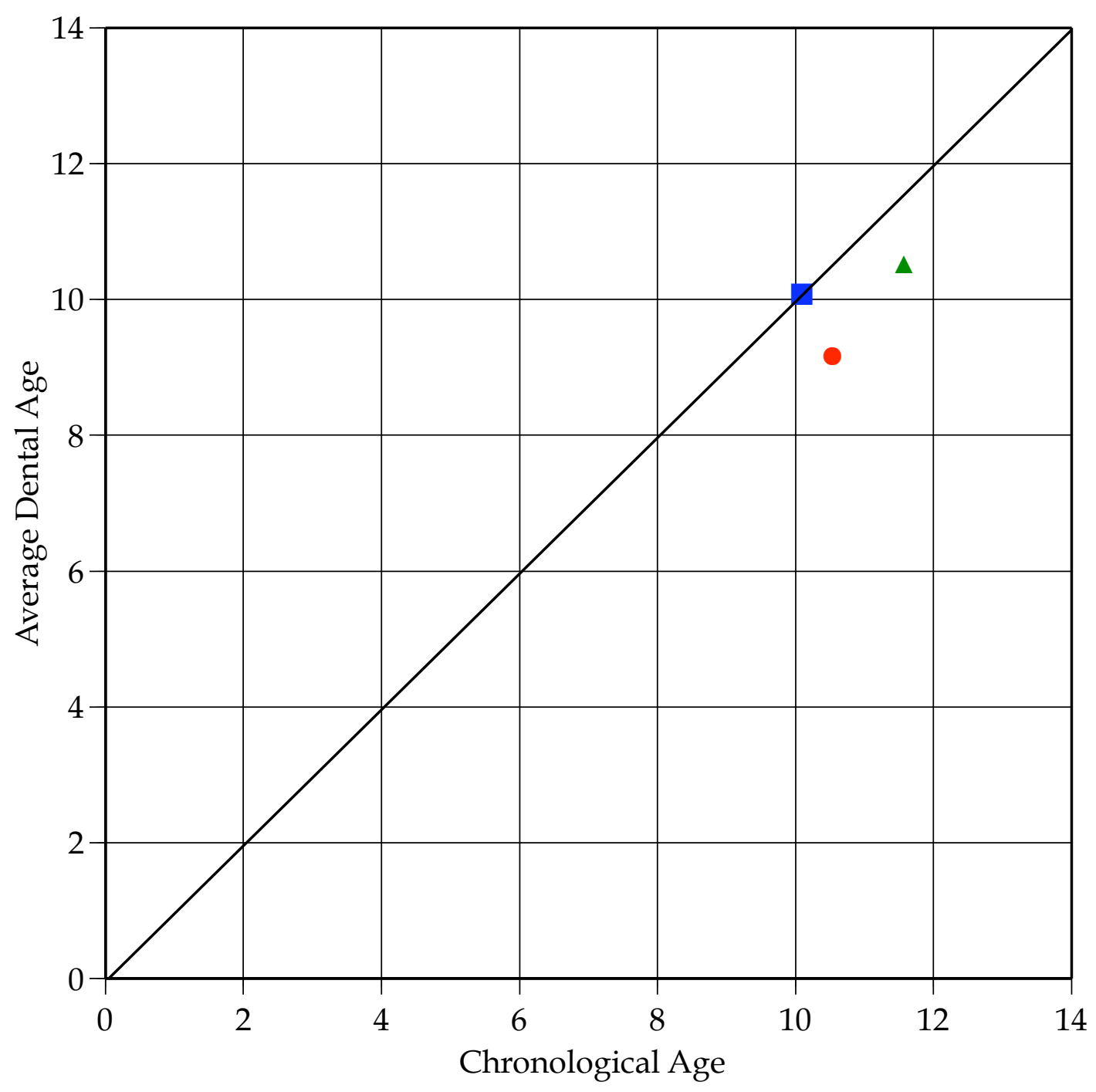

Fig. A-28. Plot of chronological age against dental age averaged across all scorable teeth (symbols) for a female (pseudo-record \#28). The square symbol denotes age at diagnosis of ALL. 


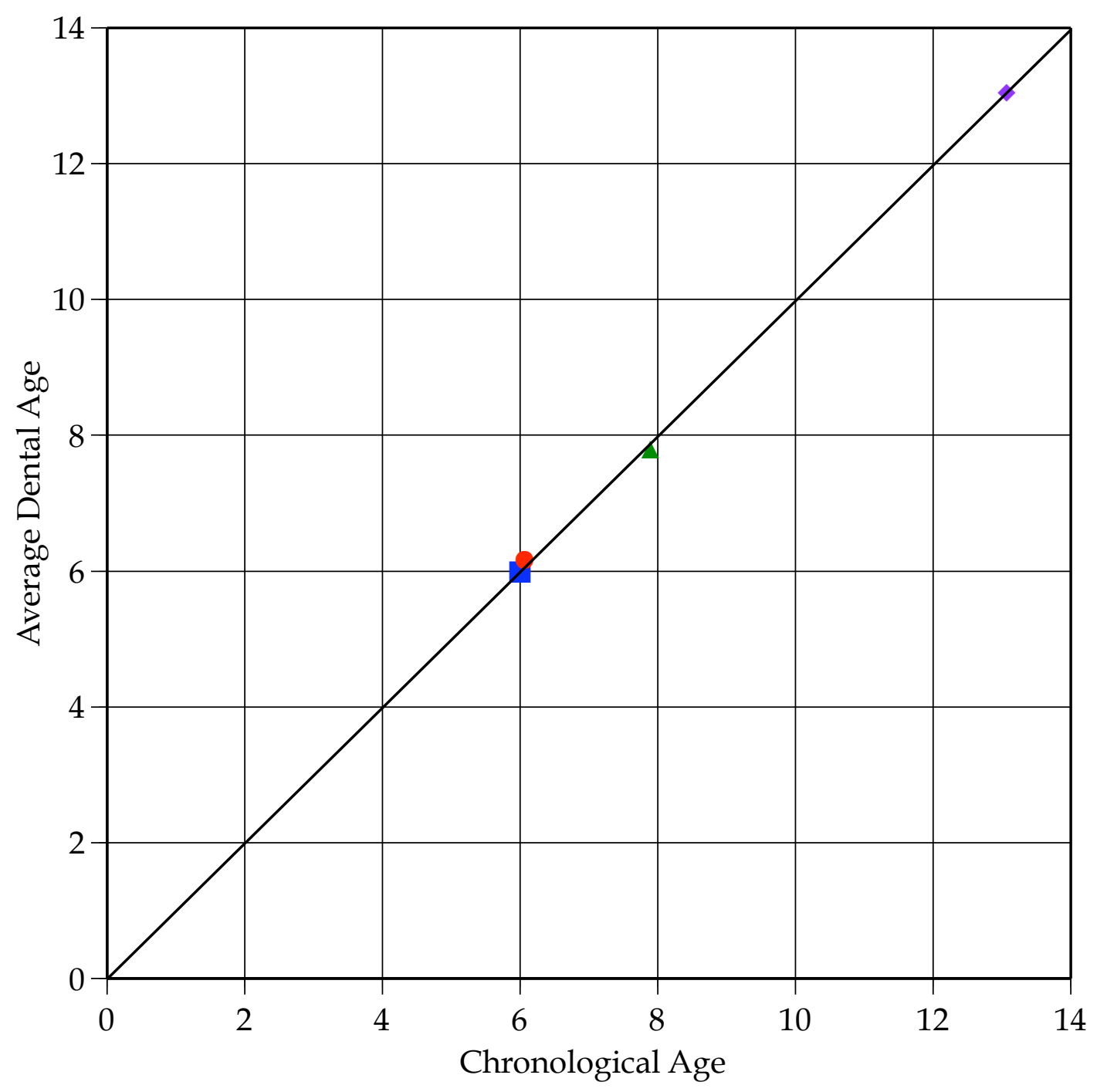

Fig. A-29. Plot of chronological age against dental age averaged across all scorable teeth (symbols) for a female (pseudo-record \#29). The square symbol denotes age at diagnosis of ALL. 


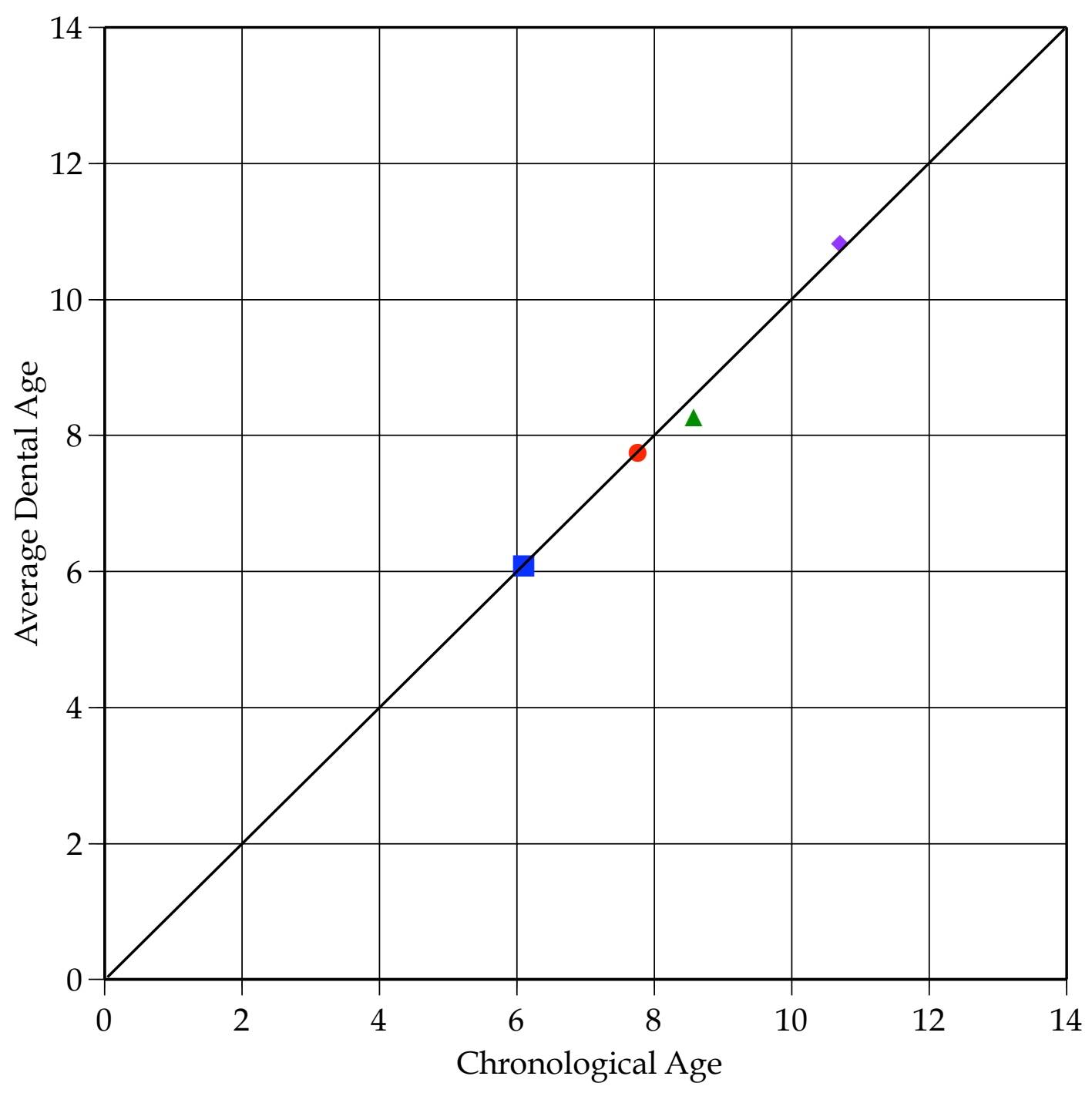

Fig. A-30. Plot of chronological age against dental age averaged across all scorable teeth (symbols) for a female (pseudo-record \#30). The square symbol denotes age at diagnosis of ALL. 


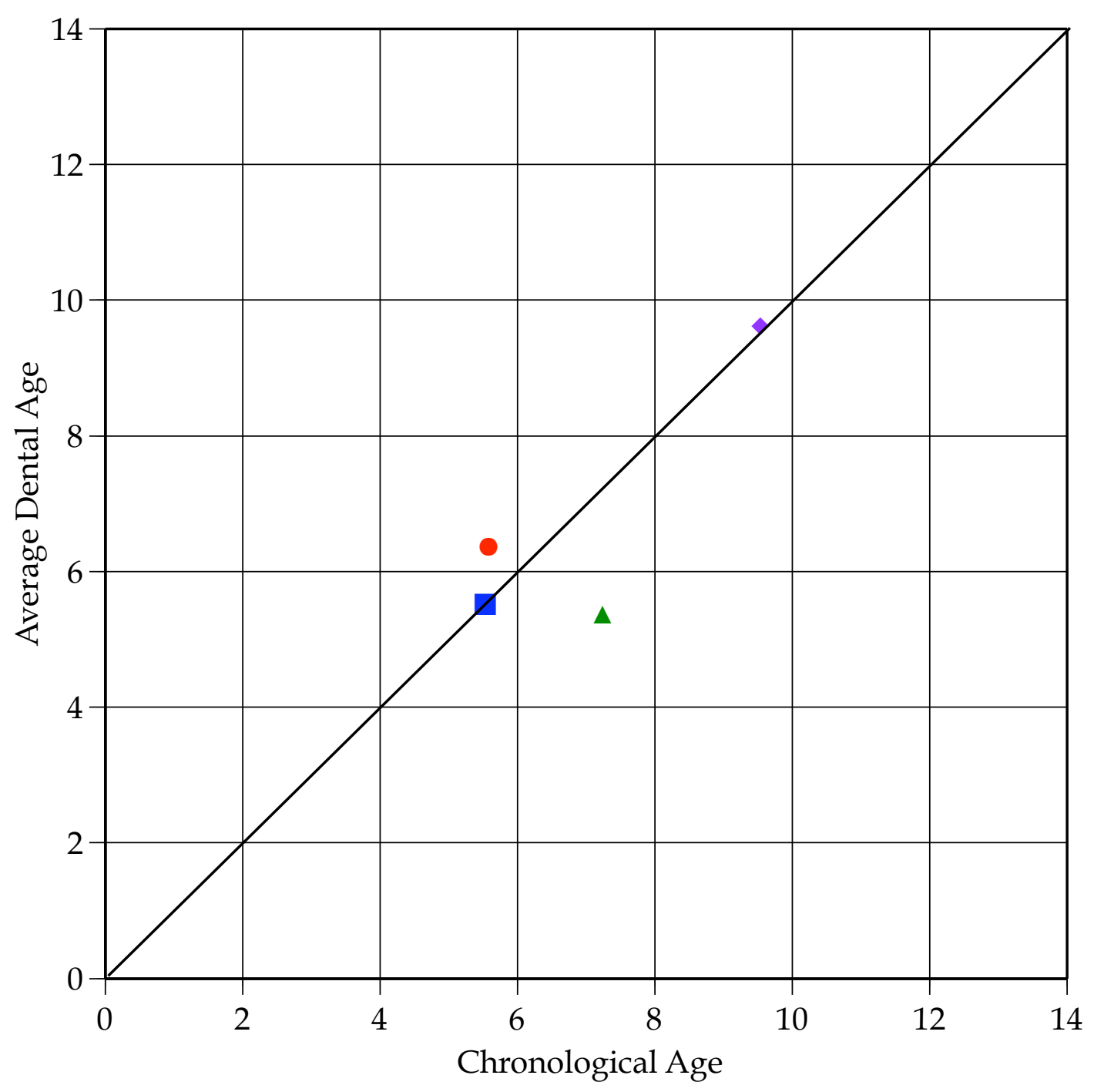

Fig. A-31. Plot of chronological age against dental age averaged across all scorable teeth (symbols) for a male (pseudo-record \#31). The square symbol denotes age at diagnosis of ALL. 


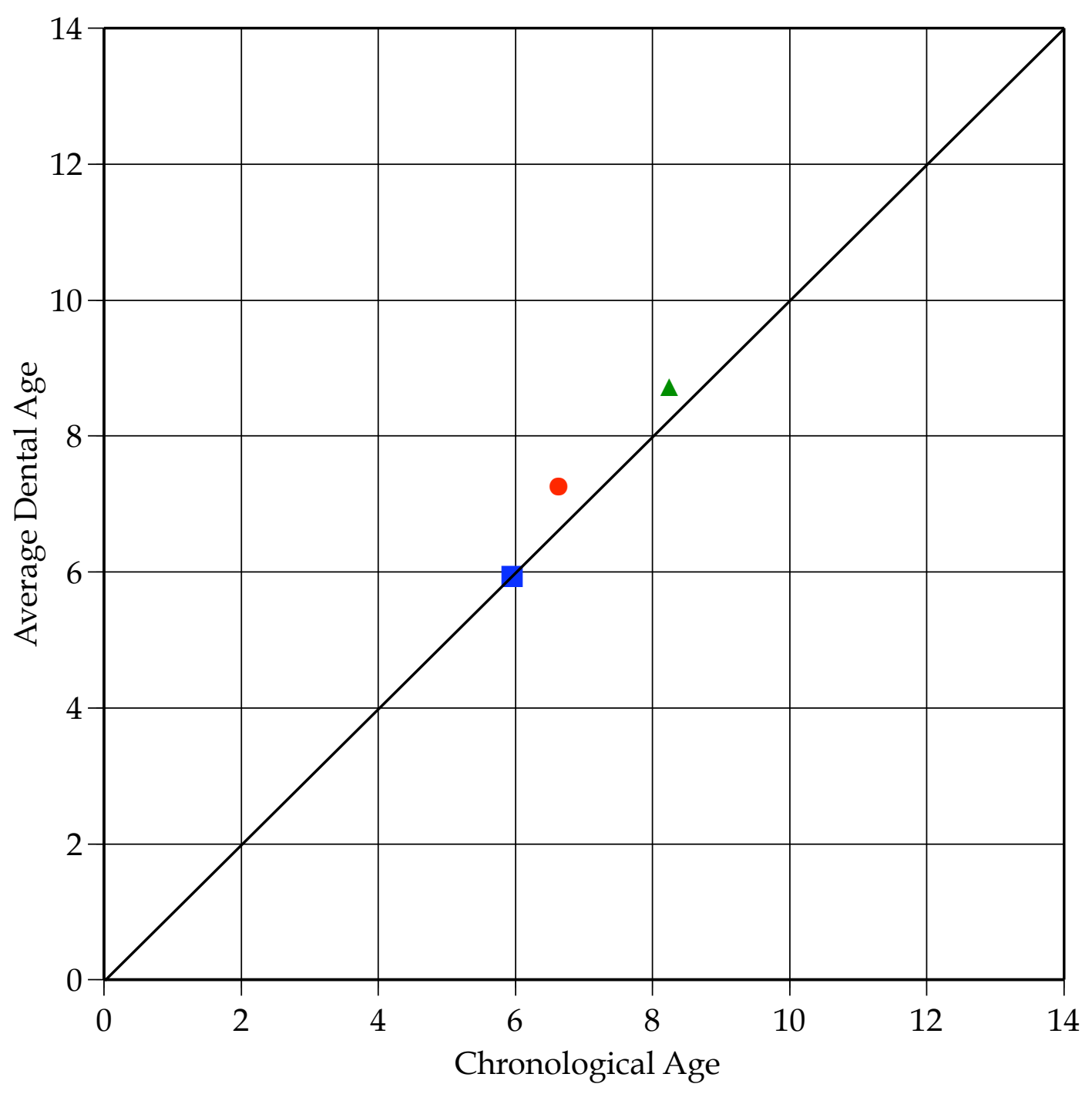

Fig. A-32. Plot of chronological age against dental age averaged across all scorable teeth (symbols) for a male (pseudo-record \#32). The square symbol denotes age at diagnosis of ALL. 


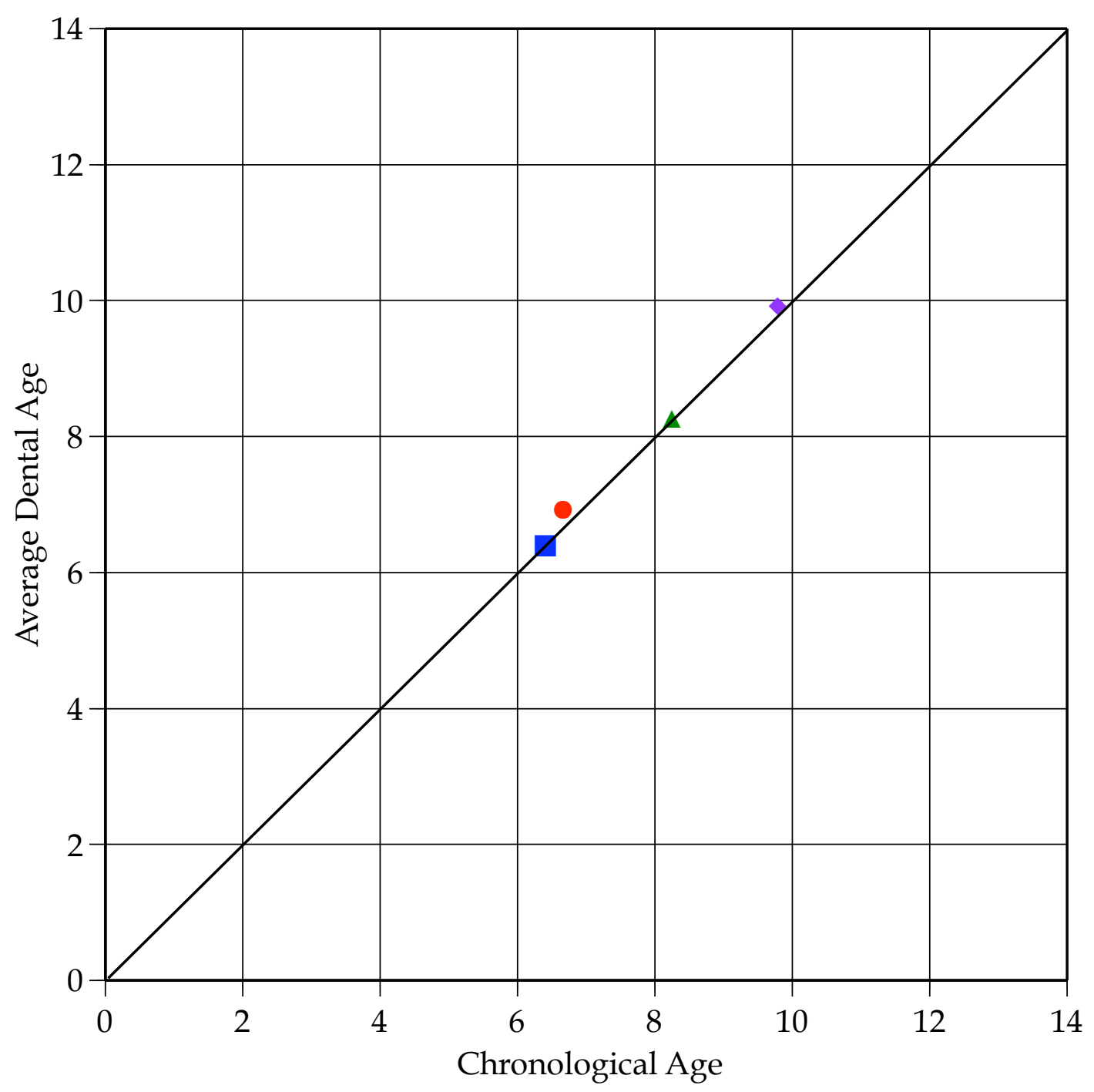

Fig. A-33. Plot of chronological age against dental age averaged across all scorable teeth (symbols) for a male (pseudo-record \#33). The square symbol denotes age at diagnosis of ALL. 


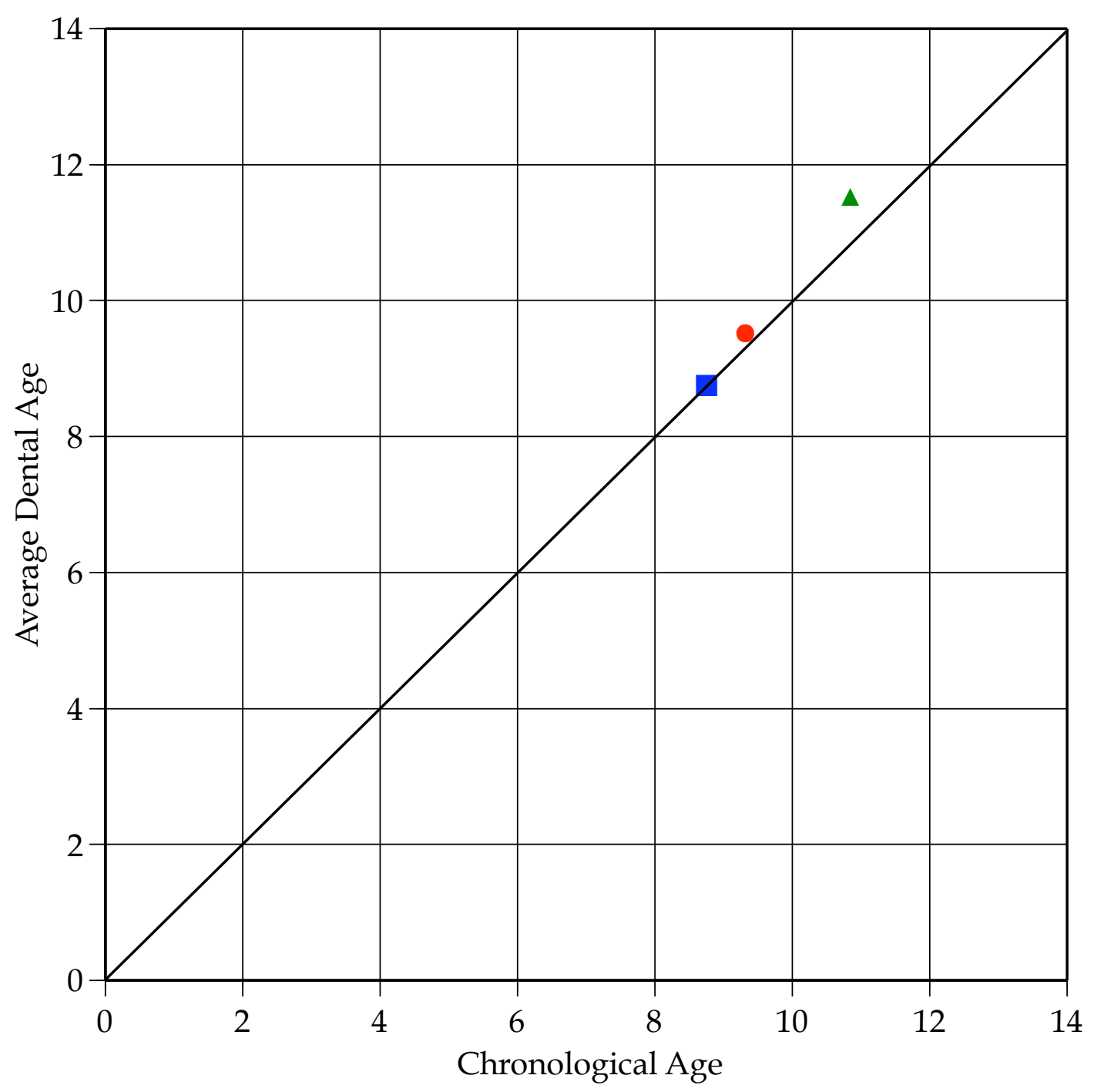

Fig. A-34. Plot of chronological age against dental age averaged across all scorable teeth (symbols) for a male (pseudo-record \#34). The square symbol denotes age at diagnosis of ALL. 


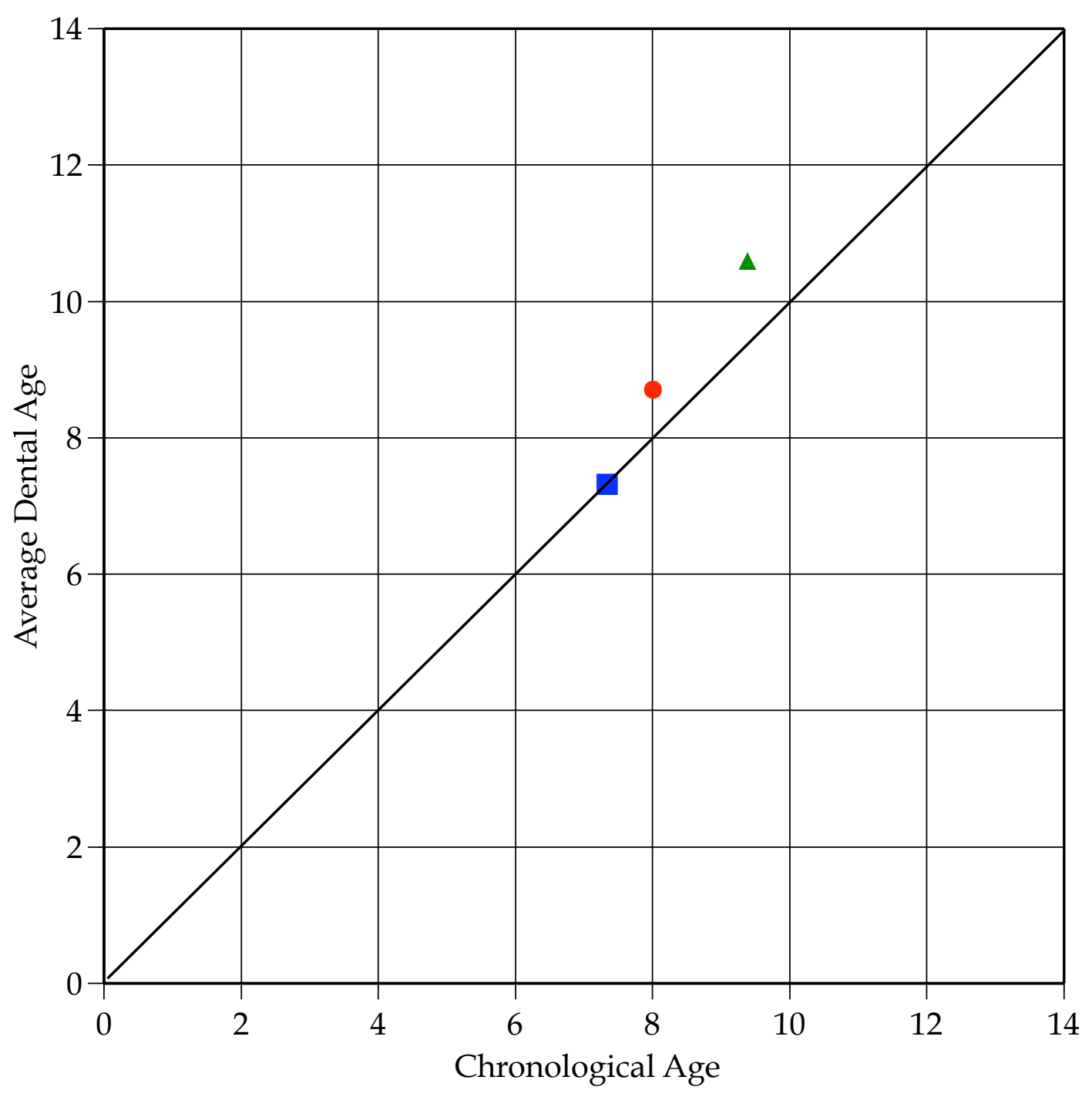

Fig. A-35. Plot of chronological age against dental age averaged across all scorable teeth (symbols) for a male (pseudo-record \#35). The square symbol denotes age at diagnosis of ALL. 


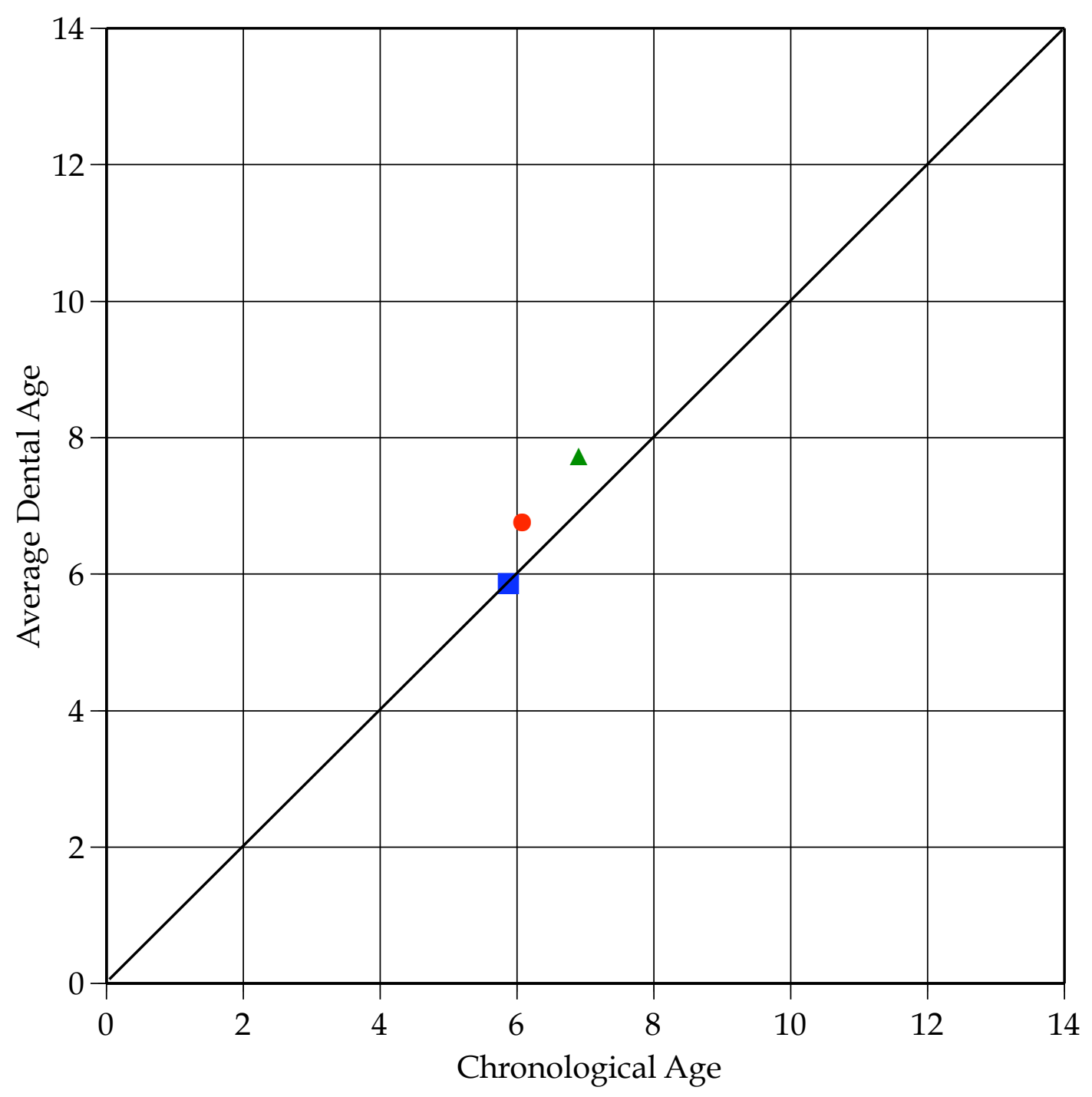

Fig. A-36. Plot of chronological age against dental age averaged across all scorable teeth (symbols) for a male (pseudo-record \#36). The square symbol denotes age at diagnosis of ALL. 


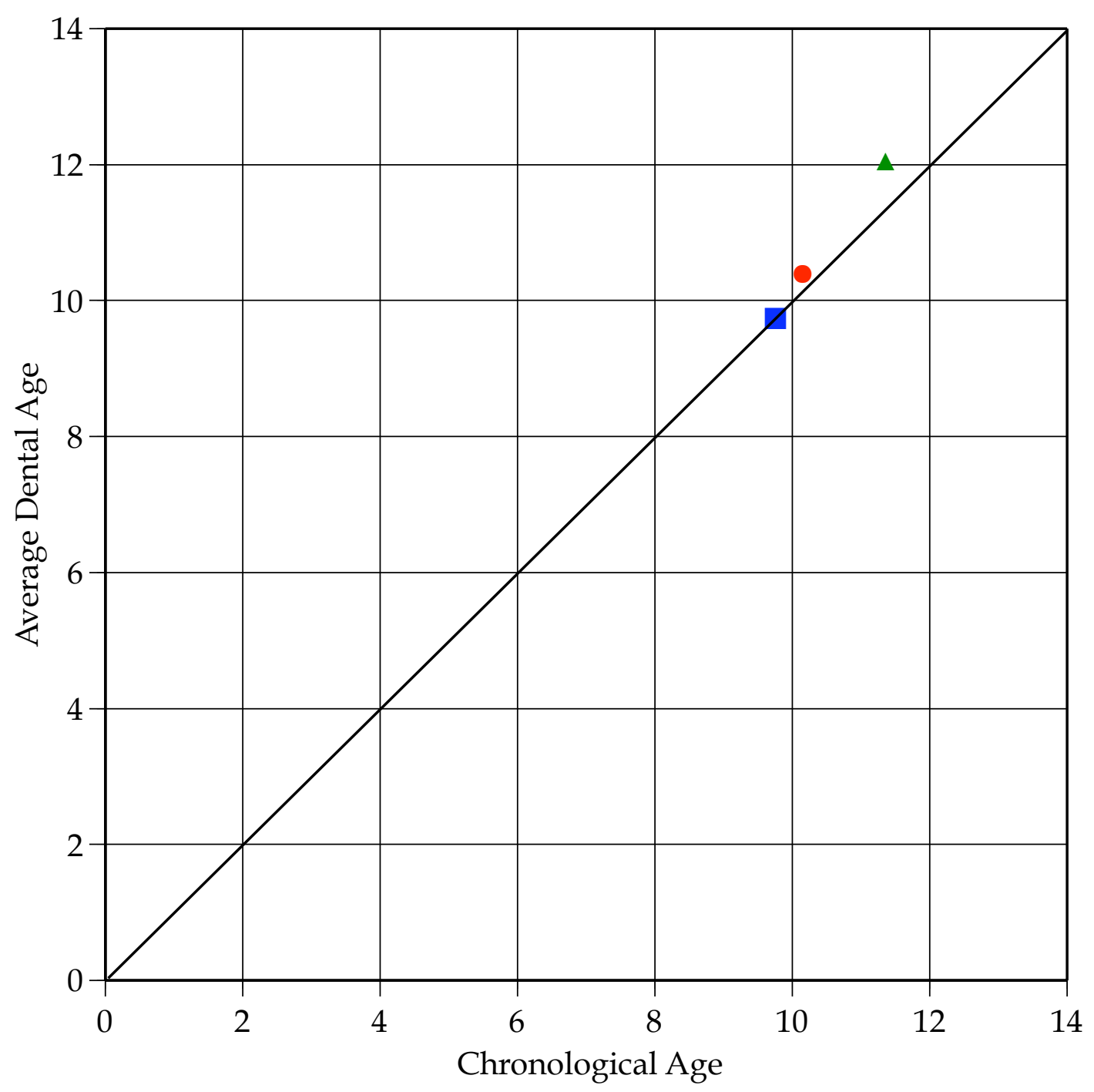

Fig. A-37. Plot of chronological age against dental age averaged across all scorable teeth (symbols) for a female (pseudo-record \#37). The square symbol denotes age at diagnosis of ALL. 


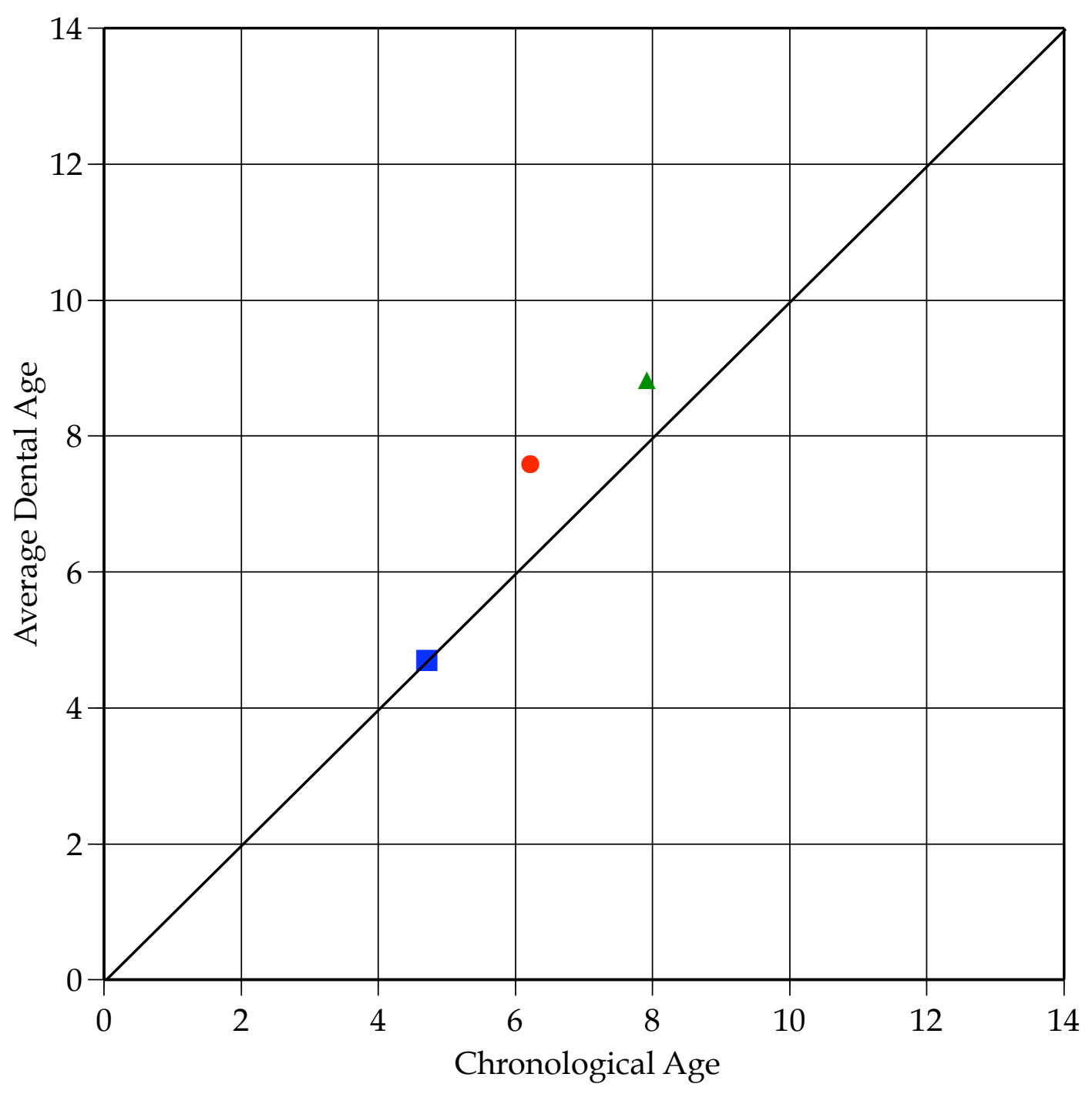

Fig. A-38. Plot of chronological age against dental age averaged across all scorable teeth (symbols) for a male (pseudo-record \#38). The square symbol denotes age at diagnosis of ALL. 


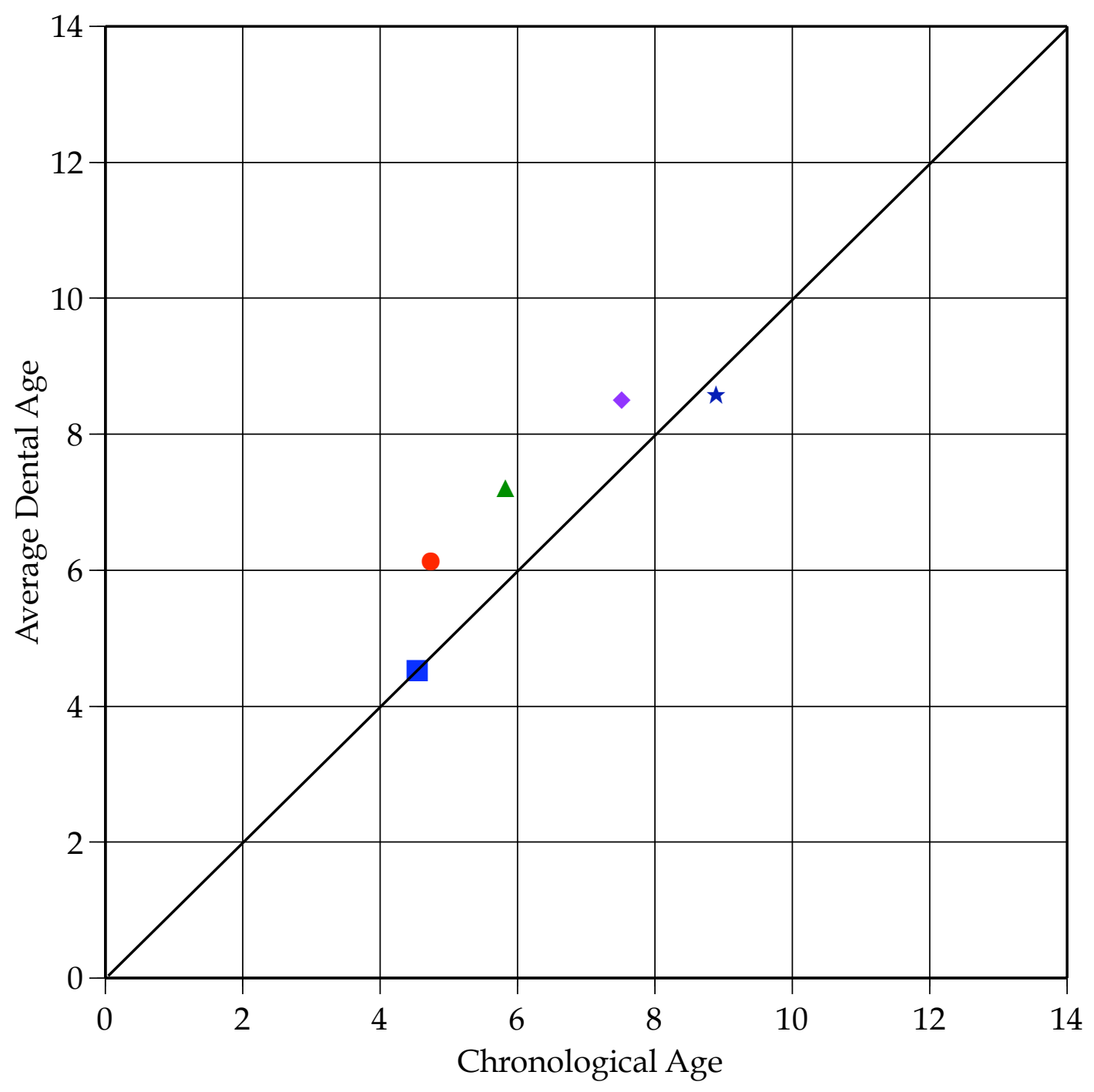

Fig. A-39. Plot of chronological age against dental age averaged across all scorable teeth (symbols) for a male (pseudo-record \#39). The square symbol denotes age at diagnosis of ALL. 


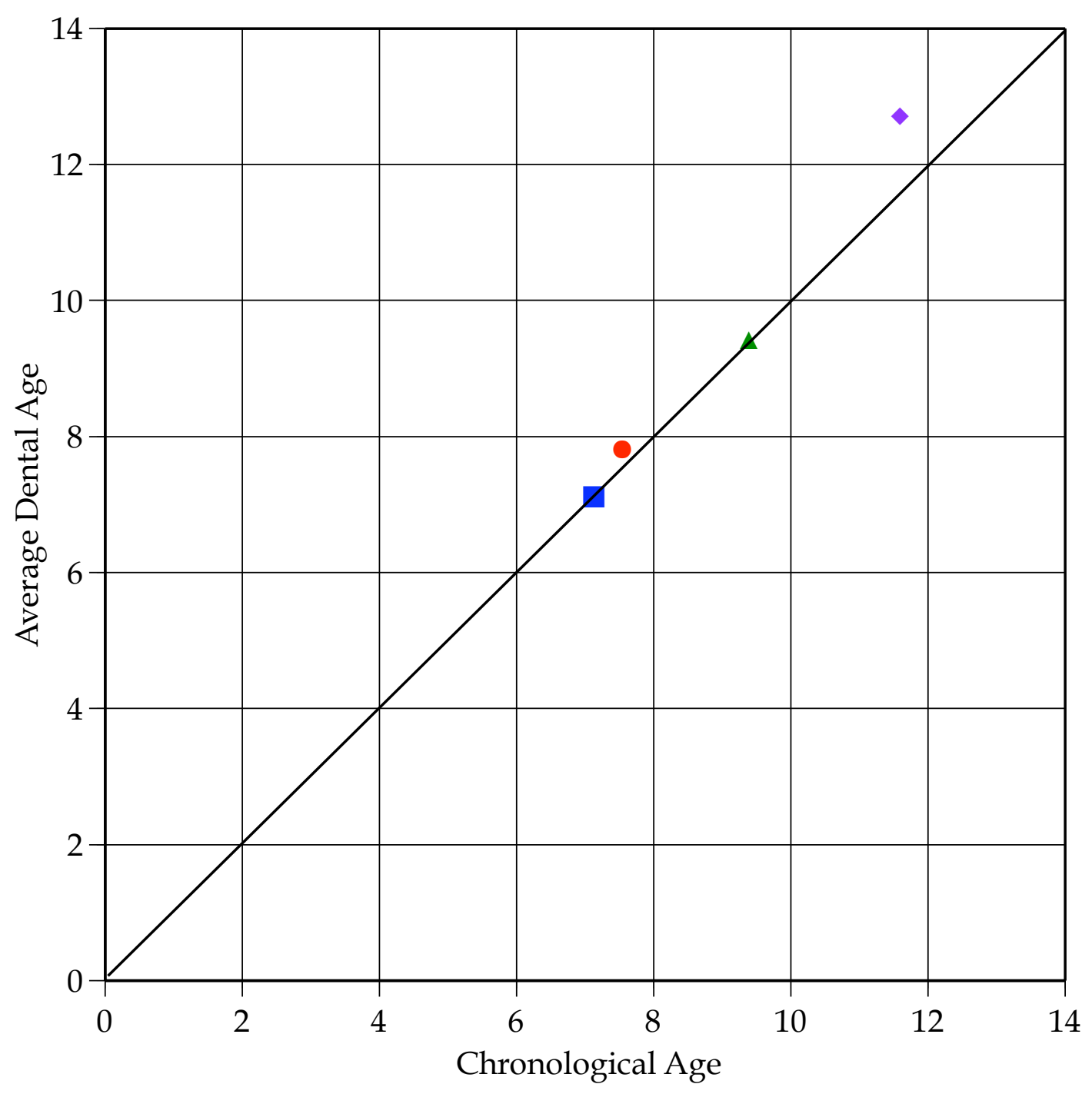

Fig. A-40. Plot of chronological age against dental age averaged across all scorable teeth (symbols) for a male (pseudo-record \#40). The square symbol denotes age at diagnosis of ALL. 


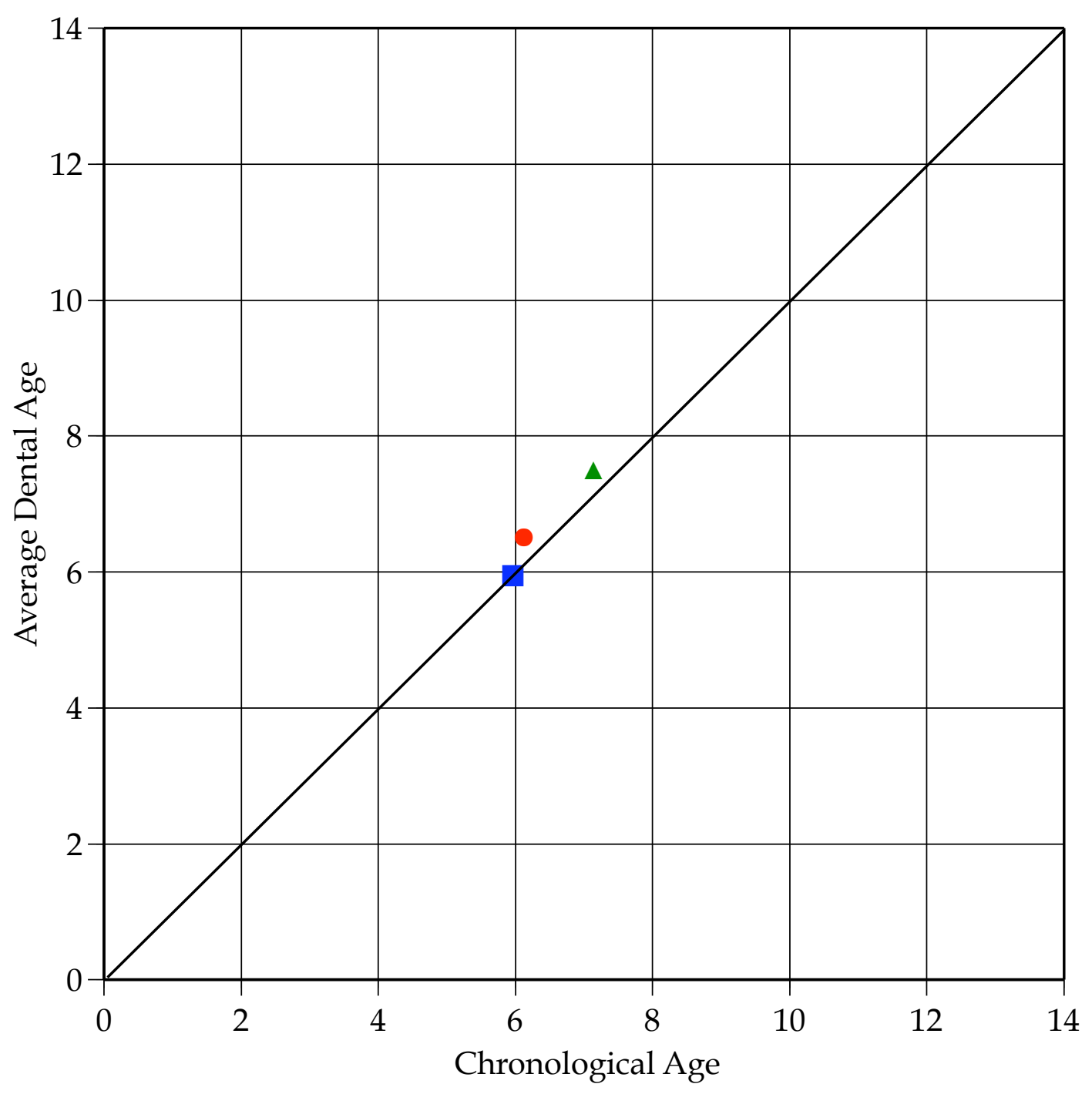

Fig. A-41. Plot of chronological age against dental age averaged across all scorable teeth (symbols) for a female (pseudo-record \#41). The square symbol denotes age at diagnosis of ALL. 


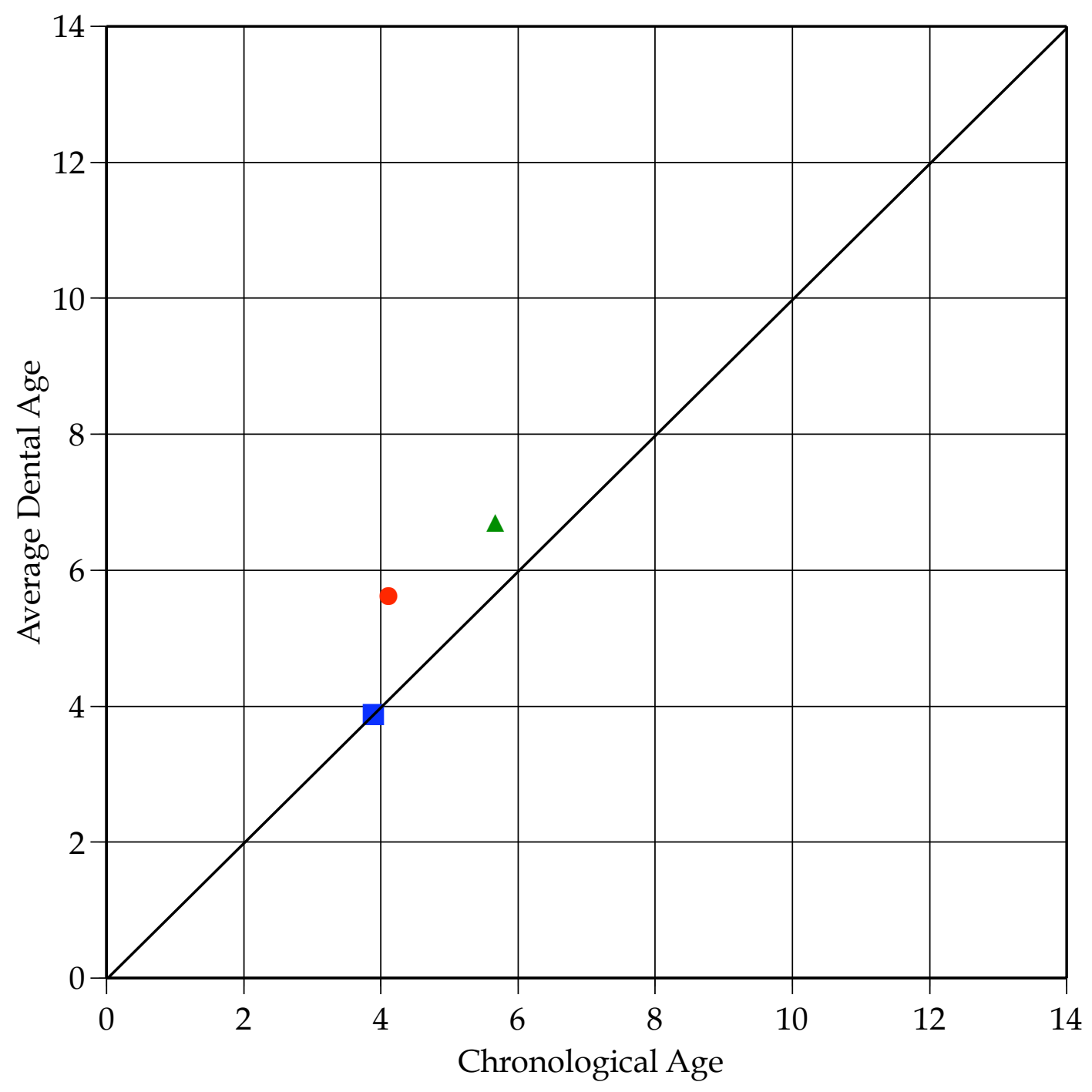

Fig. A-42. Plot of chronological age against dental age averaged across all scorable teeth (symbols) for a male (pseudo-record \#42). The square symbol denotes age at diagnosis of ALL. 


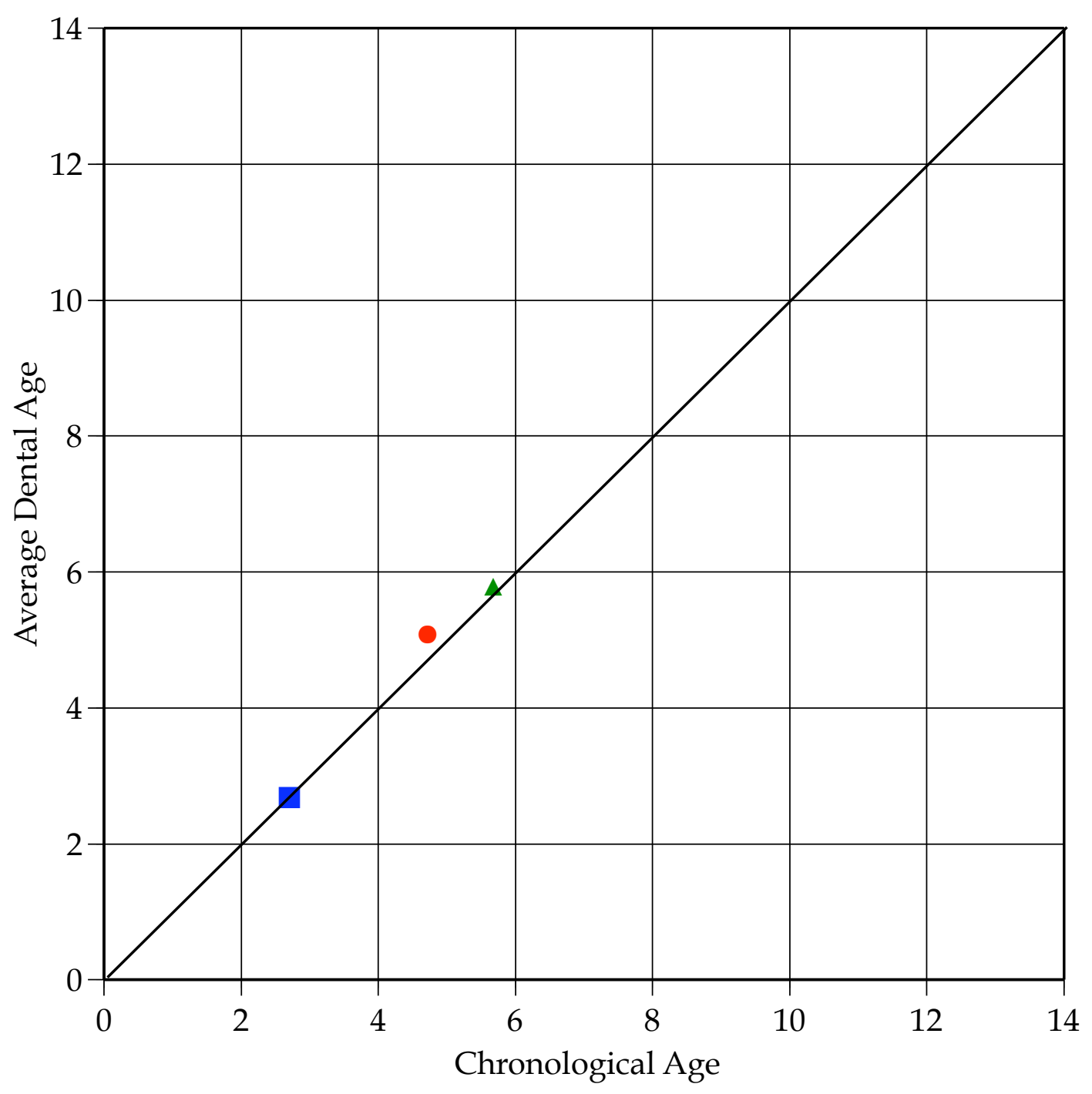

Fig. A-43. Plot of chronological age against dental age averaged across all scorable teeth (symbols) for a male (pseudo-record \#43). The square symbol denotes age at diagnosis of ALL. 


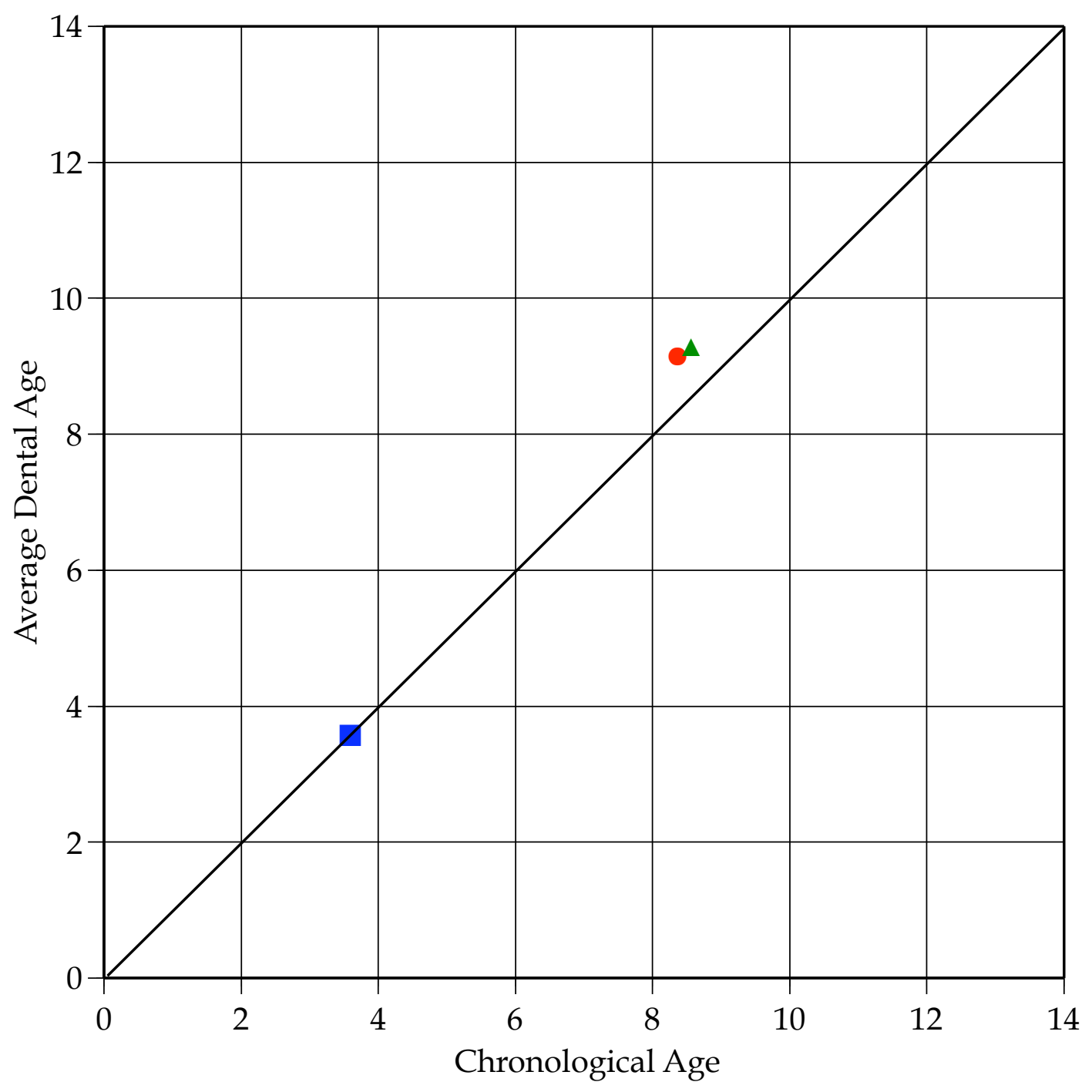

Fig. A-44. Plot of chronological age against dental age averaged across all scorable teeth (symbols) for a female (pseudo-record \#44). The square symbol denotes age at diagnosis of ALL. 


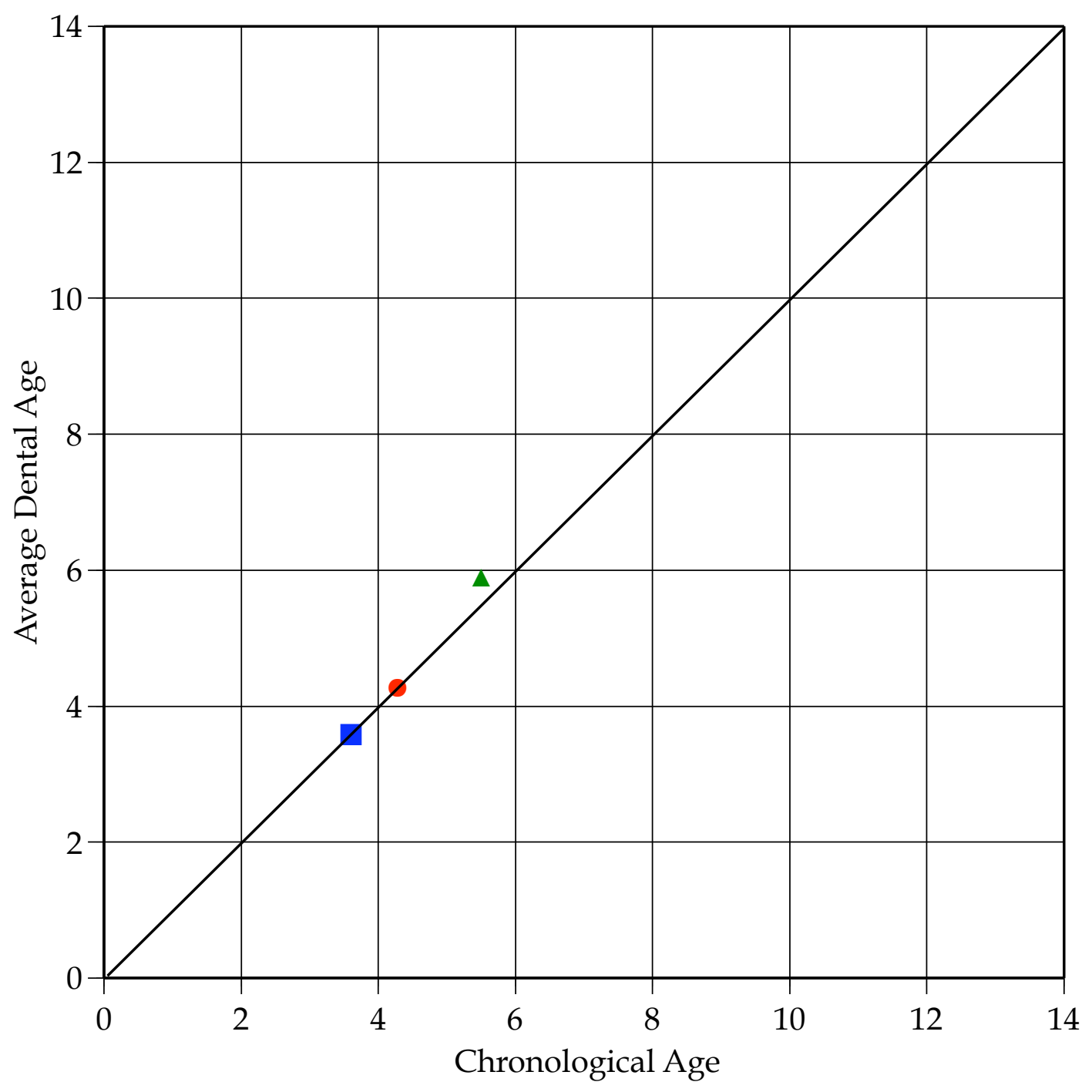

Fig. A-45. Plot of chronological age against dental age averaged across all scorable teeth (symbols) for a female (pseudo-record \#45). The square symbol denotes age at diagnosis of ALL. 


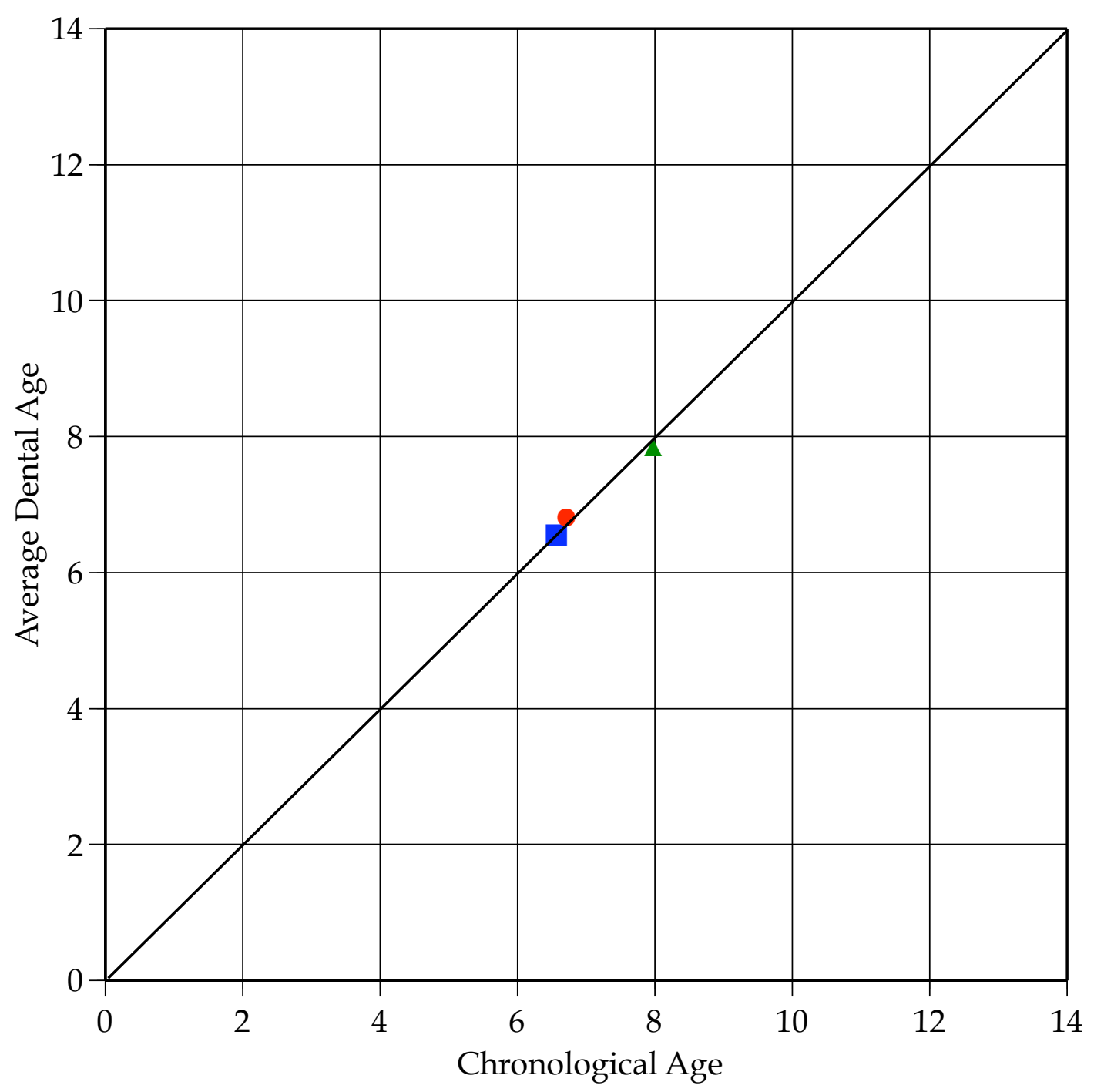

Fig. A-46. Plot of chronological age against dental age averaged across all scorable teeth (symbols) for a female (pseudo-record \#46). The square symbol denotes age at diagnosis of ALL. 


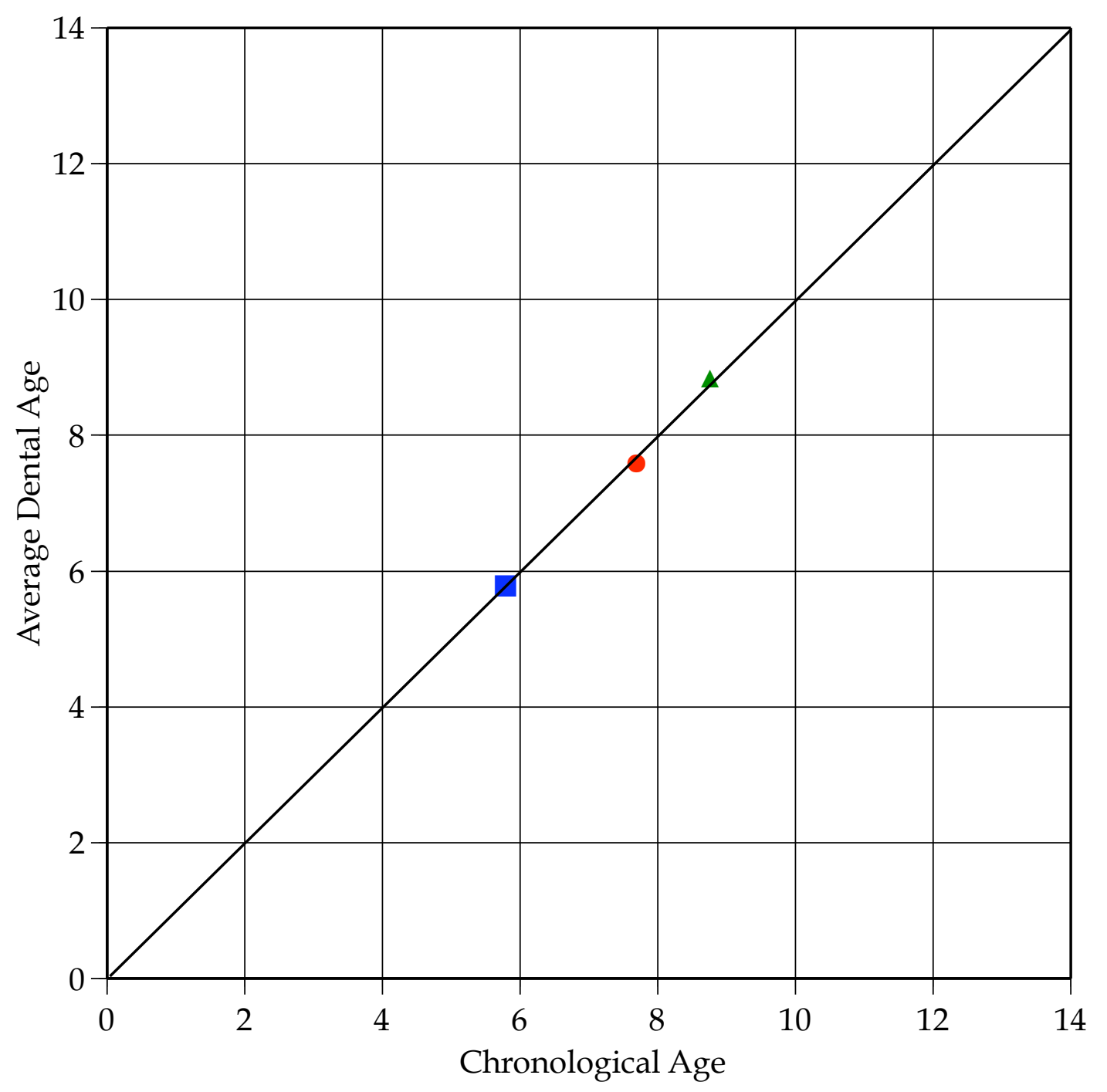

Fig. A-47. Plot of chronological age against dental age averaged across all scorable teeth (symbols) for a female (pseudo-record \#47). The square symbol denotes age at diagnosis of ALL. 


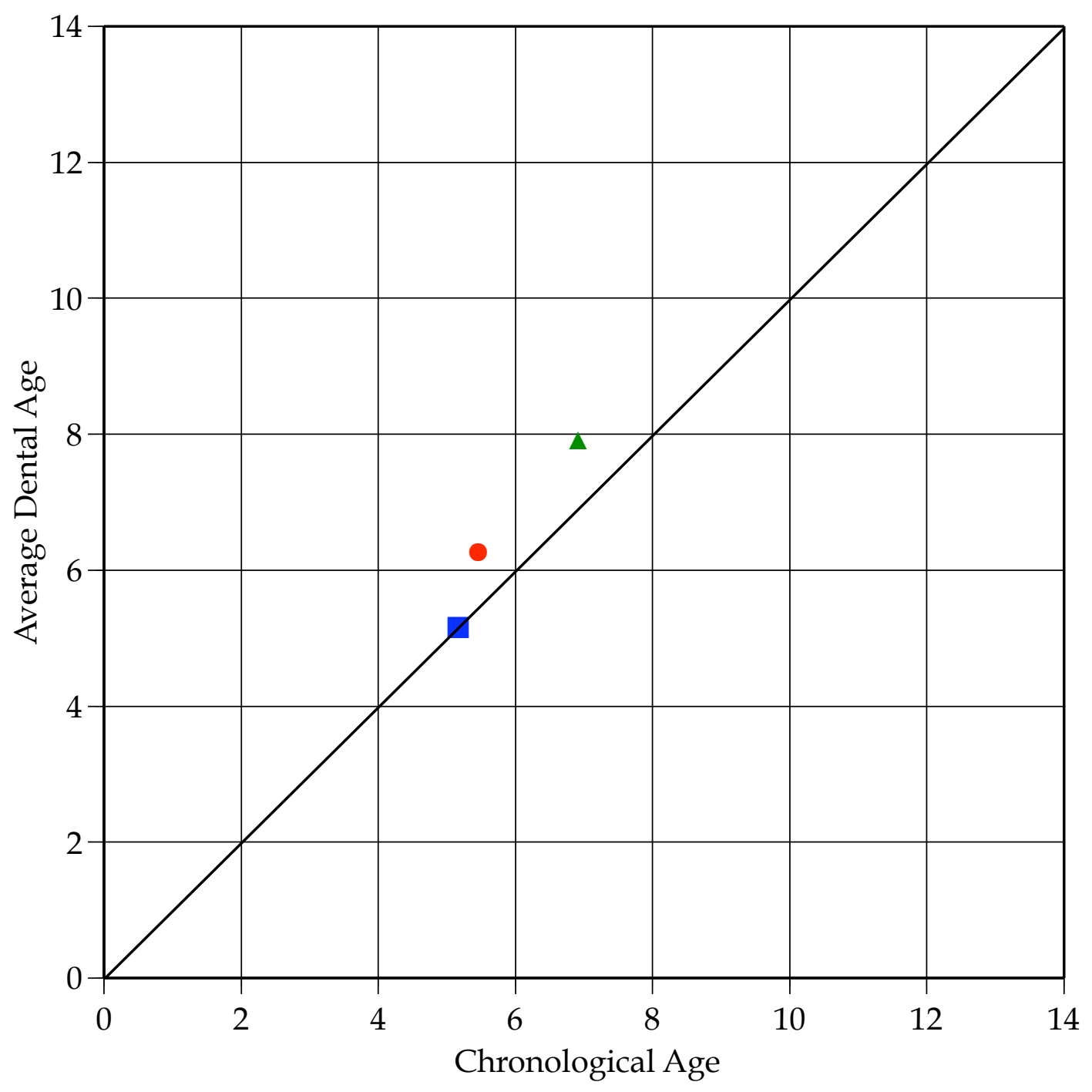

Fig. A-48. Plot of chronological age against dental age averaged across all scorable teeth (symbols) for a female (pseudo-record \#48). The square symbol denotes age at diagnosis of ALL. 


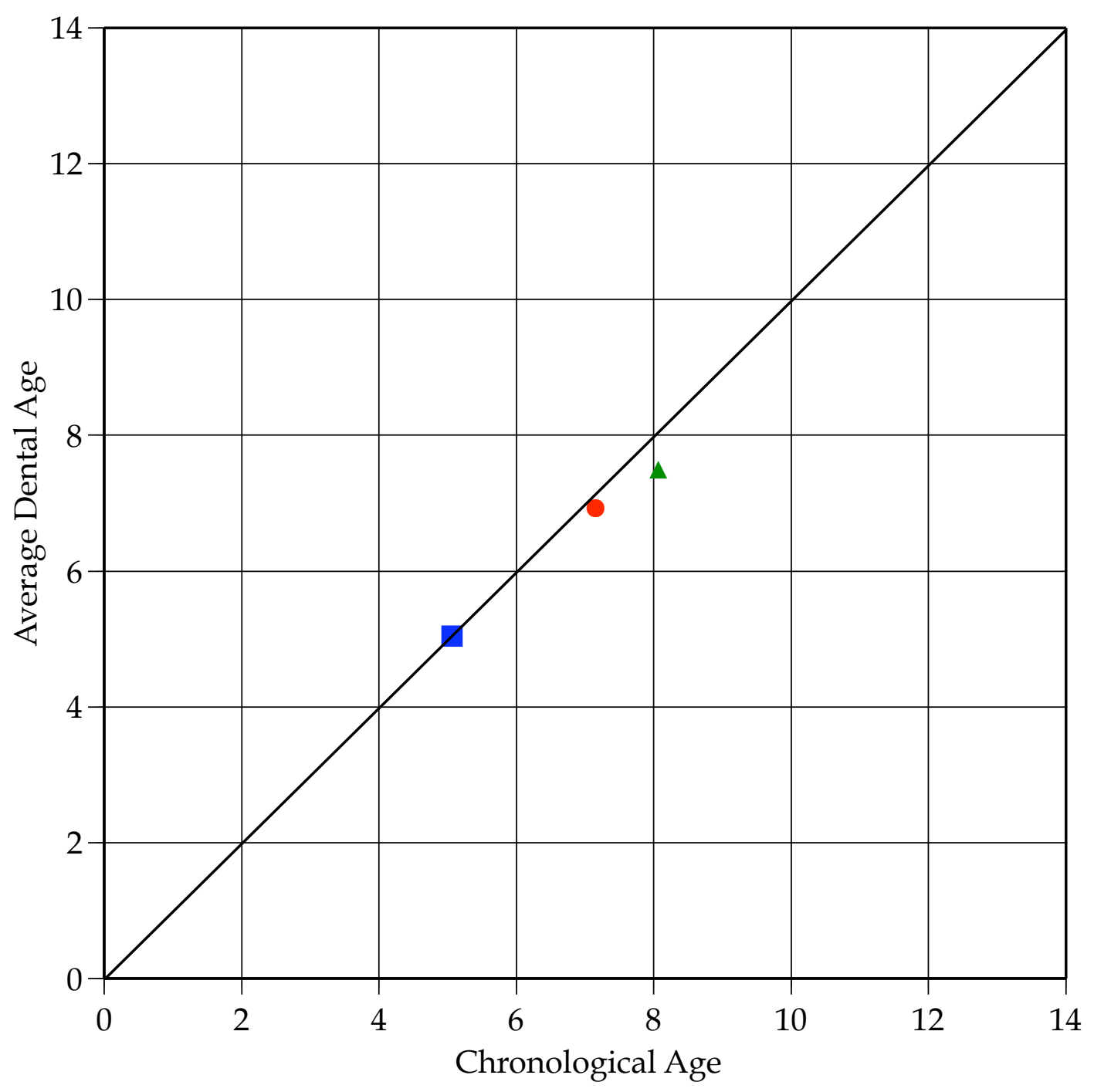

Fig. A-49. Plot of chronological age against dental age averaged across all scorable teeth (symbols) for a male (pseudo-record \#49). The square symbol denotes age at diagnosis of ALL. 


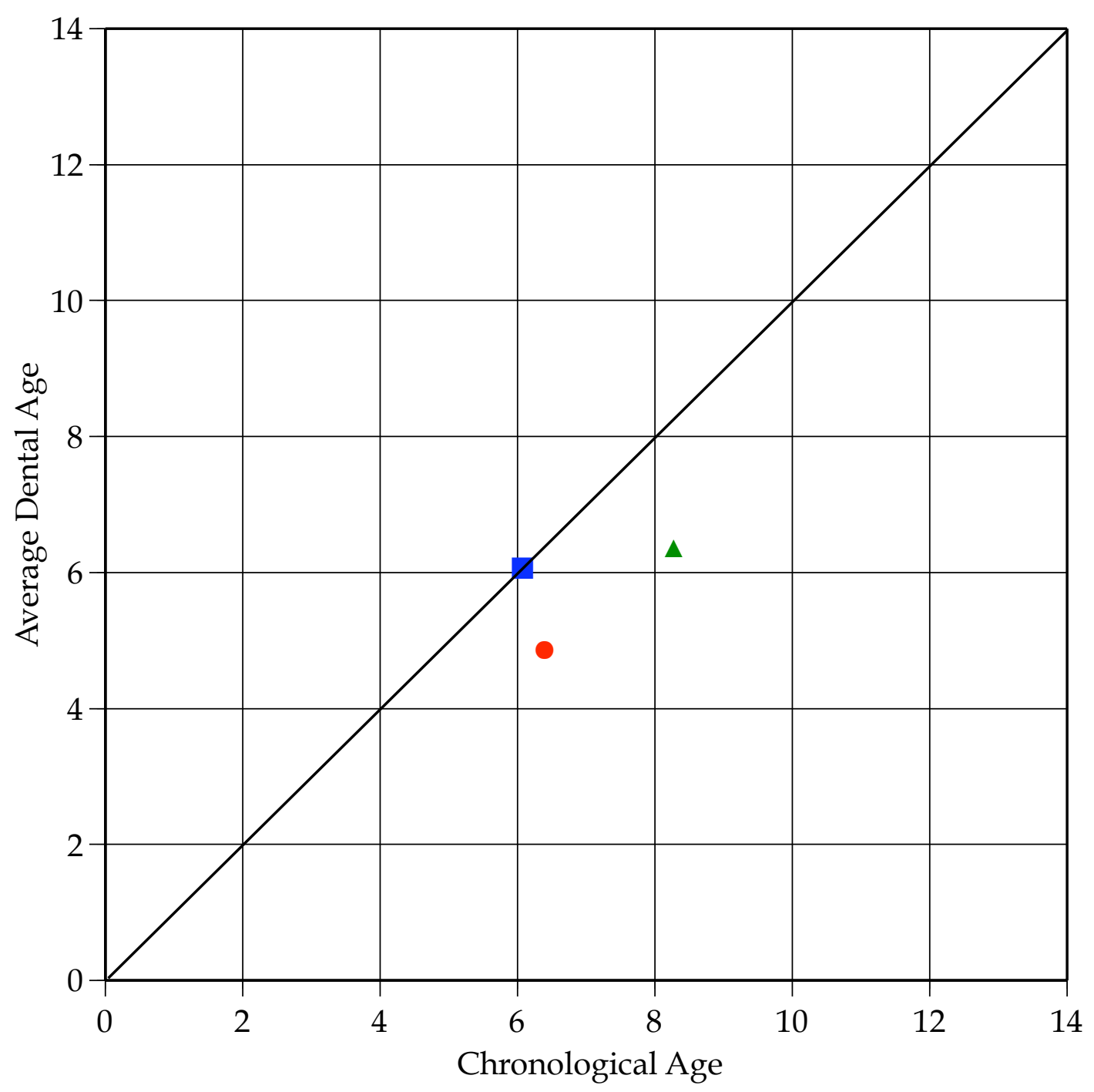

Fig. A-50. Plot of chronological age against dental age averaged across all scorable teeth (symbols) for a female (pseudo-record \#50). The square symbol denotes age at diagnosis of ALL. 


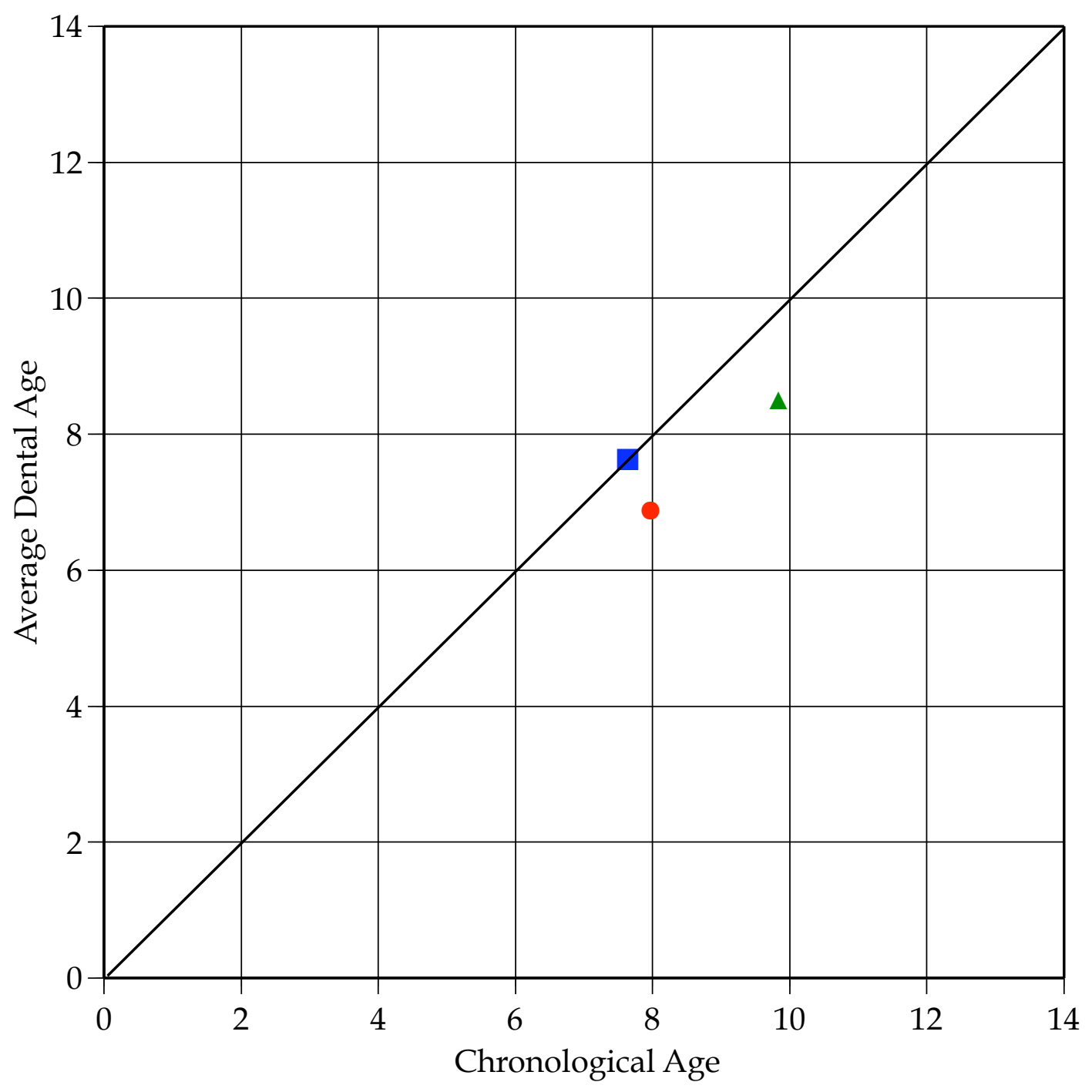

Fig. A-51. Plot of chronological age against dental age averaged across all scorable teeth (symbols) for a male (pseudo-record \#51). The square symbol denotes age at diagnosis of ALL. 


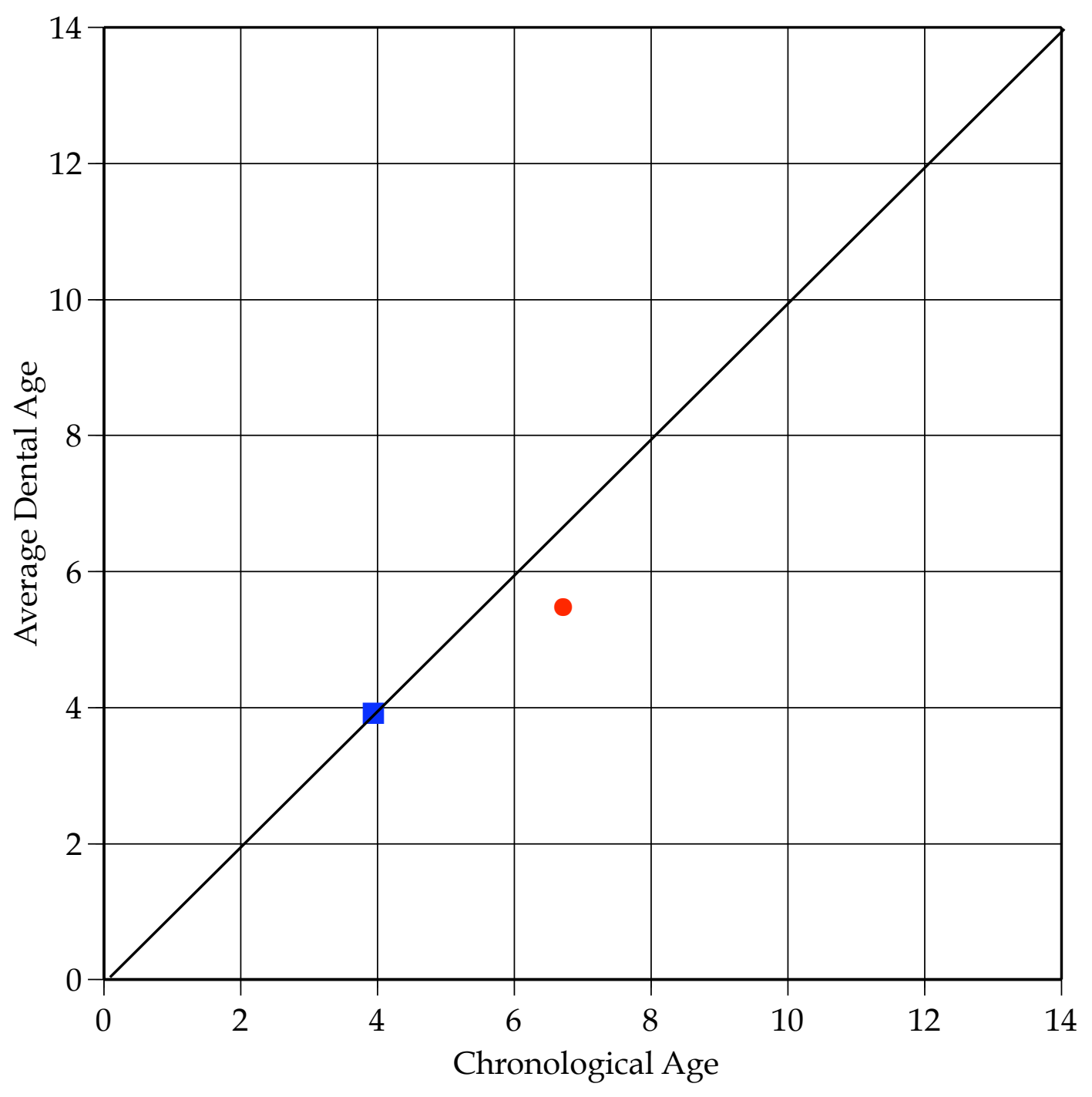

Fig. A-52. Plot of chronological age against dental age averaged across all scorable teeth (symbol) for a male (pseudo-record \#52). The square symbol denotes age at diagnosis of ALL. 


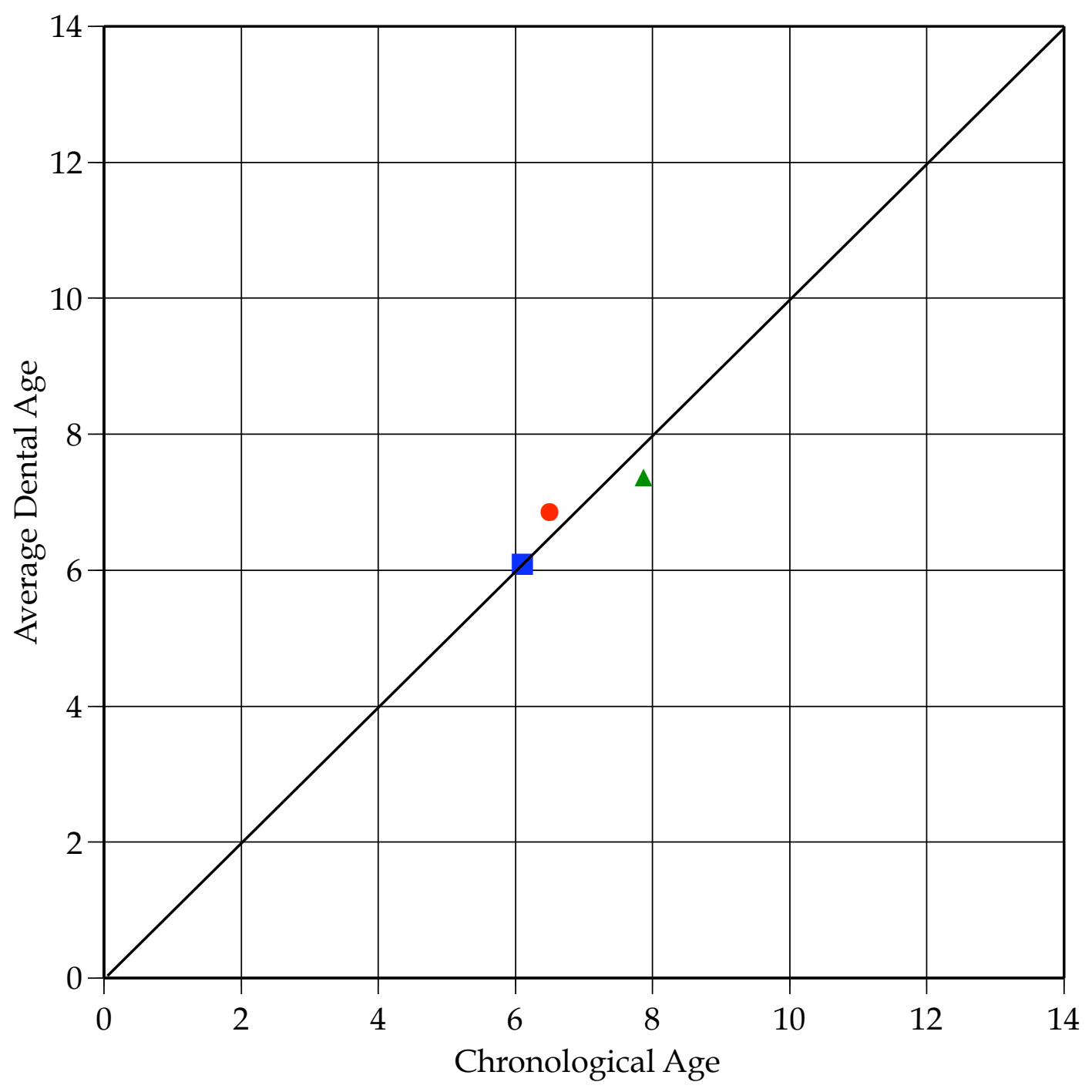

Fig. A-53. Plot of chronological age against dental age averaged across all scorable teeth (symbols) for a female (pseudo-record \#53). The square symbol denotes age at diagnosis of ALL. 


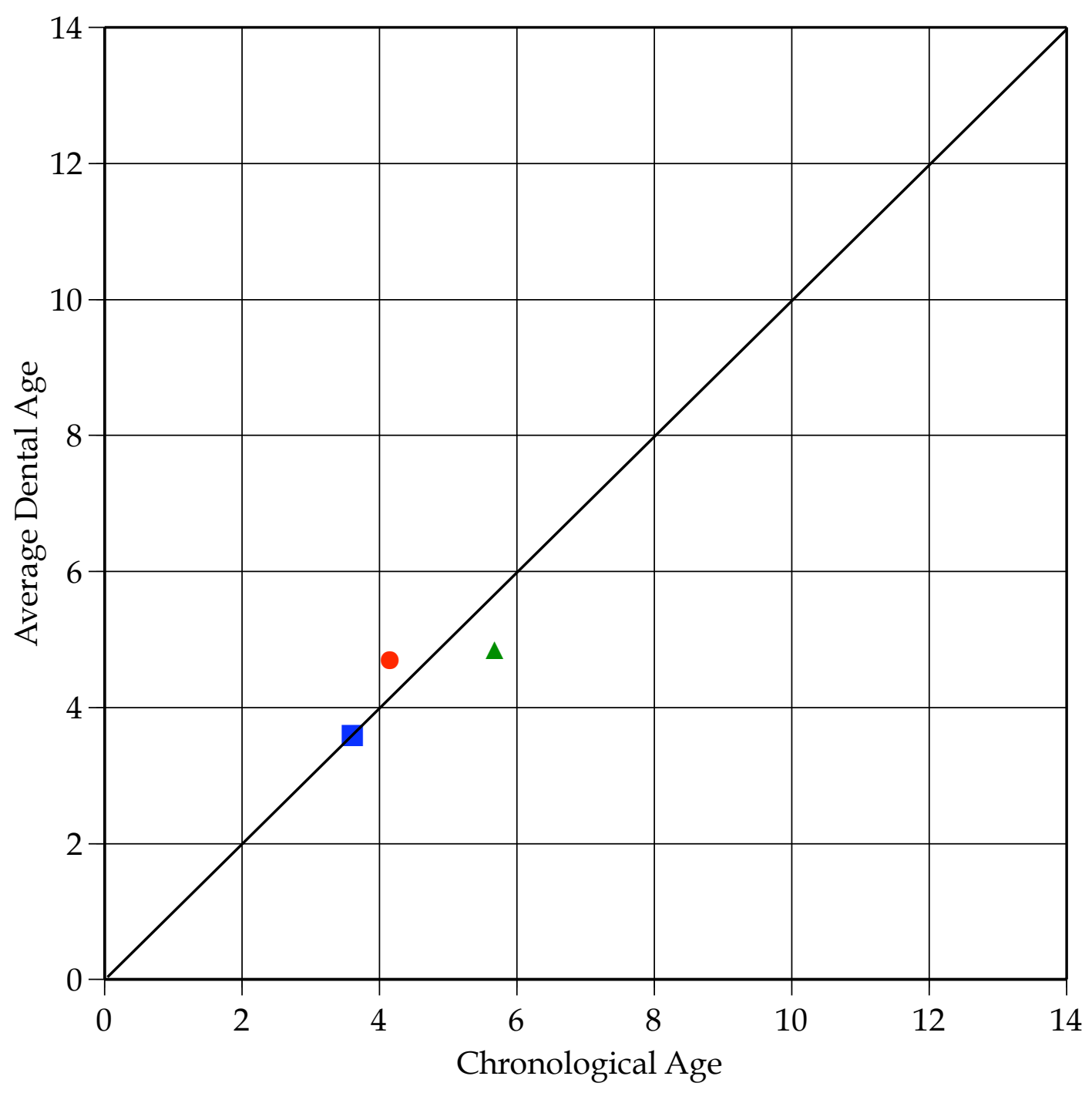

Fig. A-54. Plot of chronological age against dental age averaged across all scorable teeth (symbols) for a male (pseudo-record \#54). The square symbol denotes age at diagnosis of ALL. 


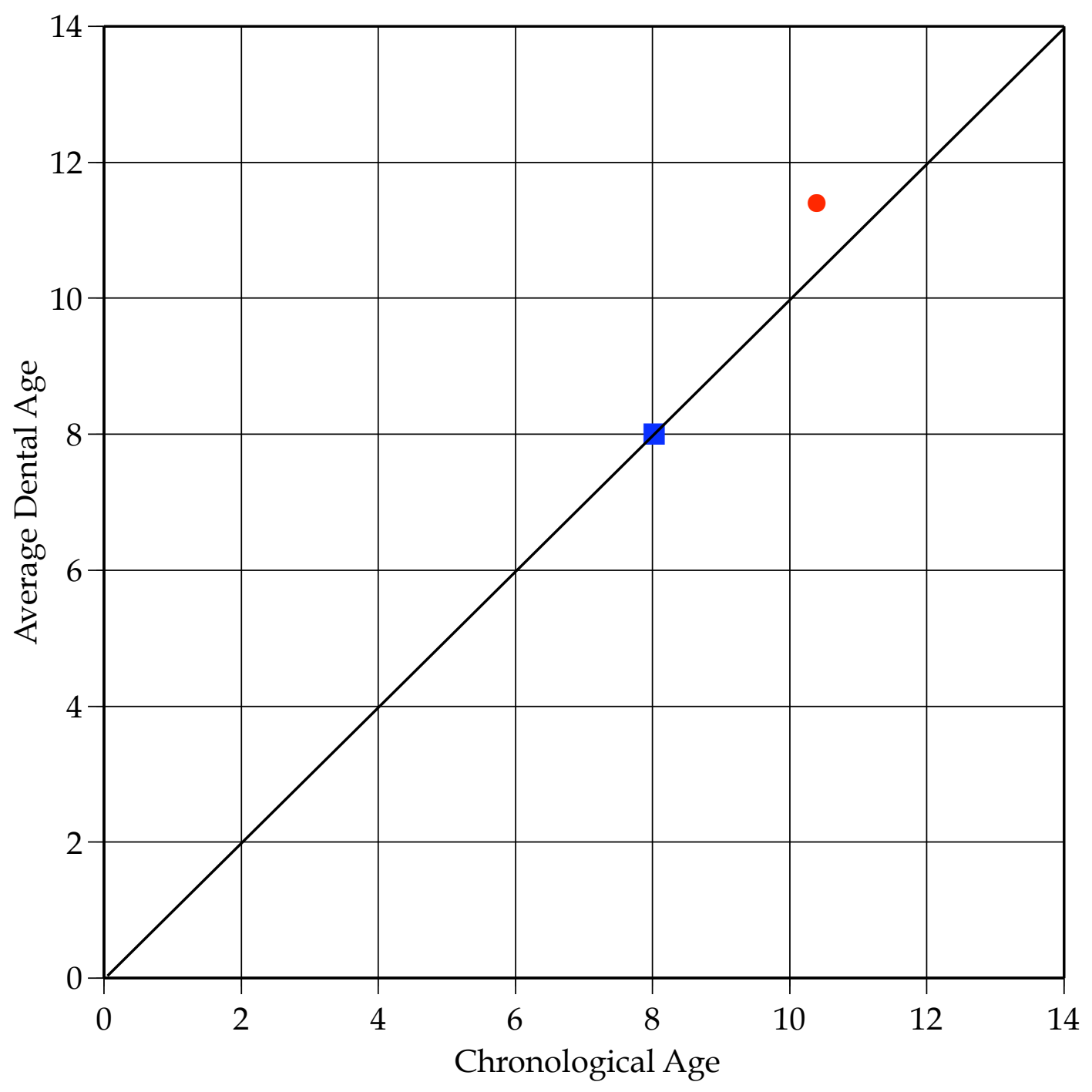

Fig. A-55. Plot of chronological age against dental age averaged across all scorable teeth (symbol) for a female (pseudo-record \#55). The square symbol denotes age at diagnosis of ALL. 


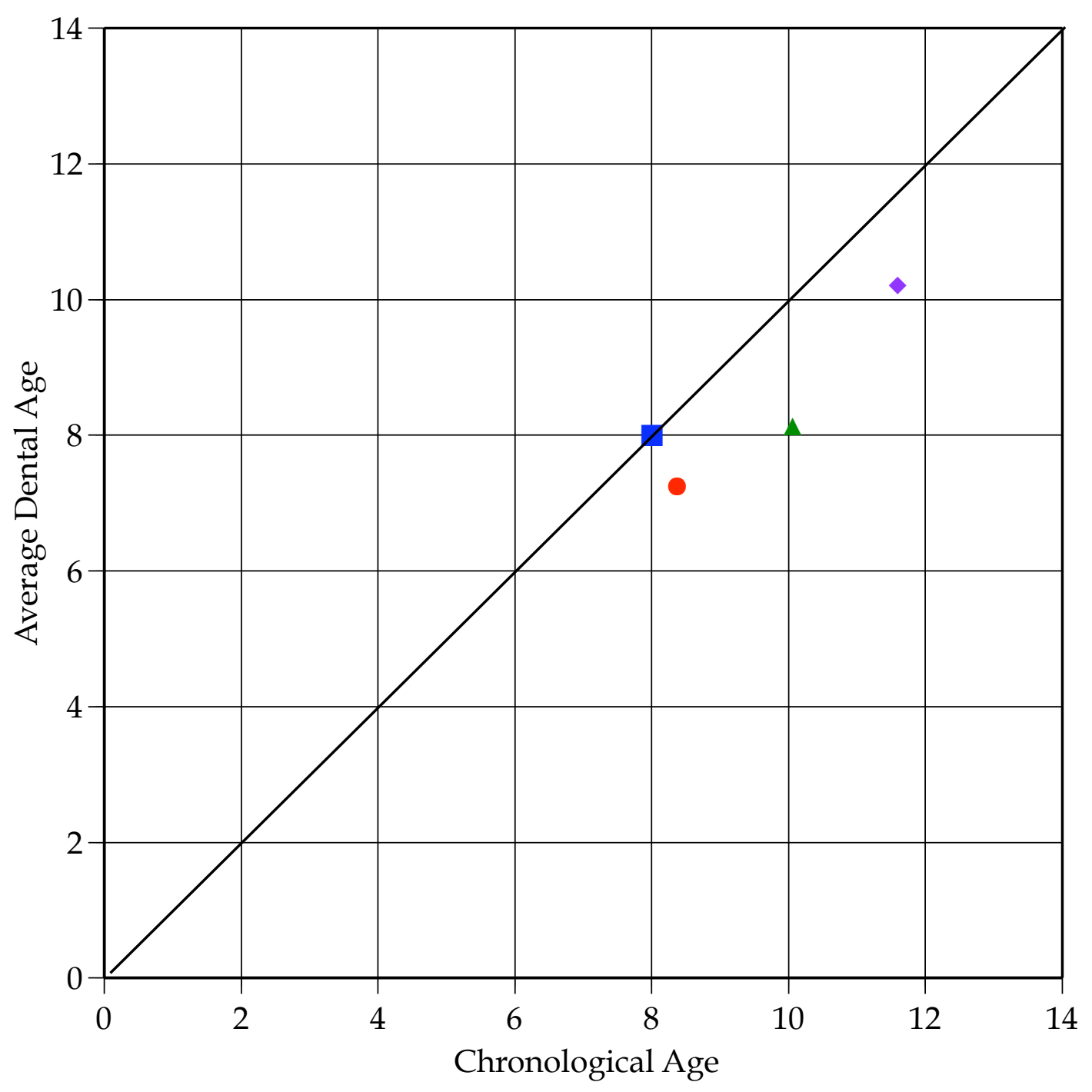

Fig. A-56. Plot of chronological age against dental age averaged across all scorable teeth (symbols) for a female (pseudo-record \#56). The square symbol denotes age at diagnosis of ALL. 


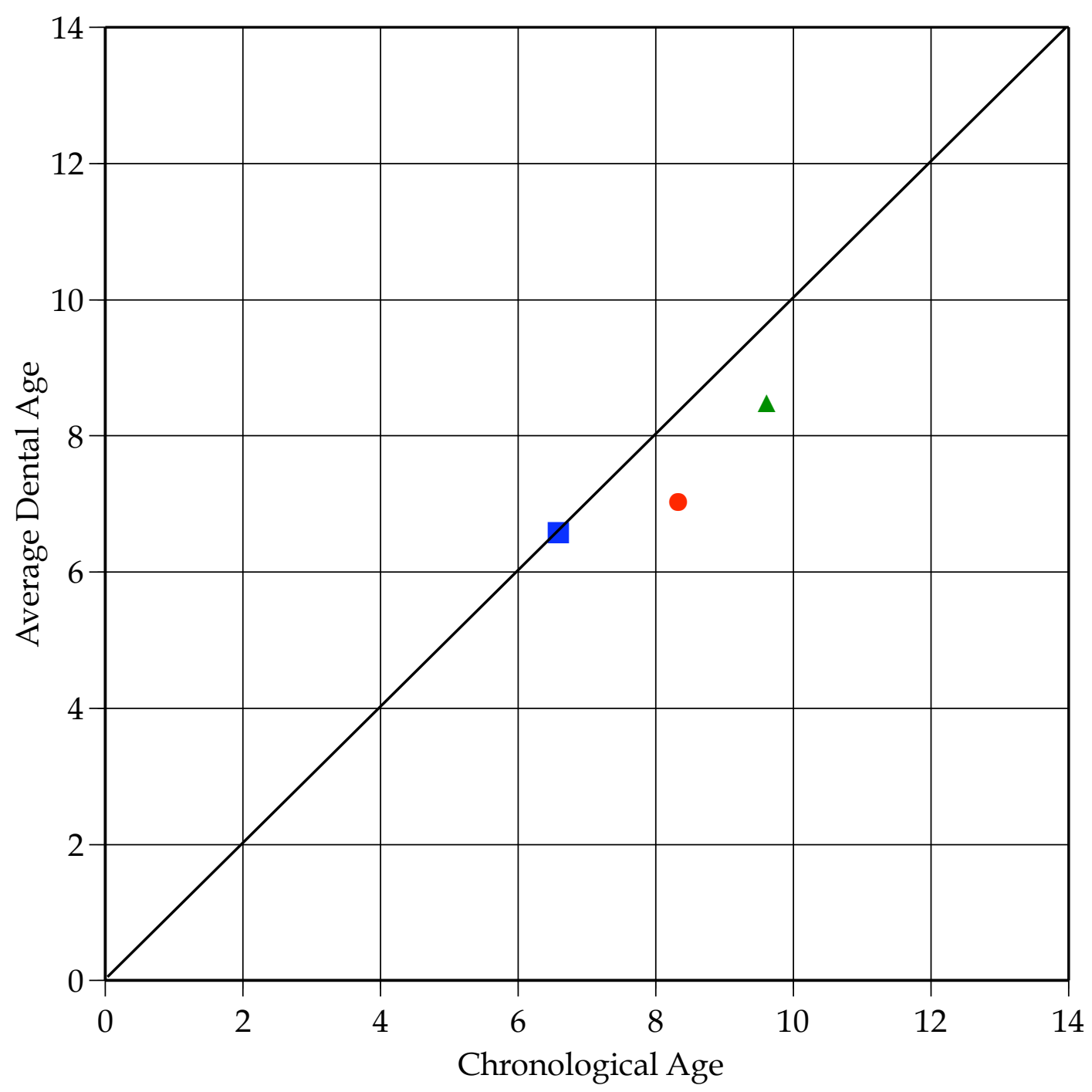

Fig. A-57. Plot of chronological age against dental age averaged across all scorable teeth (symbols) for a male (pseudo-record \#57). The square symbol denotes age at diagnosis of ALL. 


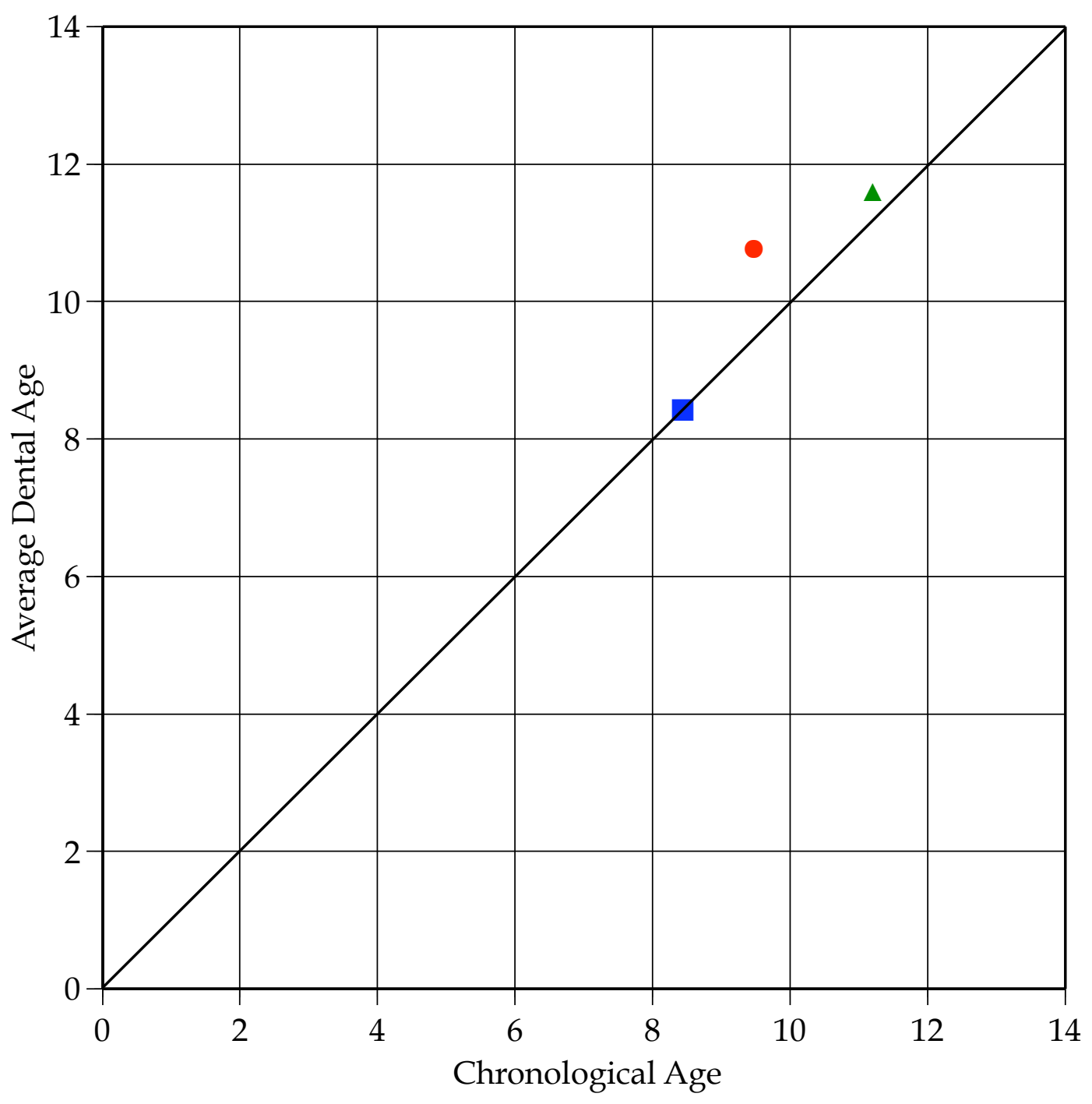

Fig. A-58. Plot of chronological age against dental age averaged across all scorable teeth (symbols) for a male (pseudo-record \#58). The square symbol denotes age at diagnosis of ALL. 


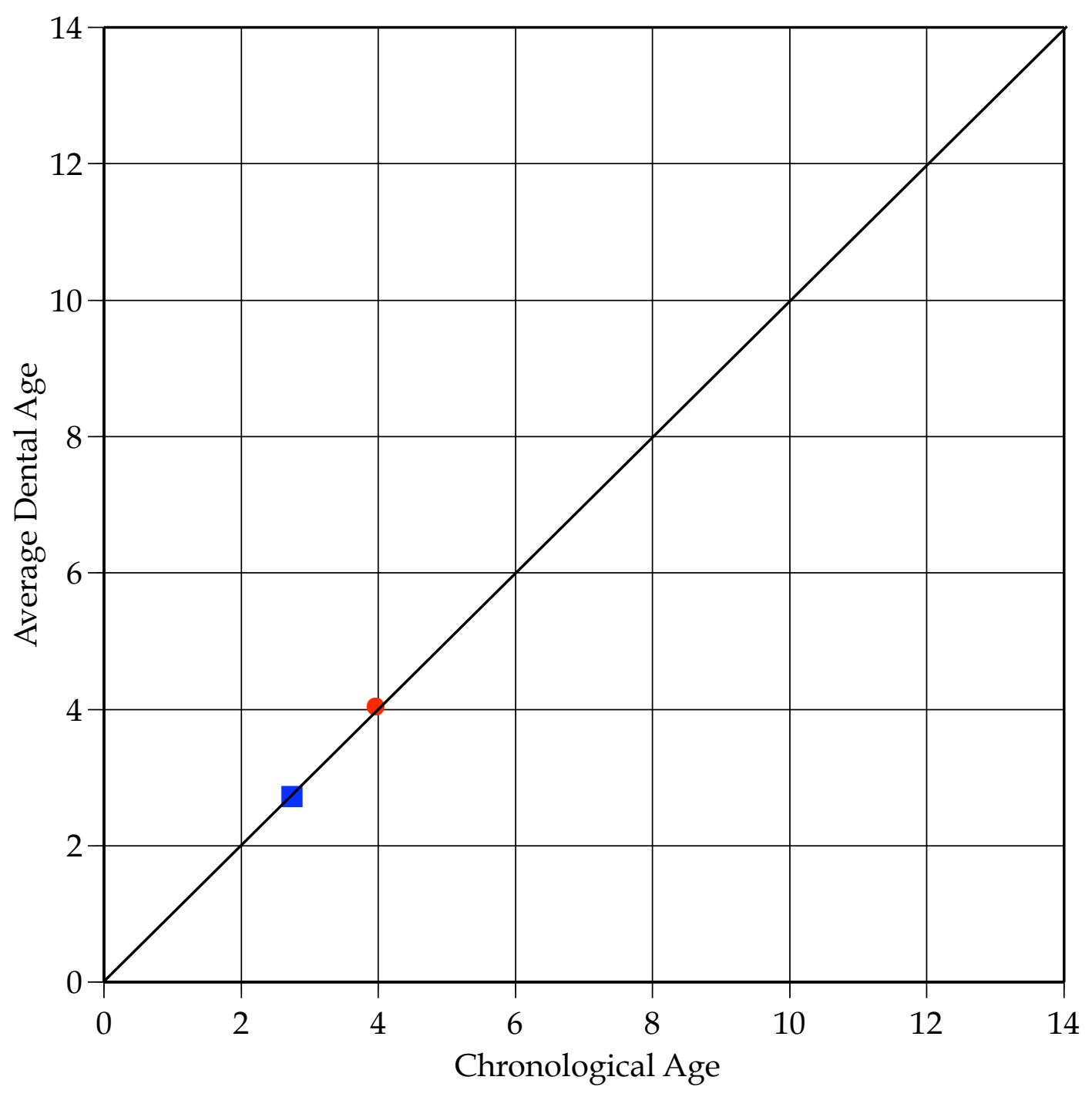

Fig. A-59. Plot of chronological age against dental age averaged across all scorable teeth (symbol) for a female (pseudo-record \#59). The square symbol denotes age at diagnosis of ALL. 


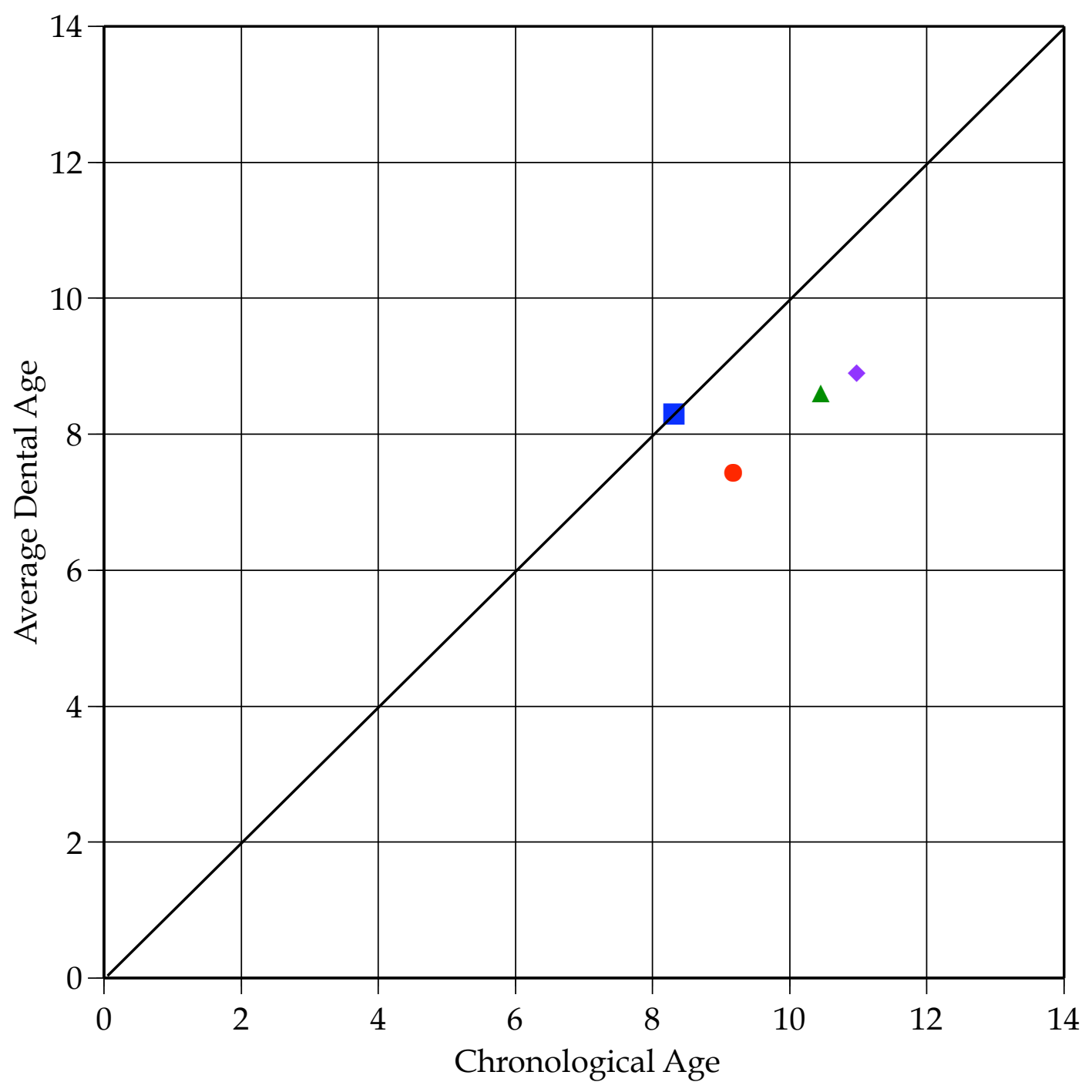

Fig. A-60. Plot of chronological age against dental age averaged across all scorable teeth (symbols) for a male (pseudo-record \#60). The square symbol denotes age at diagnosis of ALL. 


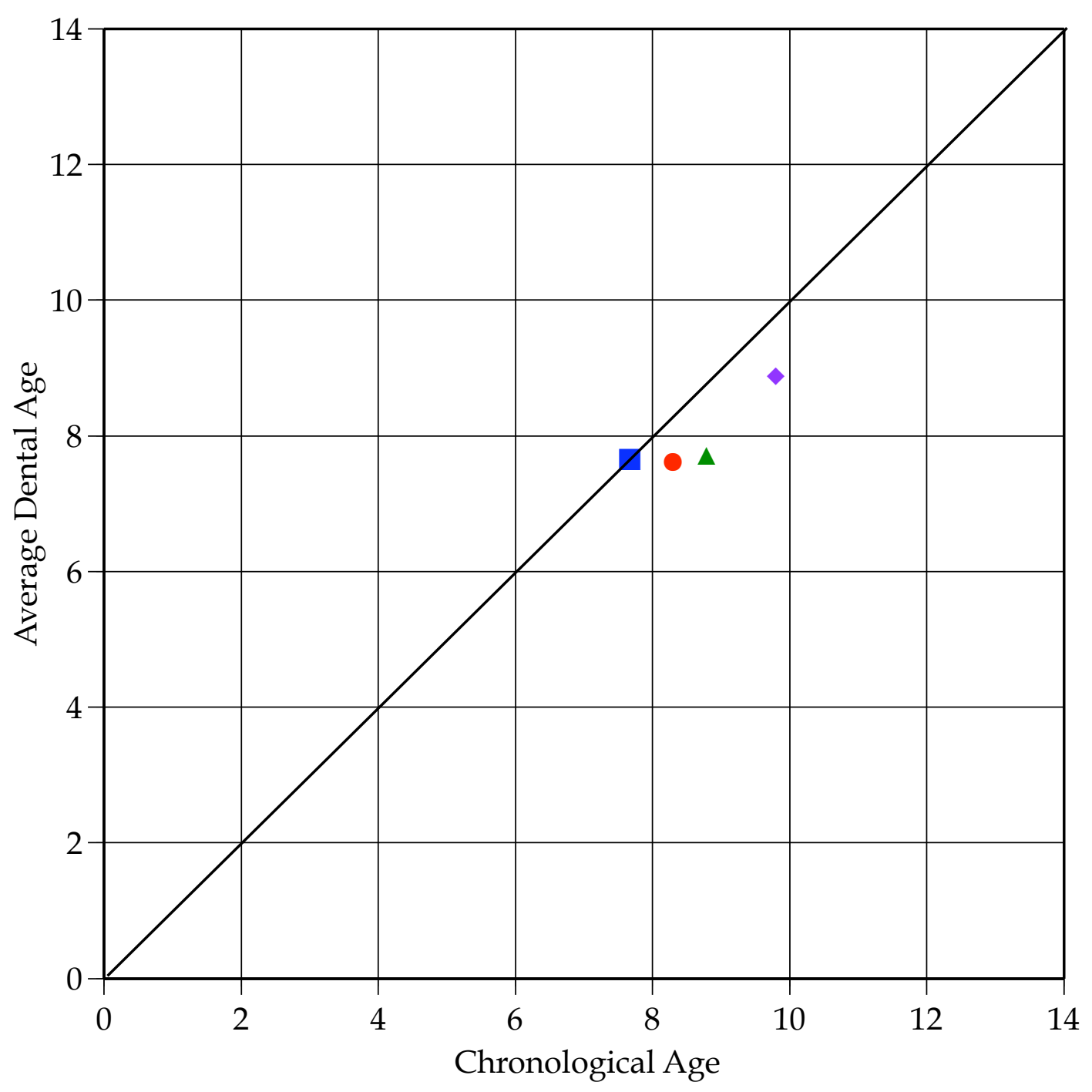

Fig. A-61. Plot of chronological age against dental age averaged across all scorable teeth (symbol) for a male (pseudo-record \#61). The square symbol denotes age at diagnosis of ALL. 


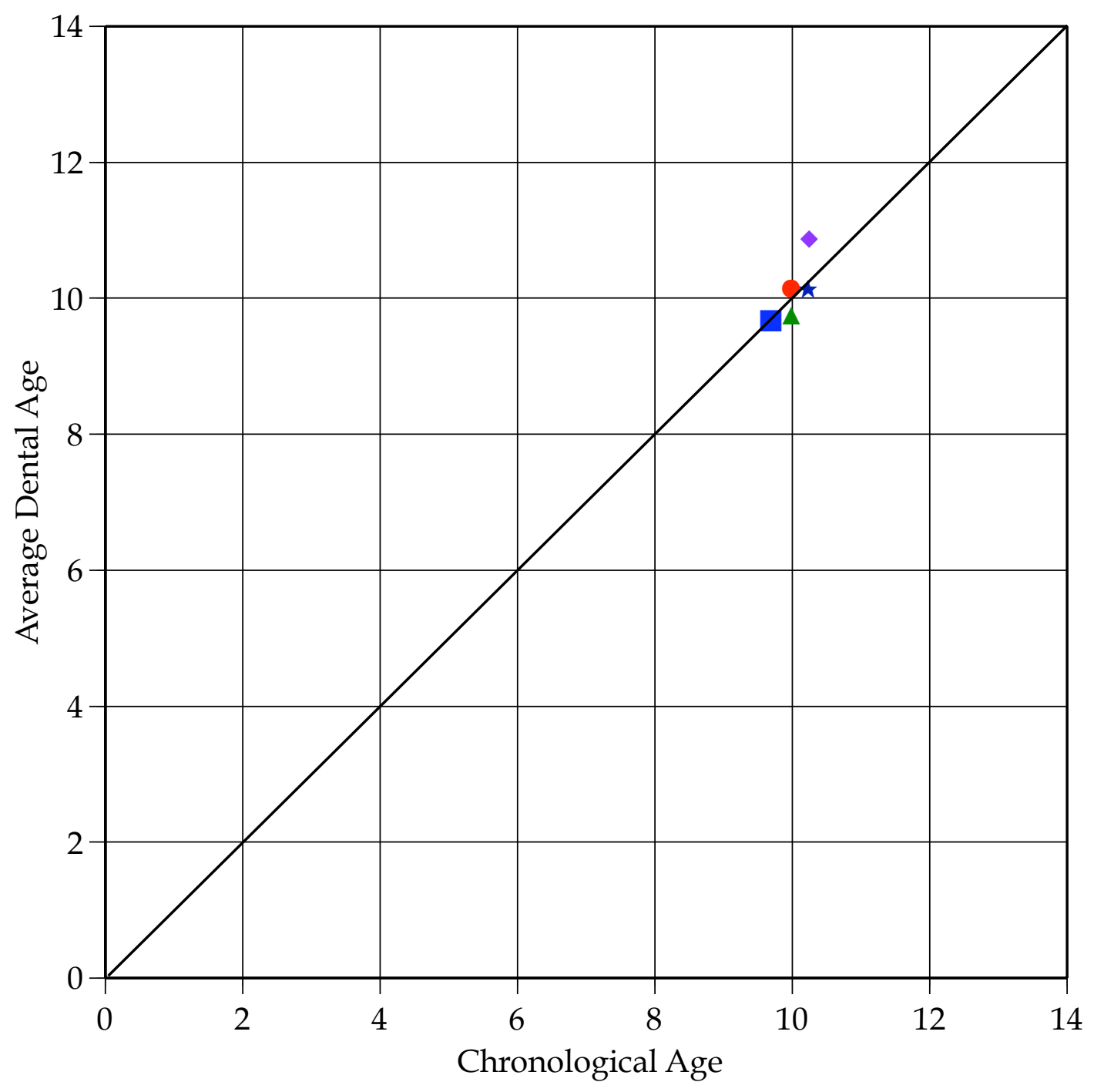

Fig. A-62. Plot of chronological age against dental age averaged across all scorable teeth (symbols) for a male (pseudo-record \#62). The square symbol denotes age at diagnosis of ALL. 


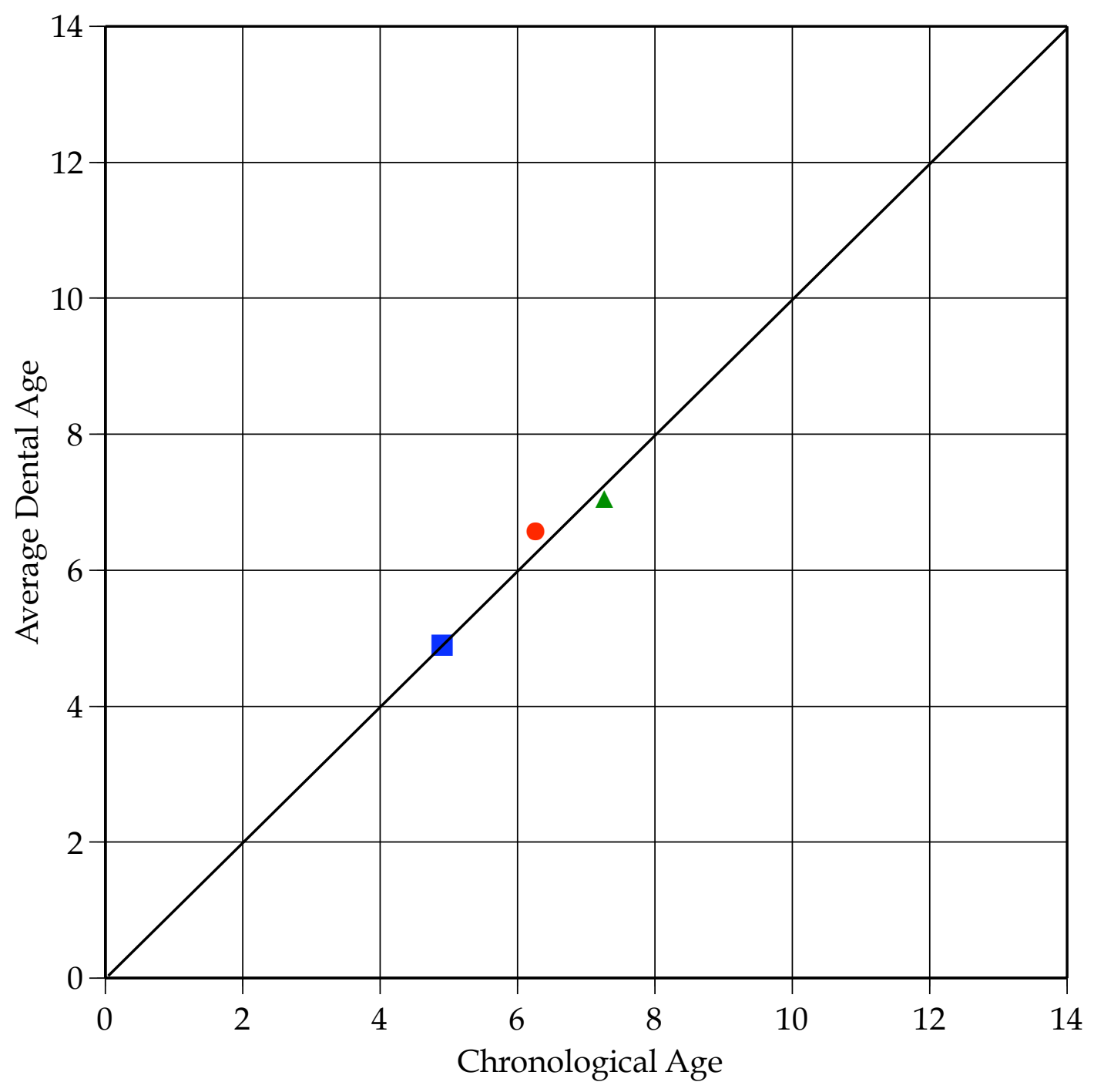

Fig. A-63. Plot of chronological age against dental age averaged across all scorable teeth (symbols) for a male (pseudo-record \#63). The square symbol denotes age at diagnosis of ALL. 


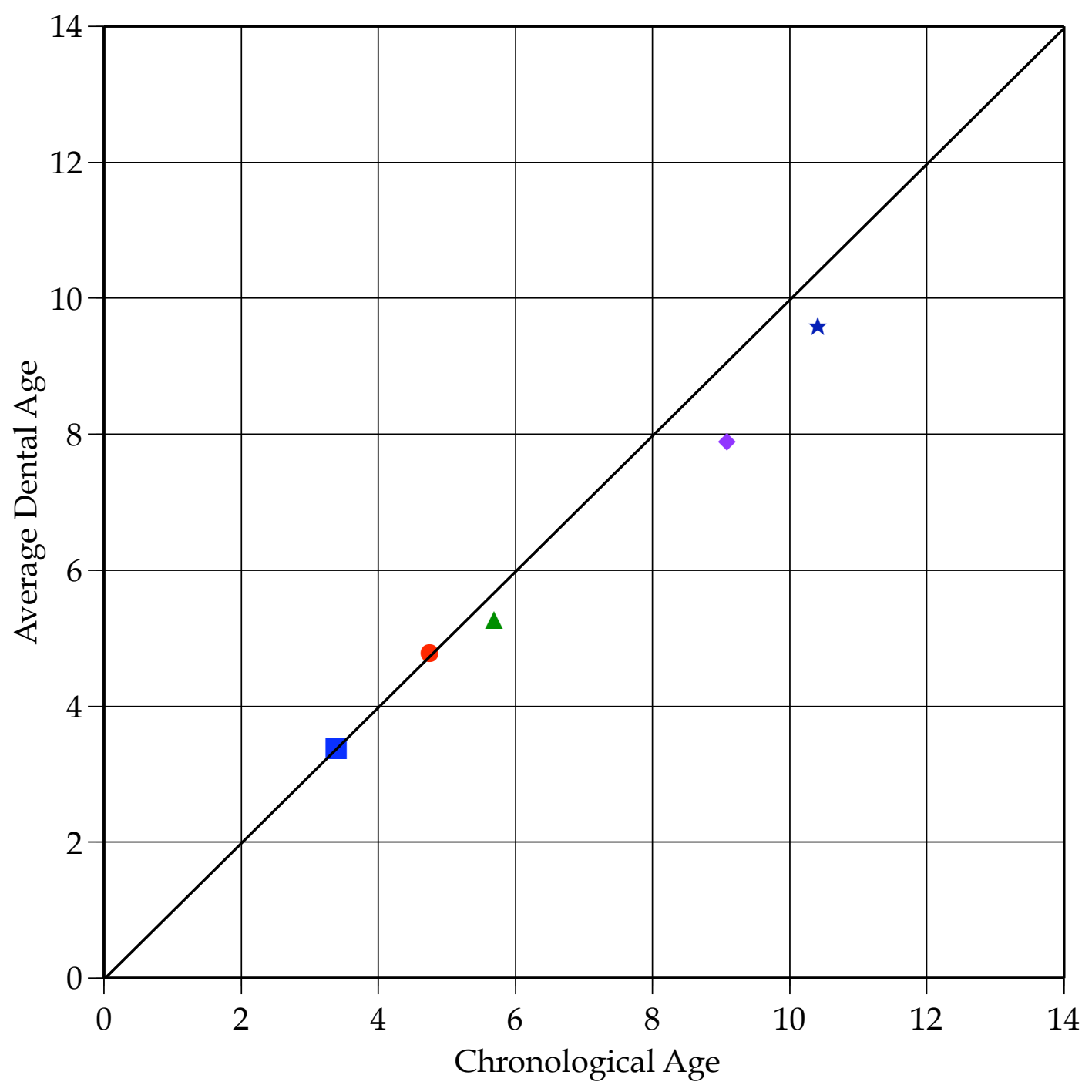

Fig. A-64. Plot of chronological age against dental age averaged across all scorable teeth (symbols) for a male (pseudo-record \#64). The square symbol denotes age at diagnosis of ALL. 


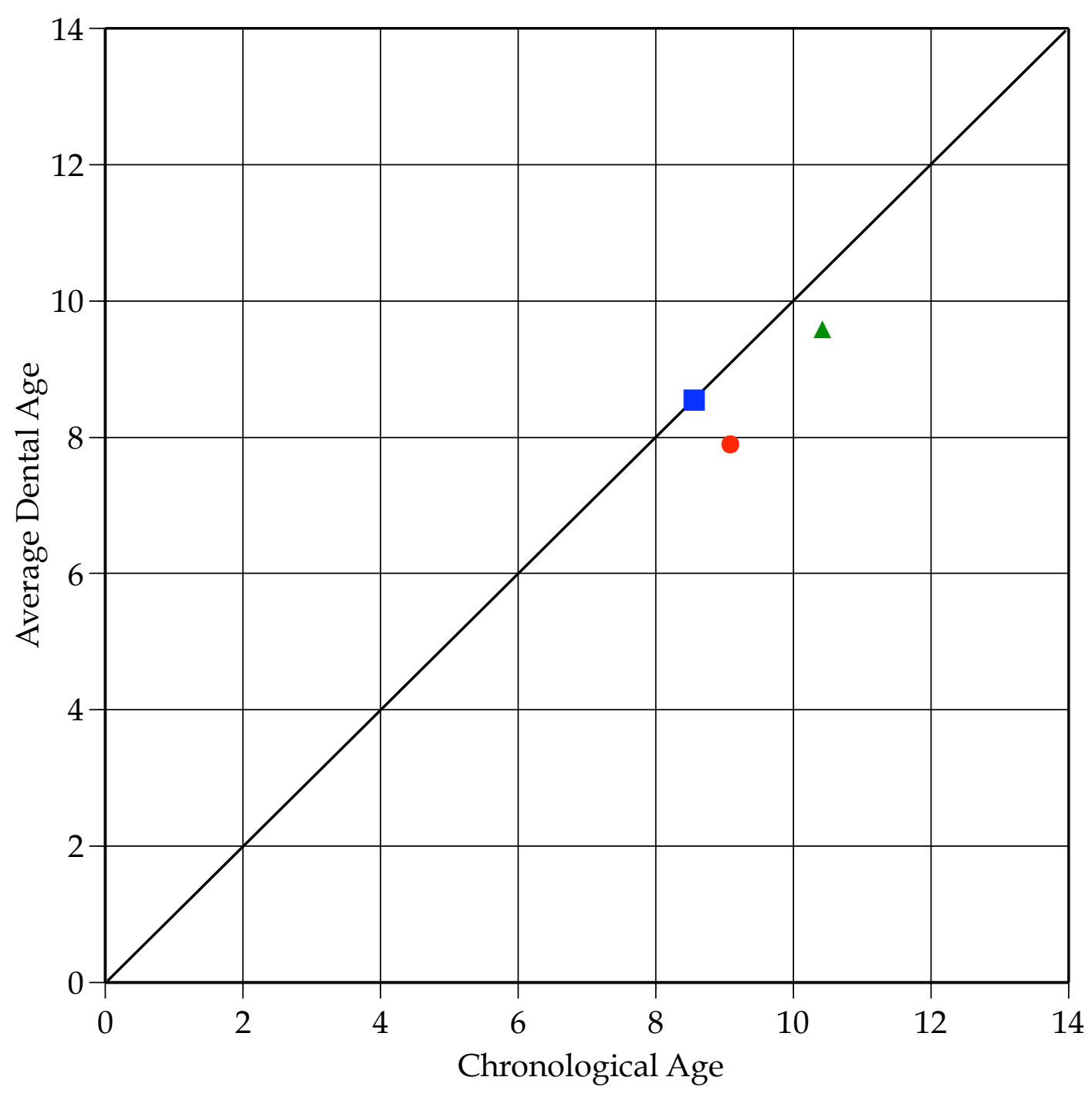

Fig. A-65. Plot of chronological age against dental age averaged across all scorable teeth (symbols) for a female (pseudo-record \#65). The square symbol denotes age at diagnosis of ALL. 


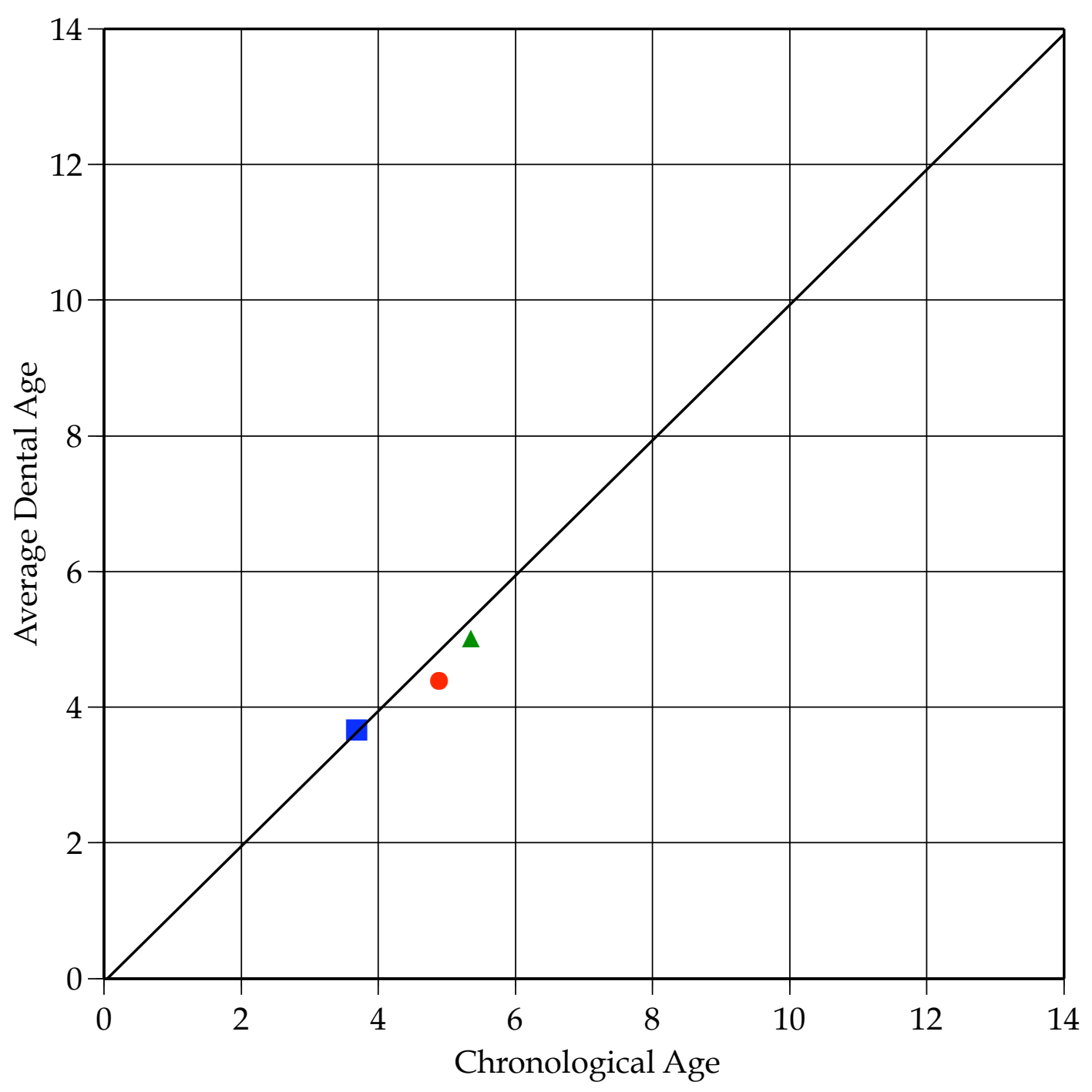

Fig. A-66. Plot of chronological age against dental age averaged across all scorable teeth (symbols) for a male (pseudo-record \#66). The square symbol denotes age at diagnosis of ALL. 


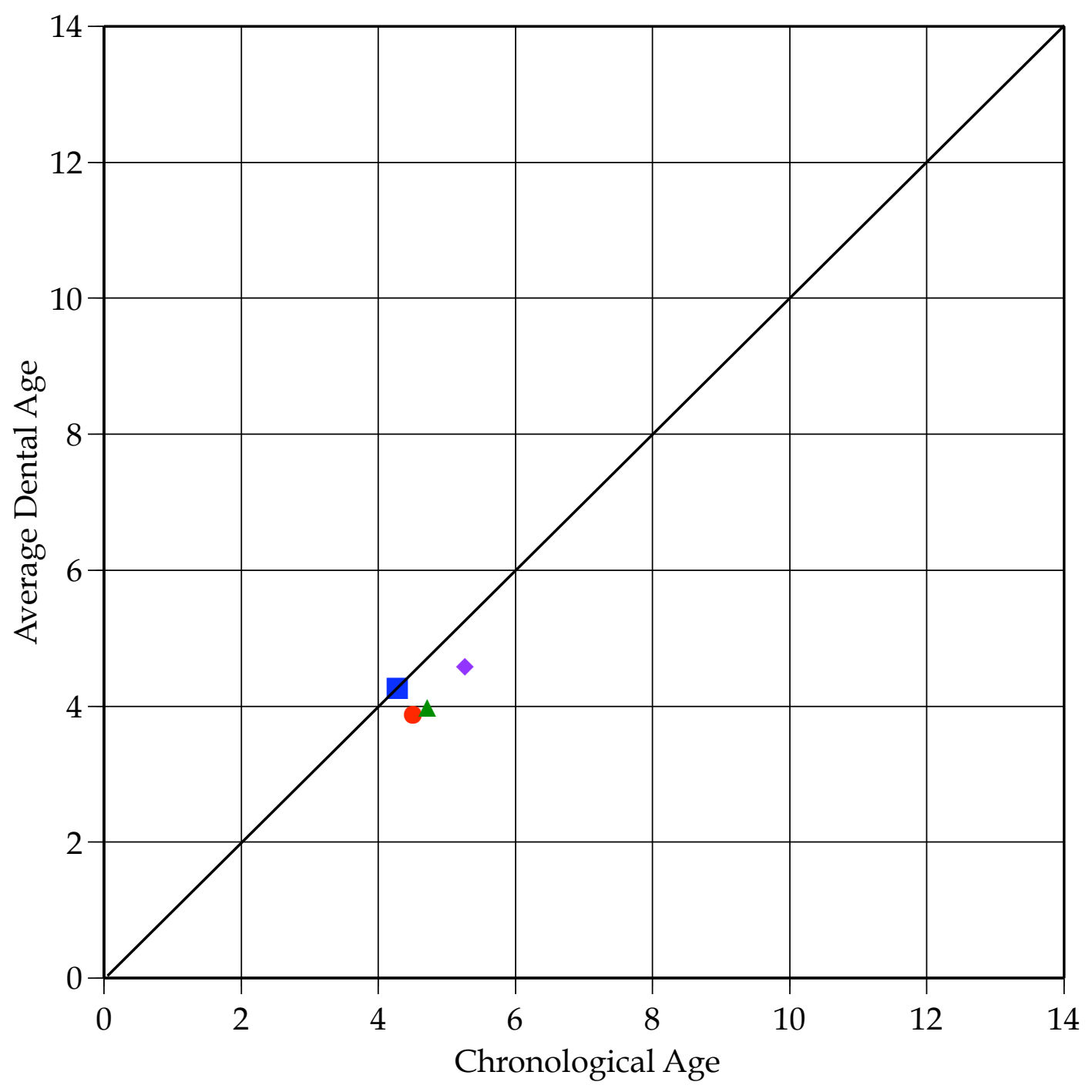

Fig. A-67. Plot of chronological age against dental age averaged across all scorable teeth (symbols) for a male (pseudo-record \#67). The square symbol denotes age at diagnosis of ALL. 


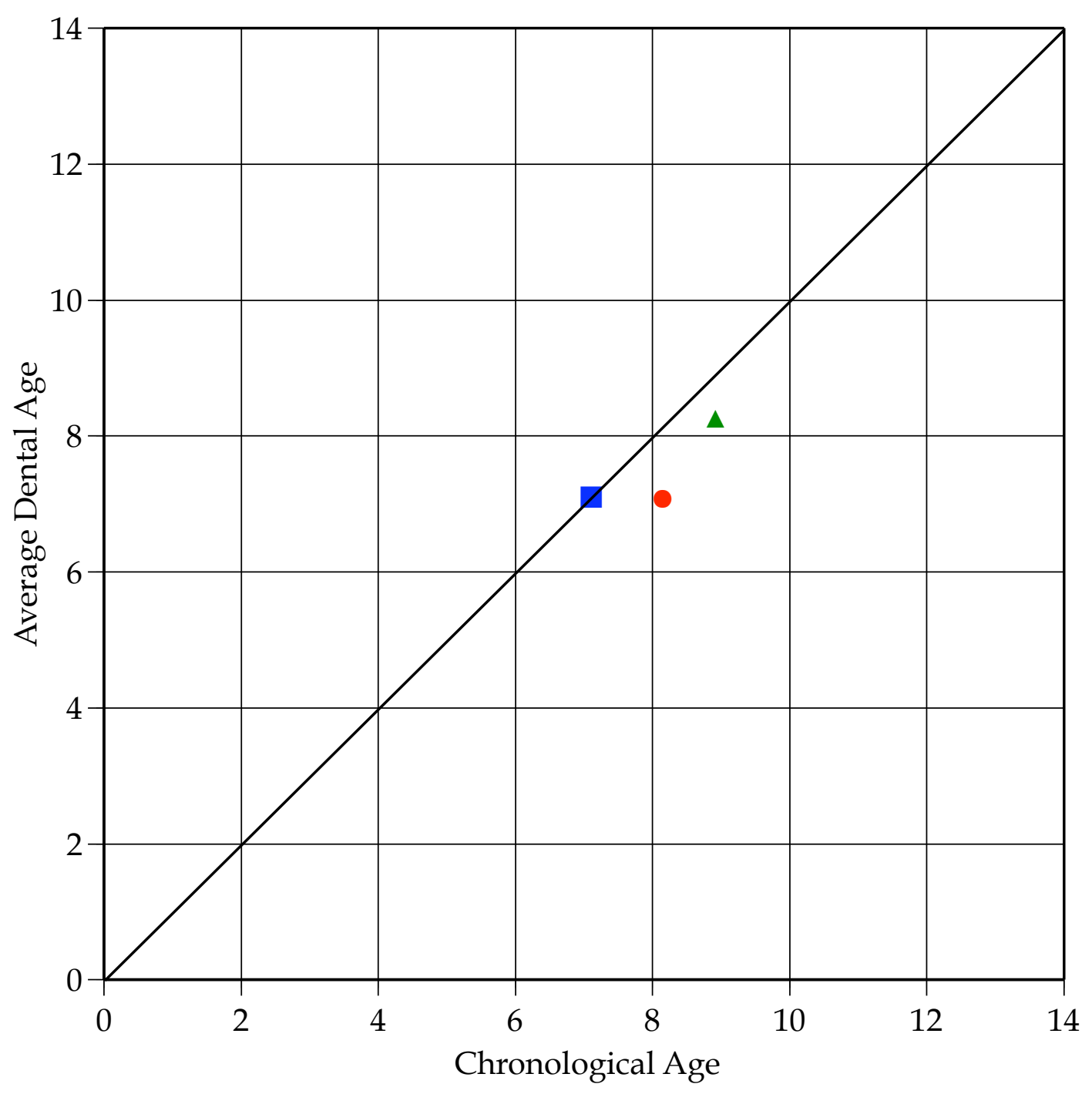

Fig. A-68. Plot of chronological age against dental age averaged across all scorable teeth (symbols) for a male (pseudo-record \#68). The square symbol denotes age at diagnosis of ALL. 


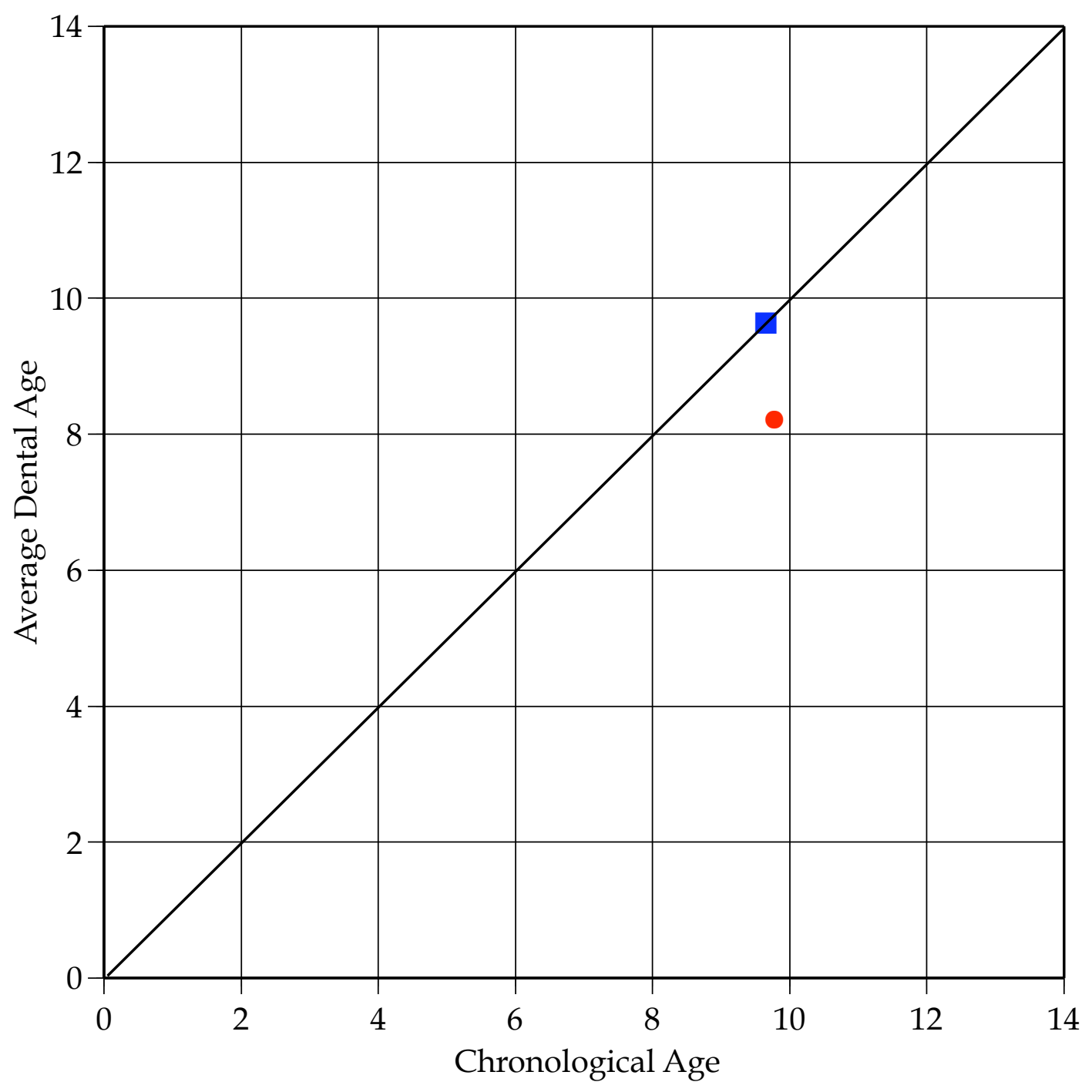

Fig. A-69. Plot of chronological age against dental age averaged across all scorable teeth (symbol) for a female (pseudo-record \#69). The square symbol denotes age at diagnosis of ALL. 


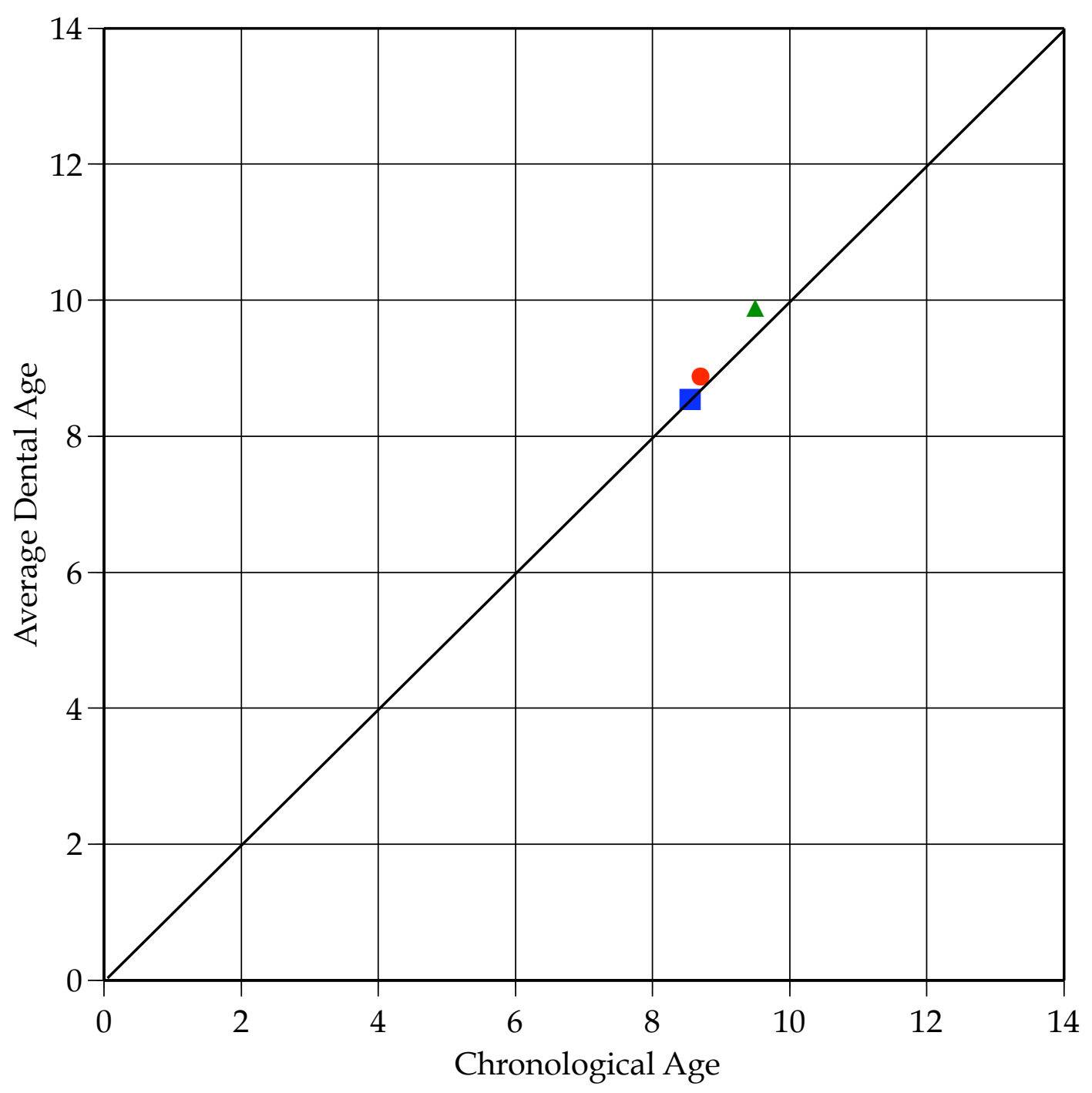

Fig. A-70. Plot of chronological age against dental age averaged across all scorable teeth (symbols) for a female (pseudo-record \#70). The square symbol denotes age at diagnosis of ALL. 


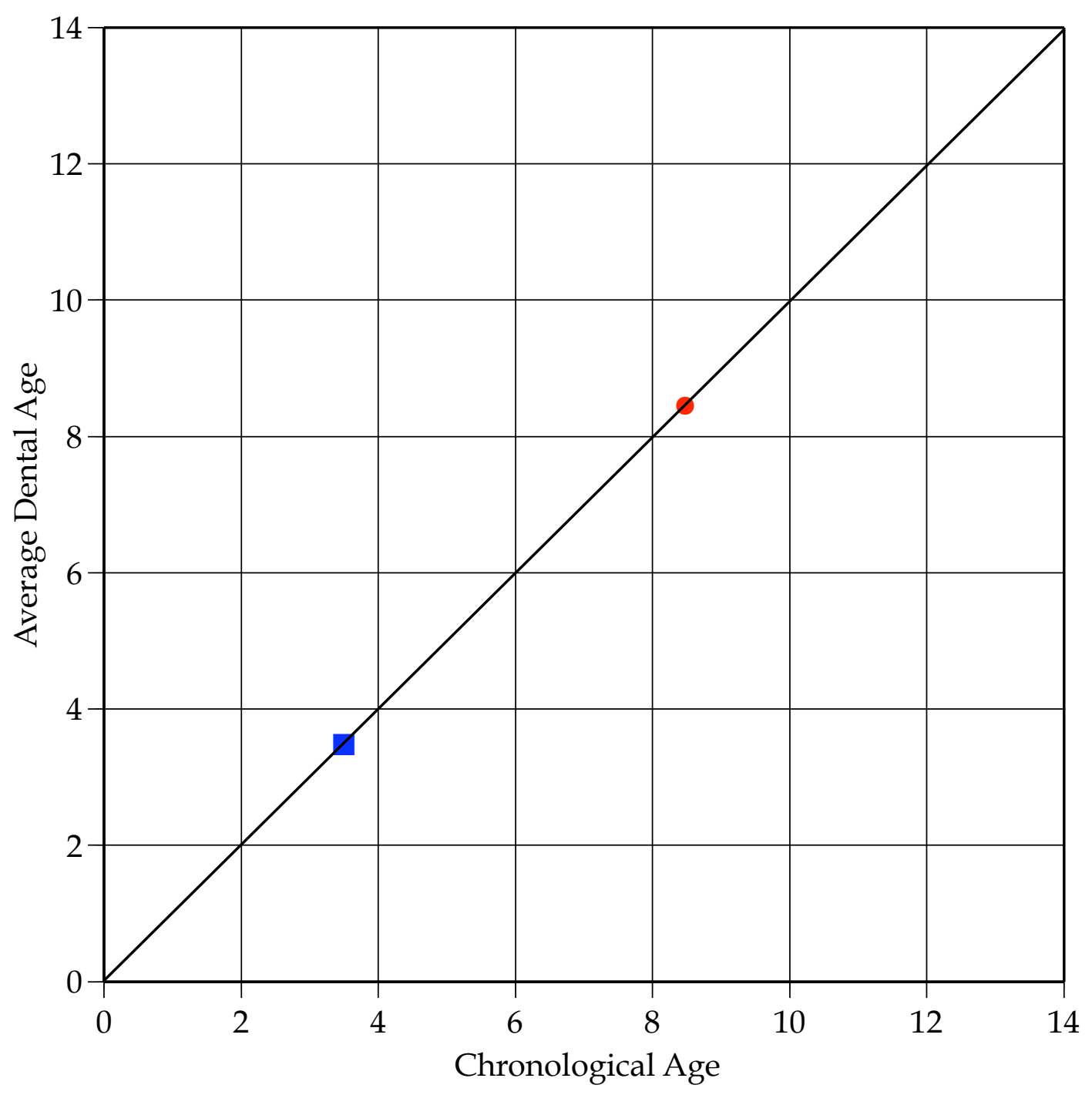

Fig. A-71. Plot of chronological age against dental age averaged across all scorable teeth (symbols) for a female (pseudo-record \#71). The square symbol denotes age at diagnosis of ALL. 


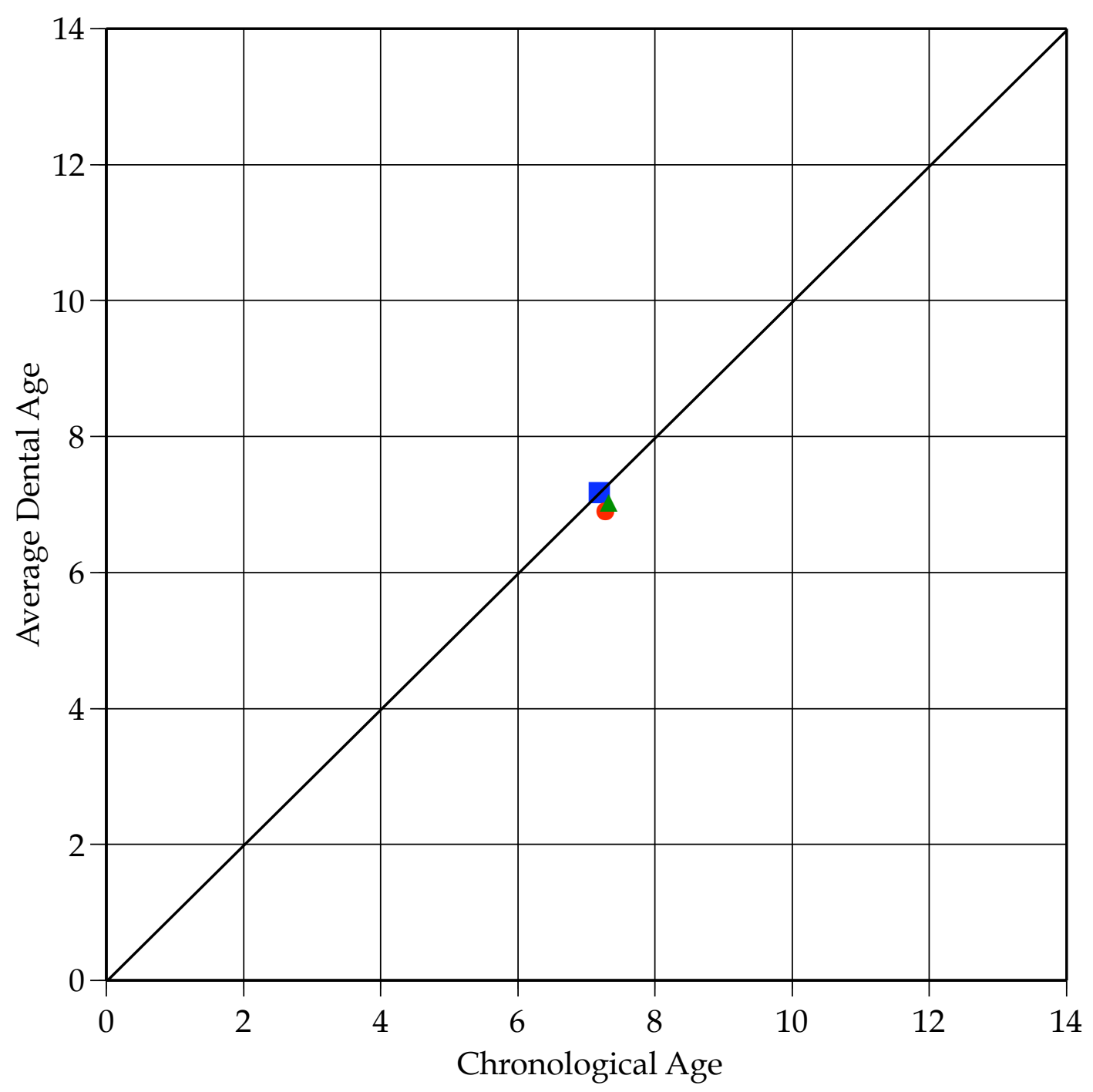

Fig. A-72. Plot of chronological age against dental age averaged across all scorable teeth (symbols) for a female (pseudo-record \#72). The square symbol denotes age at diagnosis of ALL. 


\section{Appendix B}

Plots of tooth-specific results of DA-CA for each child in this study. All examinations (i.e., all panoral radiographs) available for a patient are plotted in the graph. Note that the vertical scale (DA minus CA) normally is set to the range of +3 to -3 years, but this is exceeded for some cases with extreme values. 


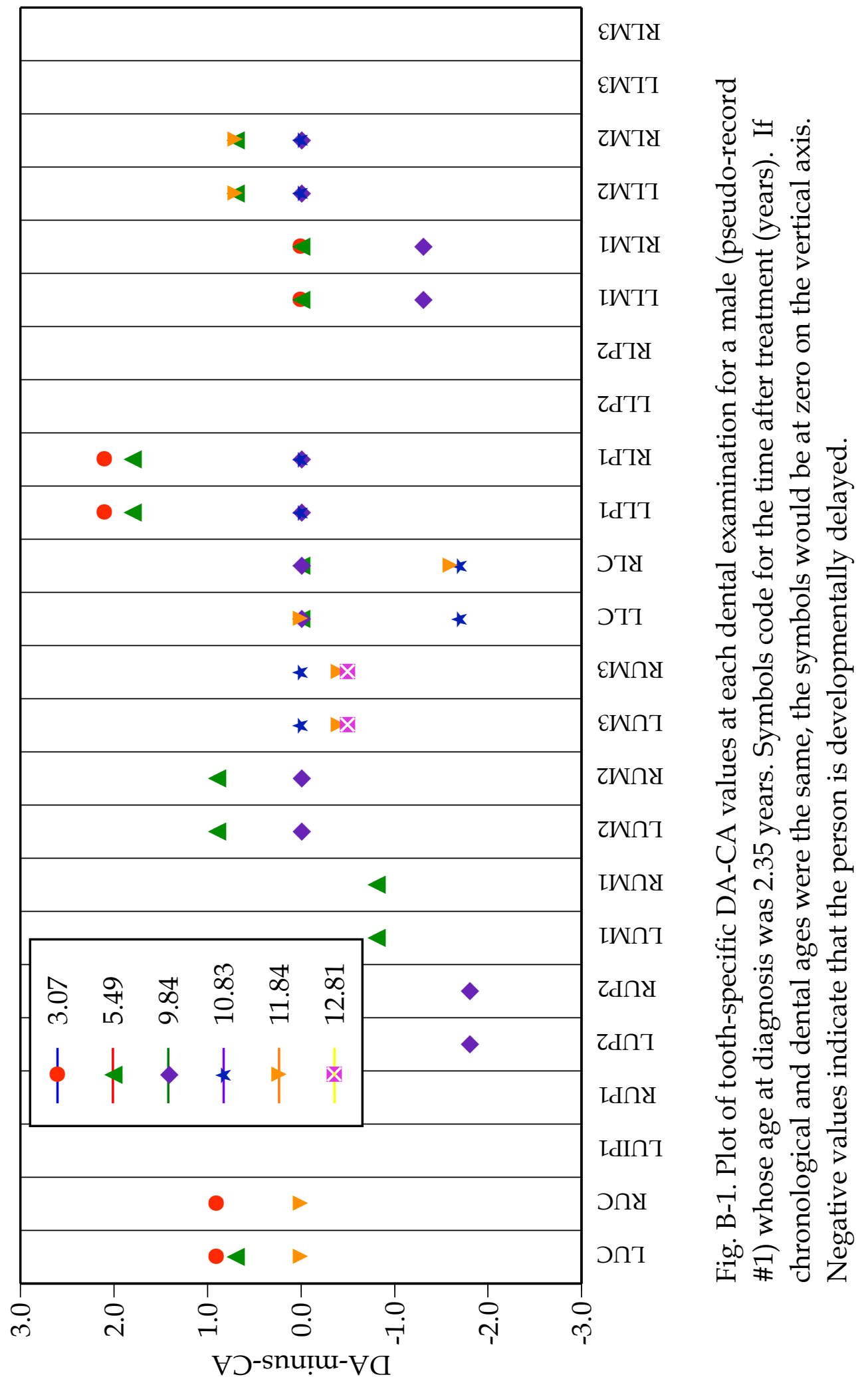




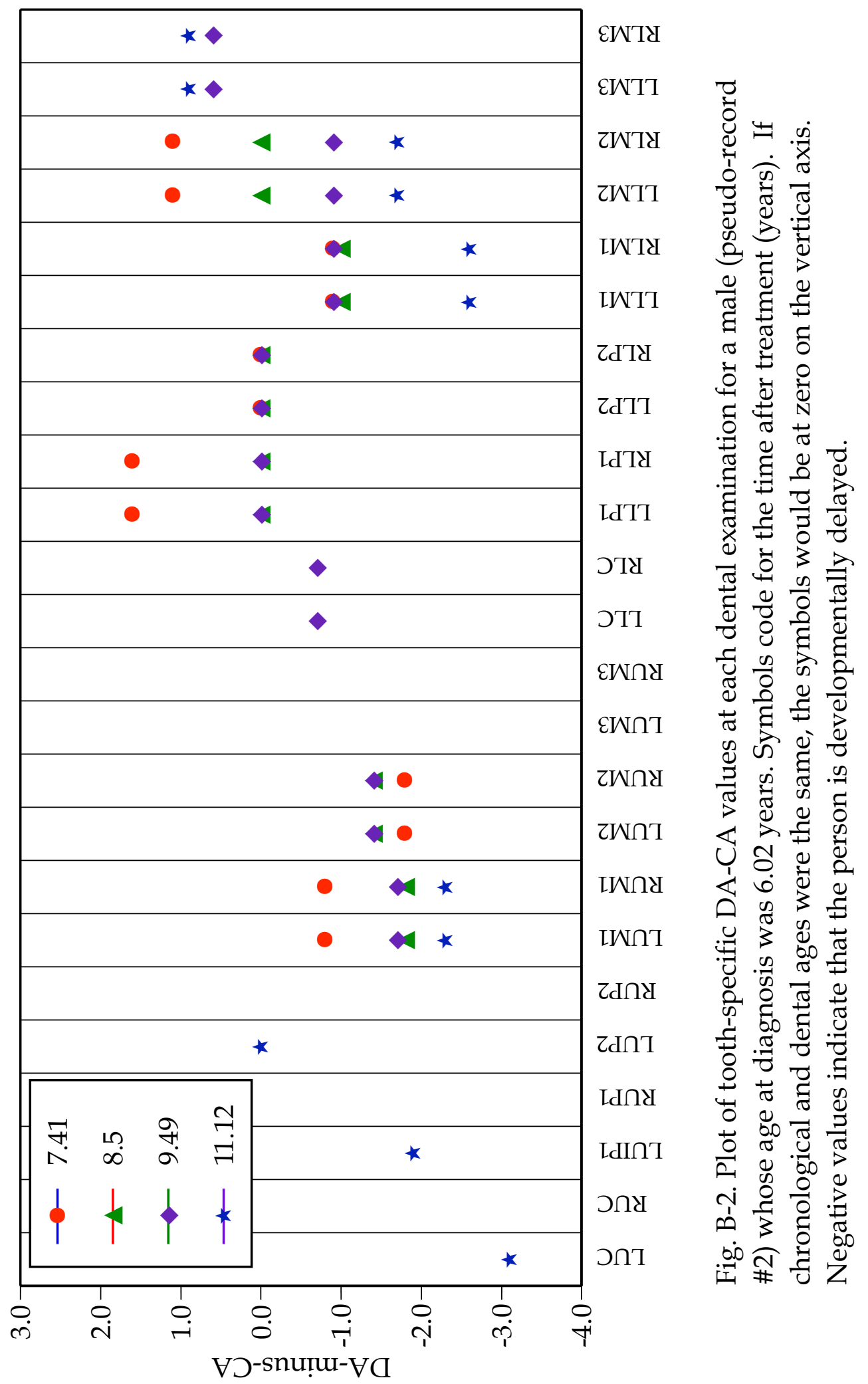




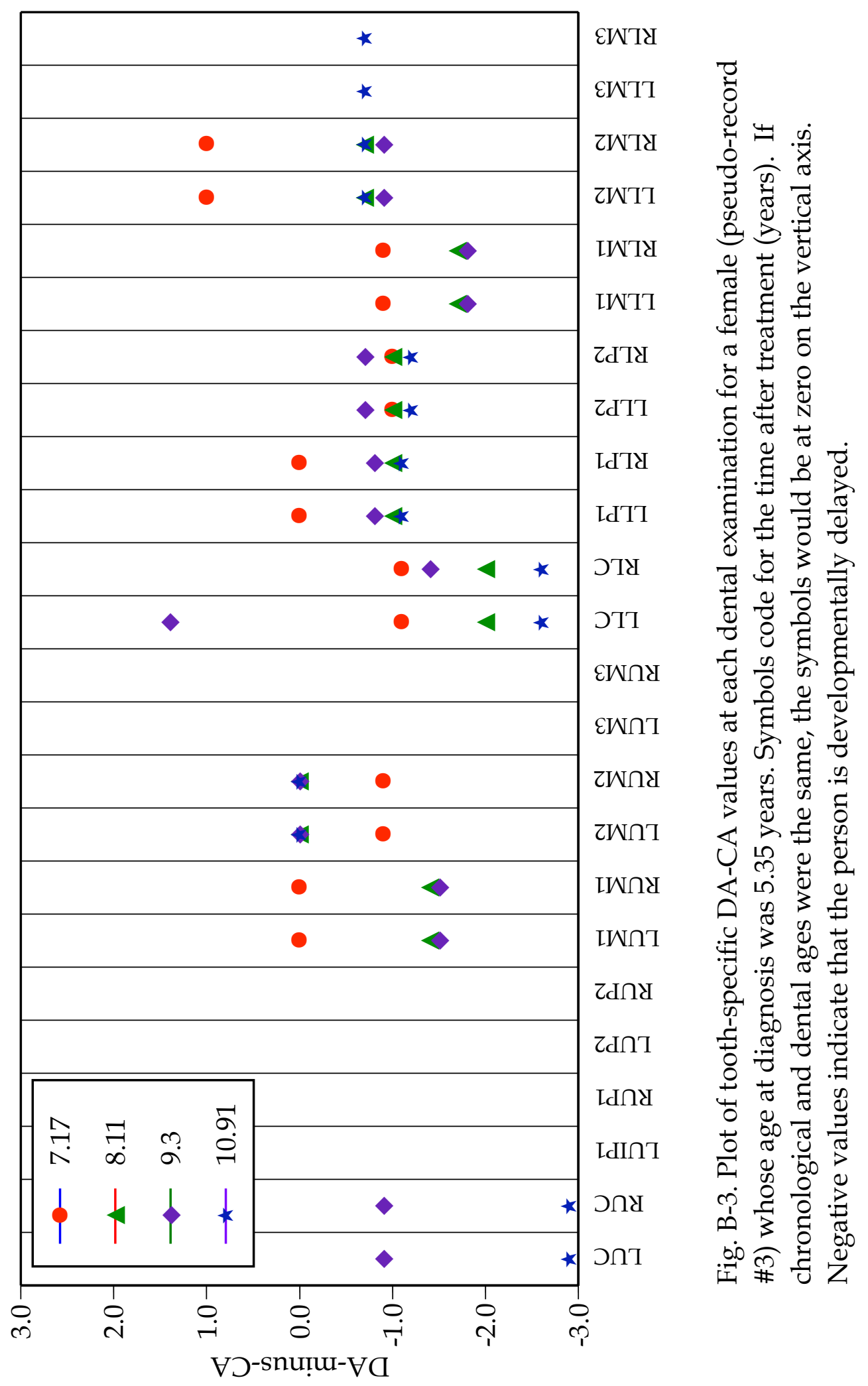




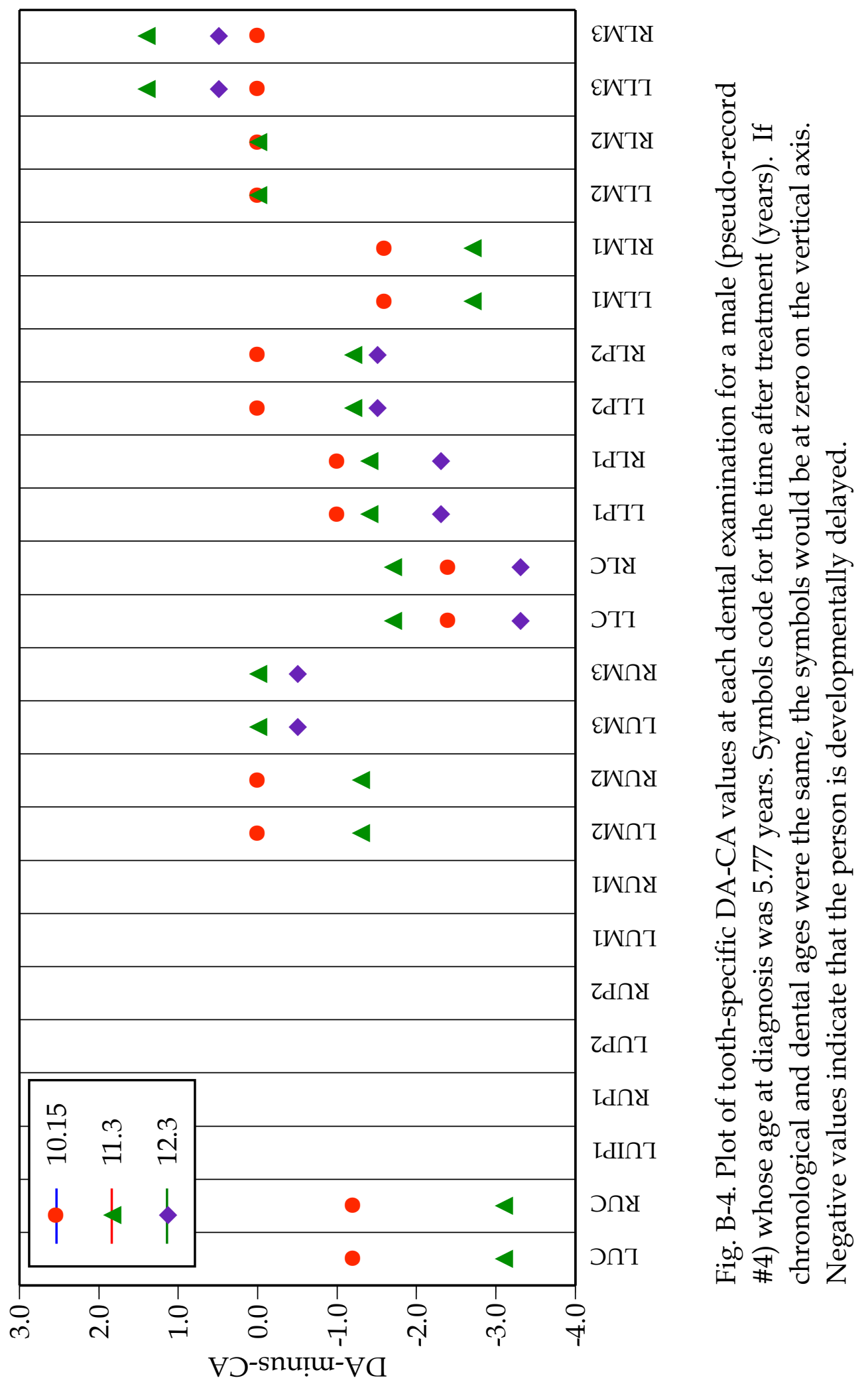




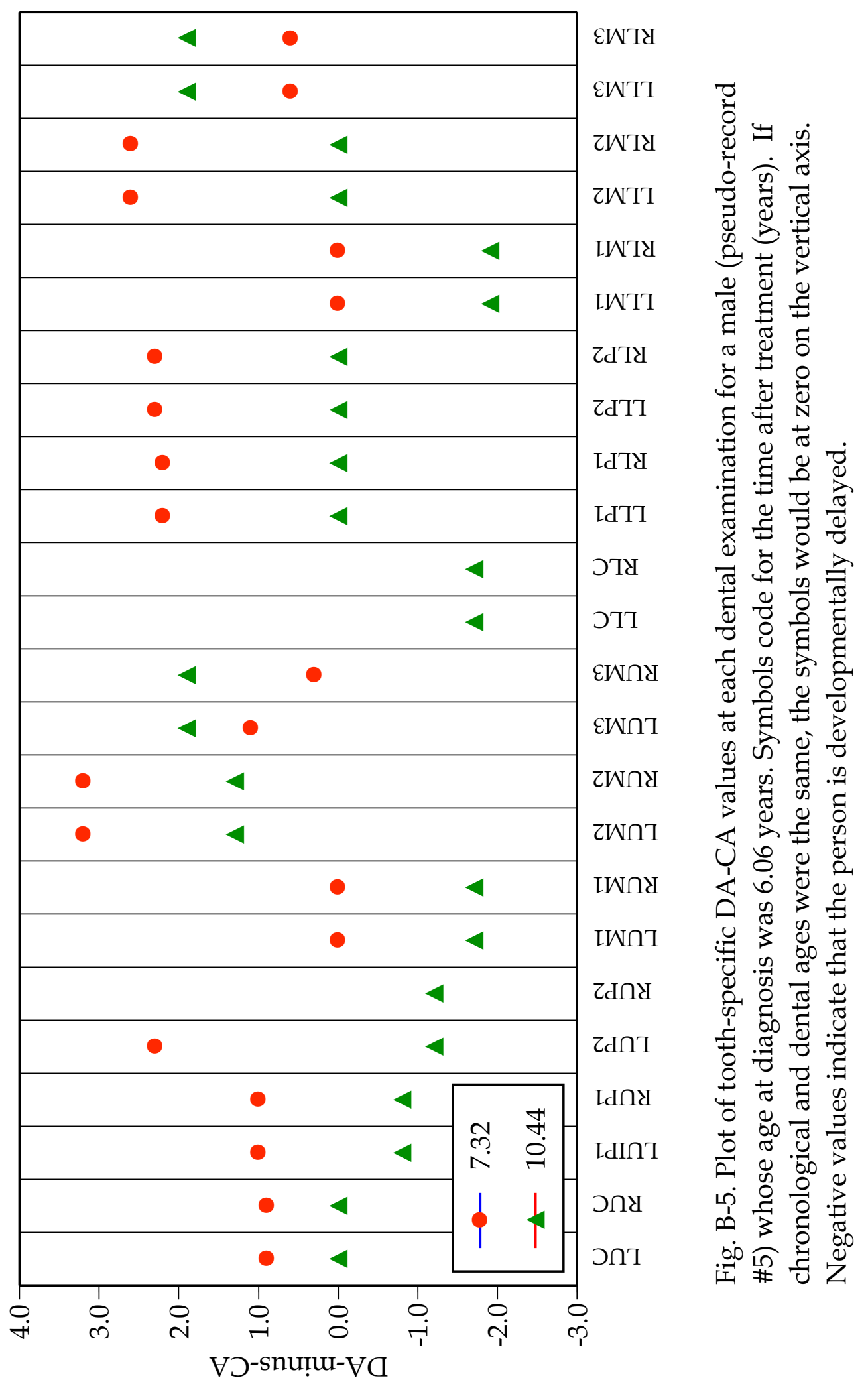




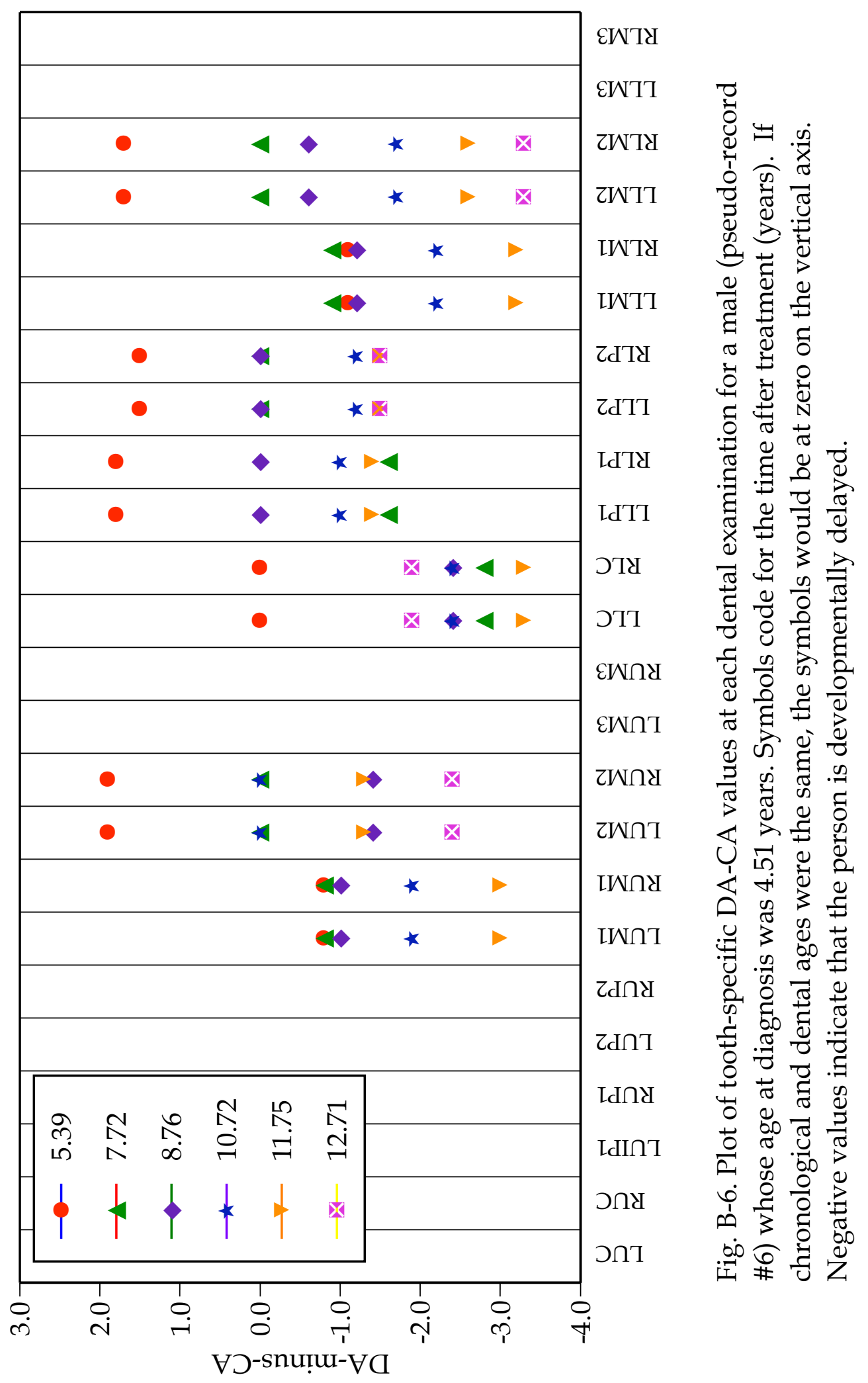




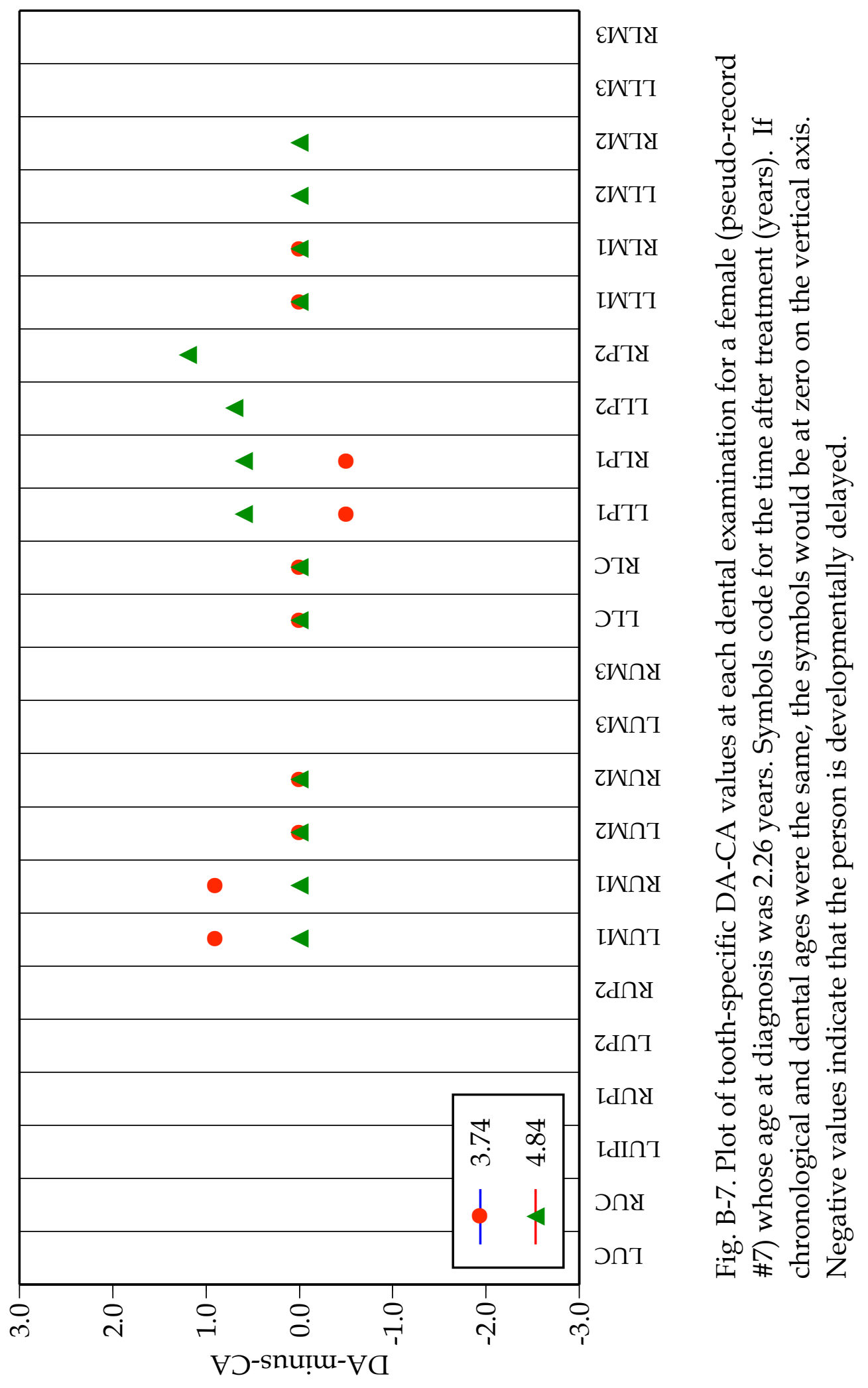




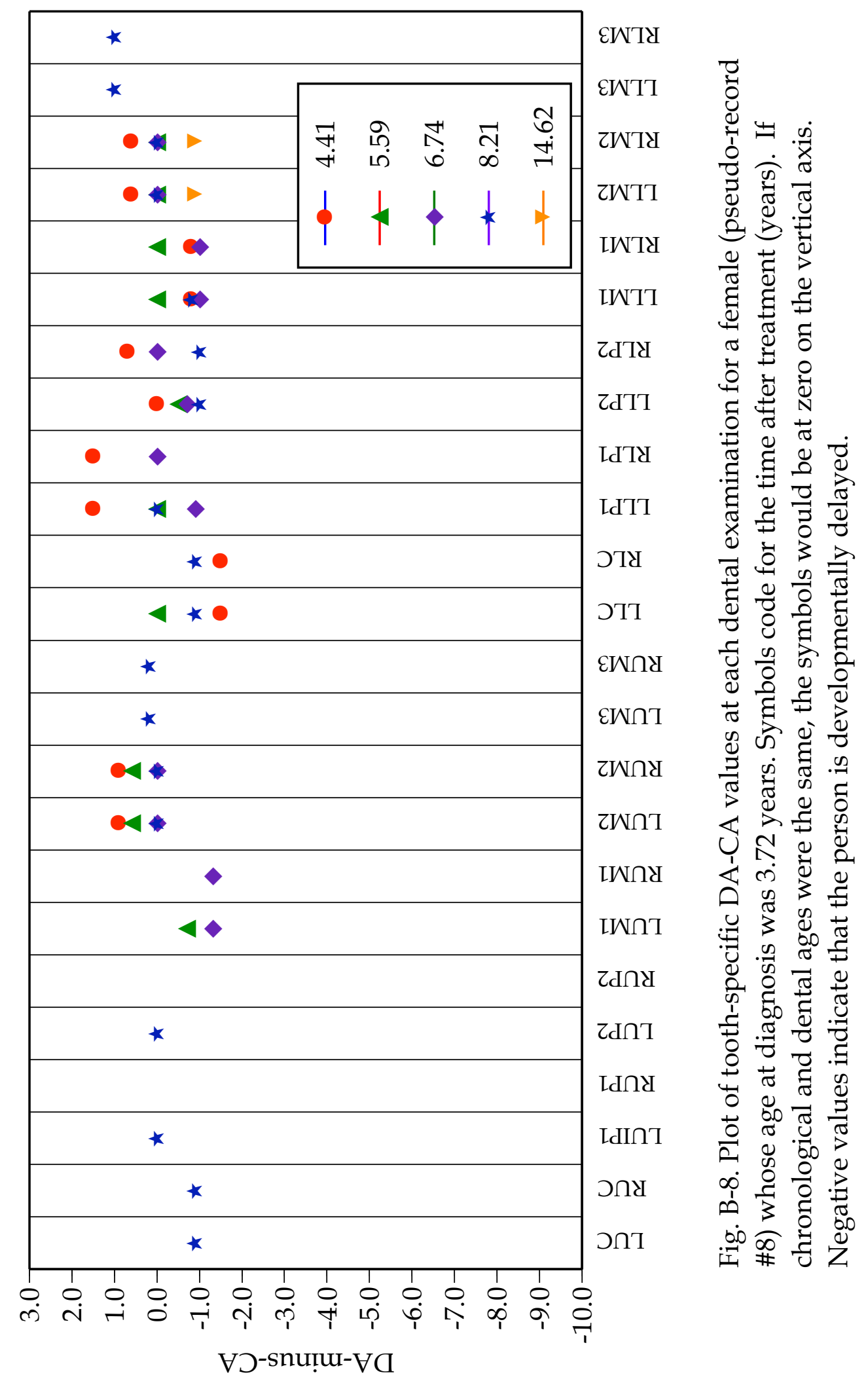




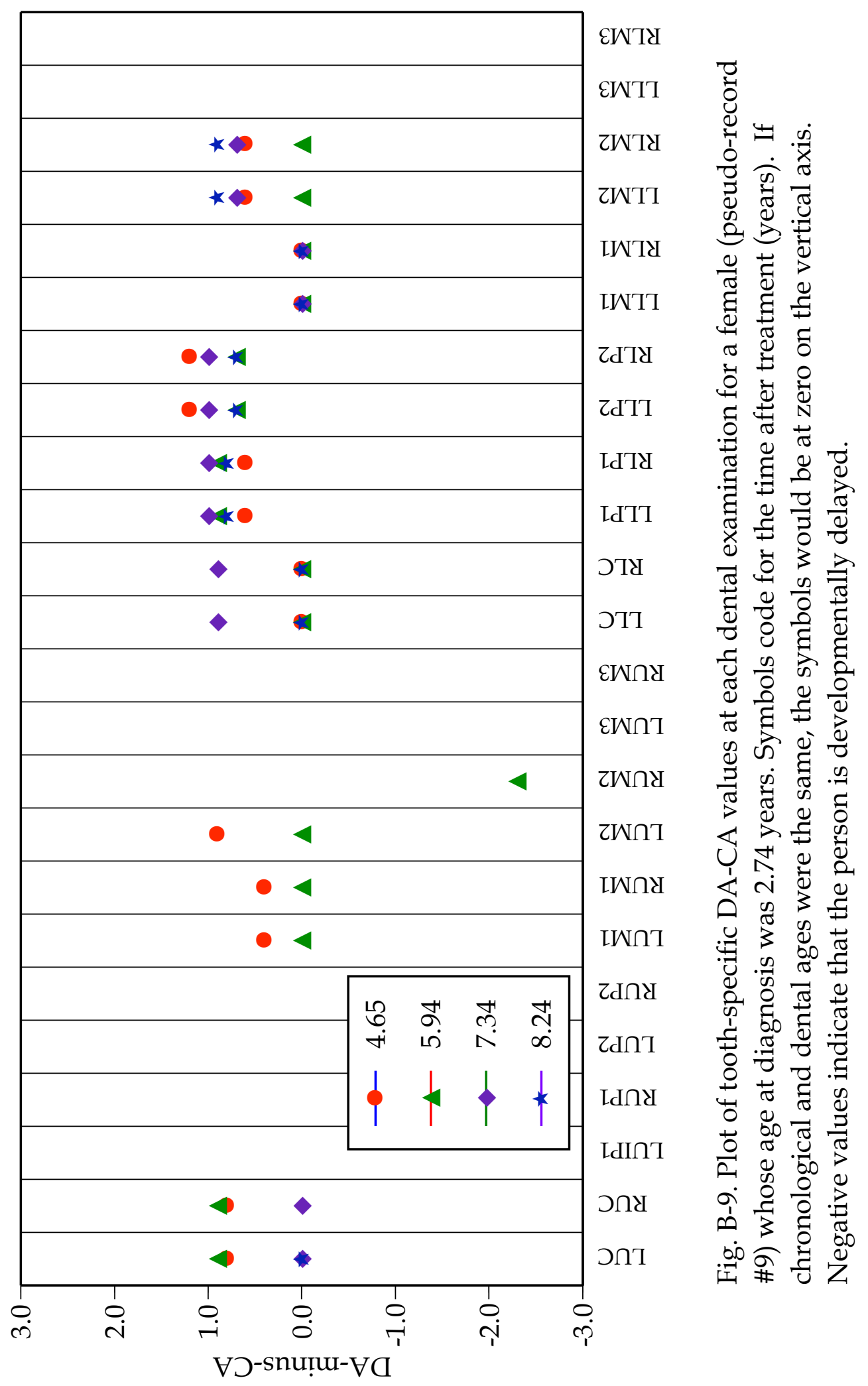




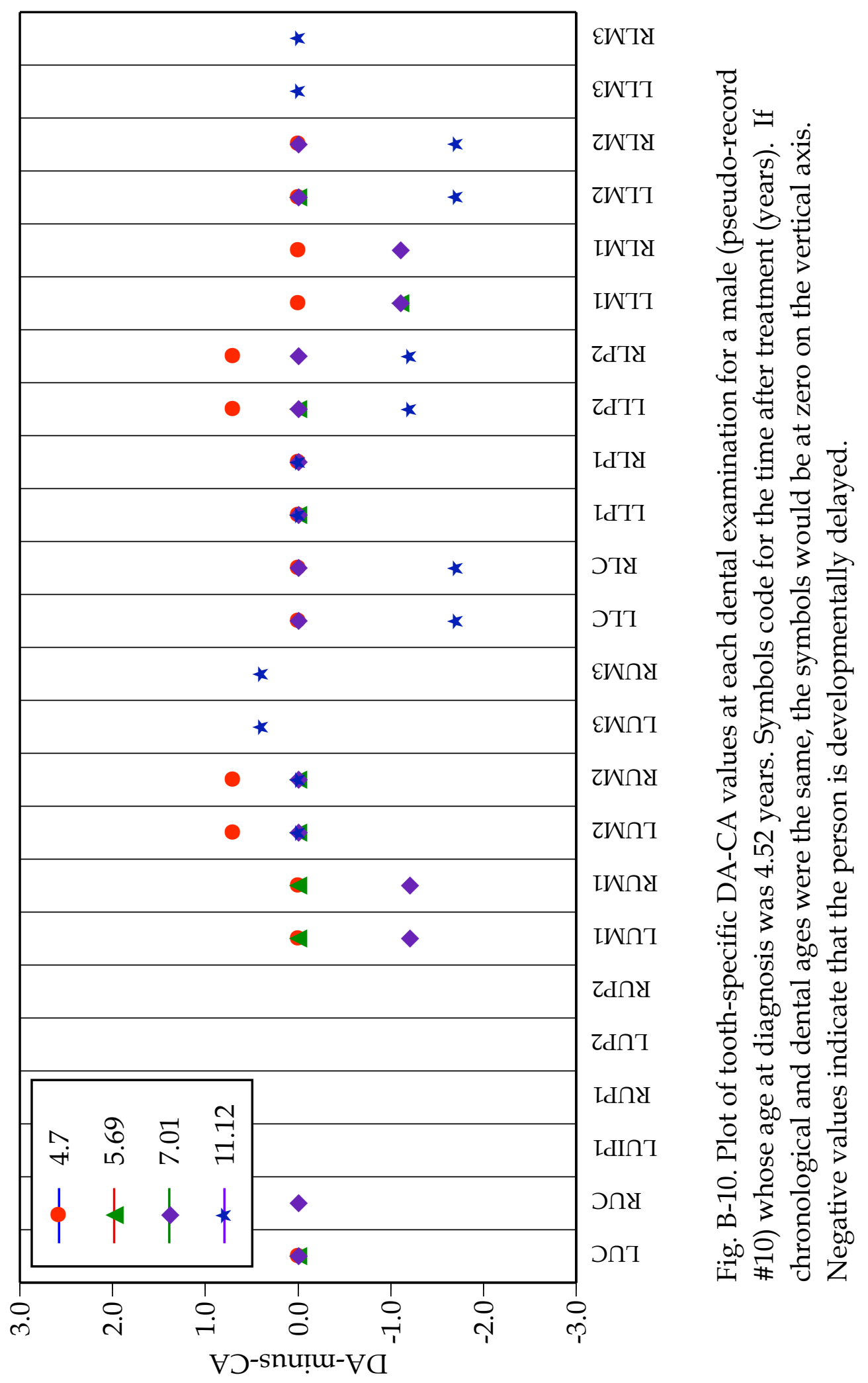




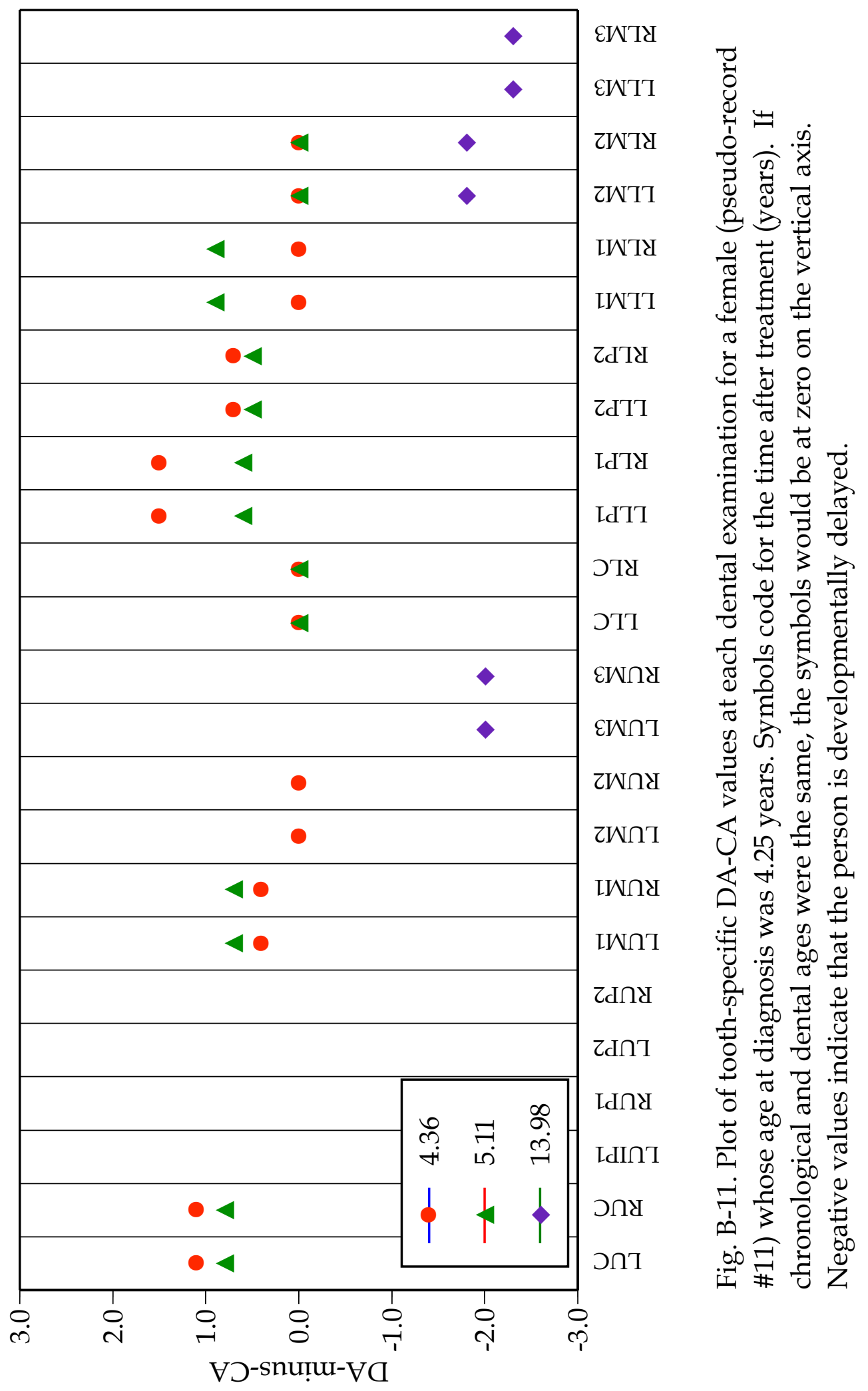




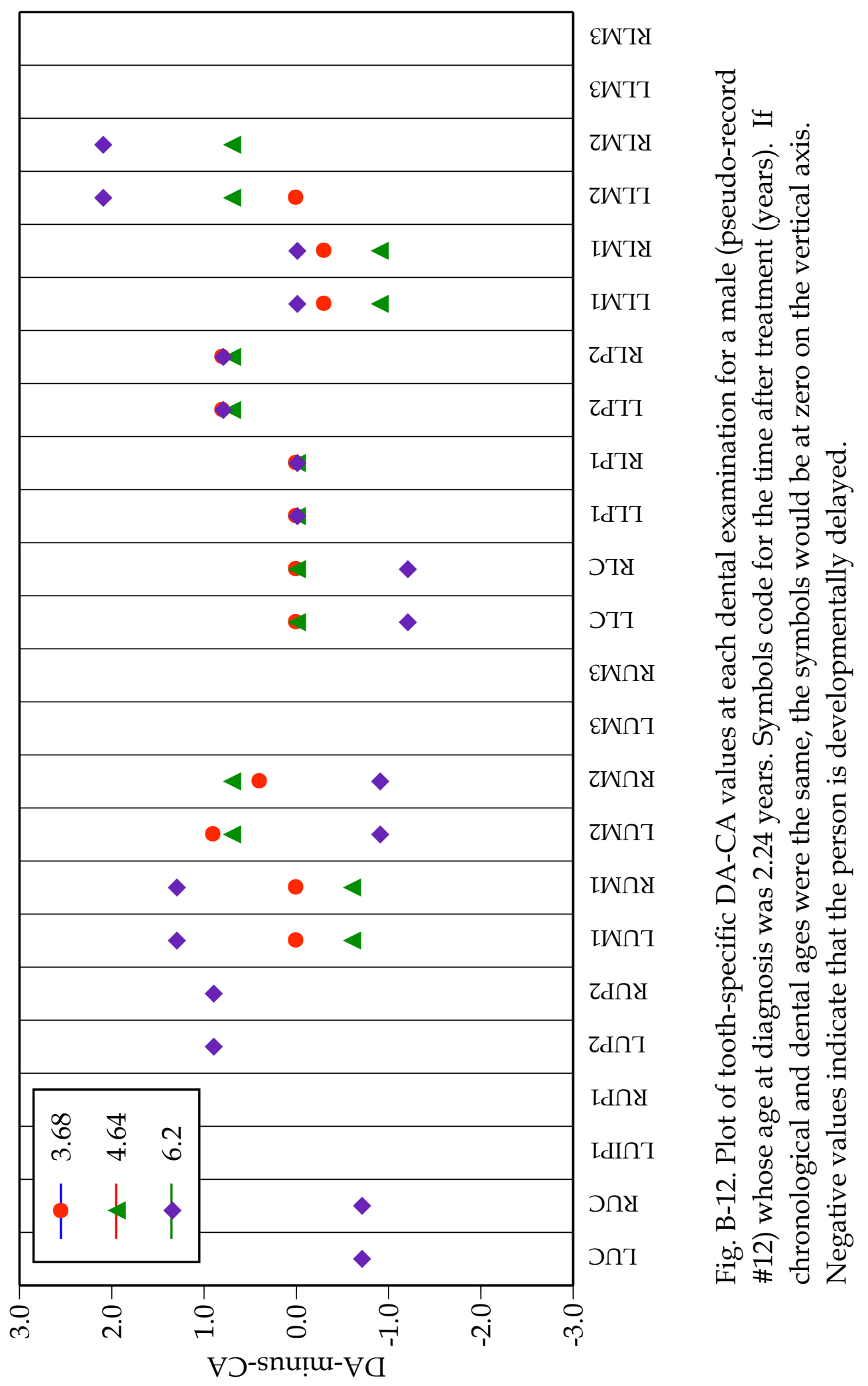




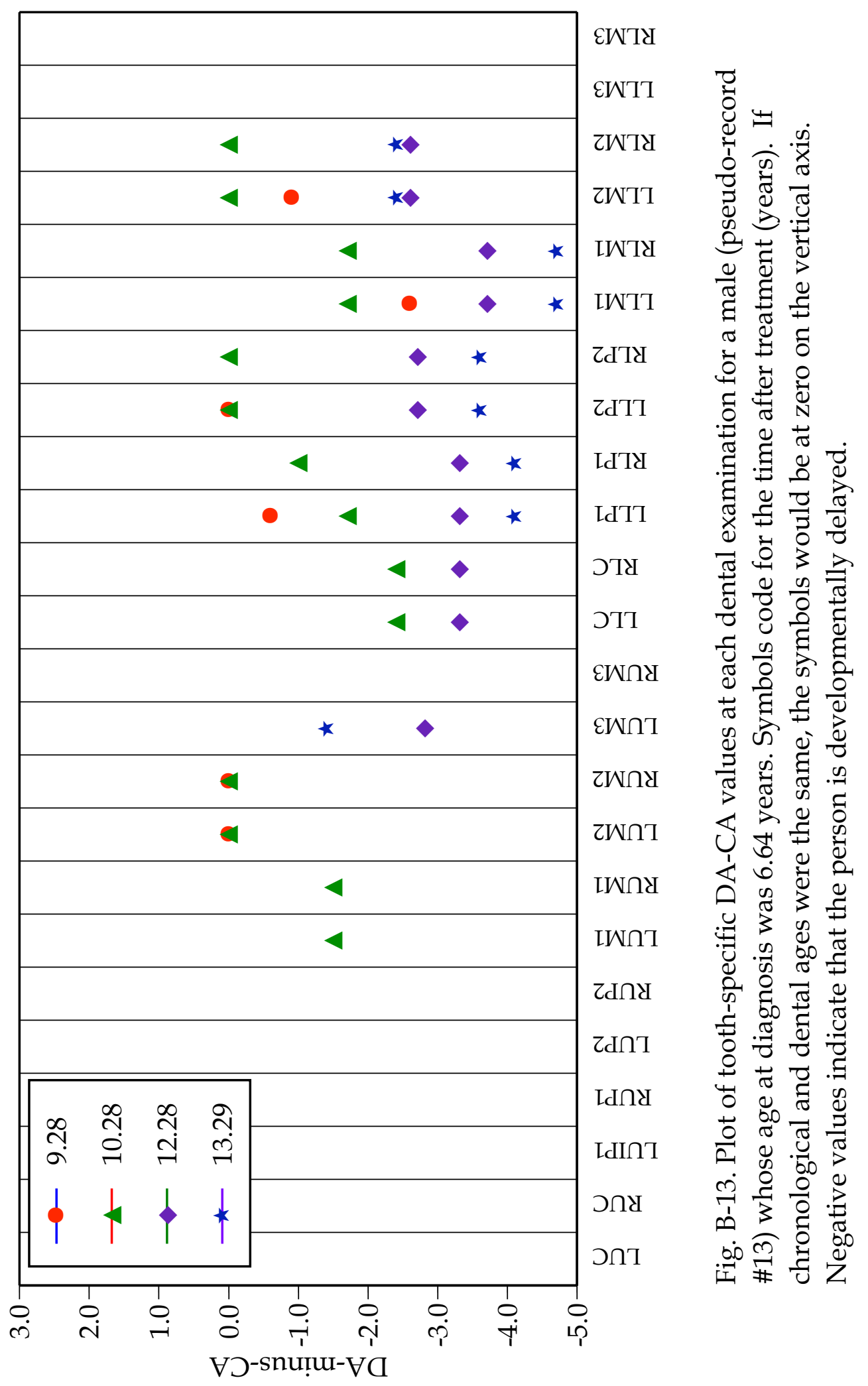




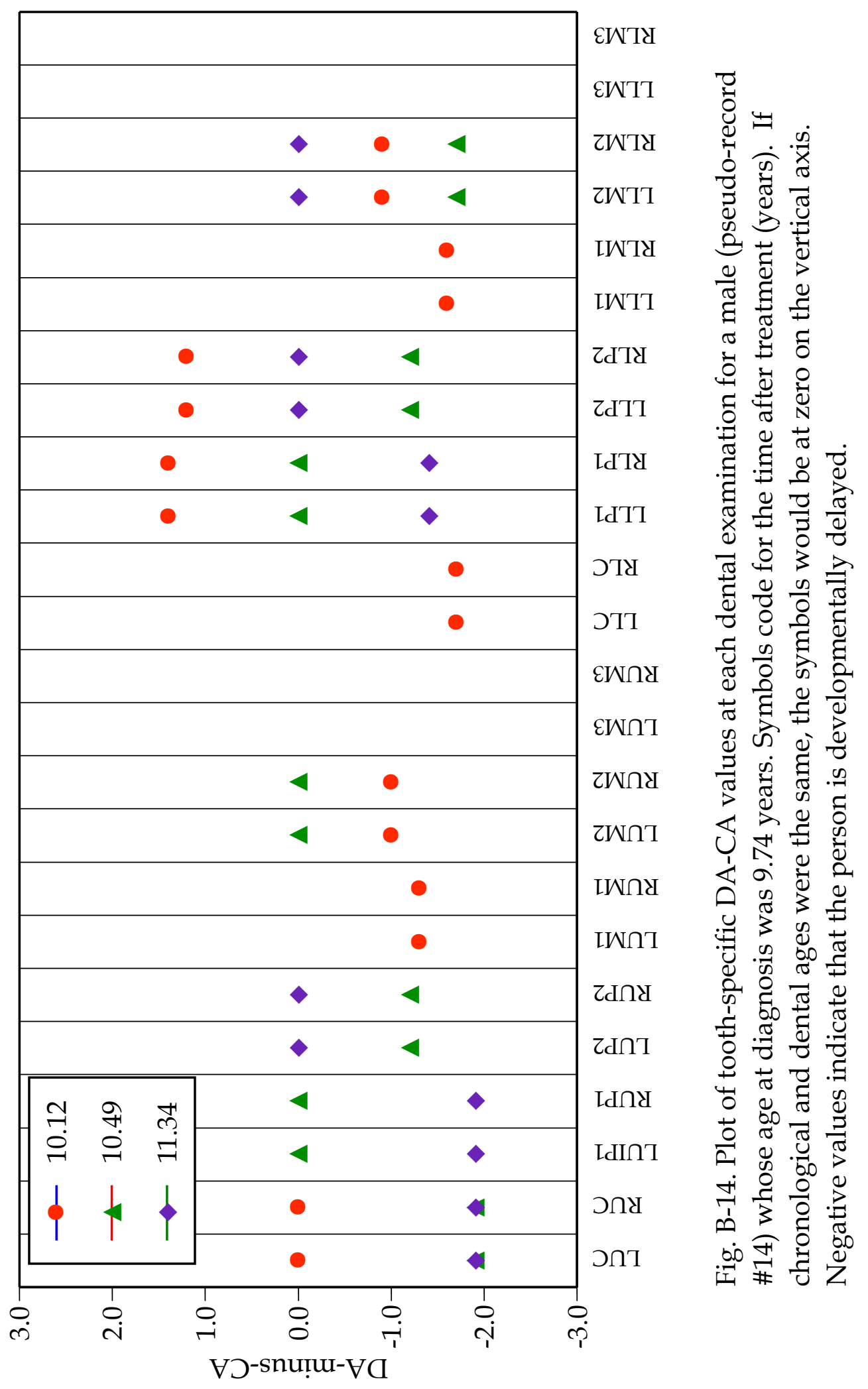




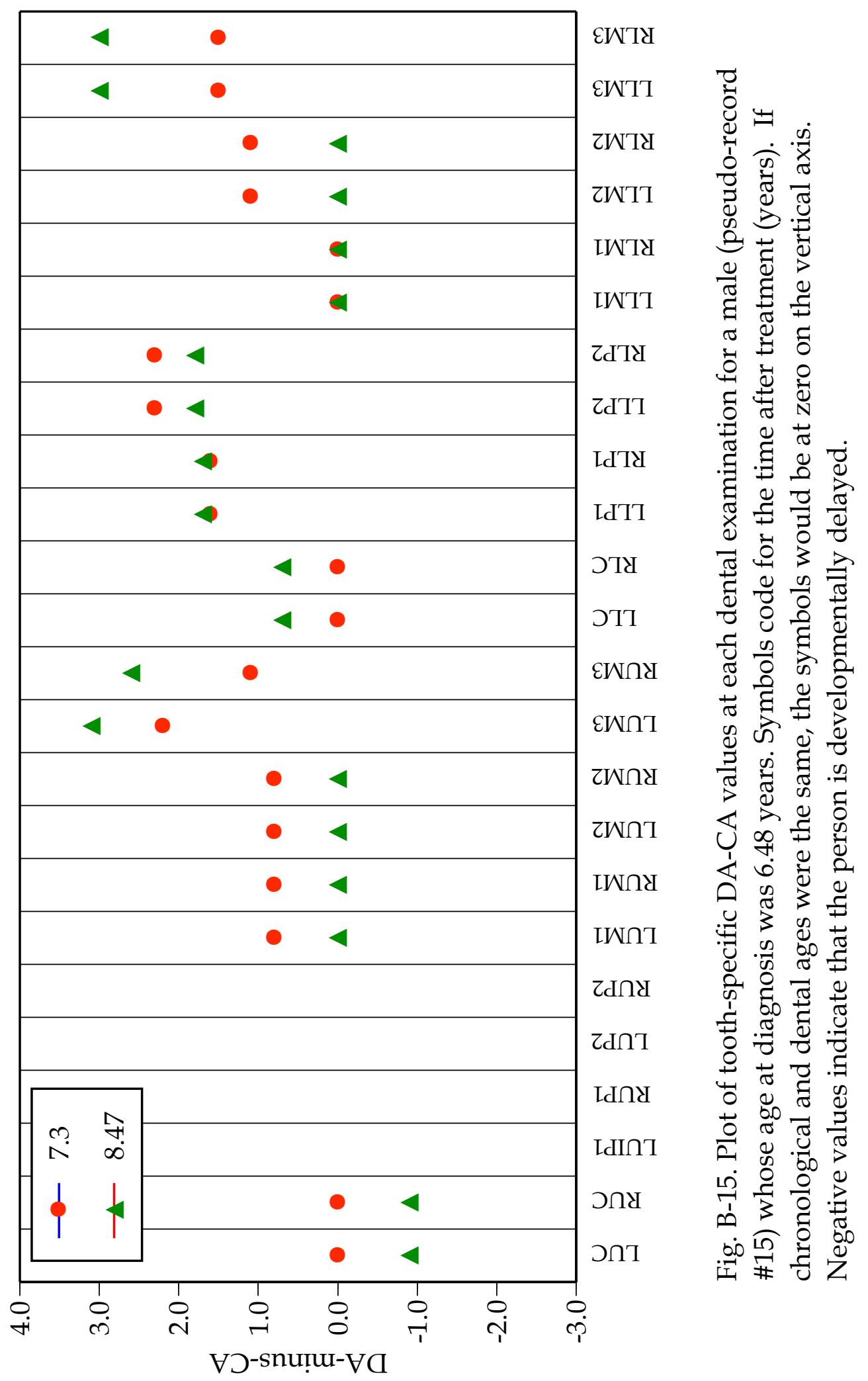




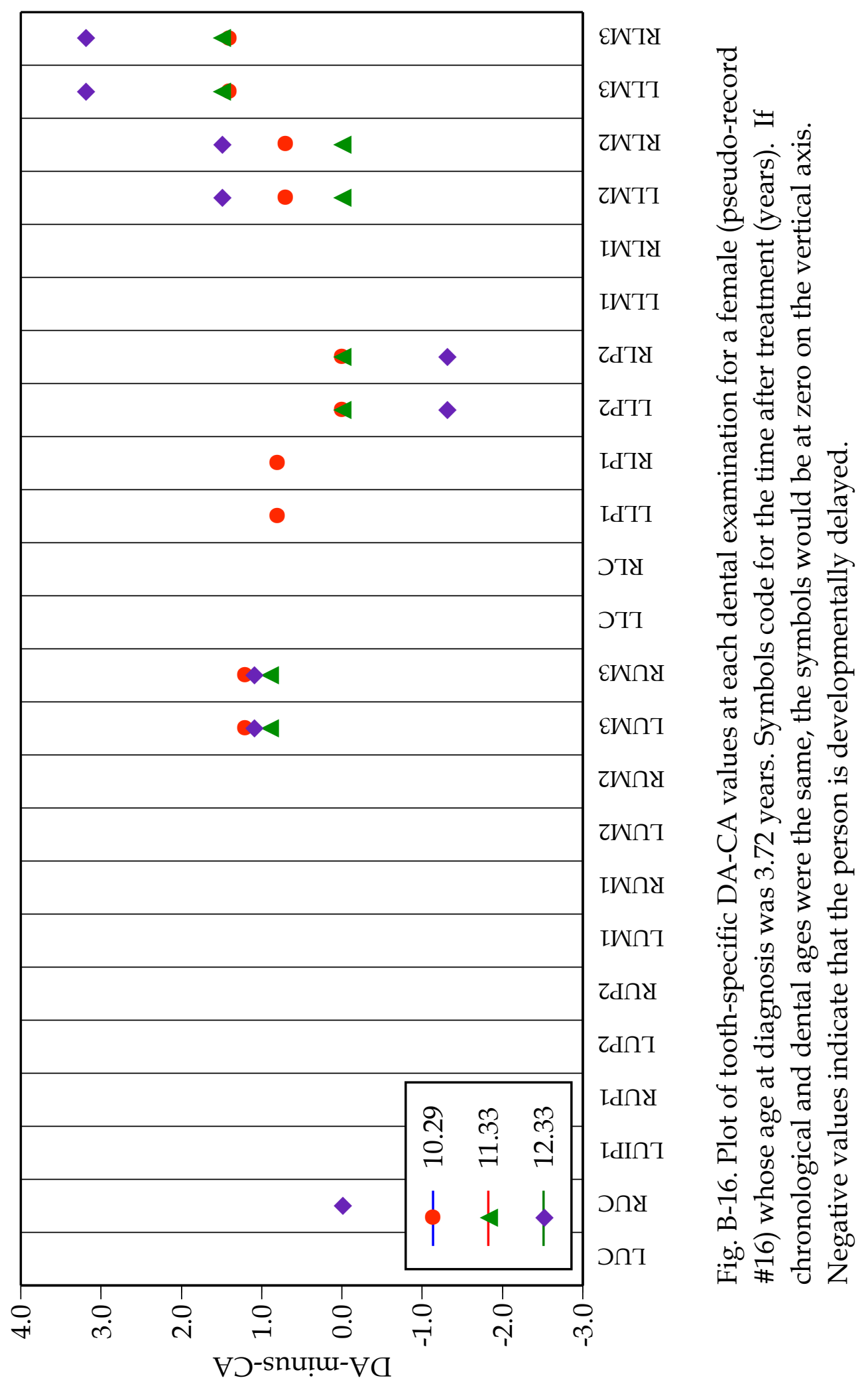




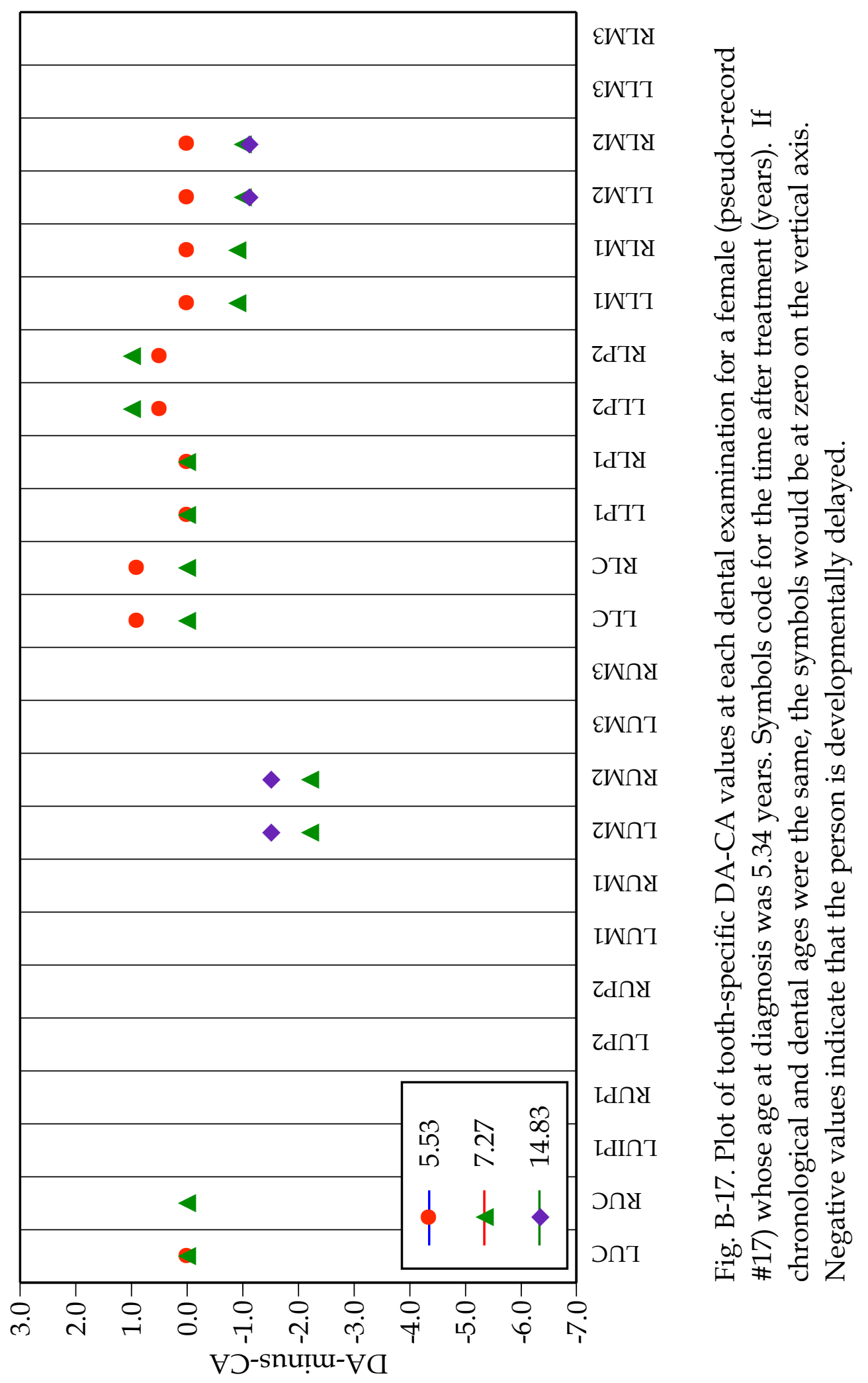




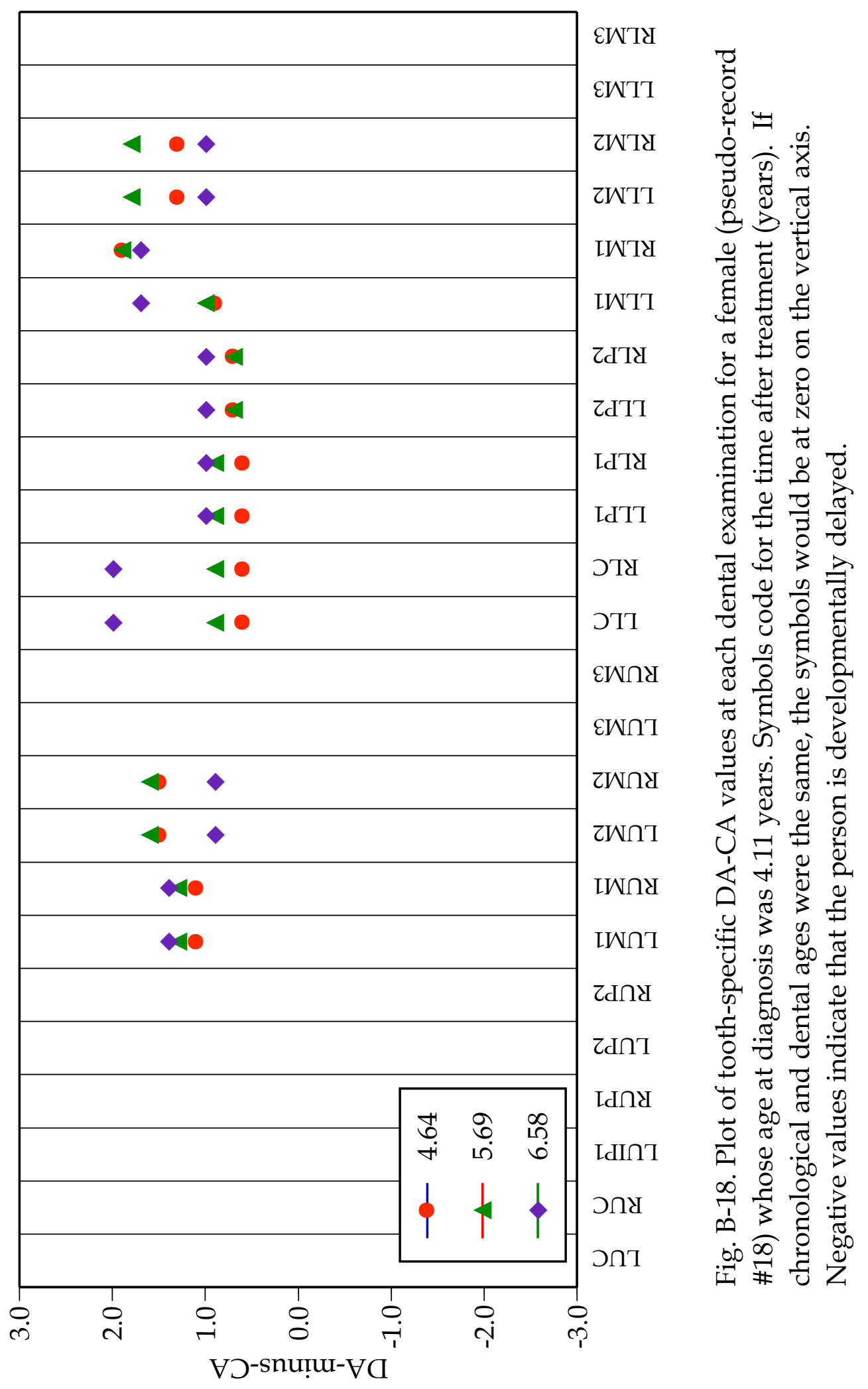




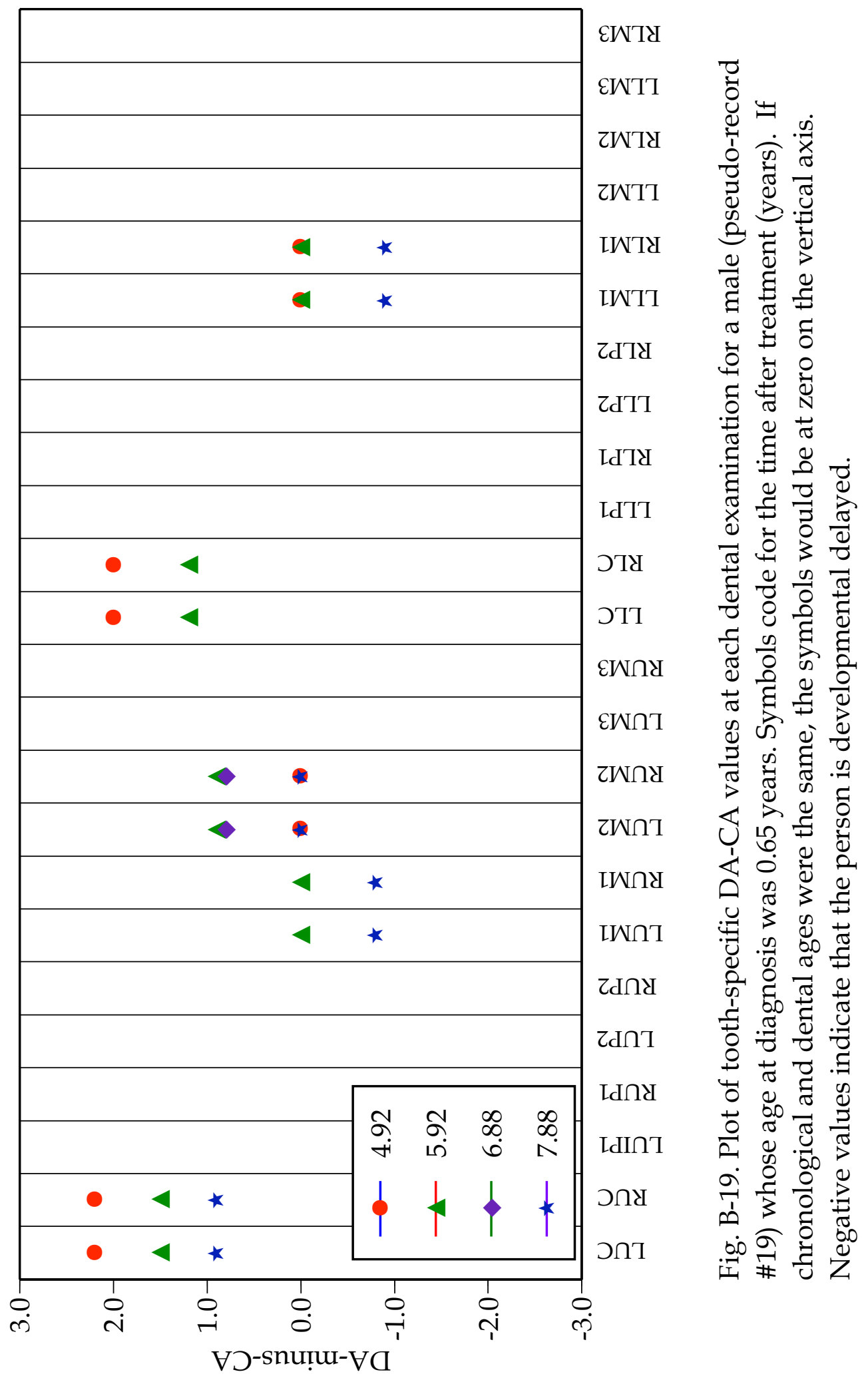




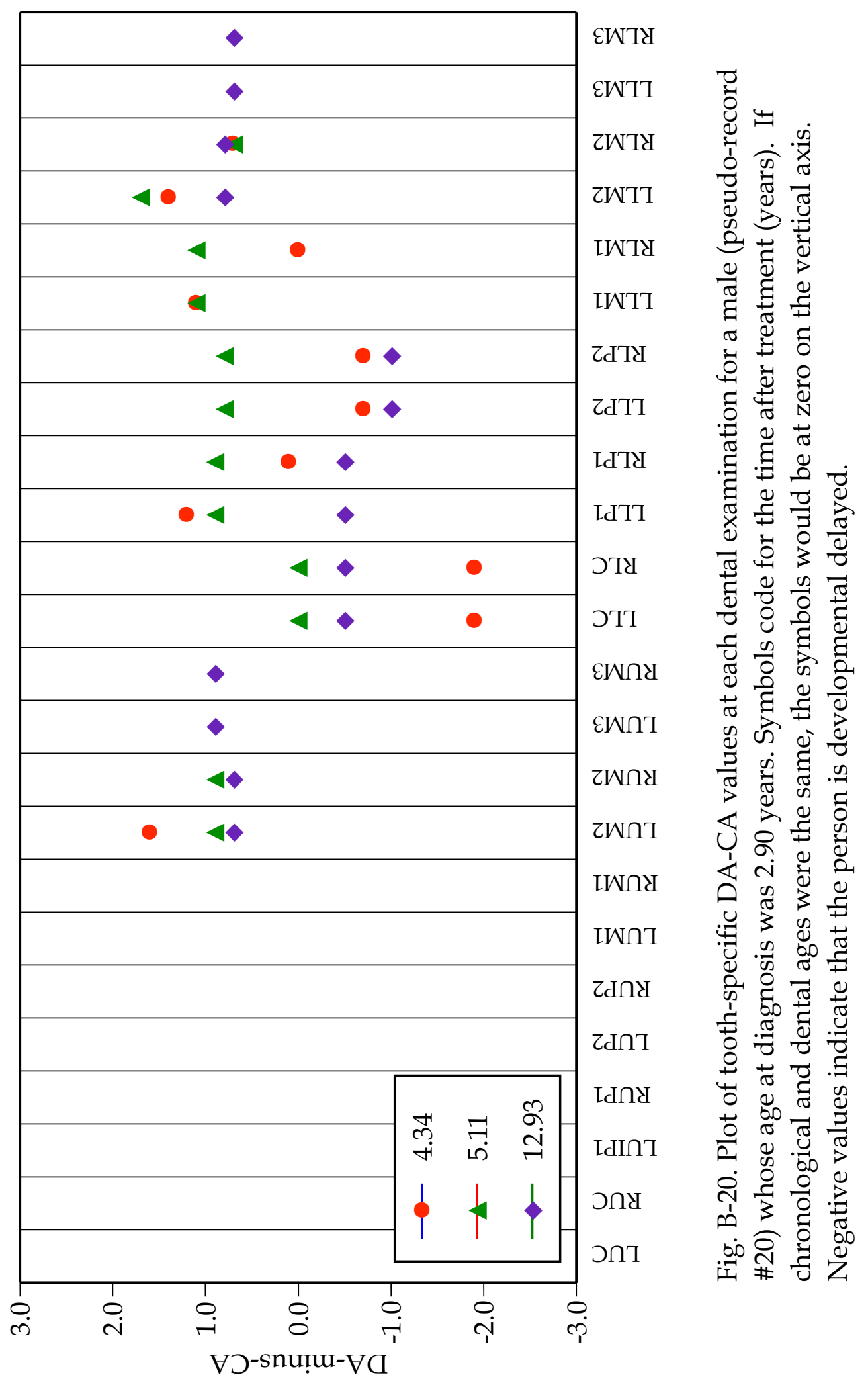




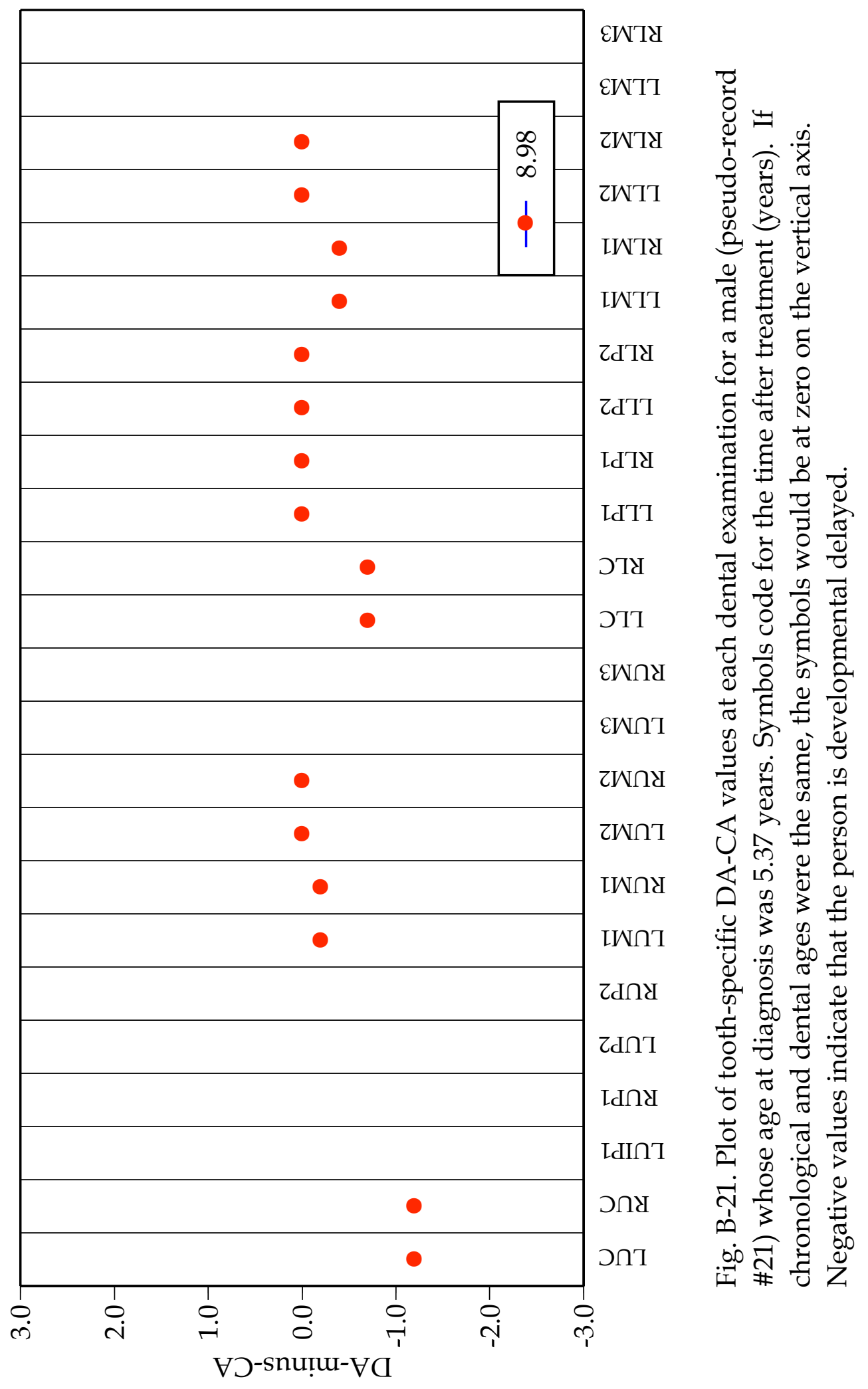




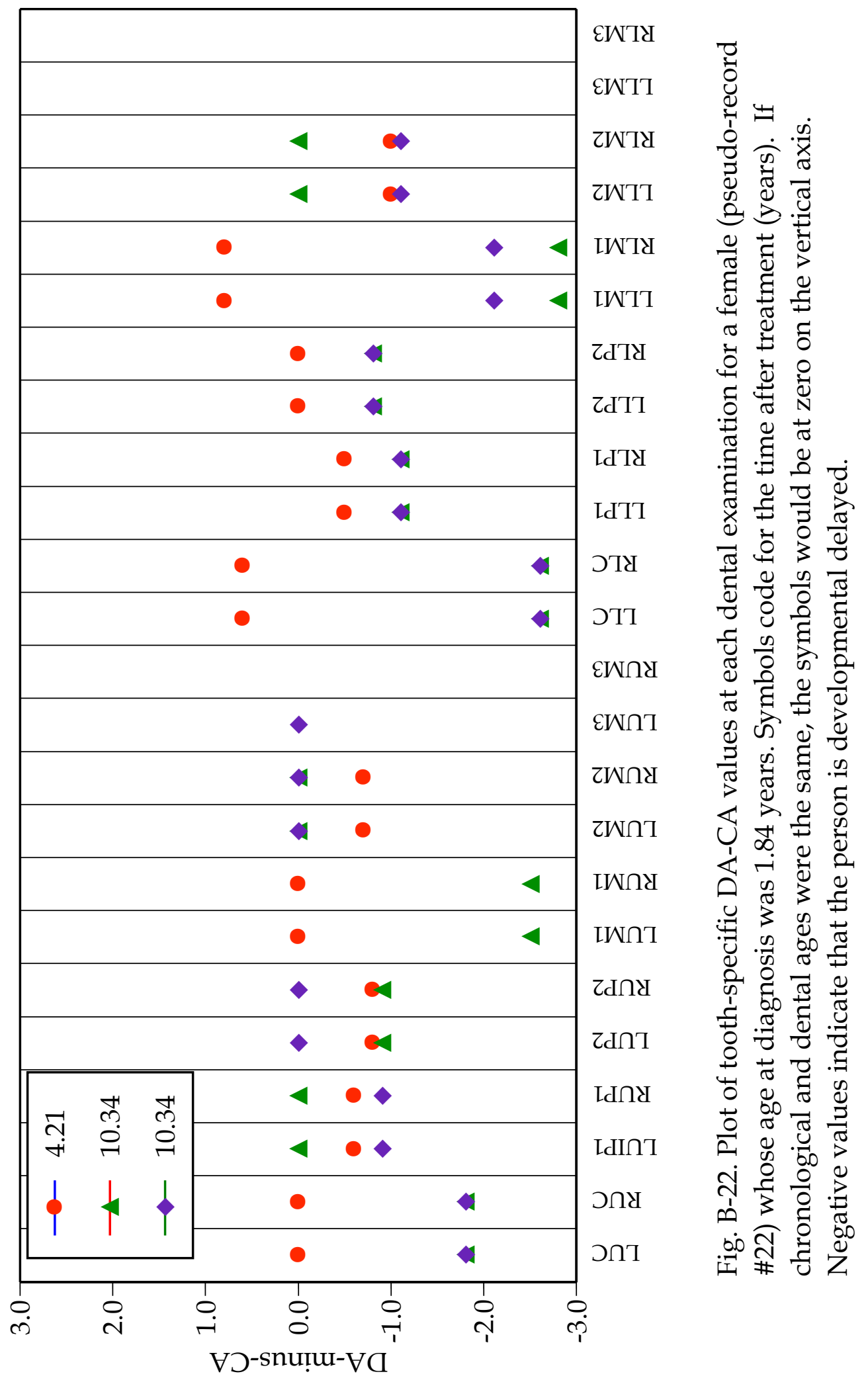




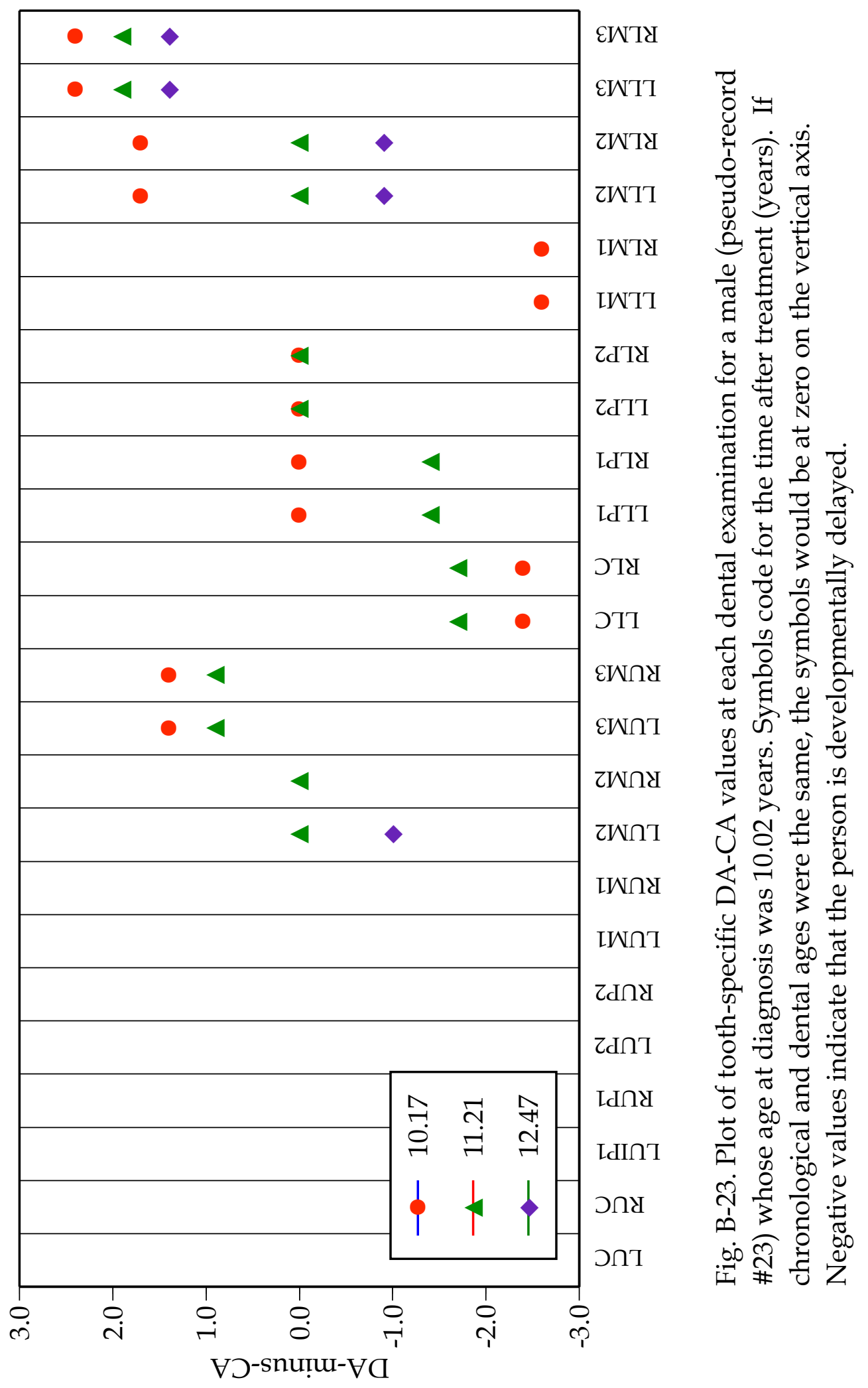




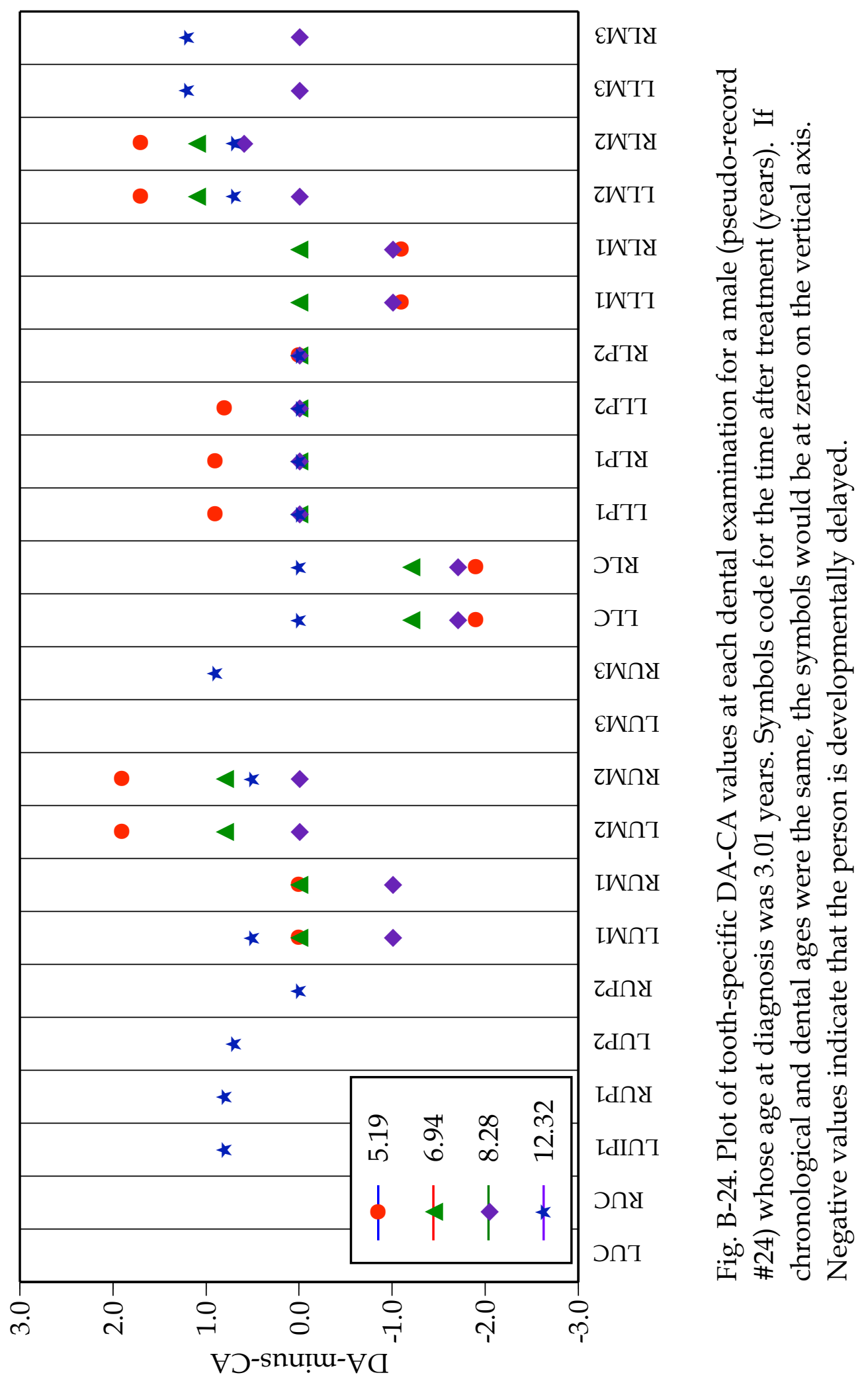




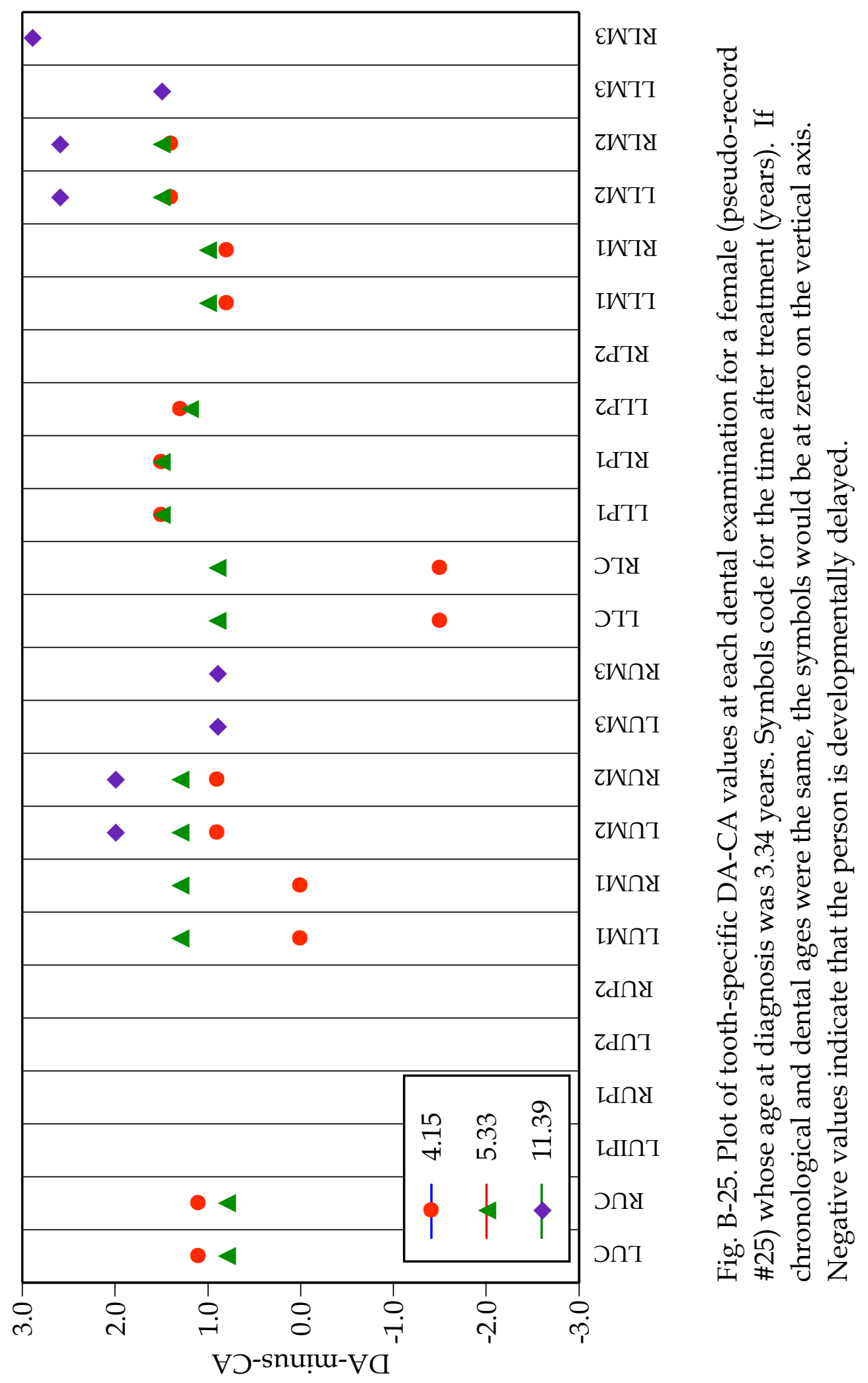




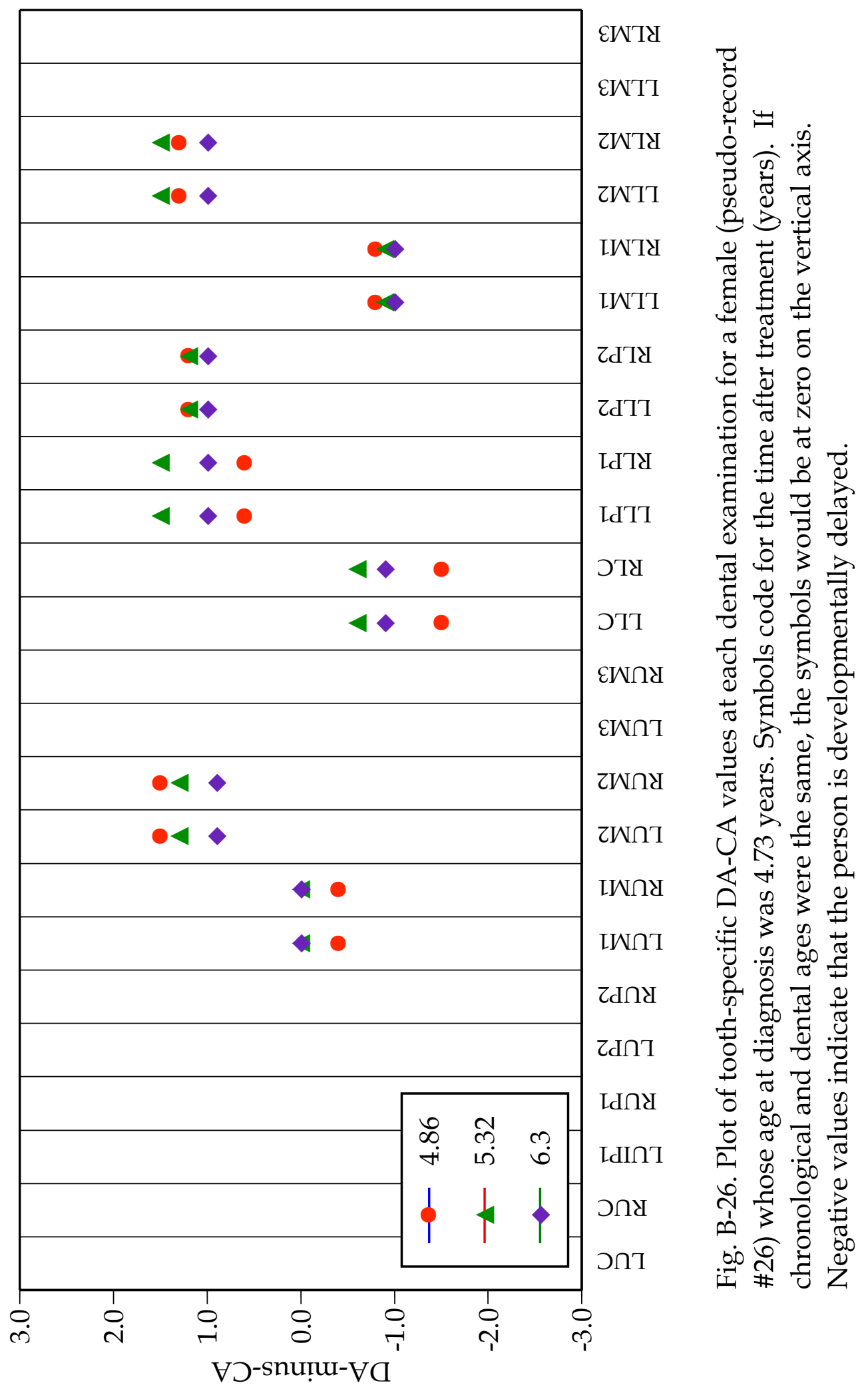




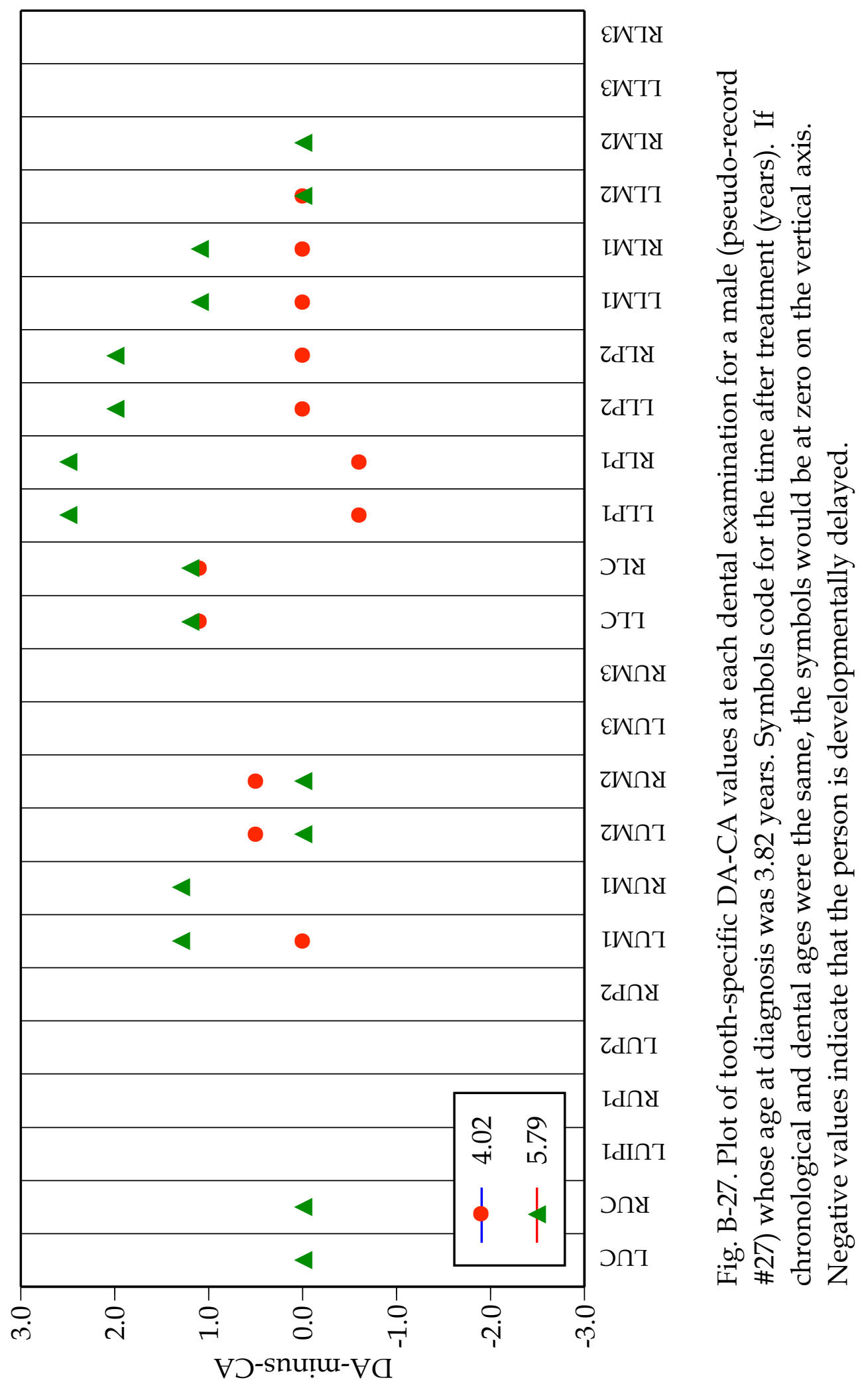




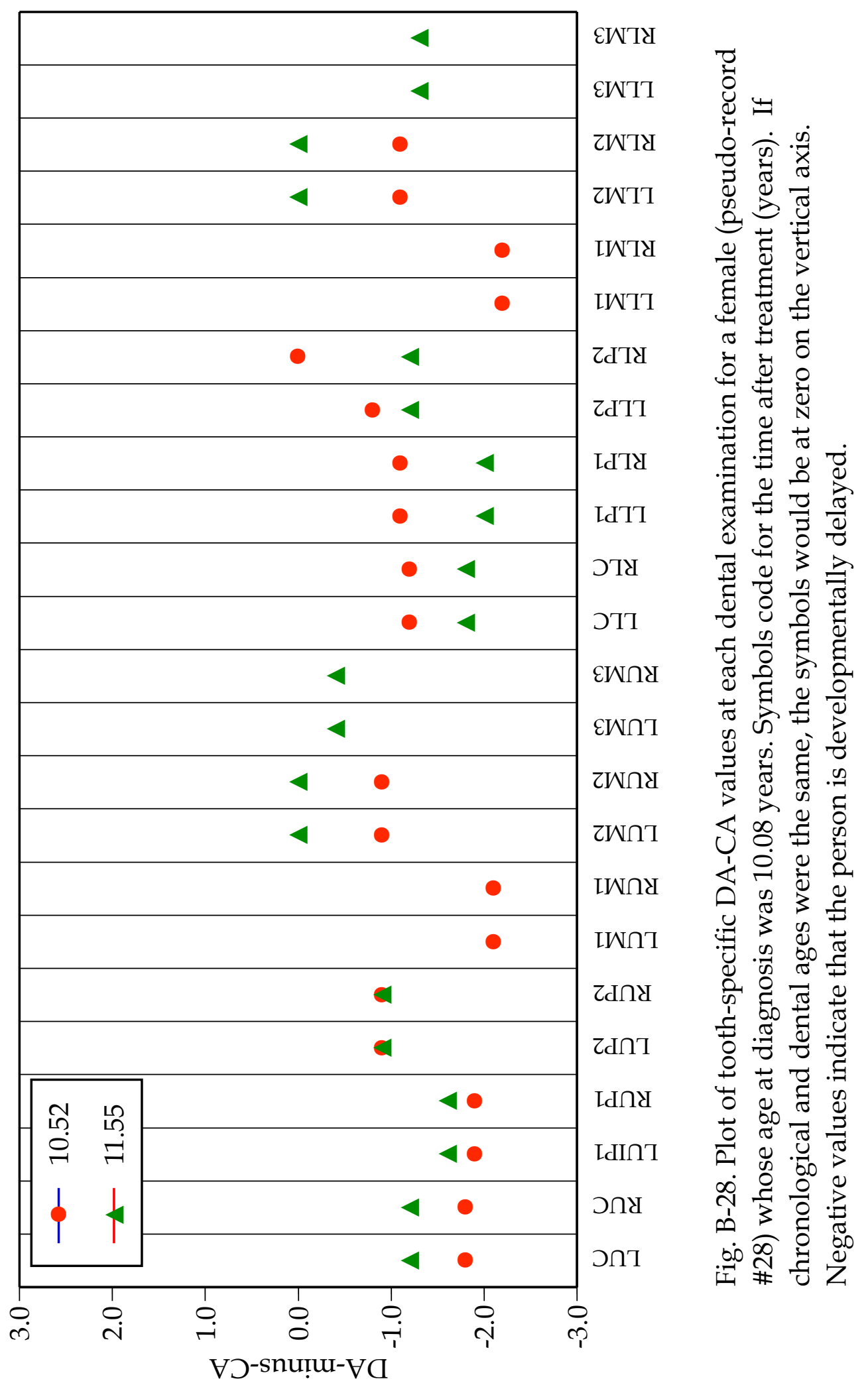




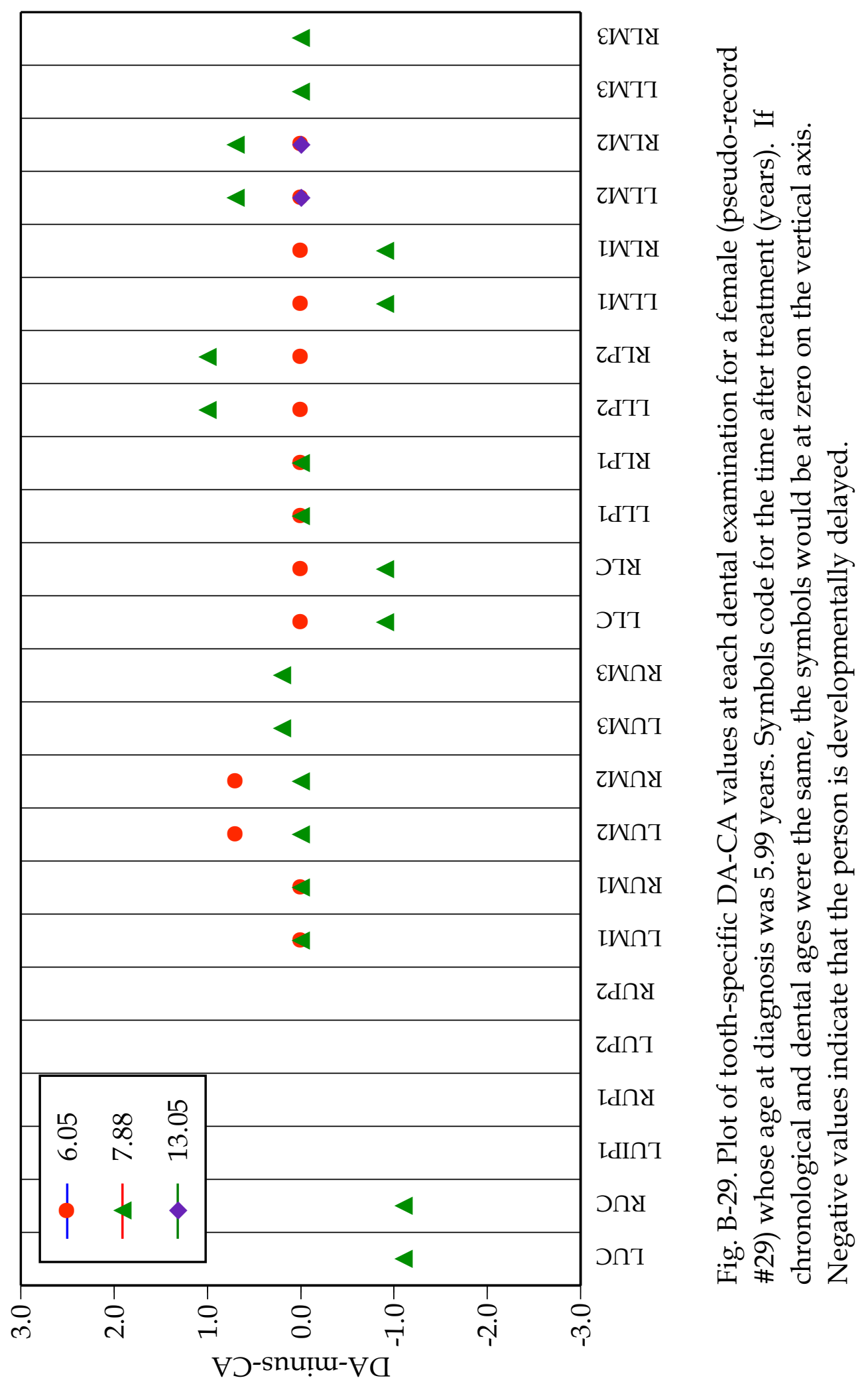




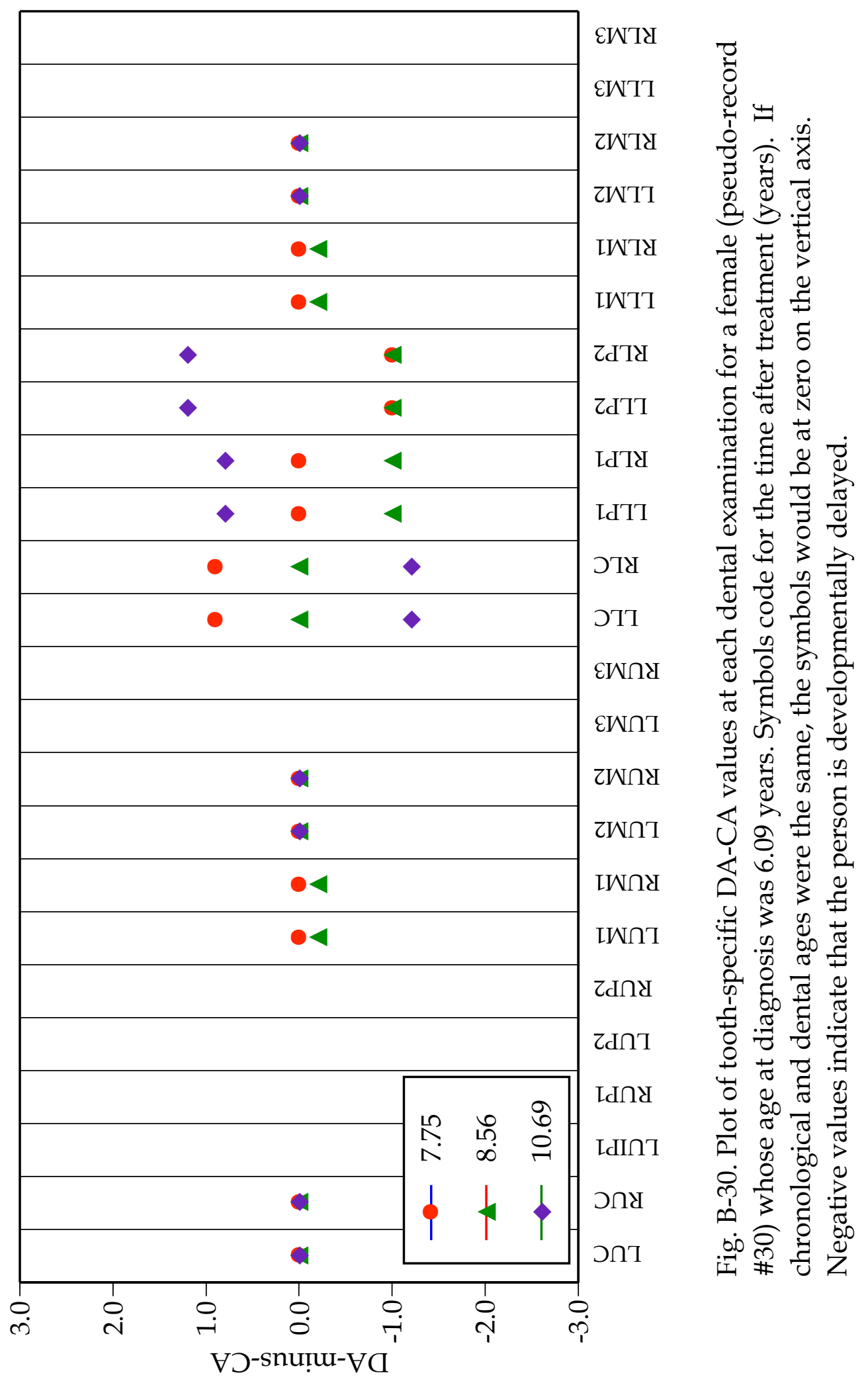




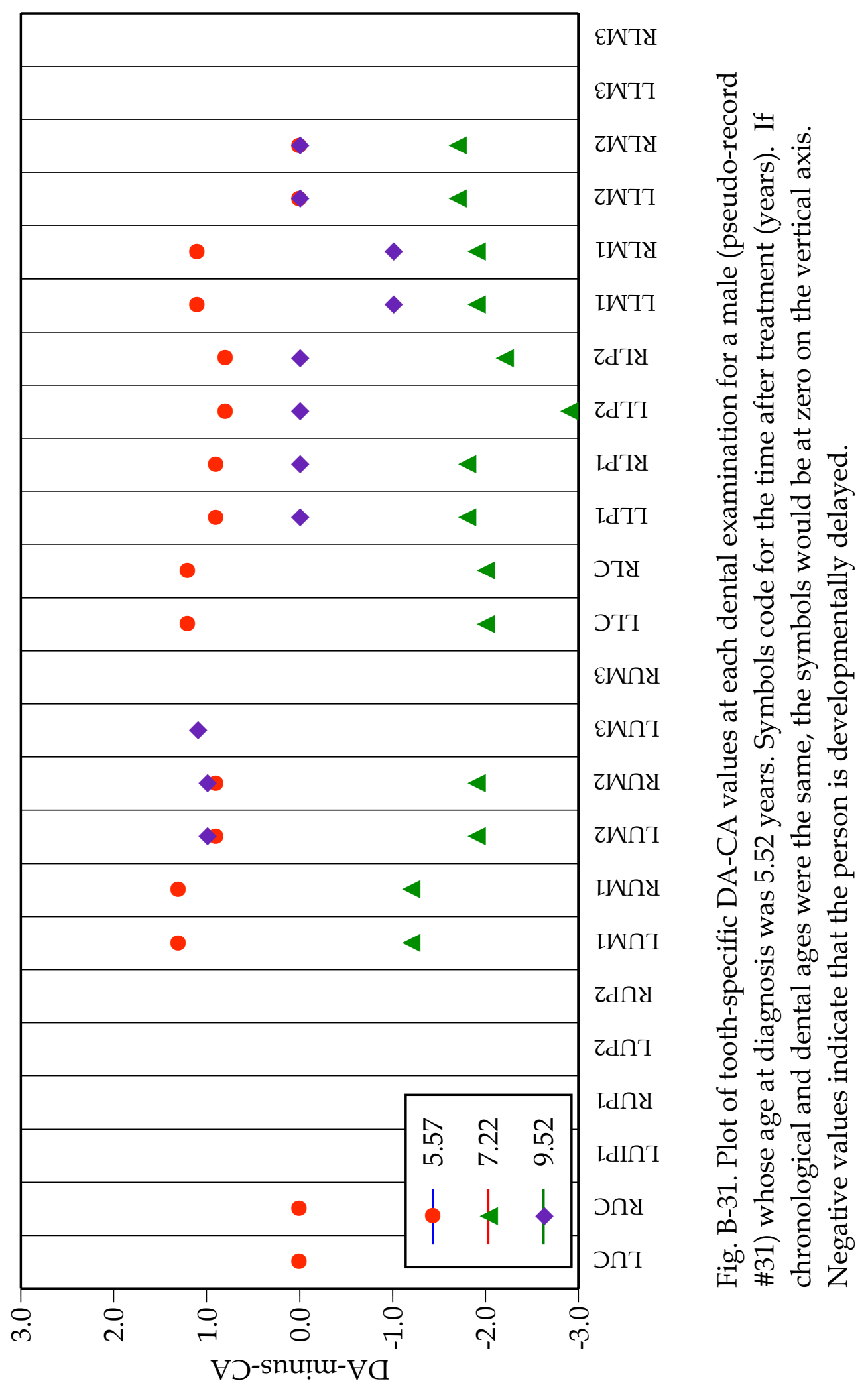




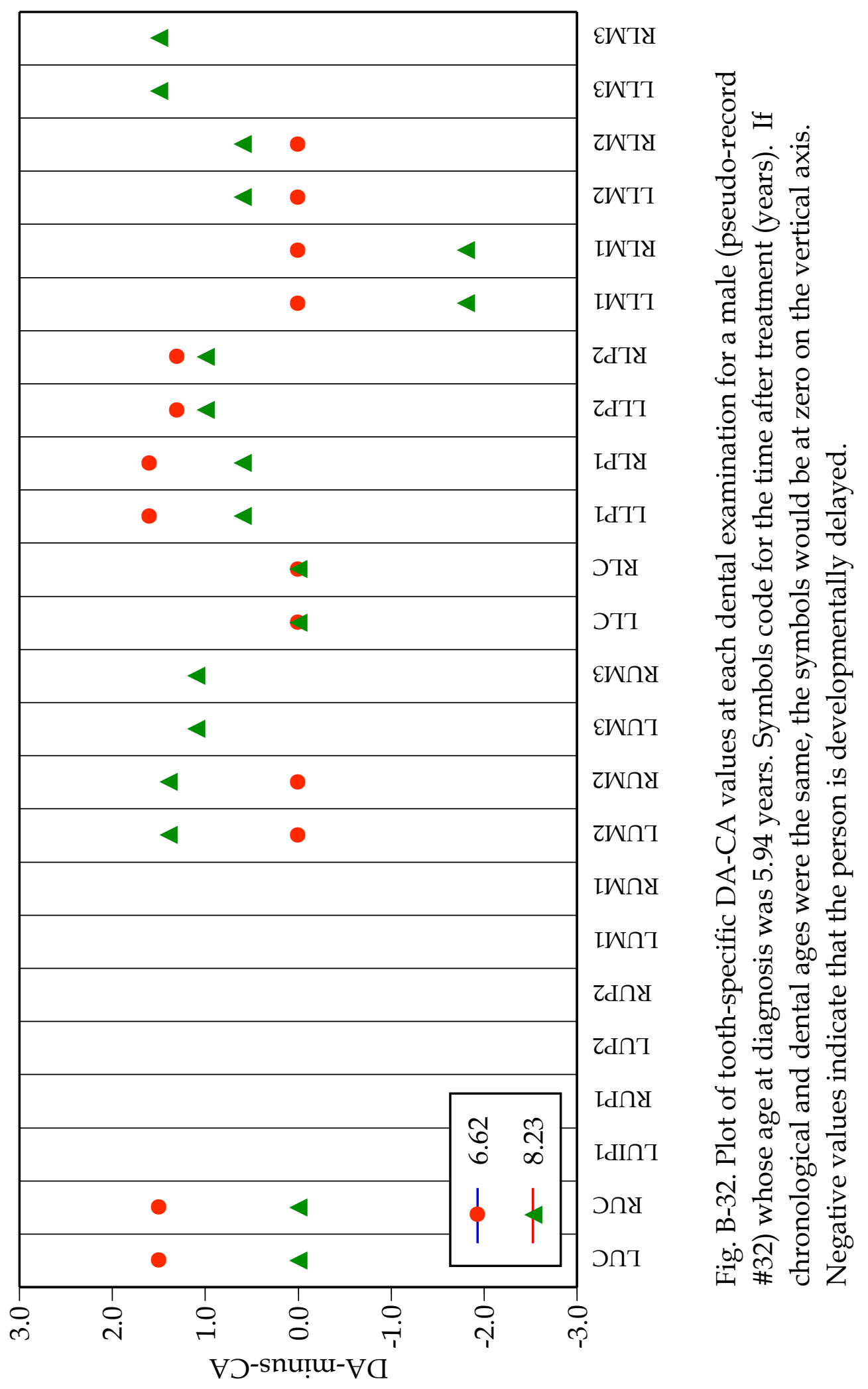




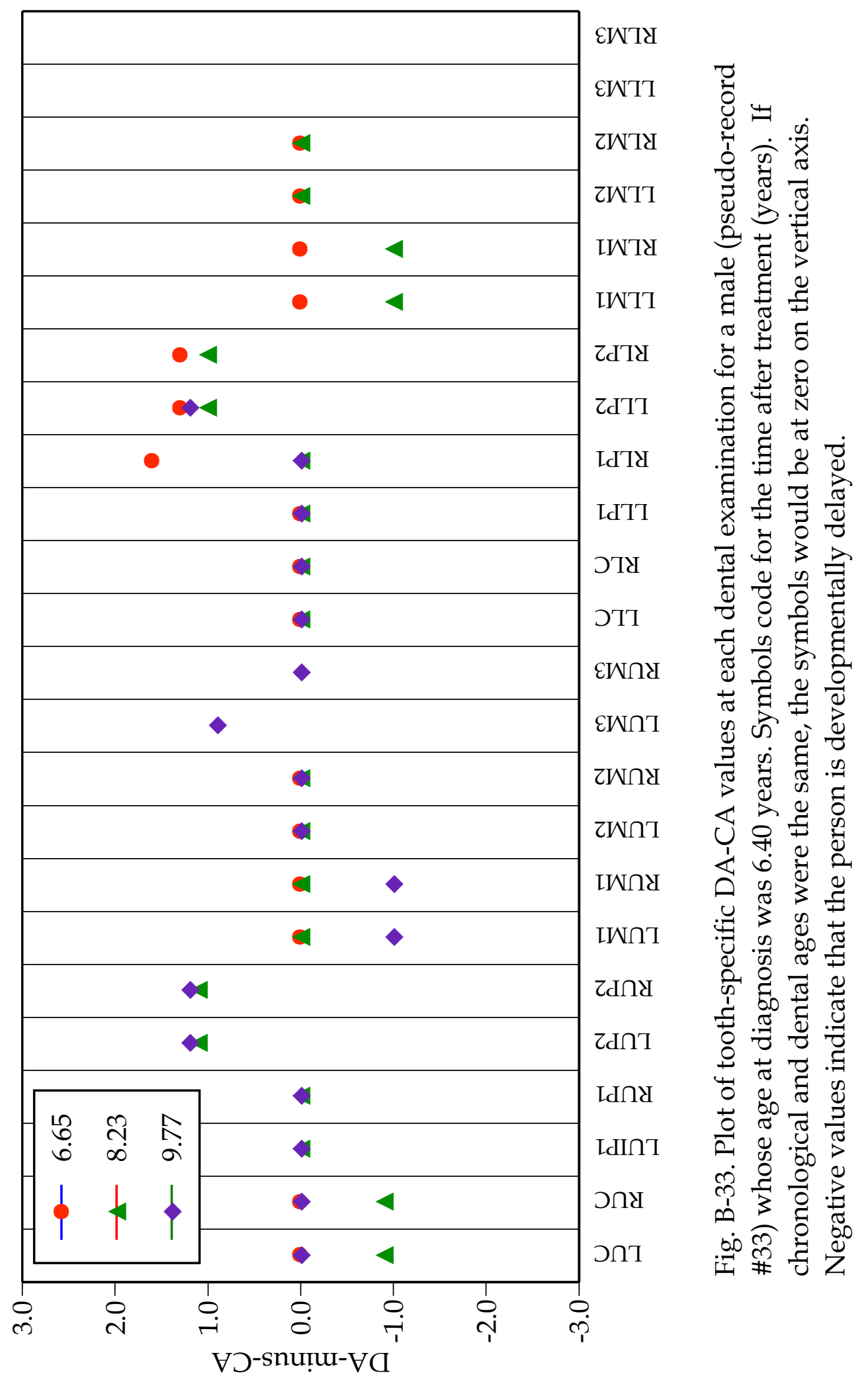




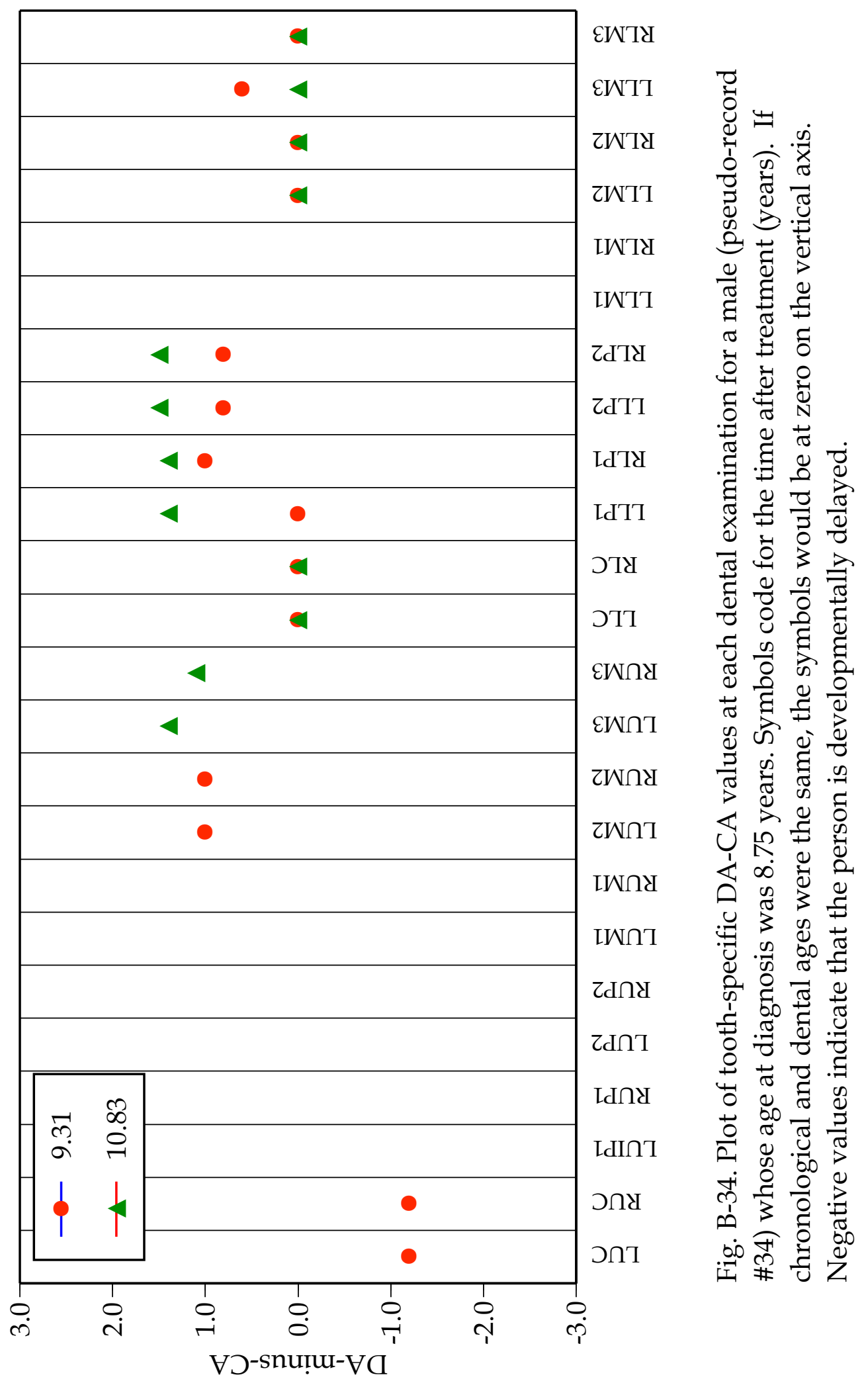




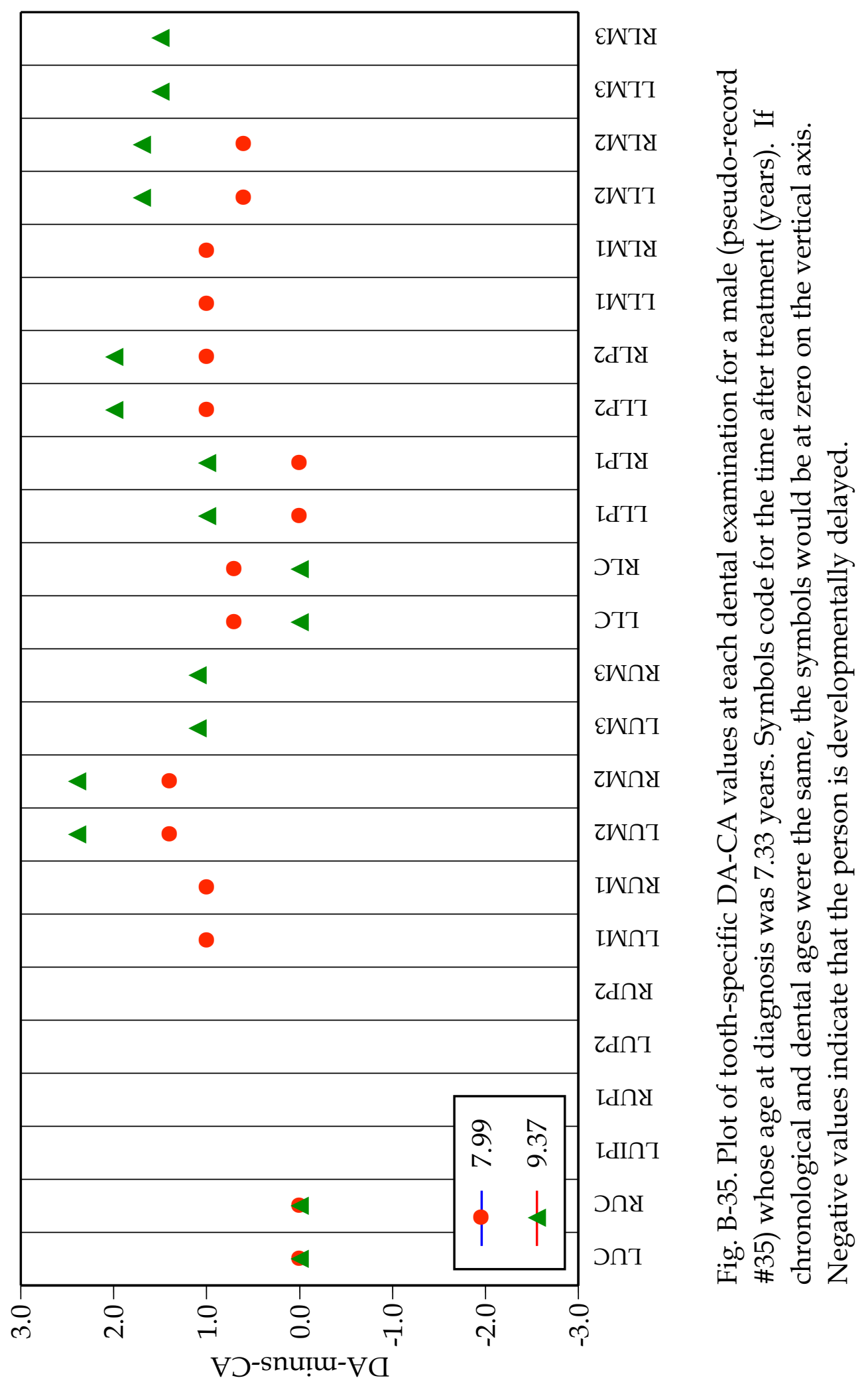




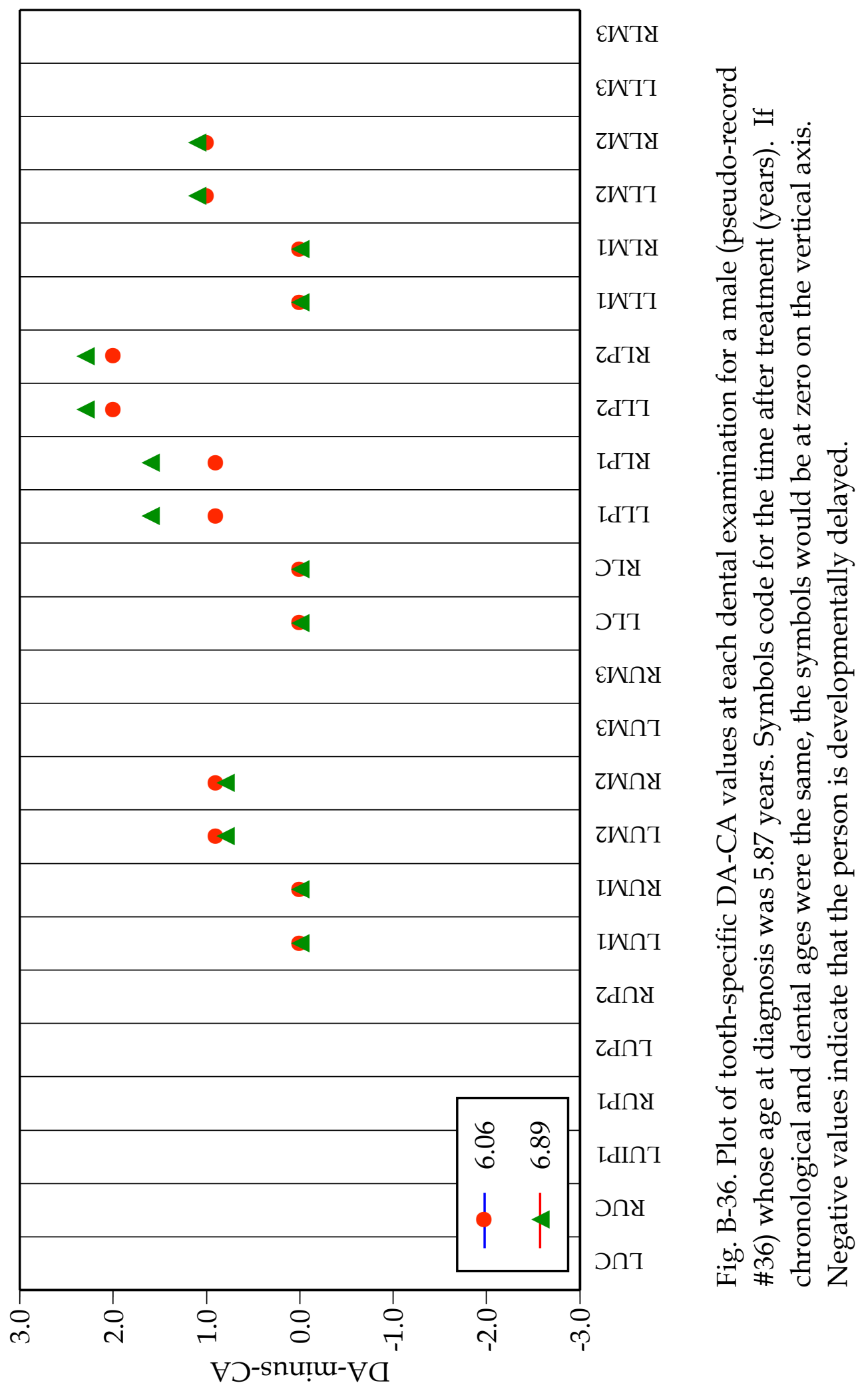




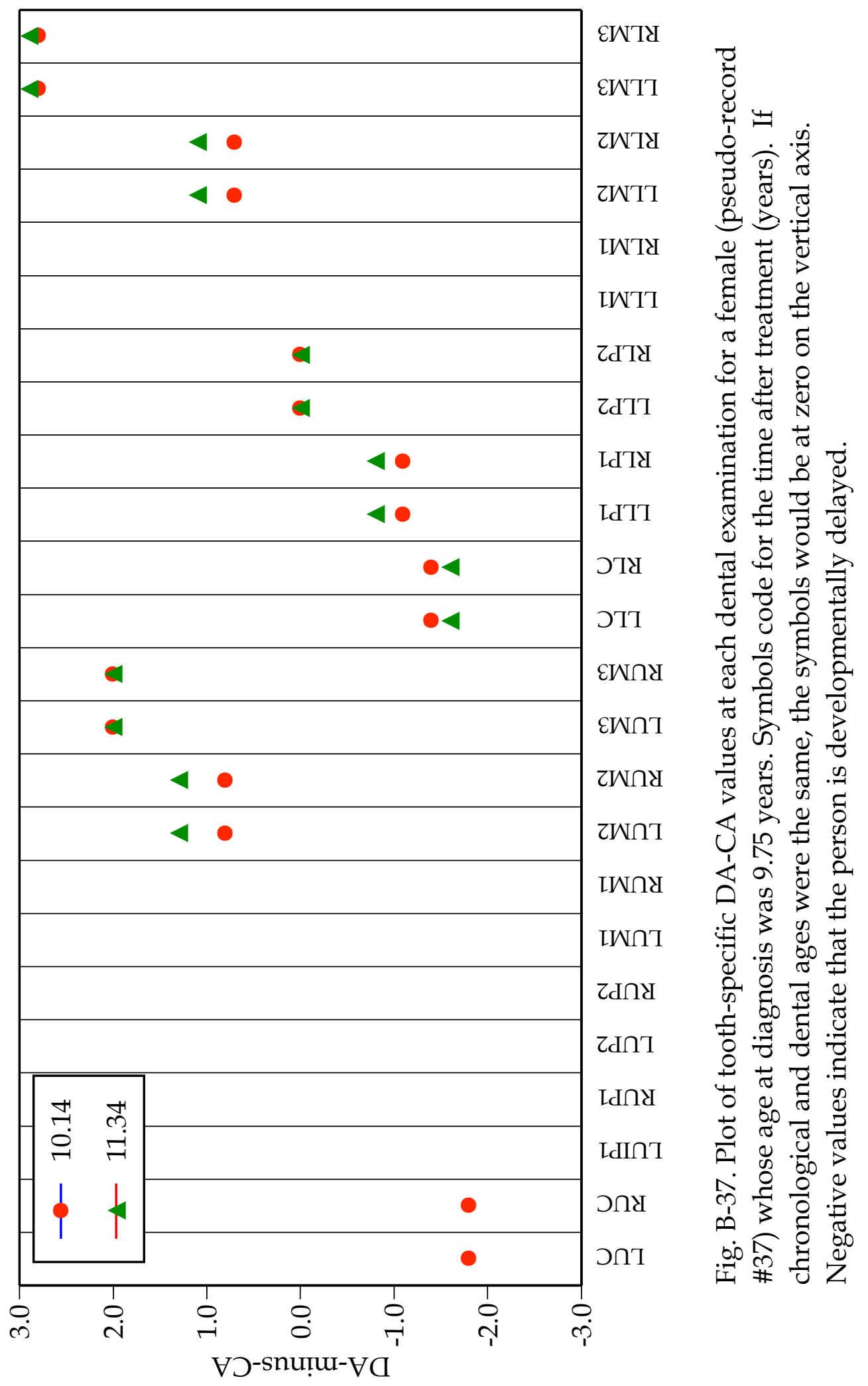




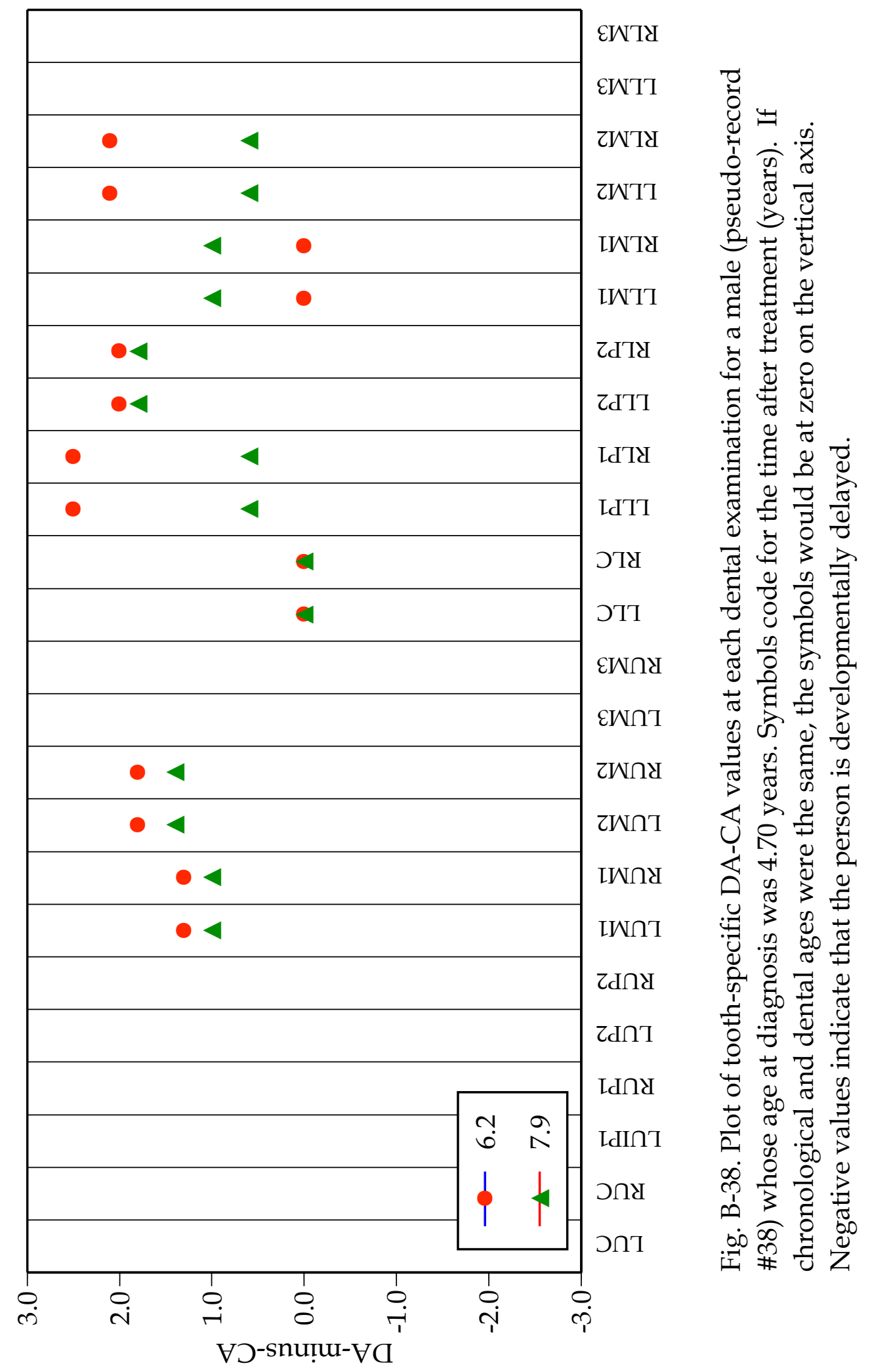




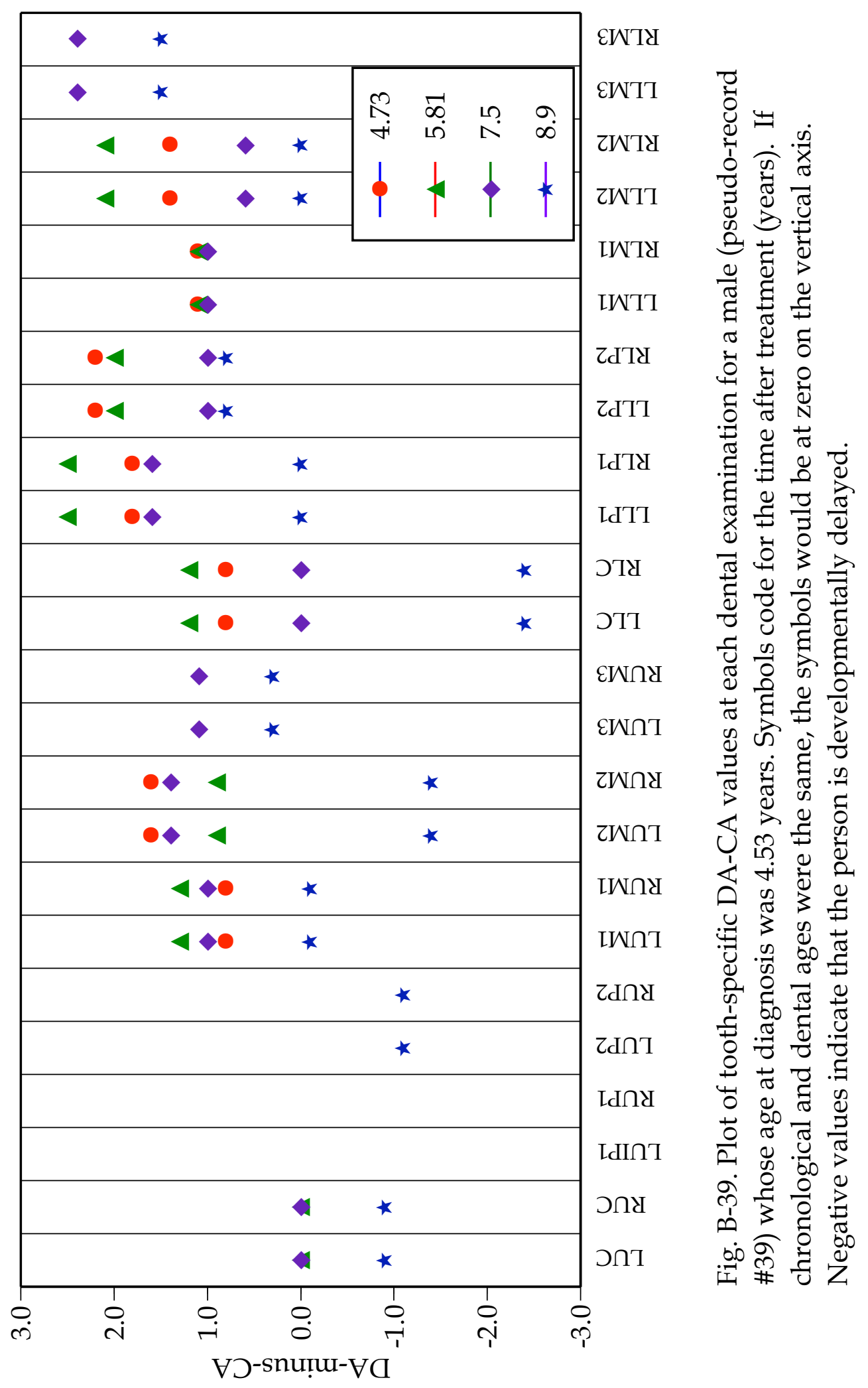




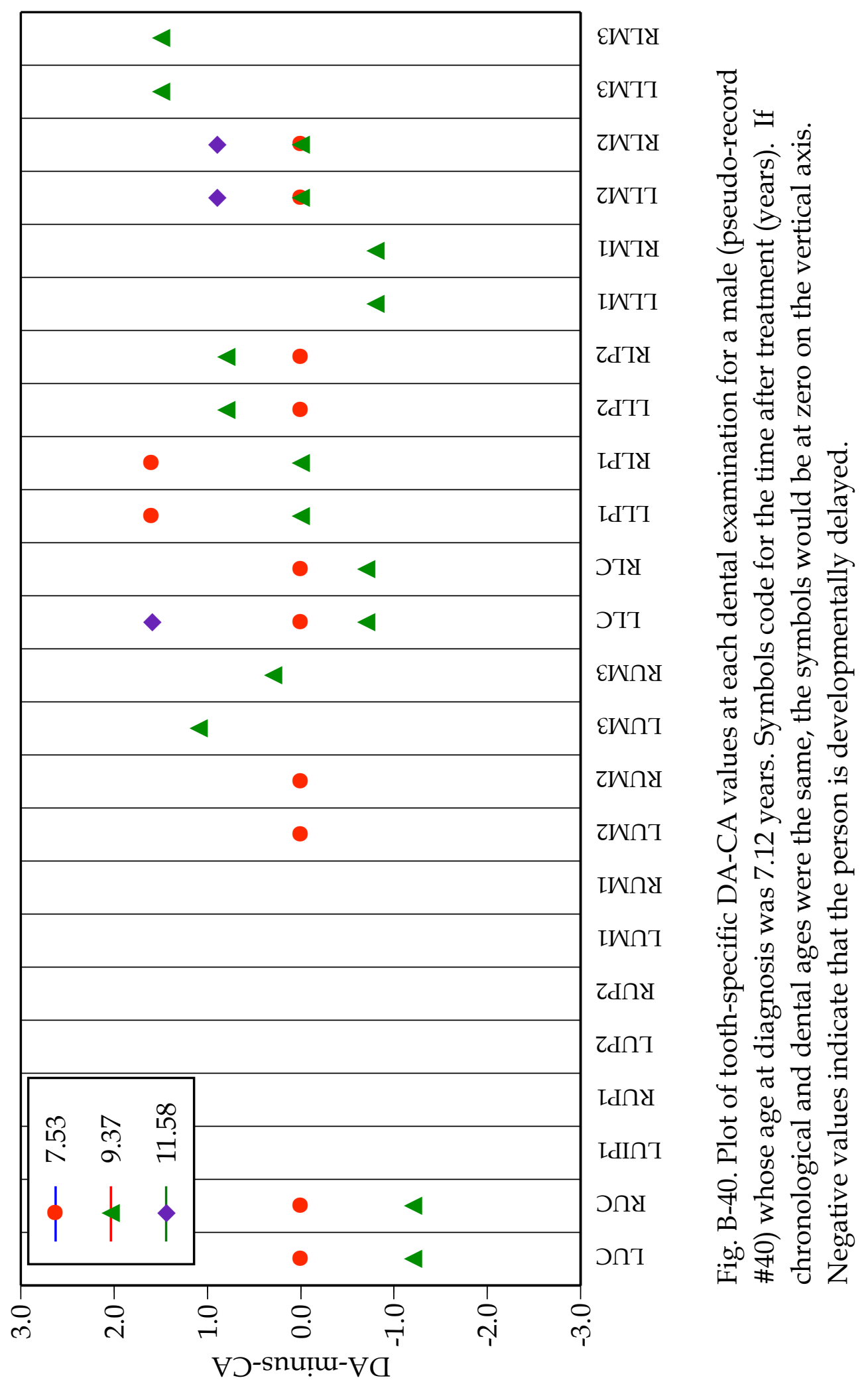




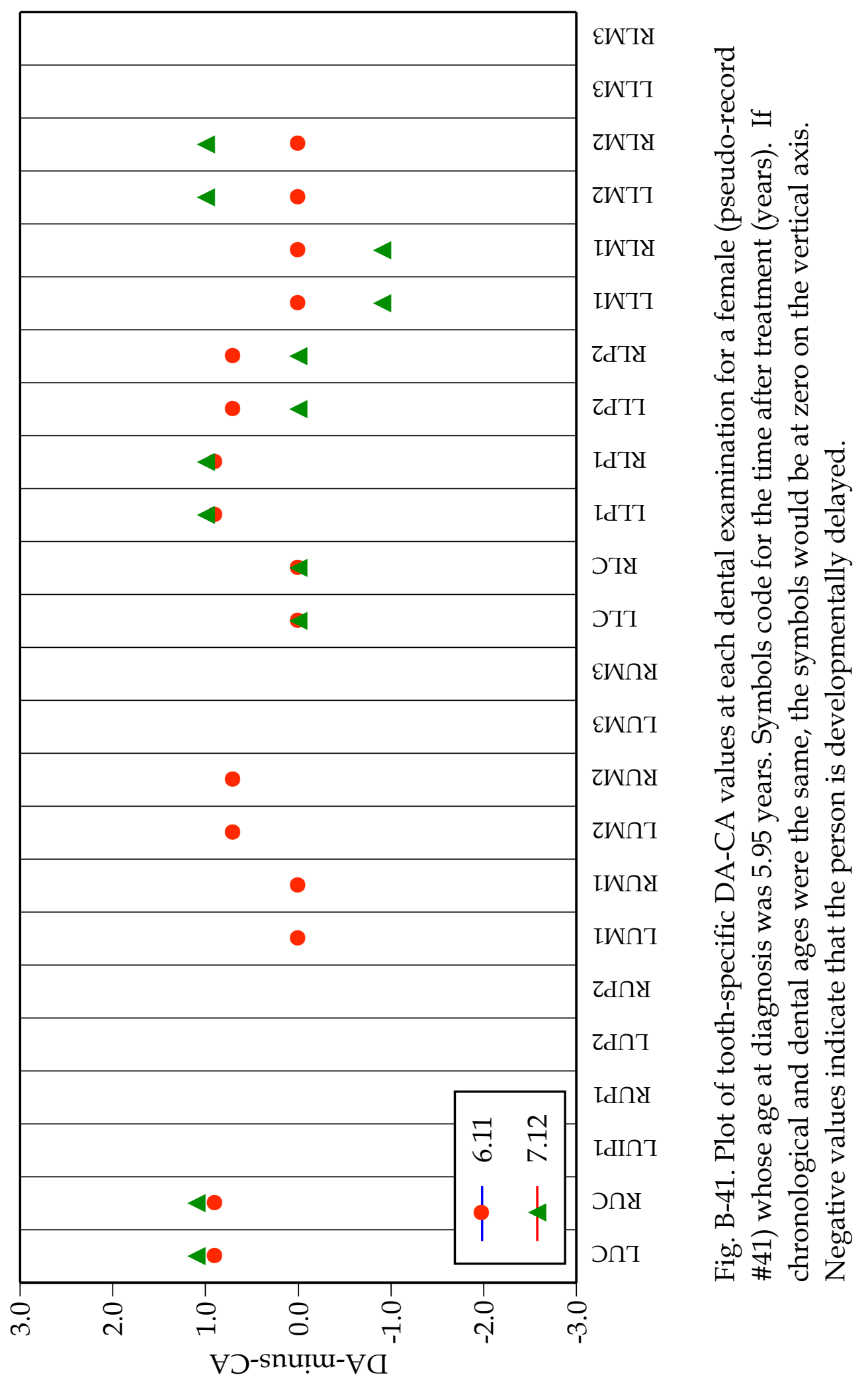




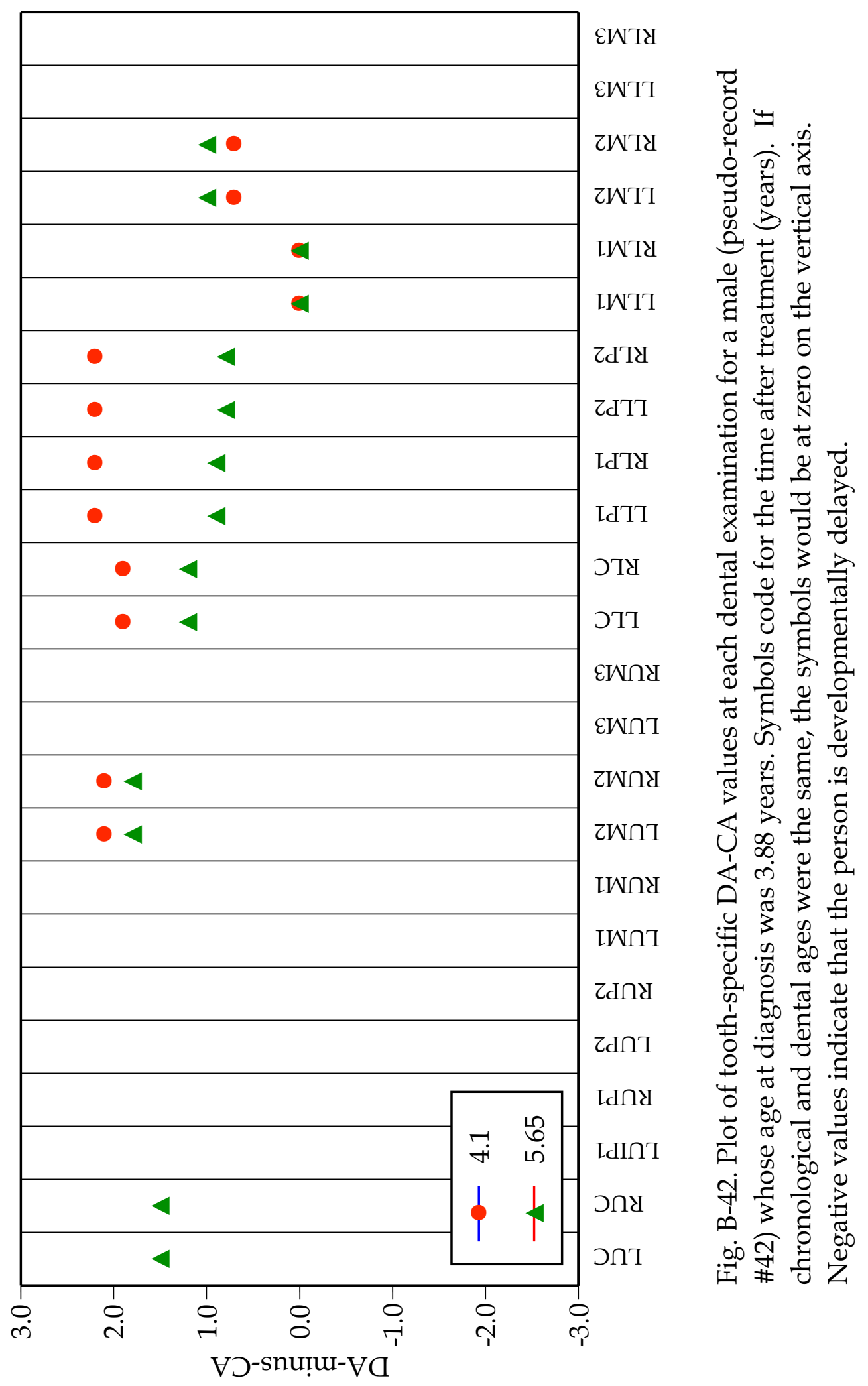




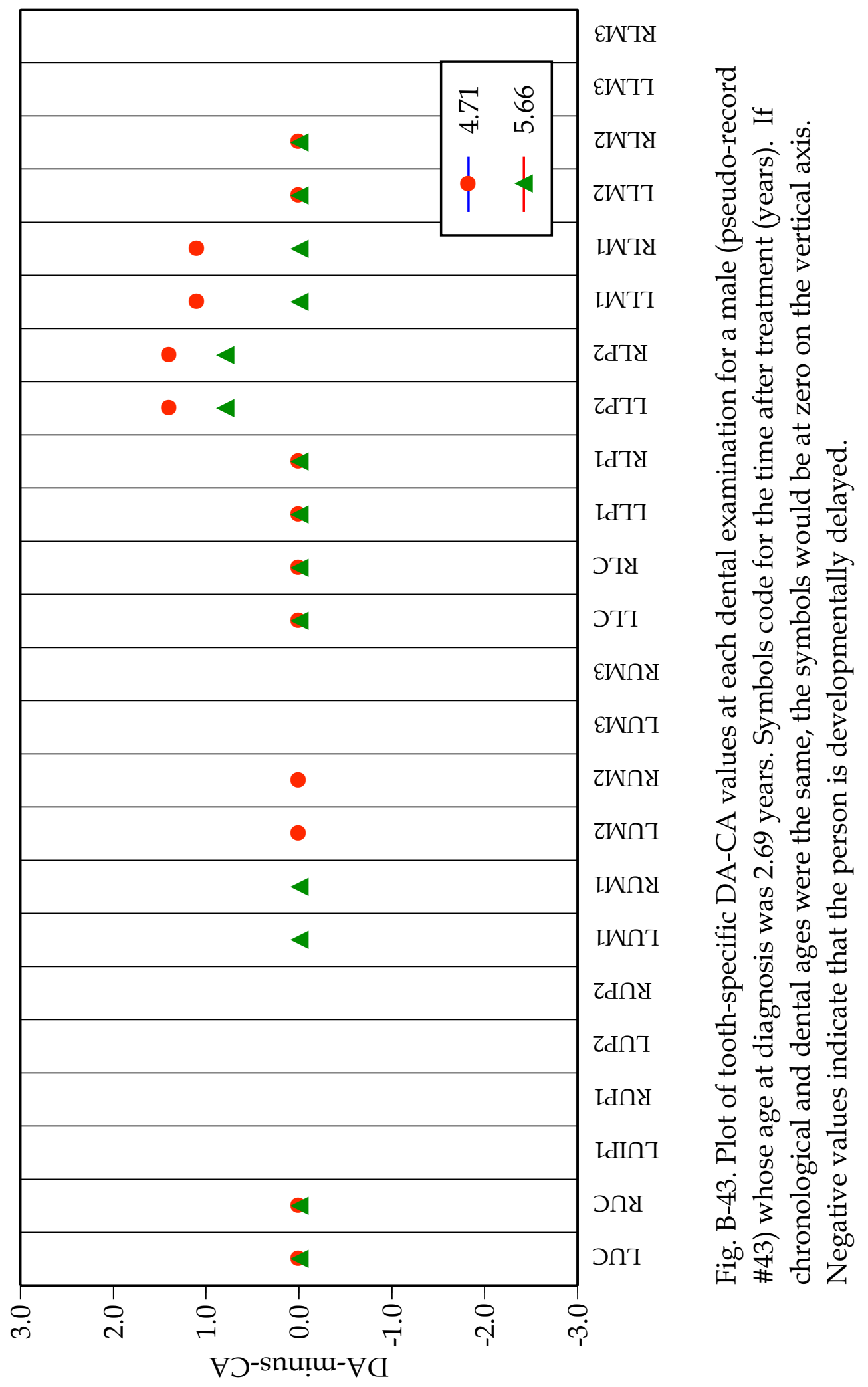




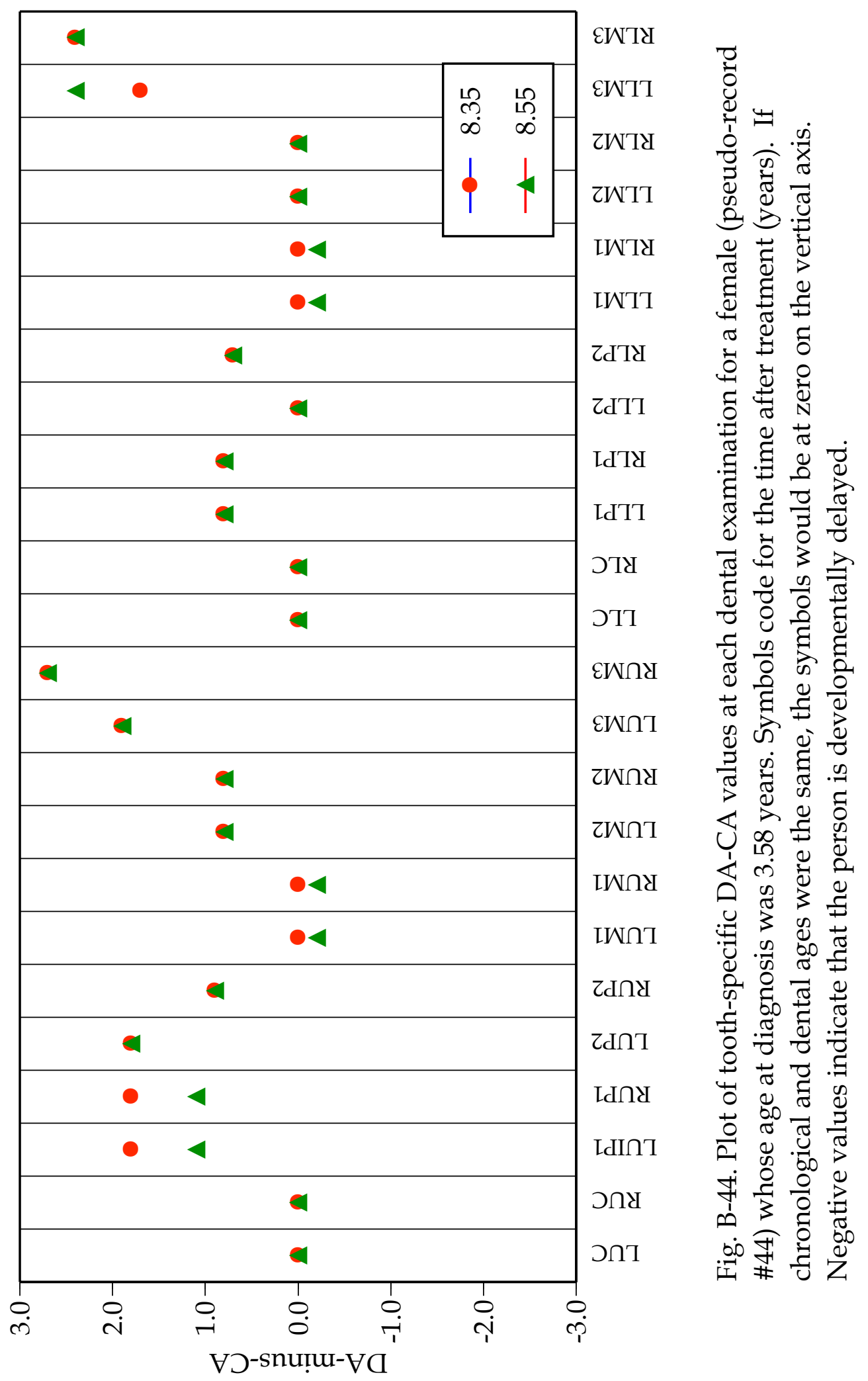




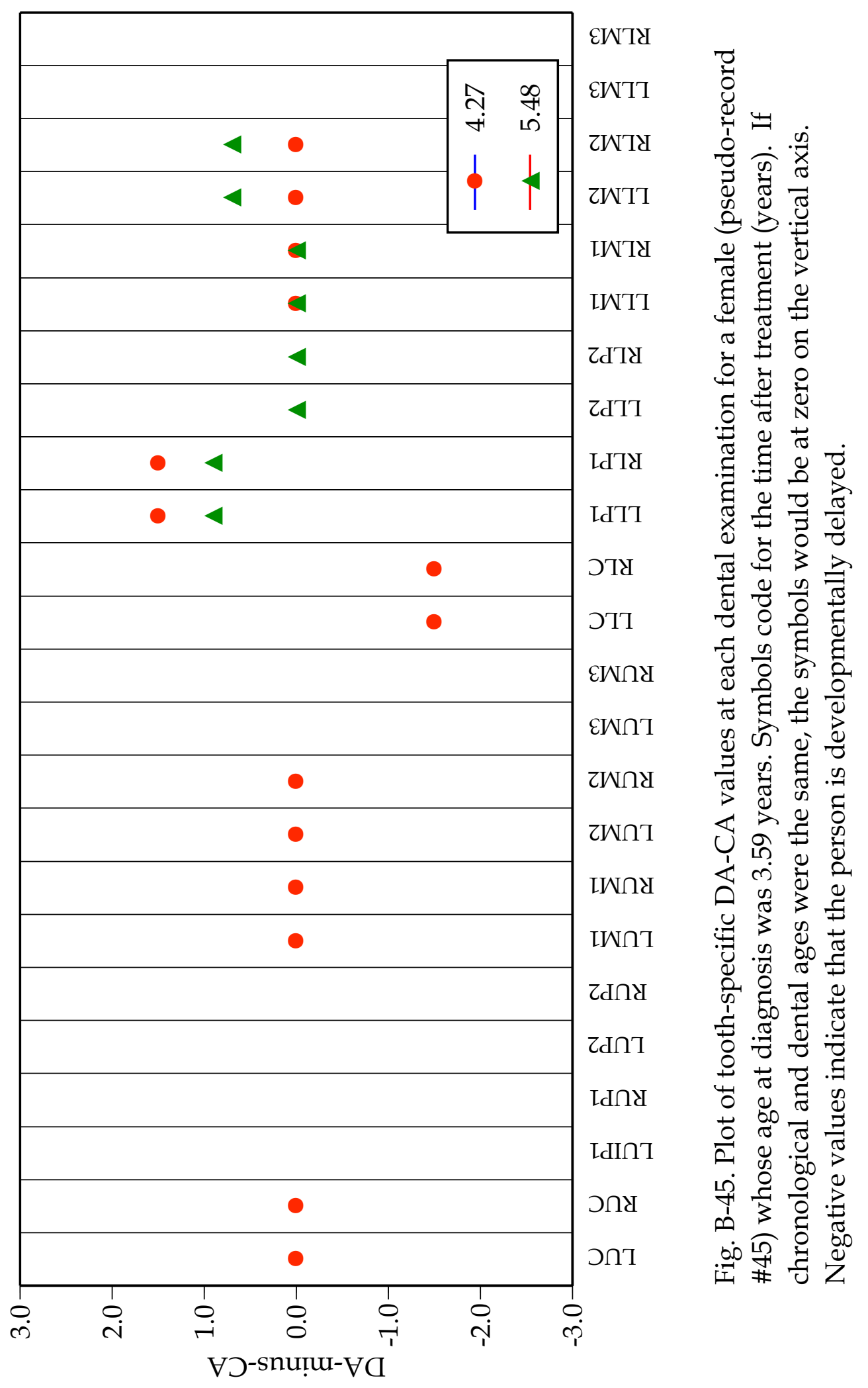




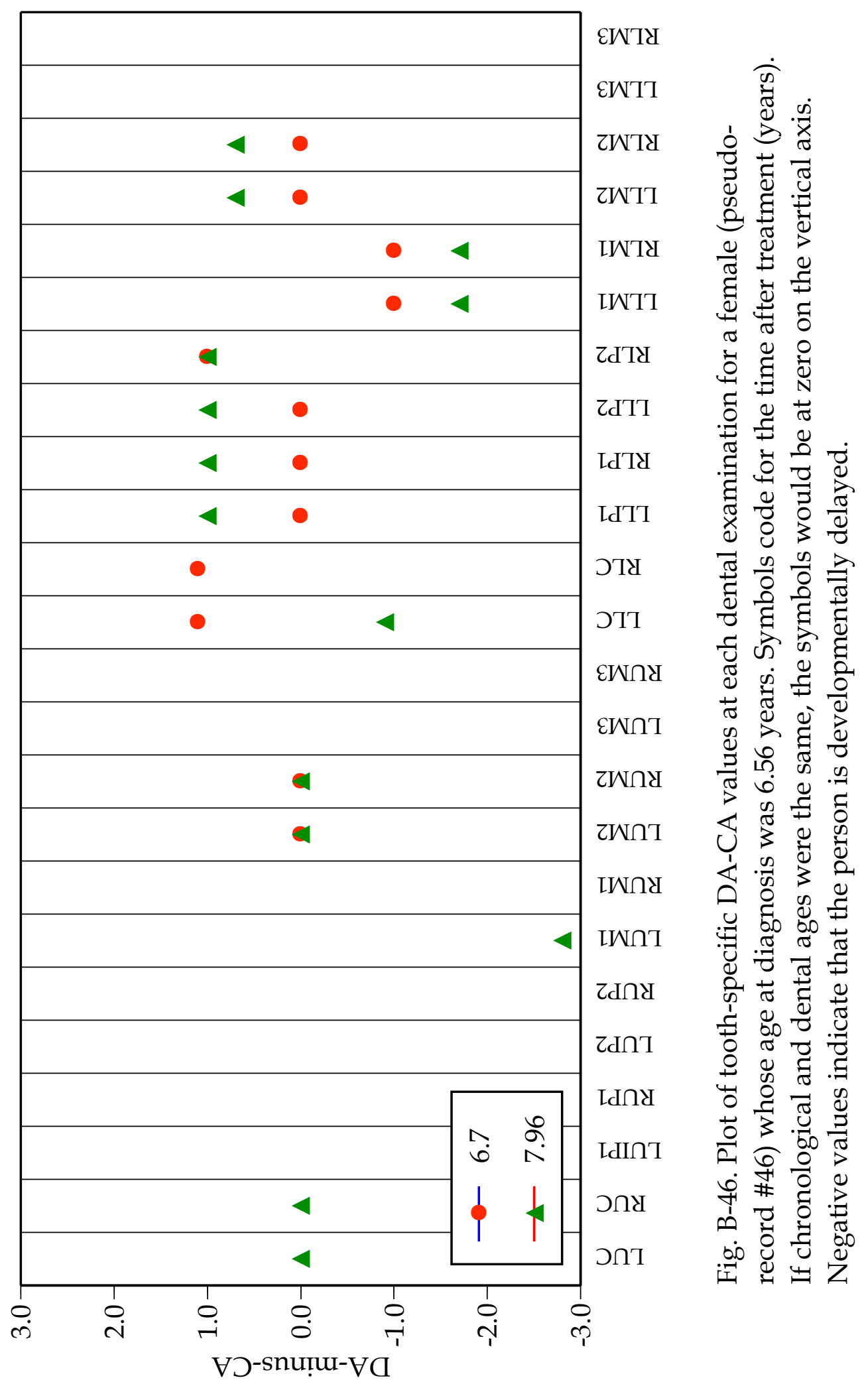




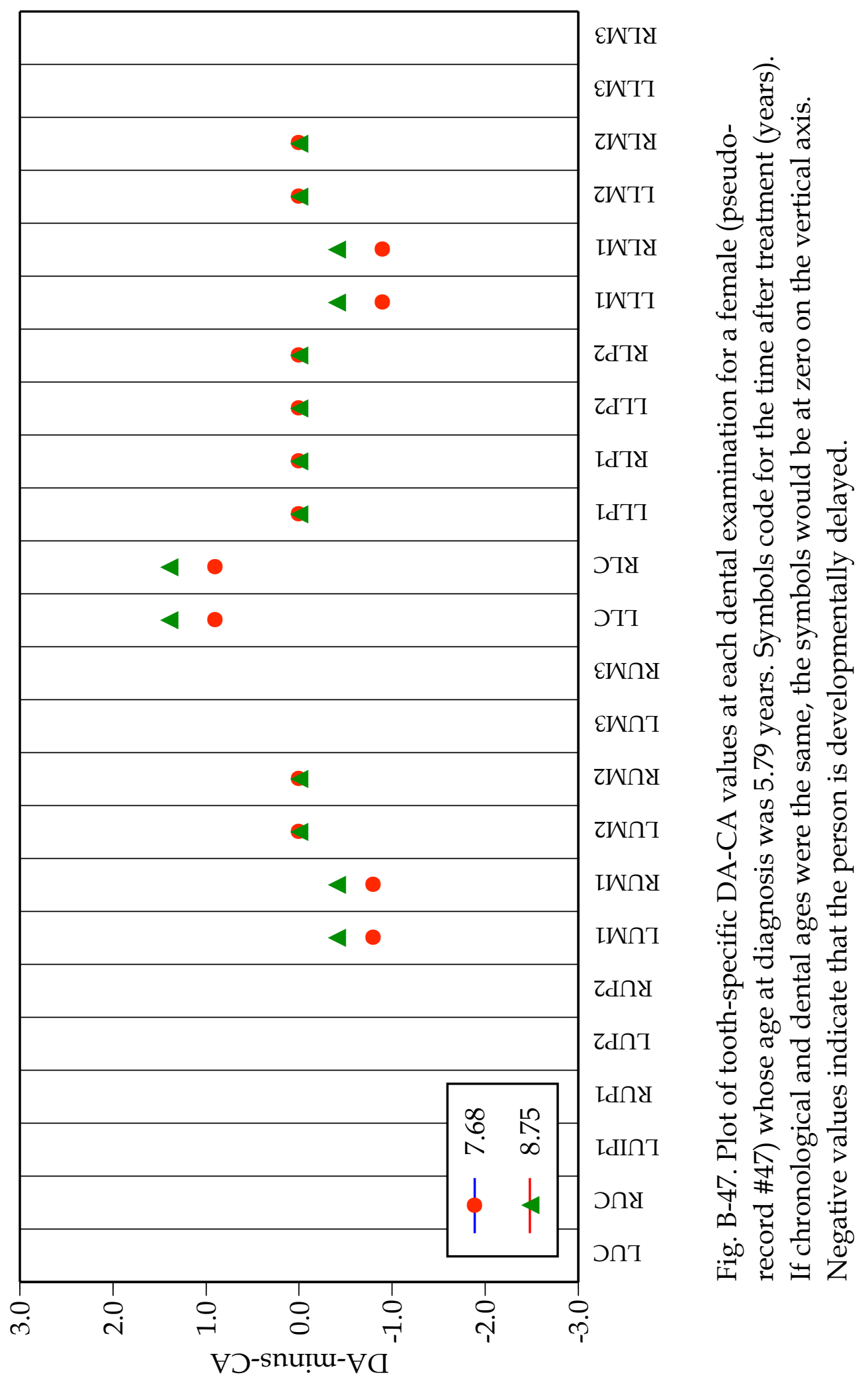




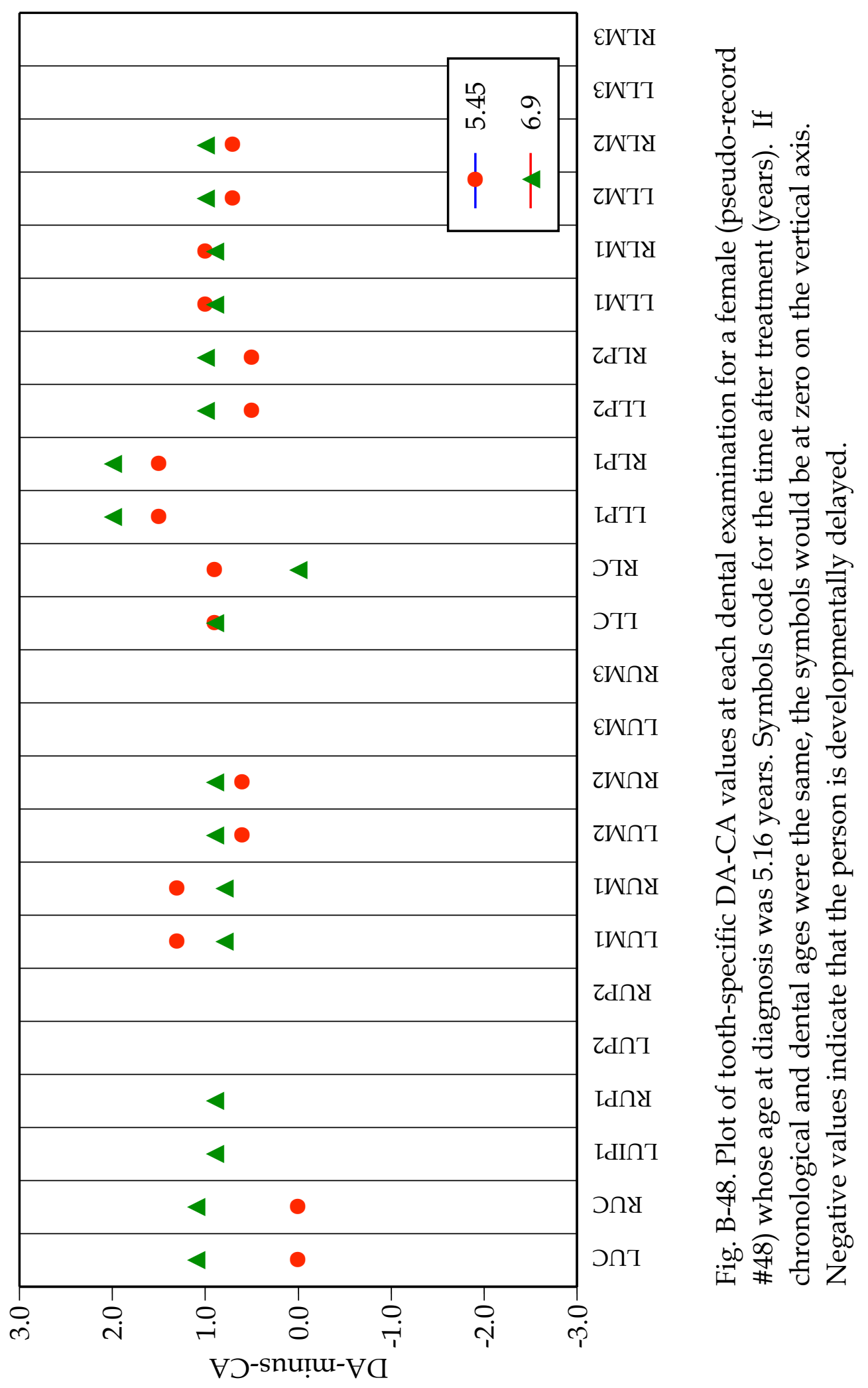




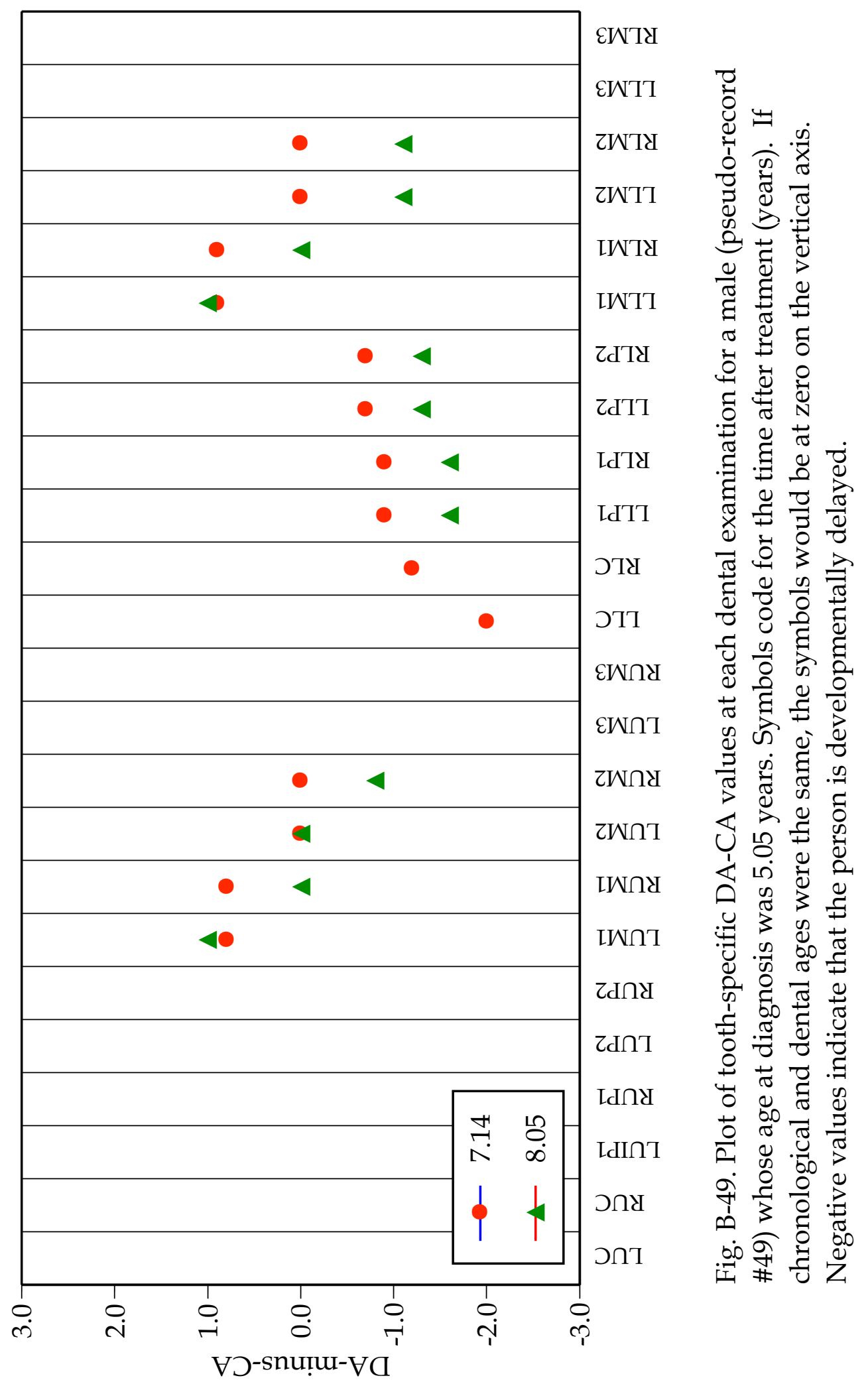




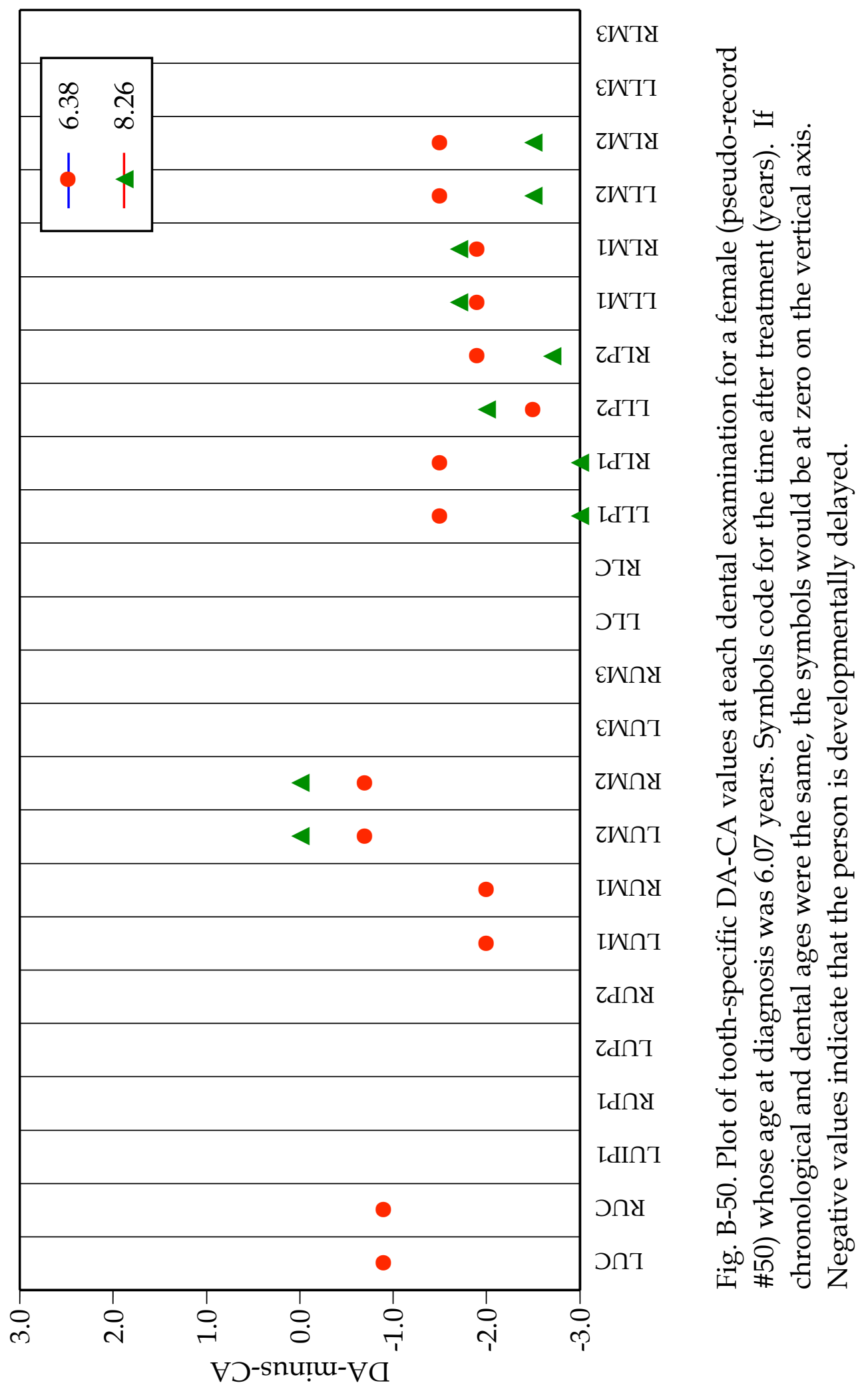




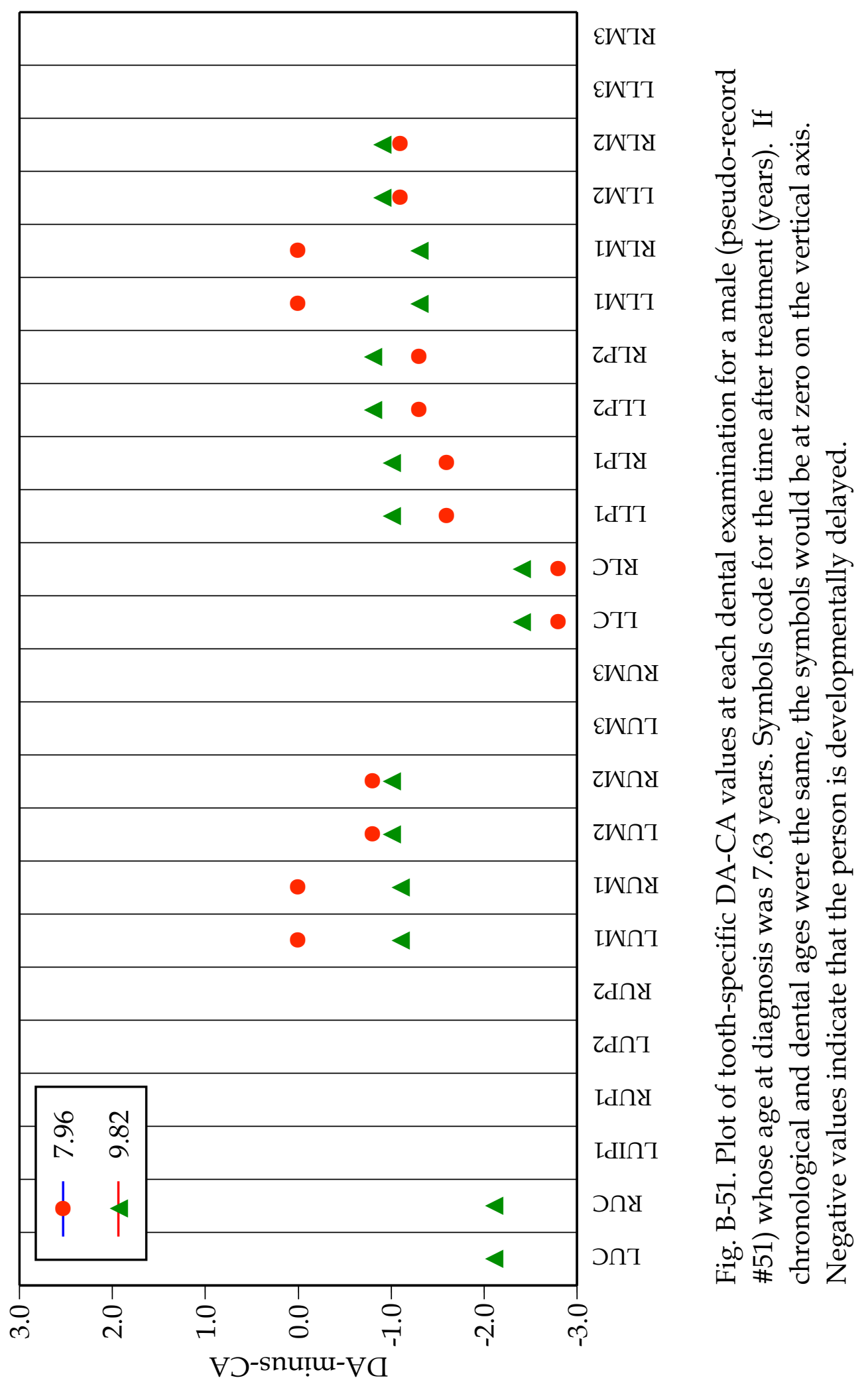




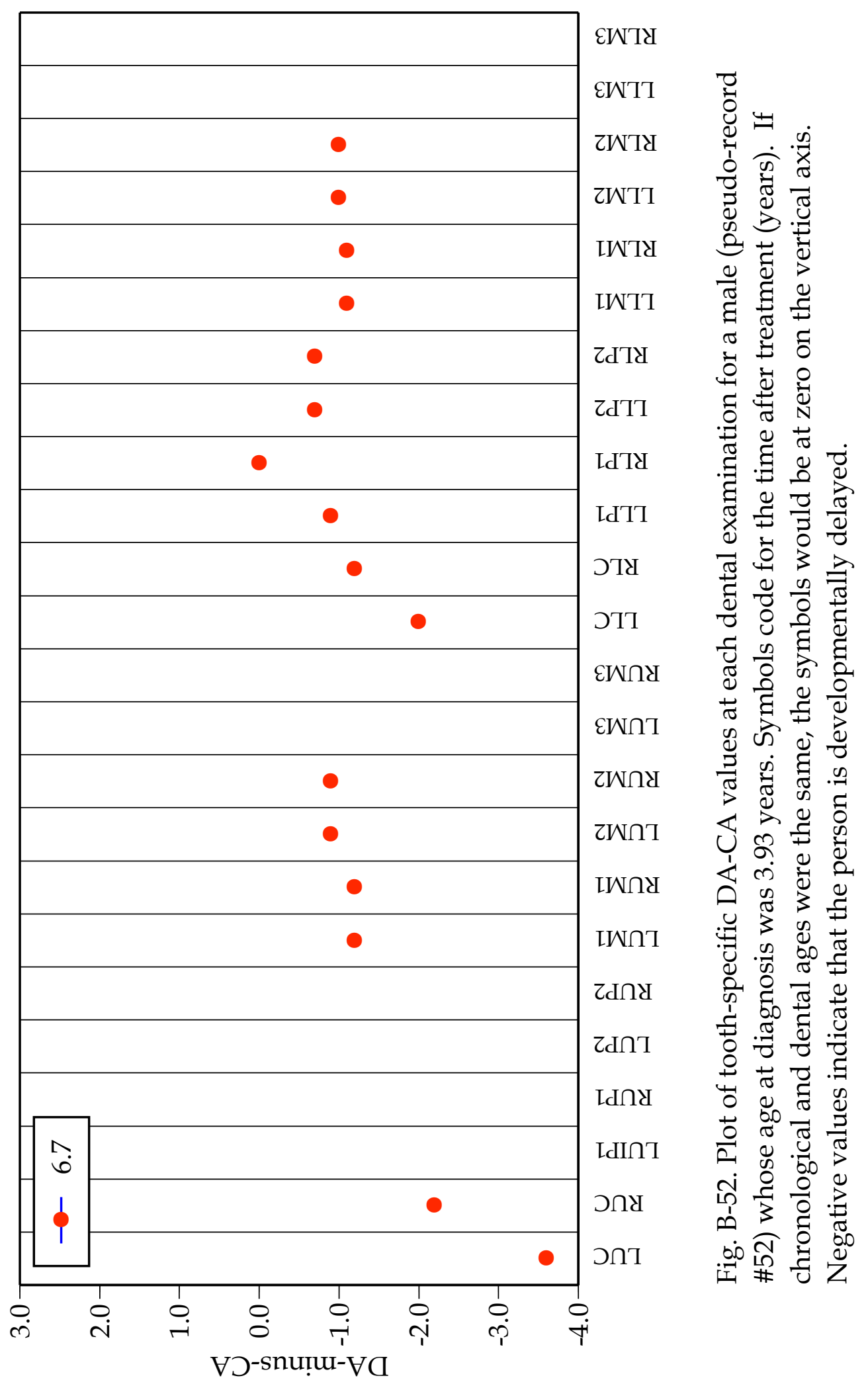




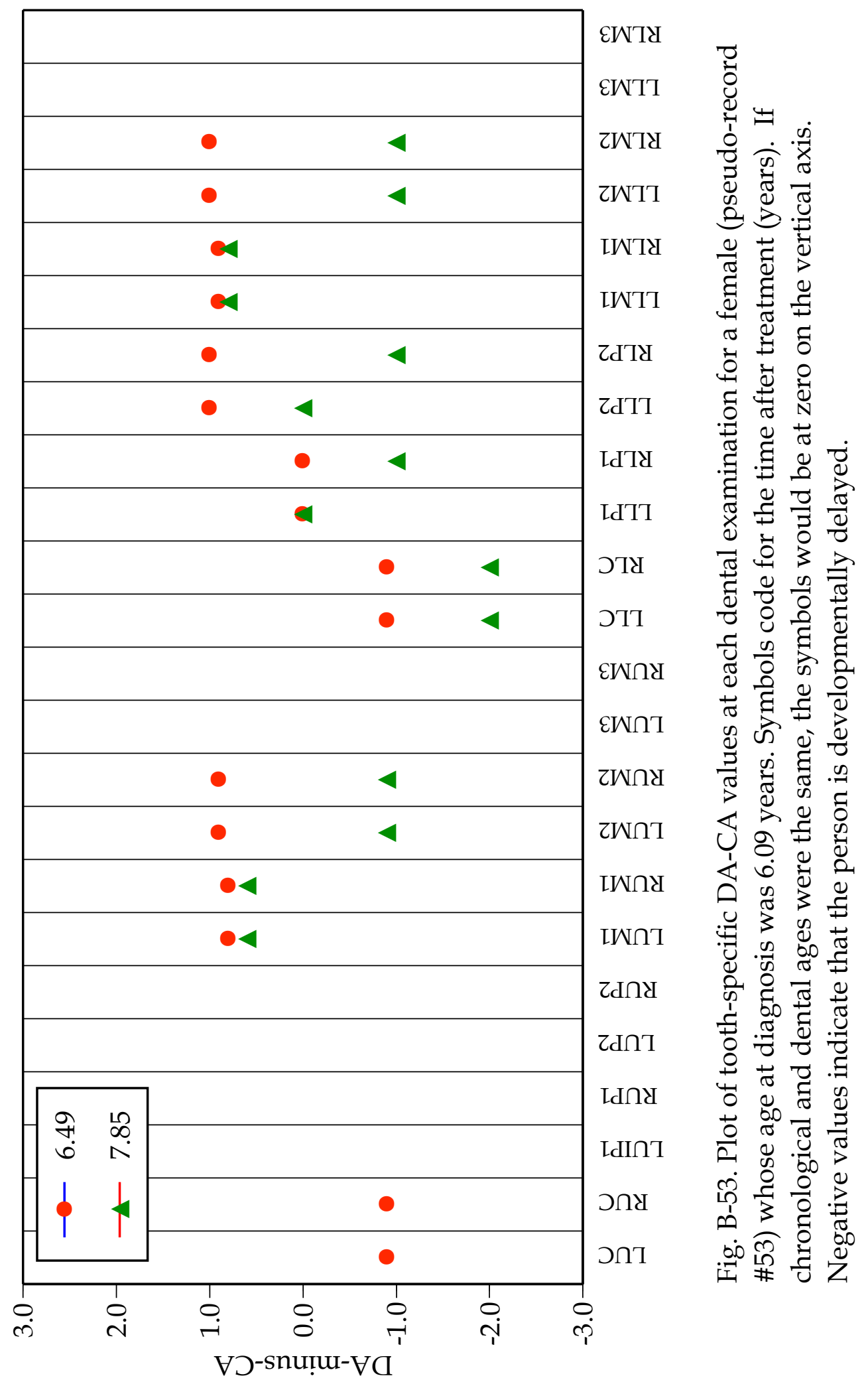




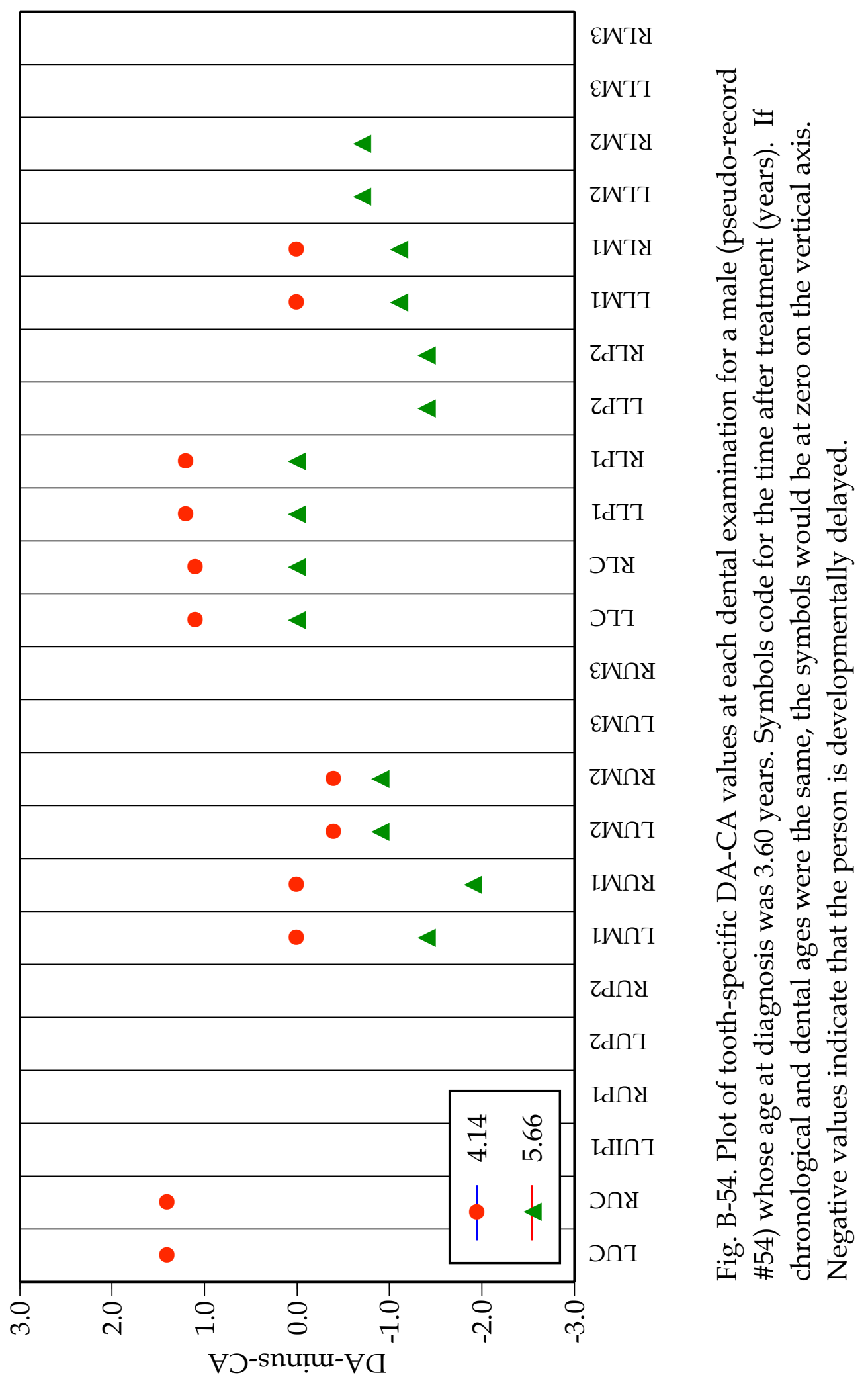




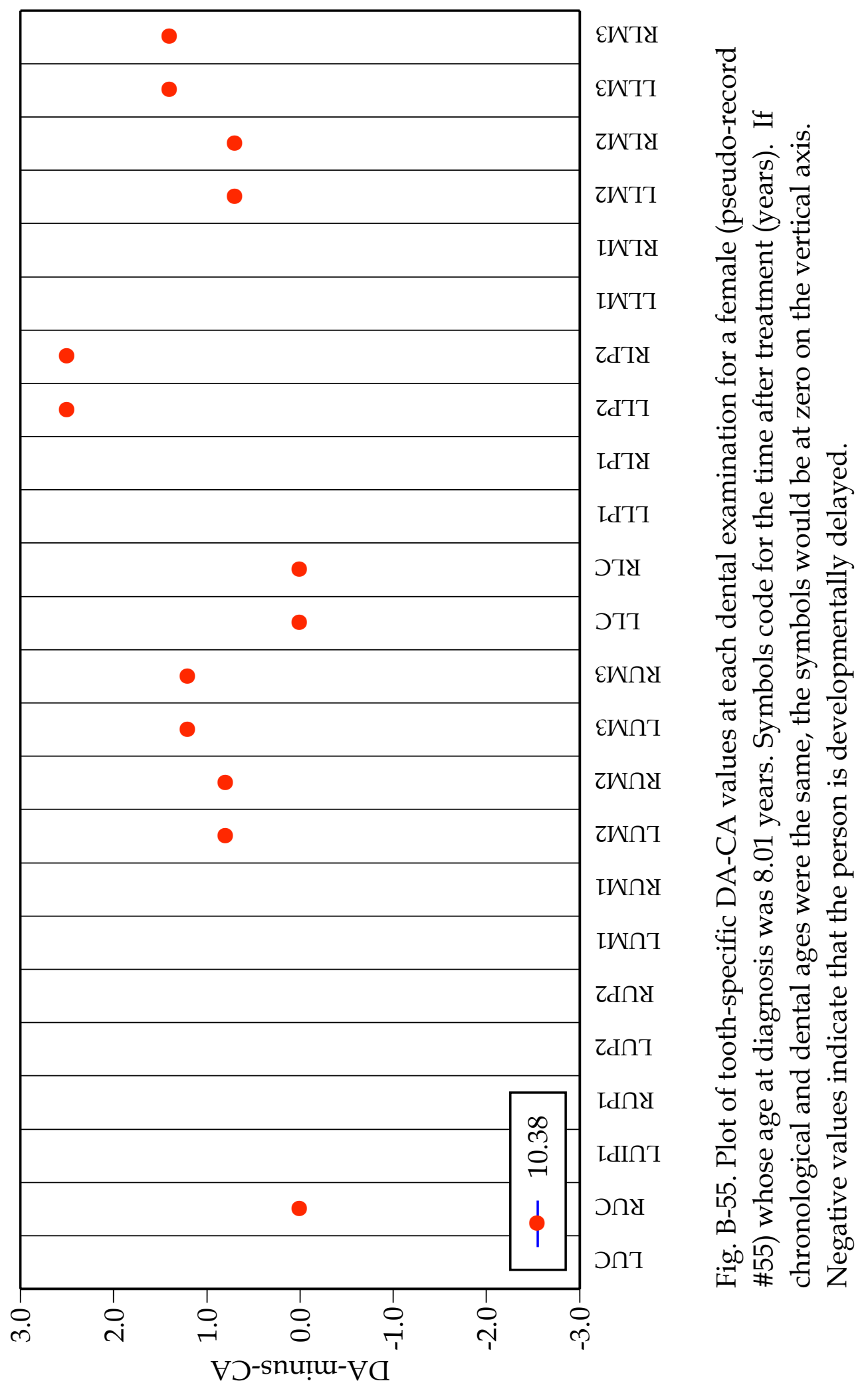




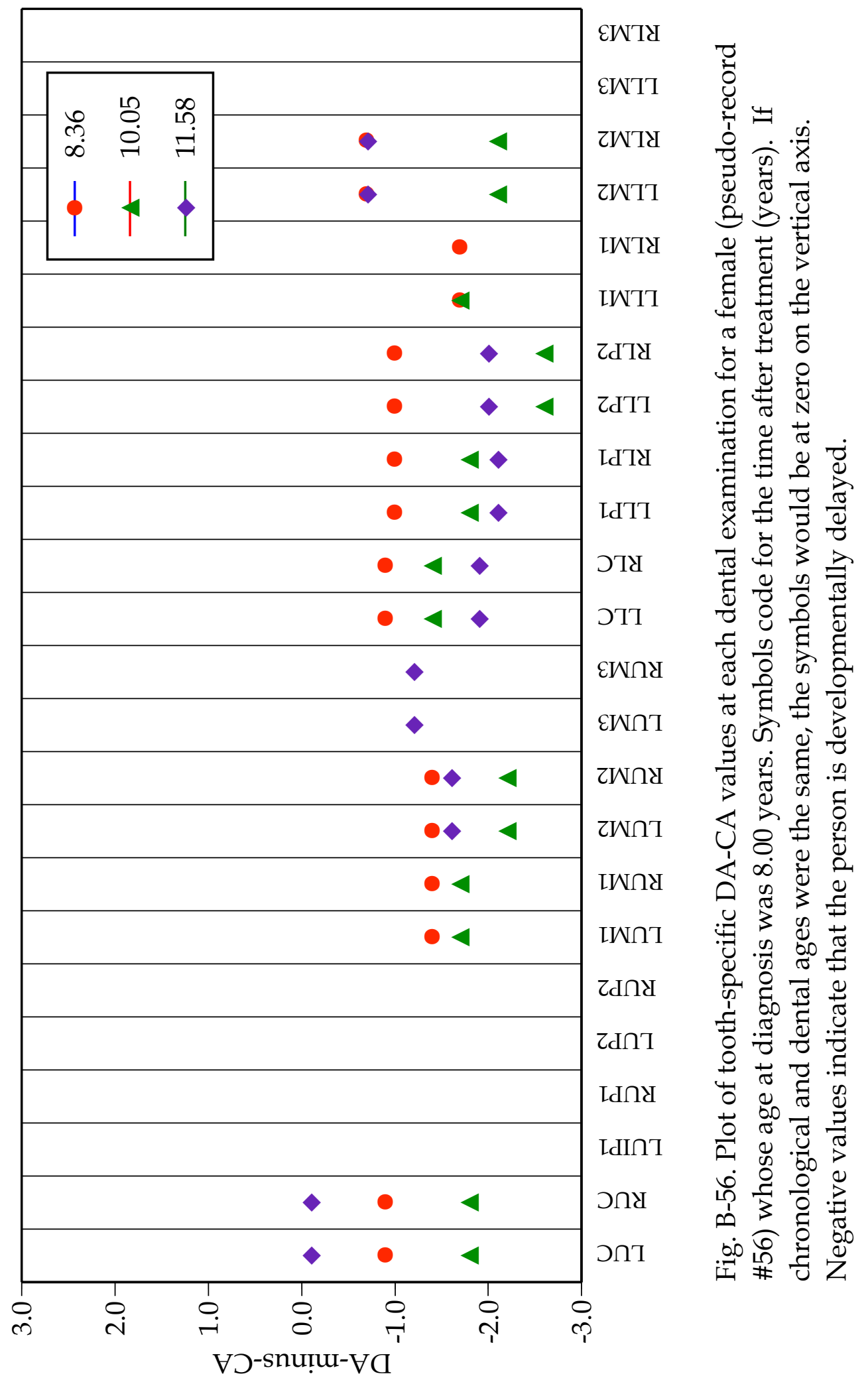




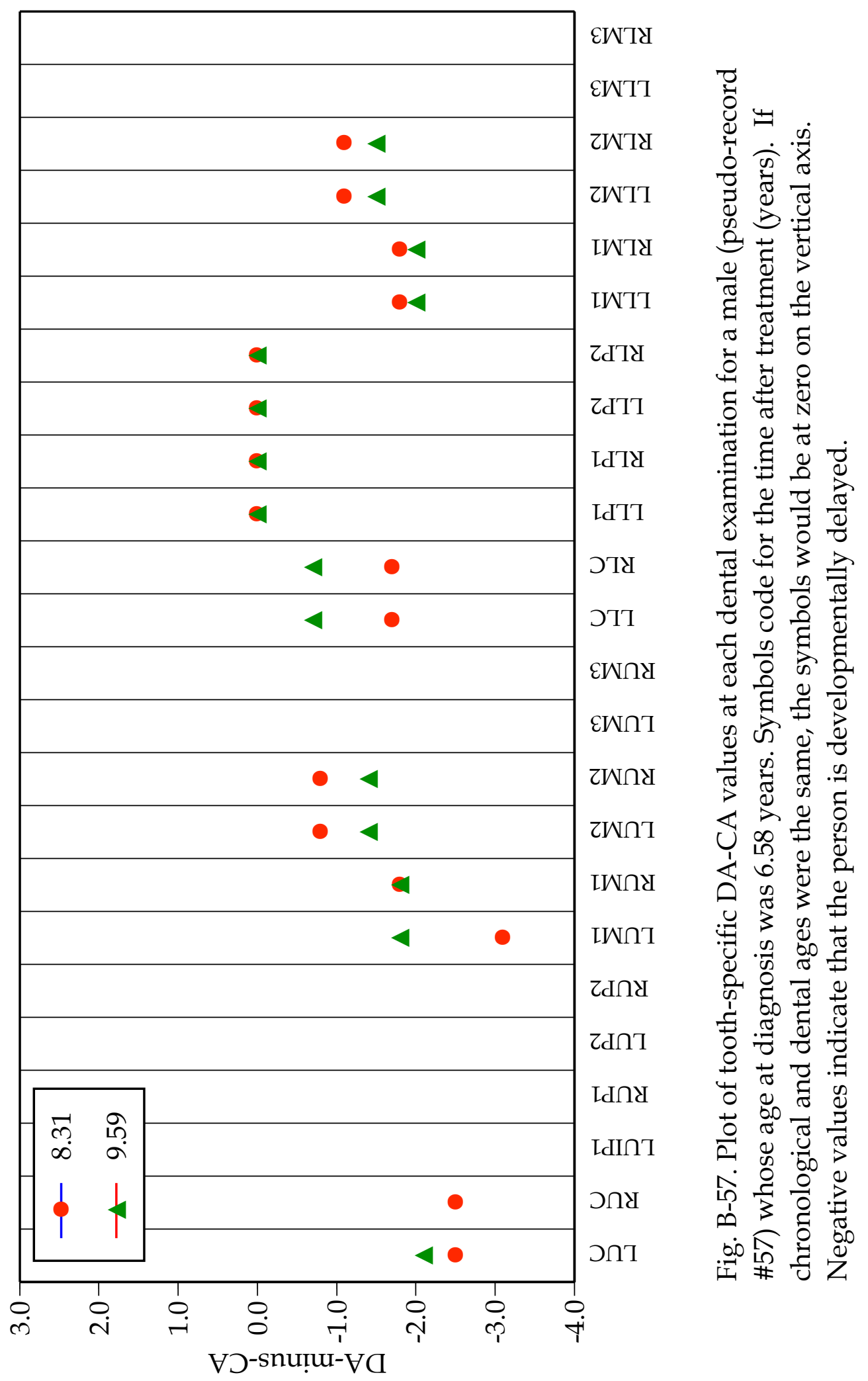




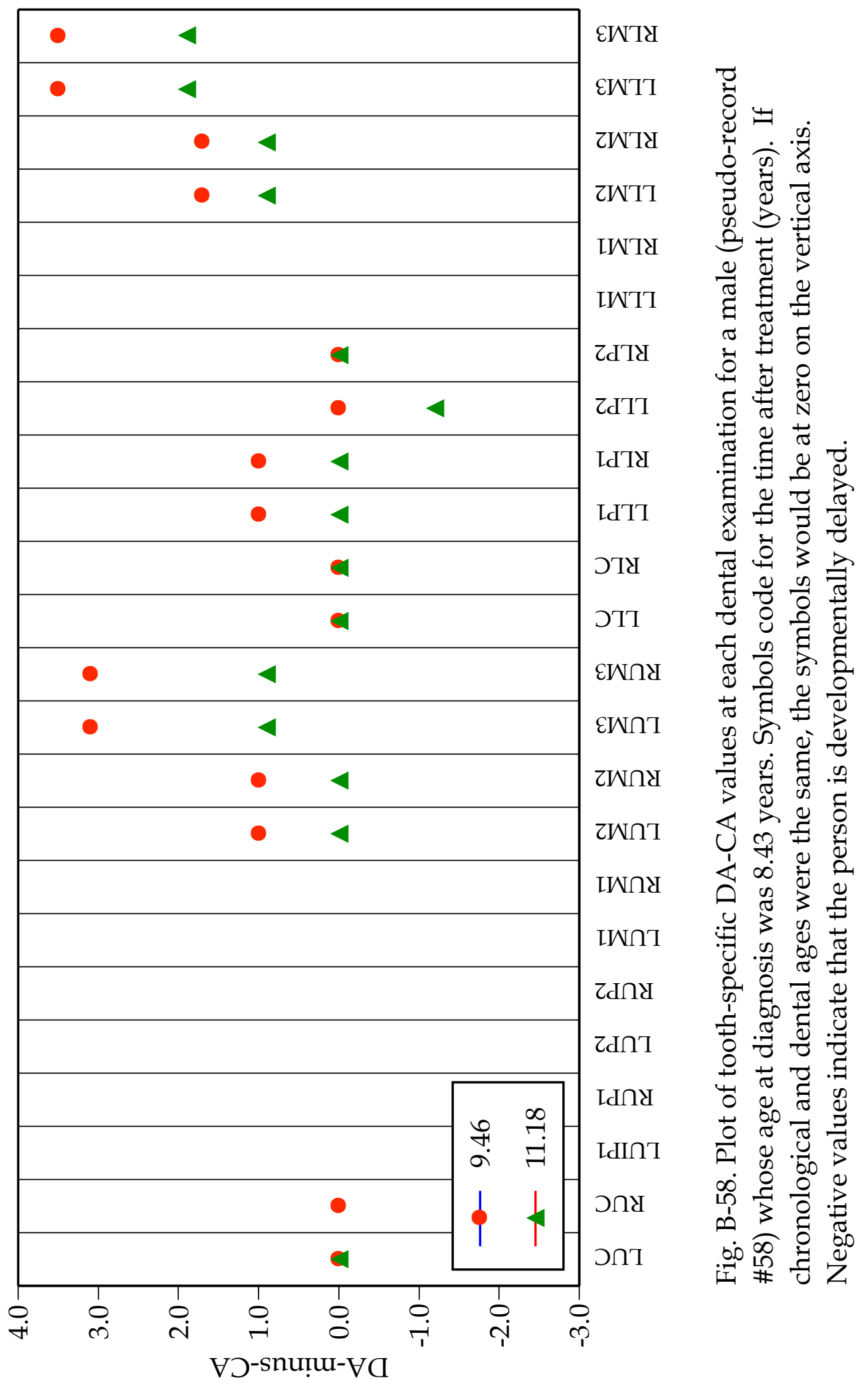




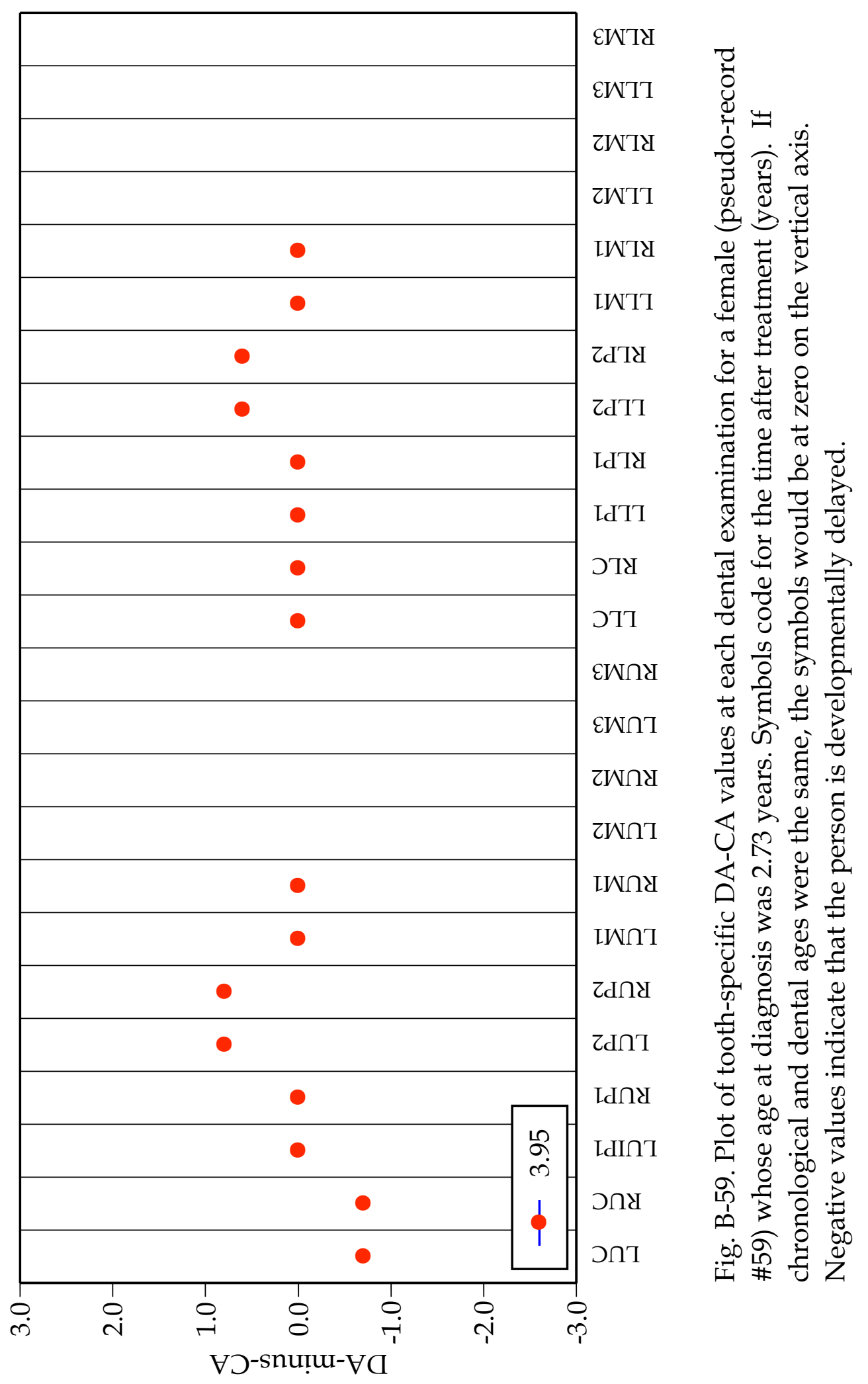




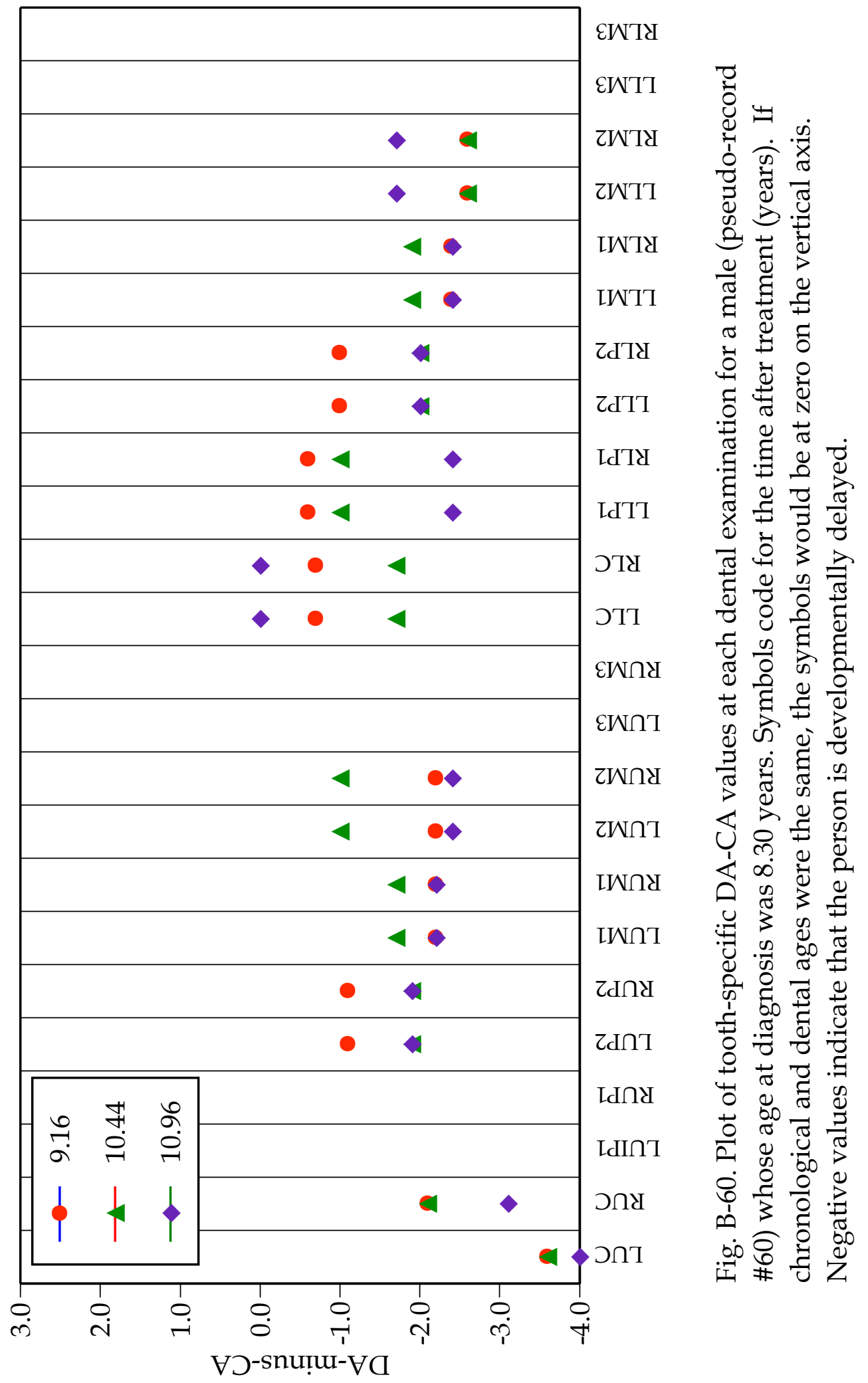




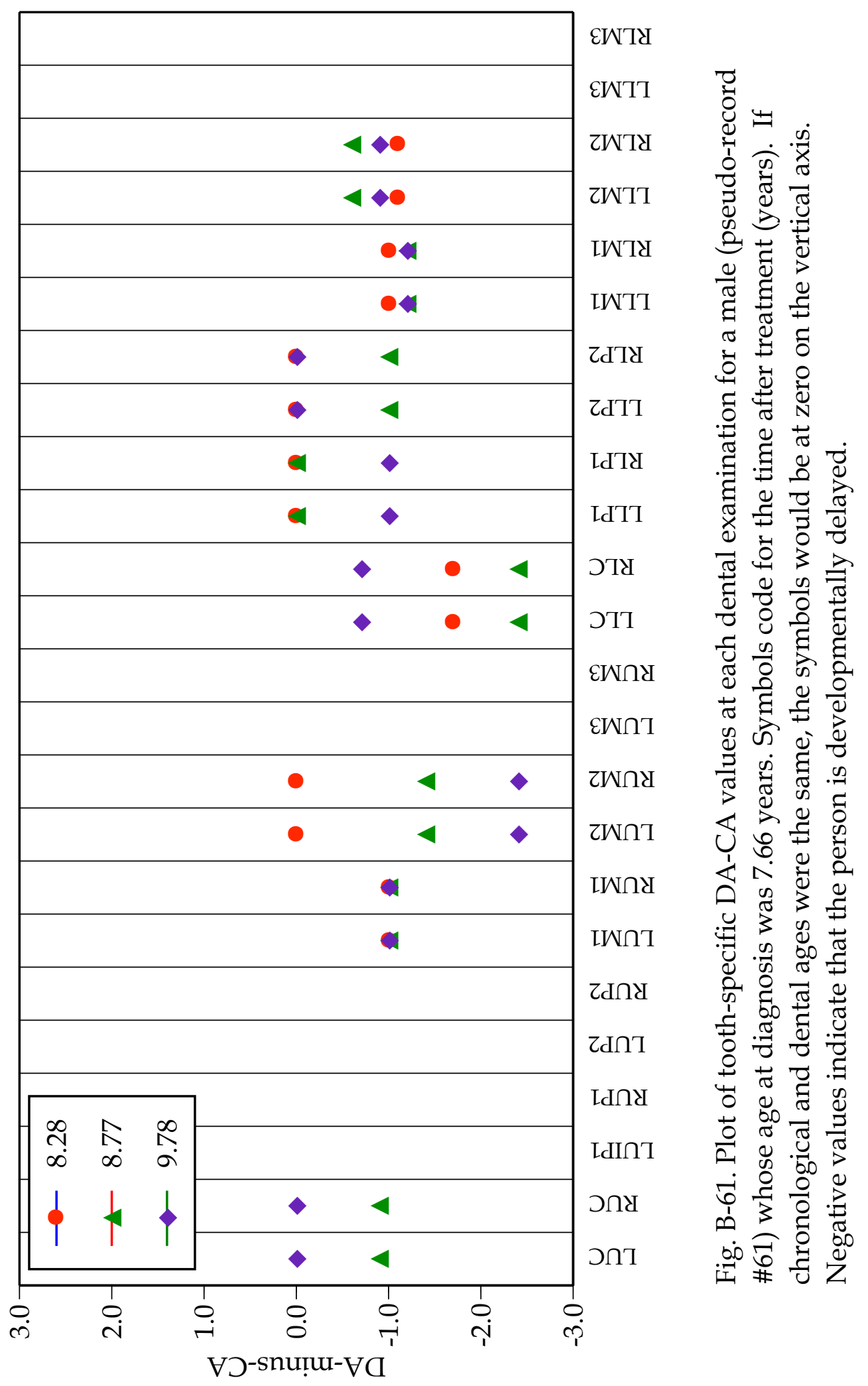




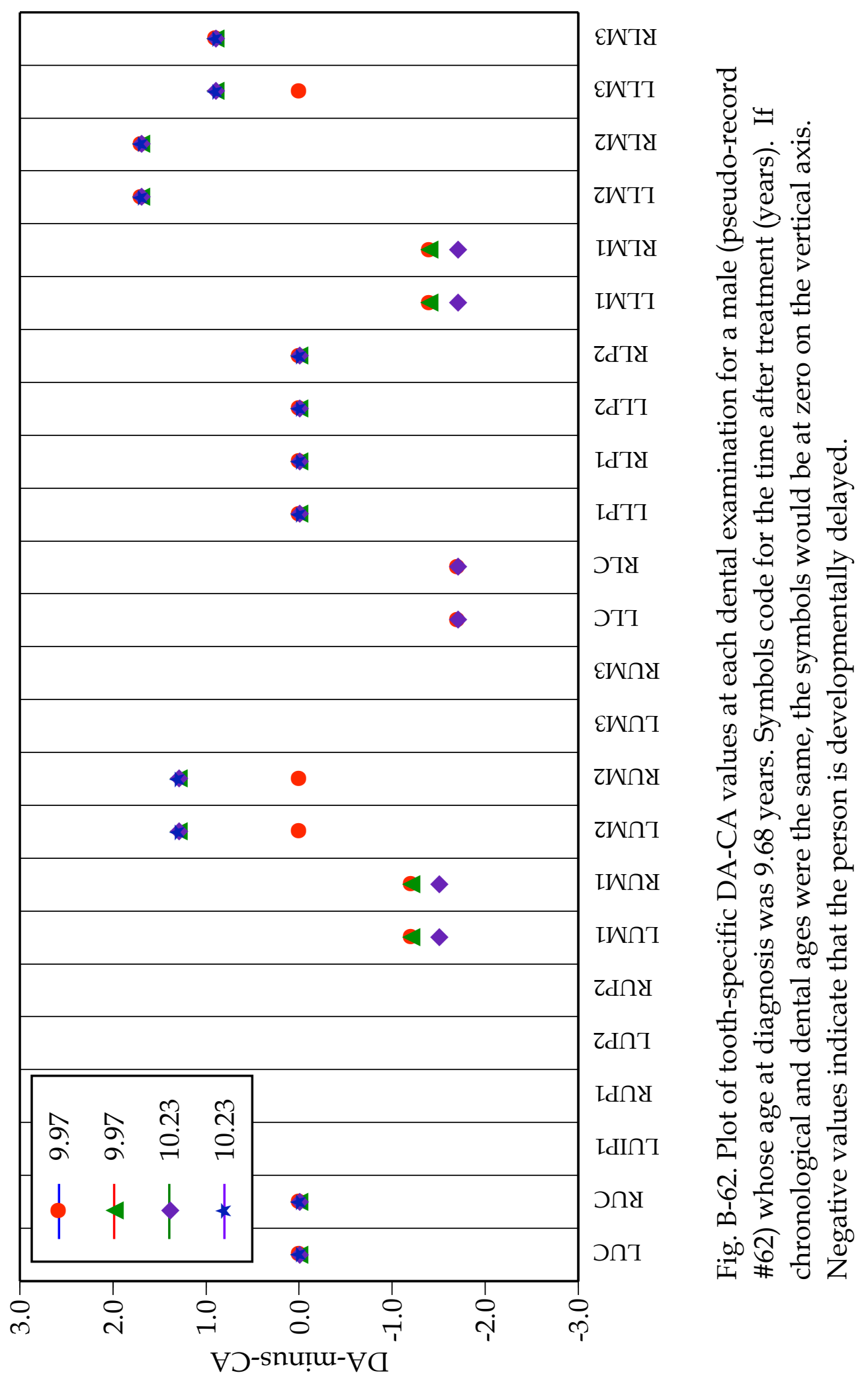




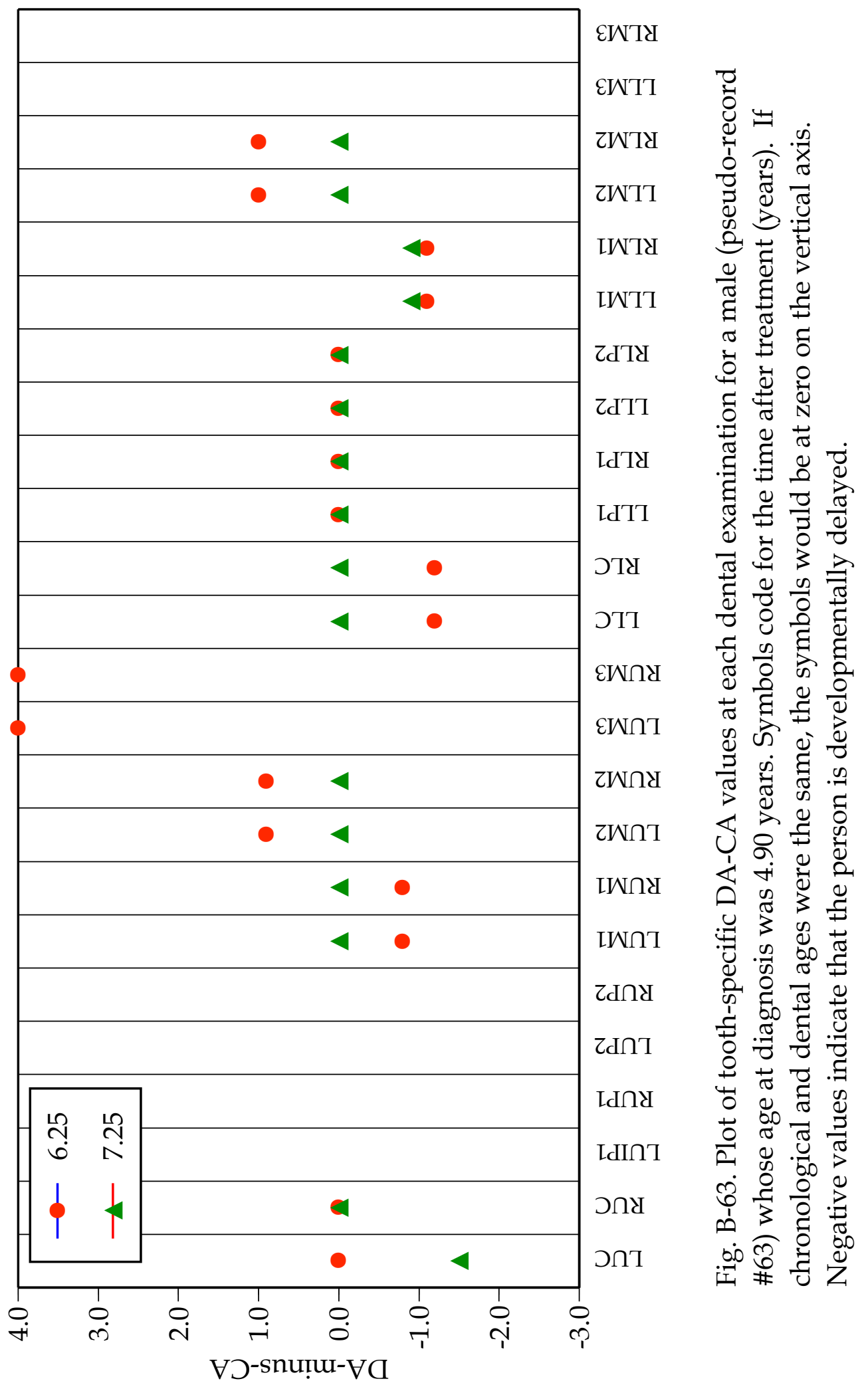




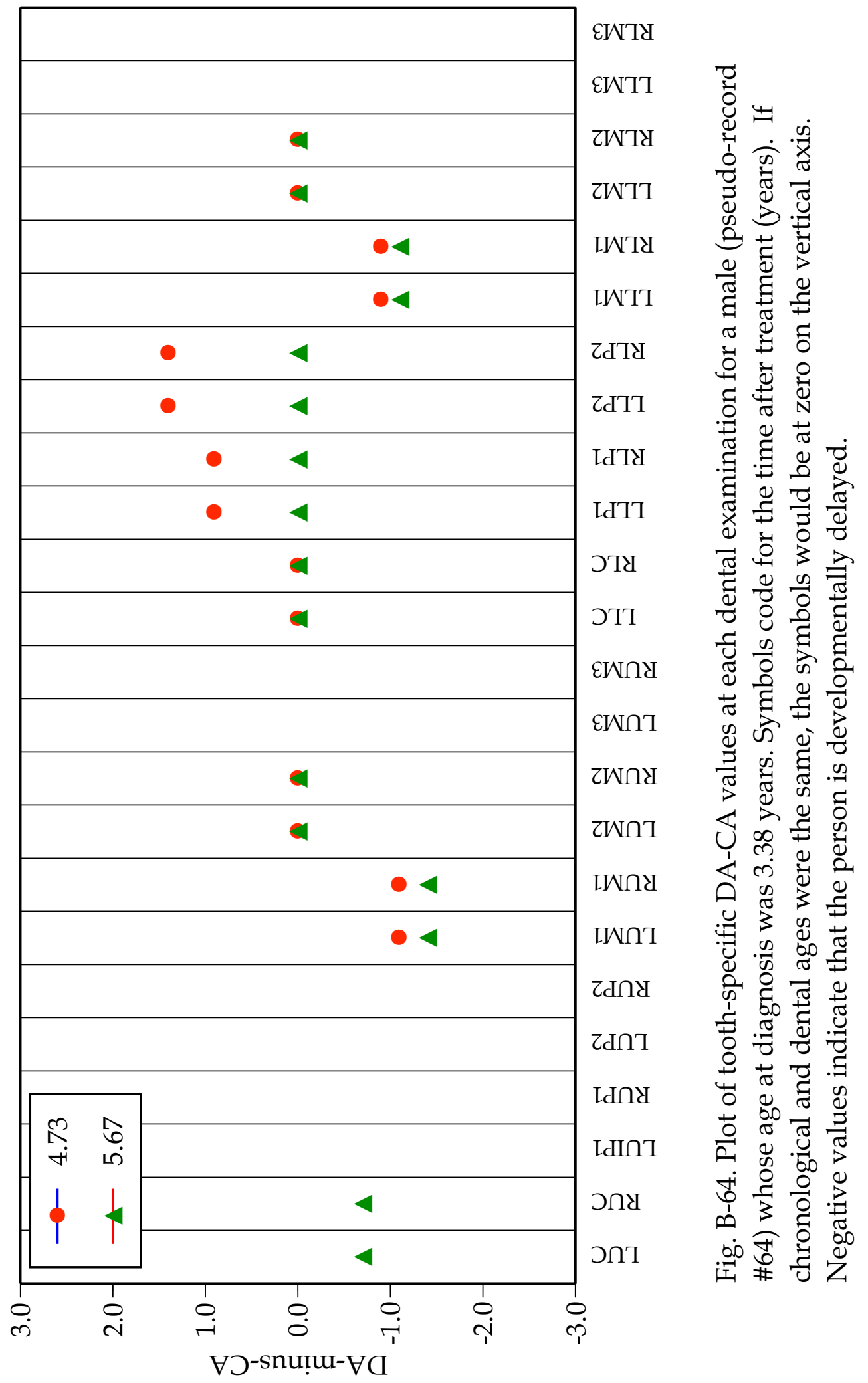




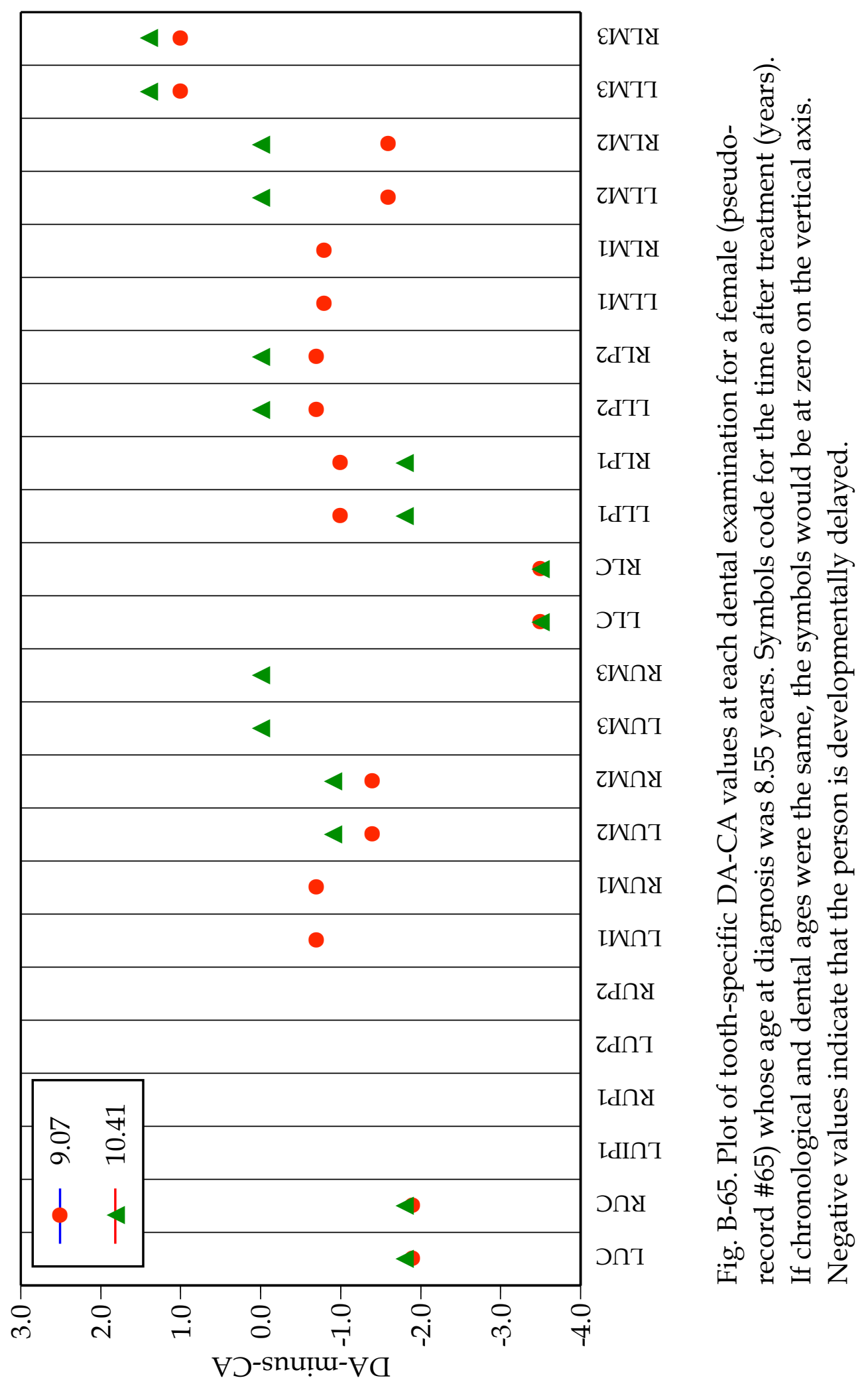




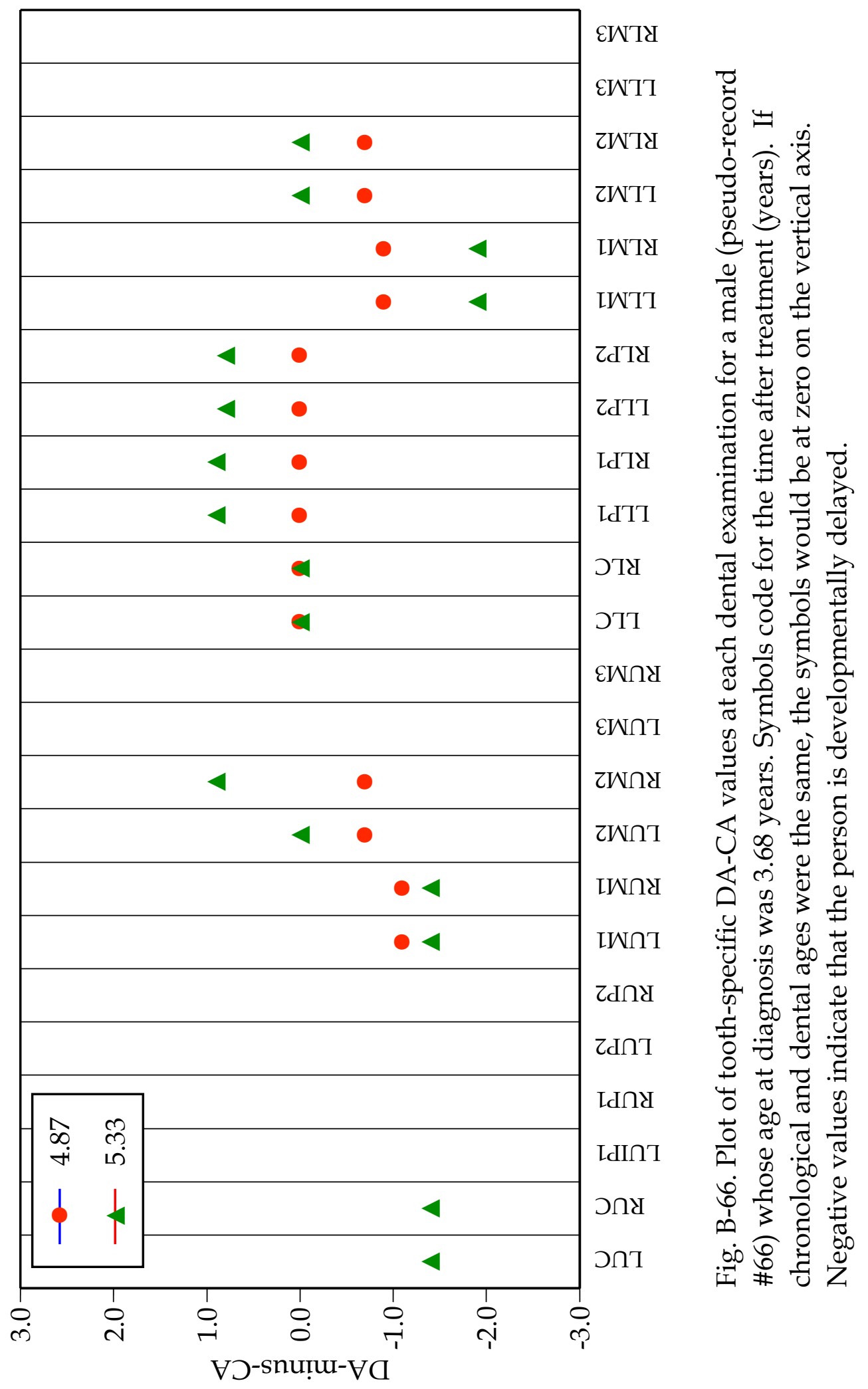




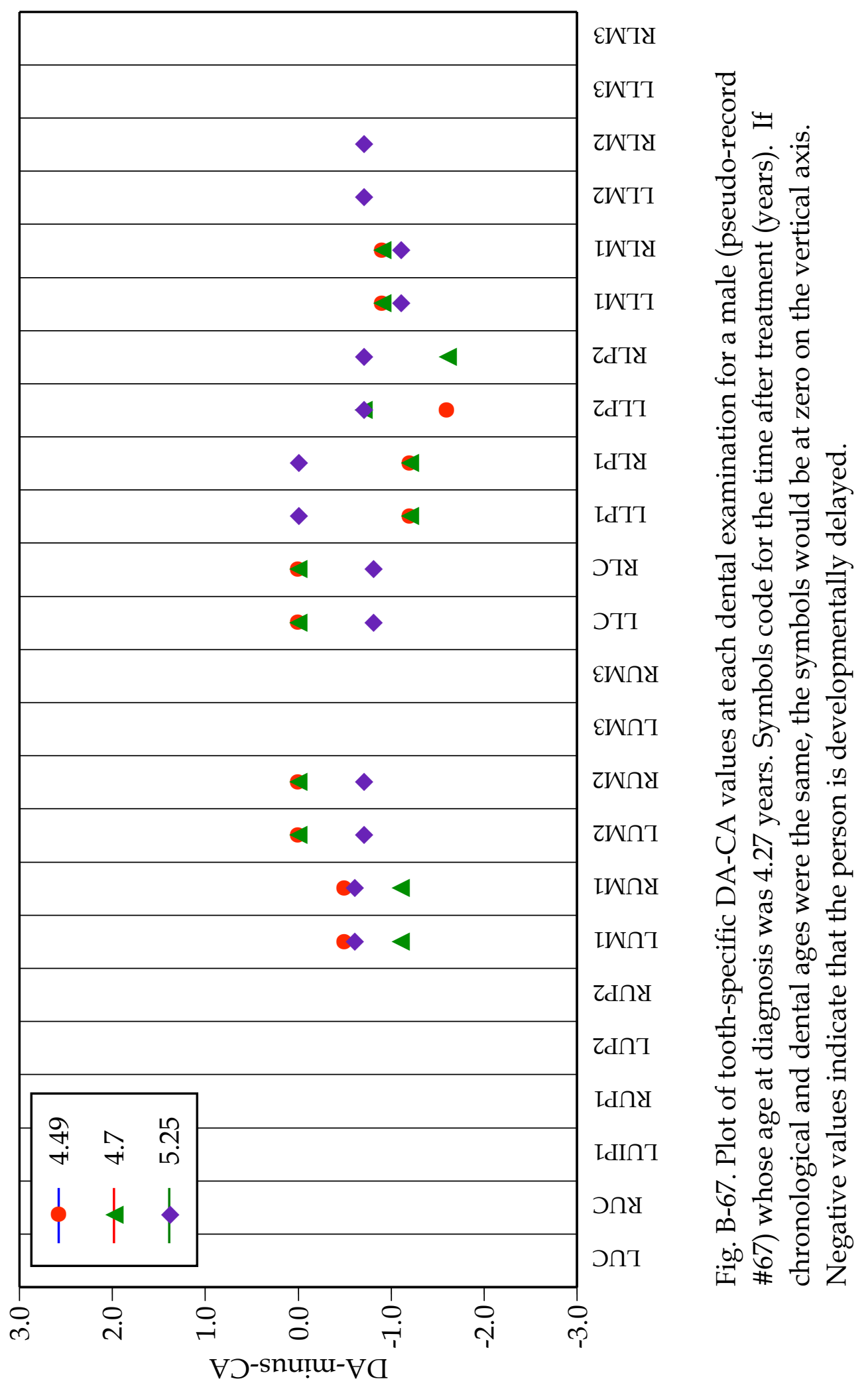




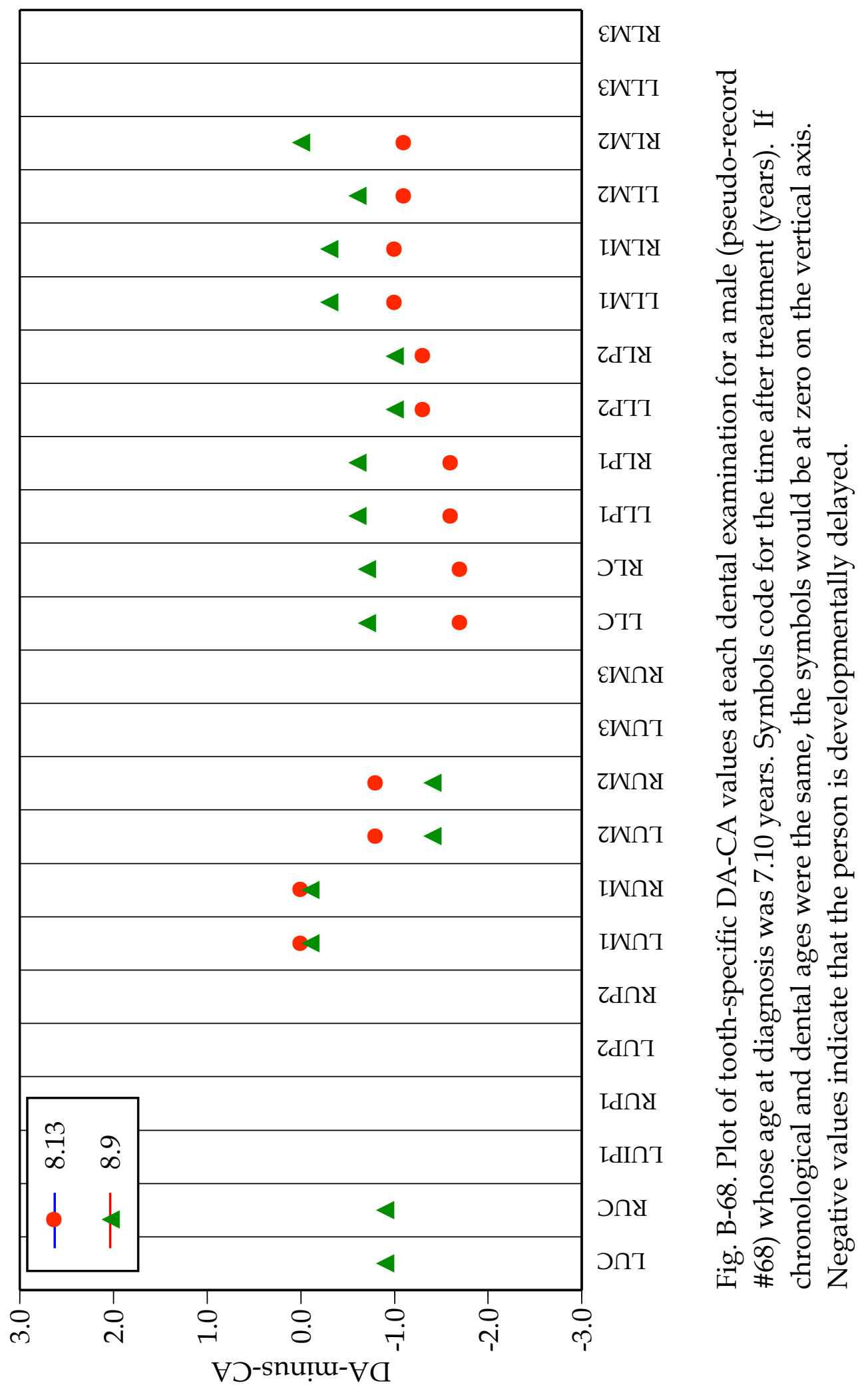




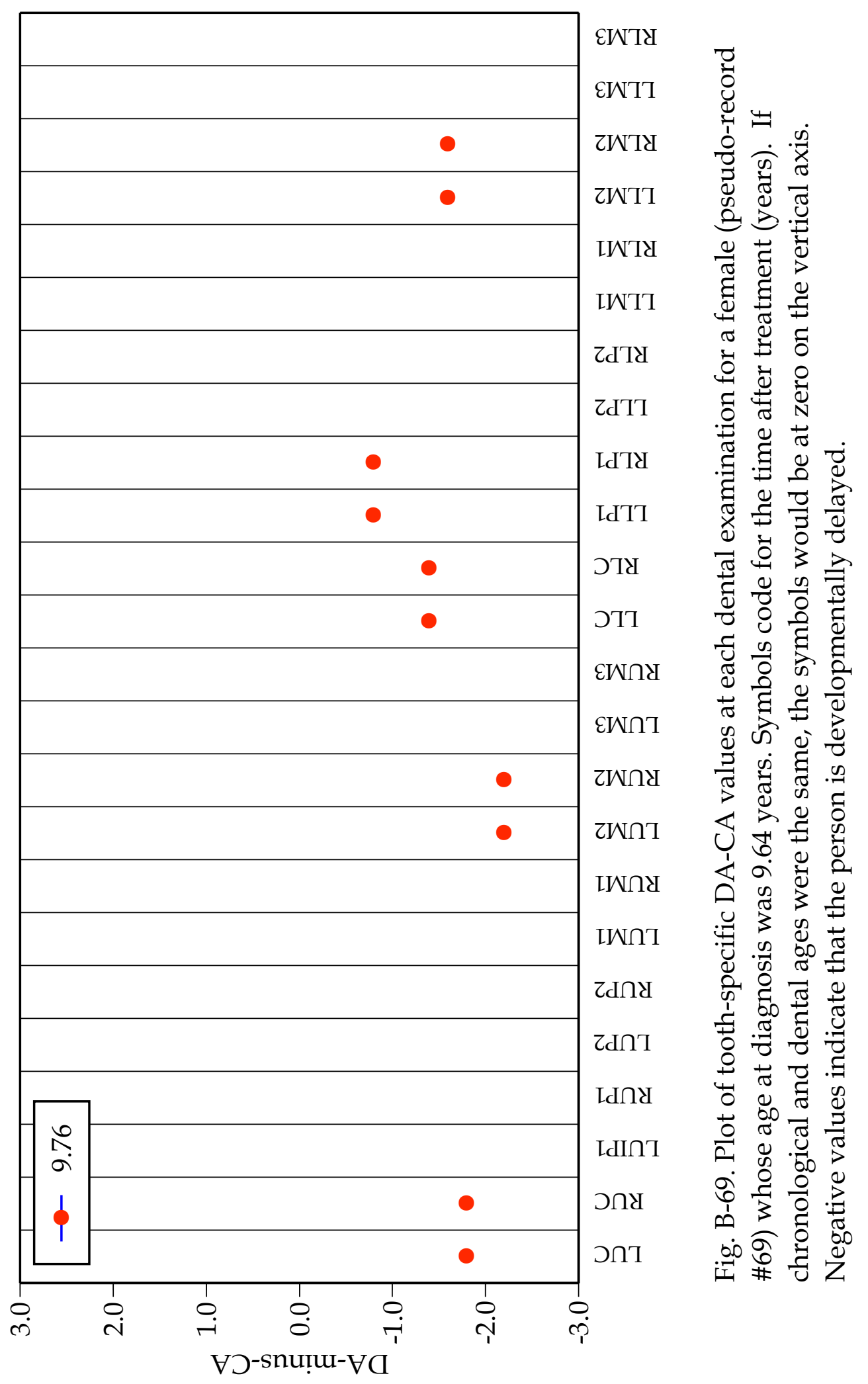




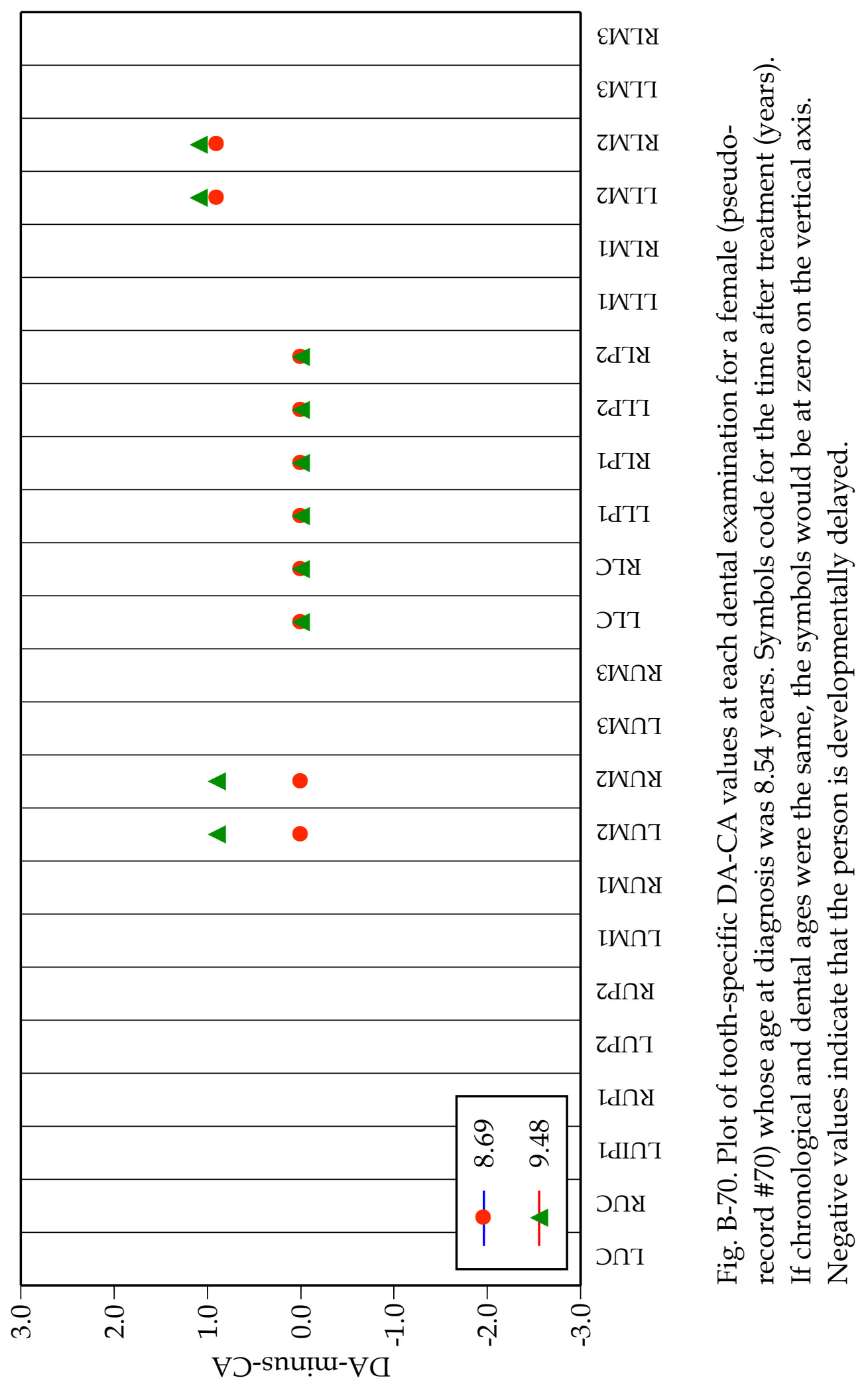




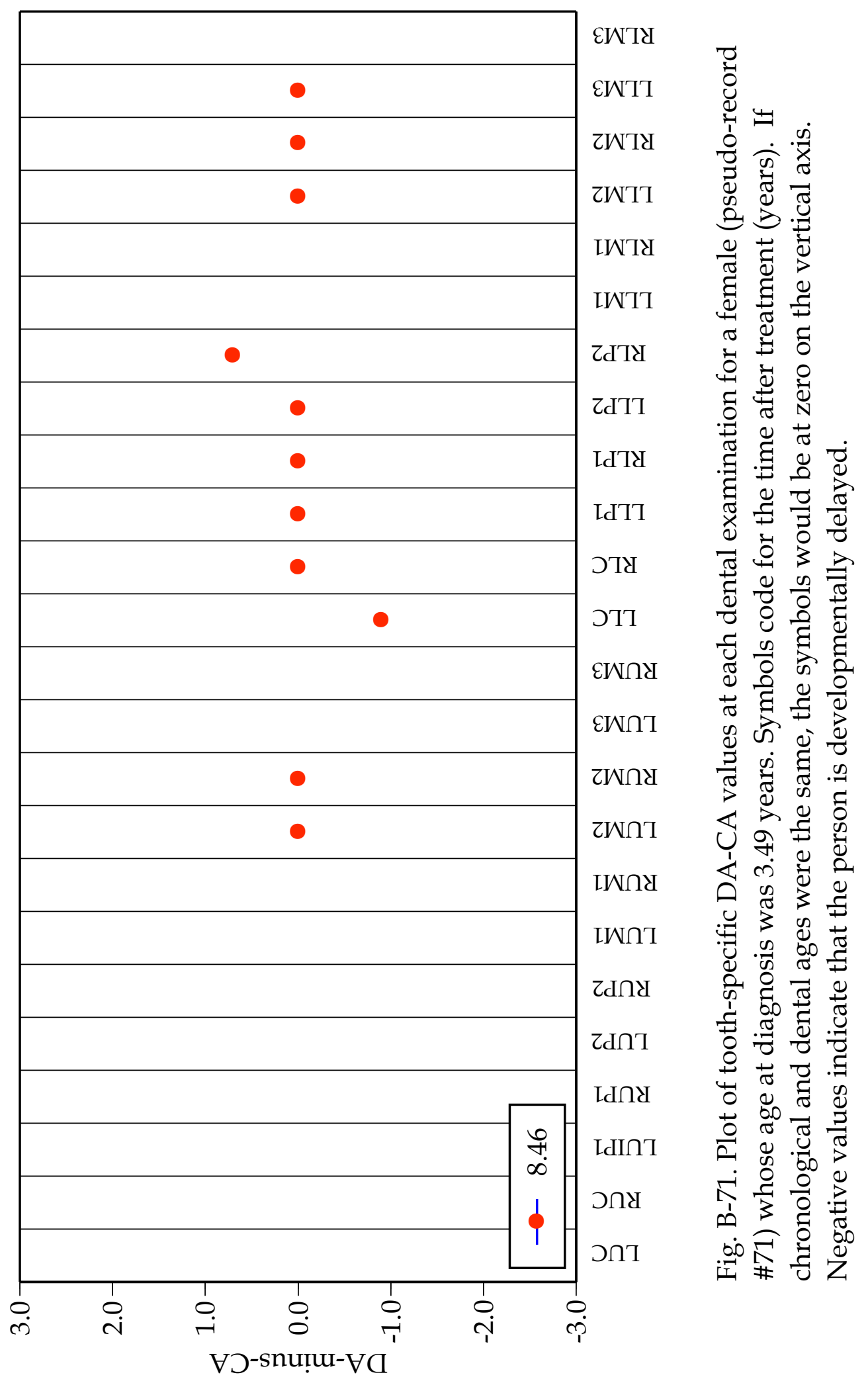




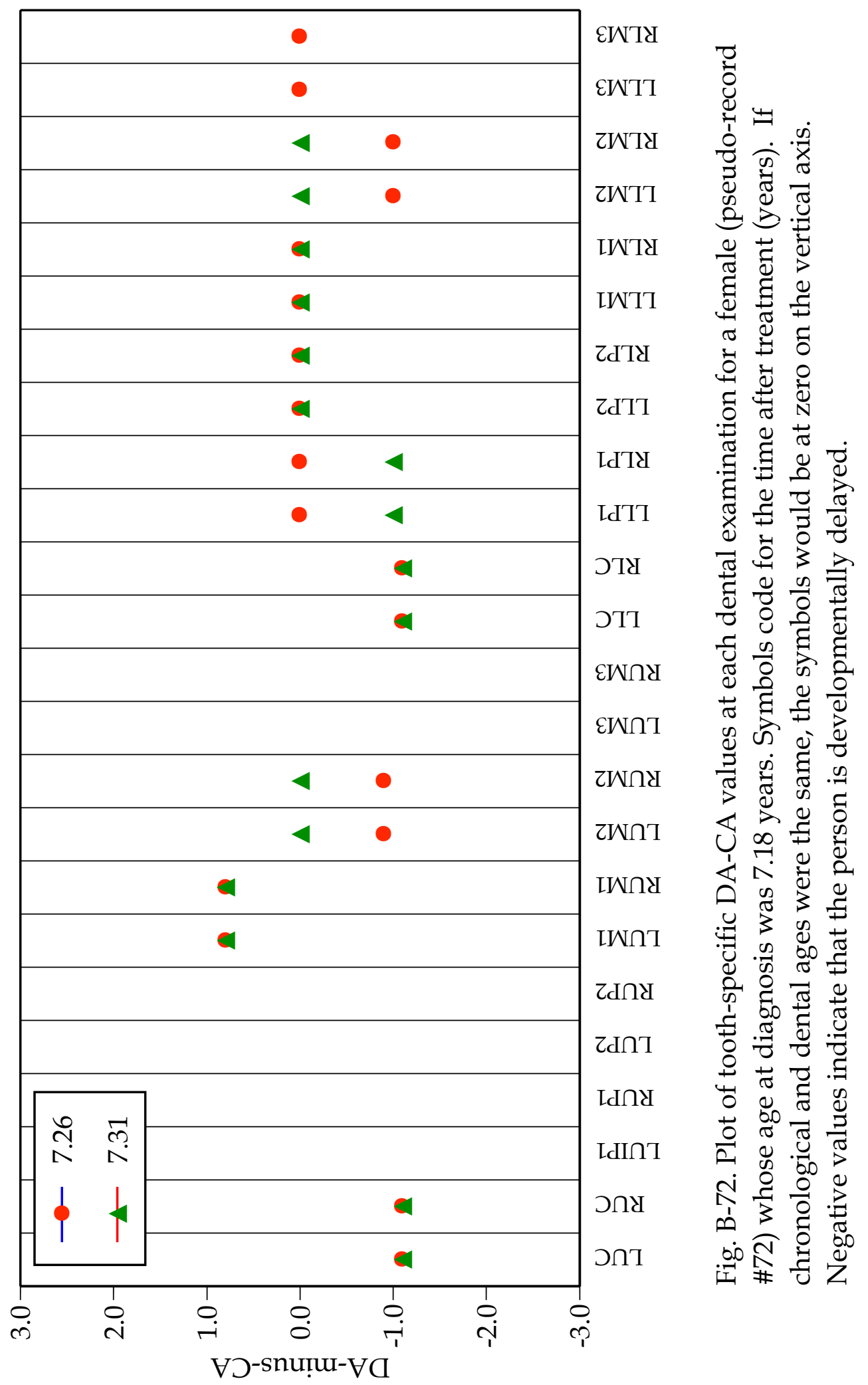


Michael Grey Weathersby was born in Columbus, Mississippi on December 15, 1975. His family moved to Chattanooga, Tennessee in 1986. He graduated from Central High School in May of 1994. Michael attended The University of Tennessee at Chattanooga where he received a Bachelor of Science degree in Biology with a Chemistry Minor and graduated Magna Cum Laude. He received his dental training and a Doctor of Dental Surgery degree from The University of Tennessee at Memphis in May of 2003. In August of 2003, he entered The University of Tennessee's Orthodontic program. He is expected to receive a Master of Dental Science degree in May 2006. Following graduation, he will move back to Chattanooga and open his private practice. 